SPRACHWISSENSCHAFT

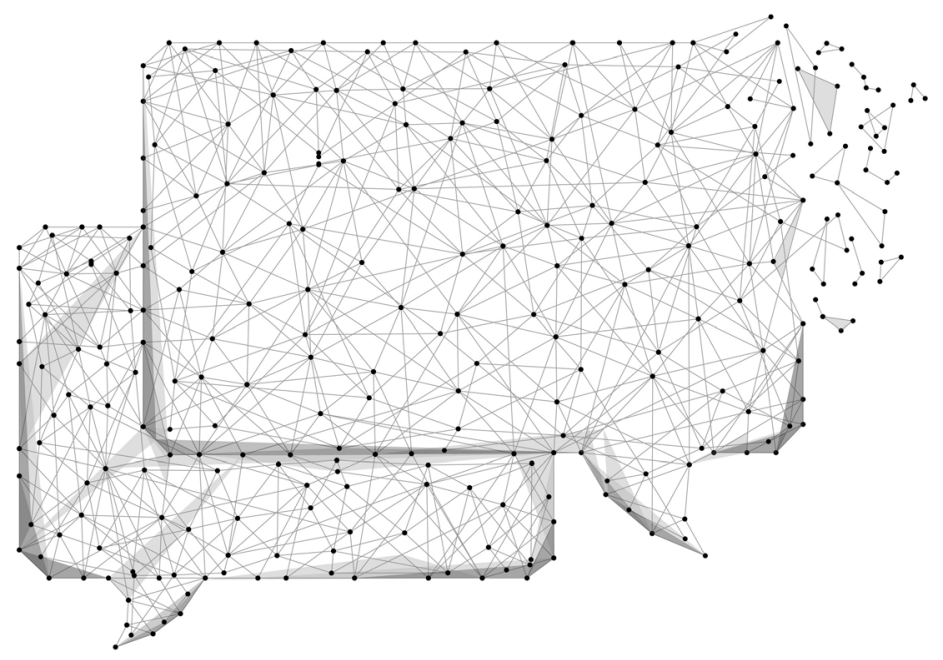

\title{
Sprachlenkung - Aspekte einer übergreifenden Theorie
}

Karolina Suchowolec

TFrank \& Timme 
Karolina Suchowolec

Sprachlenkung - Aspekte einer übergreifenden Theorie 
Sprachwissenschaft, Band 38 
Karolina Suchowolec

\section{Sprachlenkung - Aspekte einer übergreifenden Theorie}




\section{(c) (1) $(9)$}

Dieses Werk ist lizenziert unter der

Creative Commons Attribution-NonCommercial-NoDerivs 3.0 DE Lizenz (BY-NC-ND)

Diese Lizenz erlaubt die private Nutzung, gestattet aber keine Bearbeitung und keine kommerzielle Nutzung. Weitere Informationen finden Sie unter https://creativecommons.org/licenses/by-nc-nd/3.0/de/

ISBN 978-3-7329-0339-9

ISBN (E-Book) 978-3-7329-9598-1

ISBN (digital) 978-3-7329-9597-4

ISSN 1862-6149

(C) Frank \& Timme GmbH Verlag für wissenschaftliche Literatur Berlin 2018. Alle Rechte vorbehalten.

Das Werk einschließlich aller Teile ist urheberrechtlich geschützt. Jede Verwertung außerhalb der engen Grenzen des Urheberrechtsgesetzes ist ohne Zustimmung des Verlags unzulässig und strafbar. Das gilt insbesondere für Vervielfältigungen, Übersetzungen, Mikroverfilmungen und die Einspeicherung und Verarbeitung in elektronischen Systemen.

Herstellung durch Frank \& Timme GmbH, Wittelsbacherstraße 27a, 10707 Berlin.

Printed in Germany.

Gedruckt auf säurefreiem, alterungsbeständigem Papier.

www.frank-timme.de

Zugl. Dissertation Universität Hildesheim, Datum der mündlichen Prüfung 7.12.2016 Gutachter: Prof. Dr. Klaus Schubert (Hildesheim), Prof. Dr. Melanie Siegel (Darmstadt) 
für meine Mutter 



\section{Inhaltsverzeichnis}

$\begin{array}{ll}\text { Vorwort } & 11\end{array}$

$\begin{array}{ll}\text { 1. Einleitung } & 15\end{array}$

2. Forschungsstand 19

2.1. LPLP, Sprachplanung, Sprachpolitik . . . . . . . . . . . 21

2.1.1. Begriffssystematisierung . . . . . . . . . . . . . . 22

2.1.2. Geschichte . . . . . . . . . . . . . 25

2.1.3. Soziale Einbettung . . . . . . . . . . . . . . . . . . 29

2.1.4. Interdisziplinarität . . . . . . . . . . . . . . 32

2.1.5. Modelle . . . . . . . . . . . . . . . . 34

2.1.6. Autoren . . . . . . . . . . . . . . . 47

2.1.7. Erfolg . . . . . . . . . . . . . . . . . 49

2.1.8. Aktuelle Forschungsfragen . . . . . . . . . . . 51

2.2. Plansprachen .................... 54

2.2.1. Gegenstand . . . . . . . . . . . . . 55

2.2.2. Geschichte . . . . . . . . . . . . . 65

2.2.3. Erforschung von Plansprachen als Disziplin . . . . . 69

2.2.4. Autoren . . . . . . . . . . . . . . . . 78

2.2.5. Modelle . . . . . . . . . . . . . . . . . . . . 84

2.2.6. Erfolg . . . . . . . . . . . . . . . . . . 89

2.2.7. Diskussion und Zusammenfassung . . . . . . . . . . 93

2.3. Kontrollierte Sprachen . . . . . . . . . . . . . . . . . 97

2.3.1. Gegenstand . . . . . . . . . . . . . . . . . 97

2.3.2. Geschichte . . . . . . . . . . . . . . 116

2.3.3. Erforschung von Kontrollierten Sprachen als Disziplin 119

2.3.4. Autoren . . . . . . . . . . . . . . . . . . . 122

2.3.5. Modelle . . . . . . . . . . . . . . 123

2.3.6. Erfolg . . . . . . . . . . . . . . . 130

2.3.7. Zusammenfassung und Diskussion . . . . . . . . . . 134

2.4. Terminologiearbeit . . . . . . . . . . . . . . 137

2.4.1. Gegenstandsdefinition . . . . . . . . . . 137 
2.4.2. Charakterisierung . . . . . . . . . . . . . . . 139

2.4.3. Modelle . . . . . . . . . . . . . . . . . . 144

2.4.4. Einordnung der Terminologielehre (und -arbeit) als Disziplin . . . . . . . . . . . . 158

2.4.5. Geschichte . . . . . . . . . . . . . 167

2.4.6. Zielgruppen . . . . . . . . . . . . . . 172

2.4.7. Werkzeuge . . . . . . . . . . . . . . 173

2.4.8. Autoren . . . . . . . . . . . . . . . . . 180

2.4.9. Erfolg . . . . . . . . . . . . . . . . . 184

2.4.10. Zusammenfassung und Diskussion . . . . . . . . . . . 191

2.5. Forschungsstand - Fazit . . . . . . . . . . . 196

3. Konzept der Sprachlenkung 199

3.1. Einleitung . . . . . . . . . . . . . . . . . . . 199

3.2. Zusammenfassung und Verallgemeinerung der bisherigen Erkenntnisse . . . . . . . . . . . . . 200

3.2.1. Zusammenfassung der vier Gebiete bezüglich ihrer Gegenstände ... . . . . . . . . 200

3.2.2. Übertragbarkeit der theoretischen Modelle . . . . . 206

3.3. Weitere übergreifende Ansätze . . . . . . . . . . . . . 225

3.3.1. Sprachlenkung nach Ischreyt (1965) . . . . . . . . . 225

3.3.2. Kommunikationsoptimierung nach Schubert (2003, 2009, 2011a, 2014) . . . . . . . . . . . 227

3.4. Eigene Konzeptualisierung der Sprachlenkung . . . . . . . . 230

3.4.1. Festlegende Definition der Sprachlenkung . . . . . . 231

3.4.2. Charakterisierung der Sprachlenkung . . . . . . . . 241

3.5. Konzept der Sprachlenkung - Fazit . . . . . . . . . . . . . . 251

4. Umsetzung und Erfolg der Sprachlenkung 255

4.1. Einleitung . . . . . . . . . . . . . . 255

4.2. Zusammenfassung und Verallgemeinerung der bisherigen Erkenntnisse . . . . . . . . . . . . . 256

4.3. Handlungstheoretische Konzeptualisierung des Erfolges . . 258 4.3.1. Definition erfolgreicher Sprachlenkungsmaßnahmen 258 4.3.2. Erläuterungen und weiterführende Überlegungen . . 261

4.4. Forschungsannahmen . . . . . . . . . . . . . . . . 263

4.5. Identität . . . . . . . . . . . . . . . . . . . . . 264

4.5.1. Kognitive Funktion: das Selbstbild . . . . . . . . . . 265

4.5.2. Affektive Funktion: das Selbstwertgefühl . . . . . . 268 
4.5.3. Konative Funktion . . . . . . . . . . . . . . . 269

4.5.4. Theorien der Gruppenidentität . . . . . . . . . . 270

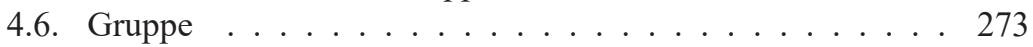

4.6.1. Was ist eine Gruppe? . . . . . . . . . . . . . . . 273

4.6.2. Gruppenprozesse in der Sozialpsychologie . . . . . 276

4.7. Mechanismen der Einstellungsänderung . . . . . . . . . 288

4.7.1. Zusammenhang von Einstellungen und Verhalten . . 289

4.7.2. Einstellungsänderung . . . . . . . . . . . . . . 293

4.7.3. Strategien zum Schutz gegen Einstellungsänderungsversuche . . . . . . . . . . . . . . . 298

4.7.4. Abschließende Bemerkungen . . . . . . . . . . . 299

4.8. Sprache und Identität in der Linguistik . . . . . . . . . . . 300

4.9. Umsetzungsorientiertes Modell der Sprachlenkung . . . . . 306

4.10. Umsetzung und Erfolg der Sprachlenkung - Fazit . . . . . . 309

5. Untersuchung 311

5.1. Datenerfassung . . . . . . . . . . . . . 312

5.1.1. Zitatauswertung . . . . . . . . . . . 313

5.1.2. Technische Umsetzung . . . . . . . . . . . . . 315

5.1.3. Abschließende Anmerkungen zur Datenerfassungsmethode . . . . . . . . . . . . . . 323

5.2. Rohergebnisse: Analyse und Interpretation . . . . . . . . . 325

5.2.1. Quantitative Vorstellung der Rohergebnisse . . . . . 326

5.2.2. Inhaltliche Vorstellung, Systematisierung und

Diskussion der Rohergebnisse . . . . . . . . . . . 327

5.3. Hauptergebnis: Zuordnungen Herausforderung-Vorgehen . . 362

5.3.1. Exemplarische Erläuterung der Analysemethode . . 363

5.3.2. Identität . . . . . . . . . . . . . . . . . 368

5.3.3. Instrumentalität . . . . . . . . . . . . . . . . . 371

5.3.4. Allgemeine außersprachliche Faktoren . . . . . . . . 373

5.3.5. Gruppe . . . . . . . . . . . . . . . 373

5.3.6. Einstellungen . . . . . . . . . . . . . . . . . 384

5.3.7. Kosten . . . . . . . . . . . . . . . . . . . . 387

5.3.8. Sprachökologie . . . . . . . . . . . . . . . . 389

5.3.9. Persuasion . . . . . . . . . . . . . . . . . . 390

5.3.10. Erlernbarkeit . . . . . . . . . . . . . . 391

5.3.11. Arbeitsprozess . . . . . . . . . . . . . . . . . . . 394

5.3.12. Machtmittel/Sanktionen . . . . . . . . . . . 397

5.3.13. Werkzeuge . . . . . . . . . . . . 400 
5.3.14. Einhalten . . . . . . . . . . . . . 401

5.3.15. Bekanntmachen . . . . . . . . . . . . 405

5.3.16. Gebrauch . . . . . . . . . . . . 405

5.4. Untersuchung - Fazit . . . . . . . . . . . . . . 406

6. Fazit 411

$\begin{array}{ll}\text { Literatur } & 415\end{array}$

$\begin{array}{ll}\text { Anhang } & 451\end{array}$ 


\section{Vorwort}

Sprache ist für die Befriedigung kommunikativer Bedürfnisse von Menschen ausschlaggebend. Zuweilen wird aber die Leistung der Sprache als nicht optimal bewertet, und man versucht in Konsequenz durch gezielte Maßnahmen den Sprachgebrauch in allgemeinen oder speziellen Kommunikationssituationen auf Dauer zu verändern. Mit anderen Worten, man versucht die Leistung der Sprache unter bestimmten Aspekten zu verbessern, wobei Verbesserung ein durchaus subjektiver Begriff ist. Beispiele für solche Eingriffe sind verschiedene Rechtschreibreformen, allgemeine Sprachpflege, Erhalt von bedrohten oder gar das Wiederbeleben von toten Sprachen, aber auch die Entwicklung von standardisierten Formen von ethnischen Sprachen sowie standardisierten Fachsprachen. Auch ich war als Linguistin an einer solchen Maßnahme aktiv beteiligt, und zwar an der Entwicklung und Umsetzung eines standardisierten Fachwortschatzes (Terminologiearbeit) und später an der Entwicklung einer Kontrollierten Sprache bei dem Druckmaschinenhersteller Koenig \& Bauer Group. Speziell in der Wirtschaft setzen sich solche Sprachstandardisierungen zunehmend durch, weil sie ein Potenzial für die Verbesserung der Texterstellung und -übersetzung in sich bergen, aber auch einen wesentlichen Beitrag zur Steigerung der Verständlichkeit von Texten für den Kunden leisten. In der Wortschatzstandardisierung geht es vor allem darum, die Synonymie und die Mehrdeutigkeit in Fachtexten zu beheben. Denn, um ein Beispiel aus meiner Praxis zu nennen, werden in einem Text, oder gar auf einer Seite, mehrere Synonyme für ein und dasselbe Bauteil einer Druckmaschine benutzt, so kann dies das Leseverstehen durch den Kunden oder aber auch durch den Übersetzer erheblich erschweren (s. Abbildung 1). Während meiner Praxis habe ich jedoch die Beobachtung gemacht, dass einem Linguisten bzw. speziell einem Terminologen recht gute Methoden zur Verfügung stehen, um aus einem Synonymenbündel das in einem bestimmten Kontext geeignete auszuwählen. Die eigentliche Schwierigkeit besteht vielmehr darin, wie man die Textverfasser dazu motivieren kann, den entwickelten Standard zu akzeptieren und ihn dann auch einzuhalten. Methodisch findet man 
also wenig Unterstützung, wie man dem Widerstand der Zielgruppe entgegenwirkt und den neuen Standard auf Dauer im Sprachgebrauch etabliert.

\author{
Feuchtduktor \\ Schöpfwalze \\ Feuchtschöpfwalze \\ Dosierwalze \\ Feuchtdosierwalze \\ Tauchwalze
}

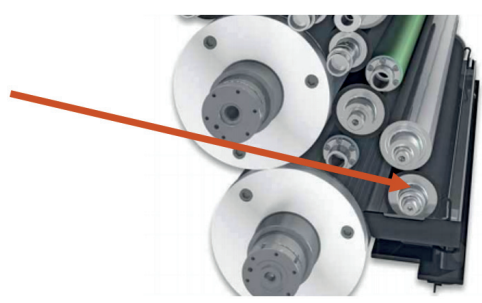

Abbildung 1.: Synonymie als Verständlichkeitsproblem am Beispiel von Koenig \& Bauer Group; Bildquelle: KBA-Sheetfed Solutions $(2016,10)$

Diese Beobachtungen aus der Praxis stellen die unmittelbare Motivation für diese Arbeit dar. Ich habe mich nämlich als Terminologin gefragt, wie andere Gebiete der Linguistik, die sich mit gezielten Sprachgebrauchsänderungen befassen, mit diesen Schwierigkeiten in der Etablierungspraxis und insbesondere dem Akzeptanzfaktor umgehen. Es lag also nahe, diese Gebiete zu identifizieren, näher zu betrachten und die festgestellten Methoden, Modelle und Best-Practice-Empfehlungen auf die Terminologiearbeit ggf. zu übertragen. Diese praktische Motivation an dieser Stelle erläutert zu haben, war mir ein wichtiges Anliegen, denn sie tritt in vorliegender Arbeit vollständig in den Hintergrund. Sie hilft aber zu verstehen, warum man sich als Linguist durchaus dazu gezwungen sehen kann, sich mit der Präskription wissenschaftlich $\mathrm{zu}$ befassen.

An dieser Stelle möchte ich mich bei allen bedanken, die mich auf dem Weg zur Umsetzung und Vollendung dieser Arbeit unterstützt haben.

Mein besonderer Dank gilt meinem Doktorvater, Herrn Professor Dr. Klaus Schubert, für sein offenes Ohr, seine Zuversicht und seine unermüdliche Betreuung.

Der Firma Koenig \& Bauer, insbesondere den Herren Jörg Hennig und Elmar Tober, für die Unterstützung im ersten Abschnitt des Promotionsprojektes. 
Meiner Familie, meiner Mutter und meinen Schwestern, für ihren Optimismus und ihre Geduld über die Jahre.

Lara und anderen Freunden aus Bonn, Hildesheim und Mannheim für kritische Diskussionen, Hilfe bei redaktioneller Arbeit und persönliche Unterstützung. Sowie allen Sportkollegen in Hildesheim und Mannheim, die zum körperlichen Ausgleich beigetragen haben.

Dem Institut für Übersetzungswissenschaft und Fachkommunikation der Universität Hildesheim danke ich ganz herzlich für die Förderung der Drucklegung dieses Buches.

Seien die genannten Personen ob der Knappheit meiner Worte unbeirrt, ich bin Ihnen allen zutiefst dankbar.

Mannheim, im Oktober 2017

Karolina Suchowolec 



\section{Einleitung}

Die vorliegende Arbeit befasst sich mit Sprachlenkung, worunter ich provisorisch den Oberbegriff für alle Maßnahmen zur intentionalen Sprachgebrauchsänderung verstehe, wie verschiedene Rechtschreibreformen, allgemeine Sprachpflege, Erhalt von bedrohten oder gar das Wiederbeleben von toten Sprachen, aber auch die Entwicklung von standardisierten Formen von ethnischen Sprachen sowie standardisierten Fachsprachen. Sprachlenkung als einen Forschungsgegenstand gilt es jedoch zunächst zu etablieren, da eine übergreifende Perspektive in der Forschung noch relativ schwach vertreten ist. Dies wird im Verlauf dieser Arbeit deutlich. Diese Arbeit leistet in erster Linie einen Beitrag zur linguistischen Theoriebildung durch Integration vorhandener Theorien, Ansätze und Erkenntnisse. Dadurch soll die Forschungslücke um den übergreifenden Begriff Sprachlenkung geschlossen und ein Austausch zwischen verschiedenen linguistischen Gebieten angeregt werden. Im Besonderen interessieren mich zwei Hauptforschungsfragen, die durch speziellere Fragen entsprechend präzisiert werden können:

1. Mit welchen Mitteln lässt sich Sprachlenkung als Gegenstand übergreifend bestimmen?

- Wie kann man Sprachlenkung definieren?

- Mit welchen Modellen, Konzepten, Kategorien und Instrumenten lässt sich Sprachlenkung übergreifend beschreiben?

2. Welche Möglichkeiten, den Erfolg der Sprachlenkung zu beeinflussen, werden in der Forschung genannt?

- Wie lässt sich der Erfolg der Sprachlenkung definieren?

- Welche Vorgehensweisen der Autoren sind bei Sprachlenkungsmaßnahmen wirksam und welche wirkungslos?

Den ersten Fragenblock beantworte ich hauptsächlich durch Synthese bestehender Erkenntnisse aus einschlägigen Gebieten der Linguistik. Hierzu 


\section{Einleitung}

wähle ich folgende Gebiete, die ein breites Spektrum an Phänomenen abdecken:

- Sprachplanung/Sprachpolitik

- Plansprachen

- Kontrollierte Sprachen

- Terminologiearbeit.

Auch die erste Spezialfrage aus dem zweiten Block beantworte ich synthetisch. Für die letzte Spezialfrage war hingegen eine Analyse geplant, in der ich die im ersten Block festgestellten Erfolgsmodelle auswerten sollte. Ohne zu viel vorwegnehmen zu wollen, zeigte es sich jedoch, dass diese Analyse aufgrund der Forschungslage nicht möglich ist. Dadurch wird sich die letzte Spezialfrage im Verlaufe dieser Arbeit ändern und entsprechend ebenfalls eine synthetische Vorgehensweise zu ihrer Beantwortung nach sich ziehen.

Der Hauptteil dieser Arbeit besteht aus vier Kapiteln. Die ersten beiden beziehen sich auf die erste Forschungsfrage, die letzten beiden auf die zweite. Im ersten Kapitel (Forschungsstand) stelle ich den Stand der Forschung in den vier linguistischen Gebieten vor, wobei ich mich auf vorab festgelegte Aspekte selektiv beschränke. Dieses Kapitel stellt die inhaltlichen Grundlagen für die Synthesen bereit. Im darauffolgenden Kapitel Konzept der Sprachlenkung erfolgt ein Vergleich der Erkenntnisse aus dem Forschungsstand über die Gebietsgrenzen hinweg, der zur Identifizierung von übergreifenden Konzepten und Modellen und schließlich $\mathrm{zu}$ einer festlegenden Minimaldefinition der Sprachlenkung und ihrer weiteren Charakterisierung führt. In Kapitel Umsetzung und Erfolg der Sprachlenkung trage ich erfolgsbezogene Erkenntnisse aus den vier Gebieten zusammen und setzte mich mit dem allgemeinen Erfolgsbegriff im Kontext der Sprachlenkung auseinander. Dies führt zu einer weiteren allgemeinen Konzeptualisierung von Sprachlenkung und zwei Konzeptualisierungen zu ihrem Erfolg. In diesem Kapitel stelle ich darüber hinaus grundlegende Konstrukte und Theorien aus der Handlungs- und Motivationspsychologie sowie der Sozialpsychologie vor, die die Strukturierungs- und Interpretationsgrundlage für die weitere Arbeit ergänzen. Hier wird auch die spezielle Forschungsfrage zum Erfolg der Sprachlenkung umformuliert. Diese neu formulierte Frage beantworte ich im Kapitel Untersuchung. Dies erfolgt methodisch durch ein Vorgehen, das ich qualitative 
Metaanalyse nenne. Diese Metaanalyse beruht auf einer Synthese, Analyse, Interpretation und Strukturierung von fragmentarischen Erkenntnissen aus der einschlägigen Literatur bezüglich der Herausforderungen und der zugehörigen Vorgehensvorschläge bei der Sprachlenkung. Das Resultat ist ein Gesamtkatalog von Best-Practice-Empfehlungen, der das aktuelle Gesamtwissen über als wirksam angenommene Handlungsmöglichkeiten für die Sprachlenkungsautoren darstellt. 



\section{Forschungsstand}

Dieses Kapitel gibt einen Überblick über die bisherige Forschung in den vier von mir betrachteten Gebieten Sprachplanung, Plansprachen, Kontrollierte Sprachen und Terminologiearbeit. Es präsentiert die Grundlagen der Gebiete, um insbesondere auch Quereinsteigern einen inhaltlichen Einblick in die Thematik zu ermöglichen.

Im Kontext dieser Arbeit soll der Forschungsüberblick speziell zeigen, inwieweit die vier Gebiete, wie angenommen, tatsächlich weitestgehend autonom sind, d. h. eigene Forschungstraditionen und -interessen verfolgen und eigene Fragestellungen, Konzepte und Modelle hervorbrachten, oder inwieweit Sprachlenkung als übergreifende Größe bereits systematisch erforscht wird. Bestehende Überschneidungen der Gebiete werden, so vorhanden, deutlich.

Auch wenn das Aufzeigen von Grundlagen einzelner Gebiete eine wesentliche Komponente dieses Kapitels ausmacht, so entscheide ich mich für einen selektiven Forschungsstand anhand folgender, deduktiv aufgestellter Punkte:

- Gegenstandsauffassung: In diesem Themenkomplex gebe ich die relevanten Gegenstandsdefinitionen aus dem jeweiligen Gebiet an, wobei ich weitestgehend sowohl die praktische Tätigkeit wie bspw. die Terminologiearbeit, das Eingriffsobjekt wie die Terminologie und das eigentliche wissenschaftliche Gebiet wie die Terminologielehre berücksichtige. Um den Umfang dieser Arbeit übersichtlich zu halten, erfolgt hier weitestgehend keine Diskussion von Einzelpositionen, sondern ich präsentiere das Resultat meiner Auswertung von verschiedenen Auffassungen.

- Geschichte: Auch dieser Themenkomplex hat einen allgemeinen einführenden Charakter und soll dabei helfen, historische Entwicklungen einzelner Gebiete besser nachvollziehen zu können, auch unter dem Aspekt ihrer Forschungsautonomie.

- Interdisziplinarität: Unter dem Schlagwort Interdisziplinarität gebe ich die Bezüge der Gebiete zu verschiedenen Wissenschaftsdisziplinen an 


\section{Forschungsstand}

und erörtere insbesondere den Bezug zur Linguistik. Ich nehme diesen Punkt in den Forschungsstand mit auf, um zu sehen, auf welche Mittel, Instrumente und Methoden die jeweiligen Gebiete für die eigene Forschung zurückgreifen. Dadurch soll deutlicher werden, ob Modellierungen und sonstige Erkenntnisse einzelner Gebiete auf ähnlichen Grundlagen beruhen und deshalb miteinander kompatibel sind oder nicht.

- Sprachauffassung: Je nach Gebiet betrachte ich unter diesem Punkt die Sprachauffassung der Disziplin selbst (und ggf. einzelner Modelle) oder aber der Autoren. Insbesondere geht es mir hier um die Frage, ob die dem Gebiet zugrunde liegende Sprachauffassung strukturalistisch oder soziolinguistisch ist. Ich betrachte diesen Themenkomplex, weil ich annehme, dass unterschiedliche Sprachauffassungen mit unterschiedlichem Verständnis von Autoren und ihren Handlungsmöglichkeiten einhergehen.

- Autoren: Dieser Punkt betrifft meine Forschungsfragen direkt. Ich bin hier zum einen an vorhandenen Typologien von Sprachlenkungsautoren, zum anderen aber an vorhandenen, expliziten Erkenntnissen zu ihren Handlungsmöglichkeiten und Erfolgsaussichten, ggf. im Zusammenhang mit den festgestellten Typologien, in den jeweiligen Gebieten interessiert.

- Erfolg: Auch dies ist ein zentraler Punkt meiner Arbeit und ich trage hier Erkenntnisse zur Wirksamkeit und zum Erfolg von Maßnahmen zusammen, wobei in diesem Kapitel mein Augenmerk vor allen auf systematischen Konzeptionalisierungen und Modellierungen liegt.

- Modelle: Dieser Themenkomplex bezieht sich ebenfalls direkt auf meine Forschungsfragen. Hier stelle ich Theorien der jeweiligen Gebiete vor als Vorbereitung auf eine übergreifende Betrachtung und Theoriebildung der Sprachlenkung.

Obwohl die Themenkomplexe deduktiv aufgestellt sind, so ist aus den Beschreibungen deutlich, dass der selektive Forschungsstand die Perspektive auf die Forschungsfragen dieser Arbeit gezielt lenken soll. Anhand dieser Punkte sollen in weiteren Teilen dieser Arbeit einerseits theoretische Aspekte einer übergreifenden Sprachlenkungsbetrachtung ausgearbeitet werden, andererseits aber Vorüberlegungen zur Untersuchung von Handlungsmöglichkeiten und Wirksamkeit/Erfolg von Sprachlenkungsautoren getroffen werden. 
Im Folgenden beschreibe ich den Forschungsstand in einzelnen Gebieten anhand der vorgestellten Punkte. Ihre Reihenfolge kann jedoch variieren und es können weitere thematische Schwerpunkte hinzukommen, wie beispielsweise der Themenkomplex Werkzeuge, den ich speziell in den Gebieten Kontrollierte Sprachen und Terminologiearbeit behandle.

\subsection{LPLP, Sprachplanung, Sprachpolitik}

In diesem Teilkapitel betrachte ich das Gebiet Sprachplanung und berücksichtige dabei vor allem auf die angelsächsische Forschungstradition. Ich orientiere mich an den vorgeschlagenen Themenkomplexen, wobei für dieses Gebiet die geschichtliche Beschreibung besonders wichtig ist, da sie in vielen Fällen den roten Faden darstellt und eine Strukturierungshilfe bietet.

Trotz dieser Selektivität und Einschränkung ist bereits die Identifizierung und eine nähere Bestimmung von Grundkonzepten wie Sprachplanung oder Sprachpolitik schwierig, was auf die Fülle an Literatur, verschiedenen Betrachtungsweisen und einer mangelnden theoretischen Konsolidierung des Gebietes zurückgeht. Aus diesem Grund entscheide ich mich bei der Gegenstandbestimmung in 2.1.1 für eine zusammenfassende, resultative Darstellung und nicht für eine umfassende Präsentation und Diskussion von Einzelpositionen.

Im Zentrum der Sprachplanung steht, wie bei den anderen von mir betrachteten Gebieten, die Beschäftigung mit einer bewusst bzw. intentional herbeigeführten Änderung einer Sprache in einer bestimmten Sprachgemeinschaft. Viele Forscher und Praktiker siedeln jedoch Sprachplanung speziell auf der makrosozialen Ebene an und betrachten primär die überregionale (z. B. Minderheitensprachen in einem Staat), staatliche (z. B. Rechtschreibreformen in Deutschland) oder supranationale (z. B. geschlechtsneutrale Sprache) Ebene (exemplarisch Ager 2001; Grin 2003). Andere weisen wiederum darauf hin, dass die meso- und mikrosozialen Ebenen wie Sprachplanung in Unternehmen oder in einer Familie ebenfalls ins Spektrum des Gebiets fallen (exemplarisch B. H. Jernudd 1983; Cooper 1989; Kaplan, Baldauf Jr u. a. 2000; Janich 2004; Spolsky 2009; Duchêne und Heller 2012). In diesem Sinn wären Terminologiearbeit in Unternehmen und die Konstruktion von Kontrollierten Sprachen ein Teil des Gebiets Sprachplanung, zu dem Tauli (1968) auch die die Plansprachenkonstruktion zählt. Sprachplanung würde somit die in dieser Arbeit gesuchte übergreifende Einheit darstellen. Obwohl diese erweiterte Perspektive postuliert oder zumindest nicht explizit ausgeschlossen wird, so 


\section{Forschungsstand}

wird in der Literatur die Makro- und Mesoebene, wie der Staat und die Gesellschaft, bevorzugt. ${ }^{1}$ In diesem Teilkapitel entscheide ich mich deshalb für die Beschreibung dieser typischen Perspektive, ohne auf die Stellung der Terminologiearbeit und der Konstruktion Kontrollierter Sprachen in der Sprachplanung einzugehen.

\subsubsection{Begriffssystematisierung}

In der Literatur findet sich eine Fülle an Definitionen von Sprachplanung und Sprachpolitik, wobei unterschiedliche Forscher unterschiedliche Schwerpunkte setzen. Mit verschiedenen Definition und Positionen setzt sich beispielsweise Cooper $(1989,29-42)$ ausführlich auseinander. Ich entscheide mich im Folgenden, mein eigenes systematisiertes Verständnis des Gegenstandes vorzustellen, um so auch den Sprachgebrauch für diese Arbeit festzuhalten.

Im Hintergrund der Beschäftigung mit Sprachplanung und Sprachpolitik steht die aktuelle Sprachsituation in einer bestimmten Gemeinschaft wie beispielsweise einem Staat. Für diese Situation gilt der Sprachgebrauch als konstituierend. Er ist ein Ergebnis von ungelenkten Sprachentwicklungsprozessen wie Sprachwandel oder auch vorangegangenen intentionalen Eingriffen (vgl. Spolsky 2004, 5; S. Wright 2004, 3). Die aktuelle Situation wird von bestimmten Personengruppen als nicht zufriedenstellend bewertet, so dass eine Entscheidung über einen Eingriff in die Sprache (und damit eigentlich in den Sprachgebrauch) getroffen wird. Eine Möglichkeit, die Konzepte der Sprachplanung und der Sprachpolitik zu definieren und voneinander abzugrenzen, ist die Unterscheidung zwischen dem Eingriff (als Prozess) einerseits, in dem der Entwurf der neuen Sprachform, aber auch die Umsetzungsrichtlinien und methodische Vorgehensweisen ausgearbeitet werden und die eigentliche Umsetzung stattfinden, sowie dem Eingriffsergebnis andererseits. Das Ergebnis kann explizit in Form einer schriftlichen Richtlinie festgehalten sein, die man als Referenz bei der Umsetzung verwenden kann. Oder es kann implizit bzw. nichtöffentlich vorhanden und nur durch das Verhalten der Umsetzer beobachtbar sein (vgl. Baldauf 1994, 83; Ager 2001, 195; Shohamy 2006, xvi, 1, 51; Tollefson 2011, 357). Den Eingriff einschließlich aller Tätigkeiten, die dabei notwendig sind, bezeichnet man in der Literatur gewöhnlicherweise als

1 Exemplarisch nenne ich hier zwei aktuellere Untersuchungen, die sich mit der Terminologiearbeit auf Meso- und Makroeben befassen. Antia (2015) beschreibt die Terminologiearbeit in Südafrika im Kontext der Sprachplanung und Chan (2015) geht auf die kanadische Terminologiearbeit im Mehrsprachigkeitskontext ein. 
Sprachplanung (language planning) (obwohl Synonyme wie language engineering, language management, language development, language regulation noch vereinzelt zu finden sind (Cooper 1989, 29)). Der statische Blick auf die aktuelle Situation, das gewünschte Ergebnis, die neue Norm oder die explizite Richtlinie für den neuen Sprachgebrauch kann hingegen mit Sprachpolitik (language policy) bezeichnet werden.

Neben dieser Konzeptualisierung finden sich in der Forschung andere Verwendungen von Sprachplanung und Sprachpolitik, beispielsweise Sprachplanung als Bezeichnung für die Korpusplanung und Sprachpolitik für die Statusplanung (bspw. Grin 2003, 28) (zu Korpus- und Statusplanung s. 2.1.5.3), Sprachplanung als normative Festlegung ,,von oben“ (top-down) und Sprachpolitik als eine nichtverbindliche Empfehlung (bspw. Ager 2001, 5-6; Shohamy 2006, 49).

In manchen Fällen erfolgt die Definition und eine nähere Begriffsbestimmung durch die Analyse der Formseite beider Komposita, was aber nachteilig ist. Versucht man nämlich vom Wort Sprachplanung auf seine Bedeutung zu schließen, ergibt sich zwangsläufig, dass Sprachplanung eine umfassende Recherche und Erfassung des Istzustands nach sich zieht und somit ein systematischer Eingriff in die Sprachsituation ist (s. dazu 2.1.5.1). Obwohl dies sicherlich in vielen Fällen zutreffend ist, wie beispielsweise in den früheren Sprachplanungsmaßnahmen in den Entwicklungsländern (s. 2.1.2), so wird dem auch entgegnet, dass Sprachplanung auch eine „spontane“ oder nicht (systematisch) geplante Tätigkeit sein kann (Cooper 1989, 41; Hornberger 2006, 25; Spolsky 2012, 5).

Aus der wörtlichen Auffassung der Sprachplanung als systematischen Planungsprozess ergibt sich eine weitere Frage, nämlich ob eine Sprachpolitik immer als Ergebnis von Sprachplanung oder auch unabhängig davon entsteht (bspw. Hornberger 2006, 25). Sicherlich kann eine neue Norm und insgesamt eine Sprachpolitik ad-hoc ohne viel Recherche festgelegt werden oder als Nebenprodukt von anderen Tätigkeiten wie Bildungspolitik entstehen. In diesem Fall sehe ich die Sprachpolitik nicht unabhängig von der Sprachplanung, sondern als ihren einzigen Inhalt. Wie bereits erwähnt, muss die Sprachplanung keine vorbereitenden Tätigkeiten umfassen, um als Sprachplanung identifizierbar zu sein. Geht man bei Sprachplanung und Sprachpolitik von Zeicheninhalt aus, ist die Sprachpolitik im Kontext der Sprachplanung zu sehen. Eine wörtliche Auslegung der Zeichenform führt hingegen zum Paradox, da die Sprachplanung in Wirklichkeit ungeplant sein kann. 
Die Aufgaben der Sprachplanung werden in der Literatur vielfältig angegeben. Eine umfassende, aber recht unsystematische, Darstellung heterogener Aufgaben findet sich bei Nahir (1984) (vgl. Hornberger 2006, 30-33). Er spricht dabei nicht von Aufgaben, sondern Zielen der Sprachplanung und nennt dabei folgende Punkte:

1. Purifizierung (intern/extern)

2. Renovation

3. Revitalisierung

4. Reform

5. Standardisierung

6. Verbreitung

7. lexikalische Modernisierung

8. terminologische Vereinheitlichung

9. stilistische Vereinfachung

10. Internationalisierung

11. Sprachpflege

12. Standardisierung von sekundären Kanälen

Jede konkrete Sprachplanungsmaßnahme kann man einer oder mehreren dieser Kategorien zuordnen. An dieser Übersicht sind die bereits erwähnten Bezüge der Sprachplanung zu den anderen Gebieten: Terminologiearbeit und Kontrollierten Sprachen (z. B. Standardisierung, terminologische Vereinheitlichung) sowie Plansprachenkonstruktion/ Interlinguistik (Internationalisierung) deutlich zu erkennen. In der Literatur zur Sprachplanung werden am häufigsten Ziele 1-7 diskutiert.

Neben der Unterscheidung zwischen Sprachplanung und Sprachpolitik, die die Praxisperspektive auf den Gegenstandes abdecken, gibt es in der Forschung einen weiteren Begriff, nämlich den der wissenschaftlichen Disziplin, die die Prinzipien der Sprachplanung untersucht (Ricento 2009, 212). Auch hierfür findet man in der Literatur verschiedene Synonyme: academic 
language policy and planning, language planning, language policy and language planning, LPP, LPLP, theory of language planning (s. bspw. Tauli 1968, 26-27; Hornberger 2006, 25; Ricento 2009, 212; Hult 2010, 8; Grin 2016). Im Folgenden verwende ich:

- Sprachplanung als Bezeichnung für die praktische Tätigkeit, den Oberbegriff für Einzelmaßnahmen,

- Sprachpolitik für die festgelegte, implizite oder explizite Richtlinie, die sowohl die neue Sprachform als auch Hinweise zu Status, Vorgehensweisen und Zielen enthalten kann,

- LPLP für die wissenschaftliche Disziplin.

Auch dort, wo ich die Arbeiten anderer Autoren (indirekt) wiedergebe, verwende ich, wenn nicht anders angegeben, diese Systematik.

\subsubsection{Geschichte}

Die wissenschaftliche Beschäftigung mit der Sprachplanung - LPLP - ist eine relativ junge Disziplin, obwohl Sprachplanung bereits in der Antike belegt ist. So nennt Haugen (1966) Grammatiker als klassisches Beispiel für Sprachplaner, da sie die Sprache(n) nicht nur erforschten und beschrieben, sondern auch die Sprachnorm festlegten und unterrichteten (Haugen 1966, 3-4).

Als eigentlicher Vorreiter von LPLP gelten Arbeiten von sog. Sprachakademien, die in der Renaissance in Europa (Italien, Frankreich, Spanien, Deutschland) entstanden (Cooper 1989, 3-6; Janich 2004, 483). Die nationalen Bewegungen im Europa des 19. Jh. stärkten die Position von Akademien, da man sich durch puristische Spracharbeit und die ihr häufig zugrunde liegende Auffassung ein Staat - eine Sprache eine Festigung der nationalen Identität und somit die Existenzberechtigung für den jeweiligen Staat erhoffte (B. Jernudd und Nekvapil 2012, 18-22; vgl. Haugen 1966, 10-11; S. Wright 2004, 8).

Von LPLP im heutigen Sinn kann man erst seit dem 20. Jh. sprechen. Bei der Entwicklung der Disziplin lassen sich drei Phasen feststellen.

\subsubsection{Erste Phase 1960er - 1970er}

Nach dem zweiten Weltkrieg und dem Zerfall der Kolonialmächte rückten die unabhängigen, postkolonialen Staaten ins internationale Interessenspektrum. Für die Etablierung von LPLP als wissenschaftliche Disziplin Ende der 


\section{Forschungsstand}

60er Jahre waren hierbei zwei Probleme von Bedeutung. Zum einen wurden mit Kaplan, Baldauf Jr u. a. (2000) die neuen Staaten häufig ungeachtet der Stammesgrenzen ,von oben“ gegründet, so dass sich verschiedene, bis dahin fremde oder gar sich bekriegende Gruppen in einer staatlichen Einheit zusammenfanden. Diese Konstellation führte oft zu Unruhen und einer gesellschaftlichen Destabilisierung (Kaplan, Baldauf Jr u. a. 2000, 135). Zum anderen bestand mit Kaplan und Baldauf (1997) zwischen den neuen Staaten und Europa/Nordamerika ein großes Gefälle, was den Zugang zu Technologie, Bildung und Austauschmöglichkeiten anging. Der Wunsch der neuen Staaten nach einem gleichberechtigten Zugang zu Ressourcen sollte vom Westen unterstützt werden (Kaplan und Baldauf 1997, 241-248).

In diesem Kontext untersuchten die Sprachplaner die linguistische Situation in postkolonialen Staaten. Dies war aber weniger als ein Beitrag zur linguistischen Forschung, sondern als eine wissenschaftlich fundierte Unterstützung der allgemeinen Entwicklung und Modernisierung zu verstehen (Ricento 2006, 13; B. Jernudd und Nekvapil 2012, 23-24). Der Schwerpunkt lag also auf der praktischen Tätigkeit der Sprachplanung und weniger auf einer Etablierung von LPLP.

Der Umgang mit der multilingualen Situation beschränkte sich oft auf die Reduktion der sprachlichen Diversität durch die Auswahl von wenigen offiziell zugelassenen Sprachen. Die sprachliche Diversität wurde nämlich als hinderlich gesehen - die sprachliche Homogenität sollte auch der gesamten Gesellschaft den Anschein von Homogenität geben (Cooper 1989, 24; Kaplan, Baldauf Jr u. a. 2000, 135; Ricento 2006, 219-220). In diesem Zusammenhang griff man auf das Diglossie-Modell zurück, bei dem eine, oft europäische, Sprache in öffentlichen Bereichen wie Bildung, Verwaltung oder Technologie eingesetzt wurde, während man im Privaten weiterhin die einheimische Sprache verwenden durfte (Ricento 2006, 13). Entschied man sich jedoch gegen das Diglossie-Modell und bestimmte für den öffentlichen Bereich eine einheimische Sprache, war häufig eine Modernisierung notwendig. $\mathrm{Zu}$ diesem Zweck entwickelten die westlichen Sprachplaner für die jeweilige Sprache normative Rechtschreibsysteme, Grammatiken, Wörterbücher etc. (Ricento 2006, 12).

Für alle diese Maßnahmen war es im Geiste der Zeit notwendig, im Vorhinein einen systematischen Vorgehensplan zu entwickeln. Er sollte die Erfassung des Istzustands, die Auswahl von geeigneten Sprachen, die konkreten Schritte, aber auch Evaluierungsmöglichkeiten enthalten (zur Evaluierung s. Rubin 1971). Alle Teilmaßnahmen sollten aufeinander abgestimmt sein, wo- 
bei auch der größere Kontext von anderen gesellschaftlichen Projekten nicht außer Acht gelassen werden sollte: ,The planning was conceived as a set (...) of rational, yet politically authorized activities (fact-finding, planning goals, strategies and outcomes; implementation; and feedback), which take place in concrete social contexts (...)“(B. Jernudd und Nekvapil 2012, 25).

\subsubsection{Kritik der ersten Phase}

Wie bei allen Projekten zur Entwicklungsunterstützung für postkoloniale Staaten herrschte auch bei LPLP und konkreten Sprachplanungsmaßnahmen eine optimistische, oft als positivistisch bezeichnete Einstellung vor, dass man durch systematisches, auf die Erkenntnisse der modernen Wissenschaft aufbauendes Planen und Handeln die Probleme der Entwicklungsländer schnell lösen könnten (Hornberger 2006, 26). Auf diese anfängliche Zuversicht folgte die Ernüchterung, da die Vorgehensweise nicht nur die alten Probleme nicht löse, sondern auch neue verursache (Cooper 1989, 21-28; Kaplan, Baldauf Jr u. a. 2000, 136). Der größte Schwachpunkt war eine unreflektierte Übernahme der bereits erwähnten europäischen Idee ein Staat - eine Sprache, was in der neuen Situation nicht adäquat war (Kaplan und Baldauf 1997, 153). Die Kritik betrifft vor allem folgende Punkte:

Die scheinbar neutrale und sachliche Vorgehensweise der Sprachplaner riss die einheimischen Sprachen aus ihrem soziohistorischen Kontext und der sozialen Funktion (Ricento 2009, 220). Durch einen fremden Eingriff entstanden Sprachen, mit denen sich die Sprecher nicht mehr identifizieren konnten. Ricento (2006) geht sogar soweit zu behaupten, dass dadurch gar neue Sprachen erschaffen wurden (Ricento 2006, 13). Diese Veränderung der Sprachen ,von oben“ aber auch die soziale Stigmatisierung von nicht offiziellen Sprachen führten zur Abwertung der muttersprachlichen Kompetenz und in Konsequenz auch des Muttersprachlers selbst (Ricento 2006, 13). Dies warf deshalb die Frage nach dem Selbstbestimmungsrecht von Gruppen auf.

Der mangelnde Erfolg sowie auch die offensichtliche Ungerechtigkeit der bisherigen Vorgehensweise hatte für LPLP die Konsequenz, dass sich die Disziplin mit ihren Ansätzen, aber auch mit der (praktischen) Sprachplanung kritisch auseinandersetzen musste. 


\section{Forschungsstand}

\subsubsection{Zweite Phase 1980er - 1990er}

Die zweite Phase von LPLP ist durch diese kritische Auseinandersetzung und den Metadiskurs gekennzeichnet. Die althergebrachten Ziele und Methoden wurden in Frage gestellt (Hornberger 2006, 23). Zwar standen die Entwicklungsländer immer noch im Fokus, aber es wurden dabei realistische Umsetzungschancen und negative Konsequenzen der Sprachplanung diskutiert. Sprache wurde verstärkt als Faktor gesehen, der Ungerechtigkeit fördert (Ricento 2006, 13).

Darüber hinaus wurde zunehmend die Erweiterung des Gebiets um solche Domänen wie Technik, Recht und Industrie, losgelöst von den Problemen der Entwicklungsländer, gefordert (B. H. Jernudd 1983, 345-349; Rubin 1983, 330-332). Die Untersuchung dieser Domänen dominiert aber die LPLP-Forschung bis heute nicht. Des Weiteren wandte man sich systematischer den Minderheiten und dem gegenseitigen Einfluss von Sprachen zu: „In LPLP, the focus on the linguistic dimension of modernisation and nation building was eclipsed, and many researchers and scholars turned their attention to the social, economic and political effects of language contact, concentrating particularly on issues of advantage/disadvantage, status and access" (S. Wright 2004, 9).

\subsubsection{Dritte Phase ab 1990}

Weitere gesellschaftliche Veränderungen wie der Zerfall der Sowjetunion aber auch die fortschreitende europäische Integration leiteten eine neue Phase in der LPLP-Forschung ein. Zum einen verstärkte sich der Einfluss der angelsächsischen, speziell der US-amerikanischen, Kultur auf andere Regionen (S. Wright 2004, 10-12). Zum anderen aber erhöhte sich die Mobilität der Menschen und eine weit gehende Annäherung fand in vielen Lebensbereichen statt.

In LPLP wandte man sich diesen aktuellen Entwicklungen zu, so dass der kritische Metadiskurs an Bedeutung verlor. Themen wie Verbreitung des Englischen, Schutz von regionalen oder Minderheitensprachen (Ricento 2006, 232) oder der Sprachimperialismus im Allgemeinen (s. 2.1.8), obwohl nicht neu, erlangten eine gleichberechtigte, wenn nicht sogar eine bevorzugte Stellung in der Forschung (S. Wright 2004, 10-12).

Weiterhin wurde die Domänenerweiterung gefordert, da diese nur unzureichend umgesetzt wurde (B. Jernudd und Nekvapil 2012, 17): ,(..) these problems and issues apply not only to 'developing' nations, but equally to 
the issues implicit in modernising languages and polities aswell, and not only at the macro (national and supra-national) level but also at the micro (local) level, in multinational corporations, among varieties of service providers, and in even smaller contexts“ (Kaplan, Baldauf Jr u. a. 2000, 137).

Für eine Übersicht über die aktuellen Forschungsfragen s. 2.1.8.

\subsubsection{Soziale Einbettung}

Theoretisch ist es möglich, eine neue Sprachform zu entwickeln, ohne dass dafür konkrete (soziale) Anreize vorhanden sind. Wie ich im Abschnitt zur Geschichte deutlich gemacht habe (2.1.2), kristallisierte sich Sprachplanung und in Konsequenz auch LPLP im Kontext einer Hilfestellung bei sozialen Problemen heraus. Ich nehme deshalb an, dass irgendeine Form von Wechselwirkung zwischen Sprachplanung und Gesellschaft in der Disziplin von Anfang an angenommen wurde. Vor allem in älteren Arbeiten findet man vereinzelt die sog. linguizentrische Position (Bezeichnung mit Spolsky 2004, ix$\mathrm{x})$, bei der der außersprachliche Hintergrund der Sprachplanung außer Acht gelassen wird (z. B. bei Tauli 1968). Diese Auffassung wurde jedoch in der Forschung relativ früh als inadäquat bewertet und deshalb abgelehnt (B. H. Jernudd und Gupta 1971, 211; B. H. Jernudd 1983; Cooper 1989, 183).

Es stellt sich somit lediglich die Frage, wie sich das Verständnis der Wechselwirkung von Sprachplanung und Gesellschaft in der LPLP-Forschung änderte. Um diese zu beantworten, analysiere ich die vorgestellten geschichtlichen Phasen und stelle fest, dass jeder geschichtlichen Phase eine veränderte Auffassung der Wechselwirkung von Sprache und Gesellschaft zugrunde liegt. Dabei gehe ich weniger von einem Bruch zwischen verschiedenen Phasen aus, da ältere Positionen nicht vollständig abgelehnt, sondern allmählich erweitert und teilweise weiter vertreten werden. Mit der darauffolgenden Beschreibung gehe ich lediglich auf die eingeführten Neuerungen ein.

\subsubsection{Erste Phase: Sprachplanung $\Rightarrow$ Gesellschaft}

Aus der Beschreibung der geschichtlichen Entwicklung von LPLP geht hervor, dass die Sprachplanung als Lösung für soziale Probleme aufgefasst wurde (s. auch Haugen 1983, 286; Cooper 1989, 33-34). Sprachplanung, als Teil der allgemeinen sozialen Planung (Tollefson 2011, 357), nimmt also Einfluss auf die Gesellschaft. Als bezeichnend für diese Auffassung sehe ich, dass eine mögliche Rückkopplung und die Beeinflussung der Sprachplanung durch die 


\section{Forschungsstand}

Gesellschaft ausgeblendet wird. Es muss sich bei der ersten Phase also weniger um eine Wechselwirkung handeln, sondern eher um eine Beeinflussung, die nur einseitig von der Sprachplanung auf die Gesellschaft gerichtet ist.

\subsubsection{Zweite Phase: Sprachplanung $\Leftrightarrow$ Gesellschaft}

Durch die beschriebene Kritik an früheren Ansätzen zum Umgang mit der Sprachsituation in den Entwicklungsländern wurde erkannt, dass die „Einbahnstraße“ Sprachplanung $\Rightarrow$ Gesellschaft eine konzeptuelle Vereinfachung darstellt und für LPLP problematisch ist.

Sprachplanung wurde zwar immer noch als Problemlösung aufgefasst, aber die Wirkung von außersprachlichen, speziell gesellschaftlichen, Faktoren auf den gesamten Prozess sollte ebenfalls berücksichtigt werden. LPLP und die Sprachplanung sind also nicht neutral und sachlich, wie ursprünglich dargestellt wurde, sondern immer in einen politischen, ideologischen und historischen Kontext eingebettet, der die Ziele, Methoden und Ergebnisse mit bestimmt (Edwards 2012, 431, 435). Wie Ricento (2006) anmerkt, spiegelt Sprachplanung die gesellschaftliche Struktur wider: „Decisions about which languages will be planned for what purposes ultimately reflect power relations among different groups and sociopolitical and economic interests" (Ricento 2006, 5-6).

Neben der Anerkennung dieser übergreifenden Einflussfaktoren schloss man die Sprechergemeinde in die Betrachtung mit ein. Auch die lokale Tradition, die Sicht der Sprecher und ihre Bedürfnisse sollten bei der Gestaltung einer konkreten Sprachplanungsmaßnahme beachtet werden (vgl. Cooper 1989, 385; Tollefson 2011, 375): „Language planning is not 'language engineering'; it must, ultimately, satisfy the speakers of all languages involved. While language planning solutions must be 'sold' to the public through conventional advertising techniques, the basic plan must be bottom-up, and must serve the interests of the community or it will not meet the conditions just enunciated for that language plan to survive" (Kaplan und Baldauf 1997, 309). Seit dieser Phase unterscheidet man deshalb zwischen der sog. Top-down- und der Bottom-up-Sprachplanung. Bei der ersten werden Vorgaben für die Maßnahme durch eine kleine Gruppe in Machtposition gemacht, ohne dass die betroffene Sprechergemeinde bei der Gestaltung mitwirkt. Bei der zweiten hingegen wird die Maßnahme durch die betroffene Gemeinde selbst durchgeführt. In der Praxis findet man zudem Mischformen. 
Schließlich wurde unter der Anerkennung des sozialen Einflusses auch die Frage nach dem Erfolg einer Maßnahme in ein neues Licht gerückt. So gelangt beispielsweise Rubin (1983) zur Erkenntnis, dass der Erfolg deshalb schwer zu erzielen ist, da er von vielen außersprachlichen Faktoren abhängt, auf die der Sprachplaner nur einen geringen Einfluss hat. Darüber hinaus kann sich auch im Laufe der Zeit die Auffassung von Problemen ändern, für die die Sprachplanung eine Lösung darstellen sollte (Rubin 1983, 329).

\subsubsection{Dritte Phase: Sprache $\Leftrightarrow$ Gesellschaft + Identität}

Die Auseinandersetzung mit den Bottom-up-Einflüssen hatte eine Neuerung in der Auffassung der sozialen Einbettung zufolge. Man wandte sich dem symbolischen Wert der Sprache und ihrer identitätsstiftenden Funktion zu (Kaplan, Baldauf Jr u. a. 2000, 137; Ager 2001, 2005; Edwards 2012, 428). Diese sind vor allem im Kontext von Minderheitensprachen und den sog. sprachlichen Menschenrechten von Bedeutung: „Language is the medium by and through which individuals define and inhabit their own identities and, in the process, assess and ascribe the identities of others. It is often these differences in identities (whether achieved or ascribed) that lead to conflicts in which language may play an important role" (Ricento 2006, 231).

Die Wechselwirkung zwischen der Sprachplanung und der Gesellschaft wird weiterhin anerkannt. Es soll lediglich die Identität als Faktor hervorgehoben werden, von dem man sich in bestimmten Kontexten eine bessere Erklärung des Sprachplanungsprozesses erhofft.

\subsubsection{Zusammenfassung}

Die Entwicklung in der Auffassung der sozialen Einbettung von Sprachplanung kann man der Sprachauffassung in der Linguistik gegenüberstellen. Wie bereits angedeutet, positionierten sich die LPLP-Forscher klar gegen die systemlinguistische Auffassung von Sprache als „(...) a self-contained entity independent of the human beings who used the language and independent of all the other languages and linguistic resources present in the environment" (Kaplan, Baldauf Jr u. a. 2000, 136). Vielmehr vertreten sie aus der sprachwissenschaftlichen Sicht die soziolinguistische Position: ,To the extent that one's primary language indexes (i.e., "points to") class, education level, ethnicity/ race in a particular context, it may reinforce negative or positive stereotypes about particular ethnolinguistic groups. This can (and often does) correlate with social hierarchies in which language competencies can be viewed as 


\section{Forschungsstand}

causes for relatively higher or lower social status, rather than as the result of sociopolitical processes associated with nationalism and state formation which position certain languages/varieties hierarchically in defined contexts" (Ricento 2009, 212).

Auch im historischen Querschnitt erkennt man deutliche Parallelen dazwischen, wie sich die Auffassung der sozialen Einbettung der Sprachplanung und der Sprachbegriff in der Soziolinguistik änderte (Frage nach der Wechselwirkung der Sprache und Gesellschaft, Stigmatisierung vs. akzeptierte Andersartigkeit als Konsequenz des Sprachgebrauchs etc.; für allgemeine Einführung in die Soziolinguistik siehe bspw. Schlieben-Lange (1991), Dittmar (1997) und Löffler (2010)).

Diese Positionierung ist insofern interessant, als sie in dieser Deutlichkeit in den anderen von mir beschriebenen Gebieten, vor allem der Terminologiearbeit und bei der Konstruktion von Kontrollierten Sprachen, nicht zu finden ist.

\subsubsection{Interdisziplinarität}

Die Einordnung von LPLP als wissenschaftliche Disziplin scheint auf den ersten Blick relativ einfach zu sein. Da es sich mit Sprache im sozialen Kontext beschäftigt, liegt die Vermutung nahe, sie als Teilgebiet der Soziolinguistik (bzw. bei manchen Autoren der Soziologie der Sprache wie beispielsweise bei Cooper $(1989,45))$ aufzufassen. Tatsächlich wird betont, dass die systematische Erforschung der Sprachplanung primär durch Soziolinguisten geschah (Janich 2004, 389; Ricento 2006, 12; B. Jernudd und Nekvapil 2012, 17-18). Allerdings ist diese Einordnung weniger eindeutig, wenn bedacht wird, dass LPLP wie bereits erörtert soziale, politische, historische und andere Faktoren berücksichtigen muss, um angemessene Aussagen zu machen. Wie Cooper (1989) anmerkt, kann die Soziolinguistik allein nicht alle Fragen aus Theorie und Praxis beantworten. Insofern wird eine interdisziplinäre Ausrichtung von LPLP für sinnvoll gehalten, sowohl was die Übertragung von Konzepten als auch Methoden angeht: ,(...) it may be useful to look at frameworks suggested by other disciplines or subdisciplines not only to understand language planning better but also to forward the development of a framework particularly suited for language planning" (Cooper 1989, 45). B. Jernudd und Nekvapil (2012) betonen allerdings, dass die Erkenntnisse, die durch einen Bezug zu anderen Disziplinen gewonnen wurden, bisher nur selten in diese Disziplinen zurückflossen. Dies scheint sich aber zurzeit zu ändern (B. Jer- 
nudd und Nekvapil 2012, 18). Aus diesem Grund kann man zum heutigen Zeitpunkt noch nicht von LPLP als einem vollständig interdisziplinären, sondern einem außerdisziplinär ausgerichteten Forschungsgebiet innerhalb der Linguistik sprechen.

Die klassischen Bezugsdisziplinen von LPLP sind außer Linguistik auch die Soziologie oder im allgemeinen Sozialwissenschaften, Politikwissenschaften und Wirtschaftswissenschaften (Grin 2009):

- Sozialwissenschaften: Sprachplanung als Teil der allgemeinen sozialen Planung (Ricento 2009, 212; Tollefson 2011, 357);

- Politikwissenschaften: Erklärung von Sprachkonflikten vor dem Hintergrund der kulturellen/politischen Konflikte (Ricento 2006, 6-7); Kontrolle und Macht durch Sprache (Cooper 1989, 85-91, 119-120; S. Wright 2004, 13; Shohamy 2006);

- Wirtschaftswissenschaften: Untersuchung von Kosten-Nutzen-Verhältnis von Sprachplanung (Janich 2004, 489); Bewertung von alternativen Maßnahmen aus wirtschaftlicher Sicht (Grin 2003, 133-137), oder im Allgemeinen wirtschaftliche Aspekte der Mehrsprachigkeit: „For example, are linguistically diverse countries more or less prosperous than unilingual ones? Is productive efficiency affected by the choice and range of languages used in a multilingual corporation? Do people really prefer goods to be advertised and sold to them in their native language?" (Grin 2009, 276).

In der neueren Literatur wird außerdem eine Vielzahl von verschiedenen Forschungsgebieten und -disziplinen genannt, die zwar weniger zentral sind, aber je nach Forschungsfrage eine Rolle spielen können: Innovationsmanagement, Marketing, Entscheidungsforschung (Cooper 1989), Kritische Theorie, Historiographie, postkolonialistische Ansätze, Governmentality Studies, Identity Studies, Diskursanalyse (Ricento 2009, 214-215), psychologische Soziologie, Ethnographie (Ricento 2006, 130-131), Rechtswissenschaften, Psychologie, Kulturwissenschaften (Janich 2004, 488-489), Sozialpsychologie (Ager 2001, 2005). Obwohl diese Fülle an Perspektiven zum besseren Verständnis des Gebiets beitragen soll, entwickelt sie sich allmählich auch zu einem Verständnishindernis. Da jede Disziplin eigene Konzepte, Gepflogenheiten und den Fachwortschatz mitbringt, ist es mitunter schwierig nachzuvollziehen, was neue Erkenntnisse sind und welche scheinbaren Neuerungen 


\section{Forschungsstand}

sich als Altbekanntes unter einem neuen Deckmantel entpuppt (s. Beispiel zum Thema „Amtssprache“ in Kaplan und Baldauf (1997, 14-18)).

Die am Anfang des Abschnitts postulierte Zuordnung von LPLP zur Soziolinguistik scheint also bei dieser Vielfalt von interdisziplinären Einflüssen nicht mehr eindeutig zu sein: ,Whether it can be claimed that language planning is a subordinate or a superordinate relative of sociolinguistics remains an unanswered question, since language planning may involve more or less than sociolinguistics depending upon a number of variables which can only be understood on a case-by-case basis" (Kaplan und Baldauf 1997, x).

\subsubsection{Modelle}

Trotz eines beachtlichen theoretischen Fundus, aus dem sich LPLP als interdisziplinär ausgerichtete Disziplin bedienen kann, gehört die eigene Theoriebildung zu den Schwachstellen des Gebiets. Obwohl versucht wird, für bestimmte Aspekte der Sprachplanung Teilmodelle zu entwickeln (z. B. ganzheitliche Betrachtung von Sprachsituation im Sinne eines ökologischen Modells, s. 2.1.5.5), sind andere weitestgehend vernachlässigt (z. B. Evaluation und Erfolgskriterien (Ricento 2009, 228)). Dem Bereich mangelt es also an einer übergreifenden Perspektive und an Modellen, die Vorhersagen über den Verlauf von Sprachplanungsmaßnahmen erlauben könnten (Cooper 1989, 41). Dieser Einwand besteht bereits seit den früheren Phasen der LPLPForschung, ist aber weiterhin aktuell (Cobarrubias 1983, 5; Spolsky 2004, ix; Ricento 2006, 6; Edwards 2012, 431).

In der Literatur werden verschiedene Gründe für diesen Mangel genannt. Häufig wird auf die Komplexität des Gebietes aufgrund einer Vielzahl an Einflussfaktoren sowie die Singularität der Fälle hingewiesen, die eine Verallgemeinerung zu einer Theorie unmöglich machen (Haugen 1966, 16; Ricento 2006, 10). Wie Cooper (1989) jedoch bemerkt, sind diese Gründe nicht die eigentliche Ursache. Die Schwierigkeiten ergäben sich daraus, dass Sprachplanung oft als Instrument zur Machtausübung eingesetzt werde. So blieben viele tatsächliche Motive, Ziele und Vorgehensweisen im Verborgenen. Erst die Berücksichtigung dieser würde eine adäquate Modellbildung voranbringen (Cooper 1989, 182).

Nicht zuletzt weist Ricento (2006) darauf hin, dass manche theoretischen Aspekte nur in interdisziplinären Forschungsgruppen ausgearbeitet werden können. (Ricento 2006, 18). Da aber die Forschung wie erwähnt vorwiegend 
von den Soziolinguisten betrieben wird, fehlt bisweilen die nötige Sachkenntnis z. B. der Politik- oder Wirtschaftswissenschaften.

Trotz dieser Umstände wird der Versuch einer Theoriebildung immer wieder gewagt. Man strebt danach, ,(...) [to] move beyond descriptive to become more theoretical, predictive, and explanatory.“ (Hornberger 2006, 33).

Im Folgenden stelle ich die wichtigsten Beiträge vor, die das Thema $L P L P$ Theorie geprägt haben. Es handelt sich dabei um die Arbeiten von Haugen (1959, 1966, 1983), Tauli (1968), Kloss (1969), Cooper (1989), Kaplan und Baldauf (1997), Ager (2001), Spolsky (2004, 2009).

\subsubsection{Haugen $(1959,1966,1983)$}

Haugens Konzeptualisierung der Sprachplanung legt den Schwerpunkt auf die Prozessbeschreibung (ursprüngliche Fassung Haugen 1959; mit wenigen Änderungen auch Haugen 1966, 1983). Sie identifiziert und beschreibt vier Phasen, die eine typische Sprachplanungsmaßnahme umfasst (hier in der Fassung aus Haugen 1983, 270-275):

1. Selektion: Auswahl der zu verändernden Sprachen, Festlegung von Zielen, Vorbereitung des Veränderungsplans,

2. Kodifikation: Konkretisierung von Änderungen am Sprachsystem und die Entwicklung einer neuen Norm: z. B. Festlegung von Rechtschreibung, Wortschatzerweiterung etc. Die Auswahl von neuen sprachlichen Mitteln soll mit Haugen (1966) diesen Prinzipien folgen: Effizienz (Wie leicht lässt sich die neue Norm benutzen?), Rationalität (Wie groß ist die Ausdruckskraft der Sprache? Wie leicht lassen sich komplexe Sachverhalte wiedergeben?) und Gemeinschaftlichkeit (Wie gut kann ein Individuum mittels der Sprache am Gemeinschaftsleben teilnehmen? - expressiver/psychologischer Wert der Sprache) (Haugen 1966, 1617),

3. Implementation (früher auch: Akzeptanz): praktische Umsetzung und Etablierung der neu entwickelten Norm durch gewählte Organisationen,

4. Elaboration: laufende Sprachpflege und Weiterentwicklung der etablierten Sprache/Norm, z. B. Wortschatzarbeit. 


\section{Forschungsstand}

Diese Konzeptualisierung, die man auch als allgemeine methodische Vorgehensweise sehen kann, ist in der Forschung weitestgehend anerkannt, obwohl auch auf ihre Schwachpunkte hingewiesen wird. Kaplan und Baldauf $(1997,30)$ stellen beispielsweise die Implikation in Frage, dass Sprachplanung immer mit einer systematischen und expliziten Vorentscheidung beginnt (Kaplan und Baldauf 1997, 30), was ich auch eingangs in diesem Kapitel angesprochen habe. Einen ähnlichen Standpunkt vertritt ebenfalls Cooper (1989): ,(...) definition of language planning as a systematic, rational, theorydriven activity suffers from the fact that one must look long and hard to find an example. Certainly none of my four defining cases exemplifies such an activity. If these cases illustrate language planning, then language planning can be a messy affair - ad hoc, haphazard, and emotionally driven" (Cooper 1989, 41). Eine Aufteilung des gesamten Prozesses in klar voneinander getrennte Phasen beschreibt also nicht jede Art von Sprachplanung.

Des Weiteren wenden Kaplan und Baldauf (1997) ein, dass Haugens Konzeptualisierung von der Schriftsprache ausgeht und die gesprochene Sprache nicht berücksichtigt (Kaplan und Baldauf 1997, 38). Ähnlich schreibt Ricento (2009), dass die unter Kodifikation aufgeführten Prinzipien das Vorhandensein einer literarischen Tradition annehmen, was offensichtlich nicht für alle Sprache zutreffend ist (Ricento 2009, 219).

Schließlich weisen B. H. Jernudd und Gupta (1971) auf die zentrale Rolle des Sprachplaners in dem so verstandenen Sprachplanungsprozess hin, wodurch aber der Bottom-up-Einfluss ungenügend berücksichtigt wird (B. H. Jernudd und Gupta 1971, 197).

Zusammenfassend ist Haugens Konzeptualisierung als eine Maximalliste von Sprachplanungsphasen zu verstehen, die sicherlich auch nicht konsekutiv ablaufen müssen. Sie eignet sich zudem vor allem zur Beschreibung von Sprachplanungsmaßnahmen, die auf eine systematisch ausgearbeitete, explizite Sprachpolitik abzielen.

\subsubsection{Tauli (1968)}

Tauli (1968) setzt sich zunächst mit den Grundkonzepten auseinander und unterscheidet zwischen der Wissenschaftsdisziplin Theorie der Sprachplanung (entspricht der in dieser Arbeit benutzten Bezeichnung LPLP) und der praktischen Tätigkeit der Sprachplanung (Tauli 1968, 26-27). Neben dieser Begriffsbestimmung stellt das Aufdecken von Prinzipien, anhand derer alternative Ausdrücke im Sprachsystem bewertet werden können, die zentrale Frage für Tauli (1968) dar, was er als Gegenstand der Theorie der Sprachplanung 
auffasst. Er kommt dabei zum Schluss, dass die sprachlichen Varianten anhand ihrer Klarheit, Ökonomie und Ästhetik evaluiert werden sollen, wobei hier gegenläufige Tendenzen wie Klarheit und Ökonomie nicht auszuschließen sind (Tauli 1968, passim).

Die Theorie der Sprachplanung befasst sich nach Tauli (1968) vorwiegend mit dem sprachlichen Zeichen selbst und ist daher weitestgehend im Strukturalismus angesiedelt. Taulis Auffassung von Sprachplanung sehe ich hingegen nicht so eindeutig strukturalistisch, wie oft von dessen Kritikern dargestellt wird (exemplarisch B. H. Jernudd und Gupta 1971, 195). Einerseits geht er in seiner Definition von Sprachplanung nicht auf die sozialen Aspekte der Tätigkeit ein: „LP [Language Planning] is the methodical activity of regulating and improving existing languages or creating new common regional, national or international languages. LP comprises all spheres of the oral and written form of the language: phonology, morphology, syntax, lexicology (...) and orthography“ (Tauli 1968, 27, Hervorhebungen im Original). Darüber hinaus marginalisiert er den symbolischen Wert der Sprache, dessen Verlust man bei der Sprachplanung bedenkenlos in Kauf nehmen könne. Die Sprache hat für ihn somit vorrangig einen instrumentellen Charakter (Tauli 1968, 151). Andererseits aber spricht er sich gegen eine zu starke Vorschreibung von Sprachmitteln aus, bei der nur eine Form und keine Alternativen zugelassen werden. Auch Sanktionen für die Abweichung von der Norm schließt er aus, da dies die individuelle Freiheit und den kreativen Umgang mit Sprache, die beispielsweise für Künstler besonders wichtig sind, zu stark einschränken würde: „S [Standardization] is also inexpedient from the ethic, social and aesthetic point of view. Nobody has the right to prescribe linguistic norms for the community. Individual freedom on linguistic matters must be guaranteed, as freedom in exercising all other human rights. Particularly essential is freedom for the artist, the writer" (Tauli 1968, 156). Stattdessen sollten Sprachberatungen als Umsetzungsmethode bevorzugt werden, in denen die Vorteile der neuen Norm mit rationalen Argumenten erklärt werden: „Instead of S [standardization], language users need information. Instead of prescriptions one should advise language users of what is better, recommendable, more efficient." (Tauli 1968, 157). In dieser Hinsicht vertritt Tauli eine durchaus moderne Sicht, die später im Kontext von sprachlichen Menschenrechten wieder aufgegriffen wird (s. dazu 2.1.8.3).

Interessant an Taulis Auffassung ist zudem, dass er die Belange der Interlinguistik, d. h. der Plansprachenkonstruktion, zur Disziplin Theorie der Sprachplanung zählt (Tauli 1968, 28). Auch weitere Punkte wie beispielsweise Par- 


\section{Forschungsstand}

allelen zu Wüsters Auffassung von Terminologiearbeit (z. B. Unterscheidung zwischen der theoretischen Terminologielehre und der praktischen Terminologiearbeit) heben Tauli als einen Forscher hervor, der einen übergreifenden Blick auf verschiedene Gebiete der Sprachlenkung behält.

\subsubsection{Kloss (1969)}

Auf Kloss (1969) geht die wohl bekannteste Unterscheidung innerhalb LPLP zurück: zwischen der Korpus- und der Statusplanung. Korpusplanung ist demnach die Tätigkeit, bei der Änderungen am Sprachsystem vorgeschlagen und festgehalten werden, z.B. im Wortschatz, in der Syntax, aber gelegentlich auch in der Morphologie. Bei der Statusplanung in der ursprünglichen Fassung handelt es sich hingegen um eine Neupositionierung einer Sprache/Varietät in der Gesellschaft oder in einer Institution. Dies soll durch die Steigerung oder Verminderung ihres Prestiges im Vergleich zu anderen Sprachen/Varietäten geschehen (Kloss 1969, 81). Kloss (1969) weist dabei darauf hin, dass beide Tätigkeiten unabhängig voneinander durch unterschiedliche Gruppen durchgeführt werden können. Für die Korpusplanung sind gewöhnlicherweise Linguisten zuständig, die Statusplanung ist die Domäne von Politikern oder Beamten (Kloss 1969, 81).

Korpus- und Statusplanung sind in der heutigen Forschung gut etablierte Konstrukte (Janich 2004, 481; S. Wright 2004, 1; Hornberger 2006, 28). Die meisten Forscher definieren die Statusplanung jedoch breiter und subsummieren darunter jede Art von Maßnahmen, die zur Umsetzung der neuen Norm eingesetzt werden: ,Kloss viewed the object of status planning to be recognition by a national government of the importance or position of one language in relation to others. However, the term has since been extended to refer to the allocation of language or language varieties to given functions, e.g. medium of instruction, official language, vehicle of mass communication" (Cooper 1989, 32; s. auch Kaplan und Baldauf 1997, 30; Tollefson 2011, 359).

Auch die bereits beschriebenen Konzeptualisierungen von Haugen (1959) und Tauli (1968) lassen sich im Rahmen von Korpus- und Statusplanung fassen. So nennt Tauli in seiner Definition von Sprachplanung nur die Aspekte der Korpusplanung. In Haugens Konzeption stellen hingegen Selektion und Implementation die Statusplanung dar, während Kodifikation und Elaboration vor allem die Korpusplanung betreffen.

Trotz einer plausiblen Trennung beider Begriffe wird in der Literatur hervorgehoben, dass diese Unterscheidung eher konzeptueller Natur ist. Beider 
Prozesse hängen stark zusammen und bedingen sich in der Praxis gegenseitig (Janich 2004, 493; Spolsky 2004, 11).

\subsubsection{Cooper (1989)}

Coopers Beitrag zur Systematisierung und Weiterentwicklung von LPLP ist vielfältig, selbst wenn er ebenfalls keine übergreifende Theorie der Disziplin aufstellt (Cooper 1989). In seiner Definition der Sprachplanung hebt er vor allem die sozialen Aspekte des Prozesses hervor, der sich weniger über die Korpus- sondern eher über die Statusplanung konstituiert: „Language planning refers to deliberate efforts to influence the behavior of others with respect to the acquisition, structure, or functional allocation of their language codes. " (Cooper 1989, 45, Hervorhebungen im Original). Darüber hinaus sieht Cooper die Sprachplanung immer als Resultat einer gesellschaftlichen Veränderung, ohne die eine Sprachplanungsmaßnahme nicht sinnvoll und erfolgreich sein kann (Cooper 1989, 164-171).

Interessant ist außerdem seine in der Pragmatik angesiedelte Vorgehensweise bei der Auswertung von älteren LPLP-Beschreibungen. Er analysiert sie anhand einer abgewandelten Lasswell-Formel (Lasswell 1948): „Who plans what for whom and how?" (Cooper 1989, 31, Hervorhebungen im Original).

Eine ähnliche Vorgehensweise wendet er beim schon erwähnten Versuch an, Sprachplanung in neue Disziplinen einzubetten. Obwohl die Einbettung in die Politikwissenschaft im Kontext der Machtausübung relativ verbreitet ist, stellen die Bezüge zur Entscheidungsforschung, aber vor allem zum Innovationsmanagement und Marketing neuartige Perspektiven dar:

- Sprachplanung und Innovationsmanagement: Die neue Sprachform ist eine gesellschaftliche (Kommunikations-)Innovation; die Vorgehensweise bei ihrer Einführung ist eine ähnliche wie bei der Einführung von Innovationen in anderen sozialen Bereichen; es geht also um die Frage: „(...) who adopts what, when, where, why, and how?“ (Cooper 1989, 6);

- Sprachplanung und Marketing: Die neue Sprachform kann als Produkt verstanden werden, das man mithilfe von geeigneten Maßnahmen vermarkten muss, wofür Marketing hilfreiche Ideen liefern kann; es geht hier um die Frage, wie man ein Produkt entwickelt sowie zum richtigen Zeitpunkt und zum richtigen Preis vermarktet (Kotler und Zaltman 1971; zit. mit Cooper 1989, 72). 


\section{Forschungsstand}

Diese neuen Einbettungen fanden in der Literatur wenig Beachtung. Sie können aber m. E. einen wichtigen Beitrag zum besseren Verständnis von Mechanismen der Sprachplanung und zur Formulierung von Praxisempfehlungen für erfolgreiche Umsetzungsmethoden leisten.

Sehr gut angenommen ist in der Literatur hingegen Coopers (1989) Erweiterung der Dichotomie: Statusplanung - Korpusplanung um einen weiteren Begriff, nämlich die Erwerbsplanung. Darunter versteht er Maßnahmen, die auf das Erlernen einer Sprache bzw. der neuen Norm gerichtet sind (Cooper 1989, 157). Die Erwerbsplanung steht nah an der Statusplanung, beide Begriffe unterscheiden sich aber in ihrer Zielsetzung: Die Erwerbsplanung soll die Anzahl der Sprecher der neuen Form erhöhen, während die Statusplanung die Häufigkeit des Gebrauchs der neuen Form unter den bestehenden Sprechern steigern soll (Cooper 1989, 33): „In other words, status planning is an effort to regulate the demand for given verbal resources whereas acquisition planning is an effort to regulate the distribution of those resources" (Cooper 1989, 120).

\subsubsection{Kaplan und Baldauf (1997)}

Die für die neue LPLP-Literatur bedeutendste Konzeptualisierung, die man auch als Modell auffassen kann, beruht auf der Berücksichtigung nicht ausschließlich der zu ändernden Sprache. In dem sog. sprachökologischen Ansatz werden Sprachen/Varietäten in einem Netz von gegenseitigen Abhängigkeiten betrachtet. So kann eine Sprache nur dann als Minderheitensprache fungieren, wenn es gleichzeitig eine Mehrheitssprache gibt. Ist eine Sprache/Varietät stigmatisiert, muss es eine andere geben, die Prestigeträger ist. Vor diesem Hintergrund erkennt man die eigentliche Komplexität der Sprachplanung. Wird eine Sprache/Varietät geändert, ist möglicherweise ein ganzes System von Sprachen betroffen, die in Beziehung zu ihr stehen (für eine Veranschaulichung des Modells s. beispielsweise Kaplan und Baldauf (1997, 311-312; 269-272)). Sprachökologische Überlegungen existierten bereits früher, Kaplan und Baldauf (1997) trugen jedoch erheblich dazu bei, dass dieses Modell in der Forschung weit verbreitet ist (Spolsky 2004, 129; Ricento 2006, 6; Hult 2010, 9; Tollefson 2011, 396).

Aus dem sprachökologischen Modell ergibt sich ein besseres Verständnis von Prozessen wie Revitalisierung, Aussterben von Sprachen und Sprachverlust (Kaplan und Baldauf 1997, 271-272; Kaplan, Baldauf Jr u. a. 2000). Es liefert auch eine theoretische Grundlage für spezielle Fragen wie nach der Verbreitung des Englischen oder nach den sprachlichen Menschenrechten (s. 
Abschnitt 2.1.8). Gleichzeitig führt das sprachökologische Modell aber dazu, dass die Trennung zwischen Sprachplanung und Sprachwandel verschwindet und LPLP sich stärker Problemen zuwendet, die traditionell in der Sprachwandelforschung behandelt werden.

\subsubsection{Ager (2001)}

In seinem Buch befasst sich Ager (2001) mit der Motivation bei der Sprachplanung und charakterisiert sie eingangs mit folgenden Fragen: Warum wird Sprachplanung durchgeführt? Welche Beweggründe stehen hinter einer expliziten Sprachplanungsmaßnahme? Was sind die Unterschiede zwischen Motivation bei Individuen, machtlosen Gruppen und Machtgruppen? Hierfür entwickelt Ager (2001) ein quantitatives Werkzeug zur Erfassung der Motivationsstruktur von Autoren einzelner Maßnahmen, mit dessen Hilfe man auch unterschiedliche Maßnahmen miteinander vergleichen kann. Ager versteht Sprachplanung als Eingriff ins Sprachverhalten von Individuen und Gruppen (Ager 2001, 5). Zur Herleitung seiner Konzeptualisierung dienen ihm Beispiele von Staaten, Gemeinschaften innerhalb eines Staates und Individuen.

Motivation ist nicht direkt beobachtbar und es handelt sich daher um ein Konstrukt ${ }^{2}$ im psychologischen Sinne. Da Ager Motivation in seinem quantitativen Werkzeug trotzdem messbar machen (operationalisieren) möchte, bricht er sie in drei weitere Konstrukte herunter: Motive, Einstellungen und Ziele.

In seinen Sprachplanungsbeispielen identifiziert Ager unterschiedliche Motive, die die Identität betreffen: Identität (z. B. Bildung der nationalen Identität), Ideologie (z. B. Unterstützung der politischen Ideologie, aber auch die Einbettung der sprachbezogenen Ansichten in jede politische Situation (Ager 2001, 137)), Image (Verbreitung der Vorstellung von eigener Identität nach außen), Unsicherheit (Verteidigung der Identität), Ungerechtigkeit (Kampf gegen Benachteiligung), Integration (in eine andere Gruppe) und Instrumentalität (Sicherung von Vorteilen). Diese Motive ordnet er zu einer Identitätssequenz mit vier Stufen (Ager 2001, 136-142; Zusammenfassung 139):

- Stufe 1

- Bildung der Identität; dabei wird angenommen, dass dieser Prozess gleichermaßen auf Individuen und Gruppen oder Organisa-

2 Zur Definition von Konstrukt s. exemplarisch Dorsch, Häcker und Stapf (1994), Tewes und Wildgrube (1999), Wenninger (2001a) und Manstead und Livingstone (2014, 31). 
tionen zutrifft, obwohl zwischen der persönlichen und sozialen Identität unterschieden werden muss (Ager 2001, 136).

- Entstehung der Ideologie

- Stufe 2

- Verbreitung des Images

- Stufe 3: Sozialer Vergleich (Vergleich mit anderen Gruppen)

- Stufe 4: Als Konsequenz des Vergleichs in Stufe 3 werden entsprechende Handlungen vorgenommen:

- Behalten der Identität

- Verteidigen der Identität

- Behalten der Ungleichheit/Benachteiligung

- Korrektur der Ungleichheit/Benachteiligung

- Integration

- Instrumentalisierung

- Verzweiflung

Mit Edwards (1994, 97) fasst Ager (2001) Einstellungen auf als Dispositionen, gegenüber einer Klasse von Objekten positiv oder negativ zu handeln (Ager 2001, 125). Des Weiteren übernimmt er aus der Sozialpsychologie die Aufteilung der Einstellungen in drei Komponenten: kognitive (Wissen um ein Einstellungsobjekt), affektive (Gefühle, die man mit einem Einstellungsobjekt verbindet) und konative (Verhalten, die man gegenüber einem Einstellungsobjekt zeigt) (s. dazu 4.7). Zwecks Operationalisierung für sein Werkzeug schlägt Ager vor, dass empirische Untersuchungen ven Einstellungen gegenüber der Sprachplanungsmaßnahmen unten genannte Inhalte abfragen. Mit anderen Worten: Das Konstrukt Einstellungen gegenüber Sprachplanungsmaßnahmen mit seinen drei Komponenten wird durch folgende vier

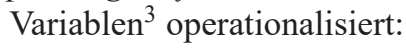

- Kognitive Komponente

3 Unter Variable versteht man ,(...) die messbare Repräsentation eines Konstrukts“ (Manstead und Livingstone 2014, 31). 
- Excellence: Diese Variable erfasst die subjektive Meinung der Probanden, wie gut ein Sprachsystem ist. Die einzelnen Items $^{4}$ der Untersuchung sollen vor allem auf folgende Aspekte eingehen: ästhetischer und literarischer Wert der Sprache sowie Effizienz, Reichtum, Präzision und Klarheit des Sprachsystems. Das vorgeschlagene Werteskala für diese Variable ist unterlegen-überlegen, wobei hier ein Vergleich mit einer anderen Sprache notwendig ist (Ager 2001, 129-130, 134).

- Vitality: Zur Bewertung der Lebendigkeit einer Sprache ist nach Ager zum einen die Art der Domänen, in denen sie benutzt wird, ausschlaggebend. So geht er von drei Domänen aus: privat (Familie/Verwandtschaft, Nachbarschaft) - mittel (z. B. Religionsgruppe, Arbeitsplatz, Geschäftswelt) - öffentlich (Regierung, Verwaltung, Bildung). Zudem nimmt er an, dass der Gebrauch einer Sprache in der jeweiligen Domäne sich auf das Prestige dieser Sprache unterschiedlich auswirkt. Zum anderen ist für die Lebendigkeit einer Sprache die Anzahl der abgedeckten Domänen wichtig. Die Variable vitality erfasst demzufolge das Wissen der Probanden um die Anzahl und die Prestigeträchtigkeit der Domänen, in denen die betreffende Sprache benutzt wird. Das vorgeschlagene Werteskala für diese Variable ist niedriges Prestige-hohes Prestige (Ager 2001, 130-131, 134).

- Affektive Komponente:

- Attractiveness: In dieser Variable werden die Gefühle der Probanden gegenüber einer Sprache erfasst, ohne dass sie einer Begründung bedürfen. Das vorgeschlagene Werteskala für diese Variable ist nicht gefallen-gefallen (Ager 2001, 132, 134).

- Konative Komponente

- Action: Diese Variable erfasst den Wunsch der Probanden, bezüglich der Korpus-, Status- und/oder Erwerbsplanung entweder selbst tätig zu werden, oder Handlungen anderer Personen zu unterstützen. Die Variable gibt insgesamt wieder, wie stark die Probanden der Ansicht sind, dass sich etwas an

4 „Einzelaufgabe oder Einzelfrage einer Testskala“ (Tewes und Wildgrube 1999), z. B. eine Frage in einem Fragebogen. 


\section{Forschungsstand}

der gegebenen Sprachsituation ändern soll. Das vorgeschlagene Werteskala für diese Variable ist entsprechend lax-präskriptiv (Ager 2001, 133, 134).

Erklären die oben beschriebenen Einstellungstheorien das Verhalten in Gruppen, so greift Ager darüber hinaus auf die goal theory zurück, um Handlungsziele von Individuen in seinem Werkzeug zu erfassen (Ager 2001, 9). Demnach lassen sich Ziele nach Abstraktheit grob in drei Klassen aufteilen, wobei sie recht vage erscheinen und die Übergänge fließend sind (Ager 2001, 8-9):

- ideals: Visionen, Intentionen (Beispiel: Frieden und Harmonie),

- objectives: Mission, Zweck (Beispiel: Bildung eines harmonischen Nationalstaates),

- targets: ,point[s] on the way towards the objective“" (Ager 2001, 8); messbar und oft quantifizierbar (Beispiel: Friedensschließung).

Abbildung 2 zeigt, wie die besprochenen drei Konstrukte zu einer Matrix zusammengestellt sind. Die Zeilen beinhalten die Motive aus der Identitätssequenz, während die Spalten die Einstellungsvariablen umfassen. Ager nimmt dabei einen (nicht deterministischen) Zusammenhang zwischen den Einstellungen und dem Identitätsmotiv an (Ager 2001, 144). Die Ziele werden hingegen getrennt erfasst und können auch im Widerspruch zu den anderen beiden Konstrukten stehen (Ager 2001, 144).

Die Einstellungsvariablen werden zudem in L1 und L2 aufgeteilt, wobei L1 für die Erstsprache der betreffenden Individuen bzw. Gruppen und L2 für die damit zu vergleichende(n) Sprache(n) steht. Warum man zur Erfassung der Motivation bei Sprachplanung (mindestens) zwei Sprachen braucht, wird von Ager nicht direkt erläutert. Ich sehe hierfür zwei Faktoren: Zum einen ist, wie Ager angibt, das konkrete Identitätsmotiv Resultat eines sozialen Vergleichs. Dies ist nicht nur für die Motive ab Stufe 3 wahr, sondern gilt auch für die Identitätsbildung, bei der die Abgrenzung von anderen Gruppen eine Rolle spielt (exemplarisch Ager 2001, 137). Zum anderen können hier auch die sprachökologischen Überlegungen, wie in Abschnitt 2.1.5.5 beschrieben, mit einfließen. Die Erfassung von Motivation muss somit durch die Gegenüberstellung der Einstellungen zu L1 und L2 erfolgen.

Ager benutzt diese Matrix, um die im ersten Teil des Buches besprochenen Beispiele zu codieren. Hierfür wird für jede Maßnahme die entsprechende 
Ryan and Giles (1982)

\begin{tabular}{|c|c|c|c|c|c|c|c|c|c|}
\hline \multicolumn{10}{|c|}{ Motivation of individuals (Ryan and Giles) } \\
\hline \multirow[t]{3}{*}{$\begin{array}{l}\text { Identity } \\
\text { sequence }\end{array}$} & \multicolumn{5}{|c|}{ Attitudinal structure } & & & & \multirow[t]{3}{*}{ Ideals/objectives } \\
\hline & \multicolumn{2}{|c|}{ Exc'ence } & \multicolumn{2}{|c|}{ Vitality } & \multicolumn{2}{|c|}{ Attract } & \multicolumn{2}{|c|}{ Action } & \\
\hline & L1 & L2 & L1 & L2 & L1 & L2 & L1 & L2 & \\
\hline $\begin{array}{l}\text { Identity } \\
\text { (personal) }\end{array}$ & & & & & & & & & \\
\hline $\begin{array}{l}\text { Identity } \\
\text { (social) }\end{array}$ & & & & & & & & & . \\
\hline Ideology & & & & & & & & & \\
\hline Image & & & & & & & & & \\
\hline Insecurity & & & & & & & & & \\
\hline $\begin{array}{l}\text { Maintain } \\
\text { identity }\end{array}$ & 2 & & 2 & & 3 & & 3 & & $\begin{array}{l}\text { coherence/improve } \\
\text { status of L1 } \\
\text { coherence/attract } \\
\text { others }\end{array}$ \\
\hline $\begin{array}{l}\text { Defend } \\
\text { identity }\end{array}$ & & & & & & & & & \\
\hline $\begin{array}{l}\text { Maintain } \\
\text { inequality }\end{array}$ & & & & & & & & & \\
\hline $\begin{array}{l}\text { Correct } \\
\text { inequality }\end{array}$ & & & & & & & & & \\
\hline Integrate & & & & & & & & & \\
\hline $\begin{array}{l}\text { Improve } \\
\text { instrument }\end{array}$ & & & & & & & & & \\
\hline Despair & & & & & & & & & \\
\hline
\end{tabular}

Abbildung 2.: Matrix zur Erfassung der Motivationsstruktur der Autoren von Sprachplanungsmaßnahmen (Ager 2001, 150)

Zeile mit dem Identitätsmotiv identifiziert. ${ }^{5}$ Anschließend werden die Einstellungsvariablen zu L1 und L2 behandelt. Da Ager (2001) qualitative Daten zur Verfügung stehen und er vorwiegend mit beschreibenden Beispielen arbeitet, benutzt er für alle Einstellungsskalen die Werte 1-3 (mit 1: schwach ausgeprägt und 3: stark ausgeprägt), ${ }^{6}$ wobei er sie nach eigener Interpretati-

5 Ich fand im Buch keinen expliziten Hinweis darauf, dass immer nur genau eine Zeile ausgewählt werden muss. Dies ist jedoch Agers Handhabung, nur das vermutlich primäre Motiv auszuwählen.

6 Ager $(2001,135)$ behandelt diese als diskrete Werte. 


\section{Forschungsstand}

on der Beispiele vergibt. Zusätzlich zu diesen quantitativen Angaben werden Ziele einer Maßnahme als Freitext, also qualitativ, angegeben.

Zusammenfassend lässt sich sagen, dass man mit Ager (2001) die Phase in der Identitätssequenz, die Spracheinstellungen und die Ziele der Autoren kennen muss, um die Motivation in einer Sprachplanungsmaßnahme zu beschreiben. Eine bestimmte Einstellungsstruktur lässt nicht auf die Ziele bzw. Strategien schließen und Einstellungen lassen nicht die tatsächliche Handlung und ihr Ergebnis vorhersagen (Ager 2001, 141, 198).

\subsubsection{Spolsky $(2004,2009)$}

Spolskys (2004) Beitrag zur Ausarbeitung einer Sprachplanungstheorie liegt in einer knappen Konzeptualisierung des Gebiets und einer Begriffsbestimmung (s. auch Spolsky 2009). Er geht von der Sprachpolitik (language policy) als einer übergreifenden Größe aus, die er selbst aber nur vage und indirekt definiert. Man kann zunächst vermuten, dass sie zunächst im klassischen Sinne als impliziter oder expliziter Regelsatz zum Sprachgebrauch zu sehen ist (Spolsky 2004, 13-15). So verstanden, besteht Sprachpolitik aus drei Komponenten: dem Sprachgebrauch (language practice) ,(...) the habitual pattern of selecting among the varieties that make up its linguistic repertoire (...)“, den Spracheinstellungen (beliefs): „(...) the beliefs about language and language use“ und der Sprachlenkung (language management): „(...) specific efforts to modify or influence that practice by any kind of language intervention, planning or management" (Spolsky 2004, 5; s. auch Spolsky 2009, 4-6; vgl. Shohamy 2006, 49). Dabei wird Sprachlenkung auch definitert als: „(...) the explicit and observable effort by someone or some group that has or claims authority over the participants in the domain to modify their practices or beliefs" (Spolsky 2009, 4). Sprachlenkung entspricht also dem klassischen Begriff von Sprachplanung. Er lehnt lediglich die Bezeichnung language planning ab, da er sie aufgrund der Probleme in der ersten Phase als negativ konnotiert bewertet (Spolsky 2009, 5).

Interessanterweise ordnet Spolsky (2004, 2009) Sprachplanung (in seiner Terminologie: Sprachlenkung) der Sprachpolitik unter. Eine Erklärung hierfür könnte sein, dass er vor allem an der Beschreibung von Sprachsituationen in verschiedenen Domänen interessiert ist, was vor allem in seiner Monographie von 2009 deutlich wird. Die Aufdeckung von Prinzipien, die die Umsetzung von Sprachplanungsmaßnahmen steuern, und dadurch die Sprachplanung als Prozess, treten somit in den Hintergrund. Spolskys Auffassung von Sprachpolitik verstehe ich also weniger als 
Regelsatz, sondern vielmehr als Beschreibung des sprachlichen Istzustands einer Gemeinschaft, wozu insbesondere das Verständnis des tatsächlichen Sprachgebrauchs, der Spracheinstellungen und die Vorstellungen von einem gewünschten Sollzustand (Sprachlenkung) aufschlussreich sein könnten.

\subsubsection{Autoren}

Bei der Entwicklung eines übergreifenden Modells der Sprachlenkung spielt die Frage nach den beteiligten Personen eine wichtige Rolle. In der LPLPLiteratur werden verschiedene (Berufs-)gruppen genannt, die an der Ausarbeitung und der Umsetzung des Sprachplans beteiligt sind: Grammatiker, Schriftsteller, Bildungselite, Linguisten, Journalisten, Politiker, Führungspersonen in einer Gemeinschaft, Berater, Beamte (Haugen 1966, 3; Kaplan und Baldauf 1997, 198; Spolsky 2004, 21). Spolsky (2004) betont aber, dass es auch Sprachplanung (in seiner Terminologie: Sprachpolitik) ohne einen Verantwortlichen geben kann, wenn die sprachlichen Entwicklungen sich selbst überlassen werden (Spolsky 2004, 14). M. E. würde es sich in diesem Fall jedoch um Sprachwandel und nicht Sprachplanung handeln.

Manche Forscher unterscheiden darüber hinaus zwischen den Auftraggebern und den Umsetzern (Edwards 2012, 419), wobei dies m. E. nur für Fragestellung in bestimmten Kontexten wie implizite/explizite Sprachpolitik mit öffentliche/nichtöffentliche Zielen, und insbesondere in Kontexten mit einem Machtgefälle relevant ist.

An die beteiligten Personen werden in der Literatur verschiedene Anforderungen gestellt. Sie müssen zum einen die übergeordneten, z. B. politischen, Ziele berücksichtigen, wissenschaftliche Anforderungen an linguistische Adäquatheit erfüllen und sozial gerecht handeln (Kaplan und Baldauf 1997, 309). Dabei bringen sie unterschiedliches linguistisches Vorwissen mit, was auch mit der Sensibilisierung für die Wechselwirkung von Sprache und Gesellschaft zusammenhängt. Dies kann zu den beschriebenen Umsetzungsproblemen führen (Cooper 1989, 176-177; Spolsky 2004, 35-36; Combs und Penfield 2012, 468). Des Weiteren haben unterschiedliche Personen unterschiedliche Motive, bei den Nichtlinguisten seien es häufig Ideologie, Purismus, Nationalismus und Gruppenzusammenhalt (Haugen 1966, 10-11).

Nur in seltenen Fällen wird die Sprachplanung von einzelnen Personen durchgeführt. Viel häufiger erfolgt die Arbeit in Gruppen, die sich entweder selbst zusammenfinden oder von einer Machtinstanz berufen werden. Im ersten Fall handelt es sich oft und die Bottom-up-, im zweiten um die Top- 


\section{Forschungsstand}

down-Sprachplanung. In der Literatur wird außerdem erwähnt, dass sprachliche Veränderungen auch durch Gruppen herbeigeführt werden können, die sich nicht primär mit der Sprache befassen (ein Beispiel ist die US-Post, beschrieben in Kaplan und Baldauf $(1997,12))$.

Typische Gruppen, die Top-down-Sprachplanung durchführen, werden häufig Sprachakademie genannt, ohne dass der Begriff klar definiert ist. ${ }^{7}$ In der Praxis wird die Bezeichnung sowohl für Gremien mit normativer Wirkung wie Académie Française als auch solche mit beratender Funktion wie beispielsweise die Deutsche Akademie für Sprache und Dichtung verwendet. Eine Sprachakademie kann (muss aber nicht) ein vom Staat beauftragtes und ggf. gefördertes Bildungsinstitut sein. Die Forschung hat diese Vagheit bei der Begriffsbestimmung bzw. die Uneinheitlichkeit in der Wortverwendung erkannt. Edwards (2012) bemerkt beispielsweise, dass manche Gruppen sich Sprachakademien nennen, lediglich um ihrer Tätigkeit einen Anschein an Kompetenz und Wissenschaftlichkeit zu verleihen (Edwards 2012, 420).

Ein entgegengesetztes Phänomen sind die sog. Sprachaktivisten, die häufig als Antwort auf die offizielle Top-down-Sprachplanung im Kontext von Minderheiten agieren: ,(...) we suggest that language activism is energetic action focused on language use in order to create, influence and change existing language policies. In this sense, language activists are individuals or groups who, through various means, actively defend their right to venerate and freely use their languages in multiple, often public domains. Language activism may develop as a reaction to larger, state-imposed efforts to suppress or discourage the use of non-dominant languages" (Combs und Penfield 2012, 462). Bezeichnend für Sprachaktivisten ist, dass sie Mitglieder der betroffenen Sprechergruppe sind und in diesem Sinn die Ideen für die Sprachplanung bottomup entstehen. Combs und Penfield (2012) fügen noch hinzu, dass die meisten Aktivisten nur über eine geringe linguistische Ausbildung verfügen (Combs und Penfield 2012, 468). Diese Form von Sprachplanung erfolgt primär ohne eine offizielle Unterstützung. Eine wichtige Aufgabe von Aktivisten ist mit Spolsky (2009) daher einerseits, die offiziellen Stellen wie staatliche Institutionen für ihre Angelegenheit zu gewinnen. Andererseits müssen häufig auch weitere Sprecher für die Belange der Spracharbeit und der Sprachplanung sensibilisiert werden (Spolsky 2009, 204).

Übersichten über Sprachakademien: Kaplan und Baldauf (1997), Edwards (2012) und Spolsky (2012), speziell zu Académie Française Cooper (1989, 3-11). 
Die Diskussion um die Top-down- und Bottom-up-Ausrichtung der Sprachplanung führte dazu, dass beide Formen als gleich wichtig gesehen werden. Es wird betont, dass eine effektive Sprachplanung nur dann möglich ist, wenn diese Aufteilung aufgehoben ist und alle Beteiligten in einem übergreifenden, demokratischen Prozess einen Beitrag zur Gestaltung der Sprachplanung leisten (Rubin 1983, 330; Tollefson 2011, 376; Combs und Penfield 2012, 471).

\subsubsection{Erfolg}

Die Frage nach dem Erfolg von Sprachplanung wird in der LPLP-Forschung recht unsystematisch behandelt. Bevor ich auf gebietsspezifische und dennoch recht allgemeine Überlegungen zum Erfolg in 2.1.7.1 eingehe, greife ich hier die m. E. relevante Frage nach der Ausrichtung der Forschung im LPLP-Rahmen auf, d. h. ob LPLP präskriptiv oder (lediglich) deskriptiv arbeitet und entsprechend nach Vorgaben für die Planung, Gestaltung und Umsetzung von Maßnahmen sucht, oder eher verschiedene Möglichkeiten ohne Untersuchung auf ihre Eignung beschreibt. Mit anderen Worten, es soll im folgenden Abschnitt erörtert werden, ob die LPLP-Forschung direkt als Hilfestellung bei der Frage nach Erfolgsfaktoren von einzelnen Sprachplanungsmaßnahmen oder allgemein der Sprachplanung angelegt ist oder nicht.

Anhand der in 2.1.2 skizzierten geschichtlichen Perspektive stelle ich fest, dass die Ausrichtung von LPLP im Laufe der Zeit wechselte und parallel zu erwähnten geschichtlichen Entwicklungsphasen zu sehen ist. Folglich war die erste Phase am stärksten normativ, sowohl was die theoretischen Modellbildungen als auch praktische Tätigkeiten betrifft. Sowohl die Theorie als auch die Praxis suchten nach verbindlichen und effizienten Methoden zur Durchführung von Sprachplanungsmaßnahmen in den Entwicklungsländern. Die Probleme aufgrund der unkritischen Vorschreibung von westlichen Vorgehensweisen, die Suche nach elaborierteren und adäquaten Konzeptualisierungen unter Berücksichtigung von sozialen Faktoren, aber auch eine stärkere Einbettung von LPLP in die gewöhnlicherweise deskriptiv angelegte Linguistik führten dazu, dass die Präskription in LPLP eher negativ belegt wurde. Aus diesem Grund wurde in der zweiten Entwicklungsphase die Berücksichtigung einer Vielzahl von unterschiedlichen, außersprachlichen Faktoren zur einzigen Vorschrift, sowohl was die Forschung und Theoriebildung als auch die Praxis betraf. Es handelte sich in der zweiten Phase also nicht um Präskriptivität im engeren Sinne. Erst neuerdings entdeckt man für die Forschung die 


\section{Forschungsstand}

Normativität der Praxis als Gegenstand wieder und wendet sich der Frage zu, was Sprachplanungsmaßnahmen erfolgreich macht (bspw. Edwards 2012).

Normativität und Deskriptivität schließen sich in der Forschung und LPLP als Disziplin keineswegs aus. Mit Kaplan und Baldauf (1997) befindet sich LPLP in einem Spannungsfeld zwischen den beiden Polen (Kaplan und Baldauf 1997, 330). Edwards (2012) bemerkt mit Kaplan und Baldauf(1997), dass auch in der Praxis der Sprachplanung beides zu vereinbaren ist und als Phasen verstanden werden soll. Sprachplaner müssen zunächst deskriptiv arbeiten und den Istzustand erfassen und erst anschließend entsprechende Vorgaben machen, was die neue Sprachform, aber auch die geeignete Umsetzungsmethoden angeht (Edwards 2012, 432).

\subsubsection{Erfolgsfaktoren}

Man könnte erwarten, dass in der Sprachplanung als einer praktischen, zielgerichteten Tätigkeit das Thema Erfolg einen zentralen Platz einnähme. Bisher mangelt es aber an einer umfassenden Darstellung von Faktoren, die eine Maßnahme begünstigen oder erschweren (mit wenigen Ausnahmen wie beispielsweise Spolsky (2004, 222)). Sicherlich ist dieser Umstand mehreren Gründen geschuldet. Zum einen fehlt eine Definition von Erfolg im Kontext von LPLP, zum anderen aber herrscht Unklarheit, wie man Erfolg, sei es mit qualitativen, sei es mit quantitativen, Methoden messen kann. Die Messbarkeit des Erfolgs scheint eng mit dem Vorhandensein von vordefinierten Zielen zusammenzuhängen und ist auch an die Evaluationsmöglichkeiten der Maßnahme gebunden (Grin 2003, 137; Ricento 2009, 227; Spolsky 2011, 22-23). Dieser Aspekt ist wichtig, denn die Erfolgsfrage kann erst an eine Maßnahme dann sinnvoll gestellt werden, wenn im Vorfeld ein Sollzustand explizit definiert wurde. Im Vergleich vom Geplanten und dem tatsächlich Erreichten kann festgestellt werden, ob eine Maßnahme erfolgreich war. So wie die Praxis bestimmte Vorgaben braucht, um Erfolg zu messen, so können in der Theorie nur dann allgemeine Erfolgsfaktoren diskutiert werden, wenn sie über die deskriptive Betrachtung hinausgeht und die präskriptive Perspektive erlaubt. Fehlende Kriterien zur Erfolgsmessung verhindern auch die Entwicklung von Best-Practice-Empfehlungen.

Generell geht man davon aus, dass der Erfolg von Sprachplanungsmaßnahmen schwer zu erzielen ist (Edwards 2012, 436; Żelazny 2012, 154) und eine gut durchdachte Sprachpolitik bedeutet nicht, dass sie beachtet wird (Spolsky 2012, 4-5). Dies kann im Allgemeinen wieder auf die Komplexität des Gebiets zurückgeführt werden, da man viele verschiedene Faktoren, die zum 
Teil nicht in den Händen von Sprachplanern liegen, kontrollieren muss. Im Umkehrschluss ist der erzielte Erfolg oder Misserfolg nicht zwangsläufig nur auf die Planungsmaßnahme zurückführbar (Rubin 1983, 329). Dies ist umso häufiger in demokratischen Gesellschaften der Fall, in denen die individuelle Freiheit stärker verankert ist (Edwards 2012, 421). Ein weiterer Grund für die mangelnde Präzisierung der Erfolgsfaktoren ist die Subjektivität des Erfolgsbegriffs: „However, moving from an existing to a presumably better linguistic environment does entail gains for some and losses for others, and the question arises of who gains, who loses, and how much, as a result of the implementation of langauge policy“ (Grin 2009, 282).

Eine umfassende Auswertung verschiedener Aussagen über den Erfolg der Sprachplanung ist in Kapitel $5 \mathrm{zu}$ finden.

\subsubsection{Aktuelle Forschungsfragen}

Aus den vorangegangenen Überlegungen kann man bereits die aktuellen Forschungsrichtungen ablesen. Für gewöhnlich sind in der Literatur Einzelfallstudien zu Sprachplanungsmaßnahmen stark vertreten. Darüber hinaus werden weitere Gesichtspunkte ausführlicher diskutiert. Ich greife in der folgenden Darstellung hauptsächlich auf die Schwerpunktsystematik sowie einzelne Aufsätze aus dem Sammelband von Hellinger und Pauwels (2009) sowie seiner Einleitung in Pauwels und Hellinger (2009) zurück, wobei ich einzelne Themenschwerpunkte granulärer betrachte sowie einzelne Schwerpunkte aus anderen Quellen hinzufüge:

1. Verbreitung und Dominanz des Englischen

2. Sprache und Macht

3. Sprachminderheiten und sprachliche Diversität

4. Sprachplanung als Regierungsinstrument

5. Sprachpolitik am Arbeitsplatz

6. Autoren und ihre Evaluierung

7. Sprachplanung und neue Medien

Die ersten drei Themenbereiche sind für die aktuelle Entwicklungsphase charakteristisch, stellen aber verschiedene Perspektiven auf ein Phänomen 


\section{Forschungsstand}

dar. Themen 4.-7. lenken den Blick auf spezielle Probleme der Sprachplanung.

\subsubsection{Verbreitung und Dominanz des Englischen}

Die Beschäftigung mit der Verbreitung von Englisch und seinem Aufstieg zum dominanten internationalen Kommunikationsmittel kann man sowohl LPLP wie auch der Sprachwandelforschung zuordnen. In LPLP wird vor allem versucht, eine Sprachpolitik aufzudecken, die zu dieser Verbreitung führte (Spolsky 2004, 76-112). Da man dahinter oft eine implizite Sprachpolitik mit nichtöffentlichen Zielen vermutet (Shohamy 2006, bspw.), wird dieses Thema in manchen Arbeiten in einen kritischen Diskurs eingebettet und Englisch zusammen mit anderen ehemaligen Kolonialsprachen diskutiert. In diesem Kontext stellte Phillipson (1992) die These des sog. Sprachimperialismus auf. Dabei handelt es sich um die Annahme, dass die Sprachplanung auch eine Form von Imperialismus sei und zur Ausbeutung und Benachteiligung der einheimischen Bevölkerung führe (Ricento 2006, 16; Phillipson 1992, vgl. 2006). Obwohl dieses Thema in der LPLP-Forschung zuletzt etwas weniger Beachtung findet, da wie Spolsky (2004) bemerkt, letztendlich nicht die Sprachplanung sondern der Imperialismus an sich das Kernproblem ist (Spolsky 2004, 85), führte die Auseinandersetzung mit den einzelnen Kolonialsprachen zu weiteren Forschungsfragen. Die Frage nach der Verbreitung des Englischen ist in der LPLP-Forschung zwar nicht mehr zentral, dient aber als Grundlage für die sprachökologischen Betrachtungen von anderen Sprachen.

\subsubsection{Sprache und Macht}

Das Thema des sprachlichen Imperialismus wurde auf andere Kontexte übertragen, in denen die Rolle der Sprache und speziell der Sprachplanung bei der Machtausübung betrachtet wird. Dabei befasst man sich mit unterschiedlichen Machtformen auf unterschiedlichen gesellschaftlichen Ebenen, von Alltagsinteraktionen bis zur offiziellen Sprachpolitik (Ricento 2006, 17). Einen speziellen Forschungsschwerpunkt bildet hier die institutionalisierte Machtausübung durch Sprachpolitik: ,Studies in this mould investigate how language practices shape if not define an institution, how ideologies influence institutional discourse in explicit and implicit ways, or how access to institutional language is influenced and sometimes regulated by socioeconomic, cultural and other non-linguistic factors“ (Pauwels und Hellinger 2009, 7). 


\subsubsection{Sprachminderheiten und sprachliche Diversität}

Die Kehrseite der Beschäftigung mit Macht ist die Frage nach dem Schutz von benachteiligen Gruppen. Obwohl dieses Thema alle benachteiligen Gruppen gleichermaßen umfasst (Ager 2001, 158-160), wendet man sich in der Forschung vor allem den ethnischen Minderheiten zu (Ricento 2006, 232). Allerdings ist der Begriff Minderheit nicht klar definiert und bezeichnet nicht nur numerische Minderheiten (Spolsky 2004, 13).

Angelehnt an die Idee der Menschenrechte, die jedem das Recht auf Selbstbestimmung gewähren, wurde in diesem Kontext der Begriff der sprachlichen Menschenrechte eingeführt. Im Allgemeinen wird hier das Recht eines Individuums auf das Erlernen einer bestimmten Sprache und den Gebrauch seiner Muttersprache verstanden (Spolsky 2004, 105; Ricento 2006, 17), obwohl beispielsweise Żelazny (2012) auf die Vagheit des Begriffes hinweist (Żelazny 2012, 169-171). In diesem Zusammenhang werden darüber hinaus weitere Rechte diskutiert, beispielsweise ob der Staat die sprachlichen Menschenrechte lediglich passiv tolerieren soll oder sich auch aktiv an ihrer Durchsetzung beteiligen muss (Skutnabb-Kangas 2009; vgl. Kloss 1998, xxv-xxvi; Spolsky 2004, 110, 115, 119, 130).

Der Beschäftigung mit den Minderheitensprachen und den sprachlichen Menschenrechten liegt darüber hinaus die Einstellung zugrunde, dass sprachliche Diversität einen hohen Wert hat und erhalten werden muss (vgl. Grin 2003, 26, 33; Spolsky 2004, 128).

\subsubsection{Sprachplanung als Regierungsinstrument}

Einen neuen Schwerpunkt findet man in der Literatur, in der die Sprachplanung als ein Instrument der kulturellen und politischen Führung betrachtet wird. In dem sog. Governmentality-Ansatz wendet man sich den öffentlichen Diskussionen um das Thema Sprachplanung/Sprachpolitik zu: „Thus, governmentality shifts attention from the details of policies and their implementation to public debates about policies and the implicit visions of society that are at stake in these debates“" (Tollefson 2011, 370; s. auch Pennycook 2006, 65).

\subsubsection{Sprachpolitik am Arbeitsplatz}

Diesem Schwerpunkt widmen sich beispielsweise Duchêne und Heller (2012). Dabei soll untersucht werden, wie durch eine bestimmte Sprachpolitik verschiedene Unternehmen oder Institutionen den Zugang zu 


\section{Forschungsstand}

Arbeit und Wissen gestalten. Im Fokus stehen vor allem mehrsprachige Individuen und die Benachteiligung, die die Sprecher von Minderheitenoder stigmatisierten Sprachen am Arbeitsplatz erfahren. Schließlich wird auch untersucht, wie man am Arbeitsplatz mehr sprachliche Gerechtigkeit erzielen kann und wie das Potential von Sprechern von seltenen oder stigmatisierten Sprachen erkannt und genutzt werden kann (Duchêne und Heller 2012).

\subsubsection{Autoren und ihre Evaluierung}

In letzter Zeit sind Beiträge erschienen, die sich mit dem historischen Querschnitt über die Tätigkeit verschiedener Autoren von Sprachplanungsmaßnahmen, vor allem der Sprachakademien, beschäftigen (Edwards 2012; s. auch Spolsky 2011). Dabei geht es weniger um eine vollständige diachrone Beschreibung, sondern um die Evaluierung ihrer Arbeit. Die Tendenz kann hierbei in die Richtung gehen, Best-Practice-Empfehlungen zu formulieren und so den Erfolg von Sprachlenkungsmaßnahmen besser voraussagen zu können.

\subsubsection{Sprachplanung und neue Medien}

2009 widmete das Journal Current Issues in Language Planning eine Ausgabe dem Einfluss von neuen Medien auf die Sprachplanung. Liddicoat (2009) identifiziert in diesem Zusammenhang drei Bereiche, die für LPLP relevant sein können: Auswirkung von neuen Medien auf die Sprachökologie [z. B. Verbreitung des Englischen], Auswirkung von neuen Medien auf Sprachsysteme einzelner Sprachen sowie der Einsatz von neuen Medien zur Unterstützung von Sprachplanungsmaßnahmen (Liddicoat 2009, 355; für Letzteres s. auch Pauwels 2012).

\subsection{Plansprachen}

In diesem Teilkapitel beschäftige ich mich mit dem Gebiet Plansprachen. Ich greife dabei weitestgehend die ausgewählten Themenblöcke auf, obwohl ihre Anordnung und inhaltliche Schwerpunktsetzung teilweise anders als im vorangegangenen Teilkapitel ausfallen. Ich steige jedoch wie gehabt mit einer Begriffsbestimmung ein, die ebenfalls aufgrund einer Fülle an Material aber 
auch unterschiedlicher Stellung verschiedener Plansprachentypen in der Forschung erschwert ist.

\subsubsection{Gegenstand}

In diesem Abschnitt beschäftige ich mich mit der Bestimmung von Plansprachen als Forschungsgegenstand. In der Auffassung dieser Arbeit umfassen Plansprachen verschiedene Sprachen wie Esperanto, Interlingua, ${ }^{8}$ aber auch Klingonisch, ${ }^{9}$ Dothraki ${ }^{10}$ oder Lojban. ${ }^{11}$ Stark vereinfachend formuliert könnte es sich bei Plansprachen um solche Sprachen handeln, die auf allen Ebenen des Sprachsystems neu entwickelt werden. Die Literatur befasst sich aber unter dieser Bezeichnung auch mit solchen Phänomenen wie Basic English (Ogden 1968; vgl. D. Blanke 1985, 70-71), das bestimmte Gebrauchsformen einer ethnischen Sprache restringiert, ${ }^{12}$ also de facto eine Kontrollierte Sprache ist. Einen Definitionsversuch erschweren zusätzlich zahlreiche Synonyme wie Kunstsprachen, künstliche Sprachen, Universalsprachen etc., die beim näheren Betrachten bestimmte Schwerpunkte setzen und deshalb oft nur quasi-synonym gebraucht werden (vgl. Duličenko 1989, 49-50; Sakaguchi 1998, 61).

Eine fest umrissene Definition von Plansprachen ist an dieser Stelle nicht möglich. Um sich einer Begriffsbestimmung trotzdem anzunähern, könnte man die Synonyme auf ihre Bedeutung und ihren Gebrauch in der Literatur hin untersuchen. So geht beispielsweise D. Blanke (1985, 51-62) vor (s. auch D. Blanke 1997). Oder man kann eine vorhandene Klassifikation von Plansprachen heranziehen. Je nach gewählter Untersuchungsperspektive können einer Klassifikation verschiedene Kriterien zugrunde gelegt werden:

- semiotische Realisierung: Unterscheidung zwischen rein auf schriftlichen Gebrauch beschränkten Systemen (Pasigraphien) (bspw. Sakagu-

8 Diese Sprache wird in der Literatur auch Interlingua (Gode) oder Interlingua (IALA) bezeichnet, um sie von Interlingua (Peano) zu unterscheiden. In dieser Arbeit verwende ich für die Sprache von Peano die Bezeichnung Latino sine Flexione.

$9 \quad$ Klingonisch wurde für die Star-Trek-Filmreihe entwickelt und umfasste zunächst einzelne Wörter und Sätze. Die Sprache wird nach und nach vom Linguisten Marc Okrand im Auftrag von Paramount Pictures zu einem vollständigen Sprachsystem entwickelt (vgl. Okrent 2009; Okrand u. a. 2011).

10 Dothraki wurde von Martin (1991-) für die Bücherreihe $A$ Song of Ice and Fire erschaffen und umfasste zunächst nur wenige Wörter. Für die Verfilmung als Fernsehserie Game of Thrones wurde es vom Linguisten David J. Peterson im Auftrag von HBO zu einem vollständigen System ausgebaut (Ingsve u. a. 2011).

11 Lojban entstand als Alternative zu Loglan. Zur Geschichte: Okrent (2009, 199-240).

12 Ob es sich bei dieser Art Eingriffen um die Veränderung des Sprachsystems oder lediglich des Sprachgebrauchs handelt, beschäftigt sich beispielsweise Schubert (2013, 55-56). 


\section{Forschungsstand}

chi 1998, 137) und solchen Systemen, die sowohl über die schriftliche als auch die lautliche Ebene verfügen,

- systemlinguistische Nähe zu einer ethnischen Sprache: Unterscheidung zwischen apriorischen und aposteriorischen Plansprachen (s. 2.2.5.1),

- tatsächlich übernommene kommunikative Funktion: Unterscheidung zwischen Sprachprojekten, Semiplansprachen und (vollständigen) Sprachen (D. Blanke 1985, 105-108); (s. 2.2.5.3),

- Konstruktionsmotive und die angedachte Funktion: Welthilfssprachen, philosophische Sprachen und künstlerische Sprachen (vgl. Ray Brown 2006, o.S. Steenbergen 2008, o.S.).

Weitere Klassifikationen sind möglich (bspw. D. Blanke 1985, 109-110; Back 1996, 883-884) und Steenbergen (2008) schlägt eine Kreuzklassifikation vor. Für meine Arbeit entscheide ich mich für die Betrachtung von Plansprachen nach ihren Konstruktionsmotiven. Im Folgenden trage ich Erkenntnisse zusammen, mit denen die Plansprachentypen Welthilfssprachen, philosophische Sprachen und künstlerische Sprachen in der Forschung allgemein charakterisiert werden, ohne dabei ein bestimmtes System ausführlich vorzustellen.

\subsubsection{Welthilfssprachen}

Die erste Gruppe der Plansprachen bilden die sog. Welthilfssprachen. Dabei handelt es sich um Sprachsysteme für Kommunikation zwischen Menschen, die keine gemeinsame Muttersprachen haben. Im Allgemeinen stehen hier also die internationale bzw. interethnische Kommunikation und die Überwindung der Sprachbarrieren und nationalen Grenzen im Vordergrund (Schubert 1989b, 10; Sakaguchi 1998, 36, 287; D. Blanke 2006, 32; Ray Brown 2006, o.S. Steenbergen 2008 , o.S.). ${ }^{13}$ Welthilfssprachen sollen die jeweiligen Muttersprachen nicht ersetzen, sondern lediglich als Zweit-/Fremdsprachen fungieren (League of Nations 1922, 565; Jacob 1948; Schild 1957, 779; Schubert 1989b, 10; Sakaguchi 1998, 282). Zu den bekanntesten Plansprachen dieser Art gehören Volapük, Esperanto oder Ido, wobei in der Literatur konventionell auch Basic English oder Interlingua dazu gezählt werden, ${ }^{14}$ bei deren

13 Zur Diskussion der Begriffe international und interlingual im Kontext der Welthilfssprachen siehe Kimura $(2011,31-32)$.

14 Vergleiche entsprechend: Janton (1978, 8), Large (1985, 167), Schubert (1989b, 10), Okrent (2009, 138142, 200), D. Blanke (und 1985, 174-183), Large $(1985,187)$ und Sakaguchi $(1998,39-40)$. 
Entstehung noch weitere Gesichtspunkte relevant waren (Janton 1978, 13; D. Blanke 1985, 146-149; Sakaguchi 1998, 50, 210; Smith 2011, 42-43).

Das Motiv der internationalen Kommunikation kann man präzisieren. An erster Stelle stehen die pazifistisch-humanistischen Aspekte (D. Blanke 2006, 32, 1985, 69-70) mit solchen Motiven wie Völkerverständigung (D. Blanke 1985, 69, 2004, 1563), internationaler Frieden (Jurkowski 1986, 46) oder Stärkung der internationalen Solidarität (Bormann 1958, 291-293). Hinzu kann man auch die „Demokratisierung der Kultur und Kommunikation" zählen, worunter der direkte Kontakt aller Menschen ohne die Vermittlung der sozialen Eliten verstanden wird (Janton 1978, 26-27; vgl. Fiedler 2006, 75). Das prominenteste Beispiel für diese Motivgruppe ist sicherlich Esperanto (Large 1985, 71), aber auch Volapük (Schleyer 1982, III-IV; vgl. D. Blanke 1985, 206).

Weitere Motive nennt beispielsweise Smith (2011): Zugang zum Wissen, wirtschaftliche Aspekte sowie Missionsunterstützung (Smith 2011, 18). Das erste Motiv aus dieser Gruppe kann man beispielsweise bei Latino sine Flexione oder teilweise auch Interlingua erkennen, wobei sich Smith (2011) vermutlich auch auf ältere Plansprachen und möglicherweise philosophische Sprachen bezieht (siehe 2.2.1.2).

D. Blanke (2006) bemerkt, dass es auch Plansprachen gibt, die zwar für den Einsatz als Welthilfssprache gedacht waren, jedoch primär aus dem Bedürfnis nach Verbesserung bestehender Projekte entstanden (D. Blanke 2006, 55-59; vgl. Smith 2011, 30-31). Darunter fallen Idiom Neutral (als Verbesserung des Volapük), Ido (als Verbesserung des Esperanto), Novial und Interlingua (als allgemeine Verbesserungen bestehender Projekte).

Nicht zuletzt sind bei Welthilfssprachen auch nationalistische Motive zu nennen: Durch eine Welthilfssprache soll eine Kultur verbreitet und ihr Einfluss weltweit gestärkt werden (z. B. Wede, Basic English, teilweise Interlingua: Pei 1966, 432; D. Blanke 1985, 71; vgl. Sakaguchi 1998, 50). ${ }^{15}$

Diese granulare Betrachtung der Konstruktionsmotive ist für ein besseres Verständnis des wissenschaftlichen Diskurses um die Welthilfssprachen notwendig. Wird nämlich allgemein die Verbesserung der internationalen Kommunikation gefordert, stellt sich eine legitime Frage nach einem möglichen Einsatz einer (toten oder lebendigen) ethnischen Sprache, zumal diese über stabilisierende Faktoren wie Sprechergemeinschaft, Kultur und ggf. politi-

15 D. Blanke (1985) nennt noch weitere Motive für die Konstruktion internationaler Plansprachen, beispielsweise das spielerische oder das philosophische. Diese Einordnung ist jedoch nur im Kontext seiner Definition der Interlinguistik und der Plansprachen zu verstehen, von der ich hier abweiche. Eine kurze Diskussion der Interlinguistik und ihres Gegenstandes nach D. Blanke (1985) führe ich in 2.2.3.2. 


\section{Forschungsstand}

sche und wirtschaftliche Bedeutung verfügt (Duličenko 1989, 59-60; vgl. Klaesi 1945, 763-764; Large 1985, 195-196). Ein großer Teil der frühen wissenschaftlichen Literatur zum Thema Welthilfssprachen beschäftigte sich genau mit dieser Frage und erörterte speziell die Notwendigkeit einer Plansprache überhaupt. Setzt man die internationale Kommunikation in den Kontext der humanistisch-pazifistischen Bestrebungen, wird klar, dass man sich von einer Plansprache Neutralität erhoffte (Schuchardt 1904, 47-49; Ölberg 1954, 249). Neutralität bedeutet hier zum einen das Losgelöstsein von einem bestimmten nationalen, kulturellen, politischen oder wirtschaftlichen Kontext. Mit Künzli (2006) soll eine Welthilfssprache kein Machtinstrument sein, sondern „sprachliche Gleichberechtigung“ fördern und bei der Überwindung der „sprachnationalistischen Gefühle“ helfen (Künzli 2006, 41). Eine ethnische Sprache hingegen würde die Muttersprachler, die sich darin freier ausdrücken könnten, bevorzugen und in Konsequenz eine Rangordnung der Völker etablieren (Klaesi 1945, 765; s. auch Jespersen 1930, 149; Mayrhofer 1969, 311-312; Back 1996, 881-882; D. Blanke 2009b, 206).

Zum anderen wurde die Neutralität einer Welthilfssprache im Sinne einer leichten Erlernbarkeit angenommen, da sie für alle gleich leicht bzw. gleich schwierig zu erlernen sei. Dieses würde beruhen auf dem Zusammenspiel von strukturellen Eigenschaften, die in der Literatur als Regelmäßigkeit, Logik, Eindeutigkeit, Präzision etc. bezeichnet werden (Mangold 1970, 129; D. Blanke 1985, 86; Harry 1997, 175-176).

Die so verstandene Neutralität ist eine Idealvorstellung. Allein die Herkunft des Sprachmaterials, aus dem die meisten Welthilfssprachen konstruiert werden (romanische Sprachen), bevorzugt einen bestimmten Sprecher- und Kulturkreis. Auch die Kategorien und die Begriffe sind kulturell geprägt, selbst wenn die starke Sapir-Whorf-Hypothese in der Linguistik nicht mehr vertreten wird (vgl. Penn 1972; Grimshaw 1987, 9). Schließlich ist auch die Frage nach einer objektiv leichten Erlernbarkeit einer Sprache weder im Kontext der Welthilfssprachen ${ }^{16}$ noch im allgemeineren Kontext des Zweit-/Fremdsprachenerwerbs eindeutig geklärt (für Letzteres s. exemplarisch Kurtz 2005).

Aus den genannten recht abstrakten Konstruktionsmotiven ergeben sich für Welthilfssprachen unmittelbar unterschiedliche Einsatzmöglichkeiten. Beispielsweise wurde Esperanto zunächst als Mittel der persönlichen oder fachlichen Kommunikation verwendet (D. Blanke 1985, 290-292). Darüber

16 S. dazu Pei (1966, 89), Large (1985, 190-191), Back (zum Ansatz der Systemgüte: 1979), D. Blanke (vgl. 1985, 77-78), D. Maxwell (1989) und Sakaguchi (1998, 147-151). 
hinaus kamen durch den Gebrauch und allgemeine Auseinandersetzungen mit der Sprache weitere Möglichkeiten hinzu, die bei der Konstruktion vermutlich nicht mit bedacht waren. So wurde im Zuge der Überprüfung der Unterrichtssituation für Esperanto durch den Völkerbund in den 1920er Jahren festgestellt, dass es sich aufgrund seiner Regelmäßigkeit als Fremdsprachenpropädeutikum gut eignet (League of Nations 1922, 568-571; vgl. D. Blanke 1998, 60; Künzli 2006, 45; Fiedler 2011a, 83-84). Des Weiteren testete man in den 1980er Jahren den Einsatz von Esperanto als Relais-Sprache für maschinelle Übersetzung (Sadler 1989, 1997; Schubert 1997).

Neben dieser Erweiterung der Einsatzdomänen erfuhren manche Projekte eine Schwerpunktverschiebung wie beispielsweise Interlingua, die sich im Laufe der Entwicklung auf die internationale Wissenschaftskommunikation spezialisierte (D. Blanke 1985, 182). Interesse der Sprecher an wiederum anderen Projekten war nie im Gebrauch als Welthilfssprache begründet. So ist beispielsweise Blissymbolics (früher auch: Semantographie, Bliss 1949) zwar von ihrem Autor als Welthilfssprache (mit philosophischen Eigenschaften) intendiert (Okrent 2009, 162; Maradan 2012, 61), fand aber erst Einsatz als Sprache für die unterstützte Kommunikation (Okrent 2009, 151-196; Maradan 2012, 62). Auch bei Esperanto verändert sich die Gewichtung der Motive, die die Menschen zum Erlernen und Gebrauchen bewegen (Sakaguchi 1998, 48; Fiedler 2006, 75).

\subsubsection{Philosophische Sprachen}

Die zweite große Gruppe der Plansprachen bilden die sog. philosophischen Sprachen. Im Vordergrund stehen hier nicht die internationale und interkulturelle Kommunikation, sondern die Wechselbeziehung zwischen Sprache und Denken und die Möglichkeit, diese Beziehung durch den Eingriff in die Sprache aktiv zu gestalten (Sakaguchi 1998, 102; Okrent 2009, 73, 201). ${ }^{17}$ Philosophische Sprachen entstehen bis heute - als Beispiele können Ithkuil und Toki Pona genannt werden (Ray Brown 2006). ${ }^{18}$ Einen wichtigen Entwicklungs-

17 Steenbergen (2008) benutzt die Bezeichnung Filosofische talen in einem anderen Sinn. Er versteht darunter Sprachen, die an eine bestimmte Philosophie gebunden sind, was eine unscharfe Erläuterung darstellt. Der Oberbegriff der Conceptuele talen entspricht weitestgehend den engelangs von Ray Brown (2006), wobei auch hier die Definition recht vage ist: ,(...) aan deze talen ligt een bepaald concept ten grondslag, dat van groter gewicht is dan manier waarop de taal uiteindelijk wordt gebruikt. Anders gezegd: het onderliggende concept is gelijktijdig het primaire doel van de taal" (Steenbergen 2008, o.S.). Bei der Auffassung von philosophischen Sprachen weiche ich deshalb von der Plansprachenklassifikation der genannten Autoren am stärksten ab.

18 Zu Toki Pona siehe: Kisa (2001-) und Blahuš (vgl. 2011). 


\section{Forschungsstand}

schub erfuhren sie jedoch im 17./18. Jahrhundert im Zusammenhang mit der Rationalisierung der Wissenschaft, neuen geographischen Entdeckungen und kaufmännischen Beziehungen (Large 1985, 14; vgl. Jermołowicz 2003, 51, 53). Die ethnischen Sprachen schienen diesen neuen Anforderungen nicht gewachsen zu sein, deshalb bemühte man sich ,(...) um die Entwicklung einer logisch-philosophischen Sprache, deren Regeln mit den Regeln des Denkens zusammenfallen sollten und die daher, von allen Mängeln, Widersprüchen und Mehrdeutigkeiten der historisch gewachsenen Sprachen befreit, leicht verstehbar sein und sich vorzüglich als universelles Instrument zur Mitteilung wissenschaftlicher Erkenntnisse eignen sollte" (Sakaguchi 1998, 101; vgl. Janton 1978, 4).

Dieses Zitat verdeutlicht, dass auch bei philosophischen Sprachen verschiedene abgeleitete Konstruktionsmotive identifiziert werden können (vgl. Sakaguchi 1998, 102; Oostendorp 2001, 210). Ich fasse diese zu drei Hauptgruppen zusammen: Je nach der vertretenen Sprachauffassung und dem geschichtlichen Kontext betreffen die Motive den Denkprozess, den Kommunikationsprozess oder die Schnittstelle, wobei der Schwerpunkt auf der Entwicklung einer optimalen Ausdrucksseite des sprachlichen Zeichens liegt: ${ }^{19}$

- Denkprozess: Sprache soll logisches Denken unterstützen, da die Ausdrucksseite des sprachlichen Zeichens als symbolischer Platzhalter für Begriffe oder komplexe Argumentationsketten den Denkprozess kognitiv entlastet. Hierfür ist ein eineindeutiges Zeichen notwendig. Dieses Motiv für philosophische Sprachen kann man beispielsweise an der Sprachauffassung von Leibniz erkennen (vgl. Święczkowska 2012).

- Kommunikationsprozess: Damit jedes Individuum seine Gedanken präzise ausdrücken, aber auch Mitteilungen anderer unmissverständlich empfangen kann, ist ebenfalls eine möglichst eineindeutige Beziehung zwischen dem Inhalt und der Form des sprachlichen Zeichens notwendig (vgl. Okrent 2009, 29).

- Schnittstelle: Sprache soll noch effizienter als Mittel der Erkenntnis eingesetzt werden. Insbesondere soll man bereits anhand der Ausdrucksseite auf den Begriff schließen können. Diese strengere Form der Eineindeutigkeit beruhte auf Kombinatorik: Ist ein Begriff

19 Loglan und später auch Lojban gehen einen Schritt weiter und binden die Eindeutigkeit auch in die Syntax ein (s. dazu Okrent 2009, 235-236). 
komplex, d.h. besteht er aus einfacheren Begriffen, soll sich dessen Ausdrucksseite aus den Ausdrucksseiten der einfacheren Zeichen kombinatorisch aufbauen lassen. ${ }^{20}$

Als vierte Gruppe von philosophischen Sprachen können diejenigen betrachtet werden, die in erster Linie zur Überprüfung einer Forschungshypothese über die Wechselbeziehung zwischen Sprache und Denken entworfen werden. Das Beispiel ist hier Loglan, das die experimentelle Überprüfung der Sapir-Whorf-Hypothese ermöglichen sollte (D. Blanke 1985, 137-139; Sakaguchi 1998, 39; vgl. Okrent 2009, 205-210). Eine weitere Sprache dieser Art ist z. B. gjâ-zym-byn (Ray Brown 2006, o.S.). Ray Brown (2006) und Steenbergen (2008) sehen diese Gruppe im größeren Kontext der sog. experimentellen Sprachen, auf die ich in 2.2.1.4 eingehe.

Viele der Versuche zur Entwicklung einer philosophischen Sprache gingen nicht über allgemeine Vorüberlegungen zu Wesen und Funktionsweise hinaus (z. B. bei Descartes oder Leibniz, s. dazu Large 1985, 19, 30, 40). Konkret umgesetzt wurden sie oft mithilfe von Klassifikationstafeln, die das Gesamtwissen der Zeit systematisiert abbildeten (Sakaguchi 1998, 99). Die wohl bekanntesten Sprachen dieser Art stammen von Dalgarno (1661) und Wilkins (1668). ${ }^{21}$ Da diese Realisierung am weitesten verbreitet war, werden philosophische Sprachen in der Literatur oft mit Klassifikationssprachen identifiziert (z. B. D. Blanke 1985, 125; Large 1985, 21; Smith 2011, 23), was aber wichtige Sprachen wie Lojban ausklammert. Auf dieser eingeengten Sicht des Gegenstandes beruht auch die häufigste Kritik an philosophischen Sprachen, die auf die Grenzen eines Klassifikationssystems eingeht. Neben den inhaltlichen Fehlern (Large 1985, 39), die teilweise auf ein übergeordnetes Problem des aktuellen Wissensstands zurückführbar sind (vgl. D. Blanke 1985, 131; Eco 1995, 255), wird darauf hingewiesen, dass es keine objektive Sicht auf die Dinge geben kann und sie deshalb in einem Klassifikationssystem nicht eindeutig ablegbar sind (Okrent 2009, 43-45, 58-60; vgl. Large 1985, 182183). Eine Mehrfachklassifikation würde aber im Widerspruch zur Annahme stehen, dass Begriffe objektiv und eindeutig sind.

Ein weiterer Kritikpunkt betrifft ihren formalen Aufbau. Da sie die Redundanz, mangelnde Präzision oder aber die kulturelle Einbettung vorhandener

20 Dieses Aufbauprinzip sieht man beispielsweise bei Urquhart (Jurkowski 1986, 25; Okrent 2009, 27-29), Dalgarno (1661), Wilkins (1668), Descartes (Large 1985, 30; Jermołowicz 2003, 55), oder bei Leibniz (Święczkowska 2012, 54; vgl. Couturat und Leau 1903, 23-28; vgl. Large 1985, 39-40; vgl. Jermołowicz 2003, 55); zum Aufbauprinzip allgemein: Sakaguchi (1998, 107-108) und Okrent (2009, 30-34).

21 Für eine ausführliche Beschreibung beider Systeme siehe Large (1985, 27-39), Okrent (2009, 21-75) und Strasser (2012). 


\section{Forschungsstand}

Sprachen korrigieren wollen, wird oft ein möglichst großer Abstand zum vorhandenen Sprachmaterial gewählt (Sakaguchi 1998, 104-105). So haben viele der realisierten Sprachen eine apriorische Form (s. 2.2.5.1). Diese führe aber zur kognitiven Überforderung beim Erlernen und Gebrauch dieser Sprachen, weshalb ihre kommunikative Tauglichkeit ganz in Frage gestellt wird (Couturat und Leau 1903, 27-28; D. Blanke 1985, 131; Sakaguchi 1998, 108-109). In der Literatur werden apriorische und philosophische Sprachen oft gleichgesetzt (Tonkin 1997b, 74; Schubert 2011b, 50; vgl. Fiedler 2006, 68), obwohl die Überlappung beider Gruppen nicht vollständig ist (Oostendorp 2001, 210; vgl. Sakaguchi 1998, 129). ${ }^{22}$

Werden philosophische Sprachen jedoch wie hier funktional definiert, so müssen auch die sog. formalen Sprachen als Untertyp gesehen werden. Bei formalen Sprachen handelt sich um ,(...) prädikatenlogische Notationen, semantische Formalismen, Symbolsysteme aus logischen Einheiten und Operationen (...)“, wobei das Gebiet recht vage umrissen ist (Schubert 1997, 124; vgl. Sakaguchi 1998, 26). Diese Sprachen können der Mensch-Mensch-Kommunikation dienen und sind dann auf einen Fachgebrauch beschränkt.

In 2.2.1.1 ging ich bereits darauf ein, dass man bei einigen Sprachen, die gewöhnlicherweise zu den Welthilfssprachen gezählt werden, auch ,,philosophische" Motive im Sinne dieses Abschnitts feststellen kann. Auch umgekehrt erwogen einige Autoren von philosophischen Sprachen durchaus den Einsatz ihrer Projekte als Mittel der internationalen Kommunikation (s. dazu Vorwort in: Wilkins 1668). Man kann aber mit Couturat und Leau (1903) konstatieren, dass hierbei der internationale Gebrauch den philosophischen Gesichtspunkten untergeordnet war (Couturat und Leau 1903, 23).

\subsubsection{Künstlerische Sprachen}

Eine weitere Gruppe bilden Plansprachen, die ich hier als künstlerische Sprachen bezeichne. Während es bei den bereits beschriebenen Plansprachentypen vor allem um die Lösung eines gesellschaftlichen Problems im weitesten Sinne geht, handelt sich bei künstlerischen Sprachen um Plansprachen, die aus ästhetischen Motiven entstehen (Ray Brown 2006, o.S. Steenbergen 2008, o.S. Okrent 2009, 282; Okrand u. a. 2011, 115; Weiner und Marshall 2011, 96). Die künstlerischen Sprachen werden also nicht dafür konzipiert, in einer realen Kommunikation eingesetzt zu werden, sondern um eine künstlerische Vision des Autors umzusetzen (Okrent 2009, 282; vgl. Fiedler 2011b, 11;

22 Ein Beispiel einer apriorischen nichtphilosophischen Sprache ist Solresol (vgl. Large 1985, 61; Sakaguchi 1998, 129-131). 
vgl. Okrand u. a. 2011, 127). Auch hier kann man abgeleitete Motive feststellen, die eine granulare Klassifikation von künstlerischen Sprachen erlauben (vgl. Steenbergen 2008, o.S.):

- fiktionale Sprachen: Sie werden in literarischen Werken eingesetzt und von ihren Protagonisten gebraucht. Sie sollen die erschaffene Welt glaubhaft machen oder andere stilistische Effekte erzielen. Die Rezipienten des literarischen Werkes sollen die Sprache nicht nur nicht erlernen, sondern sie oft gar nicht verstehen, damit ein Verfremdungseffekt entsteht (Jackson 2011, 67; Weiner und Marshall 2011, 80; vgl. Okrand u. a. 2011, 113). Zu den bekanntesten fiktionalen Sprachen gehören Quenya, Sindarin oder andere Sprachen von J. R. R. Tolkien (vgl. Weiner und Marshall 2011), Newspeak von G. Orwell (vgl. Fiedler 2011b, 18-23; vgl. Jackson 2011), Nadsat von A. Burgess (vgl. Jackson 2011), Klingonisch (vgl. Okrent 2009; Okrand u. a. 2011) sowie Dothraki (Ingsve u. a. 2011). Von allen künstlerischen Sprachen werden die fiktionalen Sprachen in der deutschsprachigen Literatur am häufigsten erwähnt. Beispielsweise geht Fiedler (2011b) auf die Parallelen zwischen den Welthilfssprachen und den fiktionalen Sprachen ein (Fiedler 2011b, 10-14).

- persönliche Sprachen: Sprachen, die aus reiner Freude an Spracherfindung entstehen und dabei aber eine bestimmte ästhetische Vision von Sprache und Kommunikation des Autors umsetzen. Sie sollen nicht von anderen Menschen erlernt werden, sondern sind höchstens zum persönlichen Gebrauch des Autors gedacht (Steenbergen 2008, o.S.). Persönliche Sprachen entsprechen also weitestgehend dem spielerischen Motiv von D. Blanke (1985). Dazu könnte die Sprache Spocanien gezählt werden (Oostendorp 2001, 216-218). Okrent (2009) beschreibt auch eine recht rege Bewegung der Plansprachenerfinder im Internet, insbesondere in der Conlang-Mailingliste (1991, 1998), wo das rein spielerische Motiv besonders stark ausgeprägt sei. Interessanterweise werden dort von vielen Autoren fiktionale Welten und Kulturen erschaffen, in die diese Plansprachen eingebettet werden, um dem gesamten Sprachprojekt einen festeren Stand zu geben (Okrent 2009, 287). Dies stellt also gegenüber den fiktionalen Sprachen eine umgekehrte Entwicklungsrichtung dar. 


\section{Forschungsstand}

- Die Klassifikation von Steenbergen (2008) umfasst noch satirische, alternative und mikronationale Plansprachen, auf die ich hier jedoch nicht näher angehe.

\subsubsection{Andere Plansprachen}

Neben den genannten Gruppen gibt es weitere plansprachliche Phänomene.

Wie in 2.2.1.2 erwähnt, schlagen Ray Brown (2006) und Steenbergen (2008) die Kategorie der experimentellen Sprachen vor: „Dit zijn in feite alle talen, die op een onderliggend concept zijn gebaseerd en niet kunnen worden gerekend tot de logische of de filosofische talen" (Steenbergen 2008, o.S.). Bei Steenbergen (2008) bilden sie zusammen mit den erwähnten Filosofische talen und den Experimentele talen die Kategorie der Conceptuele talen, während sie bei Ray Brown (2006) unter den engelangs zu finden sind. Wie angemerkt sind sie im Sprache-Denken-Paradigma stark verankert. Ray Brown (2006) bemerkt jedoch, dass diese Kategorie auch weitere Phänomene umfasst, mit denen andersartige Hypothesen überprüft werden sollen. $^{23}$

Auf eine weitere interessante Gruppe von Plansprachen macht darüber hinaus Portnow (2011) aufmerksam. Er beschreibt Plansprachen, die speziell für den Einsatz in Computerspielen entworfen sind. Als Beispiele nennt er Gargish aus der Spielreihe Ultima (speziell: Ultima VI), D'ni aus der Myst-Serie, Simlish aus der The-Sims-Serie, Al Bhed für Final Fantasy 10 sowie Logos für Tabula Rasa. Diese Sprachen sollen einerseits das Spiel ästhetisch unterstützen und glaubwürdig machen sowie das Spielerlebnis steigern. Andererseits sind sie durchaus zum Erlernen gedacht. ${ }^{24}$ Die Kenntnisse und der Gebrauch dieser Sprachen seien für das Spiel zwar nicht entscheidend, aber der Spieler könne sich dadurch Zugang zu zusätzlichen Informationen, Spielmöglichkeiten oder anderen Vorteilen verschaffen (Portnow 2011, 138). Somit stellt sich für ihre Autoren von Anfang an auch die Frage, wie sie diese Sprachen attraktiv gestalten können, so dass der potentielle Nutzer (bzw. Sprecher) erreicht werden kann (Portnow 2011, 137-140).

23 In der Sprache AllNoun gibt es beispielsweise nur Nomen als Wortart (Breton 1995a,b; vgl. Ray Brown 2006, o.S.). Das primäre Motiv war zunächst ein sprachliches, das sich aber zu einem breiteren aber vage definierten kommunikativen entwickelte: „Originally it was an experiment to see if language could be made that simple. Since it has been largely technically successful, it's [sic] purpose now is to support any advantages that can be obtained with a maximally simple grammar" (Breton 1995a, o.S. vgl. Ray Brown 2006, o.S.).

24 Dies trifft nicht auf alle der genannten Sprachen zu, da beispielsweise Gargish und Simlish vom Spieler nicht produktiv eingesetzt werden können. 
Schließlich sollen auch die Plansprachen für interstellare Kommunikation mit anderen Lebensformen erwähnt werden (Jurkowski 1986, 130-141). Der bekannteste Vertreter ist Lincos, der durch seinen prädikatenlogischen Aufbau an die philosophischen Sprachen erinnert und ein interessantes Beispiel für die didaktische Aufbereitung eines Sprachsystem ist (Freudenthal 1960; vgl. Jurkowski 1986, 139-141; Eco 1995, 308-310; Okrent 2009, 212-213).

\subsubsection{Geschichte}

Übergreifenden Untersuchungen von Plansprachen werden oft aus einer geschichtlichen Perspektive vorgenommen (bspw. Large 1985; Okrent 2009). Der soziohistorische Hintergrund ist aber nicht nur als systematisierender Faktor zu sehen, sondern kann für soziolinguistisches Verständnis aufschlussreich sein: „The way people think about language is influenced by the times they live in, and it is possible to show how changing times led, in a general way, to changes in the types of languages that inventors came up with. (...) [A]nd so, in a way, the history of invented languages is a story about the way we think about language" (Okrent 2009, 17).

Es ist im Rahmen dieser Arbeit nicht möglich, die Geschichte des Gegenstandes detailliert darzulegen, zumal Large (1985) dieses Thema ausführlich behandelte. Der historische Querschnitt der Entwicklungstendenzen verschiedener Plansprachentypen lässt sich wie folgt zusammenfassen (vgl. D. Blanke 1985, 123-124):

- Bis in das Mittelalter hinein war aus (west-)europäischer Sicht die Beschäftigung mit Welthilfssprachen nicht notwendig, da Latein als lingua franca fungierte.

- Im 17. Jh. bringt die Neuaufstellung der Wissenschaft philosophische Sprachen hervor; neue wirtschaftliche, kulturelle und kirchliche Beziehungen lösen eine Beschäftigung mit Welthilfssprachen, insbesondere der Universalschrift, aus.

- Im 18. Jh. tritt durch Nationalismus bedingt die Beschäftigung mit beiden Plansprachentypen in den Hintergrund.

- Im 19. Jh. verstärkt sich der internationale Austausch, was zur Entstehung vieler Welthilfssprachen und einer regen Welthilfssprachenbewegung um die Jahrhundertwende führt. 


\section{Forschungsstand}

- In der ersten Hälfte des 20. Jh. entstehen weiterhin Welthilfssprachen; ihre Anhänger versuchen, die Überlegenheit des eigenen Projekts zu zeigen. Zudem setzen sie sich für die offizielle Verankerung ihrer Projekte an Schulen und in internationalen Organisationen ein; auch das Interesse an philosophischen Sprachen erwacht. Schließlich setzt sich Englisch als internationale Sprache durch; Plansprachen werden in 1990er Jahren im Internet aufgegriffen, es handelt sich aber hauptsächlich um künstlerische Sprachen.

Im Folgenden gehe ich ausführlicher auf die geschichtlichen Entwicklungstendenzen der philosophischen und Welthilfssprachen ein.

\subsubsection{Philosophische Sprachen}

Eine systematische Beschäftigung mit Plansprachen als Mittel zur Unterstützung des Denkens und des präzisen Ausdrucks beginnt im 17. Jh., obwohl sich manche Autoren bereits früher mit dem Thema beschäftigten (z. B. Lullus, 13./14. Jh., vgl. Jurkowski 1986, 17; Eco 1995, 53-72). Dies war die Folge einer Neudefinition von Wissenschaft, die auf Systematik, Empirie und Experiment beruhen und die Scholastik und Spekulation verdrängen sollte (Large 1985, 4; Jermołowicz 2003, 51; vgl. D. Blanke 1985, 123; Eco 1995, 209210). Man ging davon aus, dass sich die so gewonnenen Erkenntnisse logisch organisieren und universell eindeutig darstellen ließen (Jermołowicz $2003,51)$ und nahm zudem an, dass die Begriffe und Gedanken an sich präzise seien und erst durch die Sprache verzerrt werden (Okrent 2009, 29). Eine philosophische Sprache wurde deshalb als ein wichtiger Faktor für den wissenschaftlichen Fortschritt gesehen (Jermołowicz 2003, 53). Mit dieser Entwicklungsphase sind solche Namen wie Bacon, Descartes, Dalgarno, Wilkins, Leibniz und Lodwick verbunden.

Wie Large (1985) beschreibt, verschwand Ende des 17. Jh. das Interesse an philosophischen Sprachen beinahe vollständig, da ihre Schwächen und Probleme erkannt wurden und sich bis dahin kein Projekt durchsetzen konnte. Zwar wurde das Thema Ende des 18. Jh. für einige Zeit wieder aufgegriffen, da man die unveröffentlichten Schriften von Leibniz entdeckte und erneut am Potential einer philosophischen Sprache zur Wissensvermittlung interessiert war. Aber das Interesse ließ aus beinahe denselben Gründen nach und die Beschäftigung mit philosophischen Sprachen wurde insgesamt als utopisch und unwissenschaftlich betrachtet (Large 1985, 42, 45-51). 
Im 20. Jh, insbesondere in der Zeit 1930-1950, befasste man sich erneut mit der Wechselbeziehung zwischen Sprache und Denken und speziell mit der Annahme, dass Sprache die wahren Gedanken verschleiere und als Mittel politischer oder medialer Propaganda ein gefährliches Potential berge (Okrent 2009, 139, 199). In diesem Kontext fand auch die Sapir-Whorf-Hypothese des sprachlichen Determinismus einen fruchtbaren Boden (Okrent 2009, 203 206). Umgekehrt wurde angenommen, dass man durch die Kontrolle der eigenen Sprache auch die Kontrolle über die eigenen Gedanken zurückgewinnen und so andere Probleme lösen kann (Penn 1972, 31-32; Okrent 2009, 201). Viele Sprachen, die in dieser Zeit entstanden, wie Basic English, Blissymbolics aber auch Interlingua, haben eine wesentliche philosophische Komponente, wenngleich sie nicht unbedingt als philosophische Sprachen klassifiziert werden (vgl. Okrent 2009, 139, 162).

\subsubsection{Welthilfssprachen}

Die Vorstellung, dass einst eine für alle Menschen verständliche Sprache existierte, die aber im Laufe der Zeit verloren ging, hat in verschiedenen Kulturen und Religionen eine lange Tradition (Large 1985, 3; Jurkowski 1986, 15-16; Adams 2011b, 4). Ähnlich wie bei den philosophischen Sprachen beginnt eine systematische Beschäftigung mit den Welthilfssprachen im 17. Jh. Obwohl in der Literatur auch von der Überwindung des „Turms zu Babel“ gesprochen wird (Adams 2011b, 4), waren die Beweggründe im 17. Jh. eher pragmatisch in den Bedürfnissen der Kaufleute, Missionare oder Wissenschaftler verwurzelt (Large 1985, 6-7, 15). Die Renaissance brachte nämlich nicht nur ein neues Verständnis von Wissenschaft, sondern auch neue Handelsbeziehungen und verstärkte Mission außerhalb Europas (Large 1985, 5-10; vgl. Sakaguchi 1998, 101-102). War Latein bis dahin noch die europäische lingua franca, so verlor sie zunehmend aus verschiedenen Gründen an Bedeutung und war zudem für Kommunikation mit Menschen, die die griechisch-römische Tradition nicht teilten, weniger geeignet (Large 1985, 5-8).

In der ersten Phase der Findung einer Welthilfssprache war man von der Idee des real character, einer Universalschrift, fasziniert, was aber auf einem naiven Verständnis der chinesischen Schriftzeichen und der bis dahin nicht entschlüsselten Hieroglyphen beruhte (Large 1985, 11-14). Okrent (2009) beschreibt real character wie folgt: „This was the term used by the philosopher Francis Bacon to describe Chinese writing - it was ,real“ in that the symbols represented not sounds, or words, but ideas. (...) They [missionaries] got the impression that Chinese characters bypassed language entirely, and 


\section{Forschungsstand}

went right to the heart of the matter. This impression was mistaken (...), but it encouraged a general optimistic excitement about the possibility of a universal character" (Okrent 2009, 46). Auch die Autoren von philosophischen Sprachen waren teilweise davon inspiriert (vgl. Wilkins 1668).

Im 18. Jh. bremste der verstärkte Nationalismus sowie der Aufstieg des Französischen zur Sprache der Diplomatie die Arbeiten an einer Welthilfssprache (Large 1985, 58-59, 43-45; Okrent 2009, 93), obwohl einzelne Projekte wie von Delormel weiterhin entstanden (Couturat und Leau 1903, 29 32; Okrent 2009, 79-80, 299: Liste der Projekte).

Wie Large (1985) zusammenfasst, brachte das 19. Jh. neue Impulse. Die Lebensqualität in Europa erhöhte sich und eine breitere Bevölkerungsgruppe erhielt Zugang zur Bildung. Der technische Fortschritt brachte neue Kommumnikations- und Fortbewegungsmöglichkeiten, neue internationale Organisationen entstanden. Aufgrund modernerer und effizienterer Produktion stieg die Bedeutung des internationalen Absatzmarktes (Large 1985, 87-88; vgl. Schleyer 1982, iii-iv). Die günstigen Voraussetzungen für den internationalen Austausch waren also gegeben, ein geeignetes Kommunikationsmittel fehlte jedoch. Insgesamt herrschte in der Sprachenfrage eine Aufbruchstimmung und die Zuversicht, dass eine geeignete Plansprache als Lösung des Kommunikationsproblems bald gefunden würde (Large 1985, 88). Dennoch weist Large (1985) darauf hin, dass das Nationale nach wie vor eine wichtige Identitätskomponente ausmachte und der Nationalismus in Europa stark ausgeprägt war (Large 1985, 88-89). In dieser Konstellation gelang einigen Welthilfssprachen, insbesondere Esperanto, eine verhältnismäßig rasche und weite Verbreitung. Erhebliche Schwierigkeiten stellten aber die offizielle Verankerung in einer internationalen Organisation und die Einführung als schulisches Unterrichtsfach dar. Wie bespielsweise Smith (2011) bemerkt, besserte sich unmittelbar nach den Weltkriegen kurzfristig die Situation, da die internationale Verständigung und Aussöhnung auch politisch gesucht wurden (Smith 2011, 37). Die Esperantobewegung startete jeweils aktive Kampagnen entsprechend beim Völkerbund (vgl. beispielsweise League of Nations 1922; Lapenna 1970; Large 1985, 98-99; Eichner 2012, 130-131; Lins 2012, 95-96) und den Vereinigten Nationen (UNESCO) (vgl. beispielsweise Hamburger 1954, 775-776; Schild 1957, 783; Lapenna 1971; Large 1985, 99-100; Lins 2012, 112-113), die aber nur vom bedingten 
Erfolg waren. ${ }^{25}$ Auch im Rahmen der EU ist Esperanto bis heute wenig erfolgreich (D. Blanke 2009b, 225).

In der 2. Hälfte des 20. Jh. entwickelte sich Englisch aufgrund seiner politischen, wirtschaftlichen und kulturellen Stellung zur de facto internationalen Sprache (Back 1996, 883; Künzli 2006, 43; Okrent 2009, 136-137; Smith 2011, 37). Auch der Zerfall der Sowjetunion wirkte dieser Entwicklung nicht entgegen, obwohl manche darin eine Hoffnung für die Welthilfssprachen sahen (Lins 2012, 119). Dies führte zur Verschiebung des Interessenschwerpunkts, da die Welthilfssprachen nicht mehr als Lösung des Kommunikationsproblems, sondern als ,linguistische Tatsachen“(D. Blanke 1985, 13-14), unabhängig vom verfehlten Ziel als internationale Sprache, untersucht werden. Die kreative Phase der Welthilfssprachen wird also vorerst als abgeschlossen betrachtet (Künzli 2006, 56).

\subsubsection{Erforschung von Plansprachen als Disziplin}

Der Gegenstand Plansprachen umfasst offensichtlich eine Reihe unterschiedlicher Phänomene. Es stellt sich nun die Frage, ob er in seiner vollen Breite in einer wissenschaftlichen Disziplin untersucht wird, und was seine Stellung in der Linguistik ist.

\subsubsection{Geschichtliche Grundzüge der wissenschaftlichen Beschäftigung}

Die geschichtlichen Beschreibungen in 2.2.2 fokussieren die Interessen von Plansprachenautoren. Parallel dazu kann man auch die Entwicklung des wissenschaftlichen Interesses an Plansprachen betrachten. Beide Perspektiven sind nicht immer voneinander zu trennen. Die frühen philosophischen Sprachen wurden beispielsweise von Wissenschaftlern entworfen, die sie dann allerdings auch mithilfe der Instrumente der jeweiligen Disziplin wie Mathematik, Biologie oder Philosophie behandelten. Die Herausbildung einer wissenschaftlichen, insbesondere linguistischen Disziplin, die speziell die Anforderungen der philosophischen Sprachen unter ihrem funktionalen Aspekt untersuchen würde, fand jedoch bis heute nicht statt.

Einen ähnlichen außerlinguistischen Anfang nahm auch die Beschäftigung mit den Welthilfssprachen an. Sie wurde jedoch im Laufe der Zeit immer stär-

25 Die Ergebnisse der Esperantokampagne beim Völkerbund und bei den Vereinigten Nationen werden in der interlinguistischen Literatur unterschiedlich bewertet: positiv (Jurkowski 1986, 55), verhalten (Schild 1957, 783; Lapenna 1971, 28; Okrent 2009, 126; Lins 2012, 98, 113) oder negativ (Smith 2011, 37). 


\section{Forschungsstand}

ker in den linguistischen Diskurs eingebettet. Als ersten Schritt in diese Richtung kann man die Gründung von verschiedenen Komitees sehen, die sich aufgrund der seit Mitte des 19. Jh. wachsenden gesellschaftlichen Relevanz des Themas auf der Metaebene mit den Welthilfssprachen beschäftigten. Die ersten wichtigen Gremien waren Société de Linguistique (1855) (vgl. Couturat und Leau 1903, 71-76; Large 1985, 59-60), Délégation pour l'adoption d'une langue auxiliaire internationale (1901) (Couturat und Leau 1903, viixxvi; vgl. Schild 1957, 782; D. Blanke 1985, 185-187), am Rande deren Tätigkeit das Werk von Couturat und Leau (1903) entstand, und die unter Leitung von G. Peano tätige Academia pro Interlingua (vgl. Barandovská-Frank 2002). Ihre Ansätze sind jedoch noch im vorstrukturalistischen Paradigma zu sehen. Bereits um die Jahrhundertwende (19./20. Jh.) entdeckten die ersten Linguisten die Welthilfssprachen als Forschungsgegenstand (Schuchardt 1904; Baudouin de Courtenay 1907), wobei ihn auch einige aufgrund der kontroversen Künstlichkeitsauffassung durchaus ablehnten (Eichner 2012, 127128; vgl. D. Blanke 1985, 19-22). 1911 postuliert Meysmans (1911-1912) die Interlinguistik als eine neue Wissenschaft, ,(...) die die Bildung der früheren, jetzigen und zukünftigen Hilfssprachen studieren würde“" (Meysmans 1911-1912, 112). Auch die wissenschaftliche Gremienarbeit erfährt eine zunehmende Professionalisierung und linguistische Ausrichtung, wie die Tätigkeit der 1924 gegründeten International Auxiliary Language Association (IALA) zeigt (Schild 1957; D. Blanke 1985, 167-183; Large 1985, 145-154).

Eine wichtige Rolle spielte bei den genannten Entwicklungen Esperanto, das als Auslöser und Maßstab für wissenschaftliche Vergleiche diente. Auch die Esperantobewegung gab immer wieder wichtige Impulse zur wissenschaftlichen Beschäftigung mit dem Thema. $\mathrm{Zu}$ erwähnen sind in diesem Zusammenhang die Auseinandersetzung mit Ido, die zu einer wissenschaftlichen Diskussion beider Sprachen führte, sowie die Gründung der Zeitschrift La Monda Lingvo-Problemo im Jahr 1969, als deutlich wurde, „(...) dass die sprachenpolitischen Aktivitäten der UEA [Universala Esperanto-Asocio/Esperanto-Weltbund] einer wissenschaftlichen Begründung bedürfen und eine Zusammenarbeit mit der Soziolinguistik, insbesondere mit sprachenpolitischen Fachleuten, erforderlich und nützlich ist" (D. Blanke 2009b, 227). Später in Language Problems and Language Planning umgewandelt, festigt die Zeitschrift die Position der Welthilfssprachen im allgemeinen linguistischen Diskurs (Tonkin 1997a, ix-xi; vgl. Schubert 1989b, 18; D. Blanke 2009b, 230-231). 


\subsubsection{Gegenstand der Interlinguistik}

Interlinguistik wird zuweilen auch Plansprachenwissenschaft genannt (D. Blanke 1998, 50), deshalb liegt es nahe, sie als erste Disziplin auf ihren Gegenstand zu untersuchen, um die Frage zu beantworten, ob sie die übergreifende Perspektive auf alle oben beschriebenen Plansprachentypen leistet. In der Fülle des Materials identifiziere ich grob zwei Richtungen, wie Interlinguistik bestimmt wird:

- Interlinguistik, die speziell in den Kontext der internationalen Kommunikation gesetzt wird (Back 1996, 881; D. Blanke 1998, 52-56; Künzli 2006, 48; D. Blanke 2009b, 206; Fiedler 2011b, 10).

- Interlinguistik als übergreifende Disziplin, die alle Plansprachentypen unter verschiedenen Aspekten gleichermaßen untersucht (Jurkowski 1986; Jermołowicz 2003).

Ich stelle nun die beiden Ansätze vor.

Die erste Auffassung ergibt sich unmittelbar aus den vorangegangenen geschichtlichen Beschreibungen des Gegenstands Welthilfssprachen und der Interlinguistik als Disziplin, die sich durch Auseinandersetzungen mit der internationalen Kommunikation etablierte. Seit der bereits zitierten Definition von Meysmans (1911-1912) haben unterschiedliche Forscher unterschiedliche Schwerpunkte in ihren Definitionen von Interlinguistik gesetzt. Einen inhaltlichen Überblick, in dem man auch geschichtliche Tendenzen identifizieren kann, findet man beispielsweise bei Schubert (1989b). Ich gehe hier auf die Diskussion der Interlinguistik und ihres Gegenstands bei D. Blanke (2006) ein (D. Blanke 2006, 19-34, 2011, 120-122), weil sie die Auffassung der Gesellschaft für Interlinguistik e. V. wiedergibt, die eine zentrale Stelle für diesen Themenkomplex in Deutschland ist.

D. Blanke (2006) diskutiert vier mögliche Auffassungen von Interlinguistik im Kontext der internationalen Kommunikation:

1. Interlinguistik als Wissenschaft von internationalen Hilfssprachen, wobei entweder nur Plansprachen ${ }^{26}$ oder sowohl Plansprachen als auch ethnische Sprachen mit berücksichtigt werden.

2. Interlinguistik als „(...) Wissenschaft von der internationalen sprachlichen Kommunikation und mit allen ihren Aspekten" (D. Blanke 2011, 120),

26 In diesem Abschnitt behalte ich die von Blanke verwendete Terminologie und setze mich mit ihr in 2.2.7 auseinander. 


\section{Forschungsstand}

3. Interlinguistik als Linguistik von Mehrsprachigkeit oder Kontaktlinguistik im Sinne von Wandruszka (1971),

4. Interlinguistik als Wissenschaft von der Plansprache Interlingua.

Die letzten beiden Auffassungen werden in der Forschung nicht (mehr) vertreten (Schubert 1989b, 14; D. Blanke 2006, 30-31; Fiedler 2011b, 10). In der ersten Auffassung geht es vor allem um die Erforschung der sprachlichen Mittel, während die zweite Auffassung die Perspektive um den Kommunikationsprozess mit seinen außersprachlichen Faktoren erweitert (D. Blanke 2006, 27; vgl. Fiedler 2011b, 10). Mit anderen Worten: Die erste Auffassung ist in der strukturalistischen Tradition angesiedelt, während die zweite eine eher soziolinguistische Sicht auf den Gegenstand darstellt. Aus den beiden Auffassungen leitet D. Blanke (2006) drei Bereiche der Interlinguistik ab (D. Blanke 2006, 27-30, 2011, 120):

- Allgemeine Interlinguistik, die die internationale Kommunikation in ihrer Gesamtheit erforscht. In diesem Zusammenhang „(...) muss geklärt werden, wie wirksam die internationale Kommunikation durch Ethnosprachen in der Rolle einer lingua franca war oder ist, welche politischen, ökonomischen, kulturpolitischen, psychologischen, juristischen, technologischen, informationspolitischen und andere Wirkungen eine solche Kommunikation hatte oder hat, welche Alternativen andere Kommunikationsmittel, z. B. Plansprachen, bieten können, welche Anforderungen sich aus der gesellschaftlichen Entwicklungsprozessen ergeben und wie diese zu erforschen und zu bewältigen wären (...)“ (D. Blanke 2006, 28-29). Diese Untersuchungen werden von den Linguisten bereits durchgeführt, ohne dass sie als Interlinguistik identifiziert werden (D. Blanke 2006, 29).

- Spezielle Interlinguistik (Plansprachenwissenschaft), die sich den internationalen Plansprachen zuwendet und sie unter typologischen, strukturellen und ggf. soziolinguistischen Aspekten untersucht (D. Blanke 2011, 29).

- Angewandte Interlinguistik, die die interlinguistische Erkenntnisse in anderen Bereichen wie Fremdsprachenerwerbsforschung oder Maschinelle Übersetzung anwendet (D. Blanke 2011, 30).

Aus diesen Vorüberlegungen leitet Blanke folgende allgemeine Definition der Interlinguistik ab, die sich als Arbeitsdefinition für die Gesellschaft für die Interlinguistik e. V. bewährte: „Interlinguistik kann als die Wissenschaft von der 
internationalen sprachlichen Kommunikation mit allen ihren Aspekten angesehen werden. Das impliziert die Funktion, Struktur, Entwicklung und Anwendung von Ethno- und Plansprachen als internationale Kommunikationsmittel“" (D. Blanke 2011, 121).

An dieser Stelle gehe ich auf die Auffassung von Plansprachen ein, die durch die Forscher um die Gesellschaft für die Interlinguistik e. V. vertreten wird. Diese drückt D. Blanke (1985) wie folgt aus: „(...) eine von einzelnen Personen oder Personengruppen nach bestimmten Kriterien bewußt geschaffene Sprache zum Zwecke der Erleichterung der internationalen sprachlichen Kommunikation“"(D. Blanke 1985, 53; vgl. Fiedler 2011b, 12, im Original mit Hervorhebung). Die Bezeichnung Plansprache wird also nicht wie in dieser Arbeit als Oberbegriff für verschiedene Plansprachentypen, sondern eingeschränkt für denjenigen Begriff verwendet, die ich in dieser Arbeit mit Welthilfssprache bezeichne. Diese Einschränkung wird immer wieder betont und bestätigt (D. Blanke 1985, 11, 53, 62, 1989, 63; Schubert 1989b, 10; Fiedler 2006, 67, 2011b, 12; Schubert 2011b, 47; Kimura und Fiedler 2013, 101), wobei man sich auf die Auffassung von Wüster beruft (D. Blanke 1985, 53; Kimura und Fiedler 2013, 101). Wüster führt nach eigenen Angaben die Benennung Plansprachen in seinem Buch 1931 als Erster ein (Wüster 1955, 272). Dort (1931) definiert er jedoch m. W. den Begriff Plansprache nicht. ${ }^{27} \mathrm{Er}$ verwendet allerdings folgende Ausdrücke: philosophische Plansprache, internationale Plansprache, Auswahl-Plansprache (vgl. Wüster 1931, § 851.1). Im Aufsatz von 1955 findet man eine explizitere Definition, die sich auf den eingebürgerten Gebrauch stützt, ohne den Gebrauch jedoch zu belegen oder zu erläutern: „Nach meiner Absicht ist die „Plansprache“ ein Synonym der Benennung ,,interlingvo“ in ihrer heutigen, erweiterten Bedeutung“ (Wüster 1955, 272-273). Auch in seinem Ergänzungsbericht aus dem Jahre 1966 zur 1. Auflage des Werkes von 1931 bestätigt er lediglich eine weitere Einbürgerung der Benennung, ohne den Begriff explizit zu bestimmen. Die philosophischen Plansprachen werden dort aber nicht erwähnt (Wüster 1931, § B 85).

Nach diesem Exkurs zur Auffassung von Plansprache durch die Gesellschaft für Interlinguistik e. V., die für eine abschließende Diskussion in 2.2.7 relevant ist, kehre ich zu den verschiedenen Auffassungen von Interlinguistik zurück.

27 Auch D. Blanke $(1985,53)$ und Fiedler $(2011 \mathrm{~b}, 12)$ geben keine genaue Textstelle an, was meine Annahme zu stützen scheint. 


\section{Forschungsstand}

Der auf die internationale Kommunikation eingeschränkten Auffassung der Interlinguistik steht eine weiter gefasste gegenüber. Interlinguistik als übergreifender Plansprachenwissenschaft betrachtet beispielsweise Jurkowski (1986). Er definiert sie wie folgt: „(...) dziedzin[a] wiedzy poświęcon[a] językom sztucznym, uniwersalnym i międzynarodowym, w tym także językom informatyki“ (Jurkowski 1986, 7; vgl. Jurkowski 1986, 146). ${ }^{28}$ Die von Jurkowski (1986) beschriebenen Phänomene sind breit gefächert, gehen aber weit über das Spektrum der intentionalen Mensch-Mensch-Kommunikation hinaus. In der Arbeit werden viele Quellen und Beispiele für verschiedene Plansprachentypen genannt, eine Auseinandersetzung mit der interlinguistischen Theorie findet jedoch nicht statt. Eine eigene Theorie der Interlinguistik in diesem Sinne wird ebenfalls nicht entwickelt. Eine ähnliche Auffassung findet man explizit bei Jermołowicz (2003): „(...) [interlinguistics] deals with artificial languages and aims at their development, classification and evolutional and futuristic research.“ (Jermołowicz 2003, 55) und auch teilweise bei Żelazny (2012, 95). ${ }^{29}$ Weiterhin sind auch wie erwähnt Large (1985) und Okrent (2009) an einer übergreifenden Sicht auf verschiedene Plansprachentypen interessiert, wobei sie auf den Begriff der Interlinguistik nicht explizit eingehen.

Insgesamt wird also die Auffassung von Interlinguistik als einer Disziplin, die sich übergreifend mit allen Plansprachentypen beschäftigt, entgegen der Meinung von Żelazny $(2012,95)$, in der Forschung nur wenig vertreten.

\subsubsection{Erforschung anderer Plansprachentypen}

Obwohl als Gegenstand der Interlinguistik ausgeschlossen, brachten die künstlerischen Sprachen eine aktive, sprachlich (und nicht literarisch) orientierte Bewegung hervor. Obwohl im Internet entstanden, verlässt sie zunehmend den virtuellen Raum. Seit 2006 findet alle zwei Jahre die Language Creation Conference statt, zu deren Organisation die Language Creation Society gegründet wurde. Das Profil dieser Gesellschaft hat sich im Laufe der Zeit differenziert, so dass sie heute darüber hinaus als Austauschplattform dient, Ressourcen für Interessierte bereitstellt und die

28 „Wissenschaftliche Disziplin, die sich mit künstlichen, universalen und internationalen Sprachen wie auch mit Sprachen in der Informatik befasst" (Übersetzung K.S.).

29 Żelazny (2012) präzisiert nicht, welcher Definition der Interlinguistik er sich letztendlich anschließt. Neben der erwähnten erweiterten Auffassung findet man im Buch ebenfalls Stellen, an denen er von der engeren Auffassung als Wissenschaft von Welthilfssprachen ausgeht (bspw. Żelazny 2012, 142). 
künstlerische Sprachschöpfung durch Kurzstipendien fördert (Language Creation Society 2007-). Das Hauptaugenmerk dieser Bewegung gilt vor allem verschiedenen Arten von künstlerischen Sprachen, betrachtet werden aber auch gelegentlich Beispiele aus anderen Plansprachentypen. Interessanterweise wird die Abgrenzung zu den Welthilfssprachen besonders hervorgehoben, die vor allem dann besprochen werden, wenn ihre systembezogenen Phänomene auf die Tauglichkeit im künstlerischen Kontext hin untersucht werden. Die Abgrenzung zu philosophischen Sprachen $^{30}$ ist hingegen ebenfalls per Definition vorhanden, in der Praxis aber weniger streng gehandhabt. Vor allem neuere philosophische Sprachen wie Ithkuil werden ebenfalls im geschilderten Rahmen besprochen (Boozer (2011, 3), siehe auch Posts zum Thema Ithkuil auf der Conlang-Mailingliste).

Die Bewegung entstand eher aus dem Laienbedürfnis, sie hebt aber ihr wissenschaftliches Niveau ständig an, was an den Konferenzbeiträgen oder den Artikeln in der Zeitschrift Fiat Lingua, die von der Language Creation Society veröffentlicht wird, zu erkennen ist. Trotz dieser interessanten Entwicklung ist es noch nicht zutreffend, von einer neuen wissenschaftlichen Disziplin oder Teildisziplin der Linguistik zu sprechen.

\subsubsection{Wissenschaftlichkeit der Interlinguistik}

Wie deutlich wurde, hat sich lediglich die Interlinguistik als Disziplin etabliert, die (bestimmte) Plansprachen untersucht. Im Folgenden gehe ich auf den Status der interlinguistischen Forschung insbesondere unter dem zuweilen kontrovers diskutierten qualitativen Aspekt ein.

Die Erforschung von Welthilfssprachen wird gewöhnlicherweise von den Interlinguisten selbst innerhalb der Linguistik angesiedelt (Sakaguchi 1998, 16, 312, 322; Oostendorp 2001, 207). Diese Verankerung wird immer wieder thematisiert, woraus ich schließe, dass Interlinguisten selbst viel Wert darauf legen. ${ }^{31}$ Auch aus der innerlinguistischen Perspektive wird der Gegenstand deutlich etablierter (vgl. Back 1996, 881). Dies ist zum einen die Folge einer wissenschaftlichen Auseinandersetzung mit dem naiven Verständnis von Künstlichkeit und Natürlichkeit in der Sprache, bei der die regulierenden Einflüsse in den ethnischen Sprachen gezeigt wurden (Pei 1966, 423; vgl. Mangold 1970, 130; D. Blanke 1985, 26-27;

\footnotetext{
30 Im Gebrauch rund um die Conlang-Mailingliste spricht man von engelangs, vgl. 17 und 2.2.1.4.

31 Siehe beispielsweise Erläuterungen, weshalb man sich als Linguist mit den Welthilfssprachen beschäftigen soll: Mangold (1970, 129), D. Blanke (1985, 23-24, 1989, 81-82), Schubert (1989a, 269), Dulichenko (1997, 67-69), D. Blanke (2006, 51-54), Okrent (2009, 256-258) und Eichner (2012, 149).
} 


\section{Forschungsstand}

Duličenko 1989, 51-53; Kuznecov 1989, 92-95; Schubert 1989b, 10, 18; Julià 1997, 31-33). Zum anderen aber hat sich vor allem Esperanto in der kommunikativen Praxis bestätigt, so dass es mit gewöhnlichen linguistischen Methoden untersucht werden kann (s. bspw. D. Blanke 2006, 51).

Von dieser immer weniger kontroversen Einordnung als linguistische Disziplin ist jedoch die in der Interlinguistik selbst verbreitete Annahme zu unterscheiden, die Interlinguistik werde von anderen Linguisten negativ bewertet. Die empfundene Kritik betreffe viele Aspekte: Fragestellungen, Methoden, Erkenntnisse, gelegentlich einzelne Welthilfssprachen (vgl. Eichner 2012, 123-128, 134). Ich fasse hier die wesentlichen Punkte zusammen.

Eine Zeitlang spielte für Interlinguisten ein Austausch mit anderen linguistischen Gebieten kaum eine Rolle (vgl. Sakaguchi 1998, 394-395; Eichner 2012, 134). Sie erforschten spezielle Fragen der Welthilfssprachen, manchmal ungeachtet allgemeiner linguistischer Tendenzen (vgl. Tonkin 2007, 182-183). Die Veröffentlichung ihrer Erkenntnisse in einer Plansprache (Tonkin 1997b, 82; Sakaguchi 1998, 394; Tonkin 2007, 183; Eichner 2012, 134) und die Diskussion der Erkenntnisse auf beispielsweise Esperanto-Weltkongressen statt auf regulären linguistischen Veranstaltungen (Sakaguchi 1998, 302, 394) und in Konsequenz eine allgemeine Abkopplung vom üblichen linguistischen Betrieb führte zu einer Abschottung der Interlinguistik und zur Entstehung eines hermetischen Bildes der Disziplin nach außen. Die durch den erschwerten Zugang bedingte oder aber auch gewollte Unkenntnis der Interlinguistik durch Außenstehende resultierte zudem in vielen Vorurteilen und Fehlkonzeptionen nicht nur in der Linguistik (Lapenna 1970, 99; D. Blanke 2009b, 225; vgl. Eichner 2012, 133).

In der interlinguistischen Literatur wird die Bevölkerungsmeinung thematisiert, dass Welthilfssprachen etwas Utopisches seien und sich die Frage nach ihrem Einsatz als Mittel der internationalen Kommunikation gar nicht mehr stelle (Edwards und MacPherson 1997, 109; Fiedler 2011a, 85; Eichner 2012, 133). Die Erforschung von Welthilfssprachen unter diesem Aspekt wird also als etwas Unseriöses gesehen. Die erwähnte Tatsache, dass die interlinguistische Forschung selbst eine Neuorientierung durchlebt, in der es nicht mehr um die Positionierung einer Welthilfssprache als Mittel der internationalen Kommunikation geht, wird nicht wahrgenommen.

Den Eindruck mangelnder Seriosität soll auch eine nach wie vor vorhandene starke laienhafte Komponente in der interlinguistischen Forschung erwecken, was nicht nur die Ausbildung der Beteiligten sondern auch Ansätze und 
Untersuchungsmethoden betrifft (D. Blanke 1989, 64; vgl. Tonkin 1997b, 86; Haupenthal 2012, 32). Dieses negative Bild wird zusätzlich noch durch Autoren und Anhänger einzelner Welthilfssprachen verstärkt, die manchmal als exzentrisch, aufdringlich, ideologisch geprägt oder idealistisch empfunden werden (Large 1985, 109, 200; Okrent 2009, 110, 149).

Die Interlinguistik fasst sich also selbst weitestgehend als linguistische Nischendisziplin auf. Wie Fiedler (2011a) zusammenfasst, „,...) ist die Beschäftigung mit dem Gegenstand der Karriere eines Linguisten ganz sicher nicht zuträglich“ (Fiedler 2011a, 100). D. Blanke (1985) bemerkt jedoch, dass die Linguisten nicht per se das Thema ablehnen, sondern gute wissenschaftliche Bedingungen zur Voraussetzung machen, wie sie beispielsweise von der IALA geschaffen wurden (D. Blanke 1985, 169). Heutzutage scheint es diese jedoch nicht zu geben, da Interlinguistik im wissenschaftlichen Betrieb so gut wie nicht institutionalisiert ist (Sakaguchi 1998, 397; Haupenthal 2012, 29) und über kaum Mittel aus öffentlichen Förderprogrammen verfügt (Fiedler 2011a, 100).

$\mathrm{Ob}$ es sich bei diesen Einschätzungen um Tatsachen oder (Eigen- oder Fremd-) Vorurteile handelt, kann ich an dieser Stelle nicht beantworten. Wichtig erscheint mir die Trennung der Berechtigung oder der Anerkennung der Disziplin als solchen von der (angenommenen) Bewertung der bisherigen Forschung. Wird diese nicht gemacht, so kann der Eindruck entstehen, der Status der Interlinguistik innerhalb der Linguistik sei nach wie vor unklar (vgl. Sakaguchi 1998, 15).

\subsubsection{Interdisziplinarität}

Wie in vorangegangenen Abschnitten erläutert, fasst sich die Interlinguistik selbst explizit als linguistische Disziplin auf. Obwohl Bormann (1958) sie noch als interdisziplinäres Fach mit Bezügen zu Linguistik postulierte (Bormann 1958, 293-296), ist diese Position gegenwärtig nicht weit verbreitet (vgl. D. Blanke 2006, 27) und es besteht zurzeit keine Tendenz zur Etablierung als ein von der Linguistik weitestgehend unabhängiges interdisziplinäres Fach. Die Linguistik als übergreifende Disziplin stellt somit Konzepte und Methoden zur Erforschung der Welthilfssprachen bereit. So erfuhr die Interlinguistik wie die allgemeine Linguistik eine Erweiterung der primär systembezogenen Forschungsfragen (Beschaffenheit der Plansprache auf verschiedenen Ebenen des Sprachsystems) um eher soziolinguistisch orientierte Interessen (vgl. Künzli 2006, 41; D. Blanke 2009b, 236), wodurch auch die Nähe zu anderen linguistischen Disziplinen wie Sprachplanung (D. Blanke 


\section{Forschungsstand}

2009b, 236), Pidgin-Kreol-Forschung (Back 1996, 881) und zur Fremdsprachendidaktik (Sakaguchi 1998, 147-151; 374-379) betont wird. Die soziolinguistische Tendenz ist aber ebenfalls bei Beschreibungen von einzelnen Welthilfssprachen zu erkennen, in denen die Sprechergemeinschaften verstärkt untersucht werden (vgl. Schubert 1989b, 24; Carlevaro 1998; Anton 2002; Mannewitz 2002).

Die soziolinguistische Neuausrichtung hatte selbstverständlich zur Folge, dass außersprachliche Faktoren berücksichtigt werden. Um diese besser zu verstehen, müssen stärkere Bezüge zu den jeweiligen Disziplinen (Soziologie, Philosophie, Kommunikationswissenschaft, Politikwissenschaft, Semiotik etc.) aufgebaut werden (vgl. Sakaguchi 1998, 313-314, 331-338). Somit kann man die Interlinguistik als linguistische Disziplin mit starken interdisziplinären Bezügen auffassen (vgl. D. Blanke 2006, 28-30). Sakaguchi (1998) sieht in einer starken interdisziplinären Ausrichtung der Interlinguistik jedoch die Gefahr des Wissenschaftlichkeitsverlusts, da sie leicht zur Bühne der ideologischen Propaganda werden können (Sakaguchi 1998, 313, 324).

\subsubsection{Autoren}

Nachdem ich im vorangegangenen Abschnitt die Erforschung von Plansprachen als Disziplin näher betrachtete, wende ich mich hier erneut den Plansprachen selbst als Gegenstand zu. Zunächst gehe ich auf allgemeine Charakterisierungen von Plansprachenautoren ein, die in der Literatur zu finden sind (2.2.4.1). Sie haben zumeist einen beschreibenden Charakter, fassen verschiedene Eigenschaften bisheriger Autoren zusammen und eignen sich weniger als ein Anforderungskatalog an künftige Autoren. Darauf setze ich mich mit der Sprachauffassung von konkreten Plansprachenautoren auseinander (2.2.4.2). Zu diesem Zweck stütze ich mich auf einschlägige Arbeiten zu einzelnen Plansprachen, ohne sie hier jedoch zusammenfassend darzustellen. Diesen Abschnitt schließe ich dann mit Überlegungen zur kollektiven Autorenschaft bei der Plansprachenkonstruktion ab (2.2.4.3). Die einzelnen Themen dieses Abschnittes werden mir in weiteren Kapiteln dieser Arbeit als Grundlagen dienen, auf der ich Ergebnisse der Hauptuntersuchung in Kapitel 5 diskutiere und interpretiere.

\subsubsection{Allgemeine Charakterisierung}

Im Kontext von Plansprachen werden meistens diejenigen Personen als Autoren bezeichnet, die einen Wortschatz und ggf. grammatische Regeln für eine 
Sprache explizit entwerfen. Nur bei wenigen Sprachen wie Loglan liegt die Leistung des Autors in Schaffung von Prinzipien, wie der Wortschatz aufgebaut werden kann und nicht direkt im Aufbau des Wortschatzes selbst. Anders als bei Sprachplanung oder Terminologiearbeit entstehen die meisten Plansprachen aus privater Initiative einzelner Personen. $\mathrm{Zu}$ den wenigen Sprachen, die im Auftrag entstanden, gehören beispielsweise Interlingua, Klingonisch oder Dothraki. Sprachschöpfung als gemeinschaftlicher Akt ist ebenfalls eher selten, hier können wieder Interlingua und möglicherweise Ido genannt werden (D. Blanke 1985, 187; Large 1985, 83; vgl. Ölberg 1954, 245).

Die meisten individuellen Autoren sind zwar sprachinteressiert, verfügen jedoch selten über systematische linguistische Ausbildung und unterscheiden sich bezüglich ihrer sprachtheoretischen Kenntnisse. Unter Bezug auf Golden (1981) unterteilt D. Blanke $(1985,73)$ die Autoren in folgende Gruppen (teilweise eigene Beispiele vgl. Large 1985, 171; Eichner 2012, 129):

- Wissenschaftler: Sprachwissenschaftler (Jespersen, Ogden, Gode, Okrand, Peterson); Mathematiker/Logiker (Leibniz, Couturat, Peano); Soziologen (J. C. Brown (1960)); Philosophen (Descartes); Naturwissenschaftler (Wilkins);

- gebildeter Berufsstand: geistig anspruchsvolle Berufe wie Ärzte (Zamenhof), Geistliche (Hildegard von Bingen, Wilkins, Schleyer);

- Laien mit praktischen Sprachkenntnissen; ${ }^{32}$

- Naive Projektautoren, die kaum sprachtheoretische Kenntnisse und niedriges Berufsniveau besitzen.

Carlevaro (1977) unterscheidet Autoren nach Führungsstilen: autoritär (Schleyer: Volapük), Couturat: Ido)), bürokratisch (De Beaufront: Esperanto)), demokratisch (Zamenhof: Esperanto)), anarchistisch (Peano: Latino sine Flexione)) und charismatisch (teilweise Schleyer und Couturat) (Carlevaro 1977, 97). Wie aus den genannten Personenbeispielen deutlich, berücksichtigt er nicht nur die Autoren im engeren Sinne, sondern auch weitere Mitwirkende in einer Bewegung.

Zur Tatsache, dass die Sprachwissenschaftler unterrepräsentiert sind, bemerkt Large (1985), dass sprachwissenschaftliche Expertenkenntnisse nicht

32 Die Gruppe würde viele Autoren in der Conlang-Mailingliste umfassen, auf die aber D. Blanke (1985) nicht eingeht. 


\section{Forschungsstand}

unbedingt vorteilhaft für die Entwicklung und Etablierung einer Welthilfssprache seien, weil sie zur Überbetonung der systemlinguistischen Details führen und die Praktikabilität einer Lösung in den Hintergrund stellen würden (Large 1985, 200; vgl. auch Baudouin de Courtenay in: Kotzin 1917, 144). Für Pei (1966) können Linguisten gar nur auf der Ebene der Rechtschreibung von ethnischen Sprachen einen Beitrag im Bereich der Welthilfssprachen leisten, da dies ansonsten eine politische Angelegenheit sei (Pei 1966, 436).

Die individuelle Initiative beschränkt sich nicht nur auf die Erarbeitung eines Entwurfs. Auch die Veröffentlichung, Bekanntmachung und Verbreitung des eigenen Projekts beruhen in der Regel auf privaten Bemühungen oder Kontakten des Autors, der in den meisten Fällen auch anfangs alle finanziellen Kosten trägt. ${ }^{33}$

In ihrem Buch versucht Okrent (2009) noch eine andere Eigenschaft von individuellen Plansprachenautoren zu belegen, nämlich ihre Exzentrizität. Die Beschreibungen von Joseph Schipfer, Carl Bliss, John Wolfgang Weilgart aber auch von Charles Ogden können tatsächlich ein wenig befremdlich wirken. Eine wissenschaftlich qualifizierte Aussage über die generelle Exzentrizität ist jedoch in diesem Fall unzulässig (vgl. Tonkin 2009, 292). Ebensowenig wissenschaftlich belegt ist die gesellschaftliche Wahrnehmung von Plansprachenautoren als Exzentriker.

\subsubsection{Autoren und ihre Sprachauffassung}

Wie in 2.2.2 erläutert, folgt die Schaffung von philosophischen und Welthilfssprachen bestimmten gesellschaftlichen Entwicklungen. Man kann die genannten Sprachentypen als Lösungsvorschläge für die von Autoren wahrgenommenen gesellschaftlichen Probleme sehen (vgl. Adams 2011b, 2). Zwar ist auch das jüngste Interesse an künstlerischen Sprachen gesellschaftlichtechnologischen Entwicklungen, insbesondere neuen Kommunikationsmedien wie dem Internet, zu verdanken. Diese Sprachen sind aber Ausdruck ästhetischer Bedürfnisse und m. E. nicht als eine Abhilfe für spezifische gesellschaftliche Probleme zu sehen.

Eine umfassende Beschreibung der Autoren von einschlägigen Welthilfssprache und ihrer Vorgehensweie kann in diesem Abschnitt nicht geleistet werden. Folgende Beschreibungen stützen sich auf meine umfassende Auseinandersetzung mit den Welthilfssprachen Volapük, Esperanto und Ido sowie

33 Siehe beispielsweise: Wilkins (1668) (Okrent 2009, 21-25), Esperanto (vgl. Janton 1978, 24; Forster 1982, 53-54; Okrent 2009, 97-98), Loglan (vgl. Okrent 2009, 214), Blissymbolics (vgl. Okrent 2009). 
auf allgemeinere Recherche zu weiteren Plansprachen. Für die drei genannten Welthilfssprachen beziehe ich mich vor allem auf folgende Literatur:

- Volapük: Kniele (1889) - Das erste Jahrzehnt der Weltsprache Volapük, Couturat und Leau (1903, 128-163) - Historie de la langue universelle, Abschnitt Volapük, sowie J. Schmidt (1963) und Haupenthal (2005a,b)

- Esperanto: Dr. Esperanto (1887b) - Meždunarodnyj jazyk, Forster (1982) - The Esperanto Movement sowie Zamenhof (1905), Kraśko (1972), Large (1985) und Okrent (2009). Wichtig für diese Arbeit ist die von Forster (1982) postulierte Unterscheidung zwischen der ideologisch-orientierten (value-oriented) und der instrumentell-orientierten (norm-oriented) Strömung in der Esperanto-Bewegung.

- Ido: Borgius (1908) - Warum ich Esperanto verließ, Kotzin (1917) Geschichte und Theorie des Ido sowie Peus (1913), Lehrbuch der Weltsprache Ido für Arbeiter (1923) und McPike (1925)

Aus den in den genannten Arbeiten beschriebenen Vorgehen schließe ich bei manchen Plansprachenautoren auf eine besonders stark ausgeprägte Vorstellung von Sprache als sozialem Phänomen - die Sprache wird erst durch den Gebrauch im sozialen Kontext konstituiert und nicht allein dadurch, dass das Sprachsystem veröffentlicht wird. Die zentrale Rolle der Sprecher für die Sprache nehmen beispielsweise Zamenhof aber auch LeChevalier (Lojban) an (für Letzteres s. Okrent 2009, 225). Die Herausbildung einer Sprechergemeinschaft ist aber ebenfalls ein soziales Phänomen, wie aus den ethnischen Sprachen bekannt ist (vgl. Hertzler 1965, 60); (für Plansprachen: vgl. Duličenko 1989, 52; Oostendorp 2001, 208-209; Tonkin 2001, 16). Nimmt man dies auch für Plansprachen an, so ist es möglich, die Herausbildung einer Sprechergemeinschaft von Anfang an aktiv zu unterstützen, wie das Beispiel von Zamenhof zeigt. ${ }^{34}$ Mit dem Ziel, eine offizielle Anerkennung und Etablierung der Sprache in Organisationen zu umgehen (Dr. Esperanto 1887a, 5-7, 22-27; vgl. Welger 1998, 36), etablierte Zamenhof die Sprechergemeinschaft „von unten“, die über die Jahre ihre Stabilität bewies.

34 Bei der Veröffentlichung von Esperanto meine ich insbesondere die Lernverpflichtung und das Auffordern zur aktiven Kritik und Weiterentwicklung der Sprache, die mit dem Esperanto-Manuskript erschienen sind, s. Dr. Esperanto (1887a). 


\section{Forschungsstand}

Dieses facettenreiche Verständnis von Sprache als sozialem Phänomen scheint Esperanto-spezifisch zu sein. Keiner der anderen Autoren berücksichtigt die soziale Komponente in allen Phasen der Projektumsetzung in diesem Maße: vom Entwurf des Sprachsystems, über die Etablierung der Sprechergemeinschaft bis hin zur Pflege der Gemeinschaft und der Sprache. Ich gehe davon aus, dass viele Autoren sich lediglich auf die Veröffentlichung des Projekts sowie die Aufzählung seiner Vorteile und der allgemeinen Motive beschränken, ohne die potentiellen Sprecher von Anfang an zu aktivieren (vgl. Large 1985, 140). Einige Autoren setzen, wie eingangs erwähnt, bei der Pflege an und fördern die Beteiligung der Sprecher an der Weiterentwicklung der Sprache. Andere Autoren unterschätzen wiederum die Bedeutung der sozialen Beteiligung an der Sprachentwicklung vollständig und versuchen, diese aktiv zu unterbinden (Schleyer: D. Blanke (1985, 211-212) und Large (1985, 69-70); Bliss: Okrent (2009, 173-177); teilweise auch J. C. Brown (1960): Okrent $(2009,218)$ ).

Auch die Schwerpunktsetzung in der wissenschaftlichen Literatur spiegelt dieses Missverhältnis wider. Meine Auswertung zu Plansprachen ergibt nämlich, dass die Forschung wenig Aufmerksamkeit den Fragen schenkt, welche Maßnahmen Autoren ergreifen, um die Phase des erstmaligen Gemeinschaftsaufbaus aktiv zu unterstützen, und wie diese Maßnahmen zu bewerten sind. Stärker im Fokus steht hingegen die Beteiligung der Sprecher an der Weiterentwicklung einer Plansprache, was aber das Vorhandensein einer Sprechergemeinschaft voraussetzt.

Ich nehme an, dass bei philosophischen Sprachen, die auf Klassifikation beruhen, die soziale Beteiligung an ihrer Entstehung oder Weiterentwicklung gar nicht vorgesehen ist, da sie die objektive, also auch von sozialen und individuellen Standpunkten unabhängige, „Natur der Dinge“ widerspiegeln sollen (vgl. Large 1985, 21). Bei diesen Sprachen ist außerdem relativ wenig über die Verbreitungsmaßnahmen bekannt, man kann jedoch vermuten, dass auch hier die „Richtigkeit“" als ausreichender Verbreitungsfaktor angenommen wurde.

Die künstlerischen Sprachen stellen einen Sonderfall dar. Die fiktionalen Sprachen müssen in der Regel eine fiktionale Welt glaubwürdig machen, in diesem Fall beeinflusst diese Welt die Beschaffenheit der Sprache (wie z. B. bei Klingonisch oder Dothraki). Bei den persönlichen Sprachen beeinflusst hingegen das Sprachsystem die Beschaffenheit der fiktionalen Welt, die speziell für diese Sprache erschaffen wird. Die Beziehung Sprache-Gesellschaft wird also oft nur in eine Richtung ausgearbeitet. In beiden Fällen handelt es 
jedoch um eine subjektive Modellierung und eine Vorstellung von der sozialen Einbettung der Sprache, die man nicht zur Untersuchung des reellen Phänomens heranziehen kann. Trotz dieses impliziten Verständnis von Sprache und Gesellschaft interessieren sich die Schöpfer und Anhänger von künstlerischen Sprachen vor allem für strukturelle Aspekte, wie an den Beiträgen in Fiat Lingua und der Conlang-Mailingliste zu erkennen ist.

Zusammenfassend lässt sich über die Autoren der Plansprachen sagen, dass sie ihre Projekte unterschiedlich stark in den sozialen Kontext eingebettet sehen. Die meisten Entwürfe von philosophischen und Welthilfssprachen entstanden als Lösungsvorschläge für soziale Probleme. Die wenigsten davon arbeiteten jedoch mit der Vorstellung, dass nicht nur die Sprache die Gesellschaft verändern, sondern auch umgekehrt die Sprache sozial geprägt ist. Von einer Art Wechselbeziehung kann man bei Plansprachen sprechen, die zumindest eine Rückmeldung der Sprecher und ihre Beteiligung an der Weiterentwicklung des Systems vorsehen. Dass die Sprechergemeinschaften der ethnischen Sprachen historisch in der Regel durch Übereinkunft von Individuen entstanden, scheint sich nur Esperanto zunutze gemacht zu haben, das seine Gemeinschaft gezielt eher „von unten“ als „von oben“ etabliert.

\subsubsection{Plansprachenkonstruktion und -verbreitung als kollektiver Akt}

Vor dem Hintergrund der Sprache als sozialem Phänomen kann die Selbstauffassung von Zamenhof als Initiator (und nicht als Autor) des Esperanto (Dr. Esperanto 1887a; vgl. exemplarisch D. Blanke 1985, 223) besser verstanden werden. Wenn der veröffentlichte Entwurf nur als Projekt gesehen wird, der erst durch den Gebrauch in einer Sprechergemeinschaft zu einer vollständigen Sprache wird, so müssen die Sprecher auch zu den Autoren gezählt werden. Anders ausgedrückt: Die Sprecher einer Plansprache werden zu Mitautoren, wenn sie sich aktiv an der Sprachpflege beteiligen können bzw. wenn die Sprachpflege zumindest teilweise „bottom-up“ erfolgt. Dabei sehe ich diese Beteiligung zwischen zwei Polen: induktiver Weiterentwicklung der Sprache durch den Sprachgebrauch (stark ausgeprägt bei Esperanto) und deduktiver Weiterentwicklung durch normative Vorgaben individueller Sprecher oder Sprechergruppen (stark ausgeprägt bei Ido). Entsprechend der expliziten oder impliziten Sprachauffassung des Autors ist nicht in allen Plansprachen erwünscht, Sprecher als Mitautoren zu betrachten, so beispielsweise bei Volapük oder Loglan. ${ }^{35}$

35 Das Selbstverständnis von J. C. Brown (1960) als Urheber des Loglan führte gar zu einem gerichtlichen Streit über die Sprachpflege und über die allgemeine Frage, ob man Urheberrechte an einer Plansprache 


\section{Forschungsstand}

Wenn Sprachgemeinschaften aufgrund ihrer Beteiligung an Sprachpflege als Mitautoren verstanden werden, kann nach der normativen Wirkung von Organisationen und speziellen Sprachpflegeinstitutionen, die sich für die meisten Plansprachen mit dem Status ab Semiplansprache (s. dazu 2.2.5.3) herausbildeten, gefragt werden. Die Aufgaben der bis heute tätigen Esperantoakademie können wie folgt zusammengefasst werden: „,...) (1) to conserve and protect the language according to its norms and to control its development; (2) to explore all linguistic questions concerning Esperanto; (3) to review publications from the linguistic point of view; and (4) to defend Esperanto against all competitors (...)" (Fiedler 2006, 79; vgl. D. Blanke 1985, 286; Jurkowski 1986, 53). Vor allem ist das Einhalten des Fundamento de Esperanto eine zentrale Aufgabe (D. Blanke 1985, 286). Trotz eines normativ anmutenden Eindrucks versteht sich die Esperantoakademie eher als beratendes und empfehlendes Gremium, das bei seinen Entscheidungen den etablierten Gebrauch weitestgehend berücksichtigt. Dies wirkt sich stabilisierend auf die Sprache und in Konsequenz auf die gesamte Bewegung aus (Sakaguchi 1998, 259; vgl. Fiedler 2006, 80). Eine zu geringe Präskriptivität der Esperantoakademie wird von manchen Wissenschaftlern und Sprechern kritisiert (Sakaguchi 1998, 72, 266; Fiedler 2006, 80; vgl. Piron 1989, 140-141). Die heute nicht mehr aktive Ido-Akademie verfolgte hingegen einen stark präskriptiven Ansatz (Anton 2002, 24-25; vgl. D. Blanke 1985, 197, 200).

Ich sehe die Normativitätsausprägungen von Sprachpflegeinstitutionen in Abhängigkeit von der gewünschten Beteiligung der Sprecher an der sprachlichen Weiterentwicklung: Ist die Beteiligung deduktiv oder gar nicht erwünscht/vorhanden, so zeichnen sich die Sprachpflegeinstitute durch starke Präskription aus. ${ }^{36}$ Konstituiert sich die Plansprache hingegen durch den tatsächlichen Sprachgebrauch, so arbeitet die Sprachpflegeinstitution eher deskriptiv.

\subsubsection{Modelle}

Ein weiterer Schritt zur Entwicklung einer übergreifenden Perspektive auf die Sprachlenkung ist die Identifizierung von theoretischen Konstrukten und Mo-

besitzen kann (Okrent 2009, 226-227; vgl. Adams 2011a). Im Fall von Klingonisch muss diese Frage zumindest für heute mit ,ja“ beantwortet werden (Okrent 2009, 228, 279; vgl. Okrand u. a. 2011, 126127, 132)

36 Hier mache ich keine Aussage über die Wirksamkeit dieser Normativität, sondern behaupte lediglich, dass dies die Aufgabe und die Selbstauffassung dieser Institute ist. 
dellen der Interlinguistik, die sich später ggf. auf andere Formen der Sprachlenkung verallgemeinern lassen. Ich stelle jedoch fest, dass die interlinguistische Forschung bisher kaum Theorien und Modelle hervorbrachte, die sich zudem wie erwartet speziell auf die Welthilfssprachen beziehen. Im Folgenden berücksichtige ich ausgewählte, allgemeinere Erkenntnisse und gehe auf spezielle Theorien, wie beispielsweise die zur Wortbildung des Esperanto (Saussure 1918; vgl. Schubert 1989a, 256-257) nicht ein. Als Erstes erläutere ich die Klassifikation von Welthilfssprachen/Plansprachen nach dem Grad der Anlehnung des Sprachmaterials, (insbesondere des Wortschatzes) an ethnischen Sprachen. Ferner stelle ich Empfehlungen zur Beschaffenheit naturalistischer Welthilfssprachen auf den einzelnen Ebenen des Sprachsystems vor und präsentiere schließlich die Entwicklungsphasen einer Welthilfssprache auf dem Weg zu einer vollständigen Sprache.

\subsubsection{Etymologische Plansprachenklassifikation nach Moch}

Die in der Forschung am weitesten verbreitete Plansprachenklassifikation unterscheidet zwischen apriorischen, aposteriorischen und gemischten Plansprachen. Apriorische Sprachen lehnen sich in ihrem Aufbau nicht bzw. nur in geringem Maße an ethnischen Sprachen an, wobei hier vor allem der Wortschatz und weniger die Syntax betrachtet wird (Janton 1978, 6; vgl. D. Blanke 1985, 100-101; Large 1985, 51; Sakaguchi 1998, 97; Haupenthal 2012, 17). Obwohl in der Literatur dieser Aufbau oft als willkürlich bezeichnet wird (D. Blanke 1985, 100-101; Sakaguchi 1998, 97; vgl. Janton 1978, 6), folgen die apriorischen Sprachen m.E. bestimmten Aufbauprinzipien wie Kombinatorik, Klassifikation, oder Prädikatenlogik, die aber in einer so strengen Ausprägung in den ethnischen Sprachen nicht vorkommen. Die aposteriorischen Sprachen hingegen „(...) folgen ethnosprachigen Vorbildern und entlehnen das Material für ihre Lexik aus Nationalsprachen, das sie mehr oder weniger für ihr System verändern“ (D. Blanke 1985, 101; vgl. Schuchardt 1904, 54; Mayrhofer 1969, 310-311; Sakaguchi 1998, 97, 145; Fiedler 2006, 69; Haupenthal 2012 , 18). Schließlich umfassen sog. Mischsprachen sowohl apriorische als auch aposteriorische Elemente (D. Blanke 1985, 101; vgl. Schuchardt 1904, 54; Haupenthal 2012, 19). Die Zuordnung einer Plansprache zu einer bestimmten Klasse ist nicht immer eindeutig (vgl. Large 1985, 51), obwohl sich innerhalb der Interlinguistik bestimmte Konventionen herausgebildet haben (s. bspw. die Zuordnung bei D. Blanke 1985). Deshalb wird diese Unterscheidung manchmal auch als eine Skala aufgefasst, die sich zwischen 


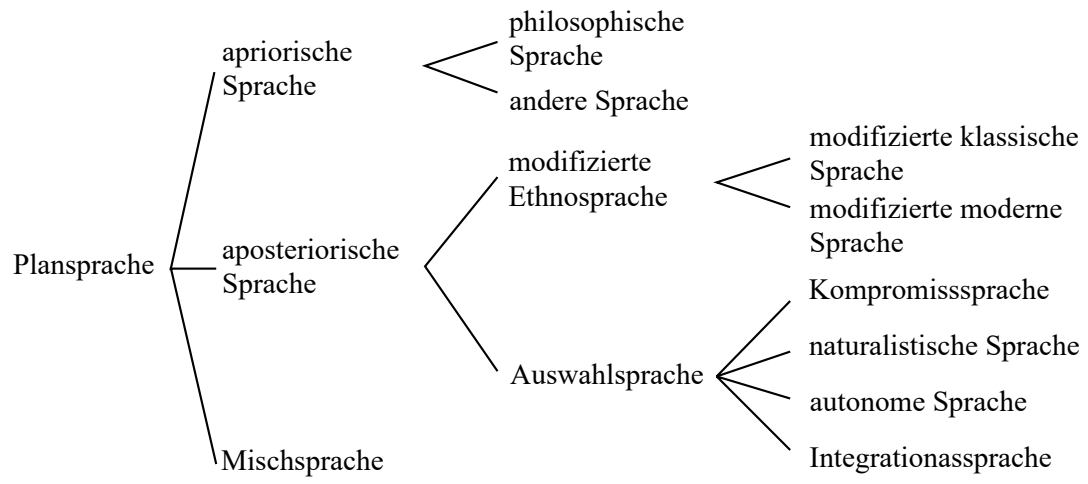

Abbildung 3.: Klassifikation von Plansprachen nach dem Grad der Anlehnung an ethnischen Sprachen (D. Blanke 1985), eigene Darstellung

den Polen „extremer Apriorismus“ und ,extremer Aposteriorismus“ bewegt (s. dazu D. Blanke 1985, 101; Sakaguchi 1998, 98-99).

Obwohl diese Klassifikation gewöhnlicherweise Moch (1897) zugeschrieben wird, (Sakaguchi 1998, 97; Haupenthal 2012, 17). ${ }^{37}$ wurde sie erst durch das Werk von Couturat und Leau (1903) einem breiterem Publikum bekannt (vgl. Schubert 2011b, 50). Im Laufe der interlinguistischen Forschung wurde die aus der Sicht der Welthilfssprachen interessanteste Kategorie der aposteriorischen Sprachen weiter differenziert. ${ }^{38}$ In der Literatur wird meistens die Klassifizierung von D. Blanke (1985) erwähnt (vgl. D. Blanke 1989, 71-78), die ich hier in Abbildung 3 zusammenfasse. Geringfügig abweichende Auffassungen sind beispielsweise bei Schild (1957, 781), Sakaguchi (1998, 225228) und Steenbergen (2008) zu finden.

\subsubsection{Empfehlungen für das Sprachsystem}

Der Konstruktion von Welthilfssprachen liegt die Suche nach einem besseren Sprachsystem zugrunde, das bestimmte kommunikative Funktionen übernehmen sollte. Eine Metadiskussion, wie ein solches System beschaffen sein sollte, spielte in der Interlinguistik eine zentrale Rolle (vgl. Large 1985, 190)) und kann noch weiter zurück verfolgt werden, wie anhand des Briefs von Des-

37 Descartes als möglicher Autor, s. Schubert (2009, 133)

38 Zur Kritik der apriorischen Plansprachen aus der interlinguistischen Perspektive s. Couturat und Leau (1903, 113-116), vgl. Large (1985, 62) und Sakaguchi (1998, 108-110). 
cartes an Mersenne diskutiert (Couturat und Leau 1903, 11-14; vgl. Large 1985, 30). Aus der sprachwissenschaftlichen Sicht ist die erste Hälfte des 20. Jahrhunderts besonders fruchtbar gewesen, was die Aufstellung von Gütekriterien für ein plansprachliches System betrifft. Diese Kriterien beziehen sich meistens auf Welthilfssprachen und sollen zum einen die Bewertung von existierenden Projekten ermöglichen, zum anderen aber auch als Empfehlung beim Plansprachenentwurf dienen. Neben den Empfehlungen der in 2.2.3.1 erwähnten Société de Linguistique wie Klarheit, Einfachheit, Logik, philosophischer Aufbau, Ausdrucksstärke, Harmonie und Flexibilität (Couturat und Leau 1903, 72) gibt es weitere, teilweise widersprüchliche, Kriterien wie Ökonomie, Redundanz, Erlernbarkeit (Mayrhofer 1969, 313-317); schematischer Aufbau (Bormann 1958, 283-286); naturalistischer Aufbau (Jespersen 1930, 158-159); Eigenschaften des phonetischen Systems (Trubetzkoy 1939). Eine ausführliche Empfehlung für die Ebenen des Sprachsystem von aposteriorischen Sprachen formulieren im Zusammenhang mit der IALA-Tätigkeit Sapir u. a. (1925) (vgl. Large 1985, 183-184; Sakaguchi 1998, 151-199):

1. einfaches (universales) Lautsystem;

2. einfache, gleichzeitig leistungsfähige Grammatik, die nicht nur logisch sondern auch leicht erlernbar ist: a) Flexionsreduktion, keine redundanten doppelten Markierungen (z. B. Adverbien zur Tempusmarkierung statt Tempora), b) Vereinfachung von Wortbildung (Komposition statt Derivation; Vermeidung von Abstrakta);

3. einfacher Transfer zwischen der ethnischen Sprache und der Welthilfssprache;

4. strukturelle Flexibilität, damit der ungewollte Transfer aus der Muttersprache nicht zu Verständigungsproblemen führt;

5. Bevorzugung von westeuropäischen Sprachen;

6. Weiterführung eines bestehenden Konzeptes, z. B. kann Latino sine Flexione zu einer analytischen Sprache ausgebaut werden;

7. leichter Einsatz der Kurzschrift;

8. Lautsystem, das beim Einsatz moderner Technik immer noch eindeutig ist 


\section{Forschungsstand}

Weitere ausführliche Empfehlungen findet man auch bei D. Blanke (1985, 88-98) und D. Maxwell (1989).

\subsubsection{Stufenmodell von Blanke}

Geht man von der strukturalistischen Sprachauffassung aus, so ist bereits ein Entwurf des Sprachsystems unabhängig von seinem tatsächlichen Gebrauch eine Sprache (vgl. Julià 1997, 30). ${ }^{39}$ Die soziolinguistische Auffassung der Sprache hingegen führt zu einer differenzierteren Betrachtung von verschiedenen plansprachlichen Systemen (vgl. D. Blanke 1985, 106; Kuznecov 1989, 90; Schubert 1989b, 19). D. Blanke (1985) schlägt neben der in Abbildung 3 dargestellten Klassifikation von Plansprachen nach etymologischen Gesichtspunkten eine weitere Unterscheidung vor. Er differenziert, ob ein System in der reellen Kommunikation tatsächlich verwendet wird oder nicht und postuliert demnach die Aufteilung aller plansprachlichen Systeme in Plansprachenprojekte, Semiplansprachen und (vollständige) Sprachen (D. Blanke (1985, 107-108), später auch in: D. Blanke (1989, 2009a) (vgl. Back 1996, 883)). Die Plansprachenprojekte sind diejenigen Plansprachen, die keine Sprechergemeinschaft herausbildeten. Ein Zwischenstadium stellen die Semiplansprachen dar, die eine Sprechergemeinschaft in Ansätzen, aber noch nicht vollständig herausbildeten. Die (vollständigen) Sprachen verfügen über eine vollwertige Sprechergemeinschaft (D. Blanke 1985, 107-108).

Aus der Sicht meiner Arbeit ist nicht die Unterscheidung von verschiedenen Plansprachen in Projekte, Semiplansprachen und Sprachen an sich interessant, sondern die Kriterien, die Blanke für die Zuordnung eines Systems zu einer der Kategorien benutzt. Er postuliert Phasen (in der ursprünglichen Fassung (1985) waren es 18, hier in der Fassung von 2006 sind es 28), die ein Plansprachenprojekt auf dem Weg zur Etablierung als (vollständige) Sprache durchläuft bzw. durchlaufen kann (D. Blanke 2006, 65-71; vgl. D. Blanke 1989, 69-70):

1. Manuskript $\rightarrow 2$. Veröffentlichung $\rightarrow 3$. Lehrmittel $\rightarrow 4$. Werbung $\rightarrow 5$. Zeitschriften $\rightarrow 6$. Korrespondenz $\rightarrow 7$. Übersetzung und Originaltexte $\rightarrow 8$. mündliche Kommunikation $\rightarrow$ 9. Organisationen $\rightarrow 10$. Zunahme der Textproduktion $\rightarrow 11$. private Kurse $\rightarrow 12$. kleine Sprachgemeinschaft $\rightarrow 13$. Diskussion sprachlicher Fragen $\rightarrow 14$. Fachkommunikation $\rightarrow 15$. Veranstaltungen $\rightarrow 16$. strukturelle Differenzierung der Sprachgemeinschaft $\rightarrow 17$. Herausbildung/Stabilisierung/Kodifizierung der Norm $\rightarrow 18$. Großveranstaltun-

39 Diese Auffassung vertritt teilweise Sakaguchi (1998, 274-277), die allerdings doch noch eine qualitative Unterscheidung zwischen nichtverwirklichten und verwirklichten Plansprachen trifft. 
gen $\rightarrow 19$. weltweite Verbreitung $\rightarrow 20$. Interlinguistik $\rightarrow 21$. heuristische Wirkung $\rightarrow 22$. externe Nutzung $\rightarrow 23$. staatlicher Unterricht $\rightarrow 24$. elektronische Medien $\rightarrow 25$. soziale Differenzierung der Sprachgemeinschaft $\rightarrow 26$. Familiensprache $\rightarrow 27$. Originalkultur $\rightarrow 28$. Sprachwandel

Diese Phasen sind zuerst beschreibend gedacht (vgl. D. Blanke 2006, 65). Ich sehe darin aber eine normative, wenn auch nicht konsekutive Reihenfolge, da die Phasen nummeriert sind und schließlich auch als Maßstab zur Einstufung eines Systems als (vollständige) Sprache von D. Blanke (1985, 2006) benutzt werden. Für jede Plansprache kann man also zum gegebenen Zeitpunkt untersuchen, auf welcher Stufe sie sich befindet und daraus auf ihren Status als Projekt, Semiplansprache oder (vollständige) Sprache schließen. Ein wenig vage erklärt D. Blanke (2006), dass die Stufen ein Versuch sind, „die wichtigsten Informationen aus der Geschichte der Plansprachenbewegung zu verarbeiten und zu verallgemeinern“(D. Blanke 2006, 65). D. Blanke (1989) konstatiert, dass Esperanto als einziges System alle Entwicklungsstufen durchlaufen hat und auch als einzige (vollständige) Sprache gesehen werden kann (D. Blanke 1989, 70). Dies ist aber nicht weiter überraschend: Obwohl die Auswahl und Anordnung der Stufen zu einem gewissen Grad für flexibel gehalten werden (D. Blanke 2006, 65, 71; Oostendorp 2001, 208 209; Schubert 2001b, 6), so sehe ich sie als erheblich durch die EsperantoEntwicklung beeinflusst. Mit anderen Worten, die Entwicklung von Esperanto wird m. E. in diesem Werkzeug zum Maßstab für andere Projekte gemacht.

\subsubsection{Erfolg}

Die Beschäftigung mit dem Erfolg von Plansprachen ist in der interlinguistischen Literatur m. E. noch recht unfokusiert. In den meisten Fällen werden einzelne Aussagen über den Erfolg im Kontext anderer Untersuchungsschwerpunkte gemacht. Ausführlicher behandelt werden lediglich die systemlinguistischen Eigenschaften (s. 2.2.5.2), was aber nur eine von mehreren Erfolgsfacetten darstellt. Umfassender wird der Erfolg des Esperanto von D. Blanke (2009a) besprochen (vgl. D. Blanke 2006, 88-98).

Im Kontext von Esperanto hat für D. Blanke (2009a, 251-252) das Attribut erfolgreich zwei Bedeutungen:

1. Die Tatsache, dass Esperanto sich von einem Plansprachenprojekt zu einer (vollständigen) Sprache entwickelte. Diese Erfolgsaufassung beruht also auf einem soziolinguistischen Faktor der Herausbildung einer vollwertigen Sprechergemeinschaft. 


\section{Forschungsstand}

2. Die Tatsache, dass Esperanto als Vollsprache weiterhin besteht, während andere (konkurrierende) Welthilfssprachen keine Rolle mehr spielen.

D. Blanke (2006, 2009a) differenziert zwischen systemlinguistischen und nichtsystemlinguistischen Faktoren, die für diesen Erfolg entscheidend waren. Im Folgenden behalte ich diese Unterscheidung, widme mich jedoch vorwiegend der Frage nach dem Sprachsystem von Plansprachen, insbesondere der Welthilfssprachen. Anschließend stelle ich kurz die nichtsystemlinguistischen Erfolgsfaktoren nach Blanke vor. Auf weitere Aussagen zum Erfolg von Plansprachen, die ich während der Literaturrecherche festgestellt habe, gehe ich an dieser Stelle nicht ein. Ich werte sie in der Untersuchung in Kapitel 5 aus.

\subsubsection{Erfolg durch systemlinguistische Merkmale}

Eine ausführliche Diskussion von systemlinguistischen Empfehlungen an Plansprachen wird in dieser Arbeit nicht geleistet, da hier der Schwerpunkt eher auf nichtsystemlinguistischen Faktoren liegt. Ich beschränke mich im Folgenden auf das Aufzeigen allgemeiner Diskussionsrichtungen.

Die in der früheren Forschung und durch die Autoren häufig vertretene Auffassung, die systemlinguistische Beschaffenheit spiele eine zentrale Rolle bei der Sprachschöpfung, habe ich bereits erörtert. Die Blütezeit der Welthilfssprachen um die vorletzte Jahrhundertwende ist durch das Bestreben charakterisiert, vorhandene Plansprachensysteme wie Volapük oder Esperanto an Neutralität, leichter Erlernbarkeit etc. (s. 2.2.5.2) zu übertreffen. Man geht hierbei davon aus, dass eine bestimmte Auswahl an morpho-syntaktischen und lexikalischen Mitteln alleine die genannten Eigenschaften verbessern und in Folge zur Etablierung des Systems als offizielles Mittel der internationalen Kommunikation führen kann (D. Blanke 1985, 107; vgl. Jespersen 1930, 158159; Ölberg 1954, 246-249; Schild 1957, 781; Bormann 1958, 283-286). Die allgemeinen Empfehlungen von Sapir u. a. (1925) habe ich bereits in 2.2.5.2 zusammengefasst. Spezielle systemlinguistische Faktoren, die den Erfolg von Esperanto fördern, erörtert D. Blanke (2009a). Er zählt dazu die Vertrautheit des sprachlichen Materials, einfache Phonologie und Rechtschreibung, morphologische Invarianz sowie eine einfache und effiziente Wortbildung, einen flexiblen Umgang mit Entlehnungen, und die nach indoeuropäischer Vorstellung eindeutigen Wortarten (D. Blanke 2009a, 253-254; vgl. Back 1996, 885; Eichner 2012, 143). 
Dass die systemlinguistischen Eigenschaften alleinige Erfolgsfaktoren ausmachen, ist jedoch nach dem heutigen Stand der interlinguistischen Forschung eine Vereinfachung. Ab einem gewissen (nicht näher bestimmten oder bestimmbaren) Grad an Regelmäßigkeit und aposteriorischer Ausrichtung ist eine weitere Systematisierung und Perfektionierung einer Welthilfssprache nicht weiter erfolgsfördernd (vgl. Large 1985, 123, 201; Sakaguchi 1998, 175). Es müssen also weitere Faktoren zum Erfolg einer Sprache beitragen (D. Blanke 1989, 69; Lo Jacomo 1989, 121-122). Andererseits werden in der Literatur explizit die systemlinguistischen Schwächen für den schnellen Untergang von Volapük mit verantwortlich gemacht: "This is not to argue that linguistic merit will automatically ensure the universal adoption of a constructed language: far from it. In the case of Volapük, however, its perceived linguistic weaknesses contributed powerfully to the clash of personalities which quickly destroyed it as an effective aspirant to the role of international language" (Large 1985, 68). Interessanterweise führt bei anderen Plansprachentypen ein komplexer und schwer erlernbarer Aufbau nicht zwangsläufig zum Misserfolg. Die Aufbaukomplexität kann einige Lerner sogar anspornen, weil sie in ihrer Beherrschung eher eine Herausforderung als Hürde sehen (insbesondere bei Lojban aber auch Klingonisch, s. entsprechend Okrent (2009, 238 und 271, 281)). D. Maxwell (1989) bemerkt hinzu, dass die individuelle Begabung und Kapazität eine wichtige Rolle beim Erlernen einer Fremdsprache spielen (D. Maxwell 1989, 102), was man als einen unkontrollierbaren Faktor auch bei der Sprachschöpfung berücksichtigen muss. Andererseits weist Pei (1966) darauf hin, dass sich beim (frühkindlichen) Spracherwerb keine Frage nach schweren oder leichten Systemen stellt und die Verankerung einer Welthilfssprache als Schulfach ihren Erfolg garantieren würde (Pei 1966, 399, 434-436; vgl. Large 1985, 190-191; s. auch Schild 1957, 780). Führt man diesen Gedanken fort, wäre diese Vorgehensweise selbst bei philosophischen Sprachen mit einem abstrakten und komplexen apriorischen Ansatz wie bei Leibniz oder Lojban m. E. viel versprechend.

Zusammenfassend lässt sich festhalten, dass ein regelmäßiges, systematisch aufgebautes Sprachsystem eine notwendige, aber keine hinreichende Bedingung für den Erfolg einer Welthilfssprache ist. Dabei geht es eher um ein bestimmtes Maß an Praktikabilität und nicht um Perfektion. Ein so aufgebautes System ist per se nicht leicht erlernbar, vermittelt jedoch zumindest einen solchen Eindruck, wodurch es sich besser für seine Verbreitung argumentieren lässt (vgl. Large 1985, 182). 


\section{Forschungsstand}

\subsubsection{Erfolg durch nichtsystemlinguistische Faktoren nach Blanke}

D. Blanke (2006) nennt eine Reihe von nichtsystemlinguistischen Erfolgsfaktoren von Esperanto, die hauptsächlich auf das sprachpolitische Handeln von Zamenhof zurückführbar sind. Die Liste ist m. W. die umfassendste Behandlung dieses Themas in der Literatur, stellt aber eine recht unsystematische Aufzählung dar (D. Blanke 2006, 91-98; vgl. D. Blanke 2009a):

1. historische Situation: günstig für Welthilfssprachen, auch schon bei Volapük;

2. Wirkung des Volapük: Abgänger, die an einer Welthilfssprache immer noch interessiert waren, wechselten zu Esperanto;

3. Persönlichkeit und Rolle Zamenhofs und anderer Personen: richtiges Verhältnis zwischen Langue und Parole und zwischen Stabilität und Entwicklung einer Sprache. Zamenhof erkannte die Bedeutung der Bindung der Sprache an die Sprecher sowie die Bedeutung der Norm und ihrer Kodifizierung;

4. ausbaufähige Sprachskizze, die einen kreativen Ausbau durch Sprecher ermöglichte;

5. Einbettung in ein humanistisches Ideal;

6. Sprache als gesellschaftliche Erscheinung: Sprache gehört den Sprechern und ist ihr Produkt;

7. universelle Verwendbarkeit: schichten- und domänenunabhängig;

8. Esperanto als Hilfssprache, nicht zur Ablösung von ethnischen Sprachen;

9. Sprache als Mittel der praktischen Kommunikation; möglichst schneller praktischer Einsatz;

10. Modelltexte mit stabilisierender Wirkung und als Orientierungshilfe für Sprecher;

11. demokratische Entscheidung über Reformen, die 1894 alle abgelehnt wurden;

12. Basisnorm fixiert; 
13. Lehren aus der Ido-Krise gezogen;

14. Entstehung der Esperantologie;

15. konservative Rolle des Lingva Komitato (später Akademio de Esperanto);

16. Ausbau organisatorischer Strukturen;

17. Identitätsgefühl der Sprachgemeinschaft, v. a. durch Auseinandersetzungen mit anderen Plansprachenanhängern;

18. Entstehung eines Quasiethos: Das organisatorische Netzwerk, die geteilte Geschichte sowie Ideale und Traditionen etc. bildeten ein stabiles Quasiethos;

19. wissenschaftliche Beschreibung.

\subsubsection{Diskussion und Zusammenfassung}

Die Unterscheidung zwischen Welthilfssprachen, philosophischen Sprachen und künstlerischen Sprachen ist in der Literatur gut etabliert, obwohl einzelne Typen teilweise unterschiedlich abgegrenzt werden. Darüber hinaus können weitere funktional bestimmte Plansprachentypen berücksichtigt werden, wie experimentellen Sprachen. Plansprachen für Computerspiele sind hingegen ein Beispiel für das von Adams (2011b) genannte Konstruktionsmotiv „Ruhm und Geld“ (Adams 2011b, 12-13). Diese Sprachen haben zwar eine wesentliche ästhetische Komponente, sollen aber m. E. hauptsächlich die Attraktivität und in Konsequenz den Verkauf des Produkts Computerspiel steigern. Die Sicherung materieller Vorteile spielt bei der Weiterentwicklung von Klingonisch eine wichtige Rolle (Okrand u. a. 2011, 121) und ich nehme sie auch für Dothraki an. Möglicherweise handelt es sich hier also um einen weiteren Plansprachentyp.

Das Problem einer eindeutigen Zuordnung einer Plansprache zu den oben genannten Plansprachentypen verdeutlicht, dass eine Plansprache aus einer Kombination von Motiven heraus konstruiert werden kann. Die einzelnen Motive können dabei unterschiedlich stark ausgeprägt sein und die angedachte Funktion kann sich mit ihrem tatsächlichen Einsatzgebiet nur teilweise oder gar nicht überlappen.

Das Phänomen einer Mehrfachzuordnung gehen einige Klassifikationen an. Ein Beispiel ist das sog. Gnoli-Dreieck (Abbildung 4), das auf 


\section{Forschungsstand}

der Conlang-Mailingliste vorgeschlagen wurde (Ray Brown 2006, o.S.). Einzelne Plansprachen können entlang der drei Dimensionen Welthilfssprache, philosophische Sprache ${ }^{40}$ oder künstlerische Sprache je nach Ausprägung eingeordnet werden (Ray Brown 2006).

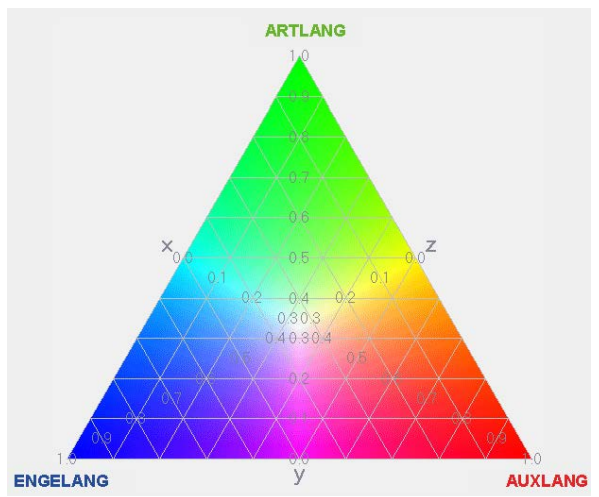

Abbildung 4.: Gnoli-Dreieck zur Einordnung einzelner Plansprachen (Ray Brown 2006)

Am Beispiel von künstlerischen Sprachen erklärt Ray Brown (2006): „The vertical lines show the amount of green (i.e. amount of artlangness); the green vertex is numbered 1.0 and the vertical lines are numbered $0.9,0.8$. 07. etc. down to the y edge which is 0 (no artlangness)“ (Ray Brown 2006, o.S.).

Eine ähnliche Veranschaulichung entlang der Pole Welthilfssprachefiktionale Sprache und geheime-öffentliche Plansprache schlägt Gobbo (2014) vor (s. Abbildung 5). Allerdings werden hier philosophische und weitere Plansprachentypen nicht berücksichtigt.

Die Stärke beider Ansätze ist ihre Multidimensionalität. Insbesondere lässt sich das Gnoli-Dreieck um weitere Motive („Ecken“) beliebig erweitern, ohne dass es an Anschaulichkeit verliert. Als nachteilig sehe ich bei beiden jedoch die mangelnde Operationalisierung, da nicht erläutert wird, wie man die Anteile einer Dimension an einer bestimmten Plansprache berechnet. Die Ansätze veranschaulichen somit das Phänomen der Mehrfachzuordnung selbst, d. h. was es bedeutet, dass eine Plansprache unterschiedliche Konstruktionsmotive besitzt. Sie können aber in der heutigen Form nicht zur Klassifikation oder zum Vergleich von Plansprachen eingesetzt werden.

40 Man beachte die abweichenden Benennungen und Begriffsdefinitionen, wie in der Fußnote 17 (S. 59) und in Abschnitt 2.2.1.4 (S. 64) beschrieben. 


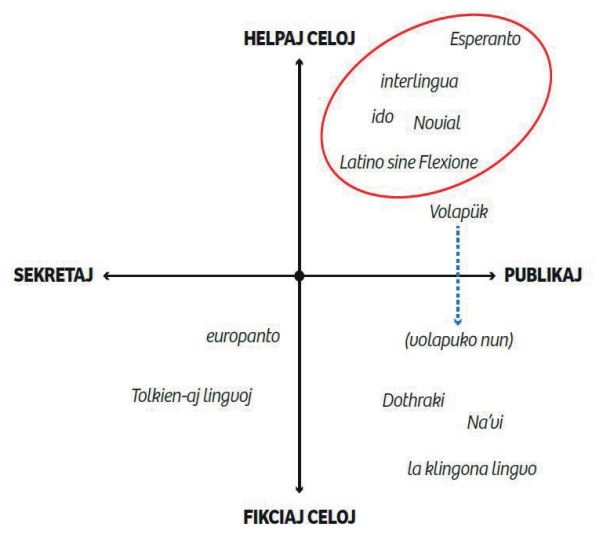

Abbildung 5.: Mehrdimensionale Plansprachenklassifikation von Gobbo $(2014,164)$

Als zweites Ergebnis möchte ich festhalten, welche Plansprachentypen ich in welchem Maße zur Sprachlenkung als Forschungsgegenstand zähle und welche in meine Untersuchung von Handlungsmöglichkeiten bei der Sprachlenkung mit einfließen.

Für Plansprachen, die im Wesentlichen als Welthilfssprachen oder philosophische Sprachen identifiziert werden können, spielen der tatsächliche Einsatz in der realen Kommunikation und der Aufbau einer Sprechergemeinschaft eine zentrale Rolle. Sie eignen sich deshalb gut zur Erforschung von Lenkungsmöglichkeiten, die die Autoren haben und tatsächlich gebrauchen. Diese Plansprachentypen gehören somit zum Kern der Sprachlenkung als Untersuchungsgegenstand.

Die künstlerischen Sprachen scheinen hingegen zunächst einmal ausgeschlossen zu sein, da hier beim Entwurf keine etablierungsfördernden Faktoren berücksichtigt werden müssen. Nach einer genaueren Literaturauswertung stelle ich jedoch fest, dass manche, vor allem fiktionale, Sprachen ungelenkt eine kleine Anzahl an Sprechern gewannen. Dies betrifft vor allem Klingonisch, aber auch in kleinerem Maße die Elbensprachen von J. R. R. Tolkien. Aus verschiedenen Gründen wurden dann von den Autoren bzw. Urhebern oder auch von den Sprechern selbst Aktivitäten initiiert, die das Ziel 


\section{Forschungsstand}

haben, die bestehende Gemeinschaft zu stabilisieren und auszubauen. ${ }^{41}$ Diese Aktivitäten können durchaus Aufschluss darüber geben, wie man die weitere Verbreitung einer Plansprache unterstützen kann. Aus diesem Grund schließe ich die künstlerischen und insbesondere die fiktionalen Sprachen nicht vollständig aus meinen Betrachtungen aus, sondern siedle sie eher am Rande der Sprachlenkung an. Entsprechende Beobachtungen zu Ausbau und Stärkung der Sprechergemeinschaft fließen auch in die Untersuchung in Kapitel 5 mit ein.

Die formalen Sprachen und die Sprachen für interstellare Kommunikation werden in der plansprachlichen Literatur nur am Rand behandelt. Obwohl sie sicherlich interessante Fragen aufwerfen und auch im Kontext der Sprachlenkung berechtigterweise anzusiedeln sind, beziehe ich sie aufgrund der Literaturlage in die Untersuchung nicht mit ein.

Abschließend gehe ich auf die wissenschaftliche Verankerung der Plansprachenforschung ein.

Im Verständnis der Gesellschaft für Interlinguistik e. V. fallen die funktional bestimmten philosophischen und künstlerischen Sprachen nicht unter den Gegenstand der Interlinguistik, da sie, im Unterschied zu den Welthilfssprachen, nicht zum Zweck der internationalen sprachlichen Kommunikation erschaffen wurden. Ich beobachte aber, dass diese Einschränkung lediglich definitorisch ist, da die betrachteten Beispiele durchaus philosophische Sprachen umfassen, die man aber ungeachtet ihrer Konstruktionsmotive als reguläre Welthilfssprachen behandelt. Diese Unstimmigkeit wird beispielsweise an der zitierten etymologischen Klassifikation von Plansprachen (Abbildung 3) deutlich, die auch philosophische Sprachen mit einbezieht. Die Inkonsequenz wird noch dadurch verstärkt, dass die fiktionalen Sprachen aufgrund der Andersartigkeit ihrer Konstruktionsmotive vom Gegenstand der Interlinguistik explizit ausgeschlossen werden (s. Fiedler 2011b, 10-11), das gleiche Ausschlussprinzip jedoch nicht auf philosophische Sprachen angewandt wird. Zwar beschäftigen sich bereits Couturat und Leau (1903) mit verschiedenen Plansprachenprojekten unabhängig von ihren ursprünglichen Entstehungsmotiven. Sie behaupten jedoch nicht, dass alle diese Projekte Welthilfssprachen sind, sondern untersuchen sie auf ihre Tauglichkeit als Welthilfssprachen hin (Couturat und Leau 1903, xxvii-xxviii). In diesem Sinn ist auch die Definition der Interlinguistik von Jespersen (1930) zu verstehen: „(...) der Zweig der Sprachwissenschaft, der sich mit der Struktur und den Grundideen

41 Zu Klingonisch-Aktivitäten von Paramount Pictures im Zusammenarbeit mit dem Linguisten Marc Okrand und der Tätigkeit von Klingon Language Institute siehe beispielsweise: Mannewitz (2002, 111114), Okrent (2009, 264-267; 278-281) und Okrand u. a. (2011, 111-115; 123-127; 132). 
aller Sprachen beschäftigt mit dem Ziel, eine Norm für Plansprachen aufzustellen (...)“ (Jespersen 1930, 148). Ich vermute, dass diese Nuancierung in der neueren interlinguistischen Literatur verloren gegangen ist. Die damit zusammenhängende Verwendung von Plansprachen als Synonym für Welthilfssprachen, wie durch die Gesellschaft für Interlinguistik e. V., aber auch schon durch Wüster (1955), halte ich aus offensichtlichen Gründen für problematisch. Diese inkonsistente Gegenstandbestimmung und auch der intransparente Benennungsgebrauch in der Interlinguistik haben Auswirkungen auf die Theoriebildung, aber auch auf die Stellung und die Bewertung anderer Plansprachentypen, insbesondere der philosophischen Sprachen, innerhalb der Interlinguistik.

Als Resümee halte ich fest, dass einzelne von mir beschriebene Plansprachentypen unterschiedlich ausführlich in der Literatur behandelt werden. Interlinguistik als Teildisziplin der Sprachwissenschaft untersucht die Welthilfssprachen aus der soziolinguistischen Perspektive. Sie wendet sich auch anderen Plansprachentypen, insbesondere den philosophischen Sprachen zu, ohne Anspruch auf eine funktionsgerechte Beschreibung. Die Beschäftigung mit anderen Plansprachentypen findet vorwiegend aus der sprachwissenschaftlichen Laienperspektive statt, wobei auch hier ein adäquates wissenschaftliches Niveau angestrebt wird. Es scheint also keine Teildisziplin der Linguistik zu geben, die sich mit allen Plansprachentypen übergreifend auseinandersetzt. Wissenschaftliche Beschäftigung mit speziellen Fragen der Plansprachen außerhalb der Interlinguistik bleiben also weitestgehend Einzeluntersuchungen.

\subsection{Kontrollierte Sprachen}

Den dritten Untersuchungsgegenstand dieser Arbeit bilden Kontrollierte Sprachen. Im Folgenden fasse ich den Forschungsgegenstand in diesem Gebiet zusammen, wobei ich wie in vorangegangenen Gebieten auf die Punkte allgemeine Gegenstandsbestimmung (2.3.1), Geschichte (2.3.2), wissenschaftliche Erforschung (2.3.3), Autoren (2.3.4), Modelle (2.3.5) sowie Erfolg (2.3.6) eingehe.

\subsubsection{Gegenstand}

In diesem Abschnitt charakterisiere ich Kontrollierte Sprachen im Allgemeinen. Auf die Definition und Abgrenzung des Gegenstandes entsprechend in 


\section{Forschungsstand}

2.3.1.1 und 2.3.1.2 folgt eine Differenzierung in textorientierte und repräsentationsorientierte Kontrollierte Sprachen, die ich jeweils bezüglich ihrer Funktionen, Einsatzbereiche, Zielgruppen und verfügbaren sprachtechnologischen Werkzeuge näher beschreibe.

\subsubsection{Definition}

Der Definition von Kontrollierten Sprachen (KS) legt man die intuitive Vorstellung von (vollen) natürlichen Sprachen wie Deutsch, Englisch etc. zugrunde. Gemäß der verbreitetsten Auffassung restringiert eine Kontrollierte Sprache eine bestimmte (volle) natürliche Sprache auf einzelnen Ebenen des Sprachsystems (Rechtschreibung-Text). Somit wird eine Kontrollierte Sprache als Teilmenge einer natürlichen Sprache aufgefasst: Da die KS nur bestimmte grammatische Regeln und bestimmte Wörter zulässt, andere aber verbietet, bilden die Aussagen, die in einer KS zugelassen sind, eine Teilmenge aller wohlgeformten Aussagen in der vollen natürlichen Sprache. ${ }^{42}$ Dabei wird betont, dass eine KS durchaus Ausdrücke zulassen kann, die standardsprachlich zwar unüblich sind, aber in einer bestimmten Varietät, meistens in einer Fachsprache, durchaus als wohlgeformt gelten (vgl. Schwitter 1998, 57).

Die Auffassung von KS als restringierte natürliche Sprachen ist anschaulich und trifft auf die meisten KS zu. Kuhn $(2014,123)$ bemerkt jedoch, dass dies in bestimmten Fällen eine Vereinfachung darstellt. So arbeiten manche KS mit Konventionen, die in einer vollen natürlichen Sprache nicht belegt sind (vgl. Pool 2006, 1). Ein Beispiel sind die farbigen Auszeichnungen in KS, die auf Grundlage der SBVR-Spezifikation definiert sind (OMG 2008; vgl. Kuhn 2014, 123). Um das ganze Spektrum von KS abzudecken, müssen also nicht nur die restriktiven, sondern in Ausnahmefällen auch die konstruktiven Elemente berücksichtigt werden (Schubert 2014, 212).

In diesem Teilkapitel schließe ich mich weitestgehend der Definition von Kontrollierten Sprachen von Kuhn (2014, 123, Hervorhebungen im Original) an, die hier in der langen Fassung angeführt ist:

„A language is called a controlled natural language if and only if it has all of the following four properties:

1. It is based on exactly one natural language (its "base language").

42 Vgl. Huijsen (1998b, 2), Schwitter (1998, 57), Kaji (1999, 37), Knops (1999, 1), Kittredge (2003, 441), Wyner u. a. (2010, 281), Pace und Rosner (2010, 227), Schwitter (2010b, 1113), Smart u. a. (2010, 207), Schubert (2011b, 52-53) und Kaljurand (2012, 110-111) 
2. The most important difference between it and its base language (but not necessarily the only one) is that it is more restrictive concerning lexicon, syntax, and/or semantics.

3. It preserves most of the natural properties of its base language, so that speakers of the base language can intuitively and correctly understand texts in the controlled natural language, at least to a substantial degree.

4. It is a constructed language, which means that it is explicitly and consciously defined, and is not the product of an implicit and natural process (even though it is based on a natural language that is the product of an implicit and natural process).“

Die meisten KS sind für schriftliche Kommunikation konzipiert und auf diesen KS liegt auch der Schwerpunkt dieser Arbeit. Mündliche KS existieren ebenfalls, für eine Übersicht s. Klassifikation von Kuhn (2014). Davon zu unterscheiden sind hingegen weitere Phänomene. Beispielsweise stellen Spaggiari und Cardey (2010) eine gesprochene, auf Englisch basierende KS vor, die das Ziel hat, für Nichtmuttersprachler besser verständlich zu sein. Dabei wird in die phonetische Ebene eingegriffen: Englische Phoneme, die in der jeweiligen Sprache keine Entsprechungen haben, werden durch ein ähnliches, in der Sprache vorhandenes, Phonem ersetzt. Es stellt sich somit die Frage, ob die so entstandene Sprache von der zugrunde liegenden natürlichen Sprache zu weit entfernt ist, und gemäß der Definition von Kuhn nicht als KS aufgefasst werden kann.

\subsubsection{Verwandte Begriffe}

Kontrollierte Sprachen basieren auf unterschiedlichen natürlichen Sprachen wie Englisch, Deutsch, Französisch, Spanisch, Portugiesisch (Marrafa u. a. 2012), Griechisch, Italienisch, Schwedisch (Almqvist und Sågvall Hein 1996), Dänisch (Andersen und Christoffersen 2006), Lettisch (Barzdins 2014), Chinesisch (Cardey, Greenfield und Wu 2004). ${ }^{43}$ Neben unterschiedlichen Benennungen im Deutschen: kontrollierte, regulierte, restringierte und vereinfachte Sprachen und im Englischen: controlled, simplified, rationalized languages (Schubert 2014, 211) existieren auch unterschiedliche Begriffe, die zwar verwandt sind, aber einen anderen

43 Wenn nicht direkt belegt, beruhen die Sprachangaben auf Hebling $(2002,61)$ und Ramírez Polo (2012,91). 


\section{Forschungsstand}

Schwerpunkt setzten. Kuhn (2014, 124-125) geht auf folgende Begriffe ein, ohne dabei eine trennscharfe Abgrenzung zu KS zu beanspruchen:

- Fachsprachen (sublanguages): Kuhn nimmt eine soziolinguistische Perspektive ein und sieht Fachsprachen als Varietät, die durch Übereinkunft im Kontext fachlicher Handlungen entsteht. Der wesentliche Unterschied zu Kontrollierten Sprachen besteht für ihn in der ungelenkten (natural) Entstehung der Fachsprachen, während KS explizit und bewusst definiert werden (Kuhn 2014, 124; vgl. Schwitter 1998, 53-54; Namahn 2002, 1; Kittredge 2003). Im Unterschied dazu diskutiert Ramírez Polo (2012, 48-58) im Kontext von Kontrollierten Sprachen eine strukturalistische Auffassung von sublanguages als Teilmenge einer natürlichen Sprache in Anlehnung an Harris (bspw. Harris 1968; vgl. Eijk, Koning und Steen 1996, 65-66). Auf eine enge Beziehung zwischen Fachsprachen und Kontrollierten Sprachen machen beispielsweise Holterhus und Meister (2001) aufmerksam. Sie benutzen die Benennungen Kontrollierte Sprache und Kontrollierte Fachsprache synonym und weisen somit indirekt darauf hin, dass eine Kontrollierte Sprache auf Grundlage einer Fachsprache und nicht der vollen natürlichen Sprache bzw. der Standardsprache definiert wird (Holterhus und Meister 2001, 16, 22; vgl. Schwitter 1998, 53-54; Ramírez Polo 2012, 60-61). Wie eingangs erwähnt, kann eine KS also Phänomene enthalten, die standardsprachlich zwar untypisch, fachsprachlich jedoch wohlgeformt sind. Kittredge (2003) betont hingegen, dass viele, vor allem syntaktische, Phänomene einer Fachsprache, die für den Außenstehenden schwer verständlich sind, bei der Definition einer KS abgebaut werden: „Thus a controlled language can be seen as the result of two operations on technical sublanguage, (1) paraphrasing technical texts into „normal“ standard language, and then (2) paraphrasing the normalized texts into a simpler form through use of a restricted set of words and structures." (Kittredge 2003, 442; vgl. Ramírez Polo 2012, 61). Demzufolge muss eine Kontrollierte Sprache die typischen (syntaktischen und lexikalischen) Elemente einer Fachsprache nicht zwangsläufig beibehalten.

- Sprachfragmente (fragments of language), gebraucht bspw. von Pratt-Hartmann und Third (2006), Bernardi, Calvanese und Thorne (2007, 196-197), Kaljurand $(2012,111)$ und Thorne, Bernardi und 
Calvanese $(2014,151)$. Kuhn $(2014,124)$ betont die Nähe des Begriffes zu Kontrollierten Sprachen, sieht aber den Unterschied in der Entstehungsweise beider Systeme: Sprachfragmente werden in der Menge der natürlichsprachlichen Aussagen identifiziert, während Kontrollierte Sprachen definiert werden (vgl. Kuhn 2010b, 16).

- Schreibregeln (style guides): Kuhn $(2014,124)$ sieht Schreibregeln eher als Empfehlungen, während Kontrollierte Sprachen neue Sprachen darstellen. Diese Sicht wird von Schubert (2009, 2013) präziser ausgedrückt: Solche Empfehlungen wie ,(...) gesetzliche und behördliche Vorschriften, Normen der Normungsinstitute oder normenähnliche Empfehlungen anderer Verbände (...)“ (Schubert 2009, 132) sind Eingriffe in die Kommunikationspraxis (parole), während Kontrollierte Sprachen, die zu sog. gestalteten Sprachen zählen, neue Sprachsysteme (langue) darstellen (Schubert 2013, 55). Für Kuhn (2014) ist zur Abgrenzung von Schreibregeln und Kontrollierten Sprachen entscheidend, dass die ersten deskriptive und ungelenkt (naturally) entstandene Best-Practice-Empfehlungen sind. Schubert (2009) weist jedoch darauf hin, dass die Verbindlichkeit solcher Empfehlungen durch Selbst- oder Fremdverpflichtung entstehen kann (Schubert 2009, 132). Als Beispiel nenne ich hier die Selbstverpflichtung zur Einhaltung bestimmter Richtlinien, die werbewirksam ist und zur Steigerung der Wettbewerbsfähigkeit genutzt werden kann. Darüber hinaus können die syntaktischen und stilistischen KS-Regeln durch schriftliches Festhalten der bereits existierenden, ungelenkt entstandenen Praxis, definiert werden, wie ich für Koenig \& Bauer Group berichtete (Suchowolec 2014). Folglich ist die Auffassung eines Phänomens als KS oder Schreibregeln oft nur synchron möglich, da ein fließender Übergang zwischen Schreibregeln und Kontrollierten Sprachen besteht (Suchowolec 2014, 121; vgl. Schaubild in Schubert 2014, 210)

- Standardisierter Wortschatz (controlled vocabularies) deckt lediglich die lexikalische Ebene einer Sprache ab und kann als Bestandteil einer Kontrollierten Sprache aufgefasst werden (Kuhn 2014, 124). Es kann sich also hierbei um das Ergebnis einer Terminologiearbeit handeln, auf die ich in Abschnitt 2.4 eingehe.

- Konstruierte Sprachen (constructed languages) sind alle Sprachen, die nicht natürlich entstehen, sondern bewusst (consciously) definiert 


\section{Forschungsstand}

werden. Somit sind für Kuhn $(2014,125)$ Kontrollierte Sprachen ein Unterbegriff von Plansprachen. Zu betonen ist jedoch, dass er zu Plansprachen ebenfalls Programmiersprachen zählt, den Begriff also nicht nur auf die Mensch-Mensch-Kommunikation beschränkt.

Des Weiteren geht Kuhn auf Phraseologie ein, die ich hier nicht näher erläutere.

\subsubsection{Charakterisierung}

Unterscheidung nach Eingriffsobjekt Die Konstruktion und der Einsatz von Kontrollierten Sprachen erfolgen vorwiegend im fachkommunikativen Kontext, vor allem bei einer asymmetrischen Kommunikation zwischen Experten unterschiedlicher Fachgebiete oder zwischen Experten und Nichtexperten/Laien (vgl. Huijsen 1998b, 1; Holterhus und Meister 2001, 16). Da eine Kontrollierte Sprache die Redundanz, Vagheit und Vielfalt der natürlichen Sprache reduziert und die (Ein-)Eindeutigkeit des Ausdrucks steigert, ${ }^{44}$ wird sie im Allgemeinen zur Verbesserung von Kommunikationsprozessen sowohl beim Sender als auch beim Empfänger eingesetzt.

Die spezifischen Konstruktionsmotive bzw. Ziele, Funktionen und Einsatzmöglichkeiten von KS gehen mit ihren sprachtypologischen Eigenschaften einher und hängen auch damit zusammen, ob eine Kontrollierte Sprache ausschließlich zur Produktion und Rezeption von Texten oder auch (vorwiegend) zur Produktion und Rezeption von formalen Repräsentationen verwendet wird. Wie in Suchowolec (o.D. $)^{45}$ unterscheide ich deshalb:

- textorientierte KS

- repräsentationsorientierte KS

Im Folgenden charakterisiere ich beide Gruppen bezüglich ihrer Funktion, Zielgruppen, Einsatzbereiche und der unterstützenden Werkzeuge.

44 Huijsen (1998b, 2), Kaji (1999, 37), Holterhus und Meister (2001, 2, 41-42), Nyberg, Mitamura und Huijsen (2003, 246), Ley (2005, 27), Schwitter und Tilbrook (2006, 2), Fiederer und O'Brien (2009, 53), Schwitter (2010b, 1113, 2010a, 36) und Ramírez Polo (2012, 45)

45 In den Abschnitten Textorientierte KS (S. 103), Funktionen (S. 103), Repräsentationsorientierte KS (S. 109) und Funktionen (S. 110) verzichte ich weitestgehend auf Beispiele und Quellenangaben, da sie in Suchowolec (o.D.) angegeben werden. 
Textorientierte KS Die textorientierten KS entstehen, um die Textproduktion und die Textrezeption im Fachkontext zu verbessern. Speziell handelt es sich aus der prozeduralen Perspektive um die Verbesserung der Texterstellung und der Textübersetzung auf der Produktionsseite und um die Verbesserung der Textverständlichkeit (d. h. des Aufbaus mentaler Modelle) und der (maschinellen) Textverarbeitung auf der Rezeptionsseite. Ich benutze hier Lesbarkeit für die textimmanente Eigenschaft, die sich aus solchen Merkmalen wie Konsistenz, Eindeutigkeit, logischer/chronologischer Aufbau etc. ergibt, und Verständlichkeit für den kognitiven Prozess, der auf den Aufbau von mentalen Modellen zielt und sowohl Bottom-up-Verarbeitung (im Wesentlichen: textgeleitet) als auch Top-down-Verarbeitung (im Wesentlichen: vorwissengeleitet) umfasst (vgl. exemplarisch Ley 2005, 8-26). Resultativ können die o.g. Motive entsprechend formuliert werden als Verbesserung der Texte und ihrer Übersetzungen an sich (also Verbesserung der Lesbarkeit) und die Verbesserung des Rezipientenwissens über die Textinhalte sowie, in Konsequenz, die Verbesserung der Rezipientenhandlung bezüglich der Textinhalte.

Funktionen Typischerweise werden textorientierte KS im Bereich der Technischen Dokumentation im weitesten Sinne verwendet (R. H. Wojcik und J. E. Hoard 1997, 238; Ramírez Polo 2012, 61). R. Wojcik, Holmback und J. Hoard (1998) sehen vor allem anleitende Texte als besonders geeignet für den KS-Einsatz, da deskriptive Texte schwieriger zu standardisieren sind: „In general, descriptive writing contains a more diverse use of language and more document domain types than procedural language. The vocabulary issue seems more difficult to manage than within the aircraft maintenance domain, and this is likely true for word senses as well. There will probably be more word sense ambiguities that arise in descriptive technical documents than in maintenance manuals" (R. Wojcik, Holmback und J. Hoard 1998, 121-122; vgl. Lehrndorfer 1996, 15). Trotzdem ist die Verwendung von KS für folgende Textsorten belegt: Bedienungs-/Betriebsanleitungen, Montageanleitungen, Serviceanleitungen, aber auch Montagepläne, Konstruktionszeichnungen, Melde- und Maskentexte in Software und teilweise Marketingtexte (wie Onlinekataloge etc.), wobei bei den letzten oft nur eine Auswahl an KS-Regeln verwendet wird.

Die Verbesserung der Textproduktion und -rezeption mithilfe einer KS kann aus der Sicht eines Unternehmens in einem breiteren Kontext gesehen werden. Da einerseits die Anzahl und die Komplexität der von Unternehmen angebotenen Produkte steigt und die Unternehmen andererseits durch 


\section{Forschungsstand}

Normen, Richtlinien etc. dazu verpflichtet sind, die Produkte in einer bestimmten Art für Kunden zu beschreiben, wird die Beherrschung des sich ergebenen Volumens und der Komplexität der Produkttexte zu einer wichtigen wirtschaftlichen Frage, welche sowohl die Textproduktion wie auch die Textrezeption betrifft.

Eine KS ist also auf der Textproduktionsseite eine der Maßnahmen zur Standardisierung der Arbeitsprozesse (vgl. Lehrndorfer 1996, 13): Alle Textproduzenten haben gleiche, schriftlich festgehaltene Vorgaben, wie die Texte, v. a. sprachlich, zu gestalten sind, so dass weniger Zeit auf die sich wiederholende Aushandlung eines einheitlichen Schreibstils verwendet werden muss. Diese Standardisierung kann darüber hinaus zusätzlich durch Modularisierungstechnologie und Single-Source-Ansätze unterstützt werden, bei denen eine KS ein reibungsloses Zusammenspiel der einzeln erstellten Informationseinheiten sicherstellt. Wird aus einzelnen Informationseinheiten ein Text zusammengestellt, sorgen der konsistente Satzbau und Wortschatz für die Textkohäsion und -kohärenz. Der Initialaufwand bei der Entwicklung und Einführung einer KS ist hoch: Die Textproduzenten brauchen anfangs mehr Zeit für die Erstellung der Texte aufgrund einer Umgewöhnung, aber auch eines notwendigen Lektoratschritts zur Überprüfung der KS-Einhaltung. ${ }^{46}$ Trotzdem wird davon ausgegangen, dass mittelfristig die Vorteile überwiegen (vgl. Hebling 2002, 10). Außerdem können die anfänglichen Produktivitätsverluste nicht immer eindeutig der KS-Einführung zugeschrieben werden, weil oft andere Maßnahmen zur Prozessstandardisierung wie ein Content Management System, ein neuer Texteditor etc. gleichzeitig eingeführt werden (vgl. Kamprath u. a. 1998, 53-54; Suchowolec 2014, 113-114).

Ein effizienterer Erstellungsprozess sowie gesteigerte Konsistenz und Eindeutigkeit des (Ausgangs-)Textes wirken sich auch positiv auf den Übersetzungsprozess und sein Ergebnis aus. Einerseits entlastet man den Übersetzungsprozess zeitlich beispielsweise durch den Einsatz von maschinellen Übersetzungssystemen (MT), bei denen der Gebrauch einer KS in der (Ausgangs-)Texterstellung als Pre-Editing aufgefasst wird. Durch abgebaute Vagheit und Redundanz werden bessere Übersetzungsergebnisse erwartet, so dass weniger Post-Editing notwendig ist. Darüber hinaus profitiert von einer KS auch der menschliche Übersetzer, da man annehmen kann, dass ein konsistenter Text nicht nur für den Produktbenutzer, sondern auch für den Übersetzer verständlicher ist. Außerdem erhöht ein konsistenter

46 Vgl. Douglas und Hurst (1996, 94), Goyvaerts (1996, 139), Huijsen (1998b, 2-4), Hebling (2002, 10, 54-56), Namahn (2002, 2), Kittredge (2003, 443), Crabbe $(2009,58)$ und Ramírez Polo $(2012,62)$ 
Text den Übereinstimmungswert von Segmenten in Translation Memorys (TM). Die Zeitersparnisse und der Einsatz von Werkzeugen sollen auch insgesamt die Reduktion von Übersetzungskosten bewirken.

Das mithilfe einer KS verbesserte Textproduktionsergebnis hat einen Einfluss auf die Textrezeption, was, wie erwähnt, sowohl auf die an der Texterstellung beteiligten Personen als auch auf die eigentliche Zielgruppe der Produktbenutzer zutrifft. Die Lesbarkeit und die Verständlichkeit sollen letztendlich zum Aufbau von korrekten mentalen Modellen, d. h. zum korrekten Wissen über das Produkt führen, und, aus der Sicht der Produktbenutzer, eine korrekte Interaktion mit dem Produkt (Kauf, Bedienung, Wartung, Pflege, Fehlerbehebung, etc.) fördern. Aus der auf dem KS-Einsatz beruhenden verbesserten Interaktion mit Produkten ergeben sich für Unternehmen weitere Vorteile wie weniger Rückfragen, weniger Fehlgebrauch, verbesserte Rechtssicherheit, höhere Kundenzufriedenheit und insgesamt gesteigerte Wettbewerbsfähigkeit. Diese Vorteile werden aber m. W. nicht als primäre Ziele oder Auslöser bei Konstruktion und Einsatz von KS betrachtet.

Einsatzbereiche Der Einsatz von textorientierten KS wird in unterschiedlichen Bereichen (Domänen) getestet und umgesetzt, die ich hier ohne Anspruch auf Vollständigkeit aufliste:

- Luft- und Raumfahrt: ASD-STE (exemplarisch: ASD-STE100 2013), Boeing Technical English (BTE) (R. H. Wojcik und Holmback 1996; R. Wojcik, Holmback und J. Hoard 1998), Français Rationalisé (Barthe 1998) (vgl. Hebling 2002, 7; Drewer und Ziegler 2011, 200-205, 213214)

- Maschinenbau

- Baumaschinen/Motoren: Caterpillar (Gallup 1993; Kamprath u. a. 1998; Nyberg, Mitamura und Huijsen 2003)

- Automobilindustrie (Haller 2000; vgl. Hebling 2002, 2): BMW (Reuther 1998; Reuther und Schmidt-Wigger 2000), Scania (Almqvist und Sågvall Hein 1996), General Motors (CASL) (L. Means und Godden 1996; Godden 1998, 2000), Ford (Rychtyckyj 2006)

- Druckindustrie: Océ (vgl. Kuhn 2014, 159), Koenig \& Bauer Group (Suchowolec 2014)

- Pumpenbau: Grundfos (Andersen und Christoffersen 2006) 


\section{Forschungsstand}

- juristische Texte: Gesetze, Richtlinien, etc. (Bünzli und Höfler 2012; Höfler 2012; vgl. Hebling 2002, 7), Patentanträge (Sheremetyeva 2006)

- internationale Notdiensteinsätze (Kommunikation zwischen Polizei, Rettungsdiensten und assozierten Institutionen, PoliceSpeak) (E. Johnson 1996; vgl. Ramírez Polo 2012, 90; Kuhn 2014, 161)

- Telekommunikation: Alcatel (Goyvaerts 1996; vgl. Kuhn 2014, 148)

- Seefahrt: Seaspeak (Strevens und E. Johnson 1983)

- Wettervorhersagen: Lawinenwarnungen (Winkler, Kuhn und Volk 2014)

- Onlineshopping (Onlinekataloge) (Lehtola, Tenni und Bounsaythip 1998)

- Informationsmanagement: bibliographische Zusammenfassungen von Literatur (Blamoutier 1977)

- Medizin (vgl. Hebling 2002, 7)

- Krisenmanagement (vgl. Kuhn 2014, 153)

Zielgruppen Aus den oben beschriebenen Funktionen und Einsatzbereichen kann man Gruppen identifizieren, die vom Einsatz einer Kontrollierten Sprache betroffen sind. Für die textorientierten KS ist es sinnvoll, zwischen Gruppen zu unterscheiden, die die KS-Spezifikation produktiv beherrschen müssen, und solchen, die lediglich mit den Ergebnissen der Textproduktion in Kontakt kommen und somit der KS rezeptiv ausgesetzt werden (vgl. Kuhn 2010b, 20; Schwitter 2010b, 1120).

Die aktive Beherrschung einer KS betrifft somit die Textproduzenten, ${ }^{47}$ in erster Linie also Technische Redakteure, (Technische) Übersetzer, Lektoren und Posteditoren (bspw. Cardey, Greenfield und Wu 2004; Allen und Hogan 2000; Drewer und Ziegler 2011, 197-198; Ramírez Polo 2012, 61). ${ }^{48}$ Gelegentlich wird zudem postuliert, dass auch andere Unternehmensbereiche wie Marketing von einer Kontrollierten Sprache (oder zumindest einer

47 Die Textproduktion und die Textrezeption bedingen sich gegenseitig, da die Produzenten den Text im Produktionsprozess ebenfalls rezipieren.

48 Zur allgemeinen Beschreibung der Berufsfelder s. beispielsweise Lehrndorfer (1996, 81-87), Schubert (2007, 102-103), Drewer und Ziegler (2011, 20-24) und Ramírez Polo (2012, 117, 120, 210-211, 216). 
stärkeren Sprachstandardisierung) profitieren können (Suchowolec 2014; vgl. Moore 2000, 61). Bei diesen Zielgruppen handelt es sich heutzutage also um Menschen, die sich der Texterstellung beruflich widmen (vgl. Grover u. a. 2000, 92). Darüber hinaus wird gefordert, dass das Personal, das KS-Schulungen durchführt, ebenfalls über Expertenkenntnisse der KS-Spezifikation verfügen muss (L. G. Means, Chapman und Liu 2000, 1, 13). Zudem wird empfohlen, dass darüber hinaus Arbeitsgruppenleiter und Übersetzungsmanager mit den KS-Regeln allgemein vertraut sein sollten, allerdings ohne Expertenkenntnisse anzustreben (L. G. Means, Chapman und Liu 2000, 5) ${ }^{49}$ Dabei sei zu betonen, dass der Einsatz einer KS nicht jede Textsorte der o.g. Produzenten gleichermaßen betreffen muss, sondern meistens für vorab festgelegte Textsorten verpflichtend ist (Lehrndorfer 1996, 5; vgl. R. Wojcik, Holmback und J. Hoard 1998, 121-122). Für andere Textsorten, wie beispielsweise unternehmensinterne E-Mails, kann der Einsatz einer KS nicht verpflichtend sein, sondern soll freiwillig erfolgen.

Darüber hinaus gibt es auch KS-Projekte, die nicht auf hauptberufliche Textproduzenten, sondern auf sog. Domänenexperten zielen, wie beispielsweise die Polizei (E. Johnson 1996), Wetterdienste (Winkler, Kuhn und Volk 2014), Softwareentwickler und Konstrukteure (Suchowolec 2014, 118). M. W. prägten solche Projekte die Literatur zu textorientierten Kontrollierten Sprachen jedoch lediglich in kleinerem Maße.

Die rezeptive Zielgruppe von textorientierten Kontrollierten Sprachen sind die eigentlichen Leser der Texte. Dabei handelt es sich abhängig vom Text um Kunden bzw. Produktbenutzer, Wartungs- und Servicemitarbeiter, etc. Diese Personen kommen zwar mit dem KS-Text in Kontakt, müssen aber die KS-Spezifikation nicht kennen, sondern lediglich die fachsprachlichen Konventionen und insbesondere die Lexik beherrschen (vgl. Bernth 1998b, 192; Fawcett 2005, o.S. Kuhn 2010b, 20; Schwitter 2010b, 1120).

Controlled Language Checker Nach der Konstruktion einer KS ist aus der Sicht der produktiven Zielgruppe vor allem die Einhaltung dieser Spezifikation entscheidend. Neben der manuellen Kontrolle durch menschliches Lektorat setzten sich in der Praxis zu diesem Zwecke die sog. Controlled Language Checker (CLC) durch. Dabei handelt es sich um Software, die die Übereinstimmung des Textes mit den KS-Regeln auf verschiedenen Ebenen des Sprachsystems prüft (Huijsen 1998b, 7; Kaji 1999, 39; Holterhus und

49 Für eine Übersicht, wie sich die Schulungsinhalte für diese unterschiedlichen Zielgruppen unterschieden, s. beispielsweise Tabelle in Hebling $(2002,146)$. 


\section{Forschungsstand}

Meister 2001, 28; Nyberg, Mitamura und Huijsen 2003, 251-252; Fawcett 2005, o.S. Møller und Christoffersen 2006, 26-27; Ramírez Polo 2012, 59, 83-84). In der Praxis hat sich die Unterscheidung zwischen der orthographischen, grammatischen, lexikalischen und stilistischen Ebene eingebürgert (Knops 1999, 43; vgl. Holterhus und Meister 2001, 28). Frühere CLC gaben bei einer nicht KS-konformen Formulierung lediglich eine Warnung aus (vgl. Eijk, Koning und Steen 1996, 68-69), die modernen Systeme bieten hingegen mehr Unterstützung, von aussagekräftigeren Meldungen mit detaillierten Fehlerbeschreibungen, über Verbesserungsvorschläge bis hin zu einer vollständigen Autokorrektur (Ramírez Polo 2012, 94; vgl. Kaji 1999, 29; vgl. Fawcett 2005, o.S.).

Neben dem Elaboriertheitsgrad der Korrekturfunktion unterscheiden sich CLC auch hinsichtlich des Zeitpunktes, wann die Konsistenzprüfung durchgeführt wird: während oder nach der Texterstellung. Hierbei unterscheiden Marrafa u. a. (2012) grob zwischen: ,(...) editors (that help users to write texts that conform to the CNL on the fly); converters (that convert natural language texts into texts in CNL format); and checkers (that check whether the text produced by the user conforms to the CNL)" (Marrafa u. a. 2012, 162). Außerdem zeichnen sich CLC durch einen hohen Grad an Kundenspezifik aus. Bei früheren Lösungen handelte es sich oft um unternehmensinterne Eigenentwicklungen für die eigene KS-Spezifikation (z. B. KANT Analyzer für CFE (Kamprath u. a. 1998, 55)). Heutzutage setzen sich zunehmend kommerzielle Lösungen durch (z. B. Acrolinx (2015-), Congree (2015-) (zu Letzterem s. auch Ramírez Polo 2012)), die auf verschiedene KS-Spezifikationen (unterschiedliche Unternehmen) oder unterschiedliche Prüfszenarien (z. B. nur Grammatikprüfung, nur Lexikprüfung in einem Unternehmen) zugeschnitten werden können (Schmidt-Wigger 1998, 76; Fawcett 2005, o.S. Ramírez Polo 2012, 95-96).

Der Einsatz eines CLC zur Konformitätsprüfung ist aus mehreren Gründen sinnvoll. In erster Linie geht es um die kognitive Entlastung bei der KS-Anwendung, weil einerseits die KS-Regeln schwer zu behalten sind (R. H. Wojcik und Holmback 1996, 22-23; Hebling 2002, 29; Marrafa u. a. 2012, 162; Ramírez Polo 2012, 93-94) und andererseits damit auch die Flüchtigkeits- oder andere Performance-Fehler (Chomsky 1972, 13-14) behoben werden können. Zusätzlich wird darauf hingewiesen, dass ein CLC Zeit und Kosten von KS-Schulungen reduziert (R. Wojcik, Holmback und J. Hoard 1998, 122; Knops 1999, 40). Des Weiteren hat ein CLC gegenüber dem menschlichen Lektorat auch einen psychologischen Vorteil, 
da sein Feedback als reproduzierbar, neutral, unpersönlich, objektiv und situationsunabhängig wahrgenommen wird (Ramírez Polo 2012, 95-96; vgl. Drewer und Ziegler 2011, 253-255). Nicht zuletzt zählen Drewer und Ziegler (2011) Prüfstatistiken und -berichte zu den Vorteilen eines CLC, wodurch Fehler im Regelwerk entdeckt werden können und eine Anpassung des Erstellungs- und Freigabeprozesses für Texte möglich ist (Drewer und Ziegler 2011, 253-255).

Abschließend lässt sich sagen, dass der Einsatz eines CLC die Einhaltung der KS-Spezifikation entscheidend erleichtern, aber nicht vollständig sicherstellen kann. Aufgrund der Beschaffenheit der Regelwerke von textorientierten KS sind die KS-Texte nicht vollständig frei von Vagheit, Redundanzen und Kontextsensitivität der natürlichen Sprache (vgl. Huijsen 1998b, 11; s. auch Schwitter 2010b, 1119). Der heutige Stand der Sprachverarbeitungstechnologie ermöglicht es aber nicht, alle Uneindeutigkeiten automatisch aufzulösen und alle Fehler aufzudecken. In Konsequenz ist ein sinnvoller Korrekturvorschlag nicht immer möglich. Neben quantitativen Qualitätskriterien von CLC wie precision, recall und convergence (Adriaens und Macken 1995, 123, 126, 140; vgl. Nyberg, Mitamura und Huijsen 2003; Ramírez Polo 2012, 138) wird deshalb postuliert, dass ein CLC eine gründliche Schulung der Textproduzenten in KS-Spezifikation nicht ersetzt (Moore 2000, 55-59) und eine Autokorrektur nicht sinnvoll ist (Knops 1999, 42; Holterhus und Meister 2001, 2930). Die Textproduzenten sollen deshalb die Entscheidungshoheit im Korrekturprozess behalten, was nicht nur aus den o.g. technischen Gründen wichtig ist, sondern sich auch auf die Akzeptanz des CLC und in Konsequenz der gesamten KS positiv auswirkt (Schwitter 1998, 51; Knops 1999, 42; Holterhus und Meister 2001, 29-30). Da der Einsatz eines CLC eine Anpassung von Arbeitsprozessen bedeutet, was, wie erwähnt, anfangs zu längeren Textproduktionszeiten führen kann (Hayes, S. Maxwell und Schmandt 1996, 86; L. Means und Godden 1996, 111; Lehrndorfer 1996, 47; Kaji 1999, 37; Ramírez Polo 2012, 62), soll er zudem in die vorhandene Softwareumgebung wie auch der Korrekturprozess in die bestehenden Arbeitsprozesse gut integriert sein (Knops 1999, 43; Bredenkamp, Crysmann und Petrea 2000, 83; Ramírez Polo 2012, 63-64).

Repräsentationsorientierte KS Repräsentationsorientierte KS entstehen, um die Produktion und Rezeption von Daten zu erleichtern, die in einer formalisierten und strukturierten Form (insbesondere also nicht als natürlichsprachliche Texte) vorliegen (vgl. Thorne und Calvanese 2010, 135; Thorne, 


\section{Forschungsstand}

Bernardi und Calvanese 2014). Einige Beispiele für solche Daten sind Ontologien, Datenbanken oder (semantische) Wikis und ich bezeichne diese Art Daten als formale Repräsentationen.

Funktionen Die Interaktion mit formalen Repräsentationen erfordert nicht nur inhaltliches Fachwissen, sondern darüber hinaus Kenntnisse in entsprechenden formalen Notationen. Repräsentationsorientierte KS sind hierbei eine Hilfestellung: Sie dienen zur Interaktion mit formalen Repräsentationen, so dass die Notwendigkeit der Kenntnisse in formalen Notationen relativiert wird. Mithilfe von Aussagen (Sätzen) in KS können formale Repräsentationen aufgebaut, erweitert, gepflegt und abgefragt werden. Darüber hinaus können auch Ergebnisse einer Repräsentationsabfrage in KS angezeigt werden (sog. Verbalisierung).$^{50}$

Hängen textorientierte KS mit dem gestiegenen Bedarf an (Fach-)Texten zusammen, so werden repräsentationsorientierte KS im Kontext des wachsenden (menschlichen) Wissens positioniert, mit dem allgemeinen Ziel, dieses Wachstum zu beherrschen. Insbesondere bedeutet dies die Verbesserung von Wissenspeicherung, -zugang, -erwerb und -anwendung durch formale Repräsentationen. Diese erfolgen zurzeit auf zwei Wegen: durch Stärkung der menschlichen Beteiligung einerseits oder durch Stärkung der Technikeinbindung und Automatisierung, die sich auf Menschen entlastend auswirken, andererseits. Die Stärkung der menschlichen Beteiligung durch KS bedeutet v. a., dass der Aufbau, die inhaltliche Prüfung und Pflege von formalen Repräsentationen nicht mehr ausschließlich durch ausgebildete Wissensingenieure, sondern zunehmend durch Fachexperten oder Fachinteressierte kollaborativ und sprachübergreifend geschehen (Smart u. a. 2010). Dies soll die Menge des formal repräsentierten Wissens vergrößern und den Erstellungsprozess zeitund kostensparender sowie demokratischer machen.

Als Konsequenz dessen, dass repräsentationsorientierte KS in logikbasierte Formen überführbar sind, ist das logische (deduktive) Schließen mit KSAussagen, bzw. ihren formalisierten Formen, möglich. Da das Schließen automatisch ausgeführt werden kann, ergeben sich sowohl für Produktion wie Rezeption unterstützende Automatisierungsmöglichkeiten, die für textorien-

50 Die KS und die Repräsentation interagieren oft nicht direkt miteinander, sondern über einen Zwischenschritt. Beim Aufbau der Repräsentation wird die KS in eine logik-basierte Form (z. B. Diskursrepräsentationsstrukuren bei ACE (exemplarisch Schwitter 1998, 106-117), für Rabbit: Denaux u. a. (2010, 253ff.)) abgebildet und erst anschließend in die Zielrepräsentation wie Ontologie, Wiki etc. überführt. Ähnlich wird bei der Verbalisierung zunächst aus der Repräsentation eine logikbasierte Form hergestellt und daraus dann ein KS-Text generiert. Diese Formalisierungsprozesse erfolgen im Hintergrund und sind für den Benutzer nicht sichtbar. 
tierte KS nicht gegeben bzw. nicht sinnvoll sind. So kann man bei Aufbau und Pflege einer Repräsentation die Konsistenz, Widerspruchsfreiheit und Vollständigkeit der Daten automatisch überprüfen (vgl. exemplarisch Schwitter 1998, 233-234; Ranta, Enache und Détrez 2012, 115) bzw. nur solche Aussagen oder Daten eingeben, die nicht im Widerspruch zum Vorhandenen stehen (Predictive Editor, s. 2.3.1.3 S. 113). Bei der Repräsentationsrezeption, insbesondere bei gezielten Abfragen, ermöglicht dieses Verfahren Inferenzbildungen, d. h. (explizite) Ausgabe von Aussagen, die durch vorhandene Daten implizit wahr sind. Aufgrund dessen ist es für den Rezipienten leichter, auf die volle Wissensbreite zuzugreifen. Dieser Aspekt ist dort besonders wichtig, wo das komplexe Wissen um die gegebene Sachlage das Vorgehen/die Handlung bestimmt, z. B. bei juristischen Angelegenheiten (wie Vertragsverpflichtungen), Kundenbetreuung (bei Fehlerbehebungen) etc. Da eine KS den Zugang zu den strukturierten formalen Repräsentationen und Automatisierungstechnologien erleichtert, führt sie auf der Rezeptionsseite schlussendlich dazu, dass geeignete Handlungen aus dem Wissen hergeleitet, Zuständigkeiten geklärt und dadurch die Interaktionsprozesse (insbesondere Geschäftsprozesse) standardisiert und effizienter gestaltet werden können.

Einsatzbereiche Im Unterschied zu textorientierten KS, die bereits auf langjährige Erfahrung im produktiven Einsatz in Unternehmen zurückblicken können, handelt es sich bei repräsentationsorientierten $\mathrm{KS}$, von denen in der Literatur berichtet wird, meistens um Testprojekte, die eine prinzipielle Einsatzmöglichkeit einer KS in einer Anwendung überprüfen und/oder KS-Details optimieren. $\mathrm{Zu}$ erwähnen seien folgende Einsatzbereiche:

- Softwarespezifikation (Organisation: ETH/Universität Zürich; KS: Attempto Controlled English, ACE) (exemplarisch Schwitter 1998; Fuchs, Schwertel und Schwitter 1999; Fuchs, Höfler u. a. 2005; Kuhn 2010b; Attempto Project 2015-), Hardwarespezifikation (Grover u. a. 2000)

- Erstellung von Landkarten (Organisation: Ordnance Survey, das britische nationale Landkarteninstitut; KS: Rabbit) (Denaux u. a. 2010; Engelbrecht, Hart und Dolbear 2010)

- Geschäftsprozesse (business rules): Klärung von Zuständigkeiten und Vorgehensweisen (KS: SBVR Structured English) (Spreeuwenberg und 


\section{Forschungsstand}

Healy 2010; vgl. OMG 2008); insbesondere öffentlicher Bereich (Institutionen für Zulassungen und Sozialleistungen, Finanzsektor (Versicherungen) (Spreeuwenberg, Grondelle u. a. 2012, 175-176; vgl. Pace und Rosner 2014); allgemeines Schlussfolgern über die zu einem Zeitpunkt gegebenen Zustände (KS: PENG Light) (Schwitter 2012)

- Mathematik: Überprüfung von Beweisen; auch als didaktisches Werkzeug zum Beweisaufbau für Mathematikstudenten (Organisationen: Universität Bonn und Universität Duisburg-Essen; KS: Naproche) (Cramer u. a. 2010; Cramer 2013)

- Wissenschaft: Erstellung von Aufsatzzusammenfassungen, die maschinell verarbeitbar sind (z. B. automatische Indexierung, besseres Retrieval) (KS: ACE, Gebiet: Proteinreaktionen) (Kuhn u. a. 2006)

- Medizin: Formulierung von Vorgehensweisen für Diagnose- und Behandlungszwecke (KS: u. a. ACE) (Cardey, Greenfield und Wu 2004; Shiffman u. a. 2010)

- Tourismus: maschinelle Übersetzung von touristischen Phrasen auf einem Smartphone (Projekt: MOLTO) (Ranta, Enache und Détrez 2012)

- Jura: Klärung von vertraglichen Zuständigkeiten und Rechtslage (Pace und Rosner 2010; Camilleri, Paganelli und Schneider 2014)

- RSS-Feeds (Zusammenfassung und Zusammenführung von Webseiten) (Organisation: Macquarie University, Australien; KS: PENG) (Schwitter und Tilbrook 2006)

- Seefahrt (Organisation: Service hydrographique et océanographique de la marine (SHOM), Frankreich; KS: INAUT) (Haralambous, SauvageVincent und Puentes 2014)

- Situationserfassung für Nachrichtendienste (Defence Science \& Technology Organisation, Australien; KS: Lexpresso)(Saulwick 2014)

Zielgruppen Der Aufbau, die Pflege und die Abfrage von formal vorliegenden Daten erfordern, wie bereits erwähnt, Kenntnisse in formalen Notationen. So erfolgt die Rezeptionsproduktion und -rezeption hauptsächlich durch sog. Wissensingenieure, die die formale Notation, Datenmodellierungs- und 
-strukturierungsansätze beherrschen. Der Einsatz von repräsentationsorientierten Kontrollierten Sprachen zielt somit nicht auf die bestehenden unmittelbaren Repräsentationsproduzenten und, de facto, -rezipienten, sondern auf Menschen, denen der direkte Zugriff auf die formal strukturierten Daten bisher nicht möglich war (vgl. Pulman 1996, 235, 239; Kuhn 2010b, 47). Diese Personen werden Domänenexperten genannt und von Denaux u. a. (2010) wie folgt charakterisiert:

„Domain experts are people who (i) have extensive knowledge of the domain to be modelled; (ii) are used to thinking at an abstract level about the domain (so they can provide descriptions and knowledge sources for concepts and relationships of the domain); (iii) have no previous experience building ontologies, but may have experience building other types of models in their specific domain; (iv) have no interest in becoming knowledge engineering experts or experts in logical formalisms; (v) have a fairly well defined idea of how the resulting ontology will be used“" (Denaux u. a. 2010, 249).

Dieses Zitat bezieht sich zwar speziell auf die Domänenexperten beim Ontologieaufbau, kann aber m. E. auch auf andere Bereiche, wie semantische Wikis etc., verallgemeinert werden. Domänenexperten sind also Menschen, die sich nicht primär mit der Entwicklung von formalisierten Daten beschäftigen; ein direkter Zugriff auf diese Daten kann jedoch für ihre Haupttätigkeit vorteilhaft sein.

Im Unterschied zu textorientierten Kontrollierten Sprachen, die ihre produktiven Zielgruppen vor allem in Unternehmen sahen, sind die Domänenexperten der repräsentationsorientierten $\mathrm{KS}$, wie im vorangegangenen $\mathrm{Ab}$ satz Einsatzbereiche aufgeführt, auch in anderen Bereichen zu finden. So fasst man auch die User von sozialen Medien, die Semantic-Web-Community (Dantuluri u. a. 2012; vgl. Smart u. a. 2010, 210), Wissenschaftler, Personal im Gesundheitswesen etc. als Zielgruppen von bestimmten KS-Projekten auf.

Ein weiterer Unterschied zu textorientierten KS besteht außerdem darin, dass die rezeptive Zielgruppe ebenfalls die Domänenexperten umfasst und sich somit mit der produktiven Zielgruppe weitestgehend deckt. Darüber hinaus erfordert eine gezielte Rezeption einer Repräsentation ebenfalls aktive Kenntnisse in KS-Spezifikation, um Abfragen formulieren zu können (vgl. exemplarisch Denaux u. a. 2010, 248). Da das Abfrageergebnis dann ein KS-Text ist, erfordert hingegen die Rezeption von diesem Text, ähnlich wie bei textorientierten KS, nur relativ wenige aktive KS-Kenntnisse (Schwitter 2010b, 1120). 


\section{Forschungsstand}

Werkzeuge Im Gegensatz zu textorientierten KS, bei denen sich der Einsatz von KS-Werkzeugen auf die Prüfung der KS-Spezifikation beschränkt und die eigentliche Textverfassung meistens in regulären Texteditoren erfolgt, setzen repräsentationsorientierte KS umfassende Werkzeugumgebungen ein, die sowohl die Erfassung der Texte in einer Kontrollierten Sprache wie auch die Überprüfung der Regeleinhaltung, Überführung der Texte in die formale Repräsentation und weitere Funktionalitäten ermöglichen. Da diese Umgebungen modular aufgebaut sind, können einzelne Module je nach Projektzielen und -anforderungen hinzukommen oder entfallen. Darüber hinaus können oft auch die hinter den Zusatzmodulen stehenden Formalismen oder ihre Implementierungen projektspezifisch angepasst werden. ${ }^{51}$ Im Folgenden stelle ich die zurzeit verfügbaren Module vor, ohne auf die technischen Details der Architektur ausführlich einzugehen. Die folgenden Informationen zu den einzelnen Werkzeugen stammen, wenn nicht anders angegeben, aus den in Fußnote 51 angeführten Quellen.

Das Herzstück der Werkzeugumgebungen ist das Authoring-Modul oft auch authoring tool oder Editor genannt. Durch das Authoring-Modul interagiert der Benutzer mit der KS und der formalen Repräsentation, die technische Umsetzung erfolgt jedoch im Hintergrund (vgl. Fuchs und Schwitter 1996, 2; Schwitter 1998, 45; Thorne und Calvanese 2010, 135) und wird durch weitere Module bereitgestellt. Die Hauptfunktion dieses Moduls ist es, den Benutzern die Erfassung von KS-Texten (Sätzen) zu ermöglichen, sowie die Spezifikationskonformität dieser Texte sicherzustellen. Die Konformitätsprüfung wird heutzutage auf dreierlei Weise umgesetzt. Die erste Methode ähnelt dem CLC-Ansatz für textorientierte KS, wobei der Text zunächst vom Nutzer verfasst und anschießend vom Werkzeug geprüft wird. Fehlermeldung und Korrekturvorschläge sollen dem User dabei helfen, den Text regelkonform zu überarbeiten (bspw. ROO: Denaux u. a. 2010; vgl. Kuhn 2010b, 20-21). In letzter Zeit setzt sich jedoch zunehmend der Einsatz von sog. Predictive Editors durch. Ein Predictive Editor wertet die Eingabe unverzögert aus und zeigt an, mit welchen Wortarten, anderen Kategorien oder konkreten Lexemen der angefangene Text fortgesetzt werden darf (bspw. ACE Editor, PENG/PENG ${ }^{A S P}$ Authoring Tool) (vgl.

51 Beispiele von etablierten Systemen sind die Werkzeuge für ACE (beispielsweise ACE Editor, RACE, AceWiki, OWL Verbalizer, für eine Übersicht s. Fuchs, Kaljurand und Kuhn (2008) und Attempto Project (2015-)), ROO Authoring Tool für Rabbit (Denaux u. a. 2010), PENG Authoring Tool, PENG $^{A S P}$ system (entsprechend Schwitter 2010b, 1115; bspw. Guy und Schwitter 2014), Werkzeuge für Grammatical Framework (Angelov und Enache 2012), CLANN-Werkzeuge (Dantuluri u. a. 2012) (für einen Überblick über Sprachen und Werkzeuge für Ontologie-KS s. auch Safwat und Davis (2014)) 
Kuhn 2010b, 21-22, 108-113; Schwitter 2010b, 1119; Guy und Schwitter 2014). Somit ist von Anfang an nur die Eingabe von KS-konformen Sätzen möglich. Damit soll erreicht werden, dass Benutzer mit geringen Kenntnissen der KS-Spezifikation bereits gültige Texte erstellen können.

Der dritte Ansatz ist das sog. conceptual authoring, das auf einer automatischen Sprachsynthese beruht (Kuhn 2010b, 22; Schwitter 2010b, 1119; für Beispiele s. Safwat und Davis 2014, 193-194).

Das Authoringmodul geht oft über die bloße Funktion als Texteditor und -checker hinaus und enthält auch andere Funktionen, wie das Nachschlagen/Blättern von bereits erfassten Daten. Das Authoringmodul wird meistens als eigenständige Webbrowser-Anwendung oder als Plug-in in einem anderen Programm umgesetzt. ${ }^{52}$

Das zweite Modul, das in jeder Werkzeugumgebung, auch bei Prototypen, vorhanden ist, ist das Sprachmodul. Es stellt die sprachlichen Ressourcen der Kontrollierten Sprache (Grammatik, Lexikon, Parser) in einer maschinenlesbaren Form zur Verfügung und verarbeitet die Texte in der Kontrollierten Sprache weiter (Syntaxanalyse/Parsing oder Generierung). Es ist also dieses Modul, das die eigentliche Konformitätsprüfung durchführt. Darüber hinaus kann es die Überführung der KS-Texte in die logikbasierten Formen (Zwischenrepräsentationen) übernehmen. Beispiele für dieses Modul sind: APE (für ACE) und Rabbit Language Processor.

Komplexere Werkzeugumgebungen verfügen oft über ein weiteres Modul - den semantischen Reasoner. Reasoner sind Programme, die über gegebene logische Aussagen schlussfolgern können (sog. Inferenzbildung). Dadurch können sie die Gesamtheit der Aussagen auf Konsistenz prüfen und implizite Aussagen explizieren (exemplarisch Endres 2007, 1; vgl. Kuhn 2010b, 123-127). Während also das sprachliche Modul die sprachliche Wohlgeformtheit der KS-Texte prüft, ermöglicht ein Reasoner die inhaltliche Prüfung. Dadurch können die neu verfassten Texte auf die Widerspruchsfreiheit mit den bestehenden Daten (z. B. in einer Ontologie) getestet werden. Darüber hinaus können so die Nutzeranfragen beantwortet werden, ob ein bestimmter Sachverhalt besteht oder nicht. Das Modul kann eine Schnittstelle zum bestehenden Reasoner enthalten oder eine Eigenimplementierung sein (z. B. RACE für ACE, GF Reasoner, entsprechend bspw. Fuchs, Kaljurand und Kuhn (2008, 116-117) und Angelov und Enache (2012, 13-15)). Dabei ist hier oft eine weitere

52 Z.B. ACE View als Plug-in für den Ontologieeditor Protégé (bspw. Fuchs, Kaljurand und Kuhn 2008, 117-119), CLANN als Plug-in für SemanticMedia Wiki (Dantuluri u. a. 2012). 


\section{Forschungsstand}

Überführung der Kontrollierten Sprache oder ihrer Zwischenrepräsentation in das vom Reasoner akzeptierte Format erforderlich.

Schließlich soll hier noch das Verbalizer-Modul erwähnt werden, mit dem die formal repräsentierten Daten, insbesondere Ontologien, als Texte in Kontrollierter Sprache angezeigt werden (bspw. ACE Verbalizer). Weitere Module umfassen u. a. Schnittstellen zum eigentlichen Zielsystem oder Zielformat, zu Grammatical-Framework-Modulen oder zu sog. Modelcheckern (bei ROO).

\subsubsection{Geschichte}

Gewöhnlicherweise wird in der Literatur BASIC English von Ogden als Vorläufer von KS im heutigen Sinne gesehen (Huijsen 1998b, 5; Schwitter 1998, 58; Kaji 1999, 37; Drewer und Ziegler 2011, 200; Doherty 2012, 22; Ramírez Polo 2012, 58; Kuhn 2014, 134). Wie in 2.2.1 erwähnt, handelte es sich dabei um eine Plansprache, die als Zweitsprache in britischen Einflussgebieten aber auch zur Verbesserung der Ausdrucksklarheit für Muttersprachler dienen sollte. Systemlinguistisch bestand sie aus 850 Grundwörtern, 100 allgemeinen Fachwörtern und je 50 Fachwörtern für einzelne Fächer (exemplarisch: Ogden 1968, 6). Es handelte sich also um eine Teilmenge des Standardenglischen (Britisch). Es sind deshalb weniger die angedachte Funktion und die Einsatzmöglichkeiten des BASIC English, sondern sein Konstruktionsprinzip - ein reduktiver Eingriff in die Mittel (genau) einer natürlichen Sprache -, das es zum Vorreiter von Kontrollierten Sprachen machte.

Abschnitt 2.2.1 kann man überdies entnehmen, dass BASIC English auch als eine Plansprache betrachtet werden kann, die in sich die Eigenschaften einer Welthilfssprache und einer philosophischen Sprache vereint. Es handelt sich hierbei auch um eine späte Plansprache. So werden die Ursprünge von KS zuweilen noch weiter zurückverfolgt. Schubert (2014) weist darauf hin, dass es vor BASIC English noch andere Welthilfssprachen gab, die auf Modifikation (genau) einer ethnischen Sprache beruhten wie Latino sine Flexione, WEDE etc. (Schubert 2014, 211-212; vgl. Lehrndorfer 1996, 29-30). In der Terminologie von D. Blanke (1985) handelt es sich also um sog. modifizierte Ethnosprachen (Abbildung 3, S. 86). Hierzu sei jedoch zu bemerken, dass diese frühen modifizierten Ethnosprachen systemlinguistisch in hohem Maße Formen benutzten, die man weder fach- noch standardsprachlich als korrekt bezeichnen kann. Somit verletzen sie eine der Bedingungen aus der ange- 
führten Definition von KS nach Kuhn (2014), nämlich dass eine KS für den Muttersprachler leicht als die (korrekte Form der) Muttersprache erkennbar ist. Die modifizierten Ethnosprachen können somit lediglich aufgrund ihres monolingualen Konstruktionsprinzips als Vorläufer von KS aufgefasst werden.

Darüber hinaus können KS als moderne Umsetzung der Ideale von philosophischen Sprachen gesehen werden. Betrachtet man die Funktionen von philosophischen Sprachen, wie in 2.2.1.2 erläutert, so steigern die KS die Eineindeutigkeit der sprachlichen Mittel in der Kommunikation zwischen Sender und Empfänger. Insbesondere setzten repräsentationsorientierte KS Leibniz' Ideen von Sprachen um, die durch Möglichkeiten zum logischen Schlussfolgern das logische Denken unterstützen (Ley 2005, S. 28, Fußnote 26; vgl. Wyner u. a. 2010, 281-282), selbst wenn die eigentliche Umsetzung nicht auf einer apriorischen Sprachform beruht, sondern maßgeblich dank modernen Technologien erfolgt.

Als erste Kontrollierte Sprache im eigentlichen Sinn gilt das Caterpillar Fundamental English (CFE) (Huijsen 1998b, 5; Kaji 1999, 37). Sie wurde 1972 mit dem Ziel entwickelt, die Verständlichkeit von Wartung- und Servicedokumentation sowohl für die Nichtmuttersprachler als auch Muttersprachler zu erhöhen, um dadurch auf die Übersetzung verzichten zu können (Gallup 1993; Kamprath u. a. 1998; vgl. Crabbe 2009, 52). Es handelte sich also um eine textorientierte KS. In Anlehnung an BASIC English bestand der Wortschatz von CFE aus ca. 850 Lexemen. Crabbe (2009) stellt fest, dass die grammatischen und stilistischen Regeln schlecht dokumentiert sind, nennt jedoch einige dank persönlicher Anfrage (Crabbe 2009, 53). CFE wurde 1982 aufgegeben, die Gründe hierfür nenne ich in 2.3.6.2. Dennoch wurden das Konstruktionsprinzip und ein enger Einsatzbereich zum Ausgangspunkt für weitere $\mathrm{KS}^{53}$

ASD-STE100 (früher auch AECMA-SE genannt) ist die KS der Luftund Raumfahrtindustrie, also, im Unterschied zu vielen anderen Projekten, ein unternehmensübergreifender und branchenweiter Standard. Sie entstand im Züge der Verständlichkeitsüberprüfung von Wartungsdokumentation, die AECMA (Association Européenne des Constructeurs de Matériel Aérospatial, heute ASD (AeroSpace and Defence)) in Zusammenarbeit mit AIA (Aerospace Industries Association of America) im Auftrag von AEA (Association of European Airlines) durchführte (Drewer und

53 Beispielsweise ILSAM (Huijsen 1998b, 5; vgl. Hebling 2002, 59), PACE (Douglas und Hurst 1996, 93), Bull (Crabbe 2009, 56-59), KS bei Kodak (Hebling 2002, 129) und, insbesondere, ASD-STE100, die heute als bekannteste KS gilt (Kittredge 2003, 442; Crabbe 2009, 55; Drewer und Ziegler 2011, 200). 


\section{Forschungsstand}

Ziegler 2011, 200). Die Untersuchungen begannen $1981^{54}$ und die erste AECMA-KS-Spezifikation wurde 1986 veröffentlicht (ASD-STE100 2013, i; vgl. Shaw 2006; s. auch Farrington 1996, 10). Seitdem wird die Sprache regelmäßig weiterentwickelt. Das Ziel von ASD-STE100 war von Anfang an die Verbesserung der Verständlichkeit von englischen Anleitungen sowohl für Muttersprachler als auch für Nichtmuttersprachler. ASD-STE100 war Vorbild für viele ähnliche Projekte, beispielsweise Français Rationalisé (bspw. Barthe 1998) und Boeing Technical English (BTE) (bspw. R. H. Wojcik und Holmback 1996; R. Wojcik, Holmback und J. Hoard 1998).

Die KS zur Verbesserung der Textverständlichkeit stellten den ersten Strang in der historischen Entwicklung von KS dar. Ungefähr zur gleichen Zeit gaben die Arbeiten an der maschinellen Sprachverarbeitung, insbesondere der Maschinellen Übersetzung, einen weiteren Entwicklungsimpuls (vgl. Eijk, Koning und Steen 1996, 67; Fawcett 2005, o.S.). Die Erfolge von Systemen wie TAUM-MÉTÉO, ${ }^{55}$ die auf fachsprachliche Anwendung beschränkt waren, gaben Anregungen zum Einsatz in Kombination mit Kontrollierten Sprachen für die Ausgangstexte. Auch hier war Caterpillar der Vorreiter. Nach der Aufgabe von CFE entwickelte man in Zusammenarbeit mit Carnegie Group Inc. und Carnegie Mellon University das Caterpillar Technical English (CTE). Die Zusammenarbeit begann 1991 und das Pilotprojekt wurde 1995 abgeschlossen (Gallup 1993, 422; Kamprath u. a. 1998, 54). Die Ziele von CTE waren vielfältig: Verbesserung der Erstellung von (Ausgangs-)Texten, sowohl was den Arbeitsprozess (Modularisierung), die Technologie, als auch die Konsistenz der Texte betrifft, darüber hinaus die Verbesserung der Textübersetzung durch Einsatz eines Maschinellen Übersetzungssystems (Gallup 1993, 422; Kamprath u. a. 1998, 53-54, 59-60; vgl. Drewer und Ziegler 2011, 212). Weitere Beispiele für KS für Maschinelle Übersetzung sind CASL, ScaniaSwedish und EasyEnglish Language (IBM) (vgl. Kaji 1999, 37).

In den 1990er Jahren folgen viele Praxisprojekte, die sich entweder als verständlichkeitsorientierte oder übersetzbarkeitsorientierte KS auffassten. Zudem intensivierte sich in dieser Zeit auch die Arbeit an CLC (für eine Übersicht s. Beiträge in: CLAW 1996; CLAW 1998; CLAW 2000). Dies führte

54 Farrington $(1996,10)$ und Drewer und Ziegler $(2011,200)$ geben 1979 als Beginn der Zusammenarbeit an.

55 Das kanadische TAUM-MÉTÉO-Projekt entwickelte seit Mitte der 1970er Jahre ein maschinelles Übersetzungssystem für Wetterberichte Englisch-Französisch (bspw. Thouin 1982; vgl. Kittredge 2003, 439440). 
zur Entstehung von kommerziellen Lösungen, die auf die Kundenspezifikation zugeschnitten werden können, so dass die Entwicklung und Einführung einer Kontrollierten Sprache für Unternehmen einfacher wird (vgl. Suchowolec 2014, 121).

Mitte der 1990er Jahre kristallisiert sich auch der dritte Entwicklungsstrang der Kontrollierten Sprachen heraus - repräsentationsorientierte KS. Vereinzelt können sie als konsequente Weiterentwicklung im Bereich der Technischen Dokumentation gesehen werden. So postulieren Mellish, Reiter und J. Levine (1996) Veränderungen im Texterstellungsprozess und, in Konsequenz, im Berufsbild der Technischen Redakteure: Die Texte in der Technischen Dokumentation sollen aus formalen Repräsentationen generiert werden, die von Technischen Redakteuren erstellt und gepflegt werden. Zum Durchbruch kommt jedoch außerhalb der Technischen Dokumentation mit dem Attempto Controlled English (ACE) (exemplarisch Fuchs und Schwitter 1996; Schwitter 1998), das zunächst das Ziel hatte, für Menschen leicht verständliche und gleichzeitig maschinell deterministisch interpretierbare Softwarespezifikationssprache zu sein. Es wurde später aber zu einer allgemeinen KS (Abschnitt 2.3.5.2, S. 125) und hatte die Konstruktion weiterer repräsentationsorientierter KS zur Folge.

Alle drei Entwicklungsstränge - KS zur Verbesserung von Verständlichkeit, Übersetzbarkeit und Interaktion mit formalen Repräsentationen - sind sowohl in der Praxis als auch in der Forschung nach wie vor vertreten. Dabei muss betont werden, dass es sich bei diesen Strängen nicht um disjunkte Entwicklungen handelt, sondern eher um pragmatische Selbsteinschätzungen seitens der Projektautoren. Eine Verflechtung dieser Entwicklungen und ihre Eignung als Grundlage für eine KS-Klassifikation diskutiere ich in Suchowolec (o.D.) und fasse die Ergebnisse in 2.3.5.4 zusammen.

\subsubsection{Erforschung von Kontrollierten Sprachen als Disziplin}

In der einschlägigen Literatur wird die Verortung der Erforschung von Kontrollierten Sprachen im wissenschaftlichen Diskurs nur vereinzelt explizit diskutiert. Lehrndorfer (1996) nimmt diese Verortung ausführlicher vor und postuliert die Erforschung von Kontrollierten Sprachen als Gegenstand der Psycholinguistik (insb. Lehrndorfer 1996, 11-12, 195-196), im Speziellen der kognitiven Linguistik (Aspekte der menschlichen Sprachverarbeitung) (Lehrndorfer 1996, passim). Gleichzeitig weist sie 


\section{Forschungsstand}

darauf hin, dass die Gegenstandsetablierung erst erfolgen muss, weil eine präskriptiv orientierte Forschung in der Linguistik eine Seltenheit darstellt (Lehrndorfer 1996, 11-12).

Um die implizite Verortung der Erforschung von Kontrollierten Sprachen in der Wissenschaft festzuhalten, zeige ich hier, ohne Anspruch auf Vollständigkeit, die Forschungsthemen und -fragen auf, die bisher prominent waren:

- Vorstellung des Regelwerks einer Kontrollierten Sprachen in einer speziellen Anwendung. Dabei identifiziere ich idealtypisch zwei Aspekte bei der argumentativen Begründung des Regelwerkes, die in der Praxis meistens eng miteinander verflochten sind:

- systemlinguistische Sicht: Wie bauen diese Regeln Ambiguität, Vagheit und Redundanz der vollen natürlichen Sprache ab (exemplarisch Bernth 1998a, 33-39; Schwitter 1998, 11-203; Schwertel 2000; Suchowolec 2014, 115-118)?

- funktionale Sicht: Wie fördern diese Regeln Verständlichkeit, (maschinelle) Übersetzbarkeit (exemplarisch Bernth 1998a, 3339), Texterstellung (exemplarisch Suchowolec 2014, 114-115) und Interaktion mit formalen Repräsentationen (exemplarisch Schwitter 1998, 221-231, 233-234), (vgl. auch Huijsen 1998b, 9-11)?

Neben der argumentativen Begründung gibt es vereinzelte empirische Studien zur Überprüfung, ob KS die ihnen zugeschriebene Funktionen erfüllen (für eine Übersicht empirischer Studien für textorientierte KS s. Link 2014, 182-195):

- Verständlichkeit (exemplarisch: Holmback, Shubert und Spyridakis 1996; Holterhus und Meister 2001)

- Übersetzbarkeit (Preux 2005)

- bei repräsentationsorientierten KS: Handhabbarkeit (Engelbrecht, Hart und Dolbear 2010), insbesondere im Vergleich zu formalen Sprachen (Kuhn 2010b,a)

Weitere empirische Studien werden gefordert (Knops 2000, 134; Ramírez Polo 2012, 46, 152; Link 2014, 196-197).

- Bei repräsentationsorientierten KS: Entwicklung und Untersuchung geeigneter Formalismen zur Überführung der KS in das Format der formalen Repräsentation, des Reasoners etc. oder zur Überführung 
von verschiedenen KS ineinander (exemplarisch: Schwitter 1998; Ranta 2004; Cramer u. a. 2010; Davis, Dantuluri u. a. 2010; Denaux u. a. 2010; Kuhn 2010b; Ranta, Enache und Détrez 2012; Smart u. a. 2010; Davis, Enache u.a. 2012; Cramer 2013; Guy und Schwitter 2014).

- Damit zusammenhängend: Vorstellung von Werkzeugen und Fragen der Werkzeugentwicklung: Funktionen, Architektur, Implementierung, Benutzerfreundlichkeit (der Benutzeroberfläche), Qualitätsbewertung (für Beispiele und Quellen s. 2.3.1.3 S. 107 und S. 113, S. 113)

- Fragen der Berechnungskomplexität von repräsentationsorientierten KS (Bernardi, Calvanese und Thorne 2007; Thorne und Calvanese 2010; Thorne, Bernardi und Calvanese 2014)

- Metadiskussion, wie Kontrollierte Sprachen, v. a. systemlinguistisch, beschaffen sein sollen (für Beispiele und Quellen s. 2.3.6.1)

- Metadiskussion, welche Typen von Kontrollierten Sprachen es gibt (für Beispiele und Quellen s. Abschnitt 2.3.5, S. 123)

Verallgemeinert man diese Themen, so erkennt man, dass die Erforschung von Kontrollierten Sprachen durch Einflüsse der Informatik, Logik, Kognitionswissenschaft (hier: insbesondere der kognitiven Linguistik), aber auch der Künstlichen Intelligenz erfolgt. Dies sind auch Disziplinen, die Amtrup (2010, 3-4) als Nachbardisziplinen der Computerlinguistik sieht. Amtrup $(2010,2)$ führt vier Möglichkeiten zur Charakterisierung der Computerlinguistik. Demnach kann sie aufgefasst werden als:

- Linguistische Disziplin, „(...) die sich (...) mit berechnungsrelevanten Aspekten von Sprache und Sprachverarbeitung beschäftigt (...), unabhängig von ihrer tatsächlichen Realisierung auf dem Computer.

- (...) Disziplin für die Entwicklung linguistik-relevanter Programme und die Verarbeitung linguistischer Daten (...).

- (...) Realisierung natürlichsprachlicher Phänomene auf dem Computer (...).

- (...) praxisorientierte, ingenieursmäßig konzipierte Entwicklung von Sprachsoftware (...) 


\section{Forschungsstand}

Nach einem Vergleich dieser Auffassungen mit den o.g. Forschungsthemen stelle ich fest, dass die bisherige Erforschung von Kontrollierten Sprachen vorwiegend aus der computerlinguistischen Sicht erfolgte (vgl. Schubert 2011b, 53), wobei man sich ebenfalls der Notwendigkeit der Forschung aus der Sicht der kognitiven Linguistik durchaus bewusst ist, wie am Anfang dieses Abschnitts bei Lehrndorfer (1996) festgestellt.

\subsubsection{Autoren}

Die Behandlung des Themas Autoren von $K S$ fällt in der Forschung eher knapp aus. Unter Auswertung der Projektberichte werden hauptsächlich drei Urhebergruppen erwähnt: Industrie (im weitesten Sinne), Wissenschaft und regierungsnahe/internationale Organisationen, was sich im autorenbezogenen Teil der KS-Klassifikation von Kuhn $(2014,125,126)$ widerspiegelt. Die Projekte entstehen meist aus der Praxis und in Zusammenarbeit der oben genannten Gruppen in unterschiedlichen Konstellationen (Kuhn 2014, 125; vgl. Ramírez Polo 2012, 91; s. auch Kittredge 2003, 444; Nyberg, Mitamura und Huijsen 2003, 245-246), wobei auch mehrere Vertreter einer Gruppe aus unterschiedlichen Gebieten zusammenarbeiten können (bspw. Wissenschaftler unterschiedlicher Fachgebiete wie Mathematik/Logik und Linguistik (KS Naproche), Medizin und Computerlinguistik/Informatik (Shiffman u. a. 2010)). ${ }^{56}$ Hierbei weist Huijsen $(1998$ b, 5) darauf hin, dass es sich bei unternehmensspezifischen KS oft um proprietäre Lösungen handelt.

In der Literatur finden sich nur wenige Hinweise darauf, welche Kompetenzen KS-Autoren haben müssen. Neben linguistischer/computerlinguistischer Kompetenz (bspw. Schubert 2011b, 53) sieht E. Johnson $(1996,116)$ auch die technische, rechtliche und Managementexpertise als wichtige Voraussetzungen, wobei es sich bei E. Johnson (1996) um ein komplexes, grenzenübergreifendes Projekt handelte.

56 Neben der erwähnten Zusammenarbeit in der Luft- und Raumfahrtindustrie (STE-1000), können hier weitere Beispiele exemplarisch genannt werden: CTE: Caterpillar und Carnegie Mellon Group; PACE: Perkins und Universität Edinbourgh (Douglas und Hurst 1996, 93); Test-Bed LinguaNet: industrielle Partner: Prolingua, Philips, Kent Constabulary, Universitäten: Cambridge, Leuven, Kopenhagen, Bordeaux II, gefördert durch die EU (E. Johnson 1996, 116); Scania und Universität Uppsala (Almqvist und Sågvall Hein 1996, 159). 


\subsubsection{Modelle}

Die Beschäftigung mit Kontrollierten Sprachen ist, wie bereits betont, anwendungsgetrieben und brachte als solche bisher verhältnismäßig wenige wissenschaftliche Theorien und Modelle hervor. Die für diese Arbeit relevanten wissenschaftlichen Metadiskussionen befassen sich vorwiegend mit verschiedenen Klassifikationen von Kontrollierten Sprachen und ihren Regeln. Im Folgenden stelle ich deshalb folgende Klassifikationen vor: mensch- und maschinenorientierte KS nach Huijsen (1998b), formalistische und naturalistische KS in Auffassungen von Pool (2006) und Clark u. a. (2010) sowie das Klassifikationsschema, einschließlich der PENS-Klassifikation, von Kuhn (2014) und seine Erweiterung durch Suchowolec (2014, o.D.). Des Weiteren gehe ich auf die Charakterisierung verschiedener Regeltypen nach O'Brien (2003) und Reuther (2003).

Andere Untersuchungen umfassen beispielsweise Überlegungen zur Beschaffenheit und Evaluation von verschiedenen Werkzeugen (Adriaens und Macken 1995; Haller 1996; Schmidt-Wigger 1998; Haller 2000; Reuther und Schmidt-Wigger 2000; Kuhn 2010b; Ramírez Polo 2012) oder zur Berechnungskomplexität von (repräsentationsorientierten) Kontrollierten Sprachen (Thorne und Calvanese 2010; Thorne, Bernardi und Calvanese 2014). Erwähnenswert ist darüber hinaus die Programmiersprache Grammatical Framework, mit der man Grammatiken natürlicher Sprachen, insbesondere also Kontrollierten Sprachen, implementieren kann. Sie findet Einsatz vor allem in multilingualen Anwendungen und kann auch zur Überführung einer (repräsentationsorientierten) $\mathrm{KS}$ in eine andere KS gebraucht werden (Ranta 2004; Ranta, Enache und Détrez 2012; Ranta 2011, 2014) Auf diese Untersuchungen gehe ich in diesem Abschnitt nicht näher ein, sie werden jedoch in die Metauntersuchung in Kapitel 5 teilweise mit einbezogen.

\subsubsection{Menschorientierte und maschinenorientierte KS}

Die Unterscheidung zwischen menschorientierten und maschinenorientierten Kontrollierten Sprachen (human-oriented und machine-oriented) geht auf Huijsen (1998b,a) zurück. Dabei werden die ersten mit dem Ziel konstruiert, die (menschliche) Verständlichkeit zu unterstützen, während die zweiten die maschinelle Verarbeitbarkeit der natürlichen Sprache verbessern sollen. Huijsen präzisiert jedoch, dass die eigentliche Einordnung einer KS nach ihrem Aufbau, also den eigentlichen Regeln vorgenommen wird (Huijsen 


\section{Forschungsstand}

1998b, 2), also nicht nach den vorher genannten Zielgruppen (MenschMaschine) oder Funktionen/Zielen (Verständlichkeit-Verarbeitbarkeit). $\mathrm{Da}$ es aber gewisse Ähnlichkeiten zwischen den beiden KS-Typen gibt - menschorientierte KS steigern auch die maschinelle Verarbeitbarkeit und andersherum (vgl. Schwitter und Tilbrook 2006, 3) - ist es deshalb in der Praxis schwierig, eine KS eindeutig zu klassifizieren (Huijsen 1998b, 2). Hierzu bemerkt Kuhn (2014, 125): „Because these types describe problems rather than languages, reusing a language in a different problem domain can change its type even if the language itself has not changed at all.", wobei ich hier problem als Funktion/Zielgruppe (wie in diesem Abschnitt definiert $)^{57}$ und language als Sprachsystem interpretiere. Ein wichtiges Unterscheidungsmerkmal ist jedoch nach Huijsen (1998b), dass die Regeln einer maschinenorientierten KS präzise (explizit) und maschinell lesbar sein müssen, während für Menschen bereits allgemeinere und vage Regelformulierungen wie „Make your instructions as specific as possible" (Huijsen 1998b, 2) in Frage kommen (vgl. Schwitter und Tilbrook 2006, 3). Ich halte deshalb fest, dass die Unterscheidung zwischen mensch- und maschinenorientierten KS zunächst nach den Zielgruppen dann nach Funktionen vorgenommen wird, letztendlich aber auf ihrer systemlinguistischen Beschaffenheit beruht.

Die mangelnde Trennschärfe ist in der Forschung gut bekannt. Beispielsweise schlägt O'Brien (2006) die Bezeichnung dualorientierte (dual-oriented) KS vor (O’Brien 2006, 6; vgl. Ramírez Polo 2012, 65), die sich in der Forschung jedoch nicht etablierte, so dass die klassische Zweiteilung nach wie vor vorherrscht (vgl. Ramírez Polo 2012, 67; Kuhn 2014, 125) und immer wieder zur Klassifikation benutzt wird (Bünzli und Höfler 2012, 22-23; Christoffersen und Møller 2010, 2; Drewer und Ziegler 2011, 196-197; Höfler 2012, 138-139; Suchowolec 2014, 119). In der neueren Literatur wird diese Zweiteilung beispielsweise von Marrafa u. a. hinterfragt, da sie heutzutage aufgrund einer Vielzahl von KS-Projekten in unterschiedlicher Anwendung nicht mehr aussagekräftig ist (Marrafa u. a. 2012, 154). Diese Behauptung interpretiere ich als eine stärkere Koppelung menschlicher Verstehensprozesse an maschinelle Abfrage- und

57 In diesem Satz bezieht sich Kuhn (2014) zunächst auf den funktionsbezogenen Teil seiner Klassifikation, die KS für (menschliche) Verständlichkeit, für Übersetzbarkeit und formale KS unterscheidet, s. 2.3.5.4. Die Klassifikation nach mensch- und maschinenorientierten Kontrollierten Sprachen sieht er als eine, gut etablierte, Vereinfachung dieser Unterscheidung, insofern kann man unter problems die Funktionen/Zielgruppen verstehen. 
Schlussfolgerungsmöglichkeiten, wie für die repräsentationsorientierte KS in 2.3.1.3 beschrieben.

\subsubsection{Regeltypen}

Wie im vorangegangenen Abschnitt diskutiert, nahm bereits Huijsen (1998b) einen Zusammenhang zwischen der Funktion einer KS und den eigentlichen KS-Regeln an. Dieser Zusammenhang wurde in der Forschung aus verschiedenen Perspektiven untersucht, ich gehe hier auf die Arbeiten von O'Brien (2003) und Reuther (2003) ein. O'Brien (2003) untersucht, ob man (für Englisch) einen Kernregelsatz identifizieren kann, der in jedem Projekt und jeder KS-Spezifikation, unabhängig von Projektzielen und KS-Funktionen, enthalten sein muss. Dieser Regelsatz würde auch eine Richtlinie für die Konstruktion neuer KS darstellen. Hierfür analysiert sie Regelsätze von acht etablierten KS u. a., ASD-STE100, CASL, Easy English (IBM) und ACE auf allen Ebenen des Sprachsystems. Als Ergebnis stellt sie fest, dass nur eine Regel in allen KS enthalten ist (zur Satzlänge) und nur sieben Regeln in der Mehrheit der KS enthalten sind. Sie schlussfolgert, dass es keinen Kernregelsatz gibt und KS-Regeln projektspezifisch sind, was mit Projektzielen, verwendeten MTSystemen und Übersetzungsrichtungen, existierenden unternehmensinternen Sprachrichtlinien, eingebürgerter Praxis in der Technischen Dokumentation und Subjektivität zusammenhängt.

Diese Ergebnisse bestätigen die bis heute gültige Beobachtungen, dass es nicht die Kontrollierte Sprache gibt und geben kann und „one-size-fits-all“ (Kittredge 2003, 446) nicht zu realisieren ist (vgl. Heald 2000, 43; Hebling 2002, 134; Nyberg, Mitamura und Huijsen 2003, 245-246; Pace und Rosner 2010, 227). Trotzdem unterscheidet man heutzutage zwischen allgemeinen $\mathrm{KS}$, die für viele Anwendungsbereiche, viele Textsorten etc. einsetzbar sind (beispielsweise ACE) und spezifischen KS, die in einem speziellen Unternehmen, einem speziellen Anwendungsbereich etc. einsetzbar sind (bspw. KS bei der Koenig \& Bauer Group) (vgl. Pool 2006, 2, 5; Schwitter 2010b, 1114; Ramírez Polo 2012, 69-70; Kuhn 2014, 125). Dabei sind die allgemeinen KS in einem Projekt nicht direkt verwendbar, sondern müssen entsprechend angepasst werden (vgl. Ramírez Polo 2012, 63-64; für Beispiele s. exemplarisch Schwitter 1998, 91-92, 121-148). Dies bedeutet, dass die Spezifikation einer allgemeinen KS genug Spielräume für eine solche Anpassung enthalten muss. Kuhn $(2014,125)$ sieht vor allem die Flexibilität der lexikalischen Ebene als wichtigste Anforderung an eine allgemeine KS. 


\section{Forschungsstand}

Reuther (2003) untersucht hingegen, ob die zwei funktional bestimmten Typen von Kontrollierten Sprachen: für (verbesserte) Verständlichkeit und für (verbesserte) Übersetzbarkeit auch wesentliche Unterschiede in den Regelsätzen aufweisen. Aus verschiedenen Regelsätzen selbst aber auch aus der wissenschaftlichen Literatur stellt sie ein Korpus aus 70 Regeln zusammen, die im Bereich Technische Dokumentation verwendet werden. Diese Regeln werden anschließend von Sprachexperten (Lektoren, Übersetzern, Posteditoren etc.) nach ihrer Relevanz für entsprechend Verständlichkeit und Übersetzbarkeit gewertet. Das Ergebnis zeigt, dass alle Regeln, die als relevant für Verständlichkeit, auch als relevant für Übersetzbarkeit gewertet wurden, aber nicht umgekehrt. Daher wird angenommen, dass die Regeln für Verständlichkeit auch die Übersetzbarkeit verbessern (vgl. Ramírez Polo 2012, 66). Diese Ergebnisse belegen zwar teilweise die gängige Annahme einer Wechselwirkung zwischen den beiden Regelsätzen, aber es muss im Auge behalten werden, dass die Ergebnisse auf Wertungen beruhen, die m. E. subjektiv sein können.

\subsubsection{Naturalistische und formalistische KS}

Neben der oben erwähnten Unterscheidung zwischen allgemeinen und speziellen KS führt Pool (2006) eine weitere ein: naturalistische und formalistische KS. Obwohl die Bezeichnungen recht verständlich erscheinen, gibt Pool keine eindeutige Definition beider Klassen explizit an. Zunächst charakterisiert er naturalistische KS als Sprachen, die eingeschränkte (natürliche) Sprachen sind, während formalistische KS formalen Notationen ähneln (Pool 2006, 1). Man könnte diese Auffassung also als ein Urteil über den Wiedererkennungswert der zugrunde liegenden natürlichen Sprache interpretieren, was aber der impliziten Handhabung beider Begriffe durch Pool (2006) nicht entspräche: Obwohl die von Pool (2006) aufgeführten Sätze in ACE im Vergleich zu Sätzen in Formalized English (FE) natürlicher erscheinen, werden beide Sprachen explizit als formalistisch klassifiziert (Pool 2006, 5, 6-8), während die naturalistische Klasse solche KS wie ASD-STE100, CASL oder ScaniaSwedish umfasst. Pool (2006) stellt im Anschluss an die erste Charakterisierung selbst fest, dass die Klassen naturalistisch und formalistisch weitestgehend den mensch- und maschinenorientierten Klassen entsprechen (Pool 2006, 1), wobei Ramírez Polo $(2012,69-70)$ formalistische KS als Unterklasse von maschinenorientierten KS sieht.

Des Weiteren kann man in den beiden Klassen Abstufungen annehmen, obwohl dies nur indirekt von Pool angedeutet wird: Die Syntax von FE wird 
explizit als naturalistischer als die Syntax von E2V (Pool 2006, 4) bezeichnet, obwohl beide Sprachen als formalistisch klassifiziert werden.

Schließlich arbeitet Pool (2006) unterschiedliche Eigenschaften für beide Klassen aus. Demnach seien formalistische KS zwar (für Maschinen) präzise, aber eingeschränkt expressiv, während naturalistische KS über eine gesteigerte Expressivität verfügen, was aber auf Kosten der Präzision (für Maschinen) geschieht. Er konstatiert, dass sich für KS für Webanwendungen ein Mittelweg herauskristallisieren könnte (Pool 2006, 5).

Das Thema des Formalismus und Naturalismus von Kontrollierten Sprachen wird von Clark u. a. (2010) systematisch aufgegriffen. Standen für Pool (2006) noch der Wiedererkennungswert bei Menschen bzw. die Zielgruppe im Vordergrund, so wird hier nach Parsing-Eigenschaften unterschieden. Demnach sind naturalistische KS solche, für die sich beim Parsing aufgrund ihrer (Rest-)Ambiguitäten mehrere Interpretationen (Strukturen) ergeben, die dann heuristisch bearbeitet werden müssen. Formalistische KS erlauben hingegen nur eine einzige Interpretation (Struktur), das Parsing erfolgt also deterministisch (Clark u. a. 2010, 65-66, 73). Beide Klassen sind mit weiteren Eigenschaften gepaart. Formalistische Kontrollierte Sprachen verwenden umständliche oder stilistisch unschöne Ausdrücke und verlangen gute Kenntnisse der KS-Spezifikation, da nur erlaubte Konstruktionen und insbesondere nur der vordefinierte Wortschatz akzeptiert werden. Im Gegenzug ist ihr Parsing-Verhalten vorhersehbar.

„In contrast, the "naturalist" language CPL [Computer-Processable Language] is more fluent and tolerant of the user: it uses WordNet to ,guess“ meanings for unknown words, will use lexical and semantic knowledge to try and perform PP attachment and semantic role labeling correctly, and attempt to resolve metonymy. However, as the CPL interpreter is more complex than that for the "formalist" language CPL-Lite, there is arguably more of a risk that CPL may not interpret the sentence in the way the user intended, and it may not be obvious to him/her how to reformulate the CPL to correct the error (if indeed the user is aware of the misinterpretation)." (Clark u. a. 2010, 70)

Eine andere Interpretation der Auffassung von Clark u.a. (2010) bieten Marrafa u. a. (2012). Naturalistische KS werden demnach durch proskriptive Regeln definiert, formalistische KS entstehen hingegen durch präskriptive Regeln (Marrafa u.a. 2012, 152). Während sich also bei den ersten die zugelassenen Ausdrücke implizit aus der vollen natürlichen Sprache ergeben, sind in den letzten nur diejenigen Ausdrücke erlaubt, die aus den explizit 
angegebenen Regeln konstruierbar sind. Folglich haben naturalistische KS zwar Vorteile für Menschen, da sie beispielsweise leichter erlernbar seien, was aber auf Kosten ihrer maschinellen Verarbeitbarkeit geschehe (Marrafa u. a. $2012,153,154)$.

An diesen weiterführenden Charakterisierungen wird deutlich, dass ein $\mathrm{Zu}$ sammenhang zwischen den Verarbeitungseigenschaften, der Beschaffenheit des Sprachsystems und der (menschlichen) Bewertung der Natürlichkeit einer KS angenommen wird. Zusammenfassend lässt sich also sagen, dass die Einteilung in formalistische und naturalistische KS nicht anhand eines einzigen Merkmals vorgenommen werden kann. Dennoch scheint das Ergebnis einer solchen Einteilung, d. h. wie konkrete KS klassifiziert werden, gleich zu sein.

\subsubsection{Klassifikationsschema nach Kuhn (2014)}

Die bisher umfang- und facettenreichste Klassifikation von Kontrollierten Sprachen wurde von Kuhn (2014) ausgearbeitet. Das Klassifikationsschema ist integrativ und fasst viele bis dahin einzeln betrachtete Eigenschaften von KS zusammen. Es besteht aus zwei Teilen, die unterschiedlich zu handhaben sind. 
Der erste Teil umfasst 9 Eigenschaften, die ich hier wie folgt gruppiere:

- Ziele

C Verständlichkeit

T Übersetzbarkeit

F Formale Repräsentation und Ausführbarkeit

- Modus

W Schriftlich

S Mündlich

- Einsatzbereich

D Domänenspezifische (fachbereichspezifische) KS (im Unterschied zu domänenübergreifenden)

\section{- Autoren}
A Wissenschaft
I Industrie (im weitesten Sinne)
G Regierung

Mit diesen Eigenschaften können einzelne KS charakterisiert werden, indem einer Sprache alle zutreffenden Buchstaben vergeben werden. So werden beispielsweise ASD-STE100 als C T W D I und ACE als F W A charakterisiert (Kuhn 2014, 148).

Der zweite Teil ist die sog. PENS-Klassifikation. PENS steht für folgende Eigenschaften:

P Präzision - Wie ambig ist eine Sprache? (Kuhn 2014, 128) Wobei hier die Logiksprachen als Bezugspunkt genommen werden.

E Expressivität - Wie viele unterschiedliche Aussagearten können in einer Sprache ausgedrückt werden? (Kuhn 2014, 129-130)

N Natürlichkeit - Wie nah steht die Sprache der natürlichen Sprache? Wird sie von den Muttersprachlern als korrekte Sprache erkannt? (Kuhn 2014, 130-131) 


\section{Forschungsstand}

S Simplizität - Wie umfangreich ist eine vollständige und explizite Beschreibung des Sprachsystems, die keine intuitiven Eigenschaften voraussetzt, und insbesondere ihre Implementierung als Computerresource? (Kuhn 2014, 131-132)

Im Unterschied zum ersten Teil muss jede Sprache anhand aller dieser Eigenschaften charakterisiert werden, wobei hier eine Gewichtung (1-5) vorgenommen wird. Die Gewichtungskriterien sind für jede Eigenschaft beschrieben. Folglich werden ASD-STE als $\mathrm{P}^{2} \mathrm{E}^{5} \mathrm{~N}^{5} \mathrm{~S}^{1}$ und ACE als $\mathrm{P}^{4} \mathrm{E}^{3} \mathrm{~N}^{4} \mathrm{~S}^{3}$ klassifiziert (Kuhn 2014, 148). Insgesamt klassifiziert Kuhn (2014) mit dem vollständigen Schema 100 existierende KS (Kuhn 2014, 147-165). Das Schema etabliert sich auch zunehmen in der Forschung (siehe beispielsweise Beiträge in Davis, Kaljurand und Kuhn (2014)).

Mit den zielbezogenen Eigenschaften (C T F) im ersten Teil des Klassifikationsschemas habe ich mich bereits in (Suchowolec 2014, o.D.) auseinandergesetzt. In Suchowolec (2014, 119-120) schlage ich die Klasse der KS für Texterstellung (authoring) als Erweiterung vor. In Suchowolec (o.D.) diskutiere ich die Konstruktionsprinzipien der Klassen (Eigenschaften) und folglich die Wohlgeformtheit dieses Klassifikationsausschnitts. Die festgestellten Probleme, vor allem eine starke Überlappung der Klassen C T A für (A für authoring) einerseits und das mit den restlichen Klassen nicht kompatible Konstruktionsmerkmal der Klasse F andererseits, führen zu einer gewichteten (1-3) und strukturierten Klassifikation der KS bezüglich ihrer Ziele, (s. Tabelle 1), wobei ich insgesamt einen beschreibenden Ansatz anstelle einer Klassifikation zur Erfassung von unterschiedlichen Zielen und Motiven der KS-Konstruktion für geeigneter halte (Suchowolec o.D., o.S.).

\subsubsection{Erfolg}

Wie auch in anderen Gebieten, die in dieser Arbeit betrachtet werden, ist das Problem einer erfolgreichen Einführung einer Kontrollierten Sprache in einem bestimmten Szenario systematisch noch beinahe unerforscht. Im Folgenden gehe ich auf die Überlegung ein, die mit den systemlinguistischen Eigenschaften des Regelwerkes zusammenhängen. Anschließend führe ich exemplarisch nichtsystemlinguistische Aspekte auf, die in der Literatur immer wieder, jedoch recht unfokusiert und unsystematisch, aufgegriffen werden. Eine ausführliche Betrachtung dieser Aspekte erfolgt jedoch erst in Kapitel 5. 


\begin{tabular}{ll|llll|llll}
\hline & & $\mathbf{T}$ & & & $\mathbf{R}$ & & & \\
\hline production & process & $T_{A}$ & $T_{T}$ & production & process & $T_{A}$ & $T_{T}$ & $R_{A}$ & \\
& result & $T_{A}$ & $T_{T}$ & & result & $T_{A}$ & $T_{T}$ & $R_{A}$ & \\
\hline reception & process & $T_{C}$ & $T_{P}$ & reception & process & $T_{C}$ & $T_{P}$ & $R_{C}$ & $R_{P}$ \\
& result & $K$ & $K_{A}$ & & result & $K$ & $K_{A}$ & & \\
\hline
\end{tabular}

Tabelle 1.: Klassifikationsschema von textorientierten (T) und repräsentationsorientierten (R) KS (Suchowolec o.D., o.S.). Das Schema benutzt folgende Symbole: $T_{A}$ : improving text authoring (T- und R$\mathrm{KS}), T_{T}$ : improving text translating (T- und R-KS), $R_{A}$ : improving representation authoring (nur R-KS), $T_{C}$ : improving text comprehension (T- und R-KS), $T_{P}$ : improving text processing (T- und R$\mathrm{KS}$ ), $R_{C}$ : improving representation comprehension (nur R-KS), $R_{P}$ : improving representation processing (nur R-KS), $K$ : improving knowledge (T- und R-KS), $K_{A}$ : improving action based on knowledge (T- und R-KS)

\subsubsection{Erfolg durch systemlinguistische Faktoren}

Neben detaillierten Aufzählungen und Beschreibungen von möglichen Regeln, aus denen man für sein Projekt die geeigneten auswählen kann, wie Leitlinie Regelbasiertes Schreiben (tekom-AG „Regelbasiertes Schreiben“ 2013; tekom-AG „Englisch für deutschsprachige Autoren“ 2014), findet man in der Literatur einige allgemeine, intuitiv fundierte Überlegung zur systemlinguistischen Beschaffenheit von Kontrollierten Sprachen. So orientieren sich Cardey, Greenfield und Wu (2004) bei der Konstruktion von einer KS für maschinelle Übersetzung Englisch-Deutsch an drei Prinzipien: Konsistenz (Widerspruchsfreiheit von Regeln, konsistenter Einsatz), Klarheit (konsequente Beachtung von Regeln) und Einfachheit (aus mehreren Ausdrückvarianten soll die einfachste bevorzugt sein) (Cardey, Greenfield und Wu 2004, 42). Heald und Zajac $(1998,125)$ fordern hingegen eine klare Form-Bedeutung-Korrespondenz (Transparenz) und Reduzierung morphosyntaktischer Ambiguität. Für eine maschinenorientierte KS hingegen fordert beispielsweise Schwitter (2010b) folgende Eigenschaften:

,(a) it should have a well-defined syntax and a precise semantics that is defined by an unambiguous mapping into a logic-based representation; (b) it should look as natural as possible and be based on a subset of a certain natural language; (c) it should be easy for humans to write and understand and easy 


\section{Forschungsstand}

for a machine to process; and (d) it should have the necessary expressivity that is required to describe a problem in the respective application domain“" (Schwitter 2010b, 1120; vgl. Schwitter 1998, 43, 45).

Darüber hinaus wurde die Entstehung von OWL Simplified English durch folgende Prinzipien geleitet:

„1. It should be possible to describe the language very briefly; as a rough guide, the basic rules should fit comfortably on a sheet of A4. 2. Any preliminary work on the lexicon should be minimised: ideally, a user should be able to type in sentences straight away, without having to list content words or entity names. 3 . The grammar should disallow sentences that people perceive as structurally ambiguous. 4 . The grammar should be finite-state, so that sentences can be parsed and interpreted efficiently by a finite-state transducer. 5. No effort should be made to guarantee that sentences are grammatical according to the conventions of normal English. Provided that the CNL makes it possible to write fluent English, adherence to conventional grammar can be left to the human author" (Power 2012, 45; typographische Auszeichnung aufgehoben).

$\mathrm{Zu}$ erwähnen ist darüber hinaus die SBVR-Spezifikation für business rules (OMG 2008; Spreeuwenberg und Healy 2010, vgl.). Sie definiert keine Kontrollierte Sprache an sich, sondern stellt ein Metamodell zur Entwicklung und Dokumentation von business-bezogenen Wortschätzen, Regeln und Tatsachensammlungen zur Verfügung, die in formale Repräsentationen (Prädikatenlogik mit modallogischen Erweiterungen) überführbar sind (OMG 2008, 3). Zudem führt diese Spezifikation das XMI-Schema als Austauschformat für business rules ein. Auf deren Grundlage sind dann solche KS wie SBVR Structured English (Spreeuwenberg und Healy 2010, 163-165) und RuleCNL (Njonko u. a. 2014) definiert.

Systematische Überlegungen dazu, wie das Sprachsystem einer KS beschaffen sein kann und soll, finden wir ebenfalls bei Kuhn2014. ${ }^{58} \mathrm{Er}$ benutzt die Klassifizierung von $100 \mathrm{KS}$ mit seinem in 2.3.5.4 vorgestellten Schema zur Metaanalyse, welche Ausprägungen des PENS-Teils mit welchen Eigenschaften aus dem ersten Teil des Schemas zusammenhängen. Hierbei stellt er beispielsweise fest, dass die KS für verbesserte Verständlichkeit im Durchschnitt folgende PENS-Klassifikation ergeben: $\mathrm{P}^{2,0} \mathrm{E}^{4,3} \mathrm{~N}^{4,7} \mathrm{~S}^{1,2}$,

58 Auch bei Kuhn (2010b, 50-52) findet man fünf Eigenschaften, die Grammatik einer KS für ein PredictiveEditor erfüllen soll (Konkretheit, Deklarativheit, Lookahead-Unterstützung, eindeutig definierter Scope und Umgang mit Anaphern, Implementierbarkeit). Es handelt sich hierbei jedoch um computerlinguistische Überlegung zur Implementierung des KS-Regelwerks als eine computerlesbare Grammatik, was nicht im Fokus dieser Arbeit steht. 
während die KS für formale Repräsentationen im Durchschnitt diesem Muster folgen: $\mathrm{P}^{4,4} \mathrm{E}^{2,3} \mathrm{~N}^{3,8} \mathrm{~S}^{3,2}$ (s. Tabelle Kuhn 2014, 142). Die Ergebnisse sind zunächst deskriptiv und zeigen die bisherigen Konstruktionstendenzen, können aber als Best Practice einen Anhaltspunkt für die Konstruktion neuer Kontrollierter Sprachen darstellen (Kuhn 2014, 143).

\subsubsection{Erfolg durch nichtsystemlinguistische Faktoren}

Auch den nichtsystemlinguistischen Erfolgsfaktoren von Kontrollierten Sprachen wurde bisher wenig systematische Aufmerksamkeit geschenkt. Eine Reflexion über die Gründe für die Aufgabe von CFE finden wir beispielsweise bei Kamprath u. a. $(1998,2)$. Die genannten nichtsystemlinguistischen Faktoren umfassen:

- Sprachlerntradition: Nichtenglischmuttersprachler müssen CFE wie jede andere Fremdsprache erlernen; das systematische Erlernen einer Fremdsprache ist nicht in jeder Lerntradition vorhanden, was auch mit mangelndem Sprachbewusstsein im Zusammenhang steht.

- Schulungsdurchführung und -kosten: hohe Fluktuation der Servicemitarbeiter erschwerte die Schulung. Zudem verursachte die steigende Komplexität von CFE steigende Schulungskosten.

- Lateinisches Alphabet: Der Einsatz von CFE statt Übersetzungen setzte Kenntnisse des lateinischen Alphabets voraus, die jedoch nicht in jedem Absatzmarkt (beispielsweise Asien) vorhanden waren.

- Nationalen Stolz: In manchen Regionen besitzen Texte in eigener Muttersprache einen hohen Stellenwert, weil sie mit der kulturellen Identität zusammenhängen.

- Sprachtechnologie: Der damalige Stand der Technik stellte keine Werkzeuge zur Verfügung, die das Einhalten der CFE-Spezifikation (maschinengestützt) überprüfen und sicherstellen könnten.

Bei diesen Faktoren handelt sich insbesondere um verschiedene soziale, psychologische, kulturelle, wirtschaftliche und technologische Aspekte und ihre Kombinationen. In der Analyse der Erfolgsfaktoren werden alle diese Aspekte berücksichtigt, der Schwerpunkt wird jedoch auf der Kombination von sozialen und psychologischen mit anderen Aspekten liegen. Eine prominente Stellung nehmen hierbei der beobachtete Widerstand der aktiven Zielgruppe 


\section{Forschungsstand}

ein, die sich in Kreativität, gewohntem Stil und Entscheidungsfähigkeit eingeschränkt fühlt (exemplarisch Lehrndorfer 1996, 47; Bernth 1998b, 192; Hebling 2002, 54-65; Namahn 2002; Crabbe 2009, 58; Ramírez Polo 2012, $62-$ 64), sowie die Überlegungen zur Benutzerfreundlichkeit von Softwarelösungen (exemplarisch Denaux u. a. 2010, 262; Kuhn 2010a). Mehr Beispiele von nichtsystemlinguistischen Faktoren, die den Erfolg bei der Einführung von Kontrollierten Sprachen fördern, finden sich in der Analyse in Kapitel 5.

\subsubsection{Zusammenfassung und Diskussion}

Anhand der angeführten Beispiele und Beschreibungen ergibt sich ein heterogenes Bild des Gegenstandes Kontrollierte Sprachen sowohl hinsichtlich ihrer systemlinguistischen Definition, Explizitheit des Regelwerkes, Konstruktionsmotive/Funktionen, Einsatzbereiche und der technischen Unterstützung. Die vorgestellte Definition des Gegenstandes von Kuhn (2014) ist zwar die facettenreichste, sie spricht aber diese Heterogenität nur unzureichend an. Darüber hinaus eignet sie sich vor allem zur Identifikation von prototypischen bzw. idealtypischen KS, bietet aber wenig Hilfestellung im Randbereich, insbesondere was die Abgrenzung zu Schreibregeln betrifft.

Zur besseren Strukturierung dieser Arbeit verwendete ich die Unterscheidung zwischen textorientierten und repräsentationsorientierten Kontrollierten Sprachen, deren Charakterisierung sich in vielen Punkten wesentlich unterscheidet. An dieser Stelle greife ich noch einmal die Zielgruppen auf, weil sie eine zentrale Rolle beim Aufbau der übergreifenden Perspektive und bei Überlegungen zu Einführungsmechanismen spielen. So zielen textorientierte KS in erster Linie auf die Veränderung des eingebürgerten Sprachgebrauchs von hauptberuflichen Textproduzenten, vor allem in der Technischen Dokumentation. Sie greifen somit in die bestehende, aufgabenbezogene Sprachpraxis sowohl von Individuen als auch, abstrakter betrachtet, von Gemeinschaften ein. Anders sieht die Situation bei repräsentationsorientierten KS aus, bei der ich anhand der Beschreibungen drei Szenarien identifiziere: 1) Bei Domänenexperten im Unternehmenskontext stellt die selbstständige Pflege einer formalen Wissensrepräsentation in den meisten Fällen eine völlig neue Aufgabe dar. Hinsichtlich dieser Aufgabe dienen KS als Werkzeug zur Bewältigung dieser Aufgabe ohne Ausbildung in formalen Notationen. Somit ist die Einführung einer KS kein Eingriff in die bestehende Sprachpraxis von Domänenexperten (sowohl als Individuen als auch als Gemeinschaft aufgefasst). Anders können sich Szenarien gestalten, bei denen der Einsatz einer formalen 
Notation eine ernstzunehmende Alternative oder gar das Mittel der Wahl ist. 2) Beherrscht man formale Notationen bereits, wie im Fall von Wissensingenieuren, so ist die Umstellung auf eine KS ein Eingriff in die bestehende (individuelle und kollektive) Sprachpraxis. 3) Beherrscht man dagegen weder formale Notationen noch Kontrollierte Sprache und steht die Wahl zwischen den beiden Ansätzen bei einer konkreten Aufgabe frei, so spielen bei der Entscheidung m. E. unter anderem solche Eigenschaften des Ansatzes wie Etabliertheit, Erfahrungen dritter, Robustheit, Funktionalitäten, Portabilität und Lernaufwand eine Rolle. Man greift also mit der KS hierbei nicht direkt in die bestehende Sprachpraxis der (künftigen) Benutzer ein, sondern ggf. in die Konventionen einer (aufgabenbezogenen) Gemeinschaft.

Die Geschichte der KS-Konstruktion verzeichnet drei Entwicklungsstränge: Verbesserung der Verständlichkeit, der (maschinellen/maschinengestützten) Übersetzbarkeit und der Interaktion mit formalen Repräsentationen. Auch die systematische Erforschung von KS orientiert sich an diesen Strängen und erfolgt vor allem aus der computerlinguistischen und kognitionslinguistischen Perspektive, wobei eine weitere empirische Sicherung der Erkenntnisse notwendig ist. Wichtig für diese Arbeit ist die Auffassung von Sprache in den vier zu untersuchenden Gebieten, wobei ich mich bei Kontrollierten Sprachen vor allem auf die implizite Auffassung stützen muss. Die Auffassung von Fachsprachen ist in der KS-Forschung durchaus eine soziolinguistische (s. 2.3.1.2). Hingegen ist die Auffassung von Kontrollierten Sprachen stark systemlinguistisch geprägt, was aus den besprochenen Gegenstandsdefinitionen klar hervorgeht. Da Kontrollierte Sprachen nicht als soziale Phänomene aufgefasst werden, fehlt in ihrer theorie- wie auch praxisgeleiteten Erforschung, und insbesondere in der Beschreibung und Beantwortung der Praxisprobleme, die soziolinguistische Perspektive. Die am stärksten vertretene computerund kognitionslinguistische Perspektive, wenn eindeutig relevant und berechtigt, reicht $\mathrm{m}$. E. nicht aus, um alle praxisrelevanten Phänomene bei der Einführung von Kontrollierten Sprachen in konkreten Projekten zu erforschen. Die zahlreichen Erfahrungsberichte sind ein großer Fundus an solchen Phänomenen, aus dem ich im Kontext dieser Arbeit an dieser Stelle das Akzeptanzproblem herausgreifen möchte. Hierfür gehe ich exemplarisch auf zwei Berichte ein.

Moore (2000) berichtet von ihrer Erfahrung mit der Einführung einer KS bei Diebold, die den Einsatz von Translation Memorys unterstützen sollte. Sie merkt an, dass Probleme mit der KS-Akzeptanz im größeren 


\section{Forschungsstand}

Kontext von allgemeineren Akzeptanzproblemen bei der Neudefinition von Arbeitsprozessen (hier: Einführung von SGML) zu sehen sind. Gleichzeitig kann die Akzeptanz von KS, speziell von CLC, dadurch beeinträchtigt sein, ob die betroffenen Technischen Redakteure am Entscheidungsprozess beteiligt werden oder nicht, und, insbesondere, ob die Einführung eines CLC von oben beschlossen wurde. Neue Technische Redakteure, die erst nach der Prozessanpassung eingestellt wurden, seien hingegen aufgeschlossener (Moore 2000, 52). Ähnliche Beobachtungen macht bereits Goyvaerts (1996), der zudem betont, dass eine aktive Beteiligung der Redakteure an der Konzeptausarbeitung, -evalutation und -verfeinerung einen akzeptanzsteigernden Effekt hat (Goyvaerts 1996, 139).

Aus dem oben Beschriebenen geht hervor, dass bei der Einführung von Kontrollierten Sprachen in der Praxis Gruppen-, Arbeits- und Organisationsprozesse eine wichtige Rolle spielen und sich auf die Akzeptanz auswirken. Somit hat diese Einführung eine wesentliche soziale Komponente, die jedoch in der systematischen Forschung weitestgehend vernachlässigt wird.

Relativ wenig Aufmerksamkeit in der Forschung wird ebenfalls der Bedeutung von KS-Autoren geschenkt. Aus der Sicht dieser Arbeit ist u. a. die Frage nach der Top-down- bzw. Bottom-up-Konstruktion und -Einführung von Kontrollierten Sprachen interessant. Anders als bei LPLP und der Erforschung von Plansprachen wird sie für Kontrollierte Sprachen auf der Metaebene m. W. nicht aufgegriffen. Auch die Projektberichte, wie in vorangegangenen Abschnitten gezeigt, gehen darauf nur vereinzelt und zwar vorwiegend dann ein, wenn ein sehr starker Top-down-Ansatz zu Akzeptanzproblemen bei der aktiven Zielgruppe führt. In diesem Fall wird eine stärkere Beteiligung dieser Zielgruppe an der Entwicklung, Umsetzung und Evaluierung der KS-Konzeption gefordert (Goyvaerts 1996; Moore 2000; vgl. Suchowolec 2014, 120-121). Eine systematische Auseinandersetzung mit oder eine empirische Überprüfung von diesen Mechanismen erfolgte bisher jedoch nicht.

Schließlich handelt es sich bei theoretischen Modellierungen des Gebietes vor allem um unterschiedliche Klassifikationen von Kontrollierten Sprachen, wobei man zurzeit stärker auf multidimensionale Charakterisierungen mithilfe von sich teilweise überlappenden Merkmalen und weniger auf eindeutige Klassifikationen oder Taxonomien setzt. Bezeichnend ist auch eine Abkehr von Beobachtungen und Beschreibungen von Einzelphänomenen hin zur Formulierung von Best-Practice-Empfehlungen, die auf Verallgemeinerung von Praxiserfahrungen beruhen. Dabei stehen auch hier vor allem systembzw. computerlinguistische Aspekte im Vordergrund. Diese Best-Practice- 
Empfehlungen befinden sich somit an der Schnittstelle zum Thema Erfolg von $K S$. Dadurch wird auch deutlich, dass sich die Erforschung von Erfolg bisher eher auf eine erfolgreiche Konstruktion und nicht eine erfolgreiche Einführung von KS konzentriert. Die Auseinandersetzungen mit Einführungsaspekten bleiben auf der Ebene von Einzelbeobachtungen und -aussagen.

\subsection{Terminologiearbeit}

In diesem Teilkapitel befasse ich mich mit Maßnahmen, die lediglich auf der lexikalischen Ebene in die Sprache eingreifen, also mit der sog. Terminologiearbeit (TA). Im Fokus steht dabei die traditionelle TA, die auf methodischen Prinzipien der Terminologielehre nach Wüster (1931) beruht. Sie zielt ursprünglich auf die Fachkommunikation und ich betrachte hier hauptsächlich Maßnahmen, die im industriellen Bereich durchgeführt werden (in Unternehmen aber auch Normungsinstituten und Fachverbänden).

In diesem selektiven Forschungsüberblick trage ich Inhalte zu gleichen Themenblöcken wie in den vorangegangenen Teilkapiteln zusammen, bespreche diese aber in einer anderen Reihenfolge. Diese abweichende Strukturierung gründet sich darin, dass die Beschreibung der traditionellen Terminologiearbeit anhand fest definierter und vorgegebener Konzepte erfolgt. Für einen Forschungsüberblick ist es deshalb notwendig, zunächst diese festgelegte Konzeptualisierung des Gebietes und seine Metasprache einzuführen. Aus diesem Grund ziehe ich im Unterschied zu vorangegangenen Teilkapiteln den Abschnitt zu (theoretischen) Modellen und methodischen Vorgehensweisen vor, so dass er direkt nach einer einleitenden Definition und einer kurzen Charakterisierung grundlegender Begriffe angeführt ist. Es folgen Abschnitte zur Einordnung der Terminologielehre als Disziplin, sowie zu Zielgruppen, Werkzeugen, Geschichte, Autoren und schließlich zum Erfolg der Terminologiearbeit.

\subsubsection{Gegenstandsdefinition}

In diesem Abschnitt gebe ich die Definition drei Hauptbegriffe - Terminologie, Terminologielehre und Terminologiearbeit - nach der Norm DIN 2342 (2011) an und erläutere diese anschließend.

- „Terminologie

Fachwortschatz 


\section{Forschungsstand}

Gesamtbestand der Begriffe (...) und ihrer Bezeichnungen (...) in einem Fachgebiet" (DIN 2342 2011, 16)

- „Terminologielehre

Terminologie (abgelehnt)

Wissenschaft von den Begriffen (...) und ihren Bezeichnungen (...) in den Fachsprachen (...)“(DIN 2342 2011, 14)

- „Terminologiearbeit

auf der Terminologielehre (...) aufbauende Planung, Erarbeitung, Bearbeitung oder Verarbeitung, Darstellung oder Verbreitung von Terminologie (...).“ (DIN 2342 2011, 14-15)

Obwohl die Norm mit Terminologie ausdrücklich den Fachwortschatz bezeichnet, findet man zuweilen auch einen abweichenden Sprachgebrauch, wie in den o.g. Definitionen mit der Bemerkung „abgelehnt“ gekennzeichnet ist. So weist auch Pearson $(1998,9-10)^{59}$ darauf hin, dass im Englischen terminology als Bezeichnung für sowohl den Fachwortschatz, für die methodische Vorgehensweise wie auch die praktische Tätigkeit gebraucht wird. Einen ähnlich mehrdeutigen Gebrauch im Deutschen stellte auch Wüster (1974, 62-63) fest.

Der Definition von Terminologielehre ist hingegen zu entnehmen, dass es sich hierbei um eine theoretische Grundlagenwissenschaft handelt, die die Gesetzmäßigkeit von Fachwortschatz erforscht und die Methoden für die praktische Tätigkeit der Terminologiearbeit bereitstellt (Wüster 1991, 1; Arntz, Picht und Schmitz 2014, 3). ${ }^{60}$ So wird Terminologielehre auch Terminologiewissenschaft genannt (Felber und Budin 1989, 1). Wichtig ist auch zu betonen, dass sie sich ausdrücklich auf Fachsprachen ${ }^{61}$ - speziell den Fachwortschatz - beschränkt (vgl. Wüster 1991, 1; vgl. Arntz, Picht und Schmitz 2014, 271). Somit sind die Methoden nicht per Definition auf den Allgemeinwortschatz anwendbar. ${ }^{62}$ Darüber hinaus führen Felber und Budin (1989) die Unterscheidung zwischen der allgemeinen Terminologielehre

59 S. auch Rey (1995, 126-128), Cabré (1999, 32), Temmerman (2000, xiii, Fußnote 1 et passim) und Schmitz und Straub (2010, 7).

60 Abweichend davon: Felber und Budin $(1989,1)$, die terminologische Grundsatzlehre als methodische Teildisziplin betrachten.

61 An dieser Stelle diskutiere ich die Auffassung von Fachsprachen in DIN 2342 (2011) nicht. Für einen allgemeinen Überblick über die Auffassung von Fachsprachen und Fachkommunikation siehe bspw. Roelcke (2014).

62 Dass terminologische Prinzipien trotz dieser Beschränkung auf Fachsprachen auch auf die Gemeinsprache angewendet werden, zeigt beispielsweise L'Homme $(2006,60)$ für Kanada, s. auch Cabré $(1999,14)$. 
(alle Fachgebiete betreffend) und der speziellen Terminologielehre (Gesetzmäßigkeiten eins bestimmten Fachgebietes wie bspw. der Medizin) ein (Felber und Budin 1989, 1), die in der heutigen Forschung und Praxis jedoch nicht etabliert ist.

Vor diesem Hintergrund handelt es sich also bei Terminologiearbeit und eine praktische Tätigkeit. Wie in der Definition angeführt, verwendet sie die Grundsätze der Terminologielehre und setzt insbesondere auch ihre Kenntnisse voraus. Auch sie beschränkt sich auf die Aufbereitung des Fachwortschatzes - Pflege des Allgemeinwortschatzes werden also gemäß DIN 2342 (2011) nicht als Terminologiearbeit aufgefasst. Zudem listet die Definition auch einzelne Prozesse auf, auf die die Terminologiearbeit heruntergebrochen werden kann wie Planung, Erarbeitung, Bearbeitung (vgl. DIN 2342 2011, 15).

Zusammenfassend lässt sich sagen, dass in der Auffassung von DIN 2342 (2011) der Fachwortschatz (Terminologie) das Eingriffsobjekt, die Terminologielehre die Eingriffsmethoden und die Terminologiearbeit den eigentlichen Eingriff in die sprachlichen Mittel darstellen. So verwende ich diese Bezeichnungen auch in dieser Arbeit. Dass diese Unterscheidung zwischen methodischen Grundsätzen und der Tätigkeit an sich in der Praxis verschwommen ist, da auch die Terminologiearbeit methodische Überlegungen hervorbrachte, die man nicht als Terminologielehre auffasst, wird anhand der Beschreibungen in Abschnitt 2.4.3 deutlich.

\subsubsection{Charakterisierung}

Um das Verständnis der Begriffe der Terminologie, Terminologielehre und Terminologiearbeit zu vertiefen, gehe ich kurz auf die verwandten Begriffe ein. Anschließend charakterisiere ich Einsatzbereiche der Terminologiearbeit und erläutere ihre Funktionen im Unternehmenskontext, d. h. aus welchen Gründen sie betrieben wird und welche Vorteile man sich von ihr erhofft.

\subsubsection{Verwandte Begriffe}

Neben den oben beschriebenen durch die Norm vorgegebenen Begrifflichkeiten Terminologie und Terminologiearbeit existieren weitere Konzepte, die vor allem bei Terminologiearbeit im Unternehmenskontext gebraucht werden.

Manche Autoren verwenden Terminologiemanagement synonym zu Terminologiearbeit (F. Mayer 2009, 15-16; Drewer und Ziegler 2011, 157). Andere unterscheiden zusätzlich Terminologieverwaltung: Während bei der Terminologieverwaltung vor allem betreut, katalogisiert und bereitgestellt wird, geht 


\section{Forschungsstand}

es beim Terminologiemanagement ums Führen, Leiten und strategische Ausrichten (Sturz 2014). Letztendlich besteht hierbei also ein konnotativer Unterschied. Schließlich behandelt DIN 2342 (2011) Terminologiemanagement und Terminologieverwaltung synonym und definiert sie als Teilprozess der Terminologiearbeit (DIN 2342 2011, 15; vgl. Schmitz und Straub 2010, 8).

Mit Terminologie ist hingegen der Begriff Corporate Language (CL) ${ }^{63}$ verwandt. In der terminologiebezogenen Literatur wird Corporate Language m.W. nicht näher bestimmt, was eine Abgrenzung schwierig macht. Lediglich aus dem Sprachgebrauch schließe ich, dass CL für manche Autoren mit Terminologie nicht deckungsgleich ist und eine übergreifende Kategorie darstellt (exemplarisch Drewer und Ziegler 2011, 157). Andere Autoren verwenden beide Bezeichnungen beinahe synonym (exemplarisch M. Mayer 2010; Weilandt 2014, 11).

\subsubsection{Einsatzbereiche}

Bei Kontrollierten Sprachen bezogen sich die Einsatzbereiche auf Fächer oder einzelne Industriebranchen (Abschnitt 2.3.1.3). Für Terminologiearbeit übernehme ich aus der Literatur eine andere Strukturierung, die für den weiteren Verlauf dieser Arbeit günstiger erscheint.

Mit Rey (1995) kann grundsätzlich zwischen zwei Perspektiven auf Terminologiearbeit unterschieden werden: nach beteiligten Fachbereichen und nach beteiligten Sprachen (Rey 1995, 158-166). Dabei handelt es sich um keine trennscharfe Gliederung sondern um unterschiedliche Blickwinkel (vgl. Cabré 1999, 16):

- Nach beteiligten Sachgebieten, wobei dieser Eingriff sowohl implizit oder explizit, als auch systematisch oder spontan erfolgen kann (Rey 1995, 159). Rey (1995) unterscheidet dabei folgende Akteure bzw. Szenarien:

- Terminologiearbeit in wissenschaftlichen und technischen Gemeinschaften oder Organisationen selbst (Rey 1995, 159-162), die sozialpolitisch unterschiedlich verankert sein können, z.B. als autonome Zusammenschlüsse oder aber als regierungsnahe Organisationen. Darüber hinaus nehme ich Unternehmen aus der privaten Wirtschaft in diesen Punkt mit auf. Alle diese Szenarien teilen das Ziel, die Terminologie des eigenen Faches, also

63 Ich gehe hier auf weitere Begriffe wie Corporate Wording (Förster, Rost und Thiermeyer 2010) oder Corporate Identity nicht näher ein. 
intern, zu vereinheitlichen und mehr oder weniger systematisch weiterzuentwickeln.

- Regierungsnahe Terminologiearbeit: Zum einen erfolgt diese Arbeit im Zusammenhang mit kulturpolitischen Maßnahmen wie Kultur- und Identitätspflege und -erhalt (Rey 1995, 162-164), also ähnlich wie in 2.1 beschrieben. Diese Sicht auf Terminologiearbeit ist $\mathrm{m}$. E. in der deutschsprachigen Literatur ein wenig zurückgedrängt, wurde aber bereits durch Wüster postuliert (Wüster 1991, 3). Zum anderen gehört für Rey (1995) auch die übersetzungsbezogene Terminologiearbeit für nationale und internationale Kommunikation beispielsweise durch EU- oder UNO-Institutionen dazu (Rey 1995, 164-165).

- Terminologiearbeit durch Normungsinstitute wie die ISO oder AFNOR, im Zusammenhang mit der Sachnormung, vor allem im ingenieur-technischen Bereich (Rey 1995, 165).

- Nach beteiligten Sprachen: Mit Rey (1995) geht es hier um terminologische Abstimmungen über die (insbesondere) nationalen Grenzen hinaus. Diese Standardisierungsbestrebungen können sowohl eine einzelne Sprache, die in mehreren Ländern gesprochen wird, betreffen (z. B. übergreifende Standardisierungen des Arabischen), als auch multilingual ausgerichtet sein (die Terminologiesammlung der EU EURODICAUTOM $)^{64}$ (Rey 1995, 165-166).

Terminologiearbeit in diesen unterschiedlichen Szenarien kann sich in vielen Punkten überschneiden und gleichzeitig unter anderen wichtigen Aspekten unterschiedlich sein. Einige Beispiele sowie Unterschiede und Parallelen zwischen der übersetzungsbezogenen und kulturpolitischbezogenen Terminologiearbeit in Kanada trägt L'Homme (2006) zusammen. So durchlaufen beispielsweise Übersetzer und Sprachplaner eine ähnliche Ausbildung in terminologischen Methoden, ihre anschließende Tätigkeit unterscheidet sich jedoch entsprechend bezüglich der Begriffsorientierung/Benennungsorientierung und der Einbeziehung der Gemeinsprache.

Im verbleibenden Teil dieses Teilkapitels konzentriere ich mich hauptsächlich auf die Methoden, Werkzeuge und Modelle der Terminologiearbeit in

64 Heute: InterActive Terminology for Europe IATE, s. Translation Centre for the Bodies of the European Union (1997-). 


\section{Forschungsstand}

Unternehmen. Die Terminologiearbeit auf der makrosozialen Ebene rechne ich dem LPLP zu, komme aber vereinzelt darauf auch in diesem Teilkapitel zurück, insbesondere in der kritischen Auseinandersetzung mit der Terminologielehre von Wüster durch Temmerman (2000) (s. Abschnitt 2.4.4.3). Auch die spätere Erfolgsanalyse für Terminologiearbeit wird nach diesem Prinzip entsprechend dem einem oder dem anderen Gebiet zugeordnet.

\subsubsection{Funktionen}

Historisch betrachtet, gründete sich die ursprüngliche Motivation für Terminologiearbeit auf die Verbesserung der Kommunikation zwischen Experten des gleichen Faches. Wüster (1931) geht insbesondere im ingenieur-technischen aber auch im naturwissenschaftlichen Bereich von wohldefinierten Gegenständen aus. Der Fachwortschatz aber, wenn seine Entstehung und Entwicklung ungelenkt verläuft, kann durch Synonymie, Mehrdeutigkeit, Nichtwohlgeformtheit etc. zu Missverständnissen, auch unter Fachexperten, führen (Wüster 1931, 1-3). Im Kontext der technologischen Entwicklungen und der industriellen Revolution des 19. Jh. bemerkt Pearson (1998): „It was becoming clear that the speed of technological progress was such that it was no longer possible to control the naming of new concepts and there was a danger that the same concept might be named differently by different communities creating confusion and communication difficulties" (Pearson 1998, 9). Durch systematische Terminologiearbeit sollte somit die Präzision des Fachwortschatzes gesteigert oder gar seine Ein(ein)deutigkeit erreicht werden. Dies würde die Kommunikation sowohl für Sender als auch Empfänger verbessern und möglichen Informationsverlusten entgegenwirken.

Um das eigentliche Interesse der Industrie an Terminologiearbeit besser nachvollziehen zu können, müssen diese grundsätzlichen Verständigungsprobleme vor dem Hintergrund der Dynamik technologischer Entwicklungen betrachtet werden. Durch das schnell anwachsende (Fach-)Wissen, und, aus Sicht der Industrie, durch höhere Varianz, kürzere Einführungszeiten und kürzere Lebenszyklen der Produkte potenzieren sich die Probleme mit dem ungelenkt entstandenen Fachwortschatz (Schmitz und Straub 2010, 8-9; Yamchi 2012, 28). ${ }^{65}$ Aus der Sicht der Unternehmen betreffen diese Probleme nicht nur die interfachliche, sondern auch die intrafachliche (z.B. Produktentwickler in interdisziplinären Projekten,

65 Für eine detaillierte Aufschlüsselung der außersprachlichen Kontextfaktoren, die eine Grundlage für Terminologiearbeit bieten, siehe Schmitz und Straub $(2010,11)$. 
Entwickler-Technische Redakteure-Übersetzer) und vor allem auch die extrafachliche Kommunikation (z. B. Unternehmen-Kunden) (vgl. Buol 2000, 1; Schmitz und Straub 2010, 15; Arntz, Picht und Schmitz 2014, 1). Schmitz und Straub (2010) fassen zusammen, dass Terminologieprobleme in verschiedenen Szenarien auftreten können: ,innerhalb eines Dokuments, innerhalb eines Unternehmensbereichs, zwischen verschiedenen Dokumenten, zwischen verschiedenen Unternehmensbereichen, zwischen Dokument und Produkt, zwischen Produkten“ (Schmitz und Straub 2010, 15, typographische Hervorhebungen aufgehoben). Zudem erhofft man sich von einem konsistenten Terminologiegebrauch ein besseres Textverständnis und, in Folge, ein besseres Produktverständnis durch die Kunden. Das verbesserte Produktverständnis spielt eine Rolle bei der Verhinderung von Fehlbedienungen (Pflaum 2009, 14), steigert also auch die Rechtssicherheit eines Unternehmens und soll sich insgesamt auf die Kundenzufriedenheit positiv auswirken.

Neben der Verständlichkeitssicherung und dem Wissenstransfer wird die Terminologiearbeit auch als Mittel gesehen, kommunikationsbezogene Arbeitsprozesse (Leitner 2014, 20) hinsichtlich der Qualität, aber auch Zeit und Kosten zu optimieren. ${ }^{66}$ Diese Überlegungen sind vor allem in der Technischen Dokumentation präsent. So ist eine einheitliche Terminologie eine Voraussetzung für den Einsatz von Content Management Systemen, oder allgemein von Modularisierungs- und Single-Source-Ansätzen zur Texterstellung (Gust 2006, 16-17; Drewer und Ziegler 2011, 157). Sie ist aber auch für den Übersetzungsprozess relevant (S. E. Wright und L. D. Wright 1997; Drewer und Ziegler 2011, 157; Leitner 2014, 18, 20; Weilandt 2014, 11): Sie entlastet den Übersetzer durch eine verkürzte Recherchezeit (Massion 2009, 27; vgl. Gust 2006, 16; Pflaum 2009, 14; Arntz, Picht und Schmitz 2014, 1), eine höhere Übereinstimmungsrate in Translation Memorys (Janke 2013, 59; vgl. Gust 2006, 17-18) oder durch verminderte Notwendigkeit von nachträglichen Änderungen (Massion 2009, 27-28) und wirkt sich insgesamt auf die Senkung der Übersetzungskosten positiv aus (Massion 2009; Drewer und Ziegler 2011, 158; Janke 2013, 59). Durch eine einheitliche Sprache in der gesamten Textpalette kommuniziert ein Unternehmen oder eine Institution ein einheit-

66 Zu einzelnen Zielen siehe exemplarisch: Verständlichkeit: Pflaum (2009, 14, 17), Drewer und Ziegler (2011, 158) und Haussteiner (2014, 24), Wissenstransfer: Drewer und Ziegler (2011, 158), Arntz, Picht und Schmitz $(2014,2)$, Haussteiner $(2014,24)$ und Weilandt $(2014,11)$, Qualität der Arbeitsprozesse: Janke (2013, 59) und Massion (vgl. 2009, 27-28), Zeit und Kosten der Arbeitsprozesse: Pflaum (2009, 17) und Gust (vgl. 2006, 17-18). 


\section{Forschungsstand}

liches Erscheinungsbild nach außen (Pflaum 2009, 17; Turhan und Collmann 2010, 36; Haussteiner 2014, 20) und profitiert von sog. Synergieeffekten.

Diese vielschichtige Perspektive auf Motive, Ziele und weitere positive Auswirkungen von Terminologiearbeit im Unternehmenskontext, die schwerpunktmäßig auf Verbesserung der (menschlichen) Verständlichkeit, Übersetzbarkeit und Optimierung der Arbeitsprozesse liegt, ist am weitesten verbreitet (vgl. auch DTT 2010, M1-2-5). Auf einen weiteren Punkt macht zudem Buol (2000) aufmerksam. Sie stellt fest, dass terminologische Konsistenz auch einen positiven Einfluss auf die Maschine-Maschine-Kommunikation hat und die Interoperabilität und Integration von unterschiedlichen Systemen ermöglicht. Eine einheitliche Terminologie verbessert also nicht nur die menschliche sondern auch die maschinelle Rezeption (Buol 2000, 26-28).

Schließlich sei noch auf das vor allem sprachbezogene Verständnis von Zielen der Terminologiearbeit von Felber und Budin (1989) hingewiesen, die die Erstellung terminographischer Sammlungen als das übergeordnete Ziel sehen. Erst diese Sammlungen schaffen eine Grundlage für die oben genannten Ziele/Funktionen wie Wissensordnung, Wissens- und Technologietransfer, Übersetzung und Informationsretrieval (Felber und Budin 1989, 206207).

\subsubsection{Modelle}

Dieser Abschnitt führt die Grundlagen der Terminologielehre nach Wüster und der darauf aufbauenden Terminologiearbeit ein.

Im ersten Teil befasse ich mich mit der Terminologielehre und stelle das elementare Modell zur Beschreibung des Fachwortschatzes - das Semiotische Dreieck - vor und erläutere anschließend das weniger verbreitete vierteilige Modell nach Wüster (1959-1960). Ferner führe ich weitere Begriffe und Instrumente der Terminologielehre wie Begriffsbeziehungen, Begriffssysteme und Definitionen ein, die es ermöglichen, die Beschreibungen zu verfeinern und den Fachwortschatz als ein zusammenhängendes System zu betrachten. Weiterhin leite ich erste methodische Vorgehensweisen ab, die hauptsächlich auf der Terminologielehre beruhen.

Im zweiten Teil des Abschnittes widme ich mich der Methoden der Terminologiearbeit, die obendrein aus der Unternehmenspraxis entstanden sind und insbesondere nichtsprachliche Aspekte wie Projekt- und Prozessmanagement für Terminologiearbeit abdecken. Insbesondere gehe ich dabei auf das 9-Stufen-Modell (bspw. Drewer und Ziegler 2011, 164) und die projektbezo- 


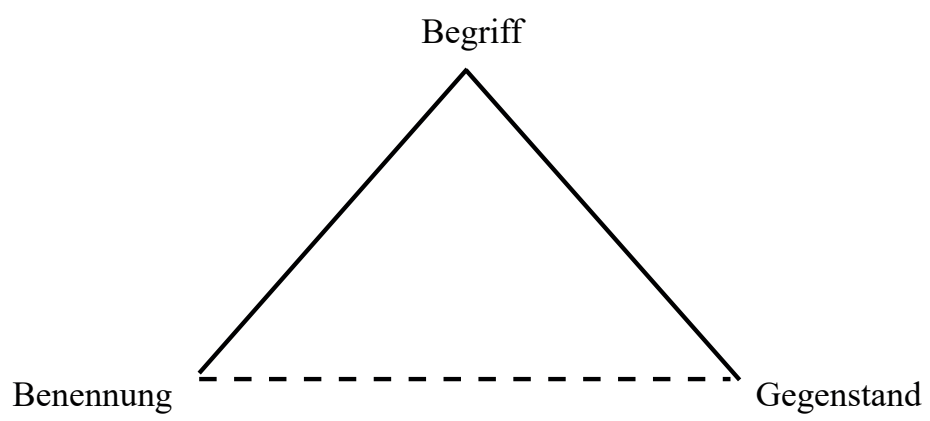

Abbildung 6.: Das semiotische Dreieck

genen Überlegungen in ISO 15188 (2001) und Deutscher Terminologie-Tag e.V. (DTT 2010, M5) ein.

\subsubsection{Terminologielehre}

Das semiotische Dreieck Die Grundlage der Terminologielehre bildet das sog. semiotische Dreieck, das die Beziehung zwischen den drei terminologischen Grundeinheiten - dem Gegenstand, dem Begriff und der Benennung veranschaulicht (s. Abbildung 6) (s. Ogden und Richards 1974).

Diese Grundeinheiten werden in DIN 2342 (2011, Hervorhebungen aufgehoben) wie folgt definiert:

- Gegenstand (Objekt): „(...) beliebiger Ausschnitt aus der wahrnehmbaren oder vorstellbaren Welt"“ (DIN 2342 2011, 5).

- Begriff: „Denkeinheit, die aus einer Menge von Gegenständen (...) unter Ermittlung der diesen Gegenständen gemeinsamen Eigenschaften mittels Abstraktion gebildet wird“ (DIN 2342 2011, 5).

- Benennung (Terminus, Fachausdruck): „sprachliche Bezeichnung (...) eines Allgemeinbegriffs (...) aus einem Fachgebiet" (DIN 2342 2011, 11).

Bei Gegenständen handelt es sich um wirkliche Objekte in der Realität, zum einen um materielle Gegenstände (also solche, die man im weitesten 


\section{Forschungsstand}

Sinne anfassen kann), zum anderen aber auch um immaterielle Gegenstände wie gegenwärtig auftretende Gefühle, Zustände, Sachverhalte etc. (vgl. Felber und Budin 1989, 62-63; DIN 2342 2011, 5; Arntz, Picht und Schmitz 2014, 45-47).

Begriffe stellen eine kognitive Verallgemeinerung über eine Gruppe von einzelnen Gegenständen zu einer Klasse dar: „Auf dem Wege vom Gegenstand zum Begriff werden diejenigen Eigenschaften zusammengefasst, die einer bestimmten Menge von Gegenständen gemeinsam sind“" (Arntz, Picht und Schmitz 2014, 49). Die Eigenschaften von Gegenständen, die zur Begriffsbildung und -abgrenzung benutzt werden, werden Merkmale genannt (DIN 2342 2011, 6). Anders ausgedrückt, abstrahiert man bei der Begriffsbildung von bestimmten Merkmalen, die als nicht wesentlich erscheinen. Des Weiteren unterscheidet DIN 2342 (2011) zwischen einem Allgemeinbegriff: „Begriff, der ausgehend von mehr als einem Gegenstand (...) durch Abstraktion gebildet wird“, Beispiele: Planet, Turm (DIN 2342 2011, 6) und einem Individualbegriff: ,Begriff, der ausgehend von einem einzelnen Gegenstand (...) durch Abstraktion gebildet wird“", Beispiele: Saturn, Eiffelturm (DIN 2342 2011, 6).

Schließlich sind Benennungen sprachliche Repräsentationen von Begriffen, insbesondere von Allgemeinbegriffen, da Individualbegriffe durch $\mathrm{Na}$ men repräsentiert werden (DIN 2342 2011, 6). Begriffe können auch mit anderen Mitteln, wie Formeln oder Symbolen, repräsentiert sein. Eine Repräsentation eines Begriffes ungeachtet dessen, ob sie sprachlich oder nichtsprachlich realisiert wird, wird Bezeichnung genannt (DIN 2342 2011, 10). Da Begriffe als kognitive Einheiten nicht direkt zugänglich sind, stellen Benennungen eine Zugriffsmöglichkeit dar. Gleichzeitig handelt es sich bei Benennungen um Zeichen, die nur durch Konvention auf Gegenstände verweisen. Dies wird im semiotischen Dreieck durch die gestrichelte Linie dargestellt: „Über die gestrichelte Linie drückt das Semiotische Dreieck aus, dass Menschen (...) [die] Gegenstände nicht direkt benennen, sondern zunächst konzeptuelle Kategorien bilden - die Begriffe - und diese dann mit Benennungen versehen (Drewer und Ziegler 2011, 159).“

Obwohl alle drei Elemente des semiotischen Dreiecks zur theoretischen Modellierung notwendig sind, so ist das Primat des Begriffs für die klassische Terminologielehre bezeichnend (Wüster 1991, 1-2). In der sog. Begriffsorientierung stellen Begriffe den Ausgangspunkt terminologischer Arbeit dar: Man ist an erster Stelle an der begrifflichen Struktur bzw. Strukturierung des Fachwortschatzes interessiert. Informationen wie Benennungen, Definitionen 
oder andere Daten werden den Begriffen zugeordnet und terminologische Ergebnisse insgesamt nach Begriffen organisiert und dargestellt (vgl. Cabré 1999, 34). Die begriffsorientierte Arbeitsweise wird in weiteren Ausführungen deutlicher. Durch Begriffsorientierung setzt sich die klassische Terminologielehre von der klassischen Lexikographie bewusst ab, die traditionell benennungsorientiert arbeitet (vgl. Wüster 1991, 1-2; Pearson 1998, 10-11; Cabré 1999, 34-36; Drewer und Ziegler 2011, 182; Arntz, Picht und Schmitz 2014, 240). ${ }^{67}$

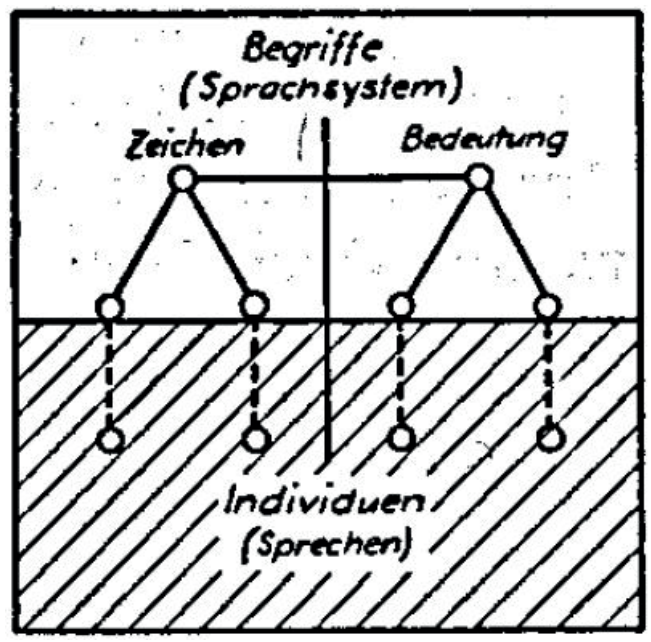

Abbildung 7.: Das vierteilige Modell (Wüster 1959-1960, 188)

Neben dem semiotischen Dreieck, das heute in der industrierelevanten Literatur zur Terminologielehre und -arbeit am weitesten verbreitet ist, existiert noch ein vierteiliges Modell des Wortschatzes nach Wüster (1959-1960), s. Abbildung 7 (vgl. Felber und Budin 1989, 20-21). ${ }^{6}$ Wüster (1959-1960, 188) erläutert das Modell wie folgt: Die horizontale Linie trennt zwischen langue (oben) und parole (unten) und die vertikale zwischen der Bedeutung (rechts) und der Form (links). Die Punkte im

67 Neben dem Wortpaar begriffsorientiert/benennungsorientiert wird auch synonym (entsprechend) onomasiologisch/semasiologisch verwendet (exemplarisch Temmerman 2000, 4-6; Costa 2006, 83; L'Homme 2006, 64, 66).

68 Das semiotische Dreieck und das vierteilige Modell sind eng verwandt und weitestgehend ineinander überführbar, wie Wüster (1959-1960, 190) erläutert. 


\section{Forschungsstand}

Quadranten rechts unten stehen für Gegenstände. Die Gegenstände werden mittels Abstraktion oder Determination zu Begriffen, die aber zunächst noch die einzelnen Gegenstände repräsentieren. Diese sog. Individualbegriffe ${ }^{69}$ sind im Quadranten rechts oben durch Punkte auf der Horizontalen repräsentiert. Erst diese Individualbegriffe werden durch weitere Abstraktion zu Allgemeinbegriffen (der obere Punkt im Quadranten rechts oben). Der Allgemeinbegriff ist durch eine Linie mit dem idealtypischen Zeichen (Quadrant links oben) verbunden. Dieses Zeichen ist ebenfalls abstrakt und Wüster nennt es Lautbegriff oder Schriftzeichenbegriff (Wüster 1959-1960, 188). Dieses idealtypische Zeichen wird durch jeden Sender ausgesprochen oder aufgeschrieben. Es handelt sich also bei Punkten im Quadranten unten links um individuelle Realisationen eines idealtypischen Zeichens durch einzelne Sprecher. In Wüsters Beschreibungen bleibt die Bedeutung der Punkte auf der horizontalen Linie im Quadranten links oben unklar.

Da im weiteren Verlauf dieser Arbeit die Sprachauffassung in der Terminologielehre und -arbeit diskutiert werden soll, gehe ich an dieser Stelle darauf ein, wie Wüster (1959-1960) das Individuelle in der Sprache, insbesondere auf der Begriffsebene, versteht.

Wüster (1959-1960) räumt ein, dass jedes Individuum zunächst subjektive Begriffe bildet, d. h. solche, die sich von Person zu Person aufgrund der Veranlagung und Erfahrung unterscheiden können. Obwohl diese subjektiven Begriffe auch bestehen bleiben, muss auch die Verständigungsfunktion der Sprache erfüllt sein. Aufgrund dieser Funktion kann man auf einen gemeinsamen begrifflichen Kern über mehrere Individuen hinweg schließen und es muss daher sog. objektive Begriffe geben. Diese objektiven Begriffe werden im Sozialisationsprozess einer Sprechergemeinschaft erworben (Wüster 1959-1960, 195). In seinem vierteiligen Modell abstrahiert daher Wüster explizit von der subjektiven Ausprägung der Begriffe und geht bei Kommunikation weitestgehend von einer begrifflichen Überlappung zwischen dem Sender und Empfänger aus (Wüster 1959-1960, 198). Dies wird auch besonders deutlich an seiner vereinfachten Darstellung des Organonmodells von Bühler (bspw. Bühler 1978), in der der nicht überlappende Teil der Zeichenbedeutung vernachlässigt wird (Wüster 1959-1960, 200). Mit anderen Worten: Für seine theoretische Modellierung ist Wüster also ausschließlich am Konventionalisierten in langue (insbesondere im Signifié) interessiert. An einer an-

69 Der Gebrauch der Bezeichnung Individualbegriff unterscheidet sich von der oben angeführten Auffassung in DIN 2342 (2011). 
deren Stelle kommt Wüster (1959-1960) auf das Subjektive zurück, wobei er hier stärker parole mit einbezieht und die im Sprachsystem konventionalisierte Zeichenbedeutung (die Bedeutung) ihrer eigentlichen Realisierung in der Kommunikation (dem Gemeinten) gegenüberstellt (Wüster 1959-1960, 199). Er geht davon aus, dass sich die Bedeutung und das Gemeinte nur im Idealfall decken und sonst aufgrund von Versprechern, mangelnden Sprachkenntnissen oder Ad-hoc-Bildungen von Begriffen, die noch nicht im Sprachsystem vorhanden sind, abweichen können (Wüster 1959-1960, 199). Diese Auflistung ist sicherlich nicht abschließend gemeint. Trotzdem ist es bezeichnend, dass Wüster (1959-1960) nicht explizit darauf eingeht, dass eine Divergenz von Bedeutung und Gemeintem auch dadurch verursacht sein könnte, dass man sich in der Kommunikation möglicherweise der subjektiven Begriffe bediene. Für Wüster (1959-1960) spielt die Subjektivität in der Darstellungsfunktion einer Sprache nur dann eine Rolle, wenn deiktische Ausdrücke (Begriffe der subjektiven Orientierung (Wüster 1959-1960, 199-200)) verwendet werden.

Aus den Ausführungen kann man schließen, dass der klassischen Terminologielehre nach Wüster eine strukturalistische Sprachauffassung zugrunde liegt.

Synonymie und Mehrdeutigkeit Mithilfe des semiotischen Dreiecks lassen sich zwei Hauptschwierigkeiten in Fachwortschätzen gut modellieren: Synonymie und Mehrdeutigkeit. ${ }^{70}$ Wie in Abbildung 8 und Abbildung 9 dargestellt, handelt es sich bei Synonymie um mehrere Benennungen, die einem Begriff zugeordnet werden können, während sich bei Mehrdeutigkeit mehrere Begriffe eine Benennung teilen (vgl. Arntz, Picht und Schmitz 2014, 135140).

Begriffsbeziehungen Wie an den eingangs angeführten grundlegenden Gegenstanddefinitionen nach DIN 2342 (2011) deutlich, interessiert sich die Terminologielehre nicht für einzelne Begriffe, sondern für das Gefüge von Begriffen in einem Fachgebiet. Eine besondere Bedeutung kommt daher der Ausarbeitung von Beziehungen zwischen einzelnen Begriffen zu, d.h. der Feststellung von Abhängigkeiten, ob es sich beispielsweise um Ober-, Unteroder nebengeordnete Begriffe handelt. Hierbei spielen die sog. abgrenzenden Merkmale eine wichtige Rolle (DIN 2342 2011, 6).

70 Manchmal wird zwischen zwei Arten von Mehrdeutigkeit, Polysemie und Homonymie, unterschieden (vgl. Drewer und Ziegler 2011, 160; Arntz, Picht und Schmitz 2014, 135). Diese Unterscheidung ist m. E. für Terminologielehre aus pragmatischen Gründen unerheblich, da die Methoden zum Abbau der Mehrdeutigkeit von ihrer Herkunft unberührt bleiben. 


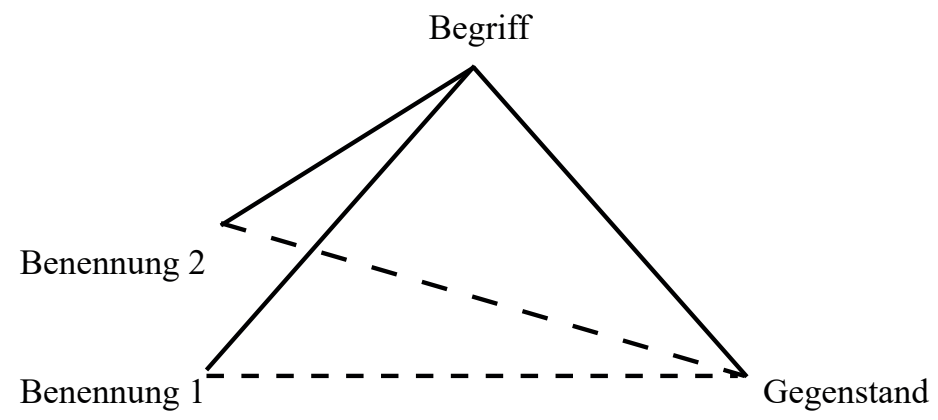

Abbildung 8.: Synonymie, modelliert mit dem semiotischen Dreieck (Drewer und Ziegler 2011, 160)

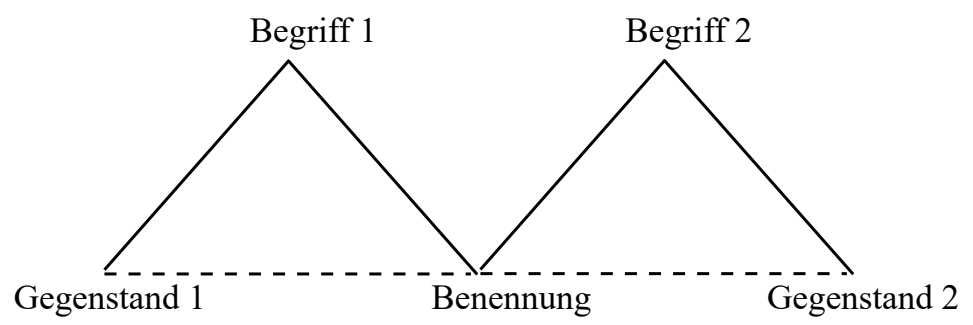

Abbildung 9.: Mehrdeutigkeit, modelliert mit dem semiotischen Dreieck (Drewer und Ziegler 2011, 160) 


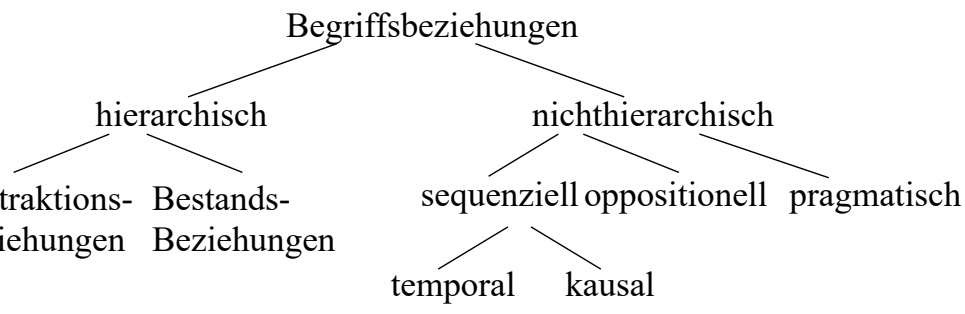

Abbildung 10.: Arten der Begriffsbeziehungen nach DIN 2342 (2011, 8-9), eigene Darstellung

Wüster (1974) unterscheidet zwischen logischen und ontologischen Begriffsbeziehungen. Die in DIN 2342 (2011) behandelten Begriffsbeziehungen stelle in Abbildung 10 zusammen (vgl. Arntz, Picht und Schmitz 2014, 7879).

Für viele Terminologieprojekte stellen Abstraktions- und Bestandsbeziehungen grundlegende Begriffsbeziehungen dar:

- Abstraktionsbeziehung: (generische Beziehung, logische Beziehungen nach Wüster (1959-1960)): ,hierarchische Begriffsbeziehung, bei der der Begriffsinhalt (...) des untergeordneten Begriffs (...) den Begriffsinhalt des übergeordneten Begriffs (...) einschließt, wobei sich der untergeordnete Begriff in mindestens einem zusätzlichen Merkmal (...) vom übergeordneten Begriff unterscheidet (Hervorhebungen aufgehoben DIN 2342 2011, 8). Dabei wird bei Abstraktionsbeziehungen zwischen Oberbegriffen (übergeordnet) und Unterbegriffen (untergeordnet) unterschieden (DIN 2342 2011, 7-8).

- Bestandsbeziehung: (Teil-Ganzes-Beziehung): „hierarchische Begriffsbeziehung, bei welcher der übergeordnete Begriff (...) sich auf einen Gegenstand (...) als Ganzes bezieht und die untergeordneten Begriffe (...) sich auf die Teile dieses Gegenstandes beziehen“" (Hervorhebungen aufgehoben DIN 2342 2011, 9). Dabei wird bei Bestandsbeziehungen zwischen Verbandsbegriffen (übergeordnet) und Teilbegriffen (untergeordnet) unterschieden (DIN 2342 2011, 7-8).

Mit den in den Normen behandelten Begriffsbeziehungen setzt sich Nuopponen (2014) auseinander. Sie weist darauf hin, dass die Normen nur einen kleinen Teil der möglichen Begriffsbeziehung abdecken und zudem typologisch nicht konsequent sind. Folglich schlägt sie eine erweiterte Typologie von Begriffsbeziehungen vor (Nuopponen 2014, 6-7). 


\section{Forschungsstand}

Begriffssysteme Sind Begriffsbeziehungen in einem Fachgebiet oder seinem Ausschnitt ausgearbeitet und festgehalten, so spricht man vom Begriffssystem, also einer „Menge von Begriffen (...) eines Begriffsfeldes (...), die entsprechend den Begriffsbeziehungen (...) geordnet sind“ (DIN 2342 2011, 7). Bei graphischen Darstellungen von Begriffssystemen spricht man von Begriffsplänen (DIN 2342 2011, 8). Die Darstellung von Begriffssystemen erfolgt nach bestimmten Konventionen, die in DIN 2331 (1980) ${ }^{71}$ beschrieben sind (vgl. Arntz, Picht und Schmitz 2014, 84-96). Zudem fordern Arntz, Picht und Schmitz (2014, 77-78) Eindeutigkeit, Verständlichkeit, Übersichtlichkeit und Ergänzbarkeit von Begriffssystemen. Der Aufbau von klassischen Begriffssystemen ist jedoch nicht immer sinnvoll oder möglich: ,[S]ind die begrifflichen Strukturen eines Gebietes so kompliziert und vielseitig, dass sich auch in der Form eines gemischten Begriffssystems nicht darstellen lassen (...), sollte man vielmehr an die Erstellung eines Begriffsfeldes denken“ (Arntz, Picht und Schmitz 2014, 109). Ein Begriffsfeld ist im Unterschied zum Begriffssystem weniger streng strukturiert und wird von der DIN 2342 $(2011,7)$ als ,Menge von Begriffen (...), die thematisch zueinander in Beziehung stehen" definiert.

Definitionen Einen Zugang zu Begriffen erhält man nicht nur durch Bezeichnungen und schematische oder bildliche Darstellungen. Man bedient sich darüber hinaus auch der Definitionen, um Begriffe zu identifizieren, voneinander abzugrenzen und ggf. begriffliche Festlegungen zu fixieren. In DIN 2342 (2011) wird Definition definiert als: „Begriffsbestimmung mit sprachlichen Mitteln“ (DIN 2342 2011, 10). In der Terminologielehre spielen vor allem drei Arten von Definitionen eine Rolle: Inhaltsdefinition (Präzisierung des Oberbegriffs durch einschränkende Merkmale), Umfangsdefinition (Aufzählung von allen Unterbegriffen) und Bestandsdefinition (Aufzählung von Teilbegriffen) (DIN 2342 2011, 10; vgl. Arntz, Picht und Schmitz 2014, 6468). Arntz, Picht und Schmitz $(2014,71)$ betonen dabei, dass Definitionen immer nur einen Blickwinkel auf einen Begriff im Begriffssystem wiedergeben, der sich am Zweck und Geltungsbereich der aktuellen Terminologiearbeit orientiert. Des Weiteren betonen sie die Notwendigkeit einer einheitlichen Verwendung von (festgelegten) Benennungen und einer laufenden Aktualisierung (Arntz, Picht und Schmitz 2014, 71-72). Sie gehen auch auf fehlerhafte Definitionen ein, die zirkulare, zu weite, zu enge und negative Definitionen umfassen (Arntz, Picht und Schmitz 2014, 72-74).

71 Die bereits 1980 erschienene DIN 2331 (1980) befindet sich zurzeit in Überarbeitung. 


\subsubsection{Methoden der Terminologiearbeit}

Deskriptive und präskriptive Terminologiearbeit Für diese Arbeit ist die Unterscheidung zwischen folgenden zwei terminologischen Arbeitsweisen notwendig: der deskriptiven und der präskriptiven Terminologiearbeit. Die deskriptive (auch: beschreibende oder feststellende (Felber und Budin 1989, 214-215)) Terminologiearbeit zielt auf die Beschreibung des aktuellen Sprachgebrauchs (Drewer und Ziegler 2011, 161; Arntz, Picht und Schmitz 2014, 4; vgl. Cabré 1999, 132). Mit anderen Worten erfasst sie den Ist-Zustand der Terminologieverwendung (Drewer und Ziegler 2011, 161; vgl. Janke 2013, 24-25). Dabei können solche Probleme wie Synonymie, Mehrdeutigkeit oder Vagheit im gegebenen Fachwortschatz identifiziert werden; sie werden jedoch nicht bewertet (Drewer und Ziegler 2011, 161). Insbesondere erfolgt bei der deskriptiven Terminologiearbeit kein Eingriff in den Sprachgebrauch.

Die präskriptive (auch: normative oder festlegende (Felber und Budin 1989, 216)) Terminologiearbeit zielt auf die Vorschreibung einer bestimmten Terminologieverwendung in einer bestimmten Kommunikationssituation, also auf die Festlegung der Soll-Norm (Drewer und Ziegler 2011, 161). Die Lehrmeinung besagt dabei, dass die präskriptive Terminologiearbeit die Kenntnis der realen Phänomene im gegebenen Fachwortschatz voraussetzt, da man problematische Aspekte durch Präskription gezielt abbauen kann. So verstanden, baut die präskriptive Terminologiearbeit auf der deskriptiven auf (Cabré 1999, 132; Drewer und Ziegler 2011, 161; Arntz, Picht und Schmitz 2014, 4, 217).

Die Deskription stellt also die grundlegende Form der Terminologiearbeit dar. Sie eignet sich vor allem zur Erstellung von Fachlexika oder anderen Wissensquellen zu einer Domäne für Experten, Nichtexperten oder Laien (Felber und Budin 1989, 214; Arntz, Picht und Schmitz 2014, 4). Als Beispiel kann hier die übersetzungsbezogene, deskriptive Terminologiearbeit genannt werden (S. E. Wright und Budin 1997, 5). Eine weitere Anwendung ist der Aufbau von deskriptiven Terminologieressourcen zur Unterstützung von Textmining (exemplarisch: Hänig und Schierle 2010; Heyer 2010; Remus und Ahmad 2010).

Der präskriptive Schritt wird hingegen für nicht immer möglich oder sinnvoll gehalten (Arntz, Picht und Schmitz 2014, 4), im industriellen Kontext jedoch als notwendig gesehen, um die Probleme der Verständlichkeit, Übersetzbarkeit und Prozessoptimierung aktiv und (mehr oder minder) systematisch 


\section{Forschungsstand}

anzugehen (Drewer und Ziegler 2011, 161; vgl. Janke 2013, 24-25). ${ }^{72}$ Zusätzlich wird beim präskriptiven Ansatz gelegentlich zwischen der sog. Terminologienormung (durch Gremien oder andere Normungsinstitute) ${ }^{73}$ und der normierenden Terminologiearbeit (intern durch einzelne Unternehmen, zuweilen mit einer Wirkung über das eigene Unternehmen hinaus) unterschieden (Arntz, Picht und Schmitz 2014, 218; vgl. DIN 2342 2011, 15). Auch übersetzungsorientierte TA kann normativ sein, wie beispielsweise im Fall von behördlichen Sprachdiensten (Arntz, Picht und Schmitz 2014, 218). Im Kontext der präskriptiven TA wird vor allem auf zwei Aspekte hingewiesen: Auf die Relevanz des Zeitpunktes, wann die Präskription ansetzt (vorzugsweiswe bereits bei der Entwicklung eines Produktes und nicht beispielsweise erst bei der Dokumentationsübersetzung) (exemplarisch: M. Mayer 2010, 38, 39; vgl. Drewer und Ziegler 2011, 165; Yamchi 2012, 28)), sowie auf die Unabdingbarkeit von Instrumenten zur Überwachung, ob die Soll-Norm tatsächlich verwendet wird (Drewer und Ziegler 2011, 161).

Auf Terminologielehre beruhende Vorgehensweise Ausgehend von Terminologielehre, insbesondere ihrer Begriffsorientierung, lässt sich für die präskriptive Terminologiearbeit folgende Vorgehensweise ableiten (vgl. exemplarisch Temmerman 2000, 5, 13-14): Begriffe werden aus einschlägigen Texten, beispielsweise aus unternehmensinternen Texten, extrahiert. Dies geschieht zunächst durch Auffinden und Sammeln von Benennungen, die aber als stellvertretend für Begriffe behandelt werden. Die identifizierten Begriffe werden in Beziehung zueinander gebracht und voneinander abgegrenzt. Es werden Begriffsfelder und nach Möglichkeit Begriffssysteme aufgebaut. Der präskriptive Schritt soll bereits an dieser Stelle erfolgen, indem ambige begriffliche Strukturen aufgelöst und ggf. bestimmte Strukturen vorgegeben werden. Gleichzeitig werden weitere Benennungen für festgestellte Begriffe gesammelt. Die vorgegebene begriffliche Strukturierung wird in Definitionen festgehalten. Erst nachdem die begriffliche Struktur festgelegt und

72 Typischerweise wird dem deskriptiven Ansatz die Eignung zur Lösung der Fachwortschatzprobleme abgesprochen (Drewer und Ziegler 2011, 161; vgl. Janke 2013, 24-25). Dennoch kann man verstärkt Projekte aus der Unternehmenspraxis verzeichnen, die vom starken präskriptiven Ansatz wieder abkehren und ausführliche und komplexe Beschreibung und rechnergestützte Modellierung des Fachwortschatzes für ausreichend befinden (exemplarisch: C. Schmidt und Mönch-Bläsing 2011; Schnieder, Stein und Schielke 2011; Wieden 2011; teilweise auch in: Stein 2013). Diese Projekte entwickeln sich oft aus den Ansätzen des Wissensmanagements und folgen deshalb anderen Prinzipien als die klassische Terminologiearbeit.

73 DIN 2342 (2011) definiert Terminologienormung als: „Normung von Begriffen (...) und ihren Benennungen (...) sowie von Begriffssystemen (...) durch autorisierte und dafür fachlich, sprachlich und methodisch qualifizierte Gremien mit dem Ziel, terminologische Festlegungen in Normen zu schaffen“ (DIN 2342 2011, 15). 
durch Definitionen untermauert worden ist, kann die eigentliche Benennungsfestlegung (Vorzugsbenennung, abgelehnte Benennung, zugelassenes Kurzwort etc.) erfolgen. Insgesamt ist zu beachten, dass die beschriebenen Schritte gleichzeitig aber auch iterativ durchgeführt werden. Diese Vorgehensweise wurde beispielsweise im Terminologieprojekt bei der Firma Koenig \& Bauer Group angewandt (vgl. Suchowolec 2009, 2014, passim).

9-Stufen-Modell Obwohl noch für Felber und Budin (1989) das Sammeln und die Erfassung von Begriffsbeziehungen, der Aufbau von Begriffssystemen und Soll-Zuordnungen sowie die Aufzeichnung der Daten den Kern der Terminologiearbeit ausmachten (Felber und Budin 1989, 206), wird heutzutage eine reine sprachliche Perspektive auf Terminologiearbeit nicht mehr vertreten. Über die bloße sprachbezogene Terminologielehre hinaus bei der Umsetzung der Terminologiearbeit im Unternehmenskontext gehen Drewer und Ziegler (2011). Hierfür schlagen sie 9 Stufen (auch Schritte genannt) der Terminologiearbeit vor. Sie integrieren in diese Schritte nicht nur die Terminologielehre, sondern werden auch der Definition der Terminologiearbeit mit ihren Teilaufgaben nach DIN 2342 (2011) gerecht (Drewer und Ziegler 2011, 164). Auch diese Stufen sind als überlappend und iterativ zu lesen:

1. Zielsetzung und Planung

2. Gewinnung der Terminologie

3. Begriffliche Systematisierung der Terminologie

4. Sprachliche Bewertung und Bereinigung der Terminologie

5. Generieren neuer Benennungen

6. Verwaltung der Terminologie

7. Darstellung und Verbreitung der Terminologie

8. Pflege der Terminologie

9. Kontrolle der Terminologieverwendung

Neben den Stufen 2-5, die aus der Terminologielehre bekannt sind, schneiden vor allem die Stufen 1, 7 und 8 die betrieblichen Aspekte der Terminologiearbeit an. So sollen in Zielsetzung und Planung die Aspekte der Zeit-, 


\section{Forschungsstand}

Personal- und Budgetplanung geklärt sowie verschiedene Rollen und Verantwortlichkeiten definiert werden (Drewer und Ziegler 2011, 165). Hingegen sollen in Darstellung und Verbreitung der Terminologie Festlegungen zu Bereitstellungsmedien und -umfang getroffen werden (Drewer und Ziegler 2011, 185). Schließlich geht Pflege der Terminologie darauf ein, wie Terminologiebestände laufend aufgebaut und ohne zeitliche Verzögerung bereitgestellt werden können und wie Terminologiearbeit in das betriebliche Tagesgeschäft angebunden werden kann (Drewer und Ziegler 2011, 185-186).

Auch Turhan (2011) orientiert sich eng am 9-Stufen-Modell von Drewer und Ziegler (2011), obwohl sie unter Auswertung einschlägiger Literatur und aufgrund ihrer Erfahrung als Terminologiemanagerin bei Fiducia IT AG mehr Details zu den betriebsbezogenen Aspekten liefert. Insbesondere geht sie bei Zielsetzung und Planung ein auf: den Rückhalt seitens der Entscheidungsträger und den Aufbau einer internen Terminologielobby, auf eine klare Herausstellung der Projektziele, Nutzen und Vorteile für das Unternehmen sowie auf die Notwendigkeit von Voruntersuchungen und vordefinierten Meilensteinen. Bei Darstellung und Verbreitung spricht sie mehr Details zu möglichen Medien an und fordert eine Definition von Szenarien z. B. für Terminologievorschläge, mehrsprachige Arbeit und Freigabeprozesse bei Pflege.

Projekt- und Prozessmanagement Die oben angeführten Beschreibungen der Terminologiearbeit gingen von terminologiespezifischen Aufgaben aus und zogen hierbei betriebliche Überlegungen hinzu. Eine umgekehrte Konzeptualisierung schlagen ISO 15188 (2001) und Deutscher Terminologie-Tag e.V. (DTT 2010) vor. Sie stellen die Terminologiearbeit in erster Linie in einen übergreifenden betrieblichen Kontext des Projekt- und Prozessmanagements und verfeinert diese Sicht durch terminologiespezifische Details. Im Weiteren stelle ich die Prinzipien des Projektmanagements von ISO 15188 (2001) vor und gehe anschließend auf die Unterscheidung zwischen Projektmanagement und Prozessmanagement von Deutscher Terminologie-Tag e.V. (DTT 2010) ein.

ISO 15188 (2001) beschäftigt sich mit den Prinzipien von Terminologieprojekten, wobei ein Terminologieprojekt als ,(...) project aimed at collecting, developing, analysing and recording the terminology of one or more subject fields" (ISO 15188 2001, 2) definiert ist. Ein solches Projekt besteht aus fünf Phasen:

1. Vorbereitung: Umfasst eine Durchführbarkeitsanalyse (Projektziele, Zielgruppen und ihre Anforderungen), den Aufbau eines organisatori- 
schen Rahmens unter Berücksichtigung von rechtlichen, finanziellen, strukturellen und sprachpolitischen Faktoren, sowie die Entwicklung von Projektspezifikationen (ISO 15188 2001, 3-4).

2. Entwurf: Umfasst die Bestimmung der Projektführung sowie den Aufbau von Arbeitsgruppen und die Bestimmung von Arbeitsplan, -methoden, -werkzeugen und Bereitstellungsformaten (ISO 15188 2001, 4-6).

3. Umsetzung: Umfasst die eigentliche Ausarbeitung der Terminologie unter Einhaltung der Terminologielehre sowie die laufende Projektevaluierung und die Metadokumentation (ISO 15188 2001, 6-7).

4. Evaluation: Umfasst die Auswertung der Projektergebnisse und des Projektes selbst (ISO 15188 2001, 7).

5. Abschluss: Umfasst den Abschluss- und Finanzbericht (ISO 15188 2001, 8).

Ähnlich geht mit der Konzeptualisierung der Terminologiearbeit in betrieblicher Praxis auch Deutscher Terminologie-Tag e.V. (DTT 2010) vor, wobei hier eine wichtige Unterscheidung zwischen Projektmanagement und Prozessmanagement getroffen wird, da beide unterschiedlich im Unternehmensalltag eingebunden werden: Bei einem Projekt handelt es sich um ,(...) ein einmaliges Vorhaben mit definierten Zielen, Ressourcen, Prozessen, Zeitrahmen und Budget“. Im Unterschied dazu wird ein Prozess definiert als ,(...) eine wiederkehrende Reihe von Aktivitäten und Maßnahmen, die in einer vordefinierten Folge stattfinden und zu messbaren Ergebnissen beitragen“" (beide Zitate: DTT 2010, M5-1).

Das Projektmanagement für Terminologiearbeit unterscheide sich demnach nicht vom betrieblichen Projektmanagement für andere Aufgaben (DTT 2010, M5-5) und beinhaltet, ähnlich wie in ISO 15188 (2001), Projektdefinition, -ausführung, -abschluss und -evaluation (DTT 2010, M5-5-6).

Das Prozessmanagement gestaltet sich für jedes Unternehmen hingegen unterschiedlich (DTT 2010, M5-7). Dennoch werden hierbei vier Phasen identifiziert:

- Produktion von Terminologie: ähnlich der Terminologielehre (DTT 2010, M5-8-9) 
- Aufbereitung und Auslieferung von Terminologie: technologische Aspekte der Terminologieverwaltung (DTT 2010, M5-10)

- Verwendung und Nutzung von Terminologie: Terminologieschulungen, Verwendungskontrolle sowie Terminologieservice und -support für alle Benutzer (DTT 2010, M5-10)

- Qualitätskontrolle von Terminologie: qualitative (inhaltliche) und quantitative (durch Metriken) Kontrolle der Terminologiebestände (DTT 2010, M5-10-11).

Typischerweise wird die Initialeinführung von Terminologiearbeit in Unternehmen als Projekt umgesetzt, in dem beispielsweise technische, personale, methodische und sprachliche Aspekte der Terminologiearbeit entworfen und etabliert werden, die man anschließend als laufenden, ins das Tagesgeschäft etablierten und zeitlich unbegrenzten Prozess weiterpflegt und ausbaut (vgl. DTT 2010, M2-3).

\subsubsection{Einordnung der Terminologielehre (und -arbeit) als Disziplin}

Die Einordnung der Terminologielehre (und in geringerem Maße auch der Terminologiearbeit) im wissenschaftlichen Diskurs wurde in der einschlägigen Literatur immer wieder thematisiert. In diesem Abschnitt fasse ich drei Hauptdiskussionspunkte zusammen: 1) an Terminologielehre angrenzende Wissenschaftsdisziplinen und -gebiete; die Positionierung gegenüber der Linguistik wird als Übergang dienen zu: 2) Unabhängigkeit der Terminologielehre als Gebiet; 3) einführende Überlegungen zur Sprachauffassung in der Terminologielehre (und in geringerem Maße auch der Terminologiearbeit), die in der Diskussion in Abschnitt 2.4.10 vertieft werden.

\subsubsection{Terminologielehre und Nachbardisziplinen}

Die Bezüge der Terminologielehre zu anderen Disziplinen werden bereits von Wüster (bspw. 1974) ausführlich behandelt, der die Terminologielehre als Grenzgebiet zwischen Logik, Ontologie, (Information und Dokumentation,) Informatik, Sachwissenschaften und Sprachwissenschaft sieht (vgl. Wüster 1991, 5-6). Diese Auffassung zog eine weitere Diskussion und Vertiefung 
nach sich (vgl. bspw. Zimmermann 1977; Felber und Budin 1989, 19-43). Im Folgenden sind die Bezüge zu klassischen Nachbardisziplinen kurz angerissen.

Logik Für Wüster ist Logik, ähnlich wie Ontologie, eine Disziplin, die sich mit Beziehungen zwischen Begriffen und zwischen Individuen beschäftigt, wobei er hier nicht nur die klassische Begriffslehre, sondern auch die mathematische Logik meint (Wüster 1974, 85; vgl. Zimmermann 1977, 18-19). Cabré (1999) erläutert diesbezüglich, dass das geteilte Interesse der Terminologielehre und der Logik an Begriffsbeziehungen den Gegenständen und ihren Beziehung in der realen Welt und nicht der semantischen Form-BedeutungKorrespondenz gilt. Da für Wüster die Abstraktionsbeziehungen die Grundlage der Logik bilden, können sie als Beitrag der Logik zur Terminologielehre verstanden werden (Wüster 1974, 84-90 et passim; vgl. Cabré 1999, 8).

Eine abweichende Auffassung von Logik vertreten beispielsweise Felber und Budin (1989). Sie sehen Logik als eine übergreifende Disziplin, der folgende Teilbereiche zugeordnet werden können: Begriffslehre, Erkenntnislehre und Methodenlehre. Diese Teilbereiche prägen die Grundlagen der Terminologielehre und können als solche einen Beitrag der Logik zur Terminologielehre verstanden werden. (Felber und Budin 1989, 23-31).

Ontologie In der klassischen Auffassung von Wüster handelt es sich bei Ontologie um die Lehre von Sein (Wüster 1974, 91). Ähnlich wie Logik steht sie aufgrund ihres Interesses an Begriffen/Gegenständen und ihren Beziehungen nah der Terminologielehre. Im Unterschied jedoch zur Logik untersucht sie nicht die Abstraktionsbeziehungen sondern alle anderen Beziehungsarten wie beispielsweise Teil-Ganzes-, chronologische oder sequenzielle Beziehungen. Diese Begriffsbeziehungen werden von Wüster ontologisch genannt (Wüster 1974, 91-98). Dank der so verstandenen Ontologie ist es der Terminologielehre möglich, eine breitere Palette von Begriffsbeziehungen in Fachsprachen zu modellieren, als es mit Logik alleine formal möglich wäre.

Manche Autoren fassen Logik und Ontologie als Teilgebiete der Philosophie auf und sprechen stattdessen von der Nähe der Terminologielehre zur Philosophie im Allgemeinen (bspw. Arntz, Picht und Schmitz 2014, 6-7).

Information und Dokumentation Obwohl Wüster (1974) im Titel nicht direkt auf das Gebiet Information und Dokumentation eingeht, so ist es im 


\section{Forschungsstand}

Abschnitt zur Informatik, insbesondere zur Thesaurusarbeit, implizit enthalten (Wüster 1974, 101-103). Bei Information und Dokumentation (auch: Informationswissenschaft, Informations- und Dokumentationswissenschaft, Ereunetik und Ichneutik (Wersig 1979, 197)) handelt es sich um eine Disziplin zum „Erschließen, Ordnen, Bewahren und Verfügbarmachen von Wissen“ (Arntz, Picht und Schmitz 2014, 7; vgl. Wersig 1979, 198), das insbesondere in Dokumentenform vorliegt. Zur Dokumenterschießung, -beschreibung und -indexierung werden größere Informationseinheiten in kleinere Einheiten aufgespaltet und sog. Thesaurusdeskriptoren entwickelt, die letztendlich als Termini aufgefasst werden können (Felber und Budin 1989, 37; für Beispiele s. Roulin 2001; Strehlow 2001a,b).

Die gegenseitigen Bezüge zwischen Terminologielehre und Information und Dokumentation sind vielfältig, wie beispielsweise Wersig (1979, 202206) und Cabré (1999, 50-52) detailliert anführen. Insbesondere fasst Wüster den Aufbau von Thesauri als eine terminologische Aktivität auf (Wüster 1974, 101-102; vgl. Cabré 1999, 8-9), wobei die Terminologielehre als eine Grundlage für Systematisierung und Strukturierung von Deskriptoren eingesetzt werden kann (F. H. Lang 1985, 171; vgl. Felber und Budin 1989, 37; Arntz, Picht und Schmitz 2014, 7).

Informatik Wüster setzte den Beitrag der Informatik zur Terminologielehre dem Einsatz von Computern zur Speicherung und Abfrage von terminologischen Ressourcen gleich (Wüster 1974, 98-99; vgl. Cabré 1999, 8). Cabré $(1999,6)$ sieht den Computer gar als eine der wichtigsten Innovationen in den Entwicklungen der Terminologiearbeit in den 1960er und 1970er Jahren. Neuerdings können noch weitere Aspekte wie automatische Termextraktion und Befüllung von terminologischen Einträgen etc. hinzugefügt werden (vgl. Cabré 1999, 52, 54). Des Weiteren kann die Informatik in den Kontext des Wissensmanagements und des Knowledge Engineering gesetzt werden (s. Abschnitt 2.4.4.1).

Sachwissenschaften Die bisher genannten Disziplinen tragen methodisch und konzeptuell zur Terminologielehre bei. Welche Begriffe aber letztendlich untersucht und strukturiert werden, wird durch die sog. Sachwissenschaften wie bspw. Physik, Elektrotechnik, Chemie etc. bestimmt (Wüster 1974, 103104; Felber und Budin 1989, 39), denn Terminologiearbeit ist kein Selbstzweck, sondern steht im Dienste der einzelnen Fächer (Cabré 1999, 9). Unter dieser Prämisse setzt die praktische Terminologiearbeit Kenntnisse fachlicher 
Zusammenhänge voraus und erfordert eine enge Zusammenarbeit zwischen den Terminologen und den Fachexperten (Wüster 1974, 103-104; vgl. Arntz, Picht und Schmitz 2014, 5).

Neben den bereits von Wüster genannten Disziplinen wurden weitere Disziplinen postuliert, die zur Erforschung von Terminologie und Verbesserung der Terminologielehre und -arbeit beitragen könnten. In diesem Zusammenhang spricht Picht $(2009,7)$ von einer Erweiterung des theoretischen Rahmens der Terminologie. Im Folgenden fasse ich die wesentlichen Erweiterungen zusammen.

Semiotik Semiotik, also die Lehre von Zeichen, wird von Wüster (1974) nicht als eine Nachbardisziplin genannt. Da aber Begriffe nur indirekt über Zeichen wie Benennungen, Formeln etc. zugänglich sind (Arntz, Picht und Schmitz 2014, 5; vgl. Zimmermann 1977, 16), kann das Zurückgreifen auf die Semiotik aufschlussreich sein (Felber und Budin 1989, 32).

Knowledge Engineering Bei Knowledge Engineering handelt es sich um ein anwendungsbezogenes Gebiet, das sich nach Buol (2000) mit folgenden Aufgaben beschäftigt: „Modellierung, Sicherung, Verwaltung und Nutzbarmachung von Wissen" (Buol 2000, 18; vgl. Meyer u.a. 1992, 957; Cabré 1999, 52-53). Des Weiteren stellt Buol (2000) fest: „Knowledge Engineering und Terminologiearbeit haben zwei Aufgaben gemein: das Aufdecken und die Repräsentation von konzeptuellen Strukturen, die in einer Domäne vorliegen“ (Buol 2000, 18).

Der Bezug der Terminologielehre zu Knowledge Engineering kann am Beispiel von Ontologien näher erläutert werden: Er beruht auf dem modernen Verständnis von Ontologien, das auf die Forschung zur Künstlichen Intelligenz (KI) zurückgeht (Zelewski 2005, 121-123, 2002, 2). Bei Ontologien im modernen Sinn handelt sich nicht um eine philosophische Disziplin, sondern um ,.... formal beschriebene Darstellungen einer definierten Menge von Begrifflichkeiten einschließlich der Beziehungen zwischen ihnen (...)“ (Arntz, Picht und Schmitz 2014, 7). Mit Zelewski (2005) modellieren Ontologien das von mehreren Akteuren geteilte Wissen in einer bestimmten Anwendung, wobei sowohl das explizit vorliegende als auch das explizit gemachte implizite Wissen mit einfließen (Zelewski 2005, 153). Bei den Akteuren kann es sich sowohl um mehrere Personen, aber auch um heterogene und verteilte Anwendungssysteme handeln (Lacy 2005, 29; vgl. Fensel 2004, 1). Die Darstellung ist zudem ein subjekt- und zweckabhängiger Realitätsausschnitt (Zelewski 


\section{Forschungsstand}

2005, 153). Zelewski (2005) betont, dass eine moderne Ontologie nicht den Anspruch hat, das Ding an sich zu wiedergeben, sondern als ein von Menschen geschaffenes Artefakt betrachtet werden muss. Es kann daher nicht die Ontologie geben, sondern man muss von Ontologien in Mehrzahl sprechen, die jeweils nur eine bestimmte Perspektive darstellen (Zelewski 2005, 121).

In der angeführten Definition von Ontologie von Arntz, Picht und Schmitz (2014) wird auf den Formalisierungsaspekt hingewiesen. Er entspringt der Anforderung an Maschinenlesbarkeit, in die der KI-Forschung eine zentrale Rolle spielt, denn Ontologien dienen im Allgemeinen dazu, „(...) Wissensbasierte Systeme (...) mit dem koordinations- und kommunikationsrelevanten Hintergrundwissen auszustatten“ (Zelewski 2005, 125). Und weiter: „,(..) Ontologien gestatten es, das koordinationsrelevante Fach- und Hintergrundwissen explizit zu spezifizieren, das inhaltliche Verständnis dieses Wissens systematisch zu analysieren, hierdurch mögliche Wissensdivergenzen aufzudecken sowie explizierungs- und artikulierungsbedingte Verständnisbarrieren des Wissensmanagements zu beseitigen“" (Zelewski 2005, 127). Die maschinelle Lesbarkeit von Ontologien soll nicht zuletzt eine Automatisierung von Wissenserwerb, -pflege und -zugang ermöglichen (Fensel 2004, 1).

Aus den Beschreibungen geht hervor, dass Ontologien als Ergebnisse des Knowledge Engineering und Begriffssysteme als Ergebnisse der Terminologiearbeit augenscheinlich gewisse Parallelen aufweisen (vgl. Meyer u. a. 1992, 957; Buol 2000, 19). Ein wesentlicher Unterschied besteht für Meyer u. a. (1992) im Umgang mit der Multidimensionalität der Daten: „While terminologists are well aware that a given domain can be subdivided in different ways, depending on the expert's point of view, they have not traditionally attempted to account for it in any serious way“ (Meyer u. a. 1992, 959). Ontologien hingegen streben danach, eine größere Komplexität heterogener Daten und Relationen (Begriffe und Begriffsbeziehungen) abzubilden (vgl. Zelewski 2005, 192; Stein 2013, 16-17). Des Weiteren bemerkt Zelewski (2005), dass Ontologien über Inferenz- und Integritätsregeln verfügen, die bei traditionellen Begriffssystemen keine Rolle spielen (Zelewski 2005, 192). Trotz dieser Unterschiede kommt es immer wieder zu einer Annäherung beider Gebiete. So beschreiben Meyer u. a. (1992) ein Projekt zum Aufbau einer Terminologiedatenbank (eigentlich: einer terminologischen Wissensdatenbank) mithilfe der Werkzeuge und Ansätze des Knowledge Engineering. Faber und Rodríguez (2012, 21-22) sprechen in diesem Zusammenhang von sog. Termontographie: „Its objective is to link ontologies with multilingual 
terminological information, and to incorporate ontologies into terminological resources“ (Faber und Rodríguez 2012, 21).

Weitere Bezüge Weiteren Nutzen für Terminologielehre sieht Cabré (1999, 39-59) durch eine stärkere Einbettung in die Kognitions- und Kommunikationswissenschaft (vgl. Picht 2009, 7), siehe dazu Abschnitt 2.4.4.3, Klassische Terminologielehre - Kritik der Sprachauffassung.

\subsubsection{Terminologielehre und Sprachwissenschaft - Eigenständigkeit des Gebiets}

Zur Einordnung der Terminologielehre und -arbeit in das wissenschaftliche Gefüge fasse ich abschließend das Selbstverständnis des Gebietes bezüglich der Sprachwissenschaft zusammen. Wüster selbst grenzt Terminologielehre ausdrücklich von der Linguistik ab und begründet dies u. a. mit den allein gültigen Merkmalen der Synchronie, Begriffsorientierung und Präskriptivität (Wüster 1974, 67-84, 1991, 1-6). An diesen Abgrenzungsmerkmalen kann man bereits erkennen, dass Wüsters Auffassung der Sprache und der Sprachwissenschaft strukturalistisch ist. Wie Cabré (1999) jedoch erörtert, hat sich die Selbstauffassung der Linguistik gewandelt. Vor allem die Angewandte Linguistik erlaubt mehrere gleichberechtigte Perspektiven auf den Gegenstand (Cabré 1999, 25-27), so dass Bezüge zu anderen Gebieten in den sprachwissenschaftlichen Rahmen besser integriert werden können. Vor diesem Hintergrund konstatiert Picht (2009), dass die Frage nach der Unabhängigkeit der Terminologielehre von der Linguistik ganz an Bedeutung verloren hat (Picht 2009, 7; vgl. Faber und Rodríguez 2012, 11). Nichtsdestotrotz stellt für Cabré (1999) die Erforschung der Terminologie aus linguistischer Sicht als Teil des Fachlexikons mit fachlichen und pragmatischen Eigenarten eine zentrale Perspektive dar. Diese Perspektive muss jedoch durch weitere, gleichberechtigte Perspektiven wie die der Sachwissenschaften ergänzt werden (Cabré 1999, 32-33). Des Weiteren geht Cabré (1999) der Frage nach, ob Terminologielehre und -arbeit ein, nicht nur von der Linguistik, sondern an sich unabhängiges Gebiet darstellt. Sie betont, dass obwohl die Konzepte und Konstrukte der Terminologielehre und -arbeit nicht originell, sondern größtenteils den o.g. Nachbardisziplinen entnommen sind, so das Zusammenspiel dieser Konzepte und ihre Verwendung zum Aufbau eigener Ansätze die eigentliche Leistung der Terminologielehre und -arbeit ist (Cabré 1999, 32). Cabré (1999) fasst zusammen, dass es eine Vielzahl an wissenschaftlichen 


\section{Forschungsstand}

Positionen gibt, die sowohl die Terminologielehre und -arbeit als auch ein lediglich angewandtes Gebiet, eine echte, unabhängige wissenschaftliche Disziplin oder etwas dazwischen sehen (Cabré 1999, 6-7). Für sie konstituieren Terminologielehre und -arbeit ein interdisziplinäres Gebiet, das zwar viele andere Gebiete für seine Zwecke heranzieht, aber auch selbst einen Mehrwert schafft, der über die Summe der Einzelbeiträge hinausgeht (Cabré 1999, 32).

\subsubsection{Klassische Terminologielehre - Kritik der Sprachauffassung}

Die bisherigen Beschreibungen, insbesondere die in Abschnitt 2.4.3 angeführten Erläuterungen zum Semiotischen Dreieck und dem vierteiligen Modell von Wüster, ergeben eine strukturalistische Fundierung der Terminologielehre, die von einem stabilen, überindividuellen (Sprach-)System von Termini ausgeht (vgl. Tabelle Temmerman 2000, 20). Der Schwerpunkt liegt also auf langue. Die (textuelle) Realität des Terminologiegebrauchs (bspw. Temmerman 2000, 7-8), die unter parole fällt, zeigt hingegen eine starke individuelle Variation im Terminologiegebrauch. Diese Variation wird von der klassischen Terminologielehre weitestgehend ausgeblendet, was einen ihrer Hauptkritikpunkte darstellt (s. beispielsweise Gerzymisch-Arbogast 1996; für einen Überblick s. Temmerman 2000, 22-37; Faber und Rodríguez 2012).

Die poststrukturalistischen Ansätze in der Terminologielehre werden beispielsweise von Faber und Rodríguez (2012) zusammengefasst. Die sog. Sozioterminologie von Gaudin (1993) sieht die Mehrdeutigkeit und Synonymie als Konsequenz von soziolinguistischen Einflussfaktoren wie Wissen, Machtverhältnisse und sozialer und beruflicher Status einer Sprechergruppe, die auch in der Fachkommunikation präsent sind (Faber und Rodríguez 2012, 14 15). Die kommunikative Terminologielehre von Cabré (bspw. Cabré 1999) ermöglicht es, den fachbezogenen Wissenstransfer mithilfe des Terminologiegebrauchs zu untersuchen (Cabré 1999, 45), was aus verschiedenen, gleichberechtigten, Perspektiven, der kognitiven, linguistischen und soziokommunikativen geschehen kann (Faber und Rodríguez 2012, 15-16). Schließlich gehen Faber und Rodríguez (2012) auf die soziokognitive Terminologielehre von Temmerman (2000) und die Frame-basierte Terminologielehre ein, die infolge der sog. kognitiven Wende entstanden sind (Faber und Rodríguez 2012, 12, 17). Den poststrukturalistischen Ansätzen ist gemein, dass sie sich vom Standardisierungsgedanken abwenden und den Schwerpunkt explizit auf eine reine Beschreibung setzen (Faber und Rodríguez 2012, 12, 18). Die klassische Terminologielehre erhebt also weiterhin als einzige einen präskriptiven Anspruch. 
Aus der oben angerissenen kritischen Literatur greife ich im folgenden Abschnitt die von Temmerman (2000) postulierte soziokognitive Sicht auf Terminologielehre heraus, da ihr eine ausführliche und systematische Auseinandersetzung mit der klassischen TL vorausgeht und sich die so festgestellten Probleme auch in anderen kritischen Ansätzen wiederfinden. Ausgehend von Temmermans Ablehnung der Eigenständigkeit der klassischen Terminologielehre als Disziplin, fasse ich ihre wichtigsten Kritikpunkte zusammen und beschreibe abschließend die Grundzüge der vorgeschlagenen soziokognitiv ausgerichteten Terminologielehre.

Temmerman (2000) spricht der klassischen Terminologielehre die Eigenständigkeit als Disziplin ab, da die Sicht auf Terminologie dem Standardisierungsgedanken unterordnet ist. Dies verhindere eine differenzierte, also adäquate, Betrachtung und Beschreibung von Terminologie und wirke sich auf die Methoden und Vorgehensweisen einschränkend aus (Temmerman 2000, 2).

Standardisierung als Ziel wird nicht an sich in Frage gestellt, sondern eine Perspektiverweiterung gefordert: Eine Sprachstandardisierung sei zwar vor allem dort sinnvoll und möglich, wo auch eine Sachstandardisierung sinnvoll und möglich ist, also vorwiegend im ingenieur-technischen Bereich (Temmerman 2000, 17-18). Andere Bereiche aber, wie am Beispiel der sog. Biowissenschaften (life sciences) illustriert wird, entziehen sich einer Standardisierung (Temmerman 2000, 4 et passim). Eine reine Beschreibung des tatsächlichen Sprachgebrauchs, die nicht als Vorbereitung auf Vereinheitlichung gemacht wird, könne mit den Konzepten und Methoden der klassischen Terminologielehre nicht zufriedenstellend bewältigt werden (für praktische Beispiele s. Temmerman 2000, 73-94). Das Selbstverständnis der klassischen Terminologielehre als allgemeine (also allgemeingültige) Terminologiewissenschaft wird deshalb angefochten (Temmerman 2000, 18, 220).

Insbesondere setzt sich Temmerman mit den fünf zentralen Prinzipien der Terminologielehre auseinander: 1) Begriffsorientierung, 2) klare Begriffsabgrenzung, 3) Begriffsdefinierbarkeit, 4) Eineindeutigkeit, 5) Synchronie (Temmerman 2000, insb. 4-16), die der tatsächlichen Verwendung der Termini in den Texten nicht entsprechen (Temmerman 2000, 220).

- Begriffsorientierung: Traditionelle Terminologielehre geht von Begriffen aus, die unabhängig von ihrer sprachlichen Realisierung existieren. Dies impliziert für Temmerman die Auffassung, dass die Sprache das Denken erschwere (Temmerman 2000, 60). Ihr wichtigster Kritik- 


\section{Forschungsstand}

punkt besteht darin, dass die Rolle der Sprache bzw. der sprachlichen Form am Verstehensprozess und an der Begriffsentstehung verkannt wird (Temmerman 2000, 21, 60). Hiernach können Benennungen vor (präzisen) Begriffen existieren. Eine Auseinandersetzung mit der Benennungsverwendung im Diskurs kann das (Sach-)Verständnis fördern und zur Präzisierung der Begriffe führen (Temmerman 2000, 5-6, 3940). Die begriffsorientierte Forderung, dass die Benennungen erst im letzten Schritt den präzise abgegrenzten und definierten Begriffen fest zugeschrieben werden, ist für Temmerman nicht haltbar (Temmerman 2000, insb. 6).

- Begriffsabgrenzung/Begriffsdefinierbarkeit: An einigen Beispielen aus den Biowissenschaften zeigt Temmerman, dass nicht alle Begriffe sich mithilfe einer Inhaltsdefinition (Abschnitt 2.4.3.1) zufriedenstellend beschreiben und von anderen verwandten Begriffen abgrenzen lassen (Temmerman 2000, 6-10, Beispiele: 74-93). Deshalb ist es nicht für alle Begriffe möglich, einen fest vorgegebenen Platz im Begriffssystem einzunehmen.

- Eineindeutigkeit: Da manche Begriffe, wie oben beschrieben, nicht eindeutig abgrenzbar und definierbar sind, ist eine eineindeutige Zuschreibung von Begriff und Benennung nicht möglich (Temmerman 2000, 10-14).

- Synchronie: Für manche Begriffe ist die Information über ihre Entstehung und den zeitlichen Bedeutungswandel wesentlich, um den Begriff zu verstehen (Temmerman 2000, 14-15, 121, 123, Beispiele: 112-115).

Eine soziokognitive Terminologielehre geht also davon aus, dass Sprachverstehen und Weltverstehen eng miteinander verbunden sind (Temmerman 2000, 62). Es gibt nämlich Termini, deren Bedeutung erst im aktuellen Diskurs (z. B. im Text) konstruiert wird, was auch unter Einbeziehung der eigenen Erfahrung erfolgt (Temmerman 2000, 40, 61). Der Objektivismus der klassischen Terminologielehre wird durch den sog. Kognitivismus abgelöst (Temmerman 2000, 66). Konkret bedeutet dies für Terminologielehre, dass zwischen zwei Arten Denkeinheiten - Begriffen und Kategorien - unterschieden werden muss (Temmerman 2000, 42-44, 223-225). Begriffe werden wie in der klassischen Terminologielehre aufgefasst und behandelt: Sie haben eine feste Bedeutung und einen festen Platz in einem Begriffssystem und lassen 
sich mit einer Inhaltsdefinition eindeutig bestimmen. Kategorien sind hingegen Denkeinheiten, deren Bedeutung unscharf ist und nicht mit klassischen Klassifikationsansätzen bestimmt werden kann. Sie lassen sich besser mithilfe prototypischer Strukturen beschreiben: Bei prototypischen Kategorien müssen nicht alle Mitglieder bestimmte Kerneigenschaften besitzen, sondern werden nach wahrgenommener Ähnlichkeit um den prototypischen Vertreter platziert. Je weiter ein Mitglied vom prototypischen Vertreter entfernt ist, umso unschärfer seine Bedeutung (Temmerman 2000, 63-64). Aus diesen fließenden Übergängen zwischen Kategorien ergeben sich wichtige Konsequenzen. Zum einen soll ihre Beschreibung keine Inhaltsdefinition sein, sondern mehr, ,enzyklopädische“ Informationen enthalten, auch unter der Angabe, wie zentral die Information für das Verständnis einer Kategorie ist. Zum anderen haben Kategorien ein hohes Mehrdeutigkeitspotenzial, so dass eine Eineindeutigkeit nicht erreichbar ist. Die Unterschiede zwischen der klassischen, strukturalistischen und der neuen, soziokognitiven Auffassung der Terminologielehre sind in Temmerman $(2000,223)$ zusammengefasst.

\subsubsection{Geschichte}

Die historischen Entwicklungen der Terminologiearbeit können aus verschiedenen Blickwinkeln betrachtet werden. Im Folgenden beschreibe ich drei Entwicklungsstränge, die sich gegenseitig bedingten und ergänzten: 1) Versuche einzelner Fachgemeinschaften, den eigenen Fachwortschatz zu vereinheitlichen, was zunächst isoliert, dann zunehmend nach fachübergreifenden Methoden geschah (2.4.5.1); 2) Entstehung von übergreifenden Organisationen, die im Kontext der Sachnormung auch die methodischen Grundsätze der (präskriptiven) Terminologiearbeit entwickelten (2.4.5.2); 3) Etablierung der Terminologielehre und Terminologiearbeit in der Wissenschaft (2.4.5.3).

\subsubsection{Beschäftigung mit den Gegenständen Terminologie und Terminologiearbeit}

Die Beschäftigung mit der Frage, wie Dinge und Begriffe korrekt zu bezeichnen sind, kann bereits in der Antike beobachtet werden (Picht 2009, 5; vgl. Rey 1995, 11). Als eigentliche Vorläufer der modernen Terminologiearbeit werden jedoch mehrheitlich die neuzeitlichen Bestrebungen der einzelnen Wissenschaftsdisziplinen gesehen, eigene Nomenklatur ${ }^{74}$ präzise zu

74 Zur historischen Entwicklung und zur Diskussion solcher Begriffe wie nomenclature, terminology und glossary siehe beispielsweise Rey (1995, 11-12, 15-16). 


\section{Forschungsstand}

definieren und festzulegen. Hierbei werden in der einschlägigen Literatur insbesondere die Arbeiten von Dürer (Mathematik, 15. Jh.), Vesalius (Anatomie, 16. Jh.), Linné (Botanik, 18. Jh.), oder Berzelius und Lavoisier (Chemie, 18. Jh.) genannt (Arntz, Picht und Schmitz 2014, 269; vgl. Felber 1979, 61; Rey 1995, 12-13). Diese und ähnliche Bestrebungen können im größeren Rahmen der Geschichte der Fachsprachenforschung betrachten werden (s. dazu bspw. Hahn 1983, 12-59).

Ursprünglich befasste man sich also mit dem Gegenstand Terminologie aus der Perspektive einzelner Fächer (Picht 2009, 5). Waren es bis einschließlich des 18. Jh. vor allem Naturwissenschaftler, wandten sich im 20. Jh. zunehmend Ingenieure und Techniker der Klärung des Fachwortschatzes zu (Cabré 1999, 1), was durch industrielle und technologische Entwicklungen bedingt war (Pearson 1998, 8-9; Arntz, Picht und Schmitz 2014, 269). An Bedeutung gewann auch die sog. Sachnormung (Normung von Gegenständen und Verfahren), bei der sich die Notwendigkeit einer vorausgehenden terminologischen Standardisierung stellte (Arntz, Picht und Schmitz 2014, 269-270; vgl. Felber 1979, 61). In diesem Kontext werden bspw. die Arbeiten des IEC (International Electrotechnical Commision; Sachnormung und Wörterbuch) und die Entstehung von Normungsinstituten wie DNA (Deutscher Normenausschuss) zu Anfang des 20. Jh. genannt (Arntz, Picht und Schmitz 2014, 270; vgl. Pearson 1998, 9; Galinski 2009, 19).

Zentral für die wissenschaftliche Fundierung von Terminologielehre und arbeit war jedoch die Erkenntnis, dass terminologische Festlegungen weitestgehend fachübergreifend gleichen methodischen Prinzipien zu folgen haben (Felber 1979, 61; Pearson 1998, 9; Arntz, Picht und Schmitz 2014, 270) und somit reproduzierbar und nachvollziehbar sein sollten. Den entscheidenden Beitrag hierfür leistete Eugen Wüster mit seiner 1931 erschienenen Dissertation Einführung in die allgemeine Terminologielehre und terminologische Lexikographie (Wüster 1979; vgl. Cabré 1999, 1). Neben Wüster, der als Begründer der modernen Terminologielehre und -arbeit gilt (Weisgerber 19591960, 93; Wersig 1979, 195; Pearson 1998, 7; Cabré 1999, 2, 5; Arntz, Picht und Schmitz 2014, 270), prägten auch zur gleichen Zeit in der Sowjetunion Drezen und Lotte das Gebiet (Picht 2009, 6; vgl. Felber und Budin 1989, 4452; Cabré 1999, 2). Ab diesem Zeitpunkt wurde angestrebt, die von Wüster aufgestellten und weiterentwickelten Methoden für die praktische Terminologiearbeit in einzelnen Fächern, aber auch im wirtschaftlichen Kontext einheitlich zu verwenden. Ein wichtiger Schritt, über die neuen Methoden zu informieren, das Bewusstsein für die Relevanz der Terminologiefrage zu 
stärken und den Austausch anzuregen, war die Gründung von Infoterm (Internationales Informationszentrum für Terminologie) im Jahre 1971 (Galinski 2009, 20-21; vgl. Felber und Krommer-Benz 1979, 71-73). Die ursprünglichen Aufgaben von Infoterm umfassten Dokumentation von und Information über terminologische Literatur, Organisationen, praktische Terminologieprojekte und Fortbildungsmöglichkeiten. Infoterm sollte außerdem ein Kontaktnetzwerk aufbauen und pflegen sowie interessierte Praktiker über terminologische Methoden beraten (Felber und Krommer-Benz 1979, 73). Da sich die Pflege des Netzwerkes und terminologische Beratung als aufwändig erwiesen, wurde 1977 mit dem Aufbau von TermNet (Internationales Terminologienetz) als Unterorganisation von Infoterm begonnen (W. Blanke 1989, 279; Galinski 2009, 21). Heute sind Infoterm und TermNet unabhängige (auch voneinander), neutrale Non-Profit-Organisationen, die durch ein Netzwerk von Mitgliedern die praktische Terminologiearbeit auf Grundlage von Terminologielehre fördern, Informationen bereitstellen und Erfahrungsaustausch anregen (Infoterm 2004, 3; TermNet 2013, 16). Dabei scheint der Hauptunterschied zwischen ihnen in der anvisierten Zielgruppe zu liegen (Arntz, Picht und Schmitz 2014, 287-288). So zielt TermNet ausdrücklich auf die Wirtschaft und bietet kommerzielle ${ }^{75}$ Schulungen/Fortbildungen, Zertifizierung von Dienstleistungen und Dokumenten an und vernetzt vor allem Partner aus der Wirtschaft (TermNet 2013, 9-15). Dieser wirtschaftlicher Schwerpunkt scheint in der Selbstauffassung von Infoterm nicht vorhanden zu sein (vgl. Infoterm 2015, About us > Mission statement) und man kann eher einen engeren Bezug zu Wissenschaft feststellen (s. beispielsweise Galinski 2009, 21).

Neben Infoterm und TermNet, die auf der internationalen Ebene agieren, unterstützen weitere Organisationen wie bspw. tekom (Gesellschaft für Technische Kommunikation - tekom Deutschland e.V.) die Bewusstseinsschaffung, Methodenverbreitung und Erfahrungsaustausch (s. Themen der Tagungen und Publikationen der tekom unter www.tekom.de).

\subsubsection{Konsolidierung der Terminologienormung}

Konzentrierten sich die ersten Versuche der internationalen Terminologiearbeit auf die eigentliche Ausarbeitung von Wörterbüchern (IEC-Wörterbuch 1938 erschienen, VDI-Technolexikon (Herzog 2009, 15, 16)), so war man nach der Veröffentlichung der Dissertation von Wüster zunehmend an Metaprinzipien der Terminologiearbeit interessiert. Zur Sachnormung entstand

75 TermNet ist zwar eine Non-Profit-Organisation, darf aber kommerzielle Aktivitäten zugunsten ihrer Mitglieder durchführen (TermNet 2006-, About us). 


\section{Forschungsstand}

1926 ISA (International Federation of the Standardizing Associations), der Vorläufer der heutigen ISO (vgl. W. Blanke 1989, 278; Galinski 2009, 19; Herzog 2009, 16; Arntz, Picht und Schmitz 2014, 270-271). Entscheidend für die Terminologiearbeit und -normung war jedoch die Gründung des TC 37 bei ISA im Jahre 1936 (W. Blanke 1989, 278; Arntz, Picht und Schmitz 2014, 271), „das die von Wüster fünf Jahre früher herausgearbeiteten Prinzipien in die internationale technische Normung einbringen sollte“ (Herzog 2009, 16). TC 37 sollte nicht die eigentliche terminographische und terminologische Arbeit auf der internationalen Ebene betreiben. Es widmete sich der Entwicklung und Standardisierung des Metawortschatzes und Methoden der Terminologielehre und -arbeit sowie den Überlegungen zur Struktur von Terminologieeinträgen in Wörterbüchern (Galinski 2009, 19; vgl. Felber 1979, 62-63). Das Sekretariat von ISA/TC 37 arbeitete eng mit Wüster zusammen (Felber 1979, 62). Viele der Aktivitäten gingen von Wüsters privatem Terminologiezentrum in Wieselburg aus (F. Lang 1959-1960).

Nach dem zweiten Weltkrieg wurde ISO als Nachfolger von ISA gegründet und TC 37 wieder eingerichtet (Felber 1979, 63; W. Blanke 1989, 279; Galinski 2009, 19). Obwohl sich ISO/TC 37 nach wie vor der Methodenstandardisierung widmete (vgl. Felber 1979), identifiziert Galinski (2009) drei historische Arbeitsphasen: Implementierung (1951-1971), in der methodische Empfehlungen zu den bereits durch ISA/TC 37 behandelten Themen herausgebracht wurden, Konsolidierung (1972-1988), in der aus den Empfehlungen Normen entstanden, und Erweiterung (1989-2008), in der neue methodische Überlegungen über die ursprünglichen ISA/TC-37-Themen hinaus, unter stärkerer Berücksichtigung der Sprachtechnologie, in die Normen mit einbezogen wurden.

\subsubsection{Etablierung der Terminologielehre und Terminologiearbeit in der Wissenschaft}

Die Geschichte der wissenschaftlichen Etablierung der Beschäftigung mit der Terminologie ist ausführlich von Picht (2009) beschrieben. Im Folgenden stütze ich mich im Wesentlichen auf diese Ausarbeitung.

Die Verwissenschaftlichung der Beschäftigung mit der Terminologie erfolgte ab den 1930er Jahren an drei Orten: Prag, Wien und in der Sowjetunion. In der Literatur wird in diesem Zusammenhang oft von drei Terminologieschulen gesprochen (s. bspw. Felber und Budin 1989, 44-52; Cabré 1999, 7, 12-13; Picht 2009, 6). Die Beschäftigung in Prag ist von Anfang an 
sprachwissenschaftlich orientiert und in den Kontext der Funktionalstile der linguistischen Prager Schule eingebettet (Felber und Budin 1989, 46-48; vgl. Cabré 1999, 13). Die anderen beiden Schulen stehen in ihren Ursprüngen näher aneinander, da sie beide von Wüsters Dissertation geprägt waren (für die Sowjetische Schule s. Felber 1998, 48; Cabré 1999, 13).

Wie Picht (2009) betont, kamen die Impulse zur wissenschaftlichen Etablierung der Terminologieforschung im Westen nicht aus der Linguistik selbst, da sie die Beschäftigung mit der Terminologie der Beschäftigung mit Fachsprachen im Allgemeinen gleichsetzte, diese aber erst Ende der 1960er Jahre zum linguistischen Untersuchungsgegenstand wurden (Picht 2009, 6; vgl. Cabré 1999, 2). Es war die sog. Wiener Schule von Eugen Wüster, die die praktischen Methoden und die wissenschaftliche Fundierung in Zentral- und Nordeuropa prägte (Cabré 1999, 13). Sie hängt eng mit dem erwähnten privaten Forschungszentrum in Wieselburg und Wüsters Tätigkeiten in internationalen Gremien wie dem ISO/TC37 zusammen (Felber und Budin 1989, 44-45). War Wüster in seiner Dissertation noch an Werkzeugen zur Lösung praktischer Fragen interessiert, so widmet er sich ab den 1950er Jahren einer wissenschaftlichen Begründung seiner Methodik und bettet diese in die in 2.4.3.1 vorgestellten Modelle ein (Wüster 1959-1960; vgl. Cabré 1999, 5; Picht 2009, 6). An der Universität Wien hält er 1972-1974 auch die erste ,zusammenhängende und theoretisch fundierte“ (Picht 2009, 7) Vorlesung zur Terminologielehre im Westen (vgl. Felber und F. H. Lang 1979, 16). Dank zunehmendem Interesse an Fachsprachen in der Linguistik, aber auch durch die Verankerung der terminologischen Inhalte in der (Fach-)Übersetzerausbildung (Picht 2009, 7) kommt es in den 1970er Jahren zu einer Etablierung der Terminologielehre in der Wissenschaft (Felber und Budin 1989, 13, 44), was auch ab der Mitte der 1970er Jahre an Themen der Abschlussarbeiten und Dissertationen und später auch an terminologischen Lehrbüchern zu erkennen ist (Picht 2009, 7).

In der Sowjetunion erfolgte die Theoretisierung und wissenschaftliche Etablierung der Terminologielehre früher (Picht 2009, 6). So wurden bereits in den 1930-40er Jahren Terminologieseminare an der Akademie der Wissenschaften in Moskau angeboten (Felber und Budin 1989, 49). In den 1950er Jahren kommt es zu theoretischen Auseinandersetzungen und zur Entstehung von ersten Terminologielehrbüchern (Felber und Budin 1989, 49; Picht 2009, 7).

Heutzutage ist die Unterscheidung zwischen den drei Terminologieschulen eher von historischem Interesse. Picht (2009) betont, dass es von Anfang an 


\section{Forschungsstand}

wesentliche Übereinstimmungen gab, die sich im Laufe der Zeit weiter vergrößerten (Picht 2009, 6-7; vgl. Felber und Budin 1989, 47-48). Es handelt sich bei den Terminologieschulen also eher um verschiedene Blickwinkel auf den Gegenstand als um konkurrierende Konzeptualisierungen (Picht 2009, 7).

\subsubsection{Zielgruppen}

Unter Zielgruppe der Terminologiearbeit verstehe ich Menschen, die in ihrer beruflichen Tätigkeit zur Einhaltung der Ergebnisse der präskriptiven Terminologiearbeit, insbesondere zur Benennungs- und, in kleinerem Maße, zur Begriffsverwendung, verpflichtet sind. Es handelt sich hier also nicht um die Zielgruppe der Terminologielehre, also Personen, die die methodischen Prinzipien beherrschen und Terminologiearbeit leisten sollen, sondern um Terminologienutzer.

Cabré $(1999,11-12)$ unterscheidet zwischen sog. direkten und indirekten Benutzern. Die direkten Benutzer sind Fachleute im eigentlichen Sinne (Ingenieure, Wissenschaftler, Konstrukteure etc.). Ihr Interesse sei begriffsorientiert, denn Terminologie diene zur Gebietskonzeptualisierung (Cabré 1999, 11). Die indirekten Nutzer sind hingegen unterschiedliche Sprachdienstleister wie Technische Redakteure, Übersetzer, Dolmetscher etc., die die Ergebnisse der Terminologiearbeit zur Erleichterung der Kommunikation benutzen (Cabré 1999, 12). Die Unterscheidung zwischen direkten und indirekten Benutzer scheint in der deutschsprachigen Literatur nicht etabliert zu sein. Gasser und Fleischmann (2008) unterscheiden hingegen nach Gebrauchshäufigkeit zwischen Gelegenheitsnutzern und Intensivnutzern (Gasser und Fleischmann 2008, 52-53; vgl. Suchowolec 2009, 48).

Die industriebezogene Literatur geht in erster Linie auf unterschiedliche Bereiche oder Abteilungen in einem Unternehmen ein, in denen die Benutzung einheitlicher Terminologie als sinnvoll erscheint. Dabei umfassen die Auflistungen beinahe alle Unternehmensbereiche wie die Technische Redaktion, Marketing/Öffentlichkeitsarbeit, Einkauf, Produktion, Produktmanagement, Schulung, Forschung und Entwicklung, Qualitätssicherung, Konstruktion (einschließlich Zeichner und Softwareentwickler (Fleischmann 2009, 5)), Verkauf, Rechtsabteilung, ausländische Vertretungen aber auch externe Zielgruppen wie Kunden, Zulieferer und externe Sprachdienste (DTT 2010, M23; vgl. Drewer 2008, 57; Pich 2008, 74; Schmitz und Straub 2010, 31, 100; Drewer und Ziegler 2011, 165; Weilandt 2014, 12). Abweichend von meinem 
am Anfang dieses Abschnittes angeführten Verständnis, wird in der Literatur meistens nicht systematisch reflektiert, ob die betroffene Zielgruppe zwingend verpflichtet ist, festgelegte Terminologie zu benutzen, oder lediglich einen Zugriff auf die terminologischen Ressourcen zu Informationszwecken haben sollte.

Obwohl man davon ausgeht, dass letztendlich möglichst viele Personen und Gruppen Terminologie nutzen sollen (Drewer 2008, 57), wird empfohlen, dass die Einführung der vorgeschriebenen Terminologie zunächst wenige Gruppen umfassen und erst allmählich auf andere erweitert werden sollte (vgl. DTT 2010, M5-2). So sehen beispielsweise Drewer und Ziegler $(2011,165)$ die Technischen Redakteure und Übersetzer als Hauptnutzer der Terminologie in Unternehmen.

Geht man von einer sukzessiven Einbeziehung mehrerer Zielgruppen aus, so stellt die Bestimmung der anfänglichen und den anvisierten Zielgruppen eine Schwierigkeit dar, da unterschiedliche Gruppen unterschiedliche Anforderungen an Inhalte stellen. So können Techniker eine andere inhaltliche Tiefe der Definitionen im Vergleich zu Technischen Redakteuren erwarten (vgl. Drewer und Ziegler 2011, 166). Zudem können bei der Auswahl von Vorzugsbenennung unterschiedliche Gesichtspunkte für die Technische Redaktion im Vergleich zu Marketing spielen (vgl. Suchowolec 2009, 61). Darüber hinaus können die Modalitäten der Terminologiebereitstellung zielgruppenspezifisch sein, so dass nicht jede Zielgruppe auf die gesamte Breite des Terminologiebestandes zugreifen muss. ${ }^{76}$

Unter den genannten Gesichtspunkten muss daher das Konzept der Terminologiearbeit von Anfang an zielgruppenspezifisch erweiterbar sein und die Ergebnisse der Terminologiearbeit eine gewisse Flexibilität besitzen. Dies wird dadurch erschwert, dass die Bestimmung der Zielgruppen bereits in der Planungsphase des Projekts- oder Prozessmanagements erfolgen muss (Arntz, Picht und Schmitz 2014, 211) und einer gründlichen Ist-/Soll-Analyse der Anforderungen bedarf.

\subsubsection{Werkzeuge}

Ähnlich wie bei Kontrollierten Sprachen wird eine effiziente Terminologiearbeit beinahe ausschließlich unter Einsatz von technischen

76 In diesem Kontext nennen Schmitz und Straub (2010, 31) den Zugriff ausschließlich auf die Ausgangssprache bei mehrsprachigen Terminologiedatenbanken für Technische Redakteure, während die Übersetzer auf die volle Sprachenbreite zugreifen sollen. Zudem erwähnen sie das Problem der Zugriffssicherheit für externe Terminologienutzer. 


\section{Forschungsstand}

Hilfsmitteln realisiert. Obwohl terminologische Werkzeuge unterschiedliche Funktionalitäten in unterschiedlichen Konfigurationen enthalten können, kann zurzeit allgemein zwischen Komponenten zur Terminologieverwaltung, -extraktion und -kontrolle sowie zur Workflowunterstützung unterschieden werden. Neben Eigenentwicklungen, die speziell auf die Anforderungen in einem Anwendungsszenario zugeschnitten sind, etablierten sich auch kommerzielle und Open-Source-Lösungen (s. bspw. Wölbrink 2008, 86-90), die einen unterschiedlichen Grad an anwendungsspezifischen Anpassungen erlauben. Im Folgenden gehe ich auf generische Überlegungen zu einzelnen Komponenten ein und verweise in einzelnen Abschnitten auf weiterführende Literatur zu konkreten Systemen. Eine detaillierte Beschreibung und Auswertung verschiedener Systeme für einzelne Komponenten findet sich in Schmitz und Straub $(2010,127-270){ }^{77}$

\subsubsection{Terminologieverwaltung}

Das Terminologieverwaltungssystem stellt die unabdingbare Komponente für eine effiziente Terminologiearbeit dar. Das Verwalten der Terminologie, d.h. die „Erstellung, Bearbeitung und Abfrage“ von terminologischen Einträgen (DTT 2010, M4-2; vgl. Thouin 1984, 272) kann zwar in Textdateien oder Tabellenkalkulationen erfolgen, wird aber bei größeren Terminologien und mehreren Beteiligten umständlich und unübersichtlich (vgl. DTT 2010, M4-2; Arntz, Picht und Schmitz 2014, 229). Daher werden gängige Office-Programme insgesamt als weniger geeignet gesehen (Seewald-Heeg 2009, 35; Arntz, Picht und Schmitz 2014, 229). Aus diesem Grund wird unter Terminologieverwaltungssystem eine speziell für die Anforderungen der Terminologiearbeit entwickelte, meist datenbankbasierte, Software verstanden (Seewald-Heeg 2009, 35; DTT 2010, M4-2; DIN 2342 2011, 17; Arntz, Picht und Schmitz 2014, 229), mit der einschlägige Begriffe, Benennungen und Zusatzinformationen wie Begriffsdefinitionen, Gebrauchsvorgaben (z.B. welche Benennung erlaubt und welche verboten ist), fremdsprachige Äquivalente etc. erfasst werden.

Drewer und Ziegler (2011, 180-185) fassen folgende Anforderungen an ein Terminologieverwaltungssystem für ein Unternehmen zusammen (vgl. Wölbrink 2008; Seewald-Heeg 2009; Schmitz und Straub 2010, 36-42):

77 Siehe auch die aktualisierte und erweiterte Darstellung in der neuen Auflage von Schmitz und Straub (2016). 
- Integrierbarkeit in die bestehende Systemlandschaft; auch Schnittstellen zu weiteren Anwendungen wie Texteditoren, Übersetzungswerkzeuge etc.

- Gutes Rollen- und Rechtesystem, um unterschiedliche Benutzergruppen zu definieren, z. B. diejenigen Nutzer, die Terminologieeinträge neu anlegen, bearbeiten und/oder löschen dürfen im Unterschied zu Nutzern, die lediglich einen Lesezugriff bekommen.

- Unterstützung von Austauschformaten (s. dazu auch exemplarisch Zerfaß 2009; Reineke 2014)

- Spezielle Anforderungen an Datenbankdefinition wie Begriffsorientierung, Benennungsautonomie, freie Eintragsstruktur, Granularität und Kombinierbarkeit der Datenfelder sowie die Konsistenzsicherung ihrer Inhalte.

Ein Terminologieverwaltungssystem soll also einerseits die Spezifik eines bestimmten Anwendungsszenarios berücksichtigen, andererseits aber die Anwendung von Prinzipien der Terminologielehre erlauben. Obwohl die Begriffsorientierung von vielen Verwaltungssystemen unterstützt wird, ist es mit den klassischen Werkzeugen nur unzureichend möglich, Begriffsbeziehungen und insbesondere Begriffssysteme festzuhalten und wiederzugeben. Die Werkzeuge neuerer Generation versuchen diese Schwäche zu beseitigen und setzen auf eine stärkere Integration der Begriffssysteme in die Datenerfassung. ${ }^{78}$

Die unterschiedlichen Lösungen unterscheiden sich hinsichtlich weiterer Punkte (vgl. Arntz, Picht und Schmitz 2014, 230-233): Ob es sich um ein eigenständiges Produkt handelt, oder ob es aber in ein anderes System, z. B. ein Übersetzungswerkzeug, eingebettet ist (Seewald-Heeg 2009, 46-57; DTT 2010, M4-2); ob man auf die Daten nur im Firmennetz oder aber im Web zugreifen kann (für webbasierte Anwendungen s. Herwatz 2009); ob es plattformgebunden oder -unabhängig ist etc. (vgl. N. Keller 2014). In Deutscher Terminologie-Tag e.V. (DTT 2010, M4-6) sind weitere Aspekte zusammengefasst, die eine Kaufentscheidung unterstützen sollen. Für einen Überblick über verschiedene Systeme, s. beispielsweise Seewald-Heeg (2009, 80-81) und Schmitz und Straub (2010, 147-193).

78 TermFactory setzt ontologische Modellierung für terminologische Daten ein (Carlson u. a. 2014); bessere Unterstützung für Begriffssysteme bieten beispielsweise Coreon (Wetzel 2014) und TermWeb (Granström 2014) an. 


\section{Forschungsstand}

\subsubsection{Terminologieextraktion}

Unter Terminologieextraktion versteht DIN 2342 (2011) den „,Teil der Terminologiearbeit (...), der darin besteht, Termini aus einem Korpus herauszufiltern“ (DIN 2342 2011, 16). Es handelt sich hierbei also um den Prozess, in dem bestehende Terminologie aus Dokumenten gewonnen wird (vgl. Eckstein 2009, 108; Arntz, Picht und Schmitz 2014, 244). Diese Aufgabe ist vor allem bei Aufbau und Pflege von Terminologiebeständen zentral, kann aber auch als Vorbereitungsarbeit für einen Übersetzungsauftrag oder aber als automatisch ausgeführter Zwischenschritt bei maschinengestützten Terminologiekontrolle (2.4.7.4) vorkommen (Arntz, Picht und Schmitz 2014, 244-245).

Im Allgemeinen kann zwischen manueller, halbautomatischer und (voll-)automatischer Terminologieextraktion unterschieden werden (Eckstein 2009, 109). Obwohl man auch bei manueller Extraktion auf generische Software wie Textverarbeitungsprogramme zurückgreifen kann, um beispielsweise Terme im Text zu markieren (Arntz, Picht und Schmitz 2014, 245-246), sind diese Mittel von den eigentlichen Werkzeugen zu Terminologieextraktion zu unterscheiden. Als Vorstufe können hier auch die sog. Konkordanzprogramme gesehen werden, die aber nicht unbedingt terminologiespezifisch sind. ${ }^{79}$

Die Extraktionswerkzeuge im engeren Sinne setzen zurzeit statistische, regelbasierte (sog. linguistische) oder gemischte Ansätze ein, um eine Liste von Termkandidaten zu erzeugen. Diese Liste enthält oft auch weitere Angaben wie den Kontextsatz. Erfolgt die Extraktion halbautomatisch, was zurzeit meistens der Fall ist, wird die Liste der Termkandidaten im Anschluss manuell gesichtet und validiert (Eckstein 2009, 109; DTT 2010, M4-10-15; Arntz, Picht und Schmitz 2014, 247-249). Setzt man hingegen eine vollautomatische Extraktion ein, erfolgt die Validierung der Termkandidaten durch das Werkzeug selbst (Eckstein 2009, 110; Arntz, Picht und Schmitz 2014, 249). ${ }^{80}$ Die Qualität der halbautomatischen und automatischen Termextraktion hängt mit den Möglichkeiten der Sprachtechnologie im Allgemeinen zusammen, wobei auch hier ähnlich wie bei Kontrollierten Sprachen (2.3.1.3) solche Maße wie precision und recall sowie die Benutzerfreudlichkeit eine Rolle spielen (Eckstein 2009, 110-111). Arntz, Picht und Schmitz (2014) stellen beim heutigen Stand der Technik fest, dass in manchen Fällen eine manuelle Bearbeitung

79 Konkordanzprogramme stellen eine einfache, meist nach Häufigkeit sortierte, Liste aller Wörter in einem Text zusammen. Die Wörter können lemmatisiert, d. h. auf ihre Grundform zurückgeführt und zusammengefasst werden, was aber nicht bei allen Programmen der Fall ist (vgl. Eckstein 2009, 112; DTT 2010, M4-9-10; Arntz, Picht und Schmitz 2014, 246-247).

80 Wie die automatische Validierung erfolgen kann, wird beispielsweise in Drouin (2006) beschrieben. 
der Termkandidatenliste sich als aufwändiger herausstellen kann, als eine rein manuelle Termextraktion (Arntz, Picht und Schmitz 2014, 249).

Auch bei Extraktionswerkzeugen stellen sich die Fragen der Eigenständigkeit oder Einbettung in andere Tools und der Austauschbarkeit der Formate (Eckstein 2009, 111). Interessant kann für manche Projekte auch die Möglichkeiten einer mehrsprachigen Extraktion sein, die aber zurzeit für mehr als zwei Sprachen noch recht begrenzt sind (Arntz, Picht und Schmitz 2014, 245). Für eine Übersicht der Extraktionswerkzeuge siehe Eckstein (2009, 113-119) und Schmitz und Straub (2010, 194-213).

\subsubsection{Terminologieworkflow}

Um die Terminologiearbeit in Unternehmenspraxis effizient zu betreiben, werden für einzelne Schritte und Aufgaben Verantwortliche bestimmt. Man spricht in diesem Zusammenhang von Rollen und Zuständigkeiten der Terminologiearbeit wie Terminologiemanager, Terminologiebenutzer, Terminologievalidierer etc. (vgl. Abschnitt 2.4.8). Vor allem bei mehreren Beteiligten ist für ein Unternehmen nicht eine statische, sondern hauptsächlich eine dynamische Sicht auf diese Rollen interessant, auf deren Grundlage bestimmte Vorgehensszenarien definiert werden, d.h. was, in welcher Reihenfolge und von wem zu tun ist, wenn beispielsweise ein Benutzer einen Fehler im Terminologiebestand meldet oder einen Erweiterungsvorschlag macht. Werden solche Szenarien, also ein sog. Terminologieworkflow, explizit definiert und festgehalten, liegen sie meistens als ein Ablaufdiagramm vor. Vor ca. 10 Jahren wurden jedoch die ersten Werkzeuge entwickelt, die solche Terminologieworkflows, insbesondere die notwendigen Kommunikationsprozesse wie Austausch und Abstimmung zwischen beteiligten Personen, aktiv unterstützen (vgl. Fleischmann 2009, 5).

Wie bereits erwähnt, unterstützen die meisten Terminologieverwaltungssysteme Vergabe von verschiedenen Schreibrechten für verschiedene Nutzer. Die Workflow-Werkzeuge sind in ein bestehendes Verwaltungssystem integriert, ${ }^{81}$ bauen auf diesen Schreibrechten auf und definiert ggf. neue Rollen, um den Workflow abzuwickeln, was ich an einem Beispiel, angelehnt an N. Keller (2013, 27-28), erläutere.

81 Beispielsweise ergänzt quickTerm das Terminologieverwaltungssystem SDL MultiTerm; Termflow von Transline arbeitet ebenfalls als Aufsatz zum bestehenden Verwaltungssystem, die Kompatibilität wird jedoch nicht konkret angegeben (N. Keller 2013). 


\section{Forschungsstand}

Das Workflow-Werkzeug kann vom Terminologiebenutzer aufgerufen werden, um einen neuen Term vorzuschlagen, der in der Terminologiedatenbank noch nicht vorhanden ist. Diese Anfrage wird an die zuständige Person, z. B. den Terminologiemanager, weitergeleitet. Der Terminologiemanager prüft allgemein die Anfrage und benachrichtigt ggf. den zuständigen Terminologen. Der Terminologe entscheidet, welche Informationen zum Term er selbst ergänzen kann und was noch einer weiteren Abstimmung, z.B. mit anderen Terminologen oder aber auch mit Fachexperten wie Entwicklern oder Konstrukteuren bedarf. Diese Personen werden ggf. benachrichtigt und tragen ihre Informationen oder Kommentare über das Werkzeug entsprechend ein. Erst wenn der im Workflow-Werkzeug vordefinierte Abstimmungsweg durchlaufen wurde und keine Stellen mehr offen sind, erfolgt ein endgültiger Eintrag in die Terminologiedatenbank, der dann für alle Nutzer sichtbar ist.

Von dem Einsatz der Worklflow-Komponenten erhofft man sich bei einer kooperativen Terminologiearbeit vielfältige Vorteile, vor allem für diejenigen, die nicht hauptberuflich an Terminologiearbeit beteiligt sind wie Techniker, Konstrukteure, Entwickler etc. Zum einen arbeiten sie nicht direkt in dem Terminologieverwaltungssystem, müssen also die Datenbankstruktur nicht kennen, sondern lediglich gezielt Informationen beitragen (vgl. Fleischmann 2009, 5). Darüber hinaus erzeugen die meisten Werkzeuge die Benachrichtigungen per E-Mail (N. Keller 2013, 28), so dass die Nichtterminologen nur anfragespezifisch involviert sind und den Eingang neuer Anfragen nicht regelmäßig selbst überprüfen müssen. Ein wesentlicher Zeitfaktor ist zusätzlich, dass in vielen einfacheren Fällen kein Zusammentreffen aller Beteiligten mehr nötig, da eine Klärung und Abstimmung per E-Mail ausreichend ist. Insgesamt erhofft man sich also durch die Workflow-Komponente eine größere Beteiligung von Fachexperten an der Erarbeitung der Terminologie und dadurch auch mehr Akzeptanz für ihre Ergebnisse, da Experten gezielt und auf zeitschonende Weise eingebunden werden.

Im Allgemeinen können Workflow-Komponenten vorteilhaft sein, da man in räumlich getrennten Teams arbeiten kann und den Fortschritt von einzelnen Aufgaben besser verfolgen und erfassen kann.

\subsubsection{Terminologiekontrolle}

Wie bereits angeführt, stellt die Sicherung der Verwendung festgelegter Terminologie eine zentrale Aufgabe in der Terminologiearbeit. Diese Kontrol- 
le kann auf vielerlei Weise erfolgen, z. B. durch menschliches Lektorat oder aber durch Einsatz der sog. Authoring-Memory-Systeme (AM), die, ähnlich wie Translation-Memory-Systeme, „Formulierungen aus Vorläuferdokumenten zur Wiederverwendung anbieten. Dabei werden sowohl völlig übereinstimmende Sätze aus früheren Texten angeboten als auch leicht abweichende Sätze (...)“ (Drewer und Ziegler 2011, 273). Terminologiekontrolle ist also eine Nebenfunktion der AM.

Spezielle Werkzeuge zur Terminologiekontrolle sind „(...) Programme oder Werkzeuge (...), die die Nichteinhaltung der vorgegebenen Terminologie und Terminologieinkonsistenzen in den Originaltexten oder in Übersetzungen melden" (DTT 2010, M4-16). Wie bei Extraktionswerkzeugen unterscheidet man zwischen statistischen und linguistischen Ansätzen. Statistische Werkzeuge prüfen gegen eine hinterlegte Benennungsliste und besitzen ggf. nur beschränkte Möglichkeiten zur Erkennung von flektierten Formen (DTT 2010, M4-17-18; Arntz, Picht und Schmitz 2014, 253). Man kehrt also insgesamt von diesem Ansatz ab (vgl. Arntz, Picht und Schmitz 2014, 253). Linguistische Prüfwerkzeuge arbeiten regelbasiert und erbringen deshalb insgesamt eine bessere Prüfleistung (vgl. DTT 2010, M4-19; Arntz, Picht und Schmitz 2014, 253). Im Gegensatz zu statistischen Werkzeugen, die auf eine reine Terminologieprüfung spezialisiert sein können, bieten die linguistischen meistens zusätzliche Prüfoptionen wie Grammatik und Stil. Es handelt sich bei kommerziellen Lösungen de facto um Controlled Language Checker für Kontrollierte Sprachen, wie in Controlled Language Checker beschrieben (DTT 2010, M4-19; M. Mayer 2010; Arntz, Picht und Schmitz 2014, 253). Auch hier muss man also bedenken, dass Sprachtechnologie lediglich eine unterstützenden Funktion haben kann und menschliche Qualitätssicherung notwendig ist, da die Technologie nicht alle Fehler finden und beseitigen kann (Yamchi 2012, 29-30).

Die Prüfwerkzeuge unterscheiden sich darüber hinaus ebenfalls bezüglich ihrer Eigenständigkeit bzw. Einbettung in anderer Systeme und ihrer Architektur (DTT 2010, M4-20; Arntz, Picht und Schmitz 2014, 252-253). Eine Entscheidungshilfe zur Anschaffung eines geeigneten Systems findet man in Deutscher Terminologie-Tag e.V. (DTT 2010, M4-18, M4-20-21), für einen Überblick über verschiedene Systeme s. beispielsweise Schmitz und Straub (2010, 213-227). 


\section{Forschungsstand}

\subsubsection{Autoren}

Als Autoren präskriptiver Terminologie verstehe ich alle Akteure, die die Terminologiearbeit konzeptionell oder inhaltlich in einem bestimmten Terminologieprojekt oder -prozess leisten. Dabei umfassen Autoren unterschiedliche Personengruppen. Im Kontext von LPLP wird Terminologiearbeit wie auch andere Maßnahmen von verschiedenen, oft regierungsnahen, Gremien und Organisationen geleistet (Humbley 1997, 263; Cabré 1999, 16). Da diese Gremien unter den Autoren von LPLP subsumiert werden können, gehe in diesem Teilkapitel nicht weiter auf sie ein.

Auch Fachgemeinschaften wie Ingenieure, Biologen, Chemiker etc. ergriffen und ergreifen selbst Initiativen zur Vereinheitlichung der eigenen Terminologie, wie in 2.4.5.1 bereits erläutert. Im industriellen Kontext, den ich in diesem Teilkapitel vor allem betrachte, schlagen sich diese Initiativen in verschiedenen Normungsinstituten auf regionaler, nationaler und internationaler Ebene nieder, die unternehmens- und branchenübergreifende Standards erarbeiten. Mit diesen Instituten, wie etwa AFNOR (Frankreich), ANIS (USA), BSI (Großbritannien), DIN (Deutschland) aber auch ISO (international) befassen sich beispielsweise Arntz, Picht und Schmitz (2014, 271-283). Das Hauptaugenmerk liegt bei dieser Autorengruppe auf der Sachnormung; die eigentliche Terminologiearbeit wird ebenfalls geleistet, ist aber der Sachnormung untergeordnet, denn sie ist notwendig, um Sachverhalte in der eigentlichen Sachnorm eindeutig und unmissverständlich beschreiben zu können. Dabei werden die terminologischen Festlegungen entweder als eigenständige Normen im Normverbund zu einem bestimmten Thema oder aber als einleitende Abschnitte in der eigentlichen Sachnorm fixiert (Arntz, Picht und Schmitz 2014, 272-273). Abhängig von den nationalen Begebenheiten, sind die Normungsinstitute unterschiedlich aufgebaut, besitzen unterschiedlichen rechtlichen Stand und somit auch die Durchsetzungsmittel (Arntz, Picht und Schmitz 2014, 272). Ausführlicher erläutert wird die Zusammensetzung eines Institutes am Beispiel von DIN. Neben hauptberuflichen Mitarbeitern bestehen seine Normen- und Arbeitsausschüsse aus (ehrenamtlichen) Vertretern von Mitgliedsorganisationen: „Mitglied des DIN können Firmen oder Verbände sowie alle an der Normung interessierten Körperschaften, Behörden und Organisationen sein (...). Diese Mitglieder tragen neben der Entrichtung der Mitgliedsbeiträge insbesondere dadurch zur Arbeit des DIN bei, dass sie Fachleute aus ihrem jeweiligen Bereich zur ehrenamtlichen Mitarbeit in die Ausschüsse entsenden (...)“ (Arntz, Picht und Schmitz 2014, 277). Die interessierten Parteien können somit nicht nur die Sachnormung sondern auch 
terminologische Festlegungen in den Normen auf nationaler Ebene aktiv mitgestalten.

Aus den bisherigen Beschreibungen geht hervor, dass Terminologiearbeit in einer Zusammenarbeit zwischen Sprachexperten, meistens Terminologen, und Fachexperten erfolgen sollte. Dies ist auch für Terminologiearbeit im Unternehmen wahr (exemplarisch Cabré 1999, 18; Drewer 2008, 57; F. Mayer 2009, 15). Für Felber und Budin (1989) ist ein Terminologe eine ,(..) sprachliche geschulte Person (...), die sich der Sammlung, Ordnung und Beschaffung von terminologischen Daten widmet" (Felber und Budin 1989, 234). Eine ähnliche Auffassung von Terminologe, die sich eng an den Aufgaben der Terminologiearbeit aus der Definition in DIN 2342 (2011) orientiert, vertritt auch Rat für Deutschsprachige Terminologie (2005, 1-2) (vgl. Arntz, Picht und Schmitz 2014, 299). Welche konkreten Aufgaben die Terminologen jedoch übernehmen, ist mit Cabré $(1999,18)$ projektspezifisch. Felber und Budin (1989) differenzieren zudem zwischen Fachterminologen (Terminologen eines speziellen Faches), Berufsterminologen (hauptberuflich, in Normungsinstituten und Gremien wie oben beschrieben) und Terminographen (Terminologen, die Terminologiebestände für die Nutzer bereitstellen) (Felber und Budin 1989, 235). Diese Unterscheidung hat sich in der Literatur jedoch nicht etabliert. Vielmehr wird anerkannt, dass ein Terminologe über ein breites Spektrum an Kenntnissen und Fähigkeiten verfügen muss. Neben der terminologiespezifischen Sachund Methodenkompetenz geht Rat für Deutschsprachige Terminologie (2005) auf weitere Fähigkeiten ein: Kenntnisse in Projektarbeit, gutes Allgemeinwissen und schnelle Auffassungsgabe, aber auch ausdrücklich verschiedene Softskills wie Verhandlungsgeschick, Überzeugungskraft, Argumentations- und Durchsetzungsvermögen, kommunikative und soziale Kompetenz sowie Problemlösungsfähigkeit (Rat für Deutschsprachige Terminologie 2005, 3; vgl. Arntz, Picht und Schmitz 2014, 300; s. auch Drewer 2008, 57; DTT 2010, M6-2).

Die zweite Gruppe der sog. Terminologieproduzenten (Arntz, Picht und Schmitz 2014, 297) sind die Fachexperten, die weitestgehend gleich wie Domänenexperten in Abschnitt 2.3.1.3 aufgefasst werden können. Es sind also zunächst terminologische Laien (Buol 2000, 2-3), die in einschlägigen Arbeitsweisen geschult werden sollen, sobald sie in Terminologiearbeit mit einbezogen werden. Hierbei handelt es sich im Unternehmenskontext projektspezifisch um Mitarbeiter solcher Abteilungen wie Konstruktion, IT, Entwicklung, Forschung etc. Nach Cabré $(1999,19)$ fällt den Fachexperten 


\section{Forschungsstand}

bei Terminologiearbeit in Unternehmen eine deutlich wichtigere Rolle als im Kontext von LPLP zu, da sie, gemäß den Prinzipien der Terminologielehre, über relevantes Wissen zur Erstellung und Validierung von Begriffssystemen verfügen (Drewer 2008, 57; vgl. ISO 15188 2001, 4) ${ }^{82}$ Fachexperten spielen aber nicht nur bei einer nachträglichen Begriffssystematisierung eine wichtige Rolle. Wie Drewer und Ziegler (2011, 165) bemerken, verringert eine enge Zusammenarbeit mit Experten in Produktforschung und -entwicklung die Notwendigkeit eines nachträglichen terminologischen Eingriffs in den ungelenkt entstandenen Terminologiegebrauch (vgl. Pich 2008, 75).

Bei der praktischen Terminologiearbeit handelt es sich also um eine kooperative Aufgabe. Die Modalitäten der Zusammenarbeit sind projektspezifisch (Buol 2000, 2, 147; vgl. F. Mayer 2009, 15). Mögliche Szenarien, wie bestimmte Aufgaben auf bestimmte Mitarbeitergruppen in Unternehmen verteilt werden können, werden ausführlich in ISO 15188 (2001) erläutert. Ich fasse sie hier in Anlehnung an Suchowolec (2009, 47-49) zusammen.

Wie in Abschnitt 2.4.3.2 beschrieben, befasst sich ISO 15188 (2001) mit projektbezogenen Gesichtspunkten der Terminologiearbeit. Insbesondere umfassen die Überlegungen zur Projektplanung wichtige personale Aspekte: Es wird gefordert, dass zum einen ein Projektmanager (eigentlich: Terminologiemanager) und eine Arbeitsgruppe, die die eigentliche Terminologiearbeit leistet, bestimmt werden (ISO 15188 2001, 4, 10). Aus Praxisbeobachtungen leitet Sturz (2014) folgende Anforderungen an den Terminologiemanager ab: Durchsetzungsvermögen über das reine Argumentieren hinaus, Entscheidungsfähigkeit sowie betriebswirtschaftliches und strategisches Denken. Die Arbeitsgruppe hingegen soll aus verschiedenen Personengruppen bestehen, die im Anhang B der ISO 15188 (2001) spezifiziert werden: Terminologiemanager, Terminologe(n), interne Fachexperten der Arbeitsgruppe, Sekretariat, externe Fachexperten. Die Auswahlkriterien sollen insbesondere folgende Punkte umfassen: Erfahrung, Motivation, Multiplikatorenqualität, Sprachwissen, Durchsetzungsvermögen und diplomatisches Geschick (ISO 15188 2001, 4).

Die Norm schlägt vier konkrete Modelle vor, wie Arbeitsszenarien in der Arbeitsgruppe aussehen können, wobei sie sich hauptsächlich in der Stellung des Terminologen unterscheiden (ISO 15188 2001, 5; vgl. Suchowolec 2009, 47-49):

82 Bei Terminologiearbeit im Kontext von LPLP spielen für Cabré $(1999,19)$ die Fachexperten im Vergleich zu Linguisten eine untergeordnete Rolle, da systematische Begriffsarbeit keine zentrale Aufgabe ist. Dies sei nicht zuletzt auch durch die Öffnung dieser Art Terminologiearbeit auf die Gemeinsprache bedingt. 
A Terminologe als externer Berater der Arbeitsgruppe

B Terminologe als Mitglied der Arbeitsgruppe

C Terminologe erarbeitet Terminologie unter Beratung von Fachexperten

D Fachexperten erarbeiten Terminologie unter Beratung von Terminologen

Unter Berücksichtigung von ISO 15188 (2001, 5, 11-12) erläuterte ich: Modelle A und B ,....) setzen eine teamzentrierte Projektarbeit voraus, bei der jeder der Projektmitglieder eine bestimme Teilaufgabe erfüllt. Dabei sind der Projektleiter und (...) [das Sekretariat] für die administrativen Aufgaben zuständig, während der Terminologe, die Arbeitsgruppe und die Fachleute die inhaltliche Arbeit leisten (...). Dies ist auch die gängigste Arbeitsform in den gegenwärtigen Terminologieprojekten (...).“ Modelle $\mathrm{C}$ und D ,(..) setzen hingegen den Schwerpunkt auf den Beitrag einer einzelnen Person, entweder des Terminologe[n] oder des Fachmanns, die die Terminologiearbeit mit allen ihren Aspekten weitestgehend selbstständig bewältigt" (Suchowolec 2009, 48). Mit Vor- und Nachteilen der Modelle setzte ich mich in Suchowolec (2011) auseinander (siehe Tabelle 2).

Obwohl ISO 15188 (2001) die Gruppe der Terminologiebenutzer nicht explizit zu den eigentlichen Terminologieproduzenten zählt, kommt ihnen in der Praxis eine wichtige Rolle im Validierungsprozess zu: „Terminologienutzer werden bei ihrer täglichen Arbeit mit den Ergebnissen der Terminologiearbeit konfrontiert und können diese auf ihre praktische Eignung überprüfen“ (Suchowolec 2009, 48-49). Dabei stellt die Homogenität der Benutzergruppe einen entscheidenden Vorteil dar (Suchowolec 2009, 48). So wird in Unternehmen versucht, dieses Potenzial der Benutzer sowohl zum Zweck der Inhaltsprüfung aber auch der Akzeptanzsteigerung durch computergestützte Feedbackmöglichkeiten verstärkt zu nutzen. De facto werden Terminologiebenutzer in der Praxis als (Mit-)Autoren der terminologischen Bestände behandelt.

Schließlich geht Deutscher Terminologie-Tag e.V. (DTT 2010) noch auf Personen ein, die IT-Support im Terminologieprojekt leisten wie Datenbankadministratoren, Tool-Entwickler und Tool-Spezialisten (DTT 2010, M6-23). Diese Personen leisten zwar keine inhaltliche Arbeit, sind aber für eine reibungslose Abwicklung der Prozesse der Terminologiearbeit unabdingbar. Ob sie somit zu den Autoren gezählt werden können, bleibt jedoch offen. 


\begin{tabular}{l|l}
\multicolumn{1}{l|}{ A-B } & \multicolumn{1}{|l}{ C-D } \\
\hline+ Weniger Sachfehler & $\begin{array}{l}\text { Weniger terminologische } \\
\text { Fehler }\end{array}$ \\
$+\begin{array}{l}\text { Kostengünstiger, denn kein } \\
\text { Vollzeitterminologe notwen- } \\
\text { dig }\end{array}$ & $\begin{array}{l}\text { Erarbeitungszeit u.U. kürzer, } \\
\text { denn Kommunikationswege } \\
\text { kürzer }\end{array}$ \\
$+\begin{array}{l}\text { Höhere Akzeptanz bei Mitar- } \\
\text { beitern } \\
-\quad \text { Mehr terminologische Fehler }\end{array}$ & $-\begin{array}{l}\text { Mehr Sachfehler (insb. C) } \\
\text { Einstieg langsamer, denn Ein- } \\
\text { arbeitung ins Sachgebiet not- } \\
\text { wendig (insb. C) }\end{array}$ \\
$-\quad$ Schulung notwendig & $-\begin{array}{l}\text { Längere Verifizierungsphase } \\
\text { Akzeptanzprobleme }\end{array}$ \\
\hline
\end{tabular}

Tabelle 2.: Vor- und Nachteile einzelner Modelle nach ISO 15188 (2001) (Suchowolec 2011)

\subsubsection{Erfolg}

Das Thema Erfolg von Terminologiearbeit nimmt in der einschlägigen Literatur eine viel prominentere Stellung ein als in den anderen von mir betrachteten Gebieten. Dennoch ist die Diskussion von erfolgsfordernden Faktoren stark praxisgetrieben, denn Terminologieliteratur versteht sich oft als Ratgeber oder Entscheidungshilfe für die Wirtschaft. Eine wissenschaftliche, theoretisierende Metadiskussion von Erfolg oder ein kritisches Hinterfragen einzelner Beobachtungen findet jedoch nicht statt.

Wie auch in anderen Teilgebieten, beschreibe ich im Folgenden die sprachbezogenen (systemlinguistischen) und die nichtsprachbezogenen (nichtsystemlinguistischen) Erfolgsfaktoren der Terminologiearbeit in Unternehmen.

\subsubsection{Erfolg durch systemlinguistische Faktoren}

Betrachtet man den Gegenstand der Terminologiearbeit aus der systemlinguistischen Perspektive, so entspricht der Begriff dem Signifié und die Benennung dem Signifiant. Wie der Begriff/das Signifié identifiziert, abgegrenzt und systematisiert werden kann, befasst sich die klassische Terminologie- 
lehre. Die Anwendung der Terminologielehre zur terminologischen Vereinheitlichung kann somit als erfolgsfördernd auf der Signifié-Seite betrachtet werden. Im Folgenden setzte ich mich deshalb mit dem Signifiant des fachsprachlichen Zeichens auseinander und gehe auf die Überlegungen zur Benennungsbewertung ein.

Wie oben dargelegt, erfolgt die Auswahl von vorgeschrieben und verbotenen Benennungen für einen Begriff erst am Ende der präskriptiven Terminologiearbeit. Aufgrund der Synonymie und Mehrdeutigkeit stellt sich in der praktischen Terminologiearbeit des Öfteren die Frage, was eine gute Benennung ausmacht. Dies bedeutet zum einen wie man zwischen verschiedenen existierenden (synonymen) Benennungen die geeignete auswählt, zum anderen aber auch, wie eine neue Benennung gebildet werden sollte. Obwohl eine isolierte Ad-hoc-Bewertung und -Bildung von Benennungen prinzipiell möglich ist, sind wie bereits für die begriffliche Ordnung auch hier Bestrebungen zu verzeichnen, feste Kriterien für die Benennungsauswahl zu entwickeln. Dies sollte zum einen den Terminologen entlasten, zum anderen aber auch die Nachvollziehbarkeit der Benennungsauswahl steigern (Haussteiner 2014, 24).

In der Literatur finden sich Auflistungen von allgemeinen Kriterien, die eine Hilfestellung bei der Beantwortung beider Fragen darstellen sollen. So nennt DIN 2330 (2013) folgende Anforderungen: sprachliche Korrektheit, Genauigkeit, Transparenz (v. a. Motiviertheit), Neutralität (v. a. Konnotationsfreiheit), Knappheit, produktive Flektierbarkeit und Bevorzugung der deutschen Sprache (DIN 2330 2013, 17-18; vgl. Drewer 2008, 59-60; Drewer und Ziegler 2011, 173-174; Arntz, Picht und Schmitz 2014, 115). Darüber hinaus werden Sprechbarkeit und sprachliche Logik (Drewer und Ziegler 2011, 174-175), Gesetzes- und Normenkonformität sowie Zielgruppenorientiertheit (DTT 2010, M3-3-21) gefordert. Diese allgemeinen Kriterien lassen in der praktischen Anwendung einen großen Interpretationsspielraum. Zudem fordern sie teilweise gegenläufige Tendenzen wie beispielsweise Knappheit-Genauigkeit, so dass in einzelnen Fällen eine Gewichtung notwendig ist (Drewer 2008, 59; Drewer und Ziegler 2011, 174; DIN 2330 2013, 19; Arntz, Picht und Schmitz 2014, 116).

Um mehr Transparenz und Objektivität zu erzielen, werden daher unternehmensspezifische Regeln aufgestellt, die orthographische und morphologische Gesichtspunkte berücksichtigen. Orthographische Regeln beziehen sich auf die sog. Schreibvarianten und legen beispielsweise die Verwendung von Bindestrichen (bspw. bereits ab 3 lexikalischen 


\section{Forschungsstand}

Morphemen), Groß- und Kleinschreibung, Schreibweise von Zahlwörtern oder Verwendung des Fugen-s fest (vgl. DTT 2010, M3-21-26; tekom-AG „Regelbasiertes Schreiben“2013, 65-78).

Noch mehr Fälle von Synonymie und Mehrdeutigkeit lassen sich durch morphologische Regeln beseitigen, die sowohl die Derivation als auch Komposition als Wortbildungsmuster berücksichtigen können. Beispielsweise entwickelte die Firma Koenig \& Bauer Group eine Rangliste von Merkmalen, die eine Benennung vorzugsweise enthalten sollte. So sind diejenigen Synonyme zu bevorzugen, die die Funktion eines Bauteiles explizit bezeichnen. Darüber hinaus sind unter anderem Regeln zur Komposition mit Ablautderivaten und Derivation mit -ung entwickelt (Suchowolec 2009; vgl. Suchowolec 2014, 115-116).

Wie aus den Beschreibungen hervorgeht, sind die einzelnen Benennungsregeln firmenspezifisch. Insbesondere bedürfen die morphologischen Regeln einer Korpusanalyse. Da im Allgemeinen auch das Kriterium der Gebräuchlichkeit der bevorzugten Formen eine zentrale Rolle spielt (DTT 2010, M316), sollen bei der Korpusanalyse vor allem unternehmensinterne Texte berücksichtigt werden. Obwohl eine allgemeingültige Empfehlung zur orthographischen und morphologischen Beschaffenheit von Termini nicht ausgesprochen werden kann, so finden sich, ähnlich wie bei Kontrollierten Sprachen, Best-Practice-Regelkataloge, die als Anregung beim Aufbau des eigenen Regelwerkes genutzt werden können (s. beispielsweise DTT 2010, M3; tekom-AG „Regelbasiertes Schreiben“ 2013).

\subsubsection{Erfolg durch nichtsystemlinguistische Faktoren}

Unter den nichtsystemlinguistischen Gesichtspunkten ist für diese Arbeit die Studie von Schmitz und Straub (2010) mit dem Titel Erfolgreiches Terminologiemanagement im Unternehmen relevant, die ich im Folgenden kurz vorstelle.

Ihrem Untertitel ist zu entnehmen, dass sich die Studie als praktische Anleitung für die Terminologiepraxis in Unternehmen versteht. Ihr Ziel ist es, eine Orientierung zu geben, was beim Entwurf eines Terminologieprojektes und bei der Einführung der Terminologiearbeit zu beachten ist. Sie stellt Argumente für Terminologiearbeit nach Prinzipien der Terminologielehre von Wüster bereit und ,(...) geht u. a. der Frage nach, wie man den Nutzen der Terminologiearbeit mit Zahlen und Fakten belegen kann“ (Brändle 2010, 71). Insgesamt soll die Studie „(...) als Grundlage für Entscheidungsprozesse im Unternehmen dienen“ (Schmitz und Straub 2010, 5). 
Die Studie besteht aus einem theoretischen und einem empirischen Teil. Im theoretischen Teil werden die Grundlagen der Terminologielehre und -arbeit erläutert, Gründe für Terminologiearbeit angeführt und eine neue Methode für quantitative Kosten-Nutzen-Analyse vorgestellt. Getreu der oben angeführten Ziele, erfolgt diese theoretische Einführung aus einer angewandten Perspektive. Der empirische Teil besteht zum einen aus einem praktischen Beispiel einer Kosten-Nutzen-Analyse und einer detaillierten Beschreibung von aktuellen Terminologiewerkzeugen: ihrer Funktionalitäten und technischen Einzelheiten. Zum anderen werden auch Ergebnisse einer Befragung und Erfahrungsberichte zum Stand und Stellenwert von Terminologiearbeit in Unternehmen vorgestellt.

Obwohl der Titel der Studie das Thema Erfolg von Terminologiearbeit explizit aufgreift, wird auf der Metaebene weder diskutiert noch konzeptionell näher bestimmt, was Erfolg ist. Trotzdem ist diese Studie aus der Sicht dieser Arbeit interessant, da sie relevante Überlegungen und empirische Daten zur Entwicklung einer übergreifenden Theorie der Sprachlenkung liefert, obwohl sie über den Text verstreut sind. Da sich die Studie als einschlägige Praxishilfe versteht, werden m. E. aus der Makroperspektive die vorgestellten Methoden wie Terminologielehre, Kosten-Nutzen-Analyse sowie projekt- und prozessbezogene Aspekte der Terminologiearbeit für Prinzipien gehalten, deren Einhaltung als erfolgsfördernd gesehen wird. Ähnlich sind die über den Text gestreute Argumentationshilfen für Terminologiearbeit aufzufassen. Für die Metauntersuchung von Handlungsmöglichkeiten in dieser Arbeit sind zudem Erkenntnisse aus der Befragung relevant, insbesondere die sechs Fallbeispiele (Schmitz und Straub 2010, 271-276) und, auf der Mikroebene, die Freitextkommentare, die als Erfahrungen und Tipps zusammengetragen sind (Schmitz und Straub 2010, 276-295).

Unter den Fallbeispielen liefert insbesondere Fallbeispiel 2 ausführlichere Überlegungen und Tipps, was man bei einem Terminologieprojekt beachten soll. Dabei sind solche Projekt- und Prozessbezogene Aspekte von besonderer Bedeutung wie eine Unterstützung durch das Management, eine klare Definition von Abläufen und Zuständigkeiten, Zusammenarbeit von mehreren Zielgruppen (Abteilungen), eine aktive Bekanntheitsteigerung und Vorrang der Qualität vor der Quantität der Terminologie. Als besonders problematisch erwiesen sich in diesem Fallbeispiel der zeitliche Aufwand allgemein und speziell der Schulungsaufwand, die Fluktuation der Mitarbeiter und persönliche Präferenzen (Schmitz und Straub 2010, 272). 


\begin{tabular}{l|l}
\hline Abstimmungsprobleme & Prozessschwierigkeiten \\
Anwendungsschwierigkeiten & Akzeptanzprobleme \\
Altlasten & Hoher Zeitaufwand \\
Hohe Kosten & Mangelnde Ressourcen und Personal \\
Mangelnde Aktualität, Probleme & Terminologielehrebezogene Schwie- \\
bei Pflege und Umfang & rigkeiten beim Bestandsaufbau \\
Zugangs- und Verfügbarkeitspro- & Fehlende Managementunterstützung \\
bleme & \\
Probleme mit Werkzeugen & Probleme durch Kunden \\
Sonstiges & \\
\hline
\end{tabular}

Tabelle 3.: Probleme und Herausforderungen der Terminologiearbeit in Unternehmen: zusammengefasste Ergebnisse einer Befragung (Schmitz und Straub 2010, 283-288)

Eine wichtige Stellung für diese Arbeit nehmen die in der Studie zusammengetragenen Freitextkommentare ein. Neben Kommentaren, die auf die positiven Effekte durch die Terminologiearbeit eingehen (Schmitz und Straub 2010, 276-282), umfassen sie auch Probleme bei der täglichen Terminologiearbeit (Schmitz und Straub 2010, 283-288) und Tipps für die Einführung eines Terminologiemanagements (Schmitz und Straub 2010, 289-295). Die letzten beiden Kommentargruppen decken sich weitestgehend mit den Schwerpunkten der Metauntersuchung in dieser Arbeit, entsprechend Herausforderungen und Vorgehensweisen, und ich übernehme sie deshalb direkt in die Untersuchung. Die in der Studie identifizierten Probleme und Herausforderungen habe ich in Tabelle 3 zusammengefasst, während Tabelle 4 Bereiche wiedergibt, für die Tipps und Vorgehensweisen angegeben sind.

In beiden Fällen enthalten die eigentlichen Listen Kommentare, die teilweise einander sehr ähneln und lediglich als Paraphrasen gesehen werden können. Darüber hinaus können ähnliche Kommentare mehreren Gruppen zugeordnet sein.

Aus den bisherigen Beschreibungen der Terminologielehre und -arbeit und aus der ersten erfolgsbezogenen Sichtung der Literatur ergeben sich verschiedene Empfehlungen für die praktische, präskriptive Terminologiearbeit in Un- 


\begin{tabular}{l|l}
\hline Zeitpunkt für die Einführung & Umfang zu Beginn \\
Dienstleister & Geschäftsführung \\
Projekt- und Prozessvorbereitung & Akzeptanz \\
Werkzeuge & Zusammenarbeit/Abstimmung \\
Bereitstellung & Terminologiearbeit \\
Projekt- und Prozessausführung & Eigentlicher Bestandsaufbau \\
Umfang und Termbestimmung & Sonstiges \\
\hline
\end{tabular}

Tabelle 4.: Tipps und Vorgehensweisen für Terminologiearbeit in Unternehmen: zusammengefasste Ergebnisse einer Befragung (Schmitz und Straub 2010, 289-295)

ternehmen, die auch als erfolgsfördernde Faktoren aufgefasst werden können. Vorläufig strukturiere ich sie nach dem idealtypischen Zeitpunkt, zu dem sie in einem Terminologieprojekt eine Rolle spielen: Zu Projektanfang, d. h. vor dem eigentlichen Aufbau des Terminologiebestandes; während des Projektes: beim Aufbau des Terminologiebestandes und nach Projektabschluss, wo es sich vor allem um eine Erweiterung und Pflege des Kernbestandes handelt. Hierbei ist jedoch zu beachten, dass bei dieser sequentiellen Strukturierung die vorangestellten Faktoren weiterhin eine Auswirkung in späteren Phasen haben können. Die unten angeführten Faktoren erheben keinen Anspruch auf Vollständigkeit und sollen lediglich bestimmte Tendenzen aufzeigen und als Arbeitsstrukturierung dienen. Eine ausführlichere Analyse erfolgt in Kapitel 5.

Vor dem Projekt Vor dem Entwurf und der Einführung eines Konzeptes für Terminologiearbeit besteht laut Literatur die größte Schwierigkeit darin, Mitarbeiter auf verschiedenen Stufen von der Notwendigkeit der Terminologiearbeit zu überzeugen (vgl. Weilandt 2014, 11). Dies betrifft zunächst vor allem diejenigen, die am Aufbau der Terminologie beteiligt sein sollten, aber auch in hohem Maße die Entscheider und Geldgeber, d. h. den Vorstand und die Manager. In der Literatur findet man deshalb verschiedene Argumentationshilfen. Beispielsweise listet Deutscher Terminologie-Tag e.V. (DTT 2010, M1) 60 unterschiedliche Argumente auf, mit denen einzelne Zielgruppen (Geschäftsleitung, Management; Controller, Finanzverantwortliche; 


\section{Forschungsstand}

Marketing, Vertrieb, Produktmanagement; Sprachdienst; Entwickler; Qualitätsbeauftrage und Kundendienst) angesprochen werden sollten. Die Liste umfasst beispielsweise Verbesserung der Kommunikationsprozesse, Steigerung der Arbeitseffizienz, Unterstützung des Wissensmanagement, sowie eine allgemeine Steigerung der Qualität und Verbesserung der Benutzerfreundlichkeit der Produkte.

Für Schmitz und Straub (2010) stehen vor allem quantitative Daten und Fakten wie das ROI zur Überzeugung der Managementebene im Vordergrund (bspw. Schmitz und Straub 2010, 3-5). Massion (2014) weist hingegen darauf hin, dass die quantitativen Daten allein nicht ausreichen, um das Management zu überzeugen, in Terminologiearbeit zu investieren. Er plädiert für die Auffassung der Terminologiearbeit als untergeordnetes strategisches Ziel, das andere, übergeordnete Ziele, wie Effizienzsteigerung, entscheidend unterstützt (Massion 2014, 133, 137; vgl. DTT 2010, M5-2). Trotz dieser prominenten Stellung der Überzeugungsarbeit in der terminologischen Literatur stellten die Ergebnisse der Befragung von Schmitz und Straub (2010) fest, dass die Notwendigkeit der Terminologiearbeit in der Industrie durchaus bekannt ist. Das eigentliche Problem scheint daher die mangelnde Bereitschaft zu sein, Terminologiearbeit in die Tat umzusetzen, was Einstellungs-Verhaltendiskrepanz genannt wird (Schmitz und Straub 2010, 3). Eine intensivere Überzeugungsarbeit mit mehr unternehmensspezifischen Beispielen und Daten wird als Abhilfe hierfür gesehen (Schmitz und Straub 2010, 3-4).

Weitere Empfehlungen für den Projektbeginn findet man beispielsweise in Deutscher Terminologie-Tag e.V. (DTT 2010, M5-1-4). Sie betreffen beispielsweise die Notwendigkeit eines planvollen und systematischen Vorgehens beim Konzeptentwurf, das auf einer Ist-/Soll-Analyse und Festlegungen von Abläufen und Rollen und Zuständigkeit beruhen soll. Zudem wird betont, dass zunächst ein kleiner Kernbestand von guter (fachlicher und terminologischer) Qualität aufgebaut werden soll, der erst nach und nach systematisch erweitert wird (vgl. Pich 2008, 78-79).

Während des Projekts Ist der Kernbestand aufgebaut, so wird vor allem darauf hingewiesen, dass das Vorhandensein des Bestandes nicht automatisch auch seine Nutzung bedeutet. Hierbei nimmt das Verfügbarmachen von benutzerfreundlichen Tools zum Terminologiezugriff eine wichtige Stellung ein (vgl. Gust 2006, 18). Des Weiteren soll der Bestand durch Multiplikatoren und gezielte Werbung bekannt gemacht werden. Mit dem Aspekt der Nutzung hängt auch das Akzeptanzproblem zusammen. Hierbei handelt es sich 
vordergründig, m. E. aber nur oberflächlich, um die Ablehnung der vorgeschriebenen Benennungen zugunsten der nicht zugelassenen Formen. Beide Probleme, der Nutzung und der Akzeptanz, versucht man zudem durch eine größere Beteiligung beim Aufbau selbst, z. B. durch Bevorzugung kooperativer Modelle der Terminologiearbeit, aber auch durch die Stärkung des sonstigen aktiven Engagements im terminologischen Geschehen durch extra Anreize wie Belohnungen für die Beteiligung an Terminologieumfragen (Lewark und Turhan 2013, 11), Informationen zum „Term des Tages“ (eine Funktionalität des quickTerm) oder Quiz-Einsendungen zu stärken (vgl. Haussteiner 2014, 24).

Nach dem Projekt Ist das einführende Terminologieprojekt abgeschlossen, besteht die Gefahr, dass der aufgebaute Bestand und die etablierten Prozesse an Aufmerksamkeit verlieren und mangels Pflege verwahrlosen. Deshalb wird empfohlen, auch nach dem Projektabschluss genug Ressourcen für das Fortbestehen und die Weiterentwicklung der Terminologiearbeit vorzusehen (Ebenhoch 2014, 7).

\subsubsection{Zusammenfassung und Diskussion}

Terminologiearbeit kann verschiedene Ausprägungen annehmen und Fachsprache oder Gemeinsprache, private Wirtschaft oder ethnische Gruppen, einsprachige oder mehrsprachige Textproduktion betreffen, um nur einige Konzeptualisierungsbeispiele zu nennen. Wie deutlich geworden ist, stellt vor allem die präskriptive Terminologiearbeit eine Form der Sprachlenkung dar und ich habe mich in diesem Teilkapitel vordergründig mit der Terminologiearbeit in Unternehmen befasst. Rein deskriptive Ansätze wie die Fachlexikographie, das Textmining oder rein deskriptive Projekte zur Erfassung und Bereitstellung des (vernetzten) Fachwortschatzes in Unternehmen habe ich nur am Rande betrachtet.

Präskriptive Terminologiearbeit in Unternehmen beruht methodisch auf der Terminologielehre von Eugen Wüster und baut auf folgenden Prinzipien auf: Begriffsorientierung, synchrone Sprachbetrachtung, Beschränkung auf (Fach-)Wortschatz, Beschränkung auf geschriebene Sprache. Terminologielehre ist von Anfang an interdisziplinär ausgerichtet und ihre Eigenständigkeit als Disziplin ist umstritten, was aber im theoretischen Diskurs eine immer kleinere Rolle spielt. Auch heute integriert sie verschiedene Ansätze aus anderen, auch neu entstandenen, 


\section{Forschungsstand}

Disziplinen und Forschungsgebieten. Mit umfangreichen Instrumenten zielt sie auf die Beschreibung und Harmonisierung der Ausdrucksund der Formseite des Fachwortschatzes. Terminologiearbeit nutzt die Methoden der Terminologielehre, um sprachbezogene Aufgaben der Wortschatzvereinheitlichung $\mathrm{zu}$ lösen. Vom einheitlichen Wortschatz verspricht man sich in Unternehmen verschiedene Vorteile, die sich unter Effizienzsteigerung der Arbeitsprozesse (v.a. der Texterstellung und der Textübersetzung), Kostenreduktion und Verständlichkeitsverbesserung der Texte subsumieren lassen.

Während Terminologielehre die sprachbezogene Methodik zur Verfügung stellt, wurde in Unternehmen erkannt, dass betriebswirtschaftliche Aspekte ebenfalls systematisch berücksichtigt werden sollten. Dies führte zur Erweiterung des Methodenrepertoires um nichtstrukturalistische Gesichtspunkte und in Konsequenz zur Umkehrung der Perspektive auf Terminologiearbeit als betriebswirtschaftliche Aufgabe mit sprachbezogenen Elementen.

Die Sprachauffassung der klassischen Terminologielehre ist strukturalistisch, mit Schwerpunkt auf langue. Ihre theoretischen Fundierungen erlauben keine Berücksichtigung und Modellierung der Sprachgebrauchsvariation zwischen Individuen und zwischen Gruppen, die jedoch eine beobachtbare Tatsache ist. Dies ist ein wesentlicher Kritikpunkt an der klassischen Terminologielehre, der Alternativansätze wie die Sozioterminographie oder die Soziokognitive Terminologiearbeit hervorbrachte, die auf anderen Sprachauffassungen beruhen.

Trotz dieses theoretischen Mangels wird in der Praxis der auf der klassischen TL beruhenden Terminologiearbeit die Tatsache der Gebrauchsvariation nicht ausgeblendet. So stellt beispielsweise Buol (2000) fest, dass präskriptive Terminologiearbeit ein hohes Konfliktpotenzial in sich birgt, da verschiedene Ansichten und Perspektiven aufeinander treffen. Dieses Potenzial sei aber für eine erfolgreiche Präskription jedoch notwendig, denn es erfordert ein Ausdiskutieren verschiedener Standpunkte und eine Kompromissaushandlung, wodurch sie letztendlich aufgelöst und zu einem gemeinsamen, allgemein akzeptierten Standard werden können (Buol 2000, 2, 147). Meine Praxiserfahrung bestätigt, dass eine Vereinheitlichung der Begriffe und Benennungen über mehrere Unternehmensbereiche (wie Technische Redaktion, Konstruktion, Softwareentwicklung) möglich ist. Dennoch bleiben andere Bereiche übrig (wie Marketing oder Vertrieb), bei denen man von einer anderen Sprachfunktion ausgehen kann, was folglich zu einer eigenen Sicht auf Begriffe, und vor allem Benennungen, führen kann. Die so begründeten Rest- 
unterschiede im Benennungsgebrauch werden oft belassen und entsprechend mit Informationen belegt, in welchem Sachgebiet bzw. in welchem Kontext welches Synonym erlaubt und welches verboten ist. Dies wird auch durch Terminologiewerkzeuge (Verwaltungssysteme und Kontrollprogramme) teilweise unterstützt. Das theoretische Variationsproblem, das auf einer strengen strukturalistischen Theoriefundierung beruht, wird also in der Praxis pragmatisch gelöst. Alternative Ansätze bieten zwar eine bessere theoretische Variationsfundierung durch Berücksichtigung der sozialen, kommunikativen und kognitiven Perspektive, zielen jedoch explizit auf die Beschreibung und nicht auf die Präskription des Terminologiegebrauchs. Sie können aber zur Erklärung der Gebrauchsvariation herangezogen werden.

Die Geschichte der Terminologiearbeit verzeichnet drei Brennpunkte: 1) isolierte Terminologiearbeit durch einzelne Fachgemeinschaften, 2) Entwicklung von übergreifenden Prinzipien der Terminologiearbeit und Terminologienormung sowie 3) Etablierung der Terminologielehre (und -arbeit) in der Wissenschaft. Bezeichnend ist, dass alle diese Entwicklungen mit der Person von Eugen Wüster und den durch ihn aufgebauten Netzwerken und Infrastruktur zusammenhängen. Durch sein persönliches Engagement, was sowohl inhaltliche Arbeit, Kontaktknüpfung und -pflege als auch materielle Unterstützung angeht, übte die sog. Wiener Schule einen wesentlichen Einfluss auf die präskriptive Terminologiearbeit aus.

Die Zielgruppe der Terminologiearbeit - die Terminologienutzer - sind eine heterogene Gruppe, die in allen Abteilungen und auf allen Hierarchiestrufen in Unternehmen präsent sind (Suchowolec 2009, 48). Als Kernzielgruppe gelten gewöhnlich Technische Redakteure und Übersetzer. Obwohl zwischen verschiedenen Nutzergruppen unterschieden wird, z. B. Gelegenheitsnutzern und Intensivnutzern, so fehlt eine Systematisierung der Nutzergruppen danach, ob die Nutzung für sie verpflichtend, empfohlen oder freigestellt ist. Eine Auseinandersetzung mit dieser Frage könnte m. E. neue Implikationen für Terminologiepraxis liefern.

Terminologiearbeit in Unternehmen kann sowohl durch einzelne Personen als auch als Teamaufgabe bewältigt werden. Verschiedene Umsetzungsszenarien habe ich vorgestellt. Die Gruppe der Terminologieautoren umfasst jedoch mehr als, in klassischer Sicht, die Terminologen und Fachexperten: Man geht davon aus, dass jeder Terminologienutzer ungeachtet dessen, wie häufig und verpflichtend sein Gebrauch ist, die Möglichkeit zur aktiven Mitwirkung an Terminologiearbeit haben sollte. 


\section{Forschungsstand}

Die Werkzeugunterstützung erfolgt im Wesentlichen für sprachbezogene Aufgaben: Terminologieverwaltung, -extraktion und -verwendungskontrolle. Darüber hinaus existieren mit Workflow-Komponenten auch Werkzeuge, die sich auf die betrieblichen Aspekte der Prozessabwicklungen spezialisieren und somit die nichtsprachbezogenen Aufgaben der Terminologiearbeit unterstützen.

Das Thema Erfolg von Terminologiearbeit spielt in den unternehmensbezogenen Literatur eine wichtige Rolle, auch wenn keine theoretische Diskussion des Konzeptes stattfindet. Die Behandlung erfolgt also eher aus einer praktischen Perspektive, die oft auf Einzelbeobachtungen beschränkt ist. Die Frage nach Erfolg von Terminologiearbeit wird oft zur Frage nach Best-PracticeEmpfehlungen umformuliert, was sich in praktischen Leitfäden, Ratgebern und Empfehlungen zur Terminologiearbeit niederschlägt.

Ohne die Ergebnisse der Metauntersuchung (Kapitel 5) vorwegzunehmen, strukturierte ich in diesem Teilkapitel Erfolgsfaktoren danach, ob sie vor, während oder nach einem Terminologieprojekt eine Rolle spielen. An dieser Stelle ordne ich diese Faktoren abschließend danach, ob sie sich auf Zielgruppen/Autoren, Werkzeuge oder Modelle beziehen.

- Zielgruppen/Autoren: Durch die Forderung nach der Beteiligung alle Terminologienutzer an der Vereinheitlichung der Terminologie verschwimmt die Grenze zwischen Nutzern und Autoren. Man kann stattdessen zwischen der Bottom-up-Vereinheitlichung (durch alle Benutzer) und Top-down-Vereinheitlichung (durch ein Terminologieteam) sprechen. Die Bottom-up-Vereinheitlichung profitiert stark von werkzeugtechnischen Entwicklungen, weil dadurch das Feedback der Terminologienutzer in Echtzeit erheblich erleichtert wird. Durch weitere Anreize, wie die erwähnten Umfragen, Quiz-Einsendungen etc., kann die Beteiligung zusätzlich gestärkt werden. Es soll sich daher jeder für die Terminologiearbeit verantwortlich fühlen, so dass eine Art Gemeinschaftsgefühl um die Terminologiearbeit entsteht. In der abgeschwächten Form kann man den Bottom-up-Einfluss auch in größeren (Normungs-)Instituten wie der DIN beobachten, wo nicht jeder Mitarbeiter der Mitgliedsorganisation, sondern lediglich einzelne Vertreter das Mitspracherecht haben. Dabei ist jedoch m. E. davon auszugehen, dass sich das Gefühl der Gemeinschaft und Mitverantwortung bei jedem einzelnen von der Norm betroffenen Mitarbeiter nur geringfügig entwickelt. 
Trotz dieser Öffnung auf den Bottom-up-Einfluss wird in der einschlägigen Literatur empfohlen, dass die Hauptlast der Terminologiearbeit von einigen wenigen Verantwortlichen (kurz: dem Terminologieteam) getragen wird, die sowohl konzeptionell als auch inhaltlich koordinieren und die Entwicklungen in eine bestimmte Richtung lenken. Wichtig ist dabei, dass zunächst ein Standardisierungskonzept ausgearbeitet wird, auf dessen Grundlage inhaltliche Arbeit erfolgt. Dies kann als Top-down-Einfluss gewertet werden. Als Erweiterung dieses Einflusses können die Versuche aufgefasst werden, das Management für die Terminologiearbeit zu gewinnen, wobei hier weniger um eine inhaltliche Beteiligung sondern um sein Wirkungspotenzial und letztendlich um eine von oben herbeigeführte Durchsetzung geht.

Für übergreifende Normungsinstitute gestaltet sich ihre normative Wirkung im Allgemeinen abhängig von der sprachpolitischen Situation im Land. So haben Gremien wie ISO, aber auch speziell DIN, einen rein empfehlenden Charakter sowohl die Sach- als auch die Terminologienormung angehend. Speziell für DIN wird dieser Charakter an seinem Status als eingetragener Verein deutlich (Felber und Budin 1989, 219; Arntz, Picht und Schmitz 2014, 277). ${ }^{83}$ Dem gegenüber stehen Terminologiegremien in Ländern, in denen seitens der Regierung Sprachpolitik aktiv gefördert oder gar betrieben wird. Hier scheint sich die Wirksamkeit der Festlegungen ähnlichen Prinzipien wie das allgemeine LPLP zu folgen (Cabré 1999, 21).

Insgesamt kann man sagen, dass in der Terminologiearbeit zunehmend auf beide Arten der Beteiligung gesetzt wird, da sich weder eine reine Bottom-up-Ausarbeitung noch eine reine Top-down-Durchsetzung in der Praxis bewährt haben.

- Werkzeuge: Werkzeuge spielen eine wichtige Rolle bei der Förderung beider Einflussrichtungen (bottom-up und top-down) und folglich auch bei der Durchsetzbarkeit vorgeschriebener Terminologie. Gleichzeitig wird darauf hingewiesen, dass ihre Einführung ein Grundverständnis von Terminologielehre und -arbeit voraussetzt. Wie bereits für Kontrollierte Sprachen beschrieben, haben Werkzeuge lediglich eine unterstützende und entlastende Funktion.

83 Dies ist $m$. E. aus der Sicht der Industrie auch sinnvoll, denn die Normen behandeln die vordergründig auf einer hohen Abstraktionsebene angesiedelten Oberbegriffe. Welche Begriffsmerkmale dann letztendlich zur Benennungsbildung von Unterbegriffen wesentlich sind (wie am Beispiel von Koenig \& Bauer Group gezeigt: Funktion, Stoff, Form), ist unternehmensspezifisch. 


\section{Forschungsstand}

- Modelle: Wie beschrieben, haben die theoretischen Unzulänglichkeiten der klassischen Terminologielehre bisher wenig Einfluss auf die Praxis der präskriptiven Terminologiearbeit in Unternehmen, da mit ihnen pragmatisch umgegangen wird. Trotzdem können alternative, deskriptive, Ansätze auch für die präskriptive Terminologiearbeit in Unternehmen interessant sein, wie beispielsweise neue Beschreibungsmittel für vage Begriffe (Kategorien), da sie adäquatere Beschreibungsergebnisse als Vorstufe der Präskription liefern können. Eine adäquatere Beschreibung des Sprachgebrauchs einzelner Sprechergruppen kann m. E. für diese Gruppen ansprechend sein und möglicherweise die Akzeptanz der auf dieser Deskription beruhenden Präskription steigern.

\subsection{Forschungsstand - Fazit}

In diesem Kapitel habe ich den Forschungsstand in den vier von mir betrachteten Gebieten beschrieben. Dabei lag der Schwerpunkt auf ausgewählten Themenkomplexen: Gegenstandsbestimmung, Geschichte, Interdisziplinarität, Autoren, Sprachauffassung, Modelle und Erfolg.

Als Erstes fällt auf, dass sich diese deduktiv aufgestellten Themenblöcke zur Bündelung der bisherigen Erkenntnisse in unterschiedlichen Gebieten unterschiedlich gut eignen. In manchen Fällen, wie bei Gegenstandsbestimmung oder Geschichte, entsprechen sie weitestgehend direkt Forschungssträngen in allen vier Gebieten, so dass ich hier die Inhalte unmittelbar zusammentragen konnte. In anderen Fällen war mehr Eigeninterpretation von vorhandenen Erkenntnissen notwendig, um die vorgegebenen Themenkomplexe abzuhandeln, da sie bis jetzt in der Forschung noch nicht auf diese Weise betrachtet wurden. Als Beispiele nenne ich hier die Sprachauffassung in Plansprachen oder die Interdisziplinarität in Kontrollierten Sprachen.

Darüber hinaus beobachte ich eine große Eigenständigkeit der Gebiete bezüglich der Auswahl und Strukturierung der Inhalte in einzelnen Themenkomplexen. Beispielsweise werden die jeweiligen Gegenstände unterschiedlich bestimmt: offen (LPLP) oder gar vage (PS) im Unterschied zu Versuchen einer präzisieren Definition einschließlich einer Abgrenzung von Nachbarphänomenen (KS) oder einer normativen Bestimmung (TA). Da der Forschungsstand eher ein beschreibendes Kapitel sein sollte, ließ ich mich weitestgehend von den gebietsspezifischen Gepflogenheiten in einzelnen Themenblöcken leiten, mit der Konsequenz, dass die einschlägigen Abschnitte im Querschnitt teilweise unterschiedlich ausfielen. 
Auch wenn ich die in der Literatur postulierten Querbezüge im Forschungsüberblick größtenteils ausgeblendet habe, so halte ich aufgrund der oben beschriebenen Heterogenität fest, dass die von mir angenommene Forschungsautonomie der einzelnen Gebiete tatsächlich besteht. Dies wird zum einen an den geschichtlichen Entwicklungen deutlich, da ich dabei nur kleine Überlappungen feststelle. Darüber hinaus macht der Forschungsüberblick die Heterogenität der identifizierten Theorien und Modelle sowie die unterschiedliche wissenschaftliche und interdisziplinäre Einbettung der Gebiete klar. Da die Überschneidungen recht klein ausfallen, sehe ich darüber hinaus die Annahme als berechtigt an, dass bisher noch relativ wenig Austausch zwischen allen Gebieten stattfindet, dessen Anregung aber m. E. für alle neue Forschungsimpulse hervorbringen kann. Ein synthetischer Aufbau einer übergreifenden Perspektive auf alle vier Gebiete, die ich in dieser Arbeit Sprachlenkung nenne, unter Berücksichtigung und Verallgemeinerung der bisherigen Instrumente, Konzeptualisierugen, Modelle und Erkenntnisse ist bei der heutigen Forschungslage durchaus sinnvoll. Mit dieser Aufgabe, die meiner ersten Forschungsfrage entspricht, befasse ich mich in nachfolgendem Kapitel 3.

Was meine zweite Forschungsfrage betrifft, so macht der Forschungsstand deutlich, dass die Frage nach dem Erfolg von praktischen Maßnahmen aus nichtsystemlinguistischer Sicht in allen Gebieten noch relativ wenig und zudem recht unsystematisch erforscht ist. Insbesondere fehlen mit wenigen Ausnahmen Modelle oder umfassende Best-Practice-Empfehlungskataloge, die eine Handreichung für die Autoren bei der Umsetzung einer konkreten Maßnahme darstellen könnten. Aufgrund dieser Forschungslücke verschiebt sich mein Schwerpunkt für die Untersuchung des Erfolges. Da es kaum geeignete und systematische Modellierungen gibt, die ich auf ihre (übergreifende) Wirksamkeit hin evaluieren könnte, entscheide ich mich für das Zusammentragen von fragmentarischen Erkenntnissen, aus denen ich in Kapitel 5 einen Überblick über die in der Literatur für möglich gehaltenen Umsetzungsvorgehen der Autoren aufbaue. Es bedeutet, dass ich meine zweite Forschungsfrage eher aus der deskriptiven Perspektive beantworte: Welche Vorgehen (also was) für möglich gehalten werden, und nicht $o b$ und ggf. in welchem Kontext diese Vorgehen tatsächlich wirksam sind. Die spezielle Forschungsfrage lautet somit: Welche Herausforderungen stellen sich bei der Sprachlenkung und welche Handlungsmöglichkeiten stehen den Autoren zur Verfügung? 



\section{Konzept der Sprachlenkung}

\subsection{Einleitung}

In diesem Kapitel leite ich das Konzept der Sprachlenkung her. Ich behandle also meine erste Forschungsfrage, genauer:

- Was ist Sprachlenkung? Wie lässt sich Sprachlenkung so definieren, dass sich Maßnahmen aus allen vier Gebieten (LPLP, PS, KS, TA) darunter subsumieren lassen?

- Was wissen wir bis jetzt über Sprachlenkung im Allgemeinen?

Um diese Fragen zu beantworten, greife ich auf verschiedene Erkenntnisse zurück. Zum einen beziehe ich mich auf den Forschungsstand, gebe aber eine isolierte Betrachtung von Gebieten zugunsten eines interpretativen Vergleichs auf. Darüber hinaus stelle ich zwei weitere übergreifende Ansätze vor, die ich im Forschungsstand nicht besprochen habe, weil sie sich keinem der vier Gebiete direkt zuordnen lassen. Schließlich ziehe ich einzelne Konstrukte aus der Handlungs- und Motivationspsychologie heran, um bestimmte konzeptionelle Unklarheiten besser in den Griff zu bekommen. Erst durch die Verschränkung dieser drei Elemente ist es möglich, Sprachlenkung zu definieren, geeignete Kategorien zur Beschreibung und zu einer weiterführenden Charakterisierung der Sprachlenkung zu finden sowie unter den in Kapitel 2 vorgestellten Modellen diejenigen zu identifizieren, die auf Sprachlenkung als übergreifenden Gegenstand anwendbar sind.

Das Kapitel ist wie folgt aufgebaut. Im ersten Teil (3.2) setze ich mich mit den Erkenntnissen aus Kapitel 2 auseinander. Ich gehe insbesondere auf die einzelnen Gegenstände ein und erörtere die Gründe für die festgestellte Forschungsautonomie. Ferner überprüfe ich die in Kapitel 2 vorgestellten Modelle auf ihre Verallgemeinerbarkeit hin.

Im zweiten Teil (3.3) stelle ich die übergreifenden Ansätze der Sprachlenkung von Ischreyt (1965) sowie der Kommunikationsoptimierung von Schubert (2003, 2009, 2011a, 2014) vor, die die Erkenntnisse aus dem Forschungsstand vervollständigen. 


\section{Konzept der Sprachlenkung}

Im letzten Teil (3.4) folgt als Resultat der vorangegangenen Auseinandersetzungen und Verallgemeinerungen die eigentliche Bestimmung des Konzeptes der Sprachlenkung. Dies umfasst zum einen eine eigene Definition von Sprachlenkung, die ich dann ausführlicher erläutere und im Kontext anderer übergreifender Definitionen diskutiere. Hier erfolgt auch der Rückgriff auf die Handlungs- und Motivationspsychologie. Zum anderen trage ich schließlich aus den vorangegangenen Erkenntnissen und Überlegungen weitere Punkte zusammen, mit denen Sprachlenkung weiterführend charakterisiert werden kann, und gebe so, auf einer recht abstrakten Ebene, den bisherigen Wissensstand über Sprachlenkung wieder.

\subsection{Zusammenfassung und Verallgemeinerung der bisherigen Erkenntnisse}

In diesem Teilkapitel unterziehe ich die deskriptiven Erkenntnisse aus Kapitel 2 einer interpretativen Analyse mit dem Ziel, eine integrative Grundlage zur Bestimmung des Gegenstandes Sprachlenkung zu schaffen. Speziell greife ich im Folgenden die Punkte Gegenstand (Abschnitt 3.2.1) und Modelle (Abschnitt 3.2.2) heraus.

\subsubsection{Zusammenfassung der vier Gebiete bezüglich ihrer Gegenstände}

Um eine erste Annäherung an den Gegenstand Sprachlenkung zu erzielen, befasse ich mich mit den einzelnen Gegenständen der vier Gebiete und gehe insbesondere auf die Fragen ihrer Konstituierung, Abgrenzung und Charakterisierung ein:

- Wodurch konstituieren sich die einzelnen Gegenstände?

- Kann eine systematische Begründung für die Aufteilung in vier Gebiete gefunden werden?

- Mit welchen Eigenschaften werden einzelne Gegenstände weiterführend charakterisiert und ggf. unterteilt? 
Zieht man die Definitionen der Sprachplanung von Tauli (1968) ${ }^{84}$ und Cooper (1989), ${ }^{85}$ die unterschiedliche Schwerpunkte setzen, stellvertretend für den Bereich der Sprachplanung heran, so stellt man fest, dass die Konstruktion von Plansprachen, Kontrollierten Sprachen und die Terminologiearbeit von diesen Definitionen durchaus abgedeckt sind. Auch unter den von Nahir (1984) besprochenen Zielen der Sprachplanung (s. Abschnitt 2.1.1) können die selben drei Gebiete identifiziert werden: Terminologiearbeit unter terminology unification, Konstruktion von KS unter stylistic purification und Konstruktion von PS unter interlingual communication. Definitorisch sind also Kontrollierte Sprachen, Plansprachen und (normativ vorgegebene) Terminologien Beispiele für Sprachpolitik, die sowohl rein sprachliche Elemente (die eigentlichen Regeln bzw. das Korpus) als auch oft die Umsetzungselemente ${ }^{86}$ umfassen.

Dennoch scheint die wissenschaftliche Disziplin von LPLP eher wenig Aufmerksamkeit den spezifischen Problemen von PS, KS und Terminologie im Unternehmenskontext zu schenken, obwohl es einzelne Beiträge aus LPLP gibt, die auf Parallelen und die Notwendigkeit eines engeren Austausches hinweisen (bspw. Wood 1979; B. H. Jernudd 1983, 345-351). Wie an den aktuellen Forschungsfragen in Abschnitt 2.1.8 zu erkennen ist, fokussiert LPLP zurzeit eher die Probleme der Mehrsprachigkeit und ethnischer Sprachen vor allem auf der makrosozialen Ebene, obwohl zuweilen auch die Mehrsprachigkeit in kleineren Gruppen wie Unternehmen zum Thema wird. Andererseits wird auch in den anderen drei Bereichen auf LPLP kaum Bezug genommen. Sie bedienen sich unterschiedlicher Konzeptualisierungen, verfolgen andere Forschungsfragen und ziehen dabei unterschiedliche Nachbardisziplinen heran, wie aus den Abschnitten zur wissenschaftlichen Einbettung und der Interdisziplinarität hervorgeht (Abschnitt 2.1.4, Abschnitt 2.2.3.5, Abschnitt 2.3.3, Abschnitt 2.4.4.1). Insbesondere fließen auch die Erkenntnisse aus der Terminologiearbeit und der Konstruktion von Kontrollierten Sprachen nicht in LPLP zurück.

84 "LP is the methodical activity of regulating and improving existing languages or creating new common regional, national or international languages. LP comprises all spheres of the oral and written form of the language: phonology, morphology, syntax, lexicology (...) and orthography" (Tauli 1968, 27, Hervorhebungen im Original).

85 "Language planning refers to deliberate efforts to influence the behavior of others with respect to the acquisition, structure, or functional allocation of their language codes" (Cooper 1989, 45, Hervorhebungen im Original).

86 Beispielsweise Terminologieworkflow in einem Terminologieleitfaden, Erläuterungen in einer KSSpezifikation, wer das Lexikon erweitern kann und wie; oder Festlegungen in Fundamento, wann und von wem die Grundregeln des Esperanto verändert werden können. 


\section{Konzept der Sprachlenkung}

Eine Ausnahme bildet hier die Interlinguistik, weil sie eine stärkere Wahrnehmung und Verankerung ihrer Forschung in LPLP anstrebt (exemplarisch s. D. Blanke 2009b; Fiedler 2011a; Tonkin 2011; Żelazny 2012, 155-159). Darüber hinaus hängt mit der Interlinguistik auch das Postulat von Wüster (1955) zusammen, die sog. gestaltende Sprachwissenschaft als linguistische Teildisziplin ins Leben zu rufen. Sie sollte über eine bloße Sprachbeschreibung hinausgehen und die Prinzipien erforschen, nach denen bewusste und systematische Sprachveränderungen herbeigeführt werden können. Sie sollte dabei sowohl die natürlichen Sprachen als auch die Plansprachen berücksichtigen (Wüster 1955, 271-272; vgl. Suchowolec 2012, 35). Eine so verstandene gestaltende Sprachwissenschaft würde also eine übergreifende Wissenschaftsdisziplin für alle vier Gebiete darstellen und hätte, im Kontext dieser Arbeit, Sprachlenkung (s. 3.4) zum Gegenstand. Eine Etablierung der gestaltenden Sprachwissenschaft erfolgte jedoch nicht.

Beim Aufbau eines übergreifenden Modells der Sprachlenkung stellt sich zwangsläufig die Frage, warum dieser Gegenstand zurzeit in einzelne Gebiete aufgeteilt ist.

Da die Sprachplanung zumindest rein definitorisch in der Lage ist, andere Gebiete abzudecken, stellt sich u. a. die Frage der Kausalität:

A Ist die Selbstständigkeit der übrigen drei Gebiete darauf zurückzuführen, dass ihre spezifischen Fragen in der LPLP-Forschung nicht behandelt werden? ODER

B Berücksichtigt die LPLP-Forschung die spezifischen Fragen der übrigen Gebiete nicht, weil sie selbstständig sind?

Die kausale Kette, falls vorhanden, lässt sich in dieser Arbeit nicht zufriedenstellend rekonstruieren und insbesondere bleibt die Antwort auf $\mathbf{B}$ offen. Aus den historischen Entwicklungen der Gebiete geht jedoch hervor, dass die Verwissenschaftlichung der Konstruktion von Plansprachen und der Terminologiearbeit im Vergleich zur Sprachplanung und LPLP entweder früher oder zeitgleich stattfand. Insbesondere zum Zeitpunkt der Definition von Tauli (1968) sind Interlinguistik und Terminologielehre etabliert und spätestens bei Cooper (1989) ist die Konstruktion von Kontrollierten Sprachen ebenfalls ein etabliertes angewandtes Gebiet. Die Aufteilung der Sprachlenkung in Gebiete kann also nicht als Abspaltung der drei Gebiete im Sinne von A verstanden werden. In diesem Zusammenhang interpretiere ich die übergreifende Ausrichtung der Definitionen der Sprachplanung von Tauli (1968) und Cooper (1989) sowie die Charakterisierung von Nahir (1984) als Versuche, In- 
strumente für eine integrative Betrachtungsweise von verschiedenen Sprachlenkungsausprägungen bereitzustellen, die in der Praxis bereits unabhängig existieren und wissenschaftlich untersucht werden. Diese Vorstöße haben jedoch wenig Auswirkung auf die LPLP-Forschung einerseits und finden auch andererseits wenig Resonanz in den anderen Gebieten.

Um die Frage zu vertiefen, wie systematisch die Verselbstständigung der drei Bereiche erfolgte, wende ich mich im Folgenden Kriterien zu, mit deren Hilfe die einzelnen Gegenstände und Bereiche definiert und abgegrenzt werden. Die aufgedeckten Kriterien sollen zudem im weiteren Verlauf dieser Arbeit zur Charakterisierung und zu einer systematischen Unterteilung der Sprachlenkung eingesetzt werden, insbesondere unter dem Umsetzungsaspekt. Ich steige mit Kontrollierten Sprachen ein.

Die Bestimmung von Kontrollierten Sprachen wird in dieser Arbeit anhand der zurzeit umfassendsten Definition von Kuhn (2014) vorgenommen (s. 2.3.1.1). Diese Bestimmung erfolgt im ersten Schritt durch die Erläuterung, wie das Sprachsystem einer Kontrollierten Sprache aufgebaut wird, wobei hier die Kombination von systemlinguistischen (hauptsächlich durch Restriktion mehrerer Ebenen eines Sprachsystems) und soziolinguistischen/varietätlinguistischen Faktoren (Vorhandensein einer natürlichen/ethnischen Sprache bzw. einer Sprechergemeinschaft, was ich zu sog. Soziolekten nach Löffler (2010) zähle) ausschlaggebend ist. Erst im zweiten Schritt werden weitere Eigenschaften von Kontrollierten Sprachen angegeben wie die soziokognitive (leichte Erkennbarkeit und Verständlichkeit für Muttersprachler) und die intentionale, um eine bessere Abgrenzung zu Nachbarphänomenen zu erzielen. Die Abgrenzung zu Plansprachen und Terminologien erfolgt explizit.

Obwohl in der Definition nicht explizit erwähnt, so stellt man die Beschränkung der Konstruktion von KS auf den fachlichen Bereich fest, was ein weiteres soziolinguistisches/varietätlinguistisches Kriterium darstellt. Diese Beschränkung kommt weniger durch eine generelle Unmöglichkeit der Konstruktion einer KS für den Allgemeingebrauch zustande, sondern ist pragmatischer Natur und maßgeblich durch die Frage des Bedarfs und der Umsetzbarkeit bedingt.

Das Kriterium der Fachsprachlichkeit reicht jedoch nicht aus, um KS von anderen Phänomenen, wie Schreibregeln oder Richtlinien für beispielsweise Einfache oder Leichte Sprache (zu beiden Begriffen s. bspw. Bock 2014) 


\section{Konzept der Sprachlenkung}

abzugrenzen. ${ }^{87}$ Diese Richtlinien greifen häufig (jedoch nicht ausschließlich) ebenfalls in eine bestimmte Fachsprache ein, werden von der KS-Forschung aber nicht als KS aufgefasst und sind deshalb eher der Sprachplanung zuzuordnen. Hier scheinen die Fragen ausschlaggebend zu sein, ob solche Eingriffe einen empfehlenden oder normativen Charakter haben und ob sie nur wenige Phänomene auf den anvisierten Ebenen des Sprachsystems abdecken, d. h. ob sie punktuell oder eher flächendeckend sind. Dabei handelt es sich weniger um absolute Werte, sondern um einen gleitenden Übergang (Schubert 2009), wobei sich KS auf der normativen und flächendeckenden Seite positionieren.

Terminologien, hier als Gegenstand der Terminologiearbeit nach der Wiener Schule, werden ebenfalls primär systemlinguistisch bestimmt, da es sich ausschließlich um die lexikalische Ebene handelt. Dies trennt Terminologien von Kontrollierten Sprachen. Des Weiteren umfasst Terminologie ausschließlich den fachsprachlichen Wortschatz, was wieder einen soziolinguistischen/varietätlinguistischen Bestimmungsfaktor darstellt. Die Fachsprachlichkeit unterscheidet Terminologiearbeit von anderen Maßnahmen, die ebenfalls ausschließlich auf die Lexik, jedoch hauptsächlich die gemeinsprachliche, gerichtet sind und gewöhnlich der Sprachplanung zugeordnet werden.

Des Weiteren unterscheidet sich auch die Terminologiearbeit von anderen, auch auf die Fachlexik gerichteten Maßnahmen dadurch, dass ihr Eingriff zu Anfang größere, zusammenhängende Wortschatzbereiche betrifft und somit von rein punktuellen Maßnahmen zu unterscheiden ist.

Schließlich ist die Terminologiearbeit nach der Wiener Schule ausgesprochen normativ, wodurch sie sich von anderen, entweder beschreibenden oder empfehlenden Maßnahmen absetzt.

Plansprachen, als Oberbegriff für Welthilfssprachen, philosophische Sprachen etc., werden ähnlich wie Kontrollierte Sprachen primär in Kombination von systemlinguistischen (Aufbau eines Sprachsystems auf allen Ebenen) und soziolinguistischen Faktoren bestimmt. Der soziolinguistische Faktor ist dabei der Soziolekt; dieses Merkmal nimmt im Vergleich zu KS eine entgegengesetzte Ausprägung an: das Nichtvorhandensein einer entsprechenden Sprechergemeinschaft bzw. einer natürlichen/ethnischen Sprache.

Obwohl die Konstruktion von Plansprachen als ein selbstständiges Gebiet behandelt wird, können die (vollen) Plansprachen zum Gegenstand von sowohl der Terminologiearbeit (D. Blanke und W. Blanke 1998, 878) als auch

87 Dass beide Phänomene - KS und Leichte Sprache - auch aus der Praxisperspektive ähnlich sind, machen beispielsweise Siegel und Lieske (2015) deutlich. Sie zeigen, wie Regeln von textorientierten KS für Leichte Sprache eingesetzt werden können, was in Konsequenz einen Einsatz von KS-typischen CLC auch für Leichte Sprache möglich macht. 


\begin{tabular}{|c|c|c|c|}
\hline \multicolumn{4}{|c|}{ Sprachplanung } \\
\hline \multicolumn{4}{|c|}{$\Downarrow \Downarrow \Downarrow$} \\
\hline & KS & TA & PS \\
\hline System & $\begin{array}{l}\operatorname{mind} \text {. Lexik und Syn- } \\
\text { tax }\end{array}$ & Lexik & alle Ebenen \\
\hline Soziolekt & $\begin{array}{l}\text { Mittel existierender } \\
\text { Sprachgemeinschaft }\end{array}$ & $\begin{array}{l}\text { (Mittel existierender } \\
\text { Sprachgemeinschaft) }\end{array}$ & $\begin{array}{l}\text { Mittel keiner Sprach- } \\
\text { gemeinschaft }\end{array}$ \\
\hline Funktionolekt & ja & ja & $\mathrm{ja} /$ nein/beides \\
\hline Umfang & $\Rightarrow$ flächendeckend & $\Rightarrow$ flächendeckend & $\Rightarrow$ flächendeckend \\
\hline Verbindlichkeit & $\Rightarrow$ normativ & $\Rightarrow$ normativ & $\Rightarrow$ normativ \\
\hline
\end{tabular}

Tabelle 5.: Kriterien zur Abgrenzung der drei Gebiete von der Sprachplanung

der Konstruktion von Kontrollierten Sprachen werden (beispielsweise Konstruktion eines kontrollierten Esperanto als Interlingua für die Maschinelle Übersetzung (s. Schubert 1992; vgl. Pool 2006)).

Aus der oberen Analyse geht hervor, dass die selbstständige Etablierung der drei Spezialgebiete auf folgenden Kriterien beruht: Sprachsystem (welche Ebenen betreffend?), Soziolekt (auf Grundlage von Sprachmitteln einer bestehenden Sprachgemeinschaft: ja/nein), Funktionolekt (fachsprachengebunden: ja/nein/beides), Umfang (punktuell-flächendeckend), Verbindlichkeit (empfehlend-normativ). Darüber hinaus wurde deutlich, dass ein einziges Kriterium nicht ausreichend ist, um eine selbstständige Etablierung zu rechtfertigen. Bezeichnend ist auch, dass jedes Gebiet eine andere Kriterienkombination benötigt und die gesamte Ausdifferenzierung daher nicht systematisch erfolgt sein kann. Tabelle 5 gibt wieder, welche Kriterien benutzt werden, um die Gebiete abzugrenzen. Diese Tabelle hat einen rein beschreibenden Charakter, der den Ist-Zustand der Forschung zusammenfasst. Sie korrigiert nicht die Randunschärfe einzelner Gebiete, die sich aus ihren aktuellen Definitionen und Charakterisierung ergibt, und erlaubt somit nicht in jedem Fall eine eindeutige Zuordnung der Grenzphänomene.

Zusammenfassend lässt sich sagen, dass in Retrospektive zwar einige Kriterien identifiziert werden können, die die Besonderheiten eines jeden Gegenstandes beschreiben können, aber die Entstehung der Gebiete weniger auf systematischen Überlegungen oder auf einer bewussten Abspaltung zu beruhen, sondern rein pragmatisch erfolgt zu sein scheint. Ich sehe hierbei vor allem die soziopolitischen und wirtschaftlichen Gegebenheiten zu einem be- 


\section{Konzept der Sprachlenkung}

stimmten Zeitpunkt als ausschlaggebend. Sie machten die Konstruktion eines bestimmten (sprachlichen) Gegenstandes (KS, PS oder Terminologien) als Lösung aktueller Probleme prominent und zogen eine systematische Beschäftigung mit genau diesem Gegenstand nach sich. Andere Gegenstände wurden gleichzeitig ausgeblendet. Ich nehme dabei an, dass die ursprünglichen Konstituierungen der drei Gebiete primär nach sprachlichen, insbesondere systemlinguistischen, Charakteristiken der jeweiligen Gegenstände erfolgte, wobei zu Beginn vor allem intuitive und vorwissenschaftliche Gegenstandsauffassungen eine Rolle spielten. Systematische Versuche von Gegenstanddefinitionen erfolgten erst post factum, nach der Etablierung der Gebiete, was m. E. die Schwierigkeiten bei einer präzisen Definition und einer trennscharfen Abgrenzung einzelner Gegenstände und somit Gebiete erklärt.

\subsection{2. Übertragbarkeit der theoretischen Modelle}

In Kapitel 2 stellte ich Konzeptualisierungen und theoretische Modelle einzelner Gebiete vor. Hier sind sie in Tabelle 6 noch einmal nach Gebieten zusammengestellt. Die Modellierungen lassen sich aber auch inhaltlich bündeln und es ergeben sich beispielsweise folgende fünf Schwerpunkte: Sprachsystem, Definition, Klassifikation, Umsetzung und Allgemeines (s. Tabelle 7). Modellierungen, die systemlinguistische Empfehlungen für Eingriffe behandeln, stehen nicht im Fokus dieser Arbeit und ich werte sie im Weiteren nicht aus. Im Unterschied dazu sind verschiedenen Definitionen (der Sprachplanung) für eine übergreifende Perspektive auf Sprachlenkung zwar wichtig, aber ich gehe auf sie erst in einem weiteren Abschnitt in diesem Kapitel ein (3.4.2).

Die unmittelbar folgenden Abschnitte sind also der Auswertung von allgemeinen Modellen (3.2.2.1, 3.2.2.2, 3.2.2.3) und der Umsetzungskonzeptualisierungen (3.2.2.4) gewidmet. Die abschließende Behandlung der Klassifikationen erfolgt mit dem Ziel, weitere relevante Kriterien zur Charakterisierung der Sprachlenkung in Abschnitt 3.4.2 zu finden.

\subsubsection{Korpus- und Statusplanung}

Die Unterscheidung zwischen der Korpus- und der Statusplanung, also entsprechend der Gestaltung der sprachlichen Mittel selbst und der Beeinflussung der Stellung der Sprachmittel in einer bestimmten Sprechergemeinschaft, stammt aus dem LPLP-Gebiet, scheint aber universell und auf die übrigen drei Gebiete übertragbar zu sein. 


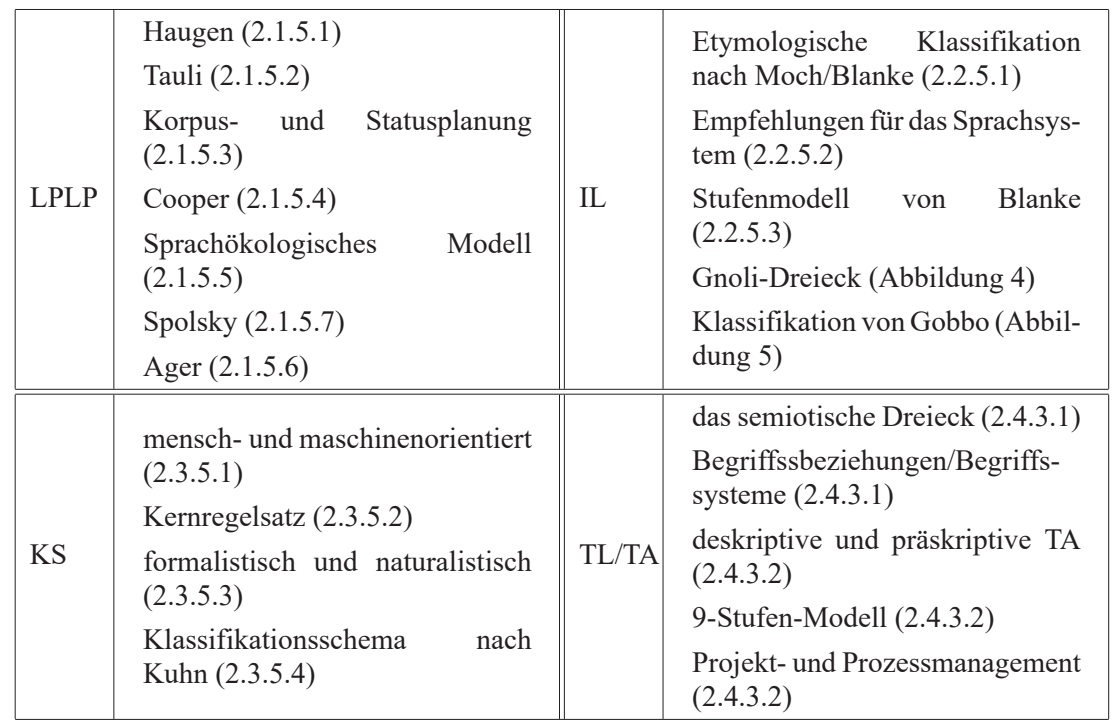

Tabelle 6.: Übersicht der Theorien und Modelle der vier Gebiete 


\begin{tabular}{|c|c|c|c|}
\hline $\begin{array}{l}\text { Sprach- } \\
\text { system }\end{array}$ & $\begin{array}{l}\text { Empfehlungen für das Sprachsys- } \\
\text { tem } \\
\text { das semiotische Dreieck } \\
\text { Begriffsbeziehungen/Begriffs- } \\
\text { system } \\
\text { Kernregelsatz }\end{array}$ & $\begin{array}{l}\text { Defi- } \\
\text { nition }\end{array}$ & $\begin{array}{l}\text { Tauli } \\
\text { Spolsky } \\
\text { Cooper }\end{array}$ \\
\hline $\begin{array}{l}\text { Klas- } \\
\text { sifika- } \\
\text { tion }\end{array}$ & $\begin{array}{l}\text { Moch/Blanke } \\
\text { Gnoli-Dreieck } \\
\text { Gobbo } \\
\text { mensch- und maschinenorientiert } \\
\text { formalistisch und naturalistisch } \\
\text { Kuhn }\end{array}$ & $\begin{array}{l}\text { Um- } \\
\text { set- } \\
\text { zung }\end{array}$ & $\begin{array}{l}\text { Haugen } \\
\text { Stufenmodell Blanke } \\
\text { deskriptive/präskriptive TA } \\
9 \text { Stufen Drewer } \\
\text { Prozess- und Projektmanagement }\end{array}$ \\
\hline $\begin{array}{l}\text { Allge- } \\
\text { meines }\end{array}$ & $\begin{array}{l}\text { Korpus- und Statusplanung } \\
\text { sprachökologisches Modell } \\
\text { Ager }\end{array}$ & & \\
\hline
\end{tabular}

Tabelle 7.: Bündelung der Theorien und Modelle der vier Gebiete

In der Interlinguistik wird die Anwendbarkeit dieser Konzeptualisierung beispielsweise von Tonkin (2011) explizit erörtert. Er zeigt für Esperanto auf, dass von Anfang an Schritte unternommen werden, die der Korpusplanung (Aufbau des Sprachsystems auf allen Ebenen) oder der Statusplanung (aktiver Aufbau der Sprechergemeinschaft, insbesondere durch Übertragung der Verantwortung für die Weiterentwicklung der Sprache auf die Sprecher) zugeordnet werden können (Tonkin 2011, 64-65). Dies schlägt sich auch in der Entstehung von Organisationen nieder, die sich speziell der Korpusplanung (Lingva Komitato) oder der Statusplanung (UEA) widmen. Des Weiteren zeigt Tonkin (2011, 65-67) die Anwendbarkeit der Konzepte Erwerbsplanung und Prestigeplanung zur Beschreibung der Entwicklungen von Esperanto.

Die Unterscheidung zwischen Korpus- und Statusplanung wird in der Terminologiearbeit nur gelegentlich getroffen. Beispielsweise prägt Picht $(2009$, 8) die Bezeichnung Terminologieplanung als einen wesentlichen, fachbezogenen Teil der Sprachplanung. Insbesondere ist die Terminologieplanung dann Bestandteil der Korpusplanung.

Die Unterscheidung zwischen Korpus- und Statusplanung ist nicht nur sinnvoll, um die Terminologiearbeit innerhalb der Sprachplanung einzuordnen, sondern auch, um die Terminologiearbeit selbst zu beschreiben. 
So stelle ich in der Definition der Terminologiearbeit von DIN 2342 (2011) sowohl die Aspekte der Korpusplanung (Planung, Erarbeitung, Bearbeitung der Terminologie) als auch der Statusplanung (Darstellung, Verbreitung der Terminologie) fest. In dieser Hinsicht stellt insbesondere die Terminologielehre die Methoden zur terminologischen Korpusplanung bereit, während die projekt- und prozessbezogenen Methoden der Terminologiearbeit die Statusplanung unterstützen.

Schließlich erscheint eine Übertragung auf Kontrollierte Sprachen ebenfalls möglich: Die Erarbeitung der KS-Regeln fällt unter Korpusplanung, während die Maßnahmen zur Etablierung dieser Regeln, z.B. in einem Unternehmen, als Statusplanung aufgefasst werden können. M.W. wird diese Unterscheidung in der KS-Literatur nicht explizit angewandt. Der Forschungsschwerpunkt scheint jedoch primär auf der Korpusplanung zu liegen, während in den Praxisberichten oft Überlegungen und Beobachtungen zur Umsetzung gemacht werden, was ich als implizite Hinweise auf die Aspekte der Statusplanung deute. Eine systematische Auseinandersetzung mit der Statusplanung scheint in der KS-Forschung vernachlässigt worden zu sein.

Wie aus den Beschreibungen deutlich, ist die Anwendung der Unterscheidung Korpus- und Statusplanung zur differenzierten Betrachtung aller vier Gebiete möglich und sinnvoll. Diese Unterscheidung stellt somit eine Konzeptualisierung zur übergreifenden Beschreibung von Sprachlenkung dar. Angewandt auf diese Arbeit bedeutet dies, dass ich hier vor allem die Aspekte der Statusplanung untersuchen möchte, die sich umsetzungsfördernd auf die Sprachlenkung auswirken.

\subsubsection{Sprachökologisches Modell}

Das sprachökologische Modell, also die Vorstellung, dass alle Sprachen in einem Netz von gegenseitigen Abhängigkeiten zu betrachten sind, entstammt ebenfalls dem LPLP-Gebiet. Seine Stärke liegt in der Beschreibung von Sprachgebräuchen. Wie der Forschungsüberblick gezeigt hat, wird das Modell in den übrigen drei Gebieten nicht explizit benutzt.

Zum einen erlaubt das Modell eine statische Sicht auf den Sprachgebrauch: Beispielsweise kann eine Sprache als Minderheitensprache nur dann fungieren, wenn eine Mehrheitssprache ebenfalls bestimmt ist. Für diese Arbeit ist jedoch interessanter, dass das Modell auch eine dynamische Sicht auf den Sprachgebrauch im Sinne einer herbeigeführten Veränderung ermöglicht: Im Kontext der Sprachplanung sagt das Modell voraus, dass der Eingriff in ei- 


\section{Konzept der Sprachlenkung}

nen bestimmten Sprachgebrauch auch andere Sprachgebräuche beeinflusst. Berücksichtigt man alle vier Gebiete und begreift man die Bereitstellung von Plansprachen, Kontrollierten Sprachen und (normativen) Terminologien als einen neuen Sprachgebrauch, so wird dieser, im Sinne des sprachökologischen Modells, mit dem existierenden Sprachgebrauch konfrontiert und muss sich gegen ihn durchsetzen. So greift die Durchsetzung einer Welthilfssprache in den bereits existierenden Gebrauch anderer (oft nationaler) Sprachen als Mittel internationaler Kommunikation ein; der Verwendung einer Kontrollierten Sprache steht die bereits existierende Verwendung einer vollen natürlichen Sprache oder ggf. einer formalen Sprache gegenüber und die normative Terminologie muss sich gegen die nicht restringierten lexikalischen Gepflogenheiten eines Individuums oder einer Gruppe durchsetzen.

Das sprachökologische Modell kann also zur Beschreibung von allen vier Gebieten eingesetzt werden und ist somit ein universelles Werkzeug zur Konzeptualisierung der Sprachlenkung. Insbesondere liegt sein Schwerpunkt auf der Statusplanung und es hat nicht nur einen beschreibenden, sondern auch einen erklärenden Charakter: Da es keine isolierte Betrachtung einzelner Sprachen erlaubt, führt es zu einem besseren Verständnis von Schwierigkeiten bei der Umsetzung der Sprachlenkung, die auf die Veränderungen der existierenden Sprachpraxis und der Sprachgewohnheiten zurückführbar sind.

\subsubsection{Ager (2001)}

In diesem Abschnitt prüfe ich, ob das Werkzeug zur Erfassung der Motivation bei Sprachplanung ebenfalls auf die Konstruktion von Plansprachen und Kontrollierten Sprachen sowie auf die Terminologiearbeit übertragbar ist. Hierfür beantworte ich vier Fragen, die die drei Gebiete betreffen:

A Lassen sich in den drei Gebieten die Ziele eines Eingriffs ebenfalls unterschiedlichen Abstraktionsstufen zuordnen?

B Lässt sich in den Maßnahmen ebenfalls mindestens ein Identitätsmotiv identifizieren?

C Was sind die betreffenden L1 und L2?

D Sind die für die Sprachplanung definierten Einstellungsvariablen in den anderen drei Gebieten verwendbar? 
A Ager (2001) unterscheidet zwischen drei Arten von Zielen, die als unterschiedliche Abstraktionsstufen aufzufassen sind: ideas, objectives und targets. Ich betrachte hier unterschiedliche sprachliche und außersprachliche Ziele der Autoren (und nicht der Sprecher) exemplarisch. Ich beziehe mich dabei auf die einschlägigen Abschnitte aus Kapitel 2.

Bei Welthilfssprachen, hier am Beispiel von Esperanto, kann die idea direkt aus Ager $(2001,8)$ übernommen werden: Frieden und Harmonie zwischen allen ethnischen Gruppen. Das entsprechende objective könnte dann die Benutzung eines gemeinsamen Kommunikationsmittels sein. Auf der Ebene der unmittelbaren targets siedele ich die eigentliche Entwicklung und Bereitstellung der sprachlichen Ressourcen, ihre Bekanntmachung und den Aufbau der Sprechergemeinschaft an.

Bei einem Unternehmen, das eine präskriptive Terminologie oder eine textorientierte KS entwickelt, könnte es sich um die idea der Steigerung oder des Erhalts der Wettbewerbsfähigkeit handeln. Das entsprechende objective ist die Optimierung der Arbeitsprozesse, was schließlich durch die konkrete Spezifikation der Terminologie oder der KS, ihre Einführung und Verwendungskontrolle oder auch die Bereitstellung der entsprechenden Software als targets realisiert wird.

Auch bei repräsentationsorientierten KS und teilweise philosophischen Sprachen könnte es sich um die letztgenannten objectives und targets handeln, während auf der abstrakten Ebene beispielsweise die Demokratisierung des Zugangs zum Wissen als idea angegeben werden könnte.

Die theoretische Unterscheidung zwischen ideas, objectives und targets ist nachvollziehbar, lässt aber in der Anwendung einen Interpretationsspielraum, was die Platzierung einzelner Ziele auf entsprechenden Ebenen angeht. Es können beispielsweise noch viel konkretere Ziele, wie Herausgabe eines Esperantowörterbuches oder Implementierung eines Reasoners (für eine repräsentationsorientierte KS) angegeben werden, so dass mehr als drei Abstraktionsebenen notwendig wären. Somit sind die hier vorgestellten Zuordnungen der Ziele lediglich Vorschläge. Ich nehme anhand der Beispiele jedoch an, dass es insgesamt für alle Maßnahmen möglich ist, Ziele auf unterschiedlichen Abstraktionsebenen anzugeben. Somit ist dieser Teil des Werkzeugs zur Erfassung der Motivation von Sprachplanung auf alle vier Gebiete anwendbar, auch wenn eine präzisere Handhabung noch entwickelt werden müsste.

B Wie in 2.1.5.6 beschrieben, postuliert Ager (2001) folgende Identitätsmotive: Identität, Ideologie, Image, Unsicherheit, Ungerechtigkeit, Integrati- 


\section{Konzept der Sprachlenkung}

on und Instrumentalität. Anhand einiger Beispiele zeige ich, dass sich diese Identitätsmotive auch in den übrigen drei Bereichen identifizieren lassen.

Aus dem Bereich Plansprachen bieten sich vor allem Welthilfssprachen und insbesondere Esperanto für eine identitätsbezogene Interpretation der Motive an. Ich zeige darüber hinaus, dass auch andere Arten von Plansprachen bei ihrer Konstruktion, Weiterentwicklung und Verbreitung eine identitätsbezogene Interpretation zulassen.

Bei Esperanto können zu verschiedenen Zeitpunkten unterschiedliche Handlungen festgestellt werden, die auf unterschiedliche Identitätsmotive schließen lassen. ${ }^{88}$ Beispielsweise spielte bei der anfänglichen Konstruktion und Verbreitung das Motiv Aufbau der Identität als tolerante, weltoffene Person bzw. Esperantosprecher die zentrale Rolle. Die Auseinandersetzung mit der Ideologiefrage des Esperanto, die Ende des 19. Jh. besonders in Frankreich vorangetrieben wurde, führte zur Entstehung zwei paralleler Strömungen unter Esperantosprechern (ideologisch- vs. instrumentellorientiert, s. Abschnitt 2.2.4.2), was als Identitätsmotiv Ideologie und Image oder aber auch Instrumentalität gesehen werden kann. Durch Konflikte in der Gruppe der Esperantosprecher um die Jahrhundertwende, aber auch durch den sozialen Vergleich mit anderen Gruppen wie Volapük- und Ido-Anhängern wurde die Verteidigung der eigenen Identität notwendig, was sich u. a. in der Festlegung des Sprachkerns in Fundamento niederschlug. Auch die Versuche, Esperanto in internationalen Organisationen und in Bildungssystemen zu verankern, können aus der Identitätsperspektive betrachtet werden als Behalten der Identität oder gar als Kampf gegen die Benachteiligung.

Bei der Konstruktion des Volapük kann ein ähnliches Motiv wie bei Esperanto festgestellt werden: Aufbau der Identität als tolerante und weltoffene Person bzw. Volapük-Sprecher. Die Reformablehnung des Volapük durch Schleyer kann als Verteidigung der bisherigen Identität gedeutet werden, während die umfassende Reform zu Idiom Neutral als Verzweiflung interpretiert werden kann.

Bei der Einführung der Interlingua (IALA) ist das Motiv Instrumentalität dominant, da sie der Verbesserung der Fachkommunikation dienen sollte. Dennoch deutet eine starke und explizite Bindung der Sprache an die zweckrationale Verwendung, wohl auch in Abgrenzung zu anderen WHS und insbesondere zu Esperanto, auch auf die Motive Ideologie und Image hin.

88 Die unteren Ausführungen beruhen auf dem Werk von Forster (1982). 
Schließlich können die Motive bei anderen Plansprachentypen ebenfalls identitätsbezogen interpretiert werden. So kann die Weiterentwicklung von Klingonisch durch den Ausbau des Sprachsystems, die Bereitstellung von Wörterbüchern oder den Aufbau des Klingon Language Institute als Behalten oder gar Stärkung/Ausbau der Identität der Star-Trek-Fans interpretiert werden. Dies sollte aber letztendlich zur Sicherung der materiellen Vorteile durch die Urheber der Sprache führen, was dem Motiv Instrumentalität entspricht. Letzteres ist beispielsweise auch bei der Entwicklung von Sprachen für Computerspiele sichtbar.

Bei der Konstruktion und Einführung von Kontrollierten Sprachen, philosophischen Sprachen sowie bei der Terminologiearbeit überwiegt das Motiv Instrumentalität. Trotzdem sind auch andere Identitätsmotive denkbar: Beispielsweise könnte ein Unternehmen durch eine verständlichere Sprache seine Identität als kundenfreundlich oder qualitätsorientiert behalten oder eine solche aufbauen. Auch das Behalten einer Position als Marktführer durch verbesserte Wettbewerbsfähigkeit, zu der eine Kontrollierte Sprache oder einheitliche Terminologie beiträgt, könnte als Motiv Identität behalten interpretiert werden.

Die beschriebenen Beispiele haben lediglich einen exemplarischen Charakter. Sie zeigen jedoch, dass sich bei Maßnahmen aus allen drei Gebieten mindestens ein Identitätsmotiv finden lässt. So erscheint es m. E. zunächst plausibel, auch diesen Teil des Werkzeugs von Ager (2001) auf alle vier Gebiete der Sprachlenkung anzuwenden. Erst durch eine praktische, flächendeckende Anwendung können ggf. Gegenbeispiele gefunden werden, was über die endgültige Validität des Werkzeugs zur Erfassung der Motivation in allen Ausprägungen der Sprachlenkung entscheiden würde.

Aus diesem Teil der Analyse halte ich für weitere Überlegungen zur Sprachlenkung darüber hinaus die Beobachtung fest, dass sich die Identitätsmotive von Autoren in zwei Gruppen einteilen lassen: Identifikation mit einer Gruppe und Instrumentalität, wie sie für die Motivation von Fremdsprachenlernern von Gardner und Lambert (1959) beschrieben wurden (vgl. Ager 2001, 108-109). Für die Gruppe der Identifikationsmotive benutze ich im Folgenden die Bezeichnung identitätsbezogene Motive/Ziele, um sie von Agers (2001) übergreifendem Konzept der Identitätsmotive zu unterscheiden.

C Die Bezeichnungen L1 und L2 werden konventionell in der Fremdsprachenerwerbs- und Multilingualismusforschung verwendet und 


\section{Konzept der Sprachlenkung}

stehen entsprechend für Erstsprache und Zweitsprache (Barkowski 1995; K. Johnson 2008, 11; vgl. Apeltauer 2006, 11-12, 15-16). Da Agers Beispiele vorwiegend aus diesen Forschungsbereichen stammen, sind die Bezeichnungskonventionen für die Matrix gut geeignet. Demnach bezieht sich L1 auf die Erstsprache der anvisierten Zielgruppe der Sprachplanung, während L2 jede andere Kontrastsprache im Sinne des ökologischen Modells bedeutet. Wie in Abschnitt 3.2.2.2 beschrieben, gilt das sprachökologische Modell auch für KS, PS und normative Terminologien und so gibt es alternative Sprachen (bzw. bei Terminologien: alternative Wortschätze), mit denen sie vernetzt sind. Aus der Perspektive des Sprachgebrauchs bedeutet die Wahl der Bezeichnungen L1/L2 durch Ager aber, dass L1 für Sprachen steht, die von der aktiven Zielgruppe bereits aktiv und bevorzugt gebraucht werden. Möchte man diese Bezeichnungen auf die übrigen drei Gebiete übertragen, so stünde L1 bei der erstmaligen Einführung von KS, PS und Terminologien für nichtkontrollierte Sprachen, nicht restringierte (Fach-)Wortschätze sowie ethnische Sprachen, während für die eigentlichen KS, PS und Terminologien die Bezeichnung L2 verwendet werden müsste. Wie aber aus dem sprachökologischen Modell deutlich wird, werden KS, PS und Terminologien nicht nur mit natürlichen Sprachen, sondern auch mit anderen KS, PS und Terminologien kontrastiert. Zur Erfassung dieser in der Matrix wäre aber weder L1 noch L2 zutreffend.

Ein weiteres Problem tritt auf, sobald die neu eingeführte Sprache im Gebrauch etabliert ist und somit eigentlich in einer bestimmten Verwendungssituation zur Erstsprache wird (z. B. eine KS zur Erfassung von Gebrauchsanleitungen) und folglich als L1 in der Matrix erfasst werden müsste.

Diese aktuelle Bezeichnungskonvention in der Matrix ist ein verhältnismäßig geringes und theoretisches Problem, das aber praktische Konsequenzen hat. Einerseits leidet die Einheitlichkeit bei der Datenerfassung, wenn in der Untersuchung mehrere Sprachlenkungsgebiete gleichzeitig berücksichtigt werden sollten. Andererseits leidet dadurch auch die Rezeption der Matrizen, insbesondere ihre Nachvollziehbarkeit, wenn für jeden Datensatz aufs Neue geprüft werden muss, wie L1 und L2 gebraucht wurden.

Zusammenfassend lässt sich sagen, dass der Kontrast der betreffenden Sprache mit den vernetzten Sprachen im Sinne des sprachökologischen Modells für alle vier Gebiete prinzipiell möglich ist und auch in den übrigen drei Gebieten neue Erkenntnisse und ein besseres Verständnis der Einstellungsstruktur erbringen kann. Eine bessere Definition der Matrixspalten, die übergreifend auf alle Gebiete zutrifft und möglicherweise 
den Verzicht auf die Bezeichnungen L1 und L2 bedeuten würde, würde aber die Handhabbarkeit der Matrix verbessern.

D In jedem der drei Bereiche wurden bereits empirische Untersuchungen durchgeführt, die (u. a.) Einstellungen erfassen, obwohl sie nicht unbedingt die Aufteilung in die kognitive, affektive und konative Komponenten berücksichtigen (beispielsweise Forster 1982; Stocker 2002; Schmitz und Straub 2010; Lewark und Turhan 2013). Es ist naheliegend, die Dreiteilung in allen vier Gebieten zu berücksichtigen. Offensichtlich können die Variablen Attractiveness und Action ohne Weiteres übernommen werden, insbesondere für Untersuchungen, die mehrere Gebiete gleichzeitig umfassen.

Bei der kognitiven Komponente müssen hingegen ein paar Besonderheiten berücksichtigt werden, die sich aus der intentionalen Beschränkung mancher Eingriffe auf einzelne Domänen (insb. Fachsprachen) ergeben:

- Zu erwarten ist eine niedrigere Vitalität einer Kontrollierten Sprache oder einer Terminologie im Vergleich zu einer (allgemeinsprachlichen) Plansprache, was insbesondere bei vergleichenden Untersuchungen beachtet werden muss.

- Aus einem ähnlichen Grund muss bei der Variable Excellence bedacht werden, dass die Erfassung des ästhetischen oder literarischen Wertes von KS, Terminologien oder auf einen fachsprachlichen Einsatz beschränkten PS nicht sinnvoll ist.

- Bei allen Variablen muss darüber hinaus auf die genaue Formulierung der Items geachtet werden, da bei Terminologiearbeit kein vollständiges Sprachsystem betroffen ist.

Auch dieser Teil des Werkzeugs - die Erfassung der Spracheinstellungen - erscheint in Verbindung mit dem sprachökologischen Ansatz auf alle Gebiete der Sprachlenkung anwendbar zu sein, wenn auch kleine Anpassungen notwendig sind.

Insgesamt ergibt die Analyse, dass Agers (2001) Instrument mit kleinen Einschränkungen durchaus übergreifend zur Beschreibungung der Motivation bei Sprachlenkung eingesetzt werden kann. Darüber hinaus halte ich für diese Arbeit zwei weitere Konzeptualisierungen fest: die Unterscheidung zwischen identitätsbezogenen und instrumentellen Motiven/Zielen sowie eine hierarchische Struktur von Sprachlenkungszielen, wobei sprachliche Ziele in übergreifende, außersprachliche Ziele eingebettet sind. 


\subsubsection{Umsetzungsmodelle: Haugen, 9 Stufen nach Drewer und Ziegler (2011), Projekt-/Prozessmanagement (TA) und Blankes Stufenmodell (PS)}

In diesem Abschnitt vergleiche ich einzelne Umsetzungskonzeptualisierungen und zeige, dass das Modell von Haugen und das Modell für Projektmanagement übergreifende Konzeptualisierungen darstellen.

Haugens Modell umfasst vier Schritte der Sprachplanung als Prozess: Selektion, Kodifikation, Implementation und Elaboration. Offensichtlich lassen sich sowohl die 19 Entwicklungsstufen einer Plansprache nach D. Blanke (2006) als auch die 9 Stufen der Terminologiearbeit (im Unternehmen) nach Drewer und Ziegler (2011) darunter vollständig subsumieren, wie in Tabelle 8 dargestellt.

Wie aus Tabelle 8 leicht zu erkennen ist, liegt der Schwerpunkt des Modells von Drewer und Ziegler (2011) auf der Kodifikation (insb. Korpusentwicklung), während die Stufen nach D. Blanke (2006) die Implementierungs- und Elaborierungsmöglichkeiten detailliert wiedergeben. Darüber hinaus stelle ich fest, dass die Stufen in Blankes Modell, die der Phase Implementation zugeordnet werden, mit den Stufen in der Phase der Elaboration verflochten und zudem nicht immer eindeutig zuzuordnen sind. Dies ist ein Hinweis darauf, dass die Phasen in Haugens Modell in der Praxis nicht konsekutiv, sondern zeitgleich ablaufen, ähnlich wie bereits bei den Schritten der Terminologiearbeit in Abschnitt 2.4.3.2 beobachtet.

Das Projektmanagement nach ISO 15188 (2001) lässt sich zwar in Haugens Modell nicht auf eine ähnliche Weise vollständig integrieren, beide Konzeptualisierungen weisen aber wesentliche Parallelen auf: Die projektbezogenen Phasen Vorbereitung und Entwurf entsprechend weitestgehend der Selektion, wobei hier vor allem die nichtsprachlichen Aspekte bestimmt werden. Die projektbezogene Phase Umsetzung umfasst sowohl den Aufbau des Terminologiebestandes als auch seine Verbreitung und entspricht daher sowohl Kodifikation als auch Implementation aus Haugens Modell. Die laufende Sprachpflege, also Elaboration, wird durch Projektmanagement hingegen nicht abgedeckt, da dies gewöhnlicherweise Bestandteil des Prozessmanagements nach dem Projektabschluss im Sinne von Abschnitt 2.4.3.2 ist. Demgegenüber geht Haugens Modell nicht auf die Aspekte der Evaluation ein, was aber für LPLP gefordert wird (Abschnitt 2.1.7.1). Dieser Punkt wird im Pro-

89 Die mit „,“" gekennzeichneten Stufen lassen sich auch der Stufe Elaboration zuordnen. 


\begin{tabular}{|c|c|c|}
\hline Phase nach Haugen & $\begin{array}{l}\text { Schritt nach Drewer und } \\
\text { Ziegler (2011) }\end{array}$ & Stufe nach D. Blanke (2006) \\
\hline Selektion & "Zielsetzung und Planung & - \\
\hline Kodifikation & $\begin{array}{l}\text { Gewinnung der Terminologie } \\
\text { Begriffliche Systematisie- } \\
\text { rung der Terminologie } \\
\text { Sprachliche Bewertung und } \\
\text { Bereinigung der Terminolo- } \\
\text { gie } \\
\text { Generieren neuer Benennun- } \\
\text { gen }\end{array}$ & Manuskript \\
\hline Implementation & $\begin{array}{l}\text { Verwaltung der Terminologie } \\
\text { Darstellung der Terminologie } \\
\text { Kontrolle der Terminologie- } \\
\text { verwendung }\end{array}$ & $\begin{array}{l}\text { Veröffentlichung } \\
\text { Lehrmittel } \\
\text { Werbung } \\
\text { Zeitschriften } \\
\text { Korrespondenz*89 } \\
\text { mündliche Kommunikation* } \\
\text { Organisationen* } \\
\text { Zunahme der Textproduktion* } \\
\text { private Kurse } \\
\text { kleine Sprachgemeinschaft } \\
\text { Veranstaltungen* } \\
\text { Großveranstaltungen } \\
\text { weltweite Verbreitung } \\
\text { staatlicher Unterricht } \\
\text { elektronische Medien } \\
\text { soziale Differenzierung der } \\
\text { Sprachgemeinschaft* }\end{array}$ \\
\hline Elaboration & Pflege der Terminologie & $\begin{array}{l}\text { Übersetzung und Originaltexte } \\
\text { Diskussion sprachlicher Fragen } \\
\text { Fachkommunikation } \\
\text { strukturelle Differenzierung der } \\
\text { Sprachgemeinschaft } \\
\text { Herausbildung/Stabilisierung/ } \\
\text { Kodifizierung der Norm } \\
\text { Interlinguistik } \\
\text { heuristische Wirkung } \\
\text { externe Nutzung } \\
\text { Familiensprache } \\
\text { Originalkultur } \\
\text { Sprachwandel }\end{array}$ \\
\hline
\end{tabular}

Tabelle 8.: Haugens Modell als übergreifende Konzeptualisierung für Modelle von D. Blanke (2006) und Drewer und Ziegler (2011) 


\section{Konzept der Sprachlenkung}

jektmanagement aufgegriffen, ebenfalls wie die formalen Aspekte des Projektabschlusses.

Der Forschungsüberblick in Kapitel 2 ergibt, dass im Unterschied zu Plansprachen und Terminologiearbeit keine Umsetzungskonzeptualisierung für den Bereich Kontrollierte Sprachen existiert. Auch hier lässt sich jedoch Haugens Modellierung im Allgemeinen anwenden, wie ich anhand von Projektbeispielen im Folgenden zeige.

Im Projektbericht zu Caterpillar Technical English (CTE) von Kamprath u. a. (1998) finden sich Beschreibungen, die sich einzelnen Phasen in Haugens Modell zuordnen lassen: Kodifikation: Extraktion, Validierung und Festlegung von Termen zum Aufbau des KS-Lexikons, Definition von Grammatikregeln, Implementation des Redaktionssystems und des Maschinellen Übersetzungssystems KANT sowie Neudefinition der Arbeitsprozesse (Kamprath u. a. 1998, 4-5); Implementation: Vorbereitungsseminare bereits ein Jahr vor der eigentlichen Einführung des CTE, eigentliche Schulungen nach der Einführung (Kamprath u. a. 1998, 6); Elaboration: Weiterentwicklung der Terminologie und der grammatischen Regeln, Aufteilung der Terminologie in mehrere Domänen, um die automatische Prüfung zu erleichtern; Wartung und Weiterentwicklung der Software, regelmäßige Updates der Schulungsmaterialien und des Redaktionsleitfadens (Kamprath u. a. 1998, 6, 8-9). Auf Selektion geht hingegen Gallup (1993, 422) ein und nennt in diesem Zusammenhang die Analyse der aktuellen Entwicklungen in der Sprachverarbeitung durch Seminar- oder Messebesuche sowie die Auswahl eines geeigneten Kooperationspartners (Carnegie Group Inc. sowie die Carnegie Mellon University). Das Modell von Haugen lässt sich ähnlich zur Beschreibung der Entwicklungen bei General Motors anwenden (s. L. Means und Godden 1996; L. G. Means, Chapman und Liu 2000).

Anders gestaltet sich die Situation, wenn Haugens Modell zur Beschreibung der KS bei der Koenig \& Bauer AG (KBA) verwendet wird. Wie in Suchowolec (2014) beschrieben, wurde kein KS-spezifisches Projekt durchgeführt und die Kontrollierte Sprache bei KBA ist das Resultat mehrerer Projekte, insbesondere zur Einführung eines Content Management Systems (CMS) und zur Einführung der Terminologiearbeit, die nicht primär die Entwicklung einer $\mathrm{KS}$ zum Ziel hatten. Insbesondere sind die morphosyntaktischen Regeln das Nebenprodukt des CMS-Projektes, während die Ergebnisse des Terminologieprojektes als KS-Lexikon aufgefasst werden. Man kann also nicht von einer systematischen, KS-bezogenen Zielfestlegung und einer planvollen Entwicklung der 
Sprachgebrauchsveränderung im Sinne von Selektion nach Haugen sprechen. ${ }^{90}$ Mit anderen Worten bleibt die Selektion bei der Entstehung der gesamten KS unbesetzt. Auch die Festlegung der morphosyntaktischen Regeln, also die Kodifikation, erfolgte recht unsystematisch und beruhte nicht auf einer linguistischen Analyse, sondern einer eklektischen Auswahl aus Standardbeispielen der PI-Mod-Spezifikation ${ }^{91}$ aus den mündlichen Absprachen unter den Technischen Redakteuren und aus der sog. guten Praxis der Technischen Dokumentation. Der Aufbau des Lexikons erfolgte hingegen unter Einsatz der im Voraus entwickelten Wortbildungsregeln und der Datenbankspezifikation. Die Implementation, also die praktische Umsetzung der KS, erfolgte mit dem produktiven Einsatz des Redaktionssystems und des CMS. Sie war begleitet durch KS-bezogene Arbeitstreffen, in denen die Technischen Redakteure die neu erstellten Texte besprachen und Formulierungsprobleme diskutierten. Die Implementierung wurde darüber hinaus durch einen Terminologieleitfaden und einen Redaktionsleitfaden unterstützt, der u. a. Formulierungsbeispiele für bestimmte Textbausteine enthielt. Zur Elaboration zähle ich die Änderungen der KS-Spezifikation. Diese Änderungen ergaben sich zum einen durch die o.g. Diskussionsrunden, zum anderen waren sie die Folge einer maschinenlesbaren Implementation der ursprünglich in Textform vorliegenden Regeln, um die Einführung eines Controlled Language Checkers zu ermöglichen. Auch Terminologie wurde weiterentwickelt, wozu ein neues Werkzeug bereitgestellt wurde, das es den Nutzern leichter machte, Änderungen in existierender Terminologie oder neue Terme vorzuschlagen.

Als Letztes betrachte ich die repräsentationsorientierte KS Attempto Controlled English $(A C E)$. Im Unterschied zu den oberen Beispielen wurde ACE nicht für eine konkrete industrielle Anwendung (im Auftrag eines Unternehmens oder einer Branche) entwickelt, sondern stammt aus dem wissenschaftlichen Bereich. Trotzdem lässt sich seine Entwicklung ebenfalls mit Haugens Modell beschreiben, wenn auch die Belegung einzelner Phasen teilweise anders als in den oben besprochenen Beispielen ist.

In seiner ursprünglichen Form sollte ACE als KS zur Verfassung von Texten dienen, die für Softwareentwickler spezifizieren, welche Funktionalitäten eine Software besitzen soll (sog. Softwarespezifikation). ACE sollte also sowohl für Menschen leichter verständlich (als eine formale Spezifikationsspra-

90 Allerdings können aller vier Phasen von Haugen zur Beschreibung des Terminologieprojektes allein vollständig eingesetzt werden. Dies würde aber nur einen Teilaspekt der KS-Entstehung beschreiben.

91 PI-Mod ist ein Informationsmodell zur semantischen Strukturierung und modularen Erfassung von Textinhalten, das vor allem im Maschinen- und Anlagenbau verwendet wird (s. PI-Mod 2015-). 


\section{Konzept der Sprachlenkung}

che), als auch maschinenlesbar sein. Eine ausführliche Beschreibung, auf die sich die vorangegangenen und folgende Beschreibungen stützen, ist Schwitter (1998) zu entnehmen.

Als Phase der Selektion sehe ich die Analyse der aktuellen Praxis der Softwarespezifikation, das Abwägen der Vor- und Nachteile der natürlichen und formalen Sprachen in diesem Kontext, was zur Formulierung der Anforderungen an eine Spezifikationssprache führte (Schwitter 1998, Kap. 2, 3). Die Konkretisierung des Sprachsystems von ACE, also die Phase der Kodifikation, erfolgte auf Grundlage einer Analyse der existierenden KS und umfasste eine detaillierte Spezifikation der KS in Kap. 4. Die erste praktische Umsetzung der KS, als Teil der Implementation, bestand in der Verfassung des sog. Bibliothekproblems in ACE (Schwitter 1998, Kap. 5). Zu dieser Phase zähle ich darüber hinaus eine Reihe von wissenschaftlichen Aufsätzen, die ACE besprachen und nach 1998 erschienen (für eine Übersicht s. Attempto Project 2015-, >Publications). Die Phase der Elaboration umfasste anfänglich die sprachliche Weiterentwicklung der ursprünglichen KS-Spezifikation, z. B. durch Behandlung der vorher nicht berücksichtigten Phänomene, wie der Pluralsemantik (Schwertel 2000). Es wurden dann auch aktualisierte ACELeitfäden bereitgestellt (bspw. Fuchs, Schwertel und Schwitter 1999). Zur Elaboration des ursprünglichen ACE zähle ich darüber hinaus die Entwicklung verschiedener ACE-Dialekte wie AceRules, AceWiki oder OWL ACE (s. Attempto Project 2015-, > Tools \& Resources), was sowohl die sprachliche Spezifikation als auch die technische Implementierung umfasste. Auch diese Dialekte stammen aus dem wissenschaftlichen Bereich. Obwohl sie aus der Sicht des ursprünglichen ACE zur Phase der Elaboration gehörten, kann die Entwicklung jeder dieser Dialekte wieder mithilfe Haugens Modell beschrieben werden, wobei sich die einzelnen Phasen ähnlich wie bei ACE gestalten. ${ }^{92}$

Aus den bisherigen Beschreibungen ergibt sich, dass Haugens Konzeptualisierung zu allgemeinen Beschreibungen der Umsetzungsphasen aller vier Gebiete weitestgehend eingesetzt werden kann. So können die Konzeptualisierungen von Drewer und Ziegler (2011) und D. Blanke (2006) als Spezifizierungen des Modells von Haugen für bestimmte Bereiche gesehen werden. Selbstverständlich sind sie in einer nicht geänderten Form nicht auf ande-

92 Selektion: (wissenschaftliche) Analyse der Ist-Situation für eine bestimmte Anwendung und die Spezifikation entsprechender Anforderungen; Kodifikation: Entwicklung des sprachlichen Regelwerkes und die Implementierung der Werkzeuge; Implementation: Umsetzung eines praktischen Beispiels zu Demonstrationszwecken; Elaboration: wissenschaftliche Publikationen, mehr Umsetzungsbeispiele, sprachliche und technische Weiterentwicklung (s. exemplarisch Kuhn 2010b). 
re Gebiete übertragbar, allein schon durch gebietsspezifische Formulierungen. Die 9 Schritte nach Drewer und Ziegler (2011) lassen sich durch leichte sprachliche und inhaltliche Änderungen beispielsweise wie folgt umformulieren:

1. Zielsetzung und Planung

2. Gewinnung des zu verändernden Materials

3. Bewertung, Bereinigung und Systematisierung des gewonnenen Materials

4. Festlegung neuer Sprachgebrauchsregeln aus dem vorhandenen Material

5. Entwicklung fehlender Regeln

6. Verwaltung der neuen Sprachgebrauchsregeln

7. Darstellung und Verbreitung des neuen Sprachgebrauchs

8. Pflege der Sprachgebrauchsregeln

9. Kontrolle des neuen Sprachgebrauchs

Auch die Konzeptualisierung von D. Blanke (2006) bedürfte einer ähnlichen sprachlichen und inhaltlichen Verallgemeinerung, um die Übertragbarkeit auf andere Gebiete zu prüfen. Im Unterschied zu den 9 Schritten nach Drewer und Ziegler (2011), die zu einem weit verbreiteten Vorgehensstandard für Terminologieprojekte in der Industrie geworden sind, ist jedoch für die Konzeptualisierung von D. Blanke (2006) unklar, ob sie sich zunächst einmal auf alle Plansprachentypen verallgemeinern lässt, da sie sich stark am (exemplarischen) Verlauf der Esperantoentwicklungen orientiert. Eine weitere Einschränkung der Allgemeinheit sehe ich zudem darin, dass sie vor allem für Maßnahmen geeignet ist, die mehrere Bereiche des öffentlichen Lebens und, linguistisch gesehen, alle Domänen des Sprachgebrauchs abdecken. So sind einige Stufen, beispielsweise Zeitschriften, mündliche Kommunikation, Familiensprache oder Originalkultur im fachsprachlichen Kontext irrelevant.

Eine ausführliche Übertragbarkeitsanalyse beider Konzeptualisierungen wird in dieser Arbeit nicht durchgeführt. Die einzelnen Elemente der Modelle werden in der Untersuchung der erfolgsfördernden Faktoren 


\section{Konzept der Sprachlenkung}

in Kapitel 5 behandelt und fließen auf diese Weise in die übergreifende Perspektive auf die Sprachlenkung mit ein.

Als Zusammenfassung halte ich fest, dass Haugens Modell zur Umsetzungskonzeptualisierung in allen vier Bereichen einsetzbar ist. Zum einen lassen sich damit reale Projekte retrospektiv beschreiben, wie am Beispiel von KS deutlich wurde. Hierbei ist zu beachten, dass nicht jede Phase aus Haugens Modell bei dieser Art Beschreibung besetzt sein muss und zudem nicht alle Handlungen einer einzigen Phase eindeutig zuordenbar sind. Dies bestätigt, dass es sich bei Haugens Modell um eine idealtypische Konzeptualisierung handelt. Sie eignet sich jedoch gut, um nachträgliche Verlaufsbeschreibungen existierender Maßnahmen zu systematisieren.

Des Weiteren habe ich gezeigt, dass sich spezielle Umsetzungskonzeptualisierugen der Terminologiearbeit und Plansprachenkonstruktion unter Haugens Modell subsumieren lassen. Dabei sind jedoch die unterschiedlichen Zielsetzungen zu beachten, denn das Stufenmodell von D. Blanke (2006) und vor allem die 9 Schritte nach Drewer und Ziegler (2011) sollen als Referenz bei der Entwicklung neuer Maßnahmen dienen und somit einen empfehlenden Charakter haben. Die problemlose Integration in Haugens Modell deutet jedoch darauf hin, dass es nicht nur für retrospektive Beschreibungen, sondern durchaus als Anhaltspunkt zur Entwicklung künftiger Maßnahmen eingesetzt werden kann, wenn eine systematische Lösung angestrebt wird. Eine zu diesem Zweck alternative Konzeptualisierung auf einer hohen Abstraktionsebene bieten die fünf Phasen des Projektmanagements für Terminologiearbeit nach ISO 15188 (2001). Parallel zu den in Abschnitt 2.4.3 festgestellten Unterschieden zwischen den 9 Schritten und dem Projektmanagement sehe ich auch den Schwerpunkt von Haugens Modell in der linguistischen Perspektive, während das Projektmanagement eher organisatorische, betriebliche und/oder wirtschaftliche Umsetzungsaspekte in den Vordergrund stellt. Somit sind beide Konzeptualisierungen nicht widersprüchlich, sondern beleuchten zum Teil unterschiedliche Facetten der Umsetzung. Ihr Einsatz, sowohl zur Beschreibung als auch Entwicklung von Projekten ist somit situations- aber auch adressatenspezifisch: In manchen Fällen eignet sich die Projektsicht weniger gut, vor allem dort, wo eine Maßnahme in keinem formellen Rahmen erfolgt und beispielsweise eine Privatinitiative ist. Im industriellen Kontext kann die Projektperspektive allerdings bevorzugt werden.

Um eine wirkliche Hilfestellung für Praktiker zu sein, sind das Modell von Haugen und die grobe Projektkonzeptualisierung jedoch zu allgemein und müssen mit konkreten Vorgehensvorschlägen gefüllt werden, so wie dies für 
TA in den 9 Schritten oder der detaillierten Ausarbeitung des Projektmanagements in ISO 15188 (2001) und für PS im Stufenmodell geschieht. Eine Verallgemeinerung und Übertragung dieser speziellen Konzeptualisierung wird in dieser Arbeit jedoch nicht direkt angestrebt. Sie erfolgt indirekt in der Untersuchung in Kapitel 5.

Angewandt auf dieses Ziel dieser Arbeit halte ich aus den oberen Ausführungen fest, dass ich in erster Linie an Vorgehen in der Implementations- und Elaborationsphase interessiert bin und die Phasen der Selektion und der Kodifikation in den Hintergrund treten.

\subsubsection{Klassifikationen}

Das Ziel dieses Abschnittes ist keine Übertragung einzelner Klassifikationen auf andere Gebiete, sondern die Identifikation weiterer Aspekte, mit deren Hilfe Sprachlenkung charakterisiert werden kann.

Bei der etymologischen Klassifikation von Plansprachen von D. Blanke (1985) in Anlehnung an Moch handelt es sich um eine Taxonomie, in der jede Plansprache genau einer Klasse zugeordnet ist. Das Hauptunterteilungskriterium ist dabei die Herkunft des sprachlichen Materials bzw. die Nähe zu einer bestehenden ethnischen Sprache. Eine Verallgemeinerung dieses Kriteriums zur Beschreibung verschiedener Sprachlenkungsmaßnahmen in den in dieser Arbeit behandelten Gebieten schlägt Schubert (2011a) (vgl. Schubert 2009, 2014) vor, was ich in Abschnitt 3.3.2 bespreche.

Benutzt man andere Unterteilungskriterien, wie das Ziel bzw. die Funktion eines Eingriffs in die Sprachmittel, so stößt man an die Grenzen einer Taxonomie zu Beschreibungszwecken, da ein Eingriff unterschiedliche Ziele gleichzeitig verfolgen kann, wie in entsprechenden Abschnitten in Kapitel 2 dargelegt wurde. Anstatt einen Eingriff einer einzigen Klasse zuzuordnen wird daher zunehmend der Ansatz gewählt, dem Eingriff bestimmte Eigenschaften zuzuordnen. Mit dem Gnoli-Dreieck werden jeder Plansprache drei Ausprägungen der Eigenschaft Funktion (WHS, philosophische Sprache, künstlerische Sprache) je nach Stärke zugeordnet. Eine Steigerung dieses Prinzips sehen wir bei Gobbo (2014), der zwei Eigenschaften (Funktion und Grad der Öffentlichkeit) mit jeweils zwei Ausprägungen gleichzeitig verwendet. In beiden Fällen bilden die Ausprägungen einer Eigenschaft die Extrema eines Kontinuums.

Auch im Bereich Kontrollierte Sprachen wurden eindeutige Klassifikationen, beispielsweise als mensch- oder maschinenorientierte bzw. naturalistische oder formalistische KS, zugunsten einer Kontinuumauffassung aufge- 


\section{Konzept der Sprachlenkung}

geben. Zunehmend setzt sich auch die Klassifikation von Kuhn (2014) durch, die ebenfalls eine Zuordnung mehrerer Eigenschaften mit mehreren Ausprägungen zu einer KS erlaubt. Im Unterschied zu PS handelt es sich bei den Ausprägungen einer Eigenschaft nicht ausschließlich um Extrema eines Kontinuums, sondern auch teilweise um verschiedene Abstraktionsebenen, wie in Abschnitt 2.3.5.4 und Suchowolec (o.D.) diskutiert. Im beschreibenden Teil der Klassifikation von Kuhn (2014) werden folgende Eigenschaften verwendet: Funktion, Modus, Domänenspezifik und Ursprungsdomäne. Der PENSTeil der Klassifikation verwendet zusätzlich die Eigenschaften Präzision, Expressivität, Natürlichkeit und Simplizität.

Aus den Ausführungen halte ich fest, dass die Charakterisierung des Gegenstandes Sprachlenkung in seiner Gesamtheit vorzugsweise nicht anhand einer Taxonomie zu erfolgen hat, weil sie sich in den einzelnen Gebieten nicht bewährt hat und bei Berücksichtigung mancher Eigenschaften wie Funktion nicht praktikabel ist. Die Beschreibung der Sprachlenkung soll zudem anhand mehrerer Eigenschaften erfolgen, um ein vollständigeres Bild zu ergeben. Neben den in Abschnitt 3.2.1 identifizierten Konstituierungs- und Abgrenzungseigenschaften:

- Ebenen des Sprachsystems,

- Soziolekt (auch: Etymologie, Natürlichkeit),

- Funktionolekt (auch: Domänenspezifik),

- Umfang und Verbindlichkeit

entnehme ich den Klassifikationen darüber hinaus die Eigenschaften:

- Funktion,

- Modus,

- Ursprungsdomäne

anhand derer ich die Charakterisierung des Gegenstandes Sprachlenkung im nachfolgenden Abschnitt 3.4.1 und Abschnitt 3.4.2 vornehme. 


\subsection{Weitere übergreifende Ansätze}

Die bisherigen Ausführungen beziehen sich auf die vier hier betrachteten Gebiete, die sich in der Forschung weitestgehend als selbstständig etabliert haben. So gehören die besprochenen theoretischen Modellierungsansätze zu einem der vier Gebiete, wobei die meisten übergreifenden von $L P L P$ bereitgestellt werden. In der Forschung existieren jedoch weitere übergreifende Betrachtungen, die entweder keinem der vier Gebiete direkt entstammen oder umgekehrt in keinem rezipiert zu sein scheinen.

In diesem Abschnitt beschreibe ich zwei Ansätze, deren Ursprung ich grob der Fachkommunikationsforschung zuordne: Sprachlenkung nach Ischreyt (1965) und Kommunikationsoptimierung nach Schubert (2003, 2009, 2011a, 2014).

\subsubsection{Sprachlenkung nach Ischreyt (1965)}

In seinem Buch untersucht Ischreyt (1965) institutionelle Terminologienormung und bettet diese zum Schluss auch in einen größeren Kontext der Sprachlenkung ein (Ischreyt 1965, 259-280). Sein Forschungsüberblick ergibt, dass Sprachlenkung (und synonym dazu auch Sprachregelung) in der Literatur zwar thematisiert wird, aber noch kein fest umrissenes Gebiet darstellt. Darüber hinaus wird Sprachlenkung oft als Instrument der Propaganda und beispielsweise staatlicher Einflussnahme negativ konnotiert. Das Ziel von Ischreyt (1965) ist daher, eine neutrale und sachliche Beschreibung der Sprachlenkung zu ermöglichen (Ischreyt 1965, 259-260).

Sprachlenkung ist demnach ,(...) eine bewußte Tätigkeit (...), die in die Entwicklung der Sprache eingreift, um sie zu beeinflussen und über einen bestimmten Weg zu lenken“" (Ischreyt 1965, 260). Sprachlenkung wird also als eine bewusste, zielgerichtete sowie beabsichtigte Handlung charakterisiert, die zudem auf Sprache und nichts anderes gerichtet ist (Ischreyt 1965, 260). Dadurch wird sie vom Sprachwandel abgesetzt. Der Sprachwandel verläuft hingegen spontan und anonym, da erst im Nachhinein Einzelhandlungen rekonstruierten werden können, die in tatsächlichen Sprachwandel resultieren (Ischreyt 1965, 260-261).

Zur Charakterisierung der Sprachlenkung übernimmt Ischreyt (1965, 261-262) weitestgehend Beschreibungskategorien von Betz (1960). Betz $(1960,99)$ unterscheidet zwischen folgenden Sprachlenkungsformen (vgl. dazu Abbildung 11): 


\section{Konzept der Sprachlenkung}

- allgemein oder individuell (mit Ischreyt - Lenkungsumfang); die erste bezieht sich auf das Sprachsystem und ich lese die zweite als Lenkung des Sprachgebrauchs.

- inhaltlich oder formal (mit Ischreyt - Lenkungsgegenstand); hier sind entsprechend die Inhaltsseite und die Formseite des sprachlichen Zeichens gemeint. Die Inhaltsseite kann entweder die denotative (Begriffsinhalt) oder die konnotative Bedeutung (Nebensinn) betreffen; zudem wird noch die affektive Bedeutung (Gefühlswert) unterschieden.

- Sprachlenkung als Lenkung der Sprache selbst oder als Lenkung der Menschen durch die Sprache (mit Ischreyt - Lenkungsziel) (Betz 1960, 99; Ischreyt 1965, 262, 263); Ischreyt $(1965,262)$ merkt dazu an, dass die beiden Lenkungsziele anhand unterschiedlicher Kriterien gebildet werden und deshalb nicht gleichwertig sind. In Abbildung 11 werden diese Ziele entsprechend Spracherhaltung (konservativ) und Manipulation (transformativ) genannt, wobei Ischreyt $(1965,262)$ auch von weiteren Zielen ausgeht und insbesondere die Kommunikationsverbesserung nennt.

- Ischreyt $(1965,262)$ erweitert die beschriebenen Formen um die Lenkungsstruktur: pluralistisch oder totalitär, bei der es sich um den Autoritätsgrad des Autors bei der Durchführung der Sprachlenkung handelt.

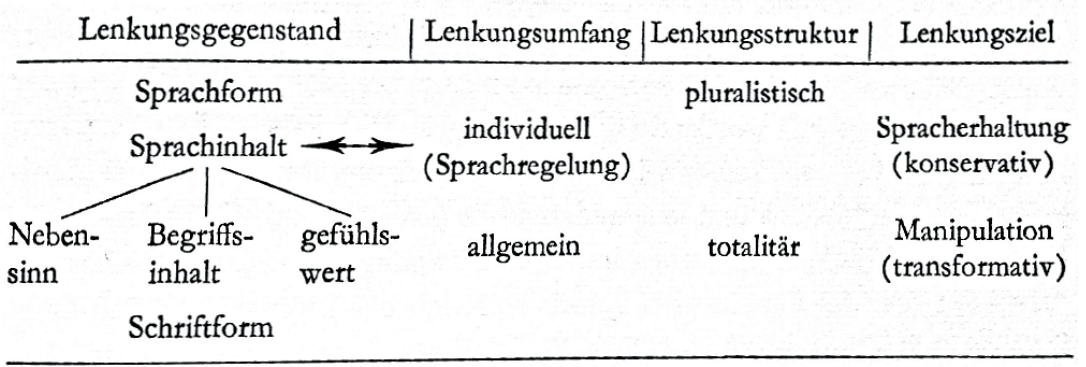

Abbildung 11.: Sprachlenkungsformen nach Ischreyt $(1965,263)$

Für Ischreyt $(1965,262,263)$ ergeben sich daraus Kombinationen wie allgemeine formale Sprachlenkung, allgemeine inhaltliche Sprachlenkung etc. Eine besondere Form der Sprachlenkung ist außerdem die individuelle inhaltliche Sprachlenkung, die er mit Sprachregelung bezeichnet. 
Schließlich geht Ischreyt (1965) auf Sprachlenkungsbeispiele ein, insbesondere im Bereich der institutionellen Terminologienormung (Ischreyt 1965, 269-272), der Sprachpflege (Ischreyt 1965, 272-275) sowie der politischen Sprachlenkung (Ischreyt 1965, 275-280).

\subsubsection{Kommunikationsoptimierung nach Schubert (2003, 2009, 2011a, 2014)}

Schubert (2003, 2009, 2011a, 2014) beschäftigt sich in seinen Aufsätzen mit Kommunikationsoptimierung. Da es sich um einen Gegenstand handelt, der im fachkommunikativen Kontext zwar immer wieder erwähnt wird, aber noch nicht ausreichend präzisiert und systematisiert ist (Schubert 2009, 116-177, 2011a, 366), geht es zunächst einmal um seine theoretische Modellierung, um auf deren Grundlage eine umfangreiche Erforschung zu ermöglichen.

Die Arbeitsdefinition der Kommunikationsoptimierung lautet: „Kommunikationsoptimierung ist bewusstes Eingreifen in das kommunikative Handeln, das mit dem Ziel vorgenommen wird, die Kommunikation im Hinblick auf bestimmte Merkmale zu verbessern. Die Kommunikationsoptimierung kann durch Einwirken auf das kommunikative Handeln selbst oder durch Einwirken auf die Kommunikationsmittel und damit mittelbar auf das kommunikative Handeln erfolgen“" (Schubert 2009, 110; vgl. Schubert 2011a, 366, 2014, 214).

Das zentrale Element der Definition ist das bewusste Eingreifen und es erhält auch eine eigene Definition: „Bewusstes Eingreifen in die Kommunikation ist ein reflektiertes und planmäßiges Vorgehen, mit dem Einzelne oder Gruppen auf die Kommunikation oder die Kommunikationsmittel absichtsvoll und zielgerichtet einwirken, um einen Effekt zu erzielen" (Schubert 2009, 111). Es handelt sich bei Kommunikationsoptimierung also um eine Handlung, die bewusst, reflektiert, absichtsvoll, planmäßig und zielgerichtet ausgeführt wird. Das Ziel ist hierbei das Optimieren, also das Verbessern, der Kommunikation, wobei das Verbessern recht vage bestimmbar und bestimmt ist. Konkreter kann es mit z. B. Kostensenkung oder Verständlichkeitssteigerung der Kommunikation angegeben werden oder aber philanthropische Gesichtspunkte betreffen (z. B. Erleichterung des Informationszugangs für Menschen mit Sinnesbehinderung) (Schubert 2014, 205).

Der Begriff Kommunikation wird vorausgesetzt (Schubert 2009, 121). Aus der Sicht dieser Arbeit ist wichtig, dass die Verbesserung der Kommunikation 


\section{Konzept der Sprachlenkung}

nicht nur das Sprachliche (S), ${ }^{93}$ sondern darüber hinaus drei weitere Dimensionen: des (fachlichen) Inhalts (I), des (technischen) Mediums (M) und der Arbeitsprozesse (A) betrifft (Schubert 2009, 127-128). Für Kommunikationsoptimierung sind also alle diese vier Dimensionen relevant und das von Schubert gesuchte übergreifende Modell soll diese Breite abdecken. Es werden verschiedene Bereiche angeführt, in denen Kommunikationsoptimierung in unterschiedlichen Dimensionen stattfindet, beispielsweise Hochsprachenbildung und Kodifizierung (S), Sprachpflege (S), Auftraggebervorgaben und Redaktionsrichtlinien (S, I, M, A), Regulierte (Kontrollierte) Sprachen (S, I), Sprachen für sprachtechnologische Anwendungen (S, M, A), Content-Management (I, S, M, A), Workflow-Management (A, M) (Schubert 2009, 128-138). $\mathrm{Da}$ dies Vorüberlegungen zu einem Modell sind, haben sie einen recht unsystematischen Charakter (Schubert 2009, 127).

In Schubert (2014) liegt der Schwerpunkt auf dem bewussten lenkenden Eingreifen in die Sprache, also der sprachlichen Dimension der Kommunikationsoptimierung. Zu betonen ist, dass hier nach wie vor das Ziel der Verbesserung/Optimierung vorliegt (vgl. Schubert 2014, 202, 205-206).

Zur Untersuchung verschiedener Formen des bewussten Eingreifens in die Sprache schlägt Schubert (2014) neben dem oben genannten Ziel folgende Merkmale vor: Gegenstand, Nachhaltigkeit, Instrument, Akteure und Wirkung (Schubert 2014, 203). Da Wirkung in dem Aufsatz nicht weiter besprochen werden soll (Schubert 2014, 203), fasse ich hier die übrigen vier Merkmale zusammen.

- Gegenstand: Der Eingriff in die Sprache kann zunächst die (sprachlichen) Kommunikationsmittel und dadurch indirekt die Kommunikationshandlung betreffen oder die Einflussnahme erfolgt auf die Kommunikationshandlung direkt (Schubert 2014, 203; vgl. Schubert 2009, 110, 139). Der Eingriff in die Mittel kann sowohl konstruktive als auch reduktive Elemente aufweisen (Schubert 2011a, 373, 2014, 206). ${ }^{94}$

- Nachhaltigkeit: Es werden drei Nachhaltigkeitsstufen des Eingriffs unterschieden (Schubert 2014, 203; vgl. Schubert 2011a, 368-369):

\footnotetext{
93 Meine Abkürzungen

94 „Reduktive Eingriffe liegen vor, wenn bestimmte Wörter, beispielsweise neuere Fremdwörter, verboten werden oder von ihrer Verwendung abgeraten wird. Konstruktive Eingriffe liegen vor, wenn etwa im Rahmen der Korpusplanung neue Wörter eingeführt werden“ (Schubert 2011a, 203).
} 
1. Werkstücksstufe, ${ }^{95}$ die nur das aktuelle Werkstück, aber keine künftigen betrifft, z. B. das Korrekturlesen eines Dokuments;

2. Arbeitsprozessstufe, „(...) auf der eine Gruppe von Dokumenten betroffen ist, wodurch eine gewisse Nachhaltigkeit für künftige Dokumente entsteht"“ (Schubert 2014, 203);

3. Systemstufe, auf der das gesamte Sprachsystem vom Eingriff betroffen ist und die Veränderung somit als nachhaltig gilt.

- (Lenkungs-)Instrumente sind die eigentlichen Regelwerke, in denen die neuen Kommunikationsregeln festgehalten sind, beispielsweise Gesetze, behördliche Vorschriften, Terminologiedatenbanken, Wörterbücher, KS-Spezifikationen (Schubert 2014, 204).

- Akteure sind weitestgehend Autoren im Sinne dieser Arbeit und umfassen unterschiedliche Gruppen wie sprachliche Laien, Behördenmitarbeiter, Teams aus Unternehmensmitarbeitern und Wissenschaftlern, Branchenverbände etc. (Schubert 2014, 205).

Schließlich ordnet Schubert $(2014,208)$ unterschiedliche Sprachformen, die sich aufgrund eines bewussten lenkenden Eingriffs ergeben, auf einer stufenlosen Skala ein, die von den Polen spontan und bewusst begrenzt ist (s. Abbildung 12).

Obwohl der Ansatz der Kommunikationsoptimierung stark durch die Fachkommunikationswissenschaft und ihre praktischen Belange geprägt ist, integriert er dennoch Erkenntnisse aus allen in dieser Arbeit besprochenen Gebieten, was zum einen an den genannten Bereichen der Kommunikationsoptimierung deutlich ist, zum anderen aber auch an den verwendeten theoretischen Elementen. So betrachtet Schubert $(2009,111)$ die Interlinguistik als den eigentlichen Ursprung des Optimierungsgedankens (vgl. Schubert 2003, 152) und verweist speziell auf die Definition der Interlinguistik von D. Blanke (1985, 17), die die Bezeichnung Optimierung verwendet (Schubert 2009; vgl. Schubert 2003, 151-152). In Anlehnung an Wüster (1955, 271-272) (s. Abschnitt 3.2.1), der sowohl in der Interlinguistik als auch Terminologielehre und -arbeit tätig war, verwendet Schubert (2011a, 369-370) außerdem für verschiedene Sprachformen, die durch Kommunikationsoptimierung entstanden sind, die Bezeichnung gestaltete Sprachen (vgl. Schubert 2001a).

95 Zur Definition von Werkstück: Schubert (2014, 203, Fußnote 2). 


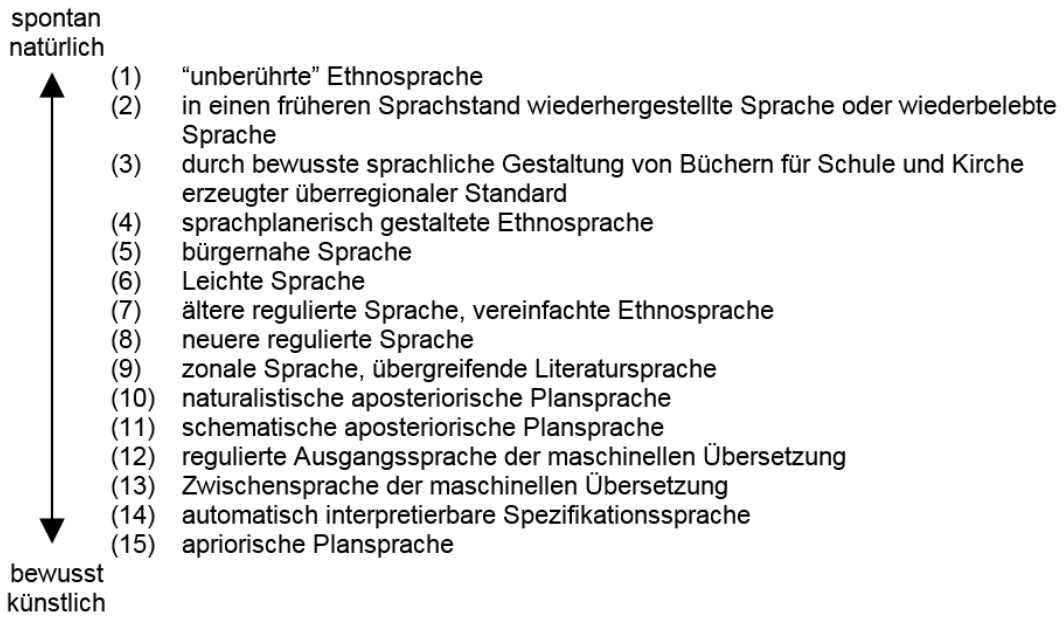

Abbildung 12.: Skala der Sprachformen durch bewussten lenkenden Eingriff (Schubert 2014, 210; vgl. Schubert 2011a, 371)

\subsection{Eigene Konzeptualisierung der Sprachlenkung}

Aus den oberen Beschreibungen geht hervor, dass alle vier Gebiete definitorisch unter Sprachplanung fallen und alle allgemeinen Konzeptualisierungen aus dem LPLP-Bereich auf die übrigen drei Gebiete entweder direkt anwendbar sind oder sich zu diesem Zweck leicht modifizieren lassen. Andererseits werden die speziellen Interessen der Konstruktion von PS, KS und der Terminologiearbeit in der eigentlichen LPLP-Forschung kaum berücksichtigt. Zudem werden Erkenntnisse aus LPLP insbesondere in der Terminologiearbeit und der KS-Konstruktion kaum wahrgenommen und die eigene Unabhängigkeit scheint für alle vier Gebiete wichtig zu sein.

Um diese Unausgewogenheit in der Forschung und Praxis nicht weiter zu vertiefen und kein bestimmtes Gebiet zu bevorzugen, verwende ich deshalb mit Ischreyt (1965) Sprachlenkung zur Bezeichnung der übergreifenden Perspektive auf alle vier Gebiete.

Im Folgenden bestimme ich eine festlegende Definition der Sprachlenkung. In anschließenden Erläuterungen lege ich fest, wie die Definition zu interpretieren ist. Schließlich gebe ich eine beschreibende Charakterisierung 
weiterer Aspekte der SL an. Diese Charakterisierung ist nicht festlegend, sondern gibt die aktuellen Erkenntnisse zum Gegenstand wieder, die sich aus dem Forschungsstand und den Ausführungen in diesem Kapitel ergeben.

Aus Übersichtlichkeitsgründen verzichte ich hier weitestgehend auf eine erneute Nennung der Quellen, die in den vorangegangenen Teilen dieser Arbeit ausführlich angegeben sind.

\subsubsection{Festlegende Definition der Sprachlenkung}

In diesem Abschnitt bestimme ich eine festlegende Definition der Sprachlenkung. Ich verfolge dabei den Grundsatz, eine Minimaldefinition des Gegenstandes zu finden, die lediglich aus konstitutiven Elementen der Sprachlenkung besteht und die beschreibenden oder charakterisierenden Elemente weglässt. Darüber hinaus definiere ich Sprachlenkungsmaßnahme. Im Anschluss gebe ich Erläuterungen, wie diese Minimaldefinition zu interpretieren ist.

Definition Sprachlenkung 1. Eingriff in die Sprache einer Gruppe von Menschen mit dem Ziel, ihren Sprachgebrauch nachhaltig zu verändern.

Definition Sprachlenkungsmaßnahme 1. Generische Bezeichnung für einzelne Instanz (tatsächliche Realisierung) der Sprachlenkung

\subsubsection{Erläuterungen zu Definition Sprachlenkung 1}

Unter Sprachlenkung verstehe ich eine (praktische) Tätigkeit und nicht ein Forschungsgebiet oder eine wissenschaftliche Disziplin. Im Unterschied zu Sprachwandel nach R. Keller $(1982,1990)$ fasse ich Sprachlenkung auch nicht als Handlungsergebnis auf. ${ }^{96}$ Meine Wahl des Wortes Eingriff weist zudem darauf hin, dass in der anvisierten (aktiven) Zielgruppe bereits eine Sprachpraxis besteht, die nun verändert werden sollte. Dies ist eine unmittelbare Bezugnahme einerseits auf die Definition des language management von Spolsky $(2009,4,6)$. Andererseits wird dadurch meine Auffassung von Sprachlenkung von Anfang an direkt ins sprachökologische Modell eingebettet.

96 Der Sprachwandel ist demnach eine notwendige, jedoch nicht beabsichtigte Folge des menschlichen Handelns. Er ist nicht auf die Handlung eines Einzelnen zurückzuführen, sondern als eine kollektive Konsequenz einer Reihe von individuellen Handlungen zu sehen, die zum Teil mit anderen Absichten ausgeführt wurden (R. Keller 1982, 9). 


\section{Konzept der Sprachlenkung}

Aus dieser Auffassung ergibt sich, dass beispielsweise die ursprüngliche Konstruktion von Klingonisch oder Dothraki nicht als Sprachlenkung aufgefasst werden kann, weil diese Tätigkeit nicht in die bestehende Sprachpraxis einer Sprechergemeinschaft eingriff. Um diese Sprachen entstanden jedoch ungelenkt Sprachgemeinschaften, die sie in bestimmten Kommunikationssituationen und zu bestimmten Zwecken benutzen. Eine (systemlinguistische) Weiterentwicklung und Verbreitung dieser Sprachen kann hingegen als Sprachlenkung aufgefasst werden, weil sie sich jeweils auf die Sprachpraxis der (ungelenkt entstandenen) Sprechergemeinschaft auswirken.

In der Literatur wird Sprachlenkung als Tätigkeit mit den Attributen intentional (intendiert), bewusst oder geplant (planvoll) versehen. Dies geschieht, um Sprachlenkung von Sprachwandel abzugrenzen. Ich verzichte in meiner Definition auf diese Attribuierungen vollständig, denn es lässt sich schwer der linguistischen Literatur entnehmen, was sie genau im Handlungskontext bedeuten und was sie voneinander unterscheidet. So können einige Beispiele für Eingriffe in den Sprachgebrauch gefunden werden, die, umgangssprachlich, scheinbar nichtintendiert bzw. nichtplanvoll verliefen.

Einen Versuch, den deutschen Sprachgebrauch von intentional, bewusst oder geplant zu beschreiben und zu ordnen unternimmt zwar R. Keller (1990, 21-25). Dies geschieht vor dem Hintergrund der Bestimmung des Sprachwandels als ein sog. Phänomen der Dritten Art (R. Keller 1990). Dieser Ansatz erwies sich aber für mich zur Bestimmung der Sprachlenkung ebenfalls als nicht weiterführend.

Um die Natur der Sprachlenkung als Tätigkeit zu beschreiben, wende ich mich deshalb handlungstheoretischen Ansätzen aus der Motivations-, Sozialund Arbeitspsychologie zu und stütze mich insbesondere auf die Auffassung von Tätigkeit und Handlung von Hacker (2010). Sie stammt zwar aus der Arbeitspsychologie, ist aber auf jede Art ergebnisorientierter Tätigkeit, nicht nur bezahlter (also bspw. Erwerbsarbeit, Hausarbeit, gemeinnützige Arbeit), anwendbar (Hacker 2010, 4).

Hacker $(2010,7)$ definiert Tätigkeiten als ,übergeordnete Verhaltenseinheiten, die ganze Handlungsketten (...) umfassen“. Tätigkeiten sind demnach abstrakte Größen, die in (mehreren konkreteren) Handlungen verwirklicht werden (Hacker 2010, 7). Handlungen bestehen ihrerseits aus Teilhandlungen (auch Operationen genannt). Diese Beschreibung trifft, wie leicht zu erkennen ist, auf Sprachlenkung zu. Aufgefasst als abstrakte Tätigkeit, wird sie in einzelnen Handlungen wie Korpusentwurf, Bereitstellung des Regelwerks etc. realisiert. Zudem gehört Sprachlenkung zu den sog. 
antriebsmittelbaren Tätigkeiten, ${ }^{97}$ bei denen dem Antriebsimpuls nicht direkt eine Handlungsausführung folgt, sondern eine zwischengeschaltete „Ziel-, Maßnahmen- und Mittelerwägung sowie -auswahl“ (Hacker 2010, 6). Wie elaboriert der zwischengeschaltete Schritt sein muss, um von antriebsmittelbaren Tätigkeiten/Handlungen zu sprechen, spezifiziert Hacker (2010) nicht. Mit Verweis auf Leont'ev (1979) identifiziert Hacker $(2010,6)$ unter den antriebsmittelbaren Tätigkeiten/Handlungen auch die sog. willentlich gesteuerten. ${ }^{98}$ In meiner Definition verstehe ich somit Sprachlenkung als eine antriebsmittelbare, willentlich gesteuerte Tätigkeit. Dieses Verständnis ersetzt somit die Attribute intentional, bewusst oder geplant.

Nach diesem Exkurs in die Handlungstheorie zur Diskussion der Begriffe intentional, geplant/planvoll und bewusst, wende ich mich wieder meiner Definition der Sprachlenkung zu und erörtere im Folgenden das Objekt des Eingriffs.

In der Definition bestimme ich allgemein Sprache als das Eingriffsobjekt der Sprachlenkung, wobei ich diese vage Formulierung mit Absicht wähle. Es kann sich nämlich sowohl um den Eingriff in die Sprachmittel im Sinne von Korpusplanung handeln. Oder aber um eine reine Statusplanung, bei der kein Eingriff in die Sprachmittel im eigentlichen Sinne erfolgt, sondern beispielsweise die bereits von der betreffenden Sprachgemeinschaft gebrauchten Mittel ab- oder aufgewertet werden. Auch hier muss beachtet werden, dass beide Planungsformen sowohl theoretisch als auch praktisch eng miteinander verflochten sind (vgl. Janich 2004, 493; Spolsky 2004, 11).

In der Definition lege ich fest, dass der Eingriff sich auf eine Gruppe bezieht. Dabei übernehme ich aus der Sozialpsychologie folgende Betrachtungsweise: „Eine Gruppe existiert, wenn zwei oder mehr Individuen sich als Mitglieder einer Gruppe verstehen“ (Nijstad und Knippenberg 2014, 440). ${ }^{99}$ Diese Festlegung in der Definition bedeutet, dass ich individuelle, nur auf sich selbst bezogene (also reflexive) Korrekturmaßnahmen nicht als Sprachlenkung auffasse. Dies stellt einen Unterschied zur Definition von Spolsky (2009, 12-13) sowie zu Beispielen von Ager (2001, 119-124) dar, der Beschlüsse von Individuen, eine Fremdsprache zu lernen, zur

\footnotetext{
97 Hacker (2010) wendet viele seiner Beschreibungen ohne Erläuterung auf beides, Tätigkeiten und Handlungen, an; so können auch einzelne Handlungen antriebs(un)mittelbar sein.

98 Hacker (2010) verwendet synonym auch zielgerichtet-willentlich reguliert und zielgerichtet-volitiv.

99 Zur sozialpsychologischen Auffassung von Gruppe s. Abschnitt 4.6.
} 


\section{Konzept der Sprachlenkung}

Sprachplanung zählt. ${ }^{100}$ Diese Trennung nehme ich vor, weil ich davon ausgehe, dass bei der Umsetzung von kollektiven Maßnahmen bestimmte Faktoren einen Einfluss haben, wie beispielsweise die Gruppenzugehörigkeit der Autoren (Handlungsträger), die bei selbstbezogenen Maßnahmen keine Rolle spielen. Ich nehme deshalb an, dass beide Tätigkeiten anderen Gesetzmäßigkeiten unterliegen.

Auch wenn Handlungen (und somit Tätigkeiten) an sich in der handlungstheoretischen Literatur nicht primär als intendiert, bewusst oder planvoll beschrieben werden, so ist das Konzept ihrer Zielgerichtetheit wesentlich: „Allgemein kann man Handeln als Verhalten charakterisieren, das auf ein bewusstes Ziel hin ausgerichtet ist (...)“ (Sonnentag, Fay und Frese 2004, 251; vgl. Six und Höcke-Pörzgen 1983, 233; Neuberger 1985, 59; Cranach, Ochsenbein und Valach 1986, 199, 202; Schüler und Brandstätter 2010, 39; J. Heckhausen und H. Heckhausen 2010, 1). Ziele können dabei definiert werden als „(...) Vorwegnahmen von Handlungsfolgen, die mehr oder weniger bewusst zustande kommen. Sie beziehen sich auf zukünftige, angestrebte Handlungsergebnisse und beinhalten zugleich auch eine kognitive Repräsentation dieser Handlungsergebnisse" (Kleinbeck 2010, 286; vgl. Hacker 2010, 7; Scheffer und Kuhl 2010, 89; Schüler und Brandstätter 2010, 41). Ziele haben vielfältige Funktionen: Sie veranlassen Handlungen, organisieren sie, richten sie aus und dienen auch zur Erfolgsevaluation (Kleinbeck 2010, 286; vgl. Schüler und Brandstätter 2010, 44). Zudem wird betont, dass Ziele dem Leben Bedeutung verleihen (Schüler und Brandstätter 2010, 44). Ziele werden nicht nur individuell verfolgt, sondern lassen sich auch auf der kollektiven Ebene untersuchen (Kleinbeck 2010, 286; Scheffer und Kuhl 2010, 90). Wie bereits aus dem Werkzeug zur Motivationserfassung bei Sprachplanung (Ager 2001) bekannt, sind Ziele hierarchisch organisiert, wobei mehr als drei Ebenen unterschieden werden können. So geht Kleinbeck (2010, 288-289) auf vier Stufen ein: Oberziele - Zielklassen - Handlungsziele - konkrete (Teil-)Ziele (s. Abbildung 13).

In den hier beschriebenen Konzeptualisierungen von Tätigkeiten und Handlungen nach Hacker (2010) verfolgen demnach Tätigkeiten das Oberziel. Da nun der Zielbegriff zur Verfügung steht, kann ich eine präzisere Handlungsdefinition von Hacker (2010) anführen: „Handlung bezeichnet eine zeitlich in sich geschlossene, auf ein Ziel gerichtete sowie inhaltlich und zeitlich gegliederte Einheit der Tätigkeit, nämlich die kleinste psychologisch

100 Selbstverständlich spielt auch bei Sprachlenkung als kollektivem Eingriff die individuelle Annahme der entwickelten Neuerung eine entscheidende Rolle. 


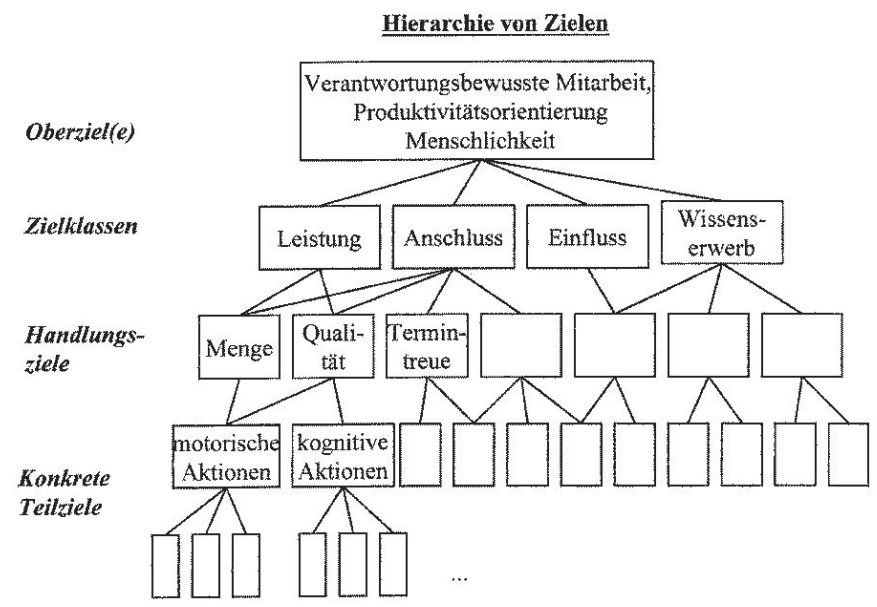

Abbildung 13.: Hierarchie von Zielen (Kleinbeck 2010, 288)

relevante Einheit willentlich gesteuerter Tätigkeiten. Die Abgrenzung von Handlungen erfolgt durch das bewusste Ziel (...). Jede Handlung (...) hat Ziele und erfüllt Aufgaben" (Hacker 2010, 7). Schließlich ist für die Bestimmung der Sprachlenkung der Unterschied zwischen Handlungen und Teilhandlungen/Operationen wichtig, bei dem das Attribut bewusst eine zentrale Rolle spielt: „Teilhandlungen oder Operationen sind nur unselbstständige Bestandteile der Tätigkeit, da ihre Resultate nicht als Ziele bewußt werden. Allerdings stellen Teilhandlungen nicht nur Mechanismen dar wie die Bewegungen, da unselbstständige, nur in bezug auf das Ziel sinnvolle kurzlebige Teilziele ihrer Regulation dienen können“ (Hacker 1985, 74, Hervorhebungen im Original).

Für die Sprachlenkung folgt aus dem Beschriebenen, dass das Ziel der Sprachgebrauchsveränderung durch die Handlungsträger (Autoren) kognitiv repräsentiert und zudem bewusst sein muss. Dabei unterscheide ich zunächst nicht, auf welcher Stufe in der Zielhierarchie dieses Ziel abgelegt ist, d. h. ob es sich um das Oberziel oder ein einfaches Ziel handelt. ${ }^{101}$ Da ich festlege, dass das Ziel der Sprachgebrauchsänderung den Handlungsträgern bewusst sein muss, schließt meine Definition Maßnahmen aus, die Sprachge-

101 Wie aus dem Forschungsstand deutlich, ist der Eingriff in die Sprache meistens in einen größeren, außersprachlichen Kontext eingebettet und die Sprachlenkung kann dann als Mittel zum außersprachlichen Zweck aufgefasst werden. Aus dieser Perspektive ist Sprachlenkung nicht mehr die Tätigkeit mit einem Oberziel, sondern eine Handlung als Bestandteil einer (außersprachlichen) Tätigkeit. 


\section{Konzept der Sprachlenkung}

brauchsänderung nicht als bewusstes Ziel verfolgen und somit als Teilhandlungen/Operationen im Sinne von Hacker $(1985,2010)$ verstanden werden.

Für das Verständnis meiner Definition der Sprachlenkung ergibt das Beschriebene, dass eine Tätigkeit genau dann als Sprachlenkung aufzufassen ist, wenn die Autoren das Ziel verfolgen, den Sprachgebrauch einer Menschengruppe zu verändern und dieses Ziel den Autoren bewusst ist.

Dies wirft automatisch die Frage auf, wie man in der Praxis entscheiden kann, ob das Ziel der Sprachgebrauchsänderung kognitiv repräsentiert (, selbstständig) und bewusst ist ${ }^{102}$ und die Maßnahme somit als Handlung (und nicht als "Teilhandlung") und in Konsequenz als Sprachlenkung aufgefasst werden kann oder nicht. Da dies aber ein methodischer und kein definitorischer Aspekt ist, gehe ich hier nicht weiter darauf ein.

Aus der sprachwissenschaftlichen Sicht erachte ich es für wichtig zu betonen, dass das Ziel des Eingriffes eine tatsächliche Herbeiführung der Sprachgebrauchsänderung sein muss, um die Tätigkeit als Sprachlenkung zu bezeichnen. Folglich können Ausarbeitung und Bereitstellung von (korpus- oder statusbezogenen) Änderungsvorschlägen lediglich Ziele von einzelnen Handlungen innerhalb der Sprachlenkung (als Tätigkeit) darstellen. Dies bedeutet, dass ich Maßnahmen, die alleine auf die Bereitstellung von Änderungsplänen abzielen, nicht als Sprachlenkung auffasse.

Schließlich gibt es Eingriffe in den Sprachgebrauch, die lediglich zeitlich begrenzt wirken. Beispielsweise sollte die neu entwickelte Plansprache Loglan in einer konkreten Untersuchungssituation kollektiv gebraucht werden, um eine wissenschaftliche Hypothese zu testen. Diese Änderung des Sprachgebrauchs sollte aber nicht über die Untersuchungssituation hinaus bestehen bleiben. Der Eingriff war somit zeitlich begrenzt. Auf ein ähnliches Beispiel weist Schubert (2011a, 369) mit der Werksstückstufe hin (Abschnitt 3.3.2). Diese und ähnliche Beispiele stellen für mich keine Sprachlenkung dar. Aus diesem Grund präzisiere ich in der Definition das Ziel, mit dem die Sprachlenkung ausgeführt wird, mit dem Attribut nachhaltig.

Anhand der oberen Ausführungen kann Definition Sprachlenkung 1 wie folgt paraphrasiert werden:

Definition Sprachlenkung 2. Eingriff in die Sprache einer Gruppe von Menschen als antriebsmittelbare, willentlich gesteuerte Tätigkeit mit dem Ziel einer nachhaltigen Änderung des Sprachgebrauchs dieser Gruppe.

102 Es gibt auch Ziele, d. h. Zustände, die kognitiv repräsentiert aber unbewusst sind (s. bspw. Kleinbeck 2010, 291). 


\subsection{Eigene Konzeptualisierung der Sprachlenkung}

\subsubsection{Vergleich von Definition Sprachlenkung 1 und Definition Sprachlenkung 2 mit Definitionen von Sprachplanung}

$\mathrm{Zu}$ Anfang dieses Kapitels habe ich aufgezeigt, dass einzelne Elemente der Theorie der Sprachplanung durchaus auf die übrigen drei Gebiete anwendbar sind und somit einen Ausgangspunkt zur Entwicklung einer Theorie der Sprachlenkung darstellen können. In diesem Abschnitt diskutiere ich die Unterschiede zwischen meinen Definitionen der Sprachlenkung 1 und 2 und Definitionen von Sprachplanung von Tauli (1968), Cooper (1989) und Spolsky (2009), um zu zeigen, warum sie den Ansprüchen einer festlegenden Definition der Sprachlenkung nicht genügen.

Alle drei Definitionen sehen Sprachplanung als eine Art Tätigkeit und verwenden Attribuierungen wie methodical (Tauli 1968), deliberate (Cooper 1989) und explicit and observable (Spolsky 2009), worauf ich aus den oben erläuterten Gründen in meinen Definitionen verzichte.

Das Ziel der Handlung ist bei Tauli (1968) systemlinguistisch; obwohl ich ebenfalls das vage Wort Sprache benutze, so stellt sie, insbesondere das Sprachsystem, für mich das Eingriffsobjekt und nicht das Eingriffsziel dar. Bei den anderen beiden Autoren ist hingegen, ähnlich wie in meiner Definition, die Veränderung des Sprachgebrauchs das Handlungsziel. Für Spolsky (2009) ist darüber hinaus die Veränderung der Spracheinstellungen ${ }^{103}$ ein weiteres Ziel. Ich sehe die Veränderung der Einstellungen als ein Mittel zur Änderung des Sprachgebrauchs und nicht als Ziel an sich. Da es ggf. noch weitere Mittel dieser Art gibt, ist für mich die Einstellungsänderung nicht konstitutiv für Sprachlenkung und deshalb ist dieser Aspekt in meiner Definition nicht enthalten. Ähnlich äußert sich für Cooper (1989) die Veränderung des Sprachgebrauchs im veränderten Korpus, Status oder einer veränderten Unterrichtssituation einer Sprache. Ich interpretiere dies so, dass diese Veränderungen Resultate der Handlung sind. Ich nenne in den Erläuterungen zu meiner Definition ebenfalls Korpus- und Statusplanung, es bestehen jedoch subtile Unterschiede zur Auffassung von Cooper (1989). Zum einen sehe ich sie nicht als Resultate sondern als Prozesse und Mittel zur Zielerreichung. Da ich zudem nicht davon ausgehen kann, dass es sich dabei um eine abschließende Liste der Mittel handelt, betrachtete ich sie eher als Beispiele. Ihre Nennung in meinen Definitionserläuterungen hat keinen konstitutiven Charakter, sondern legt die von mir angedachte Interpretation des Wortes Sprache dar.

103 Spolsky (2009) verwendet nicht das Wort attitudes sondern beliefs. Trotzdem übersetze ich dies als ,Einstellungen“, da ich vom Dreikomponentenmodell der Einstellungen ausgehe. 


\section{Konzept der Sprachlenkung}

Darüber hinaus stellt die Nennung der Ebenen des Sprachsystems durch Tauli (1968) eine weiterführende Information zur Sprachlenkung dar, da die Ausprägung und die Kombination der betroffenen Ebenen in konkreten Maßnahmen unterschiedlich sein können und sich keine abschließende Liste zufriedenstellend angeben lässt. Die Nennung unterschiedlicher Ebenen des Sprachsystems in den Erläuterungen zu meiner Definition hat aus diesem Grund keinen konstitutiven Charakter und fehlt in der eigentlichen Definition.

Cooper (1989) und Spolsky (2009) gehen zudem auf den kollektiven Charakter der Sprachplanung ein, wobei der erste Autor nicht deutlich herausstellt, ob es zwischen Individuen einen Zusammenhang geben muss und somit von einer Gruppe gesprochen werden kann. Die von Spolsky (2009) benutzte Formulierung participants in the domain deckt sich inhaltlich hingegen weitestgehend mit der von mir benutzten Gruppe von Menschen.

Die Definitionen von Tauli (1968) und Spolsky (2009) gehen noch auf Aspekte ein, die in meiner Definition nicht enthalten sind.

Mit der Nennung der Reichweite einer Sprachlenkungsmaßnahme: regional - national - international geht Tauli (1968) indirekt auf die Größe der Zielgruppe ein. Dies ist zwar ein wichtiger Aspekt, aber für Sprachlenkung an sich nicht konstitutiv. Außerdem ist diese Liste nicht abschließend, da Sprachlenkung auch auf niedrigeren Ebenen erfolgen kann, wie beispielsweise Spolsky (2009, 10-30) für Familien zeigt. Die Reichweite einer Sprachlenkungsmaßnahme stellt also eine weiterführende Information dar und ich berücksichtige sie im darauffolgenden Abschnitt 3.4.2.2.

Schließlich geht Spolsky (2009) auf die Autoren der Sprachplanung ein. Zum einen nennt er die Existenz dieser explizit in der Definition, worauf ich in meiner jedoch verzichte. Dies hat den Grund, dass ich Sprachlenkung als zielgerichtete Tätigkeit (Handlung) auffasse, bei der man automatisch von Handlungsträgern ausgehen kann. Eine (explizite) Behauptung der Trägerexistenz erschien mir deshalb als redundant. Dass die Autoren individuell oder kollektiv sein können, wie in der Definition von Spolsky (2009) angeführt, ist zudem eine erläuternde und keine konstitutive Information. Letztlich gibt Spolsky (2009) für die Autoren an, dass sie über eine tatsächliche oder angenommene Autorität in der anvisierten (aktiven) Zielgruppe verfügen müssen. Aus dem Forschungsüberblick in dieser Arbeit wurde es am Beispiel von Esperanto klar, dass dies für Sprachlenkung nicht von Anfang an der Fall sein muss und die Autoren erst im Laufe der Zeit eine gewisse Autorität erlangen können. Der Aspekt der Autorität ist zwar für die Umsetzung der Sprachlenkung rele- 
vant, da aber für die Sprachlenkung an sich nicht konstitutiv, fehlt er in meiner Definition.

\subsubsection{Vergleich der Auffassung von Sprachlenkung mit dem bewussten Eingreifen in die Sprache}

In diesem Abschnitt erörtere ich die Unterschiede zwischen meiner Auffassung von Sprachlenkung und der Kommunikationsoptimierung, speziell dem bewussten Eingreifen in die Sprache, nach Schubert (2003, 2009, 2011a, 2014).

Vergleicht man die Definitionen der Sprachlenkung und des (bewussten) Eingreifens, so stellt man fest, dass Sprachlenkung ein Spezialfall des (bewussten) Eingreifens ist, obwohl ich aus angeführten Gründen auf die Attribute bewusst, planvoll, absichtsvoll etc. verzichte.

Beim Vergleich von Sprachlenkung und Kommunikationsoptimierung wird klar, dass aus den vier Dimensionen Sprache, Inhalt, Medium, Arbeitsprozess, die vom (bewussten) Eingriff betroffen sein können, lediglich die sprachliche für Sprachlenkung relevant ist. ${ }^{104}$ Ich betrachte zudem nicht Kommunikation, sondern Sprache, wobei ich analog davon ausgehe, dass sowohl sprachliche Mittel als auch der Sprachgebrauch das Eingriffsobjekt darstellen können.

Trotz dieser Ähnlichkeit kann Sprachlenkung dem (bewussten) Eingreifen in die Sprache nach Schubert (2011a, 2014) nicht gleichgesetzt werden, da ein wesentlicher Unterschied in den Handlungszielen besteht. Das Handlungsziel der hier beschriebenen Sprachlenkung ist sprachbezogen - die Veränderung des Sprachgebrauchs. Verwendet man die Vorstellung von Zielen als Hierarchie, so liegt das Ziel der Kommunikationsoptimierung (und somit des (bewussten) Eingreifens in die Sprache) auf einer höheren Abstraktionsebene. Bei der Verbesserung/Optimierung der Kommunikation könnte es sich also um das Oberziel der Sprachlenkung handeln.

Wie jedoch aus dem Forschungsstand ersichtlich, ist die Verbesserung der Kommunikation nur eins der übergeordneten Ziele der Sprachlenkung und man kann andersartige Oberziele angeben. So lassen sich Oberziele der Weiterentwicklung von Klingonisch nur schwer als Kommunikationsverbesserung auffassen. Es handelt sich hierbei um Sicherung von materiellen Vorteilen durch eine verstärkte Bindung der Star-Trek-Fans an die Star-Trek-Marke,

104 Selbstverständlich ist dies eine starke Vereinfachung, denn aus dem Forschungsstand ergibt sich, dass auch bei sprachlichen Eingriffen, beispielsweise bei Kontrollierten Sprachen, technische Werkzeuge eine unterstützende Funktion haben können. Es geht hier also eher um das Vordergründige bzw. um das Ausschließen von Maßnahmen, die sich ausschließlich auf die übrigen drei Dimensionen beziehen. 


\section{Konzept der Sprachlenkung}

dies scheint aber nicht in eine Kommunikationsoptimierung eingebettet zu sein. Mit anderen Worten: Bei der Sicherung von materiellen Vorteilen durch Sprachlenkung muss Kommunikationsoptimierung nicht zwangsläufig zwischengeschaltet sein.

Ein anderes Beispiel, das den Unterschied zwischen Sprachlenkung und dem (bewussten) Eingreifen in die Sprache nach Schubert verdeutlicht, sind Maßnahmen, die aus dem Sprachplanungsbereich stammen. Wie Ager (2001) beschreibt, spielen beispielsweise bei machtlosen Gemeinschaften primär identitätsbezogene Motive/Ziele eine Rolle (für eine Übersicht s. bspw. Ager 2001, 200). Es geht hier also weniger um das Optimieren/Verbessern der Kommunikation, sondern um das Stärken oder Bewahren der Identität einer Gruppe.

Wie schon in Abschnitt 3.2.2.3 angesprochen, kann man sagen, dass Sprachlenkung grob in zwei Oberzielgruppen eingebettet ist: die Gruppe der identitätsbezogenen und die Gruppe der instrumentellen Ziele. ${ }^{105}$ In der Gruppe der instrumentellen (Ober-)Ziele kann man das Ziel der Optimierung/Verbesserung der Kommunikation identifizieren. Dabei muss nicht bei jeder instrumentellen SL-Maßnahme das Ziel der Optimierung auf einer Hierarchiestufe formuliert sein. Bezogen auf die sprachliche Dimension der Kommunikationsoptimierung (als Handlung), stellt Sprachlenkung somit eine übergeordnete Größe gegenüber dem bewussten Eingreifen in die Sprache dar, weil sie ein breiteres Spektrum an Maßnahmen abdeckt.

Was die kleineren Unterschiede zwischen den beiden Konzeptualisierungen betrifft, möchte ich hier noch den Nachhaltigkeitsaspekt herausgreifen. Wie in Abschnitt 3.3.2 beschrieben, stellt für mich ein textbezogener Eingriff in den Sprachgebrauch, also die Werkstücksstufe nach Schubert (2011a, 2014), keine Sprachlenkung dar, da hier keine Nachhaltigkeit per se auftritt. Die anderen beiden Stufen - Arbeitsprozess- und Systemstufe sehe ich als nachhaltig und somit als Sprachlenkung an. Ich behaupte hierbei jedoch keine Abstufung der Nachhaltigkeit, da auch Empfehlungen und punktuelle Vorschriften in bestimmten Kommunikationssituationen (und somit Registern) ${ }^{106}$ (Verwendungsbereich, Textsorte, beteiligte Personen

105 Auch diese Unterscheidung ist nicht trennscharf und kann als Skala aufgefasst werden.

106 Unter Register versteht man ein bestimmtes Sprachverhalten, das in einer Kommunikationssituation zu erwarten ist (Linke, Nussbaumer und Portmann 2001, 306; vgl. Dittmar 1997, 207-211). Dieser Begriff ist eng mit dem Begriff Varietät verbunden, stellt jedoch andere Aspekte in den Vordergrund. Mit Hymes $(1979,177)$ sind Varietäten an bestimmte soziale Gruppen, Register hingegen an bestimmte Situationen gebunden (vgl. Linke, Nussbaumer und Portmann 2001, 306). 
etc.) verbindlich und nachhaltig sein können. Sie stellen für mich hingegen den systemlinguistischen Umfang dar.

\subsubsection{Charakterisierung der Sprachlenkung}

Nach einer festlegenden Minimaldefinition von Sprachlenkung und ihrer Diskussion im Kontext anderer Definitionen gehe ich hier zu einer weiterführenden Beschreibung von Sprachlenkung über.

Im Unterschied zu Definition Sprachlenkung 1 und Definition Sprachlenkung 2 sind Beschreibungen in diesem Abschnitt nicht festlegend. Sie stellen eine Zusammenfassung der bisherigen, teilweise von mir verallgemeinerten, Erkenntnisse dar und geben somit das aktuelle Wissen über die Sprachlenkung und einzelne Maßnahmen wieder. Ich verzichte dabei weitestgehend auf Beispiele, da diese Kapitel 2 zu entnehmen sind.

\subsubsection{Sprachmittelbezogene Charakterisierung}

Hier erfolgt eine sprachmittelbezogene Charakterisierung der Sprachlenkung, wobei ich sowohl die rein systemlinguistischen, als auch soziolinguistisch bestimmte Aspekte der Sprachmittel betrachte.

Gemäß Definition Sprachlenkung 1 ist Sprachlenkung ein Eingriff in die Sprache einer Sprechergemeinschaft (Gruppe). Dabei stellt die strukturalistische Unterscheidung zwischen Sprachsystem und Sprachgebrauch einen geeigneten Ausgangspunkt zur Beschreibung der Sprachlenkung dar.

Fasst man Sprache als System auf, bedeutet dies, dass (gebräuchliche) Sprachmittel durch alternative Sprachmittel im Sinne des sprachökologischen Modells ersetzt werden.

Systemlinguistisch können einzelne Phänomene (wie Morpheme oder Wörter) oder ganze Ebenen des Sprachsystems betroffen sein. ${ }^{107}$ Die Aufzählung von Tauli (1968, 27) umfasst dabei die orthographische, phonetische, morphologische, lexikalische und syntaktische Ebene und ich ergänze sie um die textuelle Ebene, da beispielsweise Überlegungen zur Textstrukturierung zu Kontrollierten Sprachen gerechnet werden können. In den bisherigen Maßnahmen sind vor allem die lexikalische und die morphosyntaktische Ebene betroffen.

107 In diesem Abschnitt verwende ich bei Aufzählungen dieser Art aus Lesbarkeitsgründen ein inklusives Oder, d. h. ein solches, bei dem eine Kombination von einzelnen Aufzählungspunkten ebenfalls zutreffend sein kann. 


\section{Konzept der Sprachlenkung}

Interpretiert man die Anzahl der betroffenen Phänomene und Ebenen als den systemlinguistischen Umfang, nimmt eine SL-Maßnahme mit Korpusplanung zu einem bestimmten Zeitpunkt eine Ausprägung zwischen den Polen punktuell $\leftrightarrow$ flächendeckend an.

Sprachlenkung kann darüber hinaus durch die Herkunft des sprachlichen Materials charakterisiert werden; dies ist zwar eine weitere sprachmittelbezogene Eigenschaft, die jedoch durch einen sozialen Faktor bestimmt ist.

Etymologisch betrachtet, handelt es sich also in erster Linie um die Frage, ob die alternativen Mittel einer Sprache im soziolinguistischen Sinne entnommen sind oder nicht. Dabei geht es nicht um die Gebräuchlichkeit, sondern um wahrgenommene Wohlgeformtheit nach Chomsky (1972), d. h. ob die Mittel weitestgehend in der Grammatik einer gegebenen Sprache enthalten sind oder nicht. Des Weiteren kann unterschieden werden, ob diese existierende, soziolinguistisch aufgefasste Sprache die eigentliche Sprache der betreffenden Sprechergemeinschaft ist oder nicht. Zusammenfassend also: Die alternativen Mittel können der Sprache:

- der betreffenden Gruppe selbst,

- einer anderen Gruppe oder

- keiner existierenden Gruppe entstammen.

Mit den etymologischen Eigenschaften der sprachlichen Mittel hängt auch die Charakterisierung eines SL-Eingriffs als reduktiv (einschränken, von der Mittel der eigenen Sprache ausgehend) oder konstruktiv (hinzufügen, von der Mittel der Sprache einer anderen oder nicht existierenden Gruppe ausgehend) zusammen.

Die Festlegung und ggf. Ausarbeitung der neu einzusetzenden Mittel, also die Korpusplanung, erhält einen zentralen Stellenwert in einzelnen Sprachlenkungsmaßnahmen und spielt daher eine wichtige Rolle in der Sprachlenkung. Trotzdem muss Sprachlenkung, zumindest theoretisch, nicht immer eine Korpusplanung beinhalten. So sind Maßnahmen denkbar, die lediglich das Ansehen einer existierenden Sprache in der Gesellschaft anheben, ohne dabei Korpusarbeit zu betreiben, und somit eine reine Statusplanung darstellen. Nimmt mal also die strukturalistische Unterscheidung zwischen langue und parole als Ausgangspunkt, so ist nicht die Bereitstellung der sprachlichen Mittel an sich, sondern die Herbeiführung einer tatsächlichen Veränderung des Sprachgebrauchs einer Sprechergemeinschaft das oberste Ziel der Sprachlenkung aus linguistischer Perspektive. 


\subsection{Eigene Konzeptualisierung der Sprachlenkung}

\subsubsection{Außersprachliche Charakterisierung}

Die außersprachliche Charakterisierung der Sprachlenkung, auf die ich in diesem Abschnitt eingehe, umfasst Eigenschaften, die bereits aus Kapitel 2 (Forschungsstand) bekannt sind: Einsatzbereiche, aktive Zielgruppe, Autoren sowie Umsetzung.

Einsatzbereiche Als Erstes bezieht sich die außersprachliche Charakterisierung auf die außersprachlich bestimmten Sprachbereiche, die von der Sprachlenkung betroffen sein können. Spolsky $(2009,3)$ spricht in diesem Zusammenhang von verschiedenen Domänen, ich wende hingegen das soziolinguistische Varietäten-Modell von Löffler (2010, 79) an. Dieses Modell ist zwar speziell für das Deutsche entwickelt, lässt sich aber exemplarisch auch auf andere Sprachen leicht übertragen. Demnach existieren in einer Sprache verschiedene außersprachlich bestimmte Großbereiche, sog. „Lekte“, die nach Individuum (Idolekte), Medium (Mediolekte), Funktion (Funktiolekte), Areal (Dialekte), Gruppe (Soziolekte), Alter, Geschlecht (Genderlekte) und Interaktionstyp/Situation (Situolekte) unterschieden werden. In diesen Großbereichen können wiederum einzelne, nach spezifischeren Kriterien bestimmte Varietäten identifiziert werden. Zwar erfolgt die Einteilung in einzelne Großbereiche und Varietäten nach außersprachlichen Faktoren, wichtig aber ist, dass sie voneinander nach sprachlichen Kriterien unterscheidbar sind (Löffler 2010, 79-80).

Was die varietätenlinguistische Betrachtung der Sprachlenkung betrifft, lassen sich Beispielmaßnahmen für alle Großbereiche finden. ${ }^{108}$ Umgekehrt scheint sich auch jede einzelne SL-Maßnahme einem Großbereich zuordnen zu lassen, wobei manche Maßnahmen erst durch eine Kombination der Großbereiche vollständig beschreibbar sind. ${ }^{109}$

108 Idiolekte schließe ich per Definition Sprachlenkung 1 aus, da ich nur auf ein Kollektiv gerichtete Eingriffe betrachte; ansonsten können folgende Beispiele angegeben werden: Dialekte: Vereinheitlichung verschiedener Dialekte des Rätoromanischen zum Standard Rumantsch Grischun (exemplarisch Liver 1999); Soziolekte: Weiterentwicklung von Klingonisch als Gruppensprache der Star-Trek-Fans; Genderlekte: Einführung geschlechtsneutraler Ausdrucksweise (Prentice 1994); Alterssprache: Einführung einer altersgerechten Kontrollierten Sprache für Betriebsanleitungen (Christoffersen und Møller 2010); Situolekte/Textsorten: Einführung einer KS für bestimmte Textsorten der Technischen Kommunikation; Mediolekte: Einführung einer KS für gesprochene Sprache (exemplarisch Kaljurand und Alumäe 2012); Funktiolekte: alle Terminologien.

109 Dies ist eine Bestätigung der Beobachtung Löfflers zu seinem Modell, „(...) dass die Sprachwirklichkeit ein übergangsloses Kontinuum darstellt und dass alle Klassifizierungsversuche eine Frage des Standpunktes sind und immer nur unzureichend sein können. Die Übergänge sind fließend und die Unterscheidungskategorien überschneiden sich“ (Löffler 2010, 79). 


\section{Konzept der Sprachlenkung}

Ein besonderes Augenmerk schenke ich dem Großbereich der Funktiolekte, also Sprachen in der bestimmten Verwendungsfunktion, weil dieser Aspekt in Kapitel 2 eine wichtige Rolle spielt und ich ihn auch in weiteren Kapiteln wieder aufgreife. Löffler (2010, 94-96) merkt an, dass der Begriff Sprachfunktion nicht eindeutig bestimmbar ist und es folglich auch keine eindeutige Einteilung der Funktiolekte in einzelne Varietäten geben kann. Eine Grundlage für die Einteilung können die sog. Funktionalstile der Prager Schule bieten, und Löffler (2010, 96-112) gibt folgende fünf Funktiolekte an: Alltagssprache, Literatursprache, Wissenschafts- und Fachsprachen, Sprache des öffentlichen Verkehrs, Pressesprache. Auch für diese Einteilung lassen sich Beispiele für SL-Maßnahmen angeben, die entweder eine bestimmte, mehrere oder alle funktionalen Varietäten betreffen. ${ }^{110}$ Weitere Funktiolekte sind möglich, wie Löffler $(2010,96)$ selbst feststellt: „Die fünf Funktionsbereiche mit den zugeordneten Funktionalstilen decken nicht das gesamte Spektrum der Varietäten ab. Viele Varietäten sind auch mehrfunktional (...)“. Dies ist bei der umgekehrten Zuordnung der SL-Maßnahmen zu den fünf Varietäten wichtig, da sich beispielsweise die auf die religiöse Sprachpraxis bezogene SL, wie von Spolsky $(2011,31-52)$ beschrieben, nicht zuordnen lässt, was aber kein prinzipielles Beschreibungsproblem ist.

Zusammenfassend stelle ich fest, dass Sprachlenkung (mindestens) einen soziolinguistisch bestimmten Großbereich (Lekt) einer Sprache und somit (mindestens) eine Varietät betrifft. Fasst man die Anzahl der betreffenden Großbereiche und Varietäten als den soziolinguistischen Umfang auf, nimmt eine SL-Maßnahme zu einem bestimmten Zeitpunkt eine Ausprägung zwischen den Polen punktuell $\leftrightarrow$ flächendeckend an.

Aufgrund bestimmter Ausprägungen der oben genannten system- und soziolinguistischen (varietätenlinguistischen) Eigenschaften haben sich spezielle Formen der alternativen Sprachmittel etabliert, von denen insbesondere Plansprachen, Kontrollierten Sprachen und Terminologien zu erwähnen sind.

Aktive Zielgruppe Aktive Zielgruppe der Sprachlenkung sind Personen, deren Sprachgebrauch durch die Sprachlenkung geändert werden soll. In Kapitel 2 wurden einige konkrete Zielgruppenbeispiele angegeben.

$\mathrm{Zu}$ einer allgemeineren Beschreibung kann wieder das Varietätenlinguistische Modell von Löffler (2010) herangezogen werden, mit dessen Hilfe man

110 Alltagssprache: sprachpuristische Maßnahmen; Wissenschafts- und Fachsprache: jede Terminologiearbeit, aber auch bspw. Interlingua (IALA); Sprache des öffentliche Verkehrs: Leichte Sprache, Plain English; SL-Beispiele, die lediglich Pressesprache oder Literatursprache betreffen, fand ich nicht; alle Varietäten: Deutsche Rechtschreibreform, Esperanto. 
Zielgruppen aus unterschiedlichen außersprachlichen Perspektiven betrachten kann.

Einen weiteren Aspekt beleuchtet die SL-Definition von Tauli (1968), die auf unterschiedliche Geltungsbereiche konkreter Sprachlenkungsmaßnahmen wie regional - national - international eingeht. Ich interpretiere dies jedoch als einen Hinweis, aktive Zielgruppen mithilfe der Eigenschaften Gruppengröße und Proximität der Gruppenmitglieder zu charakterisieren. Dabei kann Proximität sowohl einfach als räumliche Nähe als auch die damit zusammenhängende tatsächliche Praxis eines direkten kommunikativen Kontaktes zwischen allen Gruppenmitgliedern interpretiert werden. Die beiden Gruppeneigenschaften werden einen wichtigen Platz in meiner erfolgsbezogenen Konzeptualisierung der Sprachlenkung einnehmen und ich befasse mich damit in 4.9 weiter.

Neben der Charakterisierung der aktiven Zielgruppe selbst, kann auch der Sprachgebrauch näher bestimmt werden, auf den sich die Sprachlenkung bezieht. Kapitel 2 entnehme ich, dass Sprachlenkung, mit wenigen Ausnahmen, nicht für jeden Gebrauch der Sprache durch die aktive Zielgruppe gilt, was nach pragmalinguistischen Gesichtspunkten beschreibbar ist. So kann Sprachlenkung von der Kommunikationssituation abhängen, vor allem davon, mit wem die aktive Zielgruppe kommuniziert (Rezipienten). So sollen beispielsweise Welthilfssprachen nur bei internationaler Kommunikation und textorientierte KS der Technischen Dokumentation nur für Kundendokumente verwendet werden. Darüber hinaus kann auch nach Öffentlichkeitsgrad des Sprachgebrauchs unterschieden werden, ob die Sprachgebrauchsveränderung formelle oder informelle Kommunikationssituationen betrifft. Des Weiteren kann der Faktor Textsorte relevant sein, da beispielsweise nur Bedienungsanleitungen oder Wartungshandbücher, nicht aber E-Mails oder Telefongespräche von der Sprachlenkung betroffen sein können. Außerdem kann Sprachlenkung nur einen Modus betreffen, beispielsweise den schriftlichen Sprachgebrauch, wie etwa bei Rechtschreibreformen. Ausnahmen, die jeden Sprachgebrauch der aktiven Zielgruppe, ob mündlich oder schriftlich, formell oder informell etc. betreffen, sind meistens im Bereich der politischen Sprachlenkung zu finden. Die Liste dieser pragmalinguistischen Faktoren ist nicht abgeschlossen.

Autoren Autoren von Sprachlenkungsmaßnahmen sind Personen, die den Eingriff durchführen, was oft auch eine Planung und Vorbereitung des Eingriffs mit einschließt. Autoren sind in den meisten Fällen identifizierbar. 


\section{Konzept der Sprachlenkung}

Dabei kann es sich um Einzelpersonen oder aber um kleine Personengruppen handeln, obwohl sie stellvertretend für größere Gruppen wie Regierungen, Organisationen, Unternehmen oder andere Gemeinschaften agieren können. In manchen Kontexten kann daher die Unterscheidung zwischen Auftraggebern und den Autoren im eigentlichen Sinne hilfreich sein.

Die Autoren bisheriger Maßnahmen hatten einen unterschiedlichen Bildungsstand und verfügten über verschiedenen fachlichen Hintergrund. Obwohl professionelle linguistische Kenntnisse als notwendig erscheinen können, so sind Maßnahmen zu verzeichnen, die ohne Beteiligung eines Linguisten durchgeführt wurden. M. E. erfahren die Autoren zunehmend eine Professionalisierung und die einzelnen Personen in Autorenteams bringen unterschiedliche Kompetenzen mit, die sich gegenseitig ergänzen.

Eine weitere Eigenschaft von Autoren betrifft ihre Gruppenzugehörigkeit. So gibt es sowohl Autoren (oder einzelne Personen in Autorengruppen), die Mitglieder der anvisierten aktiven Zielgruppe einer Sprachlenkungsmaßnahme sind oder aber auch solche, die von außerhalb dieser Gruppe stammen.

Autoren verfolgen mit Sprachlenkungsmaßnahmen unterschiedliche Ziele. Obwohl das Ziel einer nachhaltigen Sprachgebrauchsveränderung immer gegeben sein muss, wie in Definition Sprachlenkung 1 spezifiziert, so wird es in meisten Fällen in eine Zielhierarchie eingebettet. Dies bedeutet, dass es zu diesem sprachgebrauchsbezogenen Ziel sowohl über- als auch untergeordnete Ziele gibt. Die übergeordneten Ziele sind außersprachlich und vor allem auf die Lösung gesellschaftspolitischer oder wirtschaftlicher Fragen gerichtet. Darüber hinaus nehme ich nach meiner Analyse in 3.2.2.3 mit Ager (2001) an, dass in jeder Sprachlenkungsmaßnahme (mindestens) ein Identitätsmotiv identifiziert werden kann, auf dessen Grundlage konkrete Ziele formuliert werden.

Im Allgemeinen unterscheiden sich handelnde Subjekte bezüglich ihrer Handlungswirksamkeit, die ich hier als Autorität bezeichne. Dies trifft auch speziell auf die Sprachlenkungsautoren zu. Ich unterscheide dabei drei Ausprägungen auf einer kontinuierlichen Skala: restriktive, empfehlende und keine Autorität. Restriktive Autorität hängt mit der (formalen) Macht einer Person oder Gruppe zusammen und geht mit Möglichkeiten einer formalen Sanktionierung des unerwünschten Verhaltens einher. Diese Autorität besitzen Regierungen, aber auch andere formale Organisationen wie Unternehmen. Empfehlende Autorität hingegen besitzen beispielsweise unterschiedliche Gremien oder einzelne Fachexperten, die eine Empfehlung aussprechen können, jedoch keine Möglichkeit zur Sanktionierung haben. Wie es zur empfehlenden 
Autorität von Personen kommen kann, erklärt beispielsweise die Theorie der Erwartungszustände, die sich mit dem Status innerhalb einer Gruppe befasst (s. bspw. Nijstad und Knippenberg 2014, 462-463).

Die Autoritätsausprägung kann sich über die Zeit ändern, beispielsweise kann eine restriktive Autorität abgeschwächt werden oder ein Autor ohne Autorität kann diese im Laufe der Zeit zu einer empfehlenden ausbauen. Zudem kann es sinnvoll sein, zwischen der formellen (nominalen) und der informellen (gelebten) Autorität der SL-Autoren zu unterscheiden (zu formellen und informellen Rollen in einer Gruppe s. bspw. Nijstad und Knippenberg 2014, 460).

Mit der Frage der Autorität seitens der Autoren hängt auch die Frage der Verbindlichkeit der alternativen Sprache zusammen. Die Verbindlichkeit einzelner Maßnahmen zu einem gegebenen Zeitpunkt kann zwischen den Polen normativ $\leftrightarrow$ empfehlend erfasst werden. Bei der Beschreibung realer Sprachlenkungsmaßnahmen kann man nicht annehmen, dass beispielsweise eine restriktive Autorität immer mit einer normativen Verbindlichkeit einhergeht, da es einerseits empfehlende Maßnahmen durch Autoren mit restriktiver Autorität (bspw. Plain English) und andererseits auch Versuche gibt, einen hohen Grad an normativer Verbindlichkeit durch Autoren ohne Autorität (bspw. Blissymbolics) zu erzielen. Trotzdem kann man annehmen, dass bestimmte Konstellationen von Autorität/Verbindlichkeit besonders günstig für den Erfolg einer Sprachlenkungsmaßnahme sind.

Umsetzung Bei der Charakterisierung der Sprachlenkungsumsetzung handelt es sich um die Frage, wie das Ziel der kollektiven, nachhaltigen Sprachgebrauchsänderung von der Autoren verfolgt wird. Hier steht also das konkrete Vorgehen der Autoren im Vordergrund.

Das Modell von Haugen (1966) spezifiziert 4 idealtypische Phasen der Sprachlenkung. Die projekt- und prozessbezogene Sicht aus der Terminologiearbeit ergänzt zudem, dass in allen diesen Phasen nicht nur sprachliche, sondern auch außersprachliche Gesichtspunkte vorhanden sind und berücksichtigt werden können. Bündelt man beide Umsetzungskonzeptualisierungen, so beginnt eine idealtypische, höchstsystematische Sprachlenkung mit einer Vorbereitungsphase, in der Ziele definiert werden und eine Ist- und Soll-Analyse der linguistischen, aber auch der personellen, finanziellen, strategischen und ggf. technologischen Gesichtspunkte erfolgt. An diese Phase schließt die Kodifizierungsphase an. In erster Linie werden hier die neuen sprachlichen Mittel ausgearbeitet. Dennoch enthält auch sie idealtypisch au- 


\section{Konzept der Sprachlenkung}

Bersprachliche Komponenten, da man die in der Ist-/Soll-Analyse festgestellten personellen, finanziellen, strategischen und technologischen Mängel beheben kann. Die Umsetzungsphase stellt den eigentlichen Eingriff in die bestehende Sprache einer Gruppe dar, der mithilfe der in der vorangegangenen Phasen herausgearbeiteten Inhalte erfolgt. Diese Phase befasst sich direkt mit der Annahme der sprachlichen Alternative durch die aktive Zielgruppe und ihrer tatsächlichen Etablierung im Sprachgebrauch. Die ausgearbeiteten personalen, finanziellen und technologischen Ressourcen haben hierbei eine unterstützende Funktion. Mit der Umsetzungsphase endet die Initialeinführung der Sprachlenkung. Die Vorbereitung-, Kodifizierung und Umsetzungsphase entsprechen also weitestgehend dem Projekt im Sinne der Terminologiearbeit. Ist die Initialeinführung ansatzweise erfolgreich, d. h. etabliert sich der neue Sprachgebrauch zumindest ansatzweise, so kann Sprachlenkung als Prozess fortgeführt werden, was in Haugens Modell der Elaborierungsphase entspricht. Eine Elaborierung ist notwendig, um den neuen Sprachgebrauch einerseits zu stabilisieren und ggf. auf weitere Personen auszuweiten und dadurch den Erfolg der Maßnahme zu steigern. Andererseits sind Sprachlenkungsmaßnahmen durch die Einbettung in außersprachliche Ziele nie abgeschlossen, da sie auf die Veränderung der außersprachlichen Realität (wie etwa technologische Entwicklungen, gesellschaftspolitische Neupositionierungen) oder auf die Veränderung in der Sprachökologie (bspw. Stärkung der Position einer anderen Sprache im betreffenden Verwendungskontext) reagieren müssen. Auch eine idealtypische Elaborierungsphase umfasst Ausarbeitung, Bereitstellung und Durchsetzung von sowohl sprachlichen als auch außersprachlichen Ressourcen.

In realen Sprachlenkungsmaßnahmen variiert es, welche Umsetzungsphasen vorhanden sind und wie systematisch oder zu welchem Elaboriertheitsgrad berücksichtigt werden. Das gleiche gilt auch für die außersprachlichen Gesichtspunkte. Ich nehme jedoch an, dass sprachliche Aspekte immer auf eine Art berücksichtigt sind. Die vorhandenen Phasen sowie Systematizität und die Anzahl der außersprachlichen Aspekte sehe ich daher als nicht entscheidend dafür, ob ein Eingriff als Sprachlenkung aufgefasst wird oder nicht.

Neben der Identifizierung von idealtypischen Sprachlenkungsphasen ist für die Umsetzung zudem die Frage interessant, wie viel Beteiligung der aktiven Zielgruppe in den einzelnen Phasen durch die Autoren angedacht, erwünscht und ggf. gefördert wird. Die Extrempole sind hierbei eine reine Top-downSprachlenkung, die vollständig durch die Autoren im eigentlichen Sinne vollzogen wird und keine Einflussnahme der Zielgruppe erlaubt, sowie eine rei- 
ne Bottom-up-Sprachlenkung, bei der alle Mitglieder der aktiven Zielgruppe gleichberechtigt die Maßnahme mitgestalten und beeinflussen, ohne dabei einen Hauptverantwortlichen zu bestimmen. In der Geschichte der Sprachlenkung lassen sich leicht Beispiele für das erste Extremum finden: Schleyer mit Volapük, Bliss mit Blissymbolics oder die Sprachplanung in den Entwicklungsländern in den 1950er Jahren. Das zweite Extremum scheint eher theoretisch zu existieren. Obwohl manche Maßnahmen, wie beispielsweise Lojban, durchaus nah an eine reine Bottom-up-Ausrichtung kommen, scheint es dennoch meistens Personen zu geben, die zumindest für Organisatorisches zuständig sind. Die meisten realen Sprachlenkungsmaßnahmen bewegen sich zwischen den beiden Polen. Zusammenfassend lässt sich also sagen, dass jede SL-Maßnahme zu einem gegebenen Zeitpunkt durch den Grad der Zielgruppenbeteiligung charakterisiert werden, der sich zwischen den beiden Polen Top-down-Lenkung und Bottom-up-Lenkung befindet.

Im Zusammenhang mit dem erwünschten Beteiligungsgrad der Zielgruppe nehme ich letztlich an, dass dieser auf die Sprachauffassung der Autoren schließen lässt. Ich gehe davon aus, dass eine ausgeprägte Top-downAusrichtung für eine strukturalistische Auffassung der Sprache als System und eine ausgeprägte Bottom-up-Ausrichtung für eine soziolinguistische Auffassung der Sprache als soziales Phänomen stehen. Unter Sprache als soziales Phänomen verstehe ich, dass sich die Sprache durch die soziale Interaktion konstituiert. Im Unterschied zur Auffassung als System ist sie nicht lediglich „von oben“ gegeben, sondern beruht auch auf den Entscheidungen der Individuen, die in einem gemeinsamen situativen Kontext interagieren. Die Sprache entsteht dadurch auch ,von unten“, obwohl durch Konventionalisierung bestimmte Aspekte von „oben“ ebenfalls gegeben sind.

Auch umgekehrt nehme ich an, dass diese Sprachauffassungen entsprechende Präferenzen für Umsetzungsausrichtung mit sich bringen, wobei ich hier eher von Tendenzen und keinen absoluten Vorhersagen ausgehe. Ob der Zusammenhang zwischen Sprachauffassung und Zielgruppenbeteiligung Implikationen für den Umsetzungserfolg enthält, befasse ich mich in weiteren Teilen dieser Arbeit.

\subsubsection{Charakterisierung der wissenschaftlichen Erforschung}

Sprachlenkung wird in ihrer gesamten Breite nicht systematisch erforscht. Obwohl es einerseits die besprochen Vorstöße einer übergreifenden Definition seitens $L P L P$ und andererseits integrierende Ansätze gibt, die nicht aus LPLP stammen, so stellen sie bis jetzt nicht die Hauptströmung in der For- 


\section{Konzept der Sprachlenkung}

schung dar. Den Status Quo der SL-Forschung bildet die Aufteilung in die vier genannten Gebiete: LPLP, Plansprachenforschung (Interlinguistik), Terminologielehre und -arbeit sowie das Gebiet um Kontrollierte Sprachen. Die Selbstauffassung der Gebiete sowie ihre Positionen gegenüber der Linguistik und die interdisziplinären Bezüge behandelte ich ausführlich in Kapitel 2.

So wie ich Sprachlenkung in Definition Sprachlenkung 1 bestimme, siehe ich sie als Gegenstand der Angewandten Linguistik, die ich mit Knapp (2004, xviii) auffasse als: „(...) Disziplin, die sich mit der Beschreibung, Erklärung und Lösung von lebens- und gesellschaftspraktischen Problemen in den Bereichen von Sprache und Kommunikation befasst. Diese Probleme halten sich nicht notwendig an disziplinäre Grenzen (...)“. Auch für Sprachlenkung gilt, dass je nach Fragestellung einerseits innerlinguistische Bezüge zu Soziolinguistik, Computerlinguistik, Fachsprachenforschung oder kognitiver Linguistik erforderlich sein können. Um Sprachlenkung in ihrer vollen Breite zu erforschen, aber auch um in der Praxis auf einer wissenschaftlichen Grundlage arbeiten zu können, müssen andererseits auch Bezüge zu außerlinguistischen Disziplinen, die teilweise über die Nachbardisziplinen der genannten Bindestrichlinguistiken hinausgehen, hergestellt werden. Durch die Vielseitigkeit der Fragestellung und die inter- und intradisziplinäre Ausrichtung erachte ich es für nicht notwendig, die Konstituierung eines selbstständigen Gebietes innerhalb der Linguistik zur Erforschung der SL zu fordern.

In Definition Sprachlenkung 1 nenne ich das Kollektive als einen Bestimmungsaspekt der Sprachlenkung. An der Erforschung dieses Kollektiven und der Gruppenprozesse, die bei der Umsetzung einer Rolle spielen, bin ich in dieser Arbeit besonders interessiert. In der Forschung wurde dem Kollektiven oder Sozialen an (einzelnen Formen) der Sprachlenkung unterschiedlich viel Aufmerksamkeit geschenkt. Wie im Geschichtsabschnitt 2.1.2 beschrieben, wandten sich vor allem LPLP aber auch PS schnell von der rein systemlinguistischen Sprachauffassung ab und werden stark durch soziolinguistische Überlegungen geprägt. Am anderen Ende positioniert sich die Terminologielehre, die vollständig auf die soziale Komponente der Sprache verzichtet. Die Positionen der Terminologiearbeit und der KS-Forschung sind hingegen uneindeutig: Die angewandte Forschung wendet sich zunehmend dem Potential $\mathrm{zu}$, das eine Sprachlenkung mit verstärkter Bottom-up-Ausrichtung mit sich bringt, ohne dies jedoch in der Grundlagenforschung auf der Metaebene zu erörtern oder sich gar mit der zugrunde liegenden Sprachauffassung auseinanderzusetzen. 
Ich stelle darüber hinaus fest, dass das Kollektive in der Sprachlenkung, wenn wissenschaftlich überhaupt thematisiert, aus der soziolinguistischen Perspektive betrachtet wird (zu diesem Abschnitt siehe auch Suchowolec 2012). Dies bedeutet, dass vor allem Erkenntnisse aus der Soziologie herangezogen werden. Zur Beschreibung der Gruppenprozesse können aber auch psychologische Disziplinen wie die Sozialpsychologie oder, in speziellen Kontexten, die Arbeits- und Organisationspsychologie herangezogen werden, was aber nur in wenigen Ausnahmefällen, wie bei Ager (2001), geschieht. Beide Perspektiven sind berechtigt und stehen nicht im Widerspruch, sondern können sich sogar ergänzen, denn die Stärke der Soziologie liegt in der Erklärung der Gruppenprozesse aus der makrosozialen Perspektive, während sich die Sozialpsychologie eher mit individuelleren Erklärungsaspekten befasst (Stroebe, Hewstone und Jonas 2014, 10-11). $\mathrm{Da}$ ich mit der Vernachlässigung der psychologischen Perspektive auf die Gruppenprozesse der Sprachlenkung eine Forschungslücke feststelle, greife für meine Theorie verstärkt auf die Erkenntnisse der Sozialpsychologie und teilweise der Arbeits- und Organisationspsychologe zurück.

\subsection{Konzept der Sprachlenkung - Fazit}

In diesem Kapitel habe ich den Gegenstand Sprachlenkung als Ergebnis einer Verallgemeinerung der Erkenntnisse aus den betrachteten vier Gebieten bestimmt. Hierfür habe ich eine festlegende Definition vorgeschlagen. Diese Definition knüpft an die allgemeinen Definitionen der Sprachplanung sowie die beiden hier vorgestellten übergreifenden Ansätze an und steht somit in ihrer Tradition. Im Unterschied zu ihnen handelt es sich jedoch um eine Minimaldefinition, die nur auf Aspekte eingeht, die ich für konstitutiv für Sprachlenkung halte. Aspekte, die aus meiner Sicht für Sprachlenkung nicht konstitutiv sind, flossen in eine weiterführende Beschreibung der Sprachlenkung mit ein, die aber keinen festlegenden Charakter hat und lediglich den heutigen Wissenstand zur Sprachlenkung wiedergibt. Für diese Charakterisierung, die in 3.4.2 erfolgte, habe ich zudem im Verlauf dieses Kapitels eine Reihe von weiteren Kategorien identifiziert. Diese Kategorien können aber auch zur Beschreibung und ggf. Abgrenzung einzelner Maßnahmen genutzt werden. In Tabelle 9 trage ich alle diese Kategorien mit den in der bisherigen Forschung identifizierten Ausprägungen zusammen, die als ein praktischer Kriterienkatalog zur Charakterisierung von Maßnahmen genutzt werden kann. 


\begin{tabular}{|c|c|}
\hline Kategorie & Ausprägung \\
\hline $\begin{array}{l}\text { systemlinguistische } \\
\text { Faktoren }\end{array}$ & $\begin{array}{l}\text { Ebene des Sprachsystems } \\
\text { Etymologie des Sprachmaterials: aktive Zielgruppe | andere } \\
\text { Gruppe | keine existierende Gruppe }\end{array}$ \\
\hline $\begin{array}{l}\text { systemlinguistischer } \\
\text { Umfang }\end{array}$ & punktuell $\leftrightarrow$ flächendeckend \\
\hline Modus & schriftlich | mündlich \\
\hline Art des Eingriffs & reduktiv | konstruktiv \\
\hline $\begin{array}{l}\text { soziolinguistischer } \\
\text { Einsatzbereich } \\
\text { (-lekte) }\end{array}$ & $\begin{array}{l}\text { Mediolekte | Funktiolekte | Dialekte | Soziolekte | Alter | } \\
\text { Genderlekte | Situolekte }\end{array}$ \\
\hline $\begin{array}{l}\text { soziolinguistischer } \\
\text { Umfang }\end{array}$ & punktuell $\leftrightarrow$ flächendeckend \\
\hline Verbindlichkeit & empfehlend $\leftrightarrow$ normativ \\
\hline aktive Zielgruppe & $\begin{array}{l}\text { Gruppengröße } \\
\text { Proximität }\end{array}$ \\
\hline Autoren & $\begin{array}{l}\text { Ursprungsdomäne: Staat | Wissenschaft | Wirtschaft } \\
\text { Beteiligung: Top-down } \leftrightarrow \text { Bottom-up } \\
\text { Bildungsstand/fachlicher Hintergrund } \\
\text { Gruppenzugehörigkeit bezogen auf die aktive Zielgruppe: } \\
\text { Ingroup | Outgroup; Autorität }\end{array}$ \\
\hline Funktion/Ziel/Motiv & $\begin{array}{l}\text { identitätsbezogen | instrumentell } \\
\text { Zielhierarchie }\end{array}$ \\
\hline $\begin{array}{l}\text { pragmalinguistische } \\
\text { Faktoren }\end{array}$ & $\begin{array}{l}\text { Kommunikationssituation } \\
\text { Rezipienten (passive Zielgruppe) } \\
\text { Öffentlichkeitsgrad: formell | informell } \\
\text { Textsorte }\end{array}$ \\
\hline $\begin{array}{l}\text { Phase der Umset- } \\
\text { zung }\end{array}$ & Selektion | Kodifikation | Implementation | Elaboration \\
\hline Sprachauffassung & strukturalistisch | soziolinguistisch | kognitionslinguistisch \\
\hline
\end{tabular}

Tabelle 9.: Kriterienkatalog zur Beschreibung und Abgrenzung von Sprachlenkungsmaßnahmen 


\begin{tabular}{|l||l|}
\hline Dimension & Ausprägung \\
\hline \hline Gebrauchsdomäne & Bildung - öffentliche Verwaltung - Medien etc. \\
\hline Sphäre & staatlich - öffentlich - privat \\
\hline Gemeinwohl & Effizienz - Fairness \\
\hline Ziel & Kapazität - Gelegenheit - Wunsch \\
\hline Wirtschaftlichkeit & $\begin{array}{l}\text { Güter vs. Dienstleistungen; Kosten vs. Nutzen; Eingabe vs. } \\
\text { Ausstoß vs. Resultat }\end{array}$ \\
\hline Typ & Statusplanung vs. Korpusplanung \\
\hline $\begin{array}{l}\text { Intralinguistischer } \\
\text { Fokus }\end{array}$ & schriftlich - mündlich; Ebenen des Sprachsystems \\
\hline Art der Strategie & Anreize - Restriktionen \\
\hline
\end{tabular}

Tabelle 10.: Metatypologie zur Charakterisierung von Sprachpolitiken (Grin 2016)

Ein ähnliches Prinzip zur Beschreibung von einzelnen Maßnahmen in LPLP schlug kurzlich Grin (2016) mit seiner Metatypologie (,typology of typologies") für Sprachpolitik vor. Er bestimmt dabei acht Dimensionen und entsprechende Ausprägungen, mit deren Hilfe sich verschiedene Sprachpolitiken einordnen lassen. Wie aus Tabelle 10 ersichtlich, decken sich die Dimensionen nur teilweise mit den von mir vorgeschlagenen Kategorien und beide Ansätze ergänzen sich also.

In diesem Kapitel habe ich darüber hinaus in entsprechenden Analysen gezeigt, dass zur Beschreibung der Sprachlenkung einzelne Modelle und Konzeptualisierungen aus allen vier Gebieten eingesetzt werden können. Insbesondere eignen sich hierzu LPLP-Ansätze, wie die Unterscheidung zwischen der Status- und Korpusplanung, das sprachökologische Modell, das Werkzeug zur Motivationserfassung von Ager (2001) und die Umsetzungsphasen nach Haugen (1966). Ich habe aber auch gezeigt, dass die Ansätze aus anderen Gebieten dem übergreifenden Anspruch durchaus gerecht werden und einen Mehrwert, insbesondere für LPLP, darstellen können. So heben die TAspezifischen Umsetzungsmodellierungen auf organisatorische und betriebliche Aspekte hervor, die in den vorgestellten LPLP-Ansätzen bisher zu kurz kommen.

Eine übergreifende Sicht auf die vier Gebiete unter der Bezeichnung Sprachlenkung ist somit mithilfe der bereits existierenden Mittel möglich. Die Aufteilung des Gegenstandes auf diese vier Gebiete scheint, wie ich 


\section{Konzept der Sprachlenkung}

gezeigt habe, nicht auf theoretischen Vorüberlegungen zu beruhen, sondern eher pragmatisch als systematisch bedingt zu sein.

Abschließend möchte ich an dieser Stelle einzelne Beobachtungen aufgreifen, die ich in diesem Kapitel an verschiedenen Stellen gemacht habe, da sie für weitere Teile dieser Arbeit von Bedeutung sind.

Sprachlenkung fasse ich als Handlung bzw. als Tätigkeit auf, deren Ziele hierarchisch angeordnet sind, wobei sprachliche Ziele in außersprachliche Oberziele eingebettet sind. Im Unterschied zu Ager (2001) kann diese Hierarchie mehr als nur drei Ebenen enthalten. Die übergeordneten außersprachlichen Ziele enthalten, wie ich gezeigt habe, immer ein Identitätsziel (Identitätsmotiv) im Sinne von Ager (2001). Dies trifft auf alle Maßnahmen aus allen vier Gebieten zu. Dieses Identitätsmotiv kann zwei Ausprägungen annehmen: die identitätsbezogene oder die instrumentelle. Im weiteren Verlauf dieser Arbeit komme ich auf diese Überlegungen zu Zielen der Sprachlenkung zurück und diskutiere das Verhältnis zwischen den beiden Ausprägungen des Identitätszieles im Kontext der Sprachlenkungsumsetzung. 


\section{Umsetzung und Erfolg der Sprachlenkung}

\subsection{Einleitung}

Ab diesem Kapitel verschiebt sich mein Fokus auf die zweite Forschungsfrage dieser Arbeit und ich setzte mich mit dem Umsetzungserfolg von Sprachlenkung auseinander. In diesem Kapitel verfolge ich zwei Ziele: Zum einen fasse ich das Bekannte zum Erfolg von SL zusammen. Zum anderen bereite ich die Untersuchung von Best-Practice in Kapitel 5 konzeptionell vor.

Das Kapitel ist wie folgt aufgebaut. Im ersten Teil behandle ich das Bekannte zum SL-Erfolg. Wie sich bereits in Kapitel 2 abzeichnet, gibt es eher wenige direkte Erkenntnisse zu diesem Thema aus den einschlägigen vier Gebieten. Entsprechend fällt hier Abschnitt 4.2 eher knapp aus. In 4.3 baue ich hingegen auf den verallgemeinerten Erkenntnissen aus Kapitel 3 auf und befasse ich mich also mit dem indirekt Bekannten zum Thema SL-Erfolg. Ich verfolge dabei die handlungstheoretischen Überlegungen weiter und schlage eine Konzeptualisierung von SL-Umsetzung vor, die sich das in Abbildung 13 vorgestellte Konzept der Zielhierarchie zunutze macht. Auf dieser Grundlage sowie unter Berücksichtigung von Definition Sprachlenkung 1 und Definition Sprachlenkung 2 definiere ich die erfolgreiche Sprachlenkungsmaßnahme. Desweilen stelle ich allgemeine Überlegungen an, was geleistet werden muss, um den Erfolg oder den relativen Erfolg von Sprachlenkungsmaßnahmen zu messen.

Im zweiten Teil des Kapitels treffe ich konzeptionelle Vorbereitungen auf die Untersuchung. Eingangs (4.4) stelle ich zwei Annahmen vor, die ich über die Bedeutung der Identität von Mitgliedern der aktiven Zielgruppe für die Umsetzung der Sprachlenkung mache. Auf diese Annahmen folgt ein theoretischer Block. Zunächst stelle ich drei Konstrukte aus der Sozialpsychologie vor - Identität, Gruppe und Einstellungen. Dieser Einschub erläutert außerlinguistische Grundlagen, auf die ich später bei der Untersuchung zurückgreife. Dann gehe ich auf die Wechselwirkung zwischen Sprache und Identität aus 


\section{Umsetzung und Erfolg der Sprachlenkung}

der linguistischen Perspektive ein (4.8). Dieser theoretische Block ermöglicht mir, die oben erwähnten Annahmen zu begründen. Schließlich schlage ich ein weiteres Modell für die SL-Umsetzung vor, das stärker auf die Erfolgsbedingungen ausgelegt ist, ohne diese Bedingung jedoch direkt zu nennen. Sowohl die vorgestellten Annahmen als auch das zweite Umsetzungsmodell der Sprachlenkung sollen im Zusammenspiel mit den sozialpsychologischen Konstrukten eine konzeptionelle Grundlage für die darauffolgende Untersuchung in Kapitel 5 dienen. Die Annahmen und Modelle sollen dabei nicht als Hypothesen für die Untersuchung verstanden werden. Es handelt sich in erster Linie eher um eine Explikation meiner Forschungsperspektive, auch im Sinne der Befangenheit, mit der ich an die Untersuchung herangehe.

\subsection{Zusammenfassung und Verallgemeinerung der bisherigen Erkenntnisse}

Der Forschungsüberblick in Kapitel 2 ergibt, dass in keinem der vier Gebiete eine theoretische Auseinandersetzung mit dem Konzept des Erfolges im Allgemeinen und des Sprachlenkungserfolges im Speziellen zu verzeichnen ist. Auch der Erfolg von Sprachlenkungsmaßnahmen wird theoretisch nicht gefasst. Dies bedeutet insbesondere, dass folgende Fragen auf der Metaebene nicht gestellt oder beantwortet werden: Was ist Erfolg?, Wann ist eine SLMaßnahme erfolgreich?, Wie kann Erfolg gemessen werden?. Trotz dieser unzureichenden theoretischen Erfolgsbestimmung ist aus der Praxisperspektive die Frage nach dem Erfolg von SL-Maßnahmen latent, weil Autoren, einfach ausgedrückt, ihr Projekt in der Praxis durchsetzen wollen.

Bei der Beschäftigung mit Erfolg geht es in der einschlägigen Literatur zurzeit also weniger darum, den Erfolg theoretisch fassbar zu machen. Vielmehr bedient man sich eher eines alltagssprachlichen Verständnisses als tatsächliche Durchsetzung der sprachlichen Neuerung. Das Augenmerk liegt also auf der praktischen Frage, wie Erfolg gefördert werden kann, zumal man davon ausgeht, dass er nur schwer zu erzielen ist und seine Beeinflussung zudem nicht immer in den Händen der Autoren liegt. Darüber hinaus weist die LPLP-Literatur darauf hin, dass der Erfolg von der Perspektive abhängt. So nenne ich hier als Beispiel eine Situation, in der die Autoren eine Maßnahme als erfolgreich sehen, während sie für die aktive Zielgruppe eigentlich Nachteile bringt. Ich halte aus dieser Beobachtung fest, dass der Erfolg aus der Perspektive der Autoren, also als Top-down-Erfolg, betrachtet werden kann. 
Diese Perspektive legt eine präskriptive Auffassung des Erfolges nahe. Demgegenüber steht der Erfolg aus dem Blickwinkel der aktiven Zielgruppe, der sich nicht zwangsläufig mit dem Top-down-Erfolg decken muss. Dem Forschungsüberblick entnehme ich, dass vor allem TA, KS und weitestgehend auch die Interlinguistik den Erfolg aus der Perspektive der Autoren betrachten. In Sprachplanung/LPLP erfolgte hingegen ein Perspektivenwechsel, so dass der Erfolg verstärkt aus der Zielgruppensicht betrachtet wird, was eine Abkehr vom Präskriptivismus des Top-down-Erfolges mit sich zog.

Wie erläutert, ist in der Praxis die Frage nach erfolgsfördernden Faktoren zentral, wobei ich in Kapitel 2 zwischen systemlinguistischen und nichtsystemlinguistischen unterschieden habe. Im weiteren Verlauf dieser Arbeit sind vor allem die nichtsystemlinguistischen Faktoren von Interesse und ich halte als Zwischenergebnis die Beobachtung fest, dass man diese Faktoren unterschiedlich weiter aufteilen kann:

- Nach Großbereich: politisch, wirtschaftlich, sozial, psychologisch, kulturell, technologisch

- Nach Zeitpunkt: vor - während - nach der Maßnahme

- Punktuell: Wie in dieser Arbeit können aus den Großbereichen einzelne Punkte herausgegriffen werden. Die Erfolgsfaktoren können sich dann beispielsweise auf Zielgruppe, Autoren, Werkzeuge, Funktionen oder Modelle beziehen.

Diese Faktoren werden in der Literatur zurzeit durch Beobachtungen und Reflexion der Projekte gewonnen, wobei m. E. viele nach wie vor aus Einzelprojekten stammen. Die Erforschung von nichtsystemlinguistischen Erfolgsfaktoren von Kontrollierten Sprachen bleibt weitestgehend auf der Ebene der Einzelbeobachtungen. Im Gegensatz dazu ist in TA und Interlinguistik eine Verallgemeinerungstendenz zu verzeichnen, die eine Systematisierung der Erkenntnisse aus der Praxis bringt. In der Terminologiearbeit führt dies zur Entstehung von Best-Praxis-Empfehlungen. In der Interlinguistik ist die Datenlage verhältnismäßig schlechter als in TA, da deutlich weniger Maßnahmen als erfolgreich gelten. Trotzdem wurde die Entwicklung von Esperanto zu einem Maßstab für andere Projekte verallgemeinert. Die Aufstellung von Erfolgsfaktoren geschieht zurzeit also weitestgehend induktiv. 


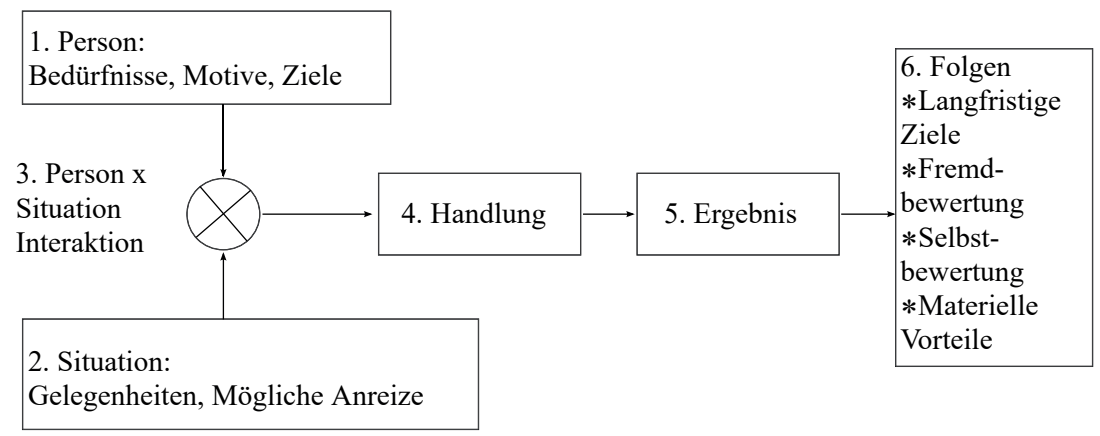

Abbildung 14.: Das Modell des motivierten Handelns von J. Heckhausen und H. Heckhausen $(2010,3)$

\subsection{Handlungstheoretische Konzeptualisierung des Erfolges}

In diesem Abschnitt setze ich mich mit der Frage auseinander, was Erfolg von Sprachlenkungsmaßnahmen ist. Hierzu entwickle ich auf der Grundlage des handlungstheoretischen Modells des motivierten Handelns nach J. Heckhausen und H. Heckhausen (2010) und in Verbindung mit Definition Sprachlenkung 1 und Definition Sprachlenkung 2 eine handlungstheoretische Konzeptualisierung, um die festgestellte Forschungslücke um den Erfolgsbegriff zu schließen.

\subsubsection{Definition erfolgreicher Sprachlenkungsmaßnahmen}

Zur Herleitung der Definition erfolgreicher Sprachlenkungsmaßnahmen nehme ich als Grundlage das Modell des motivierten Handelns von J. Heckhausen und H. Heckhausen (2010, 3), das in Abbildung 14 dargestellt ist. Das Ziel dieses Modell ist es, die Motivation zu beschreiben, die man verstehen kann als ,(...) die (...) hypothetischen, aktivierenden und richtunggebenden Vorgänge, die das individuelle Verhalten auf ein Ziel hin bestimmen und regulieren“" (Tewes und Wildgrube 1999).

Mit J. Heckhausen und H. Heckhausen (2010, 3) ist Motivation das Produkt der Person, die bestimmte Bedürfnisse, Ziele, Absichten etc. hat, und der Situation, die durch Gelegenheiten, Möglichkeiten und Anreize charakterisiert 
werden kann (s. entsprechend Punkte 3, 1 und 2 in Abbildung 14). Mit anderen Worten: „Die aktuell vorhandene Motivation einer Person, ein bestimmtes Ziel anzustreben, wird von personenbezogenen und von situationsbezogenen Einflüssen geprägt" (J. Heckhausen und H. Heckhausen 2010, 3). Die vorhandene Motivation kann zu einer Handlung führen (4). Diese Handlung liefert bestimmte Ergebnisse (5), die wiederum mit bestimmten Folgen einhergehen (5).

Das Modell des motivierten Handelns kann man der Motivationspsychologie zuordnen (vgl. J. Heckhausen 2010, vii). In der Motivationspsychologie geht es insbesondere darum zu untersuchen, wie die Motivation von Individuen zum Handeln entsteht, welche Einflussfaktoren dabei eine Rolle spielen, wie Ziele ausgewählt werden, wie aus der Motivationstendenz (J. Heckhausen und H. Heckhausen 2010, 6-7), also der Bereitschaft zum Handeln, eine tatsächliche Handlung wird sowie wie Motivation aufrechterhalten werden kann (vgl. Motivation Wenninger 2001b, 91; J. Heckhausen und H. Heckhausen 2010, 4-7). Wendet man sich der Sprachlenkung wieder zu, so sind die hier genannten Aspekte interessant, um die Motivation zur Annahme der neuen Sprachform durch die aktive Zielgruppe zu erforschen. Dieses würde eher den Bottom-up-Blick auf den SL-Erfolg bedeuten. Ich entscheide mich in dieser Arbeit aber für die vorwiegend Top-down-Perspektive. Da ich nicht untersuche, wie die Motivation der Autoren, Sprachlenkung zu betreiben, entsteht oder aufrechterhalten werden kann, so sehe ich die hier genannten Aspekte der Motivationsforschung und insbesondere ihre Systematisierung als nicht zentral für die Konzeptualisierung des Top-down-Erfolges. Ich setze das Vorhandensein der Motivation bei Autoren voraus und gehe vielmehr der Frage nach, was die Autoren aktiv unternehmen können, um erfolgreich zu sein. Auch wenn ich nicht die Motivation der Autoren an sich betrachte, so benutze ich das Modell des motivierten Handelns in abgewandelter Form als Grundlage zur Konzeptualisierung des Top-down-Erfolges der Sprachlenkung, siehe hierfür Abbildung 15.

In Abbildung 15 ist Sprachlenkung in der Mitte dargestellt. Da Sprachlenkung eine Tätigkeit (Handlung) ist, lässt sie sich mithilfe des Modells von J. Heckhausen und H. Heckhausen $(2010,3)$ weiter unterteilen. Konkret und unter Berücksichtigung von Definition Sprachlenkung 1, Definition Sprachlenkung 2 sowie Erläuterungen in 3.4.1.1 stellt die kollektive, nachhaltige Sprachgebrauchsänderung das Ziel der Sprachlenkung dar. Die Ergebnisse beziehen sich dann auf die tatsächliche Sprachgebrauchsänderung. Zwischen den Zielen und Ergebnissen erfolgt die Phase der Zielverwirklichung, 


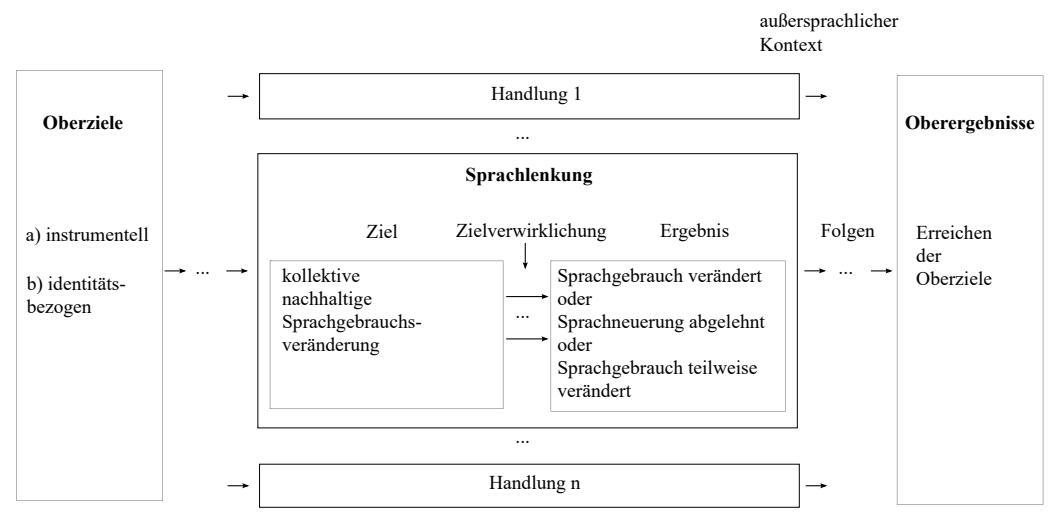

Abbildung 15.: Handlungstheoretische Fassung der Sprachlenkung und ihrer Einbettung, in Anlehnung an J. Heckhausen und H. Heckhausen $(2010,3)$

die aus mehreren Handlungen und Teilhandlungen besteht, was durch Pfeile und Auslassungszeichen angedeutet ist. Wie in Kapitel 3 zusammengefasst, ist Sprachlenkung in einen außersprachlichen Kontext eingebettet. In Abbildung 15 sind daher links die Oberziele dargestellt, die sich mit Kapitel 3 grob in instrumentelle und identitätsbezogene Ziele einteilen lassen. Die Pfeile und Auslassungszeichen zwischen den Oberzielen und der Sprachlenkung deuten an, dass es weitere, der Sprachlenkung übergeordnete, Ziele geben kann, die mittlere Stufen in der Zielhierarchie besetzen. Wie aus Abbildung 15 ersichtlich, stellt Sprachlenkung nur eine Handlung dar, um die übergeordneten Ziele zu verwirklichen. Weitere Handlungen sind daher mit Handlung 1, Handlung $n$ und Auslassungzeichen angedeutet.

Da jede Handlung nach Modell von J. Heckhausen und H. Heckhausen (2010) Folgen hat, sind diese für Sprachlenkung rechts angedeutet. Diese Folgen beziehen sich auf die übergeordneten Ziele. Die Gesamtheit der Folgen aller Handlungen trägt zum Erreichen der Oberziele bei. In Abbildung 15 sind daher rechts Oberergebnisse als Pendant zu Oberzielen dargestellt. Aus der Sicht der Sprachlenkung sind diese Oberergebnisse wiederum mittelbare Handlungsfolgen, die aber, wie erwähnt, nicht auf die Sprachlenkung allein zurückgehen.

Nach diesen handlungstheoretischen Vorüberlegungen zur Sprachlenkung ist es nun möglich, den Erfolg von Sprachlenkungsmaßnahmen zu definieren. 
Hierbei beziehe ich mich nun ausschließlich auf den Kernbereich der Sprachlenkung, wie in der Mitte der Abbildung 15 dargestellt:

erfolgreiche Sprachlenkungsmaßnahme 1. Eine Sprachlenkungsmaßnahme ist genau dann erfolgreich, wenn eine kollektive nachhaltige Veränderung des Sprachgebrauchs sowohl das Ziel als auch das Ergebnis darstellt. ${ }^{111}$

$\begin{array}{cccc} & \text { Ziel: } & & \begin{array}{c}\text { Ergebnis: } \\ \text { kollektive } \\ \text { kollektive }\end{array} \\ \begin{array}{c}\text { Sprachlenkungs- } \\ \text { maßnahme }\end{array} & & \text { nachhaltige } & \text { Veränderung } \\ \text { des Sprachgebrauchs } & & \text { nachhaltige } \\ \text { Veränderung } \\ \text { des Sprachgebrauchs }\end{array}$

Tabelle 11.: Definition Erfolgreiche Sprachlenkungsmaßnahme, formal aufgeschrieben

\subsubsection{Erläuterungen und weiterführende Überlegungen}

Wie bereits in der LPLP-Literatur angedeutet, handelt es sich bei der Frage nach dem Erfolg allgemein um einen Vergleich von dem, was erreicht werden sollte und dem, was tatsächlich erreicht wurde. Auch in meiner Konzeptualisierung mache ich mir diese Vorstellung zunutze und postuliere zur Erfolgsmessung einen Vergleich von Zielen und Ergebnissen einer Sprachlenkungsmaßnahme.

In Definition Sprachlenkung 1 gebe ich eine (kollektive, nachhaltige) Sprachgebrauchsänderung als Ziel der Sprachlenkung an. Gegen genau dieses Ziel müssen dann die Ergebnisse in erster Linie geprüft werden. Dies bedeutet insbesondere, dass ich den Erfolg der Sprachlenkung nicht durch das Erreichen von übergeordneten (außersprachlichen) Zielen bestimme. Das Ausklammern von (außersprachlichen) Folgen aus dem Erfolgsbegriff hat also zunächst theoretische Gründe, da das Sprachlenkungsziel wie in Definition Sprachlenkung 1 festgesetzt ist. Zwar setze ich die Einbettung der Sprachlenkung in außersprachliche Handlungen voraus und bringe sie auch

111 Diese Definition enthält eine Redundanz, denn aus der Tatsache, dass Sprachlenkungsmaßnahme Instanz der Sprachlenkung ist (s. Definition Sprachlenkungsmaßnahme 1), folgt unmittelbar, dass ihr Ziel eine kollektive nachhaltige Veränderung des Sprachgebrauchs ist. Dadurch kann die Ziel-Bedingung nie falsch werden (vgl. Definition Erfolgreiche Sprachlenkungsmaßnahme, formal aufgeschrieben). Ich belasse dies jedoch aus Lesbarkeitsgründen so. 


\section{Umsetzung und Erfolg der Sprachlenkung}

mit der Vorstellung in Verbindung, dass eine erfolgreiche Sprachlenkung das Erreichen von übergeordneten, außersprachlichen Zielen unterstützen soll. Für den Erfolg von Sprachlenkungsmaßnahmen im engeren Sinne ist es aber unerheblich, ob die (außersprachlichen) Oberziele erreicht werden. Eine Frage nach Wirksamkeit der Sprachlenkung zur Erreichung von außersprachlichen Zielen ist selbstverständlich legitim. Sie stellt für mich jedoch ein weiterführendes Thema dar, das in der Praxis zudem den hier behandelten rein sprachlichen Erfolg voraussetzt. Darüber hinaus vertrete ich in dieser Arbeit eine ausgesprochen Top-down-Perspektive auf den Erfolg.

Die oben angegebene Auffassung von Erfolg ist aus der Theorie hergeleitet, gibt aber noch nicht die Varianz der Praxis wieder. Wie aus Überlegungen von D. Blanke (2006, 2009a) zum Esperanto deutlich, kann auf die Erfolgsfrage einer Maßnahme oft keine simple Ja-nein-Antwort gegeben werden, da die Problematik viel differenzierter betrachtet werden muss (vgl. auch Ricento 2009, 228). Hierfür halte ich die Überlegungen zur aktiven Zielgruppe und zu pragmalinguistischen Einschränkungen des Geltungsbereiches, wie in Abschnitt 3.4.2.2 beschrieben, für hilfreich. Möchte man die Fragen nach dem Erfolg beantworten oder gar den Erfolg messen, so muss ausgehend von der oberen Konzeptualisierung präzisiert werden, wessen Sprachgebrauch und was genau an diesem Sprachgebrauch verändert werden soll. Als Zusammenfassung der Überlegungen in Abschnitt 3.4.2.2 schlage ich hierfür folgende Aspekte vor, die sich zunächst auf den Sprachgebrauch aus der Zielperspektive beziehen, aber auch zur Erfassung der Ergebnisse benutzt werden können:

- aktive Zielgruppe: Wessen Sprachgebrauch soll verändert werden?

- Situation

- Rezipienten: Für Kommunikation mit welchen Rezipienten soll der veränderte Sprachgebrauch gelten?

- Öffentlichkeit: Für welchen Öffentlichkeitsbereich soll der veränderte Sprachgebrauch gelten?

- Textsorte: Ist der veränderte Sprachgebrauch nur für bestimmte Textsorten gültig?

- Modus: Bezieht sich die Sprachlenkung nur auf den mündlichen oder schriftlichen Gebrauch?

Je präziser diese Fragen bei der Entwicklung der Maßnahme beantwortet werden, umso besser lassen sich diesbezüglich die Ergebnisse dieser Maßnah- 
me evaluieren. Dieser Vergleich stellt dann die Grundlage zur Beantwortung der Frage dar, ob eine Sprachlenkungsmaßnahme erfolgreich war oder nicht. Bereits die Evaluierung einzelner Aspekte ermöglicht eine Abstufung des Erfolges, falls nur einige Punkte erreicht wurden. Eine feinere Abstufung kann durch Abstufung einzelner Aspekte erreicht werden, z. B. durch die quantitative Auswertung, wie viele Personen in der aktiven Zielgruppe ihren Sprachgebrauch bereits tatsächlich veränderten.

Dies sind allgemeine Überlegungen, wie man den vagen Begriff des relativen Erfolges einer Sprachlenkungsmaßnahme präzisieren oder gar operationalisieren kann. Sie müssen für konkrete Praxis- oder Forschungsprojekte weiterpräzisiert werden. Ich gehe in dieser Arbeit nicht weiter auf dieses Thema ein.

\subsection{Forschungsannahmen}

In der qualitativen Metaanalyse, die in Kapitel 5 erfolgt, geht es also um die Frage, wie das Ziel der Sprachlenkung - kollektive, nachhaltige Sprachgebrauchsänderung - durch die Autoren erreicht werden kann. Mit anderen Worten: Wie können die Autoren den Weg von Zielen auf die Ergebnisse hin aktiv gestalten?

In diesem Abschnitt formuliere ich zwei Forschungsannahmen. Bei diesen Annahmen handelt es sich nicht um Forschungshypothesen, da es in der Untersuchung nicht vordergründig darum geht, diese zu bestätigen oder zu widerlegen. Vielmehr sollen sie die Auswertungs- und Interpretationsperspektive eingrenzen und fokussieren und die Betrachtung der Sprachlenkung in den sozialpsychologischen Kontext setzen.

Annahme 1. Die Gruppenidentität der aktiven Zielgruppe ist bei Sprachlenkung salient. ${ }^{112}$

Ich gehe dabei davon aus, dass die Identität der Mitglieder der aktiven Zielgruppe bereits salient ist oder durch Sprachlenkung erst salient gemacht wird. Der erste Fall kommt beispielsweise dann vor, wenn eine explizite Auseinandersetzung mit der Identitätsproblematik stattfindet, bevor die Veränderung des Sprachgebrauchs konkret diskutiert wird, d. h. wenn Sprachlenkung explizit als Mittel zur Erreichung eines identitätsbezogenen Oberzieles gesehen

112 Unter Salienz versteht man „Aktuelle Zugänglichkeit (Bewusstheit) von Kognitionen“ (Haddock und Maio 2007, 193). 


\section{Umsetzung und Erfolg der Sprachlenkung}

wird. Im zweiten Fall ruft die Sprachlenkung diese Auseinandersetzungen hervor.

Da die Identität salient ist, ergibt sich:

Annahme 2. Sprachlenkung setzt Gruppenprozesse in Gang.

Um die Sprachlenkung und ihre Umsetzung besser zu verstehen, sollen folglich die dabei ablaufenden Gruppenprozesse stärker mit betrachtet werden.

Obwohl die Annahmen in dieser Arbeit nicht empirisch gesichert werden, ist es trotzdem wichtig, sie allgemein auf Plausibilität hin zu untersuchen. Teilweise ergeben sie sich aus dem Forschungsstand. Insbesondere ist der Teil der Annahme 1 in Ager (2001) zu finden. Trotzdem bedürfen die Annahmen weiterer Erläuterungen. Aus diesem Grund stelle ich in den folgenden Abschnitten die Konzepte der Identität, der Gruppe und der Einstellungen in der Sozialpsychologie vor und gehe insbesondere auf die Arten der Identität, verschiedene Gruppenprozesse sowie Mechanismen der Verhaltens- und Einstellungsänderungen ein. Diesen theoretischen Einschub rundet dann die linguistische Perspektive auf die Wechselwirkung zwischen Sprache und Identität $a b$, die die Untermauerung beider Forschungsannahmen zusätzlich stützt.

Bei sozialpsychologischen Themen greife ich auf die Lehrwerke der Sozialpsychologie von Jonas, Stroebe und Hewstone $(2007,2014)$ sowie Werth und J. Mayer (2008) und Stürmer (2009) zurück und orientiere mich daran sowohl in Bezug auf Inhalte als auch ihre Strukturierung.

\subsection{Identität}

In der Sozialpsychologie wird sowohl von Identität als auch vom Selbst gesprochen. Mit Stürmer (2009) handelt sich dabei um keine getrennten Konzepte, sondern lediglich um einen unterschiedlichen Sprachgebrauch, der auf unterschiedliche Forschungstraditionen zurückgeht: Während Selbst aus der nordamerikanischen Kognitionsforschung stammt, kommt Identität eher aus der europäischen Forschung und wird in der Sozialpsychologie vor allem im Zusammenhang mit der Theorie der sozialen Identität verwendet (Stürmer 2009, 52). In diesem Abschnitt halte mich weitestgehend am Sprachgebrauch der angeführten Literatur.

Unter dem Selbst versteht man in der Sozialpsychologie,,(...) die Gesamtheit des Wissens über das eine Person bzgl. ihrer Selbst und ihres Platzes in der 
sozialen Welt verfügt" (Stürmer 2009, 52). Zusätzlich zu diesem sog. Selbstbild umfasst das Selbst auch ,(...) das prozesshafte Geschehen der Selbstwahrnehmung (...), Selbstaufmerksamkeit und Selbstregulation" (Werth und J. Mayer 2008, 165). Davon kann man drei Hauptfunktionen von Selbst ableiten: die strukturierende Funktion (Aufbau und Organisation des Wissens über sich selbst), die emotionale Funktion (Aufbau und Schutz des Selbstwertgefühls) sowie die konative (regulierende/ausführende) Funktion (Beeinflussung und Regulation der Informationsverarbeitung, des Verhaltens etc.) (Werth und J. Mayer 2008, 165; vgl. Simon und Trötschel 2007). Diese Aufteilung erinnert an die drei Einstellungskomponenten - kognitive, affektive und konative.

Im Folgenden fasse ich die drei Funktionen des Selbst zusammen. Ich orientiere mich dabei stark am Aufsatz von Simon und Trötschel (2007) und ziehe des Weiteren die Arbeiten von Werth und J. Mayer (2008), Fischer und Wiswede (2009) und Stürmer (2009) heran.

\subsubsection{Kognitive Funktion: das Selbstbild}

Eine wichtige Rolle in der Sozialpsychologie nahm schon immer die Erforschung der Einschätzungen einer Person über sich selbst an (Petersen, Stahlberg und Frey 2006, 40), deren Summe als Selbstbild oder Selbstkonzept bezeichnet wird. Die Einschätzungen können sowohl die äußeren Attribute wie Alter, Geschlecht, Haarfarbe betreffen wie auch die eigene Position, bspw. „Ich bin Schüler“, „Ich liebe Blumen“, beschreiben (Fischer und Wiswede 2009, 394-395).

Im Allgemeinen ist das Selbstkonzept eine „,...) kognitive Repräsentation des Selbst, die die eigenen Erfahrungen mit Kohärenz und Sinn erfüllt, einschließlich der sozialen Beziehungen, die man zu anderen Menschen hat. Das Selbstkonzept organisiert frühere Erfahrungen und trägt dazu bei, relevante Stimuli in der sozialen Umwelt zu erkennen und zu interpretieren" (Simon und Trötschel 2007, 152). Diese Definition schneidet wichtige Aspekte des Selbstbildes an, die ich im Folgenden kurz bespreche.

Wie Werth und J. Mayer (2008) erläutern, besteht das Selbstkonzept nicht nur aus der aktuellen Einschätzung, sondern ist auch durch die vorangegangenen Erfahrungen und das vergangene Selbst geprägt. Darüber hinaus ist für das Selbstbild ebenfalls das künftige Selbst von Bedeutung, d. h. die Vorstellung, wie man in Zukunft sein möchte aber auch sein sollte (Werth und J. Mayer 2008, 165; vgl. Simon und Trötschel 2007, 164). 


\section{Umsetzung und Erfolg der Sprachlenkung}

Des Weiteren machen Simon und Trötschel (2007) darauf aufmerksam, dass das Selbstbild auch die Beziehungen zu anderen Personen umfasst bis hin zur Integration von anderen Personen in das Selbstbild. Dies ist v. a. bei wichtigen Personen wie Partnern oder Familie der Fall, aber auch bei abstrakten Gegenständen wie Idealen (Simon und Trötschel 2007, 171). Diese Integration von anderen Personen und die damit verbundene Erweiterung des Selbst hat eine Auswirkung auf die Verarbeitung selbstbezogener Informationen. Die Mechanismen des Wechsels zwischen der individuellen und der sozialen Identität werden in der Selbstkategorisierungstheorie (J. C. Turner 1987) beschrieben, auf die ich in Abschnitt 4.5.4.2 eingehe.

Schließlich geht die angeführte Definition auf die kognitive, strukturierende Funktion des Selbstkonzepts ein. Wie Werth und J. Mayer (2008) präzisieren, hilft es, die selbstbezogene und umweltbezogene Information zu verarbeiten und zu organisieren (Werth und J. Mayer 2008, 165).

Aus den bisherigen Ausführungen folgt, dass das Selbstbild aus einer Vielzahl von unterschiedlichen heterogenen Einschätzungen besteht. Man geht zudem davon aus, dass diese Informationseinheiten miteinander verknüpft sind, so dass das Selbst eine Struktur ergibt. ${ }^{113}$ Normalerweise greift man jedoch nur auf einen Teil des Selbstbildes zurück oder man hat je nach Situation teilweise ein ganz anderes Bild von sich selbst (Werth und J. Mayer 2008, 163) bis hin zur Erfahrung, sich inkonsistent und widersprüchlich über die Zeit hinweg zu verhalten (Fischer und Wiswede 2009, 391). Trotz dieser hohen Variabilität ist man imstande, sich über die Zeit hinweg sowohl vergangenheits-, gegenwarts- als auch zukunftsgerichtet als dieselbe Person zu identifizieren (Simon und Trötschel 2007, 159). Man spricht deshalb von sog. Kontinuität des Selbst (Simon und Trötschel 2007, 159), die durch verschiedene Faktoren unterstützt wird (dazu bspw. Stürmer 2009, 61-62).

Entgegen einer Alltagsvorstellung ist das Selbst also weder statisch noch fest zusammengefügt, und nicht alle Selbstvarianten werden auf einmal aktiviert (Fischer und Wiswede 2009, 391; Stürmer 2009, 58-59; Werth und J. Mayer 2008, 171). Es werden in der Literatur verschiedene Faktoren genannt, die die Selbstaktivierung beeinflussen (s.a. Selbstkategorisierungstheorie). Werth und J. Mayer (2008) unterschieden hierbei zwischen chronischen und temporären Aktivierungsquellen, die folglich zusammengefasst werden können (Werth und J. Mayer 2008, 171-174):

113 Zur Struktur des Selbst s. bspw. Theorie der Selbstschemata (Markus 1977; vgl. Simon und Trötschel 2007, 152-155; Stürmer 2009, 55-57) oder Theorie der Selbstkomplexität und Selbstaspekte (Linville 1987; vgl. Simon und Trötschel 2007, 155). 
- Chronische Aktivierungsquellen: 1) offensichtliche/äußere Merkmale: z. B. Geschlecht, Rasse, Größe 2) abweichende Merkmale: z. B. Linkshänder aber auch Zugehörigkeit zu einer dominierten Gruppe wie politische/religiöse Minderheit; 3) Entwicklungsaufgaben über die Lebensspanne hinweg: z. B. Eltern sein, arbeitslos sein

- Temporäre Aktivierungsquellen: 1) Stimmungen; 2) Verhalten; 3) selbstbezogene Ziele; 4) situationsbedingte Auffälligkeit (Distinktheit/Salienz): z. B. einzige Frau unter Männern, aber auch Kleidungsstil; 5) Intergruppenvergleiche; 6) individuelle Aktivierungstendenz

Darüber hinaus erklärt die Theorie der Optimalen Distinktheit von Brewer (1991), dass Menschen sich im Spannungsfeld zwischen dem Bedürfnis nach Assimilation und dem nach Differenzierung befinden (Fischer und Wiswede 2009, 407). Für das Selbst bedeutet das, ,dass die jeweilige Identität in einer solchen Weise aktiviert wird, dass beiden Bedürfnissen in optimaler Weise Rechnung getragen wird“" (Fischer und Wiswede 2009, 407).

In der Literatur werden üblicherweise folgende Quellen genannt, die zum Aufbau des Selbstbilds herangezogen werden (Werth und J. Mayer 2008, 175-189; vgl. Simon und Trötschel 2007, 156):

- Introspektion, also die Analyse der eigenen Gedanken, Gefühle, Motive etc. (Simon und Trötschel 2007, 156; Stürmer 2009, 53), wodurch aber kein endgültiges Wissen über das Selbst entsteht (Simon und Trötschel 2007, 156).

- Beobachtung des eignen Verhaltens oder eigener Reaktionen, wie beispielsweise in der Selbstwahrnehmungstheorie von Bem (1972) beschrieben (s. dazu Simon und Trötschel 2007, 157; Werth und J. Mayer 2008, 177-183; Stürmer 2009, 53).

- Analyse von Fremdwahrnehmung und -reaktionen, was einerseits die tatsächlich geäußerten Meinungen umfasst (Simon und Trötschel 2007, 157; vgl. Werth und J. Mayer 2008, 163-189; Fischer und Wiswede 2009, 401-402) und andererseits auf dem Sich-Hineinversetzen in eine fremde Person beruht (Stürmer 2009, 54). Diese werden in der Theorie des sozialen Vergleichs erklärt, auf die ich im Folgenden eingehe. 


\section{Umsetzung und Erfolg der Sprachlenkung}

Wie Simon und Trötschel $(2007,157)$ erläutern, besagt die Theorie des sozialen Vergleichs von Festinger (1954), dass man die eigenen Fähigkeiten, Fertigkeiten oder andere Attribute mit denen der anderen Personen vergleicht, entweder um etwas Neues über sich selbst zu erfahren oder „die Gültigkeit und Angemessenheit [der] (...) Wahrnehmung, Einstellungen, [Gefühle] etc. zu überprüfen“" (Stürmer 2009, 54). Je nach Vergleichsperson, dem sog. Priming oder anderen Hinweisreizen wird entweder auf Ähnlichkeiten oder Unterschiede fokussiert (Werth und J. Mayer 2008, 184-187). Im Kontext des Selbst kann ein Vergleich zur Assimilation (Annäherung des eigenen Urteils an den Vergleichsstandard) oder zum Kontrast (Distanzierung vom Standard) führen (Werth und J. Mayer 2008, 184); wichtige und als zutreffend angesehenen Erkenntnisse werden in das eigene Selbstbild integriert (Simon und Trötschel 2007, 157). Zusammenfassend kann man sagen, dass die Neigung der Menschen zum Vergleich teilweise individuell, teilweise aber situationsabhängig ist, wenn beispielsweise ein objektiver Maßstab bezüglich eines Standards fehlt (vgl. Werth und J. Mayer 2008, 184; Stürmer 2009, 54).

\subsubsection{Affektive Funktion: das Selbstwertgefühl}

Obwohl die bisher beschriebenen Inhalte des Selbst, die sich zu einem Selbstbild zusammenfügen, eine wichtige Komponente des Selbst sind, spielt die Bewertung dieser Inhalte ebenfalls eine wichtige Rolle. Das Selbstwertgefühl ist das Resultat dieser subjektiven Bewertung einzelner Inhalte des Selbstbildes ,(...) auf einer Dimension, die von negativ bis positiv reicht (Stürmer 2009, 52; vgl. Simon und Trötschel 2007, 167; Werth und J. Mayer 2008, 165). „Das Selbstwertgefühl bezieht sich darauf, wie gut sich eine Person selbst akzeptieren kann bzw. wie sie ihren Wert in ihrer sozialen Umwelt einschätzt“ (Fischer und Wiswede 2009, 395). Dabei kann man zwei Arten von Selbstwertgefühl unterschieden: Das habituelle, das im Erwachsenalter relativ stabil ist, und das situative, das durch Umstände oder aktuelle Motive aktiviert wird (Petersen, Stahlberg und Frey 2006, 40). Die Quellen des Selbstwertgefühls sind die gleichen wie bei der Selbsterkenntnis: Selbstwahrnehmung, soziale Rückmeldung und sozialer Vergleich (Petersen, Stahlberg und Frey 2006, 41).

Das Selbstwertgefühl unterstützt die soziale Interaktion und kann zur Leistungsverbesserung führen (Simon und Trötschel 2007, 171). Zudem wirkt sich sein emotionaler und motivationaler Aspekt entscheidend auf das Verhalten aus (Werth und J. Mayer 2008, 165). Das Selbstwertgefühl spielt auch 
bei der Bedrohung des Selbst eine Rolle und wirkt bei Selbstwertschutzmechanismen mit.

In der Sozialpsychologie geht man davon aus, dass gesunde Menschen generell ein positives Selbstbild anstreben und diese Grundannahme ist in vielen Theorien wiederzufinden (z. B. Theorie der Sozialen Identität von Henry Tajfel und J. Turner (1979)). Das Streben nach einem positiven Selbstbild geht mit einem hohen Selbstwertgefühl einher (bspw. Fischer und Wiswede 2009, 408, 409, 417). ${ }^{114}$

\subsubsection{Konative Funktion}

In den vergangenen Abschnitten widmete ich mich vorwiegend dem Selbst als Ergebnis der Umweltprozesse, das zwar eine dynamische Qualität hat, aber eher als Informationsquelle zur Verfügung steht. In diesem Abschnitt verschiebt sich das Augenmerk auf die Rolle des Selbst bei der Regulierung des Verhaltens (Stürmer 2009, 62) und auf dessen Einfluss auf Entscheidungsfindung und Zielsetzung (Werth und J. Mayer 2008, 169).

Wie beispielsweise Werth und J. Mayer (2008) erläutern, stellt die sog. Selbstaufmerksamkeit eine wichtige Grundlage für die Regulationsprozesse dar, bei der sich der Fokus des Menschen von der Umwelt auf sich selbst verschiebt. Dies kann durch individuelle Veranlagung aber auch durch situative Anreize erfolgen (wie Spiegel, Aufzeichnung der eigenen Stimme oder Fragen nach dem Befinden) (Werth und J. Mayer 2008, 170; vgl. Simon und Trötschel 2007, 161; Fischer und Wiswede 2009, 396-397). Die Erkenntnisse, die aus der Selbstaufmerksamkeit gewonnen werden, können dann zur Anpassung des Verhaltens eingesetzt werden, z. B. in Form von Selbstüberwachung (Simon und Trötschel 2007, 163; Fischer und Wiswede 2009, 396; Stürmer 2009, 63), Selbstregulation (Simon und Trötschel 2007, 164-165; Werth und J. Mayer 2008, 169; Fischer und Wiswede 2009, 423; Stürmer 2009, 63-65) oder Selbstwerterhaltung (Simon und Trötschel 2007, 167-168; Stürmer 2009, 65-66). Wie diese Anpassung erfolgt, wird durch verschiedene Theorien dargestellt. Ich gehe hier nur auf die Selbstaufmerksamkeitstheorie von Duval und Wicklund (1972) ein und verweise bei anderen Theorien auf entsprechende Quellen:

114 Diese Annahme wird in verschiedenen Theorien gemacht, beispielsweise Evolutionstheorien (s. bspw. Simon und Trötschel 2007, 167), Terror Management Theory (bspw. Werth und J. Mayer 2008, 191; Fischer und Wiswede 2009, 417). 
- Selbstaufmerksamkeitstheorie (Duval und Wicklund 1972; s. bspw. Simon und Trötschel 2007, 161; Stürmer 2009, 63): Diese Theorie besagt, dass die Menschen im Zustand der Selbstaufmerksamkeit ihr Verhalten, ihre Erscheinung oder Eigenschaften an eigenen Standards oder sozialen Normen messen (Simon und Trötschel 2007, 161). Dabei entdecken sie Diskrepanzen zwischen dem Ist- und Soll-Zustand, die oft zu negativen Emotionen oder Selbstwertbedrohung führen können (Simon und Trötschel 2007, 161). Es können auch positive Diskrepanzen festgestellt werden, was zu Erhöhung des Selbstwertgefühls führt (Stürmer 2009, 62-63). Im Fall von selbstwertbedrohlichen Diskrepanzen können verschiedene Strategien eingesetzt werden z. B. ein Rückzug aus der Selbstaufmerksamkeit oder eine Verringerung der Diskrepanz durch die Anpassung des eigenen Verhaltens (Simon und Trötschel 2007, 161; vgl. Stürmer 2009, 63).

- Selbstdiskrepanztheorie (Higgins 1987; s. bspw. Simon und Trötschel 2007, 164-165; Stürmer 2009, 64-65)

- Modell der Selbstwerterhaltung (Tesser 1988; vgl. Simon und Trötschel 2007, 168; Fischer und Wiswede 2009, 414-415; Stürmer 2009, 65-66)

- Selbstwerterhaltungsstrategien: Selbstbestätigung (s. bspw. Simon und Trötschel 2007, 169), selbstwertdienliche Vergleiche (Simon und Trötschel 2007, 169; Werth und J. Mayer 2008, 194-196; Fischer und Wiswede 2009, 408), selbstwertdienliche Attributionen (Simon und Trötschel 2007, 169; Werth und J. Mayer 2008, 196-201; Fischer und Wiswede 2009,408$)$ )

\subsubsection{Theorien der Gruppenidentität}

Die beschriebenen Theorien erklären jeweils ein Phänomen, das das Selbst charakterisiert. Eine Theorie, die versucht, sowohl kognitive als auch regulierende Elemente des Selbst miteinander zu verknüpfen und das menschliche Verhalten im sozialen Kontext mithilfe des Identitätsbegriffs zu betrachten, ist die Selbstkategorisierungstheorie von J. C. Turner (1987) und ihr Vorläufer, die Theorie der sozialen Identität von Henry Tajfel und J. Turner (1979). Beide Theorien charakterisiere ich kurz im Folgenden. 


\subsubsection{Theorie der sozialen Identität}

Mit Simon und Trötschel (2007, 172-173) besagt die Theorie der sozialen Identität, dass einen wichtigen Teil der Identität die soziale Identität ausmacht. Die soziale Identität wird definiert als ,(...) der Teil des Selbstkonzepts einer Person, der sich aus dem Wissen über die Mitgliedschaft in einer sozialen Gruppe (oder sozialen Gruppen) und dem Wert und der emotionalen Bedeutung dieser Mitgliedschaft ableitet“" (Simon und Trötschel 2007, 172). Und weiter: „Soziale Identität umfasst die Selbstdefinition als austauschbares Gruppenmitglied im Sinne der Unterscheidung zwischen Eigengruppe und Fremdgruppe (,wir“ im Unterschied zu ,sie, die anderen“) (Simon und Trötschel 2007, 172).

Die Theorie eignet sich gut zur Analyse von Intergruppenbeziehungen und erklärt beispielsweise die Bevorzugung und eine positive Sicht der eigenen Gruppe, da man das Bedürfnis nach einer positiven Identität hat (Simon und Trötschel 2007, 172-173). Darüber hinaus kann die Theorie der sozialen Identität zur Beschreibung der Prozesse bei unterprivilegierten Gruppen eingesetzt werden, die eine negative soziale Identität haben (Simon und Trötschel 2007, 173).

\subsubsection{Selbstkategorisierungstheorie}

Wie Simon und Trötschel (2007) konstatieren, war die Theorie der sozialen Identität nicht als eine übergreifende Theorie des Selbst gedacht. So stellt die Selbstkategorisierungstheorie ihre Verallgemeinerung sowie eine Erweiterung um die persönliche Perspektive dar (Simon und Trötschel 2007, 173). Im Gegensatz zur sozialen Identität, die auf den Unterschieden zwischen der Eigen- und Fremdgruppe beruht (Simon und Trötschel 2007, 173), bedeutet die persönliche Identität die ,(...) Selbstdefinition als einzigartiges und unverwechselbares Individuum, die auf einer interpersonalen (oder intragruppalen Differenzierung auf der Basis individueller Merkmale beruht (,,ich“ vs. „du“ oder ,ihr“)“ (Stürmer 2009, 59; vgl. Simon und Trötschel 2007, 173).

Die Selbstkategorisierungstheorie gibt die Vorbedingungen und Konsequenzen der persönlichen und sozialen Identität an und erklärt somit das individuelle und das kollektive Verhalten (Simon und Trötschel 2007, 173). Die Grundannahmen der Theorie fassen Simon und Trötschel (2007, 173) wie folgt zusammen:

1. Persönliche und soziale Identität sind Ergebnisse von Selbstkategorisierungsprozessen; 
2. „Identitäten können auf verschiedenen Ebenen der sozialen Inklusivität konstruiert werden“" (Simon und Trötschel 2007, 173);

3. Identitäten sind relativ;

4. nicht alle Identitäten sind gleichzeitig aktiv.

1 Die Selbstkategorisierung ist ein Prozess und bedeutet „(...) die Bildung kognitiver Gruppierungen bestehend aus der eigenen Person sowie weiteren Personen in Abgrenzung zu Gruppierungen bestehend aus anderen Personen“ (Simon und Trötschel 2007, 173). Die Theorie betont die subjektivistische Konstruktion der Identität (vgl. Simon und Trötschel 2007, 173).

2 Jede Person kann sich anhand unterschiedlicher Eigenschaften charakterisieren, und Kombinationen von bestimmten Eigenschaftsausprägungen machen eine Identität aus. Dies bedeutet, dass jeder mehrere Identitäten haben kann (J. C. Turner 1987, 44; vgl. Fischer und Wiswede 2009, 421). Diese unterschiedlichen Identitäten sind hierarchisch organisiert: „Jede Kategorie [Identität] ist vollständig in einer anderen abstrakteren Kategorie zu subsumieren, ohne für diese erschöpfend zu sein" (Fischer und Wiswede 2009, 421; vgl. J. C. Turner 1987, 45). Als Beispiel nennen Simon und Trötschel (2007): Die Identität Deutscher ist in der Identität Bürger Europas enthalten; gleichzeitig ist die Identität Deutscher abstrakter als die persönliche Identität und deshalb umfasst die erste die zweite (Simon und Trötschel 2007, 176).

Darüber hinaus muss die gesamte Identität mindestens drei Ebenen umfassen: 1. als menschliches Wesen; 2. als Gruppenmitglied; 3. als einzigartiges Individuum (J. C. Turner 1987, 45-46; vgl. Fischer und Wiswede 2009, 421).

3 Identitäten sind relativ und Ergebnis eines Vergleichs: Es wird immer auf der gleichen Ebene, aber stets in Bezug auf die soziale Identität der nächst inklusiveren Ebene verglichen (Simon und Trötschel 2007, 176-177; vgl. J. C. Turner 1987, 44): Man kann ,(...) die eigene soziale Identität als Bürger Deutschlands (...) beispielsweise mit Bürgern Italiens in Bezug auf Merkmale vergleichen und bewerten, die ganz allgemein für Europäer (...) charakteristisch sind" (Simon und Trötschel 2007, 177).

4 Die Identitäten und ihre Variationen entstehen durch die Vielfalt der Gruppenmitgliedschaft. Nicht alle Identitäten sind gleichzeitig aktiviert (Fischer 
und Wiswede 2009, 178): „Die Salienz einer Identität hängt sowohl von der Bereitschaft der Person ab, eine bestimmte Identität anzunehmen, als auch davon, wie die Identität zum sozialen Kontext passt" (Simon und Trötschel 2007, 173; vgl. J. C. Turner 1987, 55). Die Bereitschaft zur Annahme einer bestimmten Identität hängt wiederum von allgemeinen Werten, sich verändernden Motiven, Zielen, Vorerfahrungen mit der Gruppe, Bedürfnissen nach Assimilation oder Differenzierung ab (Simon und Trötschel 2007, 177). Mit Fischer und Wiswede (2009) besagen dabei die wichtigsten Hypothesen, dass sich die Selbstkategorisierung zwischen zwei Polen bewegt: Annahme von ausschließlich persönlicher oder ausschließlich gruppaler Identität. Die Salienz der In-Group-Kategorisierung bedeutet die Eigenwahrnehmung als ähnliches oder gar austauschbares Mitglied einer Gruppe und führt zur sog. Depersonalisierung (Fischer und Wiswede 2009, 422; vgl. Simon und Trötschel 2007, 178). Die Depersonalisierung geht dabei einher mit der „(...) Betonung von intragruppalen Ähnlichkeiten und intergruppalen Unterschieden" (Simon und Trötschel 2007, 178; vgl. J. C. Turner 1987, 50). Die persönliche Identität unterliegt hingegen umgekehrten Prinzipien: Es werden die eigene Konsistenz akzentuiert und die interindividuellen Unterschiede betont (Simon und Trötschel 2007, 178).

Die Konsequenzen aus dieser integrativen, dynamischen Sicht auf die Identität sind für die Forschung vielfältig: „Eine wichtige Schlussfolgerung aus dieser Sichtweise besteht darin, dass das Selbst nicht im Sinne beständiger, absoluter Eigenschaften wie Selbstschemata (...) oder Selbstaspekte (...) repräsentiert wird, sondern im Sinne relationaler, wechselnder Selbstkategorien oder Identitäten“" (Simon und Trötschel 2007, 178)

\subsection{Gruppe}

In diesem Abschnitt fasse ich die Erkenntnisse über Gruppen und Gruppendynamik in der Sozialpsychologie zusammen. Ich stütze mich im Wesentlichen auf den Aufsatz von Nijstad und Knippenberg (2007) (s. dazu auch Nijstad und Knippenberg 2014) und ergänzend wieder auf die Arbeiten von Fischer und Wiswede $(2002,2009)$ und Werth und J. Mayer (2008).

\subsubsection{Was ist eine Gruppe?}

In der Alltagssprache werden unter Gruppe viele verschiedene, oft ungleichwertige Phänomene zusammengefasst, beispielsweise: Firmenabteilung, 


\section{Umsetzung und Erfolg der Sprachlenkung}

Sportverein oder aber auch Frauen oder Gaffer (Fischer und Wiswede 2002, 584; vgl. Rechtien 2003, 103-104). In der Sozialpsychologie und auch für diese Arbeit ist es notwendig, zwischen verschiedenen Formen von „Gruppierungen“ zu unterschieden, da nicht in jeder der Formen eine typische Gruppendynamik in vollem Maß stattfindet.

Die allgemeine Definition der Gruppe besagt, dass es mindestens zwei Menschen gibt, die sich selbst als zusammengehörend wahrnehmen (Fischer und Wiswede 2002, 585; Nijstad und Knippenberg 2007, 411, 2014, 440) (s. auch Abschnitt 3.4.1.1). Dabei halten es manche Autoren für erheblich, dass eine (insbesondere Face-to-Face-) Interaktion, also ein ,zielgerichtetes Miteinander" (Werth und J. Mayer 2008, 334) zwischen diesen Menschen besteht. Fischer und Wiswede $(2002,585)$ stellen fest, dass diese Gruppenauffassung den subjektivistischen Aspekt betont, da die wahrgenommene Zugehörigkeit in den Vordergrund tritt (vgl. Nijstad und Knippenberg 2007, 413, 2014, 441), und sie sich so von der Auffassung einer Gruppe als eines ,von oben“ gegebenen und unabhängig existierenden Systems absetzt (Fischer und Wiswede 2002, 585): „Ein solcher Gruppenbegriff transzendiert das übliche Gruppenverständnis der herkömmlichen Kleingruppenforschung, kann jedoch für besondere Problemstellungen zweckmäßig sein, sofern die soziale Identität für das Denken, Fühlen und Handeln der beteiligten Personen bedeutungsvoll ist"“ (Fischer und Wiswede 2002, 586). Nicht alle Autoren sehen jedoch bereits eine Dyade als Gruppe (exemplarisch Brodbeck und Guillaume 2010, 217), da dort bestimmte gruppenspezifische Phänomene, wie Bildung von Koalitionen, Synergieeffekte im Rahmen einer Diskussionsrunde oder Gruppenpolarisierung nicht vorkommen (Werth und J. Mayer 2008, 334; vgl. Rechtien 2003, 104).

Die oben angeführte Auffassung ergibt also einen recht vagen Gruppenbegriff. Demgegenüber stellen beispielsweise Fischer und Wiswede $(2002,584)$ eine ausführliche, präzisere Definition einer Gruppe im engeren Sinne vor, die sog. ,sekundäre Kriterien“ (Gruppengröße, gewisse Dauer, gemeinsames Gruppenziel) umfasst.

Gruppen können anhand einer Vielzahl von verschiedenen Kriterien eingeteilt werden, beispielsweise:

- Auf wessen Initiative die Gruppe entsteht: formelle (z. B. Verein mit expliziten Regeln) - informelle (z. B. Hobbygruppen) - natürliche Gruppen (z. B. Familien) (Rechtien 2003, 112; vgl. Fischer und Wiswede 2002, 587) 
- Nach Größe, beispielsweise Kleingruppen (in der Sozialpsychologie meistens mit ca. 25 Mitgliedern (Rechtien 2003, 104)) - Großgruppen - Quasigruppen (soziale Kategorien) - Mengen - Massen (Fischer und Wiswede 2002, 586)

- Nach Bedeutung im Sozialisationsprozess: Primärgruppe - Sekundärgruppe (Fischer und Wiswede 2002, 586)

- Nach Bezugsperson: Eigengruppe (Ingroup) - Fremdgruppe (Outgroup) (Fischer und Wiswede 2002, 587)

Für diese Arbeit nehme ich die Klassifizierung von Gruppen aus der Studie von Lickel, Hamilton, Lewis u. a. (2000) zum Ausgangspunkt (vgl. Nijstad und Knippenberg 2007, 413-415, 2014, 443-444; s. auch Lickel, Hamilton und Sherman 2001). Die Studie untersucht die Frage der Entitativität, also des Ausmaßes, ,,in dem eine Anzahl von Personen als miteinander verbundene, kohärente Einheit wahrgenommen wird (Nijstad und Knippenberg 2014, 443). Die Ergebnisse zeigen, dass je mehr Interaktion in der Gruppe stattfindet oder je wichtiger eine Gruppe für die Person selbst ist, desto geschlossener, kohärenter und ,gruppenhafter" erscheint sie; je durchlässiger und größer diese Gruppierung, desto weniger wird sie als eine typische Gruppe wahrgenommen (Lickel, Hamilton, Lewis u. a. 2000, 228, 241). Aus diesem Alltagsverständnis einer Gruppe ergab die Studie vier Gruppentypen (Lickel, Hamilton, Lewis u. a. 2000, 240, 229-230; Übersetzung der Terminologie mit Nijstad und Knippenberg 2014, 443):

- Gruppe mit emotionaler Nähe (Familie, Partner, Freunde)

- aufgabenbezogene Gruppe (Arbeitsteam, Ausschuss)

- soziale Kategorie (Frauen, Amerikaner)

- lockere Verbindung (einschließlich temporärer und Wartegruppen: Menschen, die in einer Gegend wohnen, Gaffer, Warteschlange)

Jede dieser Gruppen kann anhand von acht Merkmalen charakterisiert werden (Lickel, Hamilton, Lewis u. a. 2000, 224-225, 228; Übersetzung der Terminologie mit Nijstad und Knippenberg 2014, 444): Interaktion, Bedeutsamkeit (der Gruppenmitgliedschaft für das Mitglied), gemeinsame Ziele, gemeinsame Handlungsergebnisse, Ähnlichkeit (der Gruppenmitglieder), Dauerhaftigkeit, Durchlässigkeit und Größe. Dabei geben insbesondere die Merkmale Interaktion, gemeinsame Ziele und gemeinsame Handlungsergebnisse 


\section{Umsetzung und Erfolg der Sprachlenkung}

Aufschluss über die Interdependenz der Gruppenmitglieder. Mithilfe dieser Merkmale lassen sich die identifizierten Gruppen wie folgt charakterisieren (Lickel, Hamilton, Lewis u. a. 2000, 229):

- Gruppe mit emotionaler Nähe: klein, hohes Interaktionsniveau, dauerhaft und weitestgehend undurchlässig

- aufgabenbezogene Gruppe: klein, mittlere Dauerhaftigkeit und Durchlässigkeit, hohes Interaktionsniveau

- soziale Kategorie: groß, dauerhaft, undurchlässig

- lockere Verbindung: mittel/groß, geringe Interaktion und Dauer, große Durchlässigkeit

\subsubsection{Gruppenprozesse in der Sozialpsychologie}

Wie Nijstad und Knippenberg (2007) beschreiben, geht man in der Sozialpsychologie davon aus, dass die Neigung der Menschen zur Gruppenbildung mit dem Vorteil zusammenhängt, der einem aus dem Zusammenschluss entspringt. Dies kann beispielsweise aus soziobiologischer, kognitiver oder instrumenteller Sicht erklärt werden (Nijstad und Knippenberg 2007, 412; vgl. Fischer und Wiswede 2009, 654). Im Allgemeinen kann man sagen, dass der Nutzen, den sich ein Individuum von einer Gruppenmitgliedschaft erhofft, sowohl materiell (gemeinsame Ziele verwirklichen) als auch psychologisch (Anschlussmotiv, Selbstwertgefühl) sein kann (Werth und J. Mayer 2008, 337).

Das Verhalten eines Menschen in der Gesellschaft trägt sowohl individuelle Züge, wenn das Individuum ,(...) mit bestimmten idiosynkratischen Merkmalen und auf dem Hintergrund einzigartiger persönlicher Beziehungen $\mathrm{zu}$ anderen (...)“ handelt (Rupert Brown 2002, 545), als auch kollektive Eigenschaften, wenn das Individuum beim Handeln als Mitglied einer bestimmten (sozialen) Gruppe im weitesten Sinne wahrgenommen wird (Rupert Brown 2002, 545). Die Gruppenforschung geht davon aus, dass sich Menschen in Gruppen anders verhalten, als sie sich alleine verhalten würden (Rechtien 2003, 108-109): Man nimmt an, dass das Verhalten in der Gruppe von eigenen Prozessen gesteuert wird, so dass man von Gruppendynamik sprechen kann (Rechtien 2003, 105-106).

Die Gruppenprozesse teilen Nijstad und Knippenberg (2007) in drei Ebenen ein: Individuelle Ebene - das Individuum interagiert mit einer Gruppe, 


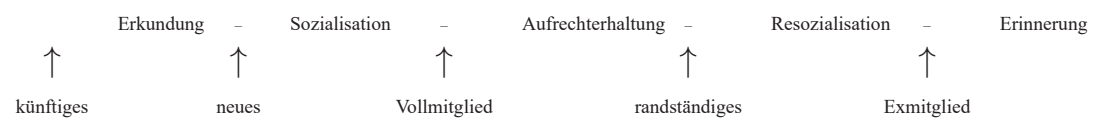

Tabelle 12.: Das Modell der Gruppensozialisation von Moreland und J. M. Levine (1982), eigene Darstellung nach Nijstad und Knippenberg (2007)

Intragruppenebene - die Gruppenmitglieder interagieren untereinander und Intergruppenebene - zwei Gruppen interagieren miteinander. Im Folgenden fasse ich die typischen Gruppenprozesse zusammen, die auf diesen Ebenen stattfinden. Dabei muss beachtet werden, dass sich die Gruppenforschung in der Sozialpsychologie häufig auf die Kleingruppen mit hohem Interaktionsniveau beschränkt (Gruppen mit emotionaler Nähe, aufgabenbezogene Gruppen), so dass sich die hier beschriebenen Prozesse nur bedingt auf die sozialen Kategorien oder lockere Verbindungen übertragen lassen (Fischer und Wiswede 2002, 583).

\subsubsection{Individuelle Ebene: Entwicklung der Gruppenmitgliedschaft}

Für Nijstad und Knippenberg $(2007,416)$ steht auf dieser Analyseebene die Gruppenmitgliedschaft eines Individuums im Vordergrund. Zentral ist dabei die Mitgliedschaftsentwicklung, bei der ein Individuum einer Gruppe betritt, zum Gruppenmitglied wird und die Gruppe wieder verlässt. Zur Beschreibung dieser Prozesse ziehen Nijstad und Knippenberg (2007, 417-425) das Modell der Gruppensozialisation von Moreland und J. M. Levine (1982) heran, in dem fünf Stadien der Gruppenmitgliedschaft enthalten sind und dazwischen jeweils ein Rollenübergang stattfindet (siehe Tabelle 12).

Die Stadien unterscheiden sich durch die Festlegung des Mitglieds auf die Gruppe, wobei die Festlegung definiert wird als „Das Ausmaß, in dem sich ein Mitglied mit der Gruppe und ihren Zielen identifiziert und seine Gruppenmitgliedschaft beibehalten möchte" (Nijstad und Knippenberg 2007, 416). Im Folgenden fasse ich, Nijstad und Knippenberg (2007) folgend, die einzelnen Stadien zusammen.

Stadium Erkundung In diesem Stadium ist das Individuum noch kein Mitglied der Gruppe, sondern erwägt erst seine Mitgliedschaft. Die Auswahl einer Gruppe wird durch Sympathie, Ähnlichkeit, räumliche Nähe sowie dem 


\section{Umsetzung und Erfolg der Sprachlenkung}

Nutzen, den man sich von einer Gruppe erhofft, bestimmt (Werth und J. Mayer 2008, 338). Die Sympathie kann sich auf die ganze Gruppe oder aber nur auf einzelne Mitglieder beziehen (vgl. Fischer und Wiswede 2002, 589). Wie Werth und J. Mayer (2008) zusammenfassen, hängt die Nutzenerwartung einerseits mit der Vorerfahrung mit dieser oder einer ähnlichen Gruppe zusammen (materieller Nutzen). Sie kann aber andererseits auch durch die Ähnlichkeit bedingt sein, da man als ein typisches Mitglied mehr Anerkennung erwarten kann (psychologischer Nutzen) (Werth und J. Mayer 2008, 338). Welcher dieser beiden Nutzenarten entscheidend ist, hängt von der Gruppe ab: Bei Gruppen mit emotionaler Nähe ist das Ähnlichkeitsprinzip wichtiger, bei aufgabenbezogenen Gruppen die Zielunterstützung (Nijstad und Knippenberg 2007, 417). Dabei soll beachtet werden, dass beim Suchen und Auswählen sowohl das Individuum als auch die Gruppe aktiv sind (Fischer und Wiswede 2002, 597).

In der Regel kann man sich die Gruppe, deren Mitglied man werden möchte, frei wählen, außer in Sonderfällen wie Familien oder anderen Gruppen mit geringer Durchlässigkeit (Fischer und Wiswede 2002, 589). Wie Nijstad und Knippenberg (2007) anmerken, reicht in manchen Fällen die Beitrittsentscheidung nicht aus, um Mitglied zu werden, und der Eintritt wird oft von Ritualen begleitet. Diese sind oft unangenehm und haben die Funktion, die Mitglieder stärker an sich zu binden, da man eher ungern etwas aufgibt oder für unattraktiv hält, wofür man sich einer harten Prüfung unterziehen musste (Nijstad und Knippenberg 2007, 417-418).

Der Eintritt in eine Gruppe markiert den Übergang vom Status künftiges Mitglied zu neues Mitglied sowie die Vollendung des Stadiums Erkundung.

Stadium Sozialisation Die Gruppensozialisation wird definiert als „Die Anstrengung der Gruppen, neue Mitglieder an die bestehenden Gruppennormen und -praktiken zu assimilieren“" (Nijstad und Knippenberg 2007, 416). In diesem Stadium lernen die neuen Mitglieder die Gruppennormen und ihre eigenen Rollen innerhalb der Gruppe kennen (Nijstad und Knippenberg 2007, 419). Mit Nijstad und Knippenberg $(2007,419)$ ist auch die Sozialisation keine Einbahnstraße und bedeutet auch für die Gruppe eine Anpassung. ${ }^{115}$ Im Stadium Sozialisation bewegt sich das neue Mitglied in

115 Ein Beispiel hierfür ist es, dass die neuen Mitglieder oft die Möglichkeit haben, Vorschläge zu machen, wie man das Geschehen in der Gruppe verändern kann. Hierbei geht Nijstad und Knippenberg (2007) auf die Studie von Choi und J. M. Levine (2004) ein, die zeigt, dass Neulinge in der Gruppe durchaus Änderungsvorschläge machen können, deren Erfolg jedoch von folgenden Faktoren abhängt (Nijstad und Knippenberg 2007, 420-422): 
Richtung Vollmitglied. Seine Festlegung auf die Gruppe steigt und erreicht ihr Maximum beim Übergang zwischen diesen beiden Stadien, der durch Akzeptanz markiert ist (Nijstad und Knippenberg 2007, 416). Wie schnell dieser Prozess verläuft, hängt u. a. von der Entfernung der Gruppengröße vom Optimum ab: Ist die Gruppe überfüllt, so wird man nur langsam zum Vollmitglied. Hat die Gruppe hingegen zu wenige Mitglieder, so wird man schneller zum Vollmitglied (Nijstad und Knippenberg 2007, 419). Das Ende des Sozialisationsstadiums kann man daran erkennen, dass dem neuen Mitglied keine besondere Aufmerksamkeit mehr geschenkt wird (Nijstad und Knippenberg 2007, 418).

Stadium Aufrechterhaltung Wie Nijstad und Knippenberg (2007) beschreiben, wird in diesem Stadium die Mitgliedschaft in der Gruppe sowohl vom Mitglied als auch von der Gruppe selbst bewertet. Diese Bewertung erfolgt im Hinblick auf die Ziele und führt dazu, dass das Mitglied entweder seine Mitgliedschaft in der Gruppe aufrechterhält, oder seine Rolle verhandelt oder sich langsam von der Gruppe entfernt und sich in Konsequenz seine Festlegung auf die Gruppe verringert (Nijstad und Knippenberg 2007, 422). Die Divergenz markiert den Übergang zwischen den Stadien Aufrechterhaltung und Resozialisierung sowie die Veränderung im Status vom Vollmitglied zum randständigen Mitglied (Nijstad und Knippenberg 2007, 416)

Stadium Resozialisierung Im Stadium Resozialisierung verringert sich die Festlegung des Mitglieds auf die Gruppe weiter: Entweder wird es selbst weniger aktiv oder die Gruppe trennt es von wichtigen Informationen, so dass es nicht mehr als Vollmitglied funktionieren kann (Nijstad und Knippenberg 2007, 422). Wenn das Mitglied, dem ein Ausschluss aus der Gruppe droht, noch einmal versucht, sich an die Gruppe wieder anzupassen, beginnt die

- War die Gruppe vor dem Eintritt des neuen Mitglieds ohnehin erfolgreich, d. h. die eingesetzten Methoden und Vorgehensweisen haben sich bewährt, wird dem Vorschlag eines Neulings wenig Aufmerksamkeit geschenkt.

- War die Gruppe hingegen nicht erfolgreich in der Vergangenheit, hat der Vorschlag mehr Chancen, wahrgenommen zu werden.

- Ist die Aufgabe und die Arbeitsweise von der Gruppe selbst gewählt, gibt sie die Gruppe nicht schnell auf, weil sie darauf stärker festgelegt ist.

- Ist die Aufgabe/Arbeitsweise hingegen fremd bestimmt, ist die Gruppe für den Vorschlag eines Neulings offener. 


\section{Umsetzung und Erfolg der Sprachlenkung}

Resozialisationsphase (Nijstad und Knippenberg 2007, 424). Gelingt die Resozialisierung nicht, kommt es zum Ausschluss, der den Übergang zwischen den Stadien Resozialisierung und Erinnerung sowie den Status randständiges Mitglied und Exmitglied markiert (Nijstad und Knippenberg 2007, 416).

Stadium Erinnerung Im Stadium Erinnerung verringert sich die Festlegung auf die Gruppe weiter, bleibt aber trotzdem größer als vor dem Beitritt (Nijstad und Knippenberg 2007, 416).

Nijstad und Knippenberg (2007, 424-425) gehen in diesem Zusammenhang auf die Arbeiten von Williams (2001) sowie ein Eisenberger, Lieberman und Williams (2003) ein, die herausfanden, dass der Ausschluss aus einer Gruppe schwerwiegende Folgen wie negative Stimmung und Ärger haben und zu geringerem Selbstwertgefühl führen kann.

\subsubsection{Intragruppenebene: die Gruppe selbst}

Die Betrachtung dieser Ebene erlaubt es, die Entstehung und Entwicklung von Beziehungen zwischen den Mitgliedern einer Gruppe zu beschreiben. Nijstad und Knippenberg (2007) bedienen sich hierfür des Modells der Gruppenentwicklung von Tuckman (1965) sowie der einzelnen Merkmale der sog. Gruppenstruktur.

Gruppen entstehen aus einem bestimmten Anlass und haben ein bestimmtes Ziel. Sie bleiben jedoch nicht statisch, da sich die Beziehungen zwischen ihren Mitgliedern stets verändern (Nijstad und Knippenberg 2007, 426). Das Modell der Gruppenentwicklung von Tuckman (1965) umfasst fünf Stadien: Herausbilden - Stürmen - Normenbilden - Leisten - Abschließen, die ich hier nach Nijstad und Knippenberg (2007, 426-427) wiedergebe:

1. Herausbilden: Die Neigung der Menschen zur Gruppenbildung habe ich bereits allgemein erläutert. Die Entstehung einer Gruppe wird dabei nicht nur von Bedürfnissen und Zielen der Beteiligten bestimmt. Fischer und Wiswede (2002, 590-591) nennen weitere Bestimmungsfaktoren:

- Aufgabe/Arbeit: Arbeitsteilung erfordert eine Strukturierung der Gruppe. Sowohl der Arbeitsinhalt als auch die Arbeitsprozesse müssen aufgeteilt werden, unter Berücksichtigung dessen, dass die Einzelergebnisse wieder zusammengeführt werden müssen. 
- Merkmale der Beteiligten: Fähigkeiten, persönliche Vorlieben, Interessen, Zielvorstellungen, Verantwortungsübernahme, Erfahrung

- Umgebung: Bei der Gründung einer Gruppe müssen die gegebenen Strukturen berücksichtigt werden, z. B. bei der Einbettung einer neuen Arbeitsgruppe in eine bestehende Organisation.

Mit Nijstad und Knippenberg $(2007,427)$ lernen sich in Stadium Herausbilden die Mitglieder kennen. Deshalb ist die Interaktion durch Unsicherheit geprägt und die Mitglieder verhalten sich oft höflich. In diesem Stadium entwickelt sich zudem die gemeinsame Identität.

2. Stürmen: In diesem Stadium kommt es zum Kampf um Einfluss und Führung. Es bilden sich Rollen und weitere Gruppenstrukturen heraus (Nijstad und Knippenberg 2007, 427).

3. Normenbilden: In diesem Stadium entstehen Gruppennormen, die die gemeinsamen Ziele bestätigen und stärken. Es entsteht eine starke Bindung der Mitglieder untereinander, die Festlegung auf die Gruppe steigt (Nijstad und Knippenberg 2007, 429).

4. Leisten: Der Aufbau der Gruppe und die Festlegung der Interaktionsmuster sind weitestgehend abgeschlossen. Nun ist die Arbeit auf das Ziel hin möglich. Das Handeln ist überwiegend aufgabenbezogen (Nijstad und Knippenberg 2007, 427).

5. Abschließen: Die abgeschlossene Arbeit wird bewertet. Die Mitglieder sind zufrieden mit oder enttäuscht von dem Ergebnis. Da das Ziel erreicht wurde, verlassen die Mitglieder die Gruppe (Nijstad und Knippenberg 2007, 427).

Wie Nijstad und Knippenberg (2007) erläutern, stellen diese fünf Stadien einen Maximalverlauf der Beziehungsentwicklung dar. Es gibt durchaus Gruppen, bei denen einzelne Stadien nicht vorkommen, z. B.: Ist bei der Gruppenbildung von vorn herein klar, wer welche Aufgabe/Rolle übernimmt und wer die Führungsposition hat, dann entfällt das Stadium Stürmen. Darüber hinaus muss die Gruppenentwicklung nicht wie im Modell angedeutet linear verlaufen. Beispielsweise kann es in Stadium Leisten erneut zu Einflusskämpfen oder zur Rollenverhandlung kommen, die für das Stadium Stürmen charakteristisch sind (Nijstad und Knippenberg 2007, 428). 
Wie eingangs erwähnt, beschreiben Nijstad und Knippenberg (2007) die Intragruppenebene nicht nur anhand des beschriebenen Modells von Tuckman (1965), sondern auch anhand der sog. Gruppenstruktur. Für jede Gruppe soll es nämlich nicht nur möglich sein anzugeben, in welchem Stadium der Beziehungsentwicklung sie sich befindet, sondern auch wie die Beziehungen zwischen den Mitgliedern aussehen und wodurch sie geregelt werden. Deshalb nennen Nijstad und Knippenberg (2007, 429-435) folgende Merkmale, die ,(...) den kleinsten gemeinsamen Nenner der Analyse von Gruppenprozessen" darstellen (Fischer und Wiswede 2002, 590) und somit die sog. Gruppenstruktur ausmachen:

- Normen

- Rollen

- Status

- Kohäsion

- Interaktionsstruktur

- Kommunikationsstruktur

Brodbeck und Guillaume (2010) nennen zudem die Gruppenkomposition und die Gruppengröße als Merkmale der Gruppenstruktur, auf die ich hier nicht näher eingehe.

Normen Gruppennormen werden definiert als ,(...) (ungeschriebene) Regeln, die von den Mitglieder[n] einer Gruppe geteilt werden und die vorschreiben, welche Einstellungen, Verhaltensweisen und Überzeugungen im Kontext der Gruppe angemessen und welche unangemessen sind“ (Nijstad und Knippenberg 2007, 429; vgl. Brodbeck und Guillaume 2010, 229). Normen sind ungeschriebene soziale Erwartungen, die aber im Gegensatz zu den Rollen (s. unten) nicht an eine bestimmte Position innerhalb der Gruppe gebunden sind, sondern alle Mitglieder gleichermaßen betreffen (Rechtien 2003, 117; Werth und J. Mayer 2008, 340).

Die Leistung der Normen ist vielfältig. Im Allgemeinen tragen sie zur reibungslosen Interaktion innerhalb einer Gruppe bei, weil das Verhalten vorhersehbar wird (Nijstad und Knippenberg 2007, 429; Werth und J. Mayer 
2008, 340). Da Normen internalisiert werden können (Nijstad und Knippenberg 2007, 430), so dass über die Interaktionskonvention nur noch selten diskutiert werden muss, setzen sie produktive Kapazitäten frei, die für die Arbeit auf das Ziel hin eingesetzt werden können (Werth und J. Mayer 2008, 341). Diese Übereinkunft über die Arbeitsweise und Aufgabenteilung führt dazu, dass das sog. „transaktive Gedächtnis“ entstehen kann, d. h. „Gemeinsames Wissen innerhalb einer Gruppe darüber, welches Gruppenmitglied welches Wissen hat" (Nijstad und Knippenberg 2007, 430). Es ist somit nicht mehr notwendig, dass jedes Mitglied das Gesamtwissen einer Gruppe besitzt, sondern dass es lediglich die Wissensstruktur der Gruppe kennt.

Die regulierende Funktion der Normen ergibt sich daraus, dass jedem Mitglied die Reaktion auf das normkonforme oder nichtkonforme Verhalten bekannt ist (Nijstad und Knippenberg 2007, 429). Die Sanktionen stellen gleichzeitig einen Mechanismus zur Einhaltung der Normen dar. Werth und J. Mayer $(2008,341)$ fassen zusammen, dass Normen unter diesen Bedingungen besonders wirksam sind:

- Wenn eine Sanktion besonders stark ist.

- Wenn die Gruppenmitgliedschaft für das Mitglied besonders wichtig ist und einen Teil seiner sozialen Identität ausmacht.

- Wenn die Gruppe klein ist. Ist die Gruppe groß, kann das nichtnormkonforme Verhalten häufiger unentdeckt und dadurch unsanktioniert blieben.

Andererseits festigt das normkonforme Verhalten auch die Normen und deutet darauf hin, dass sich das Mitglied auf die Gruppe festgelegt hat (Nijstad und Knippenberg 2007, 430). Will man Normen ändern, muss man frühzeitig in die Gruppenentwicklung eingreifen (Rechtien 2003, 117), da Normen nach dem Modell von Tuckman (1965) relativ früh entstehen (vgl. Nijstad und Knippenberg 2007, 430). Eine Änderung der Umgebung oder der Zusammensetzung der Gruppe können aber auch eine nichtintendierte Normänderung auslösen (Nijstad und Knippenberg 2007, 430).

Rollen Rollen sind, ähnlich wie Normen, Verhaltenserwartungen, die jedoch nicht für alle Mitglieder einer Gruppe gelten, sondern mit einer bestimmten Position innerhalb der Gruppe zusammenhängen (Rechtien 2003, 115; Werth und J. Mayer 2008, 342; Brodbeck und Guillaume 2010, 231; vgl. Nijstad und Knippenberg 2007, 433). Die Rolle ist nicht nur von außen durch 


\section{Umsetzung und Erfolg der Sprachlenkung}

Erwartungen anderer bestimmt, sondern auch von den Vorstellungen des betroffenen Mitglieds (sog. Rollenselbstbild) (Rechtien 2003, 115).

Wie Werth und J. Mayer (2008) erläutern, haben Rollen eine ähnliche Funktion wie Normen. Zum einen strukturieren und sanktionieren sie das Verhalten einzelner Mitglieder und machen es vorhersehbar (Werth und J. Mayer 2008, 343; vgl. Brodbeck und Guillaume 2010, 231). Darüber hinaus sorgen sie dafür, dass die Aufgaben nach Möglichkeit gleichmäßig verteilt werden, um die Überlastung einzelner Mitglieder zu vermeiden. Sie tragen auch in hohem Maße zur sozialen Identität der Mitglieder bei (Werth und J. Mayer 2008, 343). Die Rollen geben zwar einen Rahmen für das individuelle Verhalten vor, in dem sich die Mitglieder jedoch frei bewegen können, weil sie durch die Rolle geschützt werden (Werth und J. Mayer 2008, 343-344).

Wie Werth und J. Mayer (2008) weiter ausführen, können Menschen verschiedene Rollen gleichzeitig übernehmen. Dies betrifft nicht nur Rollen in verschiedenen Gruppen, sondern auch innerhalb einer Gruppe (Werth und J. Mayer 2008, 343). Neben den offiziellen Rollen bilden sich in Gruppen oft sog. Quasirollen heraus, ${ }^{116}$ die nicht an eine bestimmte Position, sondern an das individuelle Verhalten gebunden sind, beispielsweise eines sachlichen und eines emotionalen Gruppenführers (Rechtien 2003, 116-117; vgl. Brodbeck und Guillaume 2010, 231). Rechtien (2003) betont, dass man ein besseres Verständnis der Gruppenprozesse durch den Vergleich von den offiziellen Rollen und den Quasirollen erzielen kann, weil sie zuweilen im Konflikt zueinander stehen (Rechtien 2003, 116-117).

Status Unter Status versteht man ,(...) die sozial bewertete Stellung [einer Person] aus Sicht der Gruppenmitglieder (...)“(Werth und J. Mayer 2008, 346; vgl. Fischer und Wiswede 2002, 598), wobei sich der offizielle Status einer Person/Rolle von dem tatsächlichen Status unterscheiden kann (Werth und J. Mayer 2008, 346). Die Gesamtheit aller Status ergibt die Machtstruktur einer Gruppe.

Zur Erklärung der Statusunterschiede innerhalb einer Gruppe wird in der Literatur die Theorie der Erwartungszustände von Berger, Rosenholtz und Morris Zelditch (1980) herangezogen (Fischer und Wiswede 2002, 598; Nijstad und Knippenberg 2007, 433; Werth und J. Mayer 2008, 346-347). Sie „[v]ertritt die Auffassung, dass Statusunterschiede innerhalb einer Gruppe Ergebnis unterschiedlicher Erwartungen sind, die Gruppenmitglieder anein-

116 Sie werden auch entsprechend formale und informelle Rollen genannt (exemplarisch Brodbeck und Guillaume 2010, 231). 
ander haben“ (Nijstad und Knippenberg 2007, 434). Im Allgemeinen kann man sagen, dass die Erwartungen aufgabenbezogen sind: Wird von einer Person erwartet, dass sie einen großen Beitrag zum Erreichen des Gruppenziels leisten kann, ist ihr Status innerhalb der Gruppe hoch (Werth und J. Mayer 2008, 346). Diese Erwartungen entstehen durch die Auswertung von verschiedenen Faktoren (Nijstad und Knippenberg 2007, 434-435; vgl. Werth und J. Mayer 2008, 342) wie:

- aufgabenbezogener Erfolg in der Vergangenheit

- Senioritätsstatus

- spezifische Statusmerkmale: Fähigkeiten, Fertigkeiten

- diffuse Statusmerkmale: Alter, Geschlecht, ethnische Abstammung, Titel etc.; hierbei handelt es sich nicht um aufgabenbezogene Merkmale, sondern um Eigenschaften einer Person

- Persönlichkeit

Die diffusen Statusmerkmale sind besonders dann wichtig, wenn die spezifischen nicht bekannt sind (Werth und J. Mayer 2008, 346). Zudem sind mit Nijstad und Knippenberg (2007) Erwartungen teilweise kulturell bzw. gesellschaftlich bedingt. Die Erwartungen sind meistens fest etabliert, so dass sie „explizit widerlegt werden müssen, bevor sie an Einfluss verlieren“ (Nijstad und Knippenberg 2007, 435).

Kohäsion Ein weiteres Merkmal der Gruppenstruktur ist die Kohäsion, d. h. „Zusammenhalt einer Gruppe, der aus allen Kräften resultiert, die die Mitglieder motivieren, in der Gruppe zu bleiben“(Werth und J. Mayer 2008, 347; vgl. Brodbeck und Guillaume 2010, 230). Quantitativ kann man die Kohäsion als „Ausmaß des Zusammenhalts innerhalb der Gruppe“ definieren (Werth und J. Mayer 2008, 340).

Nijstad und Knippenberg (2007) unterscheidet zwischen aufgabenbezogener Kohäsion, d. h. einer solchen, die aus der Attraktivität der Aufgabe resultiert, und der interpersonellen Kohäsion, ,die auf der Sympathie bezüglich der Gruppe und gegenüber ihren Mitgliedern beruht" (Nijstad und Knippenberg 2007, 431).

Die Kohäsion hängt auch von weiteren Faktoren ab; unter Berücksichtigung der Arbeit von Cartwright und Zander (1968) nennen Fischer und Wiswede $(2002,595)$ beispielsweise: 
- Motivation einer Person, in der Gruppe zu sein (Kontaktbedürfnis)

- „Erwartungen, daß eine Mitgliedschaft tatsächlich günstige Ergebnisse erbringt (...)“ (Fischer und Wiswede 2002, 595)

- Aussicht auf und Vergleich mit Alternativen

- Vorherige Erfahrung mit dieser oder einer ähnlichen Gruppe

Obwohl Kohäsion oft als etwas Positives gesehen wird (Werth und J. Mayer 2008, 347), machen Fischer und Wiswede $(2002,596)$ auf Probleme und Konflikte aufmerksam, zu denen eine starke Kohäsion innerhalb einer Gruppe, aber auch beim Kontakt mit anderen Gruppen führen kann (vgl. Werth und J. Mayer 2008, 348).

Interaktionsstruktur Viel über die Gruppe und die Gruppenprozesse erfährt man darüber hinaus durch die Betrachtung der Interaktionsstruktur, d. h. wer mit wem und wie oft interagiert.

Zur Erfassung der Interaktion eignet sich die Interaktionsprozessanalyse (IPA) von Bales (1950), die definiert wird als „(...) formales System zur Erfassung von Beobachtungen der Interaktion unter den Mitgliedern von Kleingruppen. Die Methode beinhaltet Kategorien und Verfahren zur Kodierung von Interaktionen im Hinblick auf diese Kategorien“ (Nijstad und Knippenberg 2007, 427). Das so erfasste Interaktionsverhalten erleichtert die Ermittlung von Quasirollen und erlaubt indirekte Rückschlüsse auf Status, Hierarchie oder Stadium der Gruppenentwicklung (Nijstad und Knippenberg 2007, 433-434, 428) aber auch auf die Affekt- und Sympathiestruktur (vgl. Fischer und Wiswede 2002, 594).

Kommunikationsstruktur Bei der Kommunikationsstruktur handelt es sich vorwiegend um Kommunikationsformen und nicht -inhalte. $\mathrm{Zu}$ den Gruppenprozessen, die mit der Kommunikationsstruktur zusammenhängen, gehören der Effekt des gemeinsamen Wissens, die Gruppenpolarisierung und das Gruppendenken, auf die ich hier nicht näher eingehe. Für einen Überblick verweise ich entsprechend auf Werth und J. Mayer (2008, 364-369, 370-373, 373-375). 


\subsubsection{Intergruppenebene: Interaktion von verschiedenen Gruppen}

Nach der Beschreibung der Prozesse innerhalb einer Gruppe wende ich mich der Charakterisierung der Prozesse, die beim Kontakt zwischen zwei Gruppen stattfinden, sowie der Auswirkung, welche der Intergruppenkontext auf das Verhalten der Gruppenmitglieder hat (vgl. Nijstad und Knippenberg 2007, 435). Die Forschung auf der Intergruppenebene beschränkt sich nicht nur auf Kleingruppen, sondern umfasst auch soziale Kategorien (vgl. Rechtien 2003, 119).

Nijstad und Knippenberg (2007) machen darauf aufmerksam, dass eine Gruppenmitgliedschaft nicht automatisch bedeutet, dass das Mitglied in jeder Situation diese Mitgliedschaft bewusst wahrnimmt. Es ist u.a. die Leistung des Intergruppenkontextes, diese Selbstkategorisierung als Gruppenmitglied kognitiv zu aktivieren (salient zu machen) (Nijstad und Knippenberg 2007, 436). Eine wichtige Folge der Theorie der sozialen Identität (Abschnitt 4.5.4.1) ist hierbei, dass man die Eigengruppe positiver als die Fremdgruppe bewertet, obwohl Fremdgruppen oft als homogener und geschlossener erscheinen (Rechtien 2003, 120; Nijstad und Knippenberg 2007, 437; Spears und Tausch 2014, 519). Diese Prozesse werden nicht mehr ausschließlich als Fremdgruppendiskriminierung sondern zunehmend auch als Eigengruppenfavorisierung aufgefasst (vgl. Rupert Brown 2002, 541-544, 546-550).

Eine Erklärung für diese positivere Bewertung der Eigengruppe ist mit Rupert Brown (2002), dass die Eigengruppe einerseits als die beste für das Erreichen der eigenen Ziele gesehen wird. Darüber hinaus ermöglicht die Eigengruppenfavorisierung in uneindeutigen Situationen die Unterscheidung zwischen fremd und eigen (sog. kategoriale Differenzierung) und die Verwischung der Unterschiede innerhalb der eigenen Gruppe (Rupert Brown 2002, 556). Eine integrative Erklärung liefert darüber hinaus die Theorie der sozialen Identität von Henri Tajfel (1982). Den Mechanismus fasst Rechtien (2003, 120) wie folgt zusammen (eigene Darstellung):

1. Selbstkonzept $=$ persönliche + soziale Identität

2. soziale Identität $=$ Gruppenzugehörigkeit + Bewertung der Gruppe

3. Die Bewertung ergibt sich aus dem Vergleich mit anderen Gruppen

4. Aufwertung der Eigengruppe $\Rightarrow$ Verbesserung des Selbstkonzepts 
5. Relevanz der Gruppenzugehörigkeit ist situationsspezifisch und hängt von der gerade angenommenen Rolle ab

6. Je wichtiger die Mitgliedschaft in einer bestimmten Gruppe, umso mehr prägt sie die soziale Identität (Rupert Brown 2002, 563)

Wie bereits in Abschnitt 4.5.4.1 erwähnt, neigen Menschen nach der Theorie der sozialen Identität dazu, ein positives Selbstbild zu haben. Rupert Brown (2002) erläutert: Fällt der Vergleich mit anderen Gruppen ungünstig aus, was negative Auswirkungen auf die soziale Identität haben kann, versucht man der Eigengruppe andere positive Merkmale zuzuschreiben, um eine positive Distinktheit zu schaffen und die soziale Identität zu schützen (Rupert Brown 2002, 560-561). An einer anderen Stelle erläutert Rupert Brown (2002): Die Bedrohung der sozialen Identität wird oft durch einen wahrgenommenen Konflikt und nicht durch einen bloßen Kontakt mit anderen Gruppen hervorgerufen. Der Konflikt hängt mit den Gruppenzielen zusammen. Ist das Ziel beider Gruppen unvereinbar, so dass ,das Ziel der einen Gruppe zu Lasten der anderen geht" (Rupert Brown 2002, 547), dann befinden sie sich in Konflikt und die soziale Identität ist bedroht. Bleibt der Konflikt aus, weil die Ziele übereinstimmen und die Gruppen aufeinander angewiesen sind, ist die Identität nicht bedroht und das Verhalten ist freundlicher (Rupert Brown 2002, 547).

Die Annahmen der Theorie der sozialen Identität führen zur Erkenntnis, dass der soziale Vergleich für Gruppen mit niedrigem Status (wie Minderheiten) in der Regel immer ungünstig ausfällt, wodurch ein positives Selbstbild nie erreicht werden kann (Rupert Brown 2002, 566). Strategien, die der Gruppe mit dem niedrigen Status zur Verfügung stehen, gehen auf Henri Tajfel und J. Turner (1986) zurück und sind beispielsweise in Rupert Brown $(2002,566-$ 568) zusammengefasst. Darüber hinaus stellt Rupert Brown (2002, 570-573) weitere Strategien zusammen, die gegen Diskriminierung solcher Gruppen eingesetzt werden können.

\subsection{Mechanismen der Einstellungsänderung}

Das Thema der Einstellungen habe ich in dieser Arbeit bereits an mehreren Stellen aufgegriffen (Abschnitt 2.1.5.6, Abschnitt 3.2.2.3, Abschnitt 3.4.1.2). In der Vorbereitung auf die Metaanalyse der erfolgsfördernden Faktoren behandle ich in diesem Abschnitt speziell die Wechselbeziehung zwischen Ein- 
stellungen und Verhalten. ${ }^{117}$ Insbesondere gehe ich hier auf die Frage ein, welche Möglichkeiten zur Herbeiführung von Einstellungsänderungen bei anderen Personen einem zur Verfügung stehen und ob dies dann tatsächlich in einer Verhaltensänderung resultiert.

Zur Rekapitulation: Eine Einstellung ist aus sozialpsychologischer Sicht „(...) eine mentale Repräsentation, die aus einer zusammenfassenden [subjektiven] Bewertung eines Einstellungsobjektes besteht" (Werth und J. Mayer 2008, 206). Einstellungen umfassen drei Komponenten: kognitive, affektive und konative. Sie erfüllen unterschiedliche Funktionen, beispielsweise nennen Haddock und Maio (2007, 199-200) mit Katz (1960) Wissen, Instrumentalität, Ich-Abwehr und Werteausdruck.

\subsubsection{Zusammenhang von Einstellungen und Verhalten}

Aus der Sicht dieser Arbeit ist vor allem interessant, die Wechselwirkung von Einstellungen und Verhalten zu beleuchten. Den Ausgangspunkt bildet hier die Annahme, dass Einstellungen bestimmten Verhaltensentscheidungen zugrunde liegen (Stürmer 2009, 69). Eine lange Zeit nahm man einen direkten Zusammenhang zwischen Einstellungen und Verhalten an und erwartete, einerseits aufgrund von Einstellungen mehr oder weniger präzise Verhaltensvorhersagen von Individuen machen zu können (Haddock und Maio 2007, 214-215; Werth und J. Mayer 2008, 255; Stürmer 2009, 79), andererseits aber auch durch eine Einstellungsänderung direkt eine Verhaltensänderung herbeizuführen. Metastudien ergaben jedoch, dass zwischen Einstellungen und Verhalten weniger Zusammenhang besteht als vermutet (Haddock und Maio 2007, 214; Werth und J. Mayer 2008, 256; Stürmer 2009, 79) und so bestätigten sie auch Alltagsbeobachtungen, dass man häufig im Widerspruch zu geäußerten Einstellungen handelt (Werth und J. Mayer 2008, 256).

Allgemein kann man festhalten, dass es von einer Vielzahl von Faktoren abhängt, ob Einstellungen mit bestimmtem Verhalten einhergehen. Exemplarisch nennen Haddock und Maio (2007, 216-218) hierzu:

- Verhaltensbereich: Aus dem Einstellungsobjekt an sich lässt sich zuweilen schließen, ob eine Verhaltensvorhersage prinzipiell möglich ist oder nicht. Beispielsweise kann meistens ein Zusammenhang zwischen politischen Ansichten (Einstellung) und der Wahl einer Partei (Handlung) erwartet werden, wohingegen z. B. beim Blutspenden eher ein ge-

117 Ich orientiere mich im Sprachgebrauch weitestgehend an den angeführten Quellen und differenziere in diesem Abschnitt nicht zwischen Verhalten und Handeln. 
ringer Zusammenhang zwischen Einstellung (Blut spenden ist wichtig) und Verhalten (tatsächlich Blut spenden) vorliegt (Haddock und Maio 2007, 216).

- Einstellungsstärke: Tendenziell erlauben starke, leicht zugängliche Einstellungen eine bessere Verhaltensvorhersage als schwache, wobei dies, wie am Blutspendebeispiel deutlich, nicht absolut gilt (Haddock und Maio 2007, 217; vgl. Werth und J. Mayer 2008, 259).

- Persönliche Faktoren beeinflussen den Zusammenhang zwischen Einstellungen und Verhalten in dreierlei Hinsicht. Zum einen sind starke Einstellungen oft zentrale Bestandteile des Selbst und haben dadurch einen stärkeren Einfluss auf das Verhalten (Stürmer 2009, 81). Außerdem spielt hier noch ein weiterer Aspekt des Selbst eine Rolle, nämlich die Tendenz zur Selbstüberwachung. Bei starken Selbstüberwachern beobachtet man gewöhnlich keinen oder ganz schwachen Zusammenhang zwischen Verhalten und Einstellungen, weil sie sich beim Handeln nicht an eigenen Einstellungen, sondern an vorweggenommenen Erwartungen der anderen oder an sozialen Normen orientieren (Haddock und Maio 2007, 217-218).

Zu persönlichen Faktoren gehört auch eigene Erfahrung mit dem Einstellungsgegenstand, die zur Entstehung von besonders starken, verhaltenssteuernden Einstellungen führt (Werth und J. Mayer 2008, 259).

- Zudem werden von Werth und J. Mayer (2008) situative Faktoren genannt und die daraus resultierende Einstellungssalienz: „Die Verhaltensvorhersage ist ebenfalls besser, wenn Aspekte des Einstellungsobjekts salient sind, die seiner primären Einstellungskomponente entsprechen (...)“(Werth und J. Mayer 2008, 259), z. B. Geschmack bei Schokolade, technische Merkmale bei einer Waschmaschine (Werth und J. Mayer 2008, 259).

Eine Systematisierung der Bedingungen, wann eine Einstellung ein bestimmtes Verhalten erwarten lässt, bieten einige Theorien.

Stürmer $(2009,79)$ geht auf die Arbeit von Ajzen und Fishbein (1977) ein. Sie postulieren, dass die gemessenen Einstellungen und das Verhalten in folgenden vier Punkten übereinstimmen müssen, um eine Verhaltensvorhersage zu erlauben (vgl. Haddock und Maio 2007, 215): 
- Target: „Auf welches Objekt bzw. Ziel ist das Verhalten gerichtet?“ (Stürmer 2009, 79)

- Action: „Welches Verhalten soll untersucht werden?“ (Stürmer 2009, 79)

- Context: „In welchem Kontext wird das Verhalten ausgeführt?“ (Stürmer 2009, 79)

- Time: „Zu welchem Zeitpunkt soll das Verhalten ausgeführt werden?" (Stürmer 2009, 79)

Wie Werth und J. Mayer (2008) erläutern: Je konkreter Bedingungen abgefragt werden, umso besser lässt sich aus Einstellungen das Verhalten vorhersagen. ${ }^{118}$ Andersherum gilt das Aggregationsprinzip: „Globale Einstellungen sind dann ein guter Prädikator, wenn man unterschiedliche Verhaltensrealisierungen der Einstellung zu einem Index bündelt, die einzelnen Items also aggregiert (...)“(Werth und J. Mayer 2008, 261).

Wie von Haddock und Maio (2007, 218-220) erläutert, stellt das Modell des überlegten Handels von Fishbein und Ajzen (1975), das später von Ajzen (1991) zum Modell des geplanten Verhaltens weiterentwickelt wurde, eine integrative Sicht auf verschiedene Faktoren dar (s. Abbildung 16) (s. auch Haddock und Maio 2014, 223-226). Da sich beide Modelle nur in der wahrgenommenen Verhaltenskontrolle unterscheiden, gehe ich unten nur auf das neuere Modell ein.

Die unmittelbare Determinante des Handelns ist die Handlungsintention. Sie wird unter drei Faktoren geformt: Einstellungen, der subjektiven Norm und der wahrgenommenen Verhaltenskontrolle (s. Haddock und Maio 2007, 218-219; Stürmer 2009, 82-84; Haddock und Maio 2014, 223-224). Die Einstellungen werden mithilfe des sog. Erwartungen-mal-Wert-Modells (Fishbein und Ajzen 1975) berechnet. Die subjektive Norm bedeutet die subjektive Vorstellung eines Individuums, was andere Individuen (insbesondere wichtige Bezugspersonen) von ihm erwarten. Sie ist zusätzlich an die Motivation gebunden, diese Erwartungen zu erfüllen. Schließlich hängt die Herausbildung der Handlungsintention von der wahrgenommenen Verhaltenskontrolle ab, d.h. der ,(...) Wahrnehmung einer Person (...),

118 Soll beispielsweise das Verhalten in klassische Konzerte gehen vorausgesagt werden, sollte nicht nach einer generellen Einstellung zur klassischen Musik (da Verstoß gegen Target und Action), sondern nach der Einstellung zum Besuch von klassischen Konzerten z. B. in der bestimmten Stadt zum bestimmten Zeitpunkt gefragt werden (Werth und J. Mayer 2008, 260-261). 

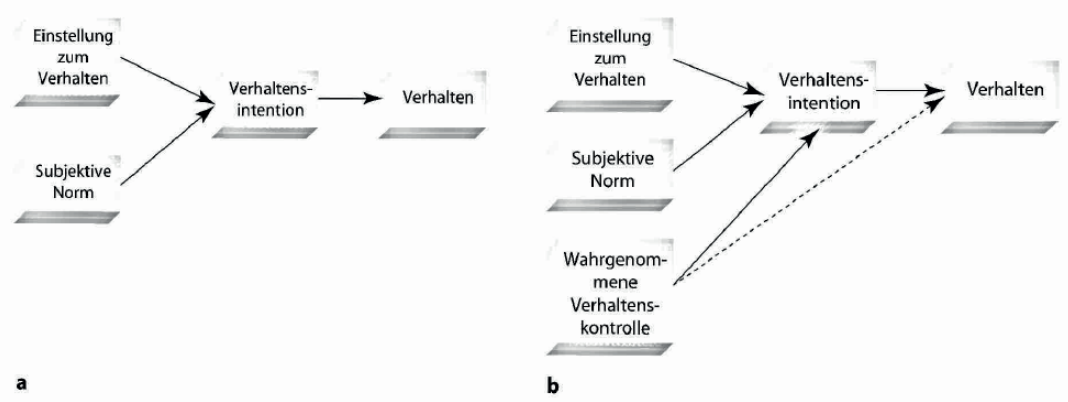

Abbildung 16.: Die Modelle des überlegten Handelns (a) (Fishbein und Ajzen 1975) und des geplanten Verhaltens (b) (Ajzen 1991) in Darstellung von Haddock und Maio $(2014,224)$

über die erforderlichen Fähigkeiten und Ressourcen zu verfügen, um ein bestimmtes Verhalten auszuführen“ (Stürmer 2009, 83).

Das Modell des geplanten Verhaltens ermöglicht eine gute Vorhersage, wenn das Verhalten frei wählbar und kontrollierbar ist und man genug Zeit zum Überlegen und Abwägen hat. Stürmer $(2009,84)$ fasst zusammen, dass die Grenzen des Modells beim spontanen Verhalten sowie beim Handeln, das routinemäßig ausgeführt wird, besonders deutlich werden, da dort die Gewohnheiten eine wichtigere Rolle spielen als Einstellungen (vgl. Haddock und Maio 2007, 221, 2014, 227).

Das spontane Verhalten wird im MODE-Modell von Fazio (1990) erklärt: „Es nimmt an, dass Motivation und Gelegenheit erforderlich sind, um die verfügbaren Informationen durchdacht zu berücksichtigen" (Haddock und Maio 2007, 221). Verfügt man nicht über ausreichend Motivation und Gelegenheit, ein Verhalten zu durchdenken, wird es nur aus leicht zugänglichen Einstellungen ausgelöst (Haddock und Maio 2007, 221; vgl. Stürmer 2009, 84).

Schließlich gehen Haddock und Maio $(2007,222)$ auf das integrative Modell von Eagly und Chaiken (1993) ein, das alle Faktoren berücksichtigt, die für eine Verhaltensvorhersage notwendig sind: Gewohnheit, Einstellung zum Verhaltensgegenstand, instrumentelle Handlungsergebnisse, normative Handlungsergebnisse, Konsequenzen für die eigene Identität. 


\subsubsection{Einstellungsänderung}

Aus dem beschriebenen bedingten Zusammenhang zwischen Einstellungen und Verhalten folgt, dass eine Einflussnahme auf das Verhalten durch Einflussnahme auf Einstellungen ebenfalls nur bedingte Erfolgsaussichten hat. In diesem Abschnitt gehe ich trotzdem auf Theorien der Einstellungs- und Verhaltensänderungen ein, um ein besseres Verständnis dieser Mechanismen zu erreichen und, in Folge, im Kontext der Sprachlenkung verschiedene Handlungsmöglichkeiten und ihre Erfolgsaussichten besser evaluieren zu können.

Die Literatur beschriebt unterschiedliche Wege zur Einstellungsänderung: Stroebe (2007) beschreibt den argumentativen durch Persuasion und den durch Verhaltensanreize wie Belohnung oder Bestrafung. Darüber hinaus geht Stürmer (2009, 85) auf die Kontaktförderung mit dem Einstellungsobjekt als Möglichkeit zur Einstellungsänderung ein, da dadurch neue Erfahrungen mit dem Objekt gemacht werden können. Im Folgenden fasse ich diese Möglichkeiten zusammen.

\subsubsection{Persuasion}

Unter Persuasion versteht Stroebe $(2007,227)$ den „,...) Einsatz von Botschaften, um die Meinungen, die Einstellungen und das Verhalten anderer Menschen zu ändern“" (Stroebe 2014, s. auch; vgl. Werth und J. Mayer 2008, 239). Die wichtigsten Fragen beziehen sich in diesem Zusammenhang darauf, welche Argumente besonders wirksam sind, welche Mechanismen der Persuasion zugrunde liegen sowie welche Faktoren das Ergebnis einer Persuasion beeinflussen.

Wie Stroebe $(2007,228)$ resümiert, gingen die ersten Modelle davon aus, dass die Wirkung der Persuasion auf einer detaillierten (systematischen) Verarbeitung der Botschaft beruht. Das Informationsverarbeitungsmodell der Persuasion von McGuire (1969) nahm beispielsweise an, dass die persuasive Wirkung das Ergebnis von fünf Verarbeitungsschritten ist: Aufmerksamkeit, Verstehen, Akzeptanz, Behalten, Verhalten (Stroebe 2007, 228, 2014, 233). Nur wenn der Botschaftsempfänger alle fünf Schritte durchläuft, wird die Persuasion Erfolg haben, was das häufige Scheitern der Persuasion erklären würde. Diese Theorie fand jedoch empirisch nur wenig Bestätigung (Stroebe 2007, 228-229, 2014, 233-234).

Als zweites stellt Stroebe $(2007,2014)$ das Modell der kognitiven Reaktionen von Greenwald (1986) vor. Es betont die Bedeutung von Gedanken, die bei der Rezeption der Botschaft hervorgerufen werden: Löst die Botschaft 


\section{Umsetzung und Erfolg der Sprachlenkung}

zustimmende Gedanken aus, so wird die Persuasion Erfolg haben. Bei ablehnenden Gedanken sind die Erfolgsaussichten eher gering. Darüber hinaus sind auch solche Faktoren wie Ablenkung und Wiederholung in das Modell integriert, da z. B. die Ablenkung die ablehnenden oder die zustimmenden Gedanken unterbrechen kann. Die wichtigste Neuerung des Modells ist somit die Fokussierung auf die interne Informationsverarbeitung des Rezipienten, die mit dem Aktivieren und Heranziehen des Vorwissens einhergeht, sowie die Verminderung der Senderrolle (Stroebe 2007, 229-230, 2014, 235-237).

Die neueren Modelle, wie das Modell der Elaborationswahrscheinlichkeit (ELM) von Petty und Cacioppo (1986) oder das Heuristisch-SystematischeModell (HSM) von Chaiken, Liberman und Eagly (1989) verbinden die Theorien der systematischen und nichtsystematischen Verarbeitung (Stroebe 2007, 233). Da sich beide Modelle im Wesentlichen überlappen, stelle ich hier wie Stroebe $(2007,233)$ nur das erste Modell vor. Die Unterschiede zwischen den beiden Modellen sind ebenfalls von Stroebe (2007, 243) erläutert (vgl. Stroebe 2014, 247-248).

Modell der Elaborationswahrscheinlichkeit Das Modell der Elaborationswahrscheinlichkeit (elaboration likelihood model, ELM) beschreibt, wann Menschen bei einem Persuasionsversuch auf die Qualität der Argumente achten und wann sie sich durch andere Faktoren beeinflussen lassen. Es nimmt an, dass die Verarbeitung einer Persuasionsbotschaft auf zwei Wegen erfolgen kann:

- zentrale Route: „Sorgfältiges und kritisches Abwägen der Argumente, die zur Unterstützung einer bestimmten Position vorgebracht werden“" (Stroebe 2007, 234), wodurch eine Einstellungsänderung erfolgt.

- periphere Route: „Umfasst diejenigen Persuasionsprozesse, die nicht auf aufwändigem themenrelevantem Denken beruhen" (Stroebe 2007, 234). Die Einstellungsänderung erfolgt als „Reaktion auf periphere Hinweisreize eines Persuasionsversuchs (...), beispielsweise auf dem Expertenstatus des Kommunikators basierend" (Werth und J. Mayer 2008, 245) und ist also „(...) relativ unabhängig von der Qualität der dargebotenen Argumente“ (Stürmer 2009, 85).

Die zentrale Route entspricht dem beschriebenen Modell der kognitiven Reaktionen von Greenwald (1986) (Stroebe 2007, 234). Durch eine intensive Auseinandersetzung mit den „Pro- und Kontra-Argumenten 
überzeugt sich die Person quasi selbst“" (Stürmer 2009, 85), so dass die Einstellungsänderung nachhaltiger und beständiger ist (Stroebe 2007, 241; Werth und J. Mayer 2008, 246-247; Stürmer 2009, 86). Bei der peripheren Route spielen z.B. die klassische Konditionierung (Stroebe 2007, 234) aber auch verschiedene Heuristiken ${ }^{119}$ wie Expertenheuristik (äußere Expertenmerkmale, Glaubwürdigkeit), Attraktivitätsheuristik (äußere Erscheinung, Sympathie), Botschaftsheuristik (Länge, Reihenfolge der Argumente, wahrgenommene Beeinflussungsabsicht) eine Rolle (Werth und J. Mayer 2008, 240-242; Stürmer 2009, 86).

Eine wichtige Leistung des EML ist die Vorhersage, wann welche Route von den Botschaftsempfängern beschritten wird (Werth und J. Mayer 2008, 245). Es wird angenommen, dass die wichtigsten Determinanten der Elaborationstiefe die Motivation und die Fähigkeiten zur Verarbeitung darstellen (Stroebe 2007, 236-237; Werth und J. Mayer 2008, 245-246). Unter Fähigkeiten versteht man verschiedene Merkmale des Rezipienten wie Intelligenz, Bildungsgrad, Alter, Selbstwertgefühl etc. (Werth und J. Mayer 2008, 243-244). Relevant ist auch das individuelle Kognitionsbedürfnis, d. h. das Bedürfnis, sich mit verschiedenen Inhalten intellektuell zu beschäftigen: „Bei der Konfrontation mit persuasiven Botschaften (...) neigen Personen mit einem starken Kognitionsbedürfnis eher zu inhaltsrelevantem Nachdenken als Personen mit einem niedrigen Kognitionsbedürfnis“" (Stroebe 2007, 237). Eine Verarbeitung über die zentrale Route ist also bei Menschen mit hohem Kognitionsbedürfnis wahrscheinlicher. Zur Fähigkeit zählen Werth und J. Mayer $(2008,243)$ außerdem auch die Ablenkung, da abgelenkte Menschen an einer tiefen Verarbeitung gehindert werden und lediglich die peripheren Hinweisreize auswerten können.

Die Verarbeitungsroute wird jedoch am stärksten durch die Motivation des Rezipienten bestimmt. Der wichtigste Motivationsfaktor ist dabei die persönliche Relevanz: Ist das Thema für den Rezipienten relevant, so ist es ihm besonders wichtig ,korrekte“ Einstellungen zu haben. Folglich verarbeitet er die Botschaft über die zentrale Route und achtet vor allem auf die Qualität der Argumente (Werth und J. Mayer 2008, 247). Ist die persönliche Relevanz niedrig, achtet man eher auf die peripheren Hinweisreize (Stroebe 2007, 237; Stürmer 2009, 86-87).

Die Motivation kann darüber hinaus durch Emotionen und Stimmungen beeinträchtigt sein: „Menschen in guter Stimmung lassen sich eher von pe-

119 Stroebe (2014) definiert Heuristiken als ,,(..) einfache Regeln wie „Statistiken lügen nicht“, ,Auf Experten kann man sich verlassen“ oder „Konsens impliziert Korrektheit“““ (Stroebe 2014, 240). 


\section{Umsetzung und Erfolg der Sprachlenkung}

ripheren Hinweisreizen beeinflussen (...)“ (Werth und J. Mayer 2008, 248), weil sie die Konfrontation mit stimmungsdämpfenden Faktoren meiden wollen (Werth und J. Mayer 2008, 248-249; vgl. Stroebe 2007, 240-241).

\subsubsection{Anreize}

Einstellungsänderung ist nicht nur durch Persuasion möglich, sondern auch durch den Einsatz von verschiedenen Anreizen wie Bedrohung, Bestrafung aber auch Belohnung (Stroebe 2007, 253-254). Diese Anreize werden meistens durch verschiedene Institutionen eingesetzt, nicht um primär Einstellungen, sondern das Verhalten zu beeinflussen (Stroebe 2007, 261). Um das Einhalten des gewünschten Verhaltens zu gewährleisten, bedarf es häufig einer kontinuierlichen Überwachung. Deshalb haben Institutionen Interesse an der Einstellungsänderung, weil man dadurch erwartet, dass die ständige Überwachung entfallen könnte (Stroebe 2007, 254).

Wie Sanktionen oder Belohnungen die Veränderung von Einstellungen bewirken können, erklären Stroebe (2007, 255-259) sowie Werth und J. Mayer (2008, 219-239) anhand von zwei Theorien, die ich bereits im Zusammenhang mit Selbst/Identität (Abschnitt 4.5.1) erwähnt habe:

- Dissonanztheorie (Festinger 1957)

- Selbstwahrnehmungstheorie (Bem 1972)

Dissonanztheorie Im Allgemeinen streben Menschen nach Konsistenz (Werth und J. Mayer 2008, 225). Auch bei Einstellungen besteht das Bedürfnis, dass „,...) sich (...) Einstellungen sowie die Komponenten einer Einstellung in einem harmonischen, spannungsfreien (...) Zustand zueinander befinden" (Werth und J. Mayer 2008, 225). Ist dies nicht der Fall, kann eine sog. Dissonanz entstehen, die als negativer emotionaler Zustand empfunden wird (Stroebe 2007, 255).

Die Dissonanztheorie im Kontext von Einstellungen geht davon aus, dass der Einsatz von Sanktionen ein einstellungskonträres Verhalten bewirkt, d. h. ein solches, „das mit der Einstellung und den Meinungen des Handelnden nicht im Einklang steht“ (Stroebe 2007, 254). Menschen sind jedoch im Allgemeinen bestrebt, Dissonanzen zu meiden oder zu reduzieren (Stroebe 2007, 255). Stroebe (2007) entnimmt der Selbstwahrnehmungstheorie, dass nicht jedes Handeln gegen die eigenen Einstellungen gleichermaßen eine 
Dissonanz hervorruft. Entscheidend sind dabei die (wahrgenommene) Entscheidungsfreiheit, die negativen Konsequenzen des einstellungskonträren Handelns und die (nicht) vorhandene Rechtfertigung sowohl bei Sanktionen als auch bei Belohnungen (Stroebe 2007, 256-257; vgl. Werth und J. Mayer 2008, 231-232). Wie Stroebe (2007) weiter erläutert: Kann man sein einstellungskonträres Verhalten gut rechtfertigen, weil man beispielsweise unter Zwang (z. B. Bestrafungsdrohung) handelt, keine negativen Folgen antizipiert oder eine Belohnung in Aussicht gestellt bekommt, dann ruft das einstellungskonträre Verhalten wenig Dissonanzen hervor. Dabei kommt es bei der Bestrafung oder Belohnung auf ihre Stärke an: „Wenn sich eine Person (...) einstellungskonträr verhält, um einer Bestrafung zu entgehen oder irgendeinen Nutzen daraus zu ziehen, wird die Dissonanz größer sein, wenn die Strafe bzw. der Nutzen eher klein als groß ist" (Stroebe 2007, 256). Auch beim Ausbleiben der versprochenen Belohnung oder bei einer anderweitigen Enttäuschung mit dem Resultat können Dissonanzen auftreten (Werth und J. Mayer 2008, 231).

Den Zusammenhang zwischen Dissonanzen und Einstellungsveränderung erklären beispielsweise Werth und J. Mayer (2008): Bei Dissonanzen steigt die Wahrscheinlichkeit, dass man sein nichteinstellungskonformes Verhalten nachträglich aufwertet (oder bagatellisiert), was in Konsequenz zur Einstellungsänderung führen kann, obwohl auch andere Dissonanzreduktionsstrategien möglich sind (Werth und J. Mayer 2008, 228-230; vgl. Stroebe 2007, 255).

Zusammenfassend kann man sagen, dass man Dissonanzen auch gezielt herbeiführen kann, um Einstellungen und in Konsequenz das Verhalten dauerhaft zu verändern.

Selbstwahrnehmungstheorie Auch die Selbstwahrnehmungstheorie von Bem (1972) kann zur Erklärung von Einstellungsänderungen eingesetzt werden. Wie Stroebe $(2007,2014)$ erläutert, ist sie besonders dann hilfreich, wenn Menschen die Aufmerksamkeit auf sich selbst richten, beispielsweise wenn sie über eigene Einstellungen im Unklaren sind (Stroebe 2007, 257, 2014, 262). Die unter (milden) Anreizen ausgeführten Handlungen können vom Individuum selbst als einstellungskonform gedeutet werden, da es sonst sein Verhalten als Quelle der selbstbezogenen Information abwerten müsste (Stroebe 2007, 257-258). Ist jedoch der Zwang zu offensichtlich, so dass man sein Verhalten nicht auf die inneren Zustände, sondern auf äußere Begebenheiten attribuiert, dann ist die eigene Wahrnehmung 


\section{Umsetzung und Erfolg der Sprachlenkung}

als Informationsquelle über das Selbst und insbesondere die eigenen Einstellungen ungeeignet (Stroebe 2007, 259). Diesen Mechanismus kann man zur Herbeiführung einer Einstellungsänderung nutzen. Die Veränderung der Einstellung aufgrund der Selbstwahrnehmung ist vor allem dort zu erwarten, wenn das (unter mildem Anreiz) gezeigte Verhalten nur geringfügig von den Einstellungen abweicht, so dass die Einstellungsveränderung kaum merkbar ist (Stroebe 2007, 258).

\subsubsection{Mere-Exposure-Effekt}

Beim Mere-Exposure-Effekt handelt es sich eigentlich um einen Mechanismus der Einstellungsentstehung, den man aber auch gezielt zur Einstellungsänderung einsetzen kann (Werth und J. Mayer 2008, 223). Wie in Haddock und Maio $(2007,191)$ erläutert, wird hierfür eine Person über einen Zeitraum hinweg einem Einstellungsobjekt immer wieder ausgesetzt, wobei eine zu häufige und zu offensichtliche Darbietung diesen Effekt wiederum verhindern kann (Werth und J. Mayer 2008, 224). Durch die bloße Steigerung der Vertrautheit wird dieses Objekt immer positiver bewertet (Haddock und Maio 2007, 191; Stürmer 2009, 72-73). Dank des Mere-Exposure-Effekts reicht also eine allmähliche Gewöhnung, um eine positive Einstellung zu einem Objekt auszulösen.

\subsubsection{Strategien zum Schutz gegen Einstellungsänderungsversuche}

Um das Bild über die Möglichkeiten einer Einflussnahme auf das Verhalten durch Einstellungsänderungen zu vervollständigen, gehe ich abschließend auf die Perspektive von den zu beeinflussenden Personen ein und stelle kurz Strategien vor, mit deren Hilfe man sich vor Einstellungsänderungen schützen kann. Zu den bekanntesten gehören (Werth und J. Mayer 2008, 252):

- Einstellungsimpfung

- Vorwarnung

- Reaktanz

Bei der sog. Einstellungsimpfung setzt man eine Person im Vorfeld eines Einstellungsänderungsversuchs Argumenten gegen ihre Einstellungen aus, wobei meistens nur einzelne Argumente dargeboten werden. Auf 
diese Weise kann sie eine Gegenargumentation vorab entwickeln, die ihre eigene Position festigt und gegen eine systematische und umfangreiche Argumentation resistent macht (Werth und J. Mayer 2008, 252).

In manchen Fällen schützt vor einer Einstellungsänderung bereits eine Vorwarnung, also „das Wissen über die persuasive Absicht eines anderen (...)“ (Werth und J. Mayer 2008, 252).

Schließlich gibt es eine automatische Reaktion auf einen Einstellungsänderungsversuch - die Reaktanz. Darunter versteht man den ,(...) Widerstand, welcher gegen Einschränkungen der eigenen Handlungsfreiheit gerichtet ist" (Werth und J. Mayer 2008, 253). Nach der Reaktanztheorie von Brehm (1966) ist man in diesen Fällen stark bestrebt, „(...) diese Freiheit wiederherzustellen“" (Stroebe 2007, 260). „Folglich sind zu starke, offensichtliche Beeinflussungsversuche kontraproduktiv und führen eher zur Verfestigung der ursprünglichen Einstellungen (...)“(Werth und J. Mayer 2008, 254).

\subsubsection{Abschließende Bemerkungen}

Zusammenfassend lässt sich sagen, dass eine Einstellungsänderung schwer zu erzielen ist und auch dann nicht zwangsläufig zur gewünschten Verhaltensänderung führt. Außerdem liegt einem veränderten Verhalten nicht zwangsläufig eine Einstellungsänderung zugrunde. Stroebe (2007) stellt beispielsweise fest, dass die durch Sanktionen oder Belohnungen motivierte Verhaltensänderung ,selten mit einer Einstellungsänderung einhergeht (...)“ (Stroebe 2007, 259), weil das Verhalten auf externe Gründe attribuiert wird. Die Einstellungsänderung durch verhaltensändernde Maßnahmen ist aber auch aus anderen Gründen beschränkt: aus Mangel an Macht und Überwachung sowie aufgrund individueller Unterschiede in Bezug auf die Befolgung von Regeln (Stroebe 2007, 261). Stroebe (2007) spricht sogar von ,paradoxen Wirkungen" der Anreize auf Einstellungen, da z. B. das Verbotene attraktiver wird, das Phänomen der Reaktanz auftritt oder die intrinsische Motivation unterminiert wird (Stroebe 2007, 260). Stroebe (2007) resümiert, dass außer in Fällen, in denen sich das neue Verhalten als nicht so unangenehm wie befürchtet zeigt und in Konsequenz zur Entwicklung von positiven Einstellungen führt (Stroebe 2007, 255), es häufig ratsam ist, Anreize mit Persuasion zu verbinden, damit der Sinn einer Maßnahme erkannt wird (Stroebe 2007, 262). 


\subsection{Sprache und Identität in der Linguistik}

Die Frage nach dem Zusammenhang zwischen Sprache und Identität wird vor allem in der Soziolinguistik, aber auch in Sprachlern- und erwerbsforschung sowie in der Mehrsprachigkeitsforschung im weitesten Sinne erörtert. Ich identifiziere dabei zwei unterschiedliche Grundannahmen, die zu unterschiedlichen Fragestellungen und Schwerpunktsetzungen in der Forschung führen:

- Sprache als Ausdruck der Identität(en),

- Sprache als Teil der Identität.

Im Folgenden erläutere ich kurz beide Auffassungen.

Eine Zusammenfassung der ersten Annahme findet sich beispielsweise in Crystal (1998, 17-79), auf die ich mich hier hauptsächlich beziehe. Demnach manifestieren sich viele verschiedene Merkmale einer Person in ihrer Sprache. Bei den Merkmalen kann sich um relativ stabile physische Merkmale wie Geschlecht, Statur, oder auch Alter, Intelligenz oder andere idiosynkratische Merkmale handeln, aber auch solche, die mit der geographischen, ethnischen, sozialen (schichtbezogenen, statusbezogenen) etc. Prägung zusammenhängen.

Vor diesem Hintergrund wird noch einmal deutlich, dass jede Varietät z. B. im Sinne von Löffler (2010) als Ausdruck einer bestimmten Identität gedeutet werden kann, wobei sich diese Identitäten auch in der Linguistik zwischen den Polen der persönlichen und gruppalen (sozialen) Identität einordnen lassen. Darüber hinaus halte ich aus dem Obigen fest, dass in der Linguistik ebenfalls davon ausgegangen wird, dass Identität nicht ein unteilbares Ganzes ist, sondern verschiedene Teile umfasst, die, wie Crystal $(1998,17)$ anführt, durch situative Anreize wie Umgebung, Teilnehmer oder aktuelle Handlung aktiviert werden.

Eine Veranschaulichung dieser multiplen, situativ aktivierten, sprachbezogenen Sicht auf Identität bieten beispielsweise das Modell der multiplen Sprachidentität und das Modell der dialogischen Identitätskonstruktion von Kresic (2006), die entsprechend in Abbildung 17 und Abbildung 18 dargestellt sind.

Das Modell der multiplen Identität (Kresic 2006, 227-233) besagt, dass durch eine bestimmte Sprachverwendung, also insbesondere auf Grundlage der beherrschten Varietäten und Register, eine sprachliche (Teil-)Identität im Diskurs konstruiert wird. Die Gesamtidentität besteht also aus mehreren Teilidentitäten, die durch das Individuum unterschiedlich gewichtet sind. Diese 


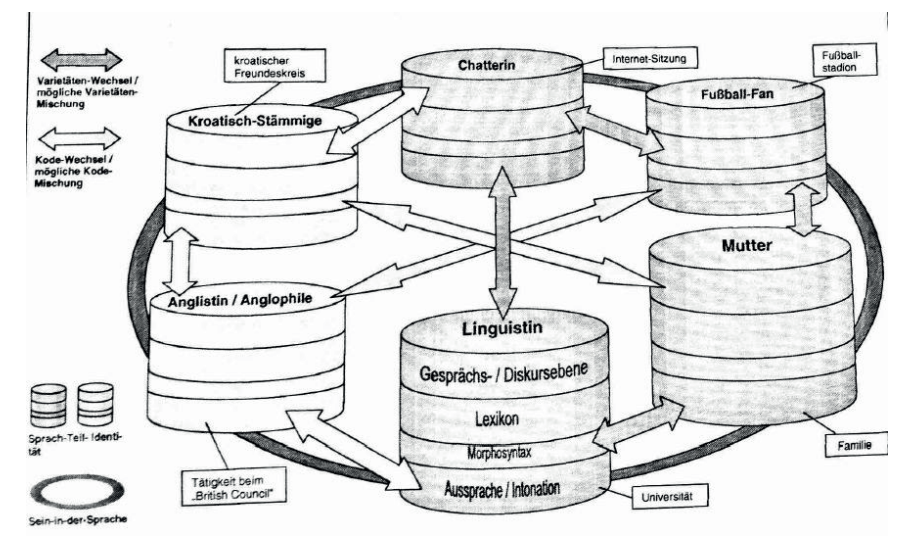

Abbildung 17.: Modell der multiplen Sprachidentität (Kresic 2006, 228)

Zusammensetzung und Gewichtung sind dabei nichts Festes und können sich im Laufe der Zeit ändern. Andererseits betont Kresic (2006, 229), dass die Teilidentitäten nicht nebeneinander existieren, sondern vielfältig miteinander verflochten sind und eine Art Struktur ergeben (Kresic 2006, 230). Im zweiten Modell (Kresic 2006, 233-236) wird hingegen der erwähnte Prozess der Identitätskonstruktion im Diskurs näher erläutert. Demnach wird die Identität im Dialog zwischen dem Sprecher und dem Hörer ausgehandelt. Hier ist vor allem der Hörer entscheidend, da er anhand der rezipierten Sprache eine Annahme über die Identität des Sprechers macht. Da die Rollen im Dia$\log$ wechseln, wird eine Identität für jeden Kommunikationspartner im Dialog konstituiert. Der Sprecher kann hingegen beispielsweise durch bewusste Auswahl von Sprachmitteln oder Selbstdarstellungsstrategien auf diese hörerseitige Identitätskonstruktion einen Einfluss nehmen. Für Kresic (2006) ist bei der Identitätskonstruktion also zentral, dass es ein Gegenüber gibt, in dessen Anwesenheit die eigene Identitätskonstruktion erst möglich ist. Die Identität wird also immer in Abgrenzung zu anderen konstruiert (Kresic 2006, 234). Insgesamt postuliert Kresic (2006) auch eine Erweiterung des Organonmodells von Bühler (1978, exemplarisch) um die Zeichendimension der Identitätskonstruktion (Kresic 2006, 234).

Wenn man aufgrund sprachlicher Merkmale eine Identität konstruiert, wie dies in den beiden Modellen von Kresic (2006) der Fall ist, so stellt sich eine naheliegende Frage, ob jede Identität (mindestens) ein sprachliches Merkmal besitzt, das diese Identität repräsentiert oder ob es auch (Teil-)Identitäten ge- 


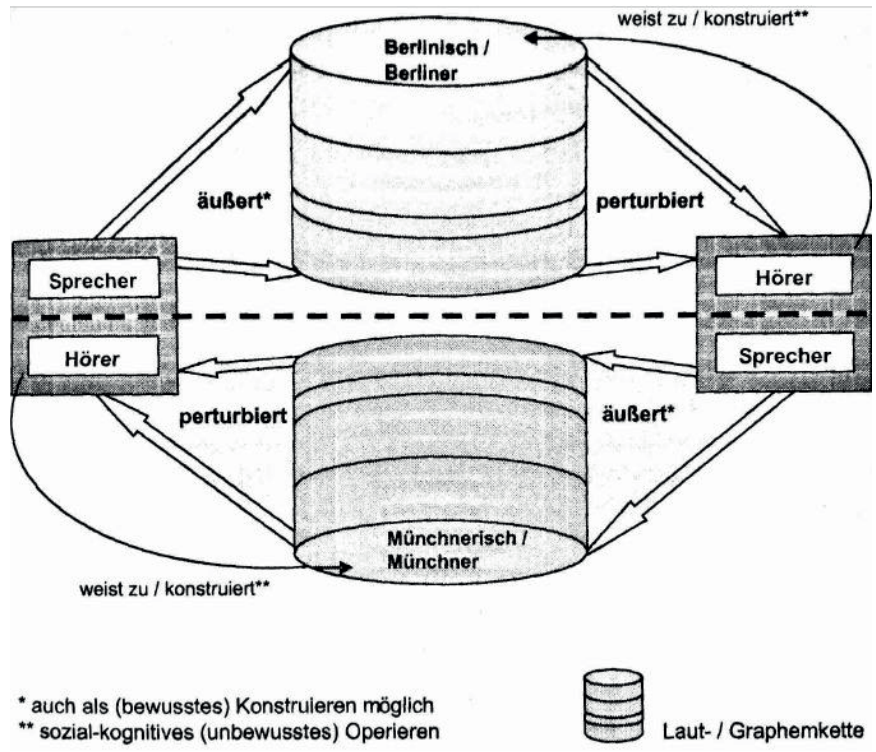

Abbildung 18.: Modell der dialogischen Identitätskonstruktion (Kresic 2006, 235)

ben kann, die sprachlich nicht markiert sind. Obwohl ich diese Frage im Rahmen dieser Arbeit nicht direkt und abschließend beantworten kann, so stellt sie eine Überleitung zur zweiten Auffassung des Zusammenhanges zwischen Sprache und Identität dar: Sprache macht einen festen Teil der Identität aus.

Diese Auffassung wird vor allem im Zusammenhang mit der ethnischen Identität diskutiert, wobei Schnar (2010) hier auch auf die Unterschiede zwischen den europäischen und den angelsächsischen Forschungstraditionen hinweist (Schnar 2010, 19, 22). Hiernach ist Sprache neben anderen Symbolen wie Kultur oder Religion konstitutiv für die ethnische Identität. Obwohl diese Symbole nicht für alle Gruppen gleich sind und sich ihre Ausprägung und Gewichtung auch unterscheiden kann (vgl. Schnar 2010, 18, 24), so geht man dennoch generell davon aus, dass Sprache ein unabdingbarer Bestandteil der ethnischen Identität ist.

Mit dieser Auffassung polemisiert Haarmann (1986), und ich gebe hier die einschlägigen Gedanken seiner Untersuchungszusammenfassung wieder (Haarmann 1986, 257-265). Haarmann (1986) sieht die Rolle der Sprache in der Konstruktion und Erhaltung der ethnischen Identität in der Soziolinguis- 
tik als übertrieben an. Am Beispiel der Ischoren-Gemeinschaft zeigt er, dass die ethnische Identität trotz eines Sprachverlustes erfolgreich weiterbestehen kann (Haarmann 1986, 259). Die Sprache kann also nicht generell als unerlässlich für die ethnische Identität betrachtet werden und folglich ist die gegenseitige Beeinflussung beider Faktoren unterschiedlich ausgeprägt: Sprache als Abgrenzungsmittel steht immer im Dienste der (ethnischen) Identität. Andersherum aber: Die ethnische Identität kann (muss aber nicht) an die Sprache gebunden sein (Haarmann 1986, 260-261). Ob die Sprache einen unerlässlichen Teil der ethnischen Identität ausmacht, wird alleine durch die Gruppe bestimmt und ist somit einzelfallspezifisch: „The relevance of language in ecological relations depends on the role it is assigned by ethnic groups in interethnic relations“" (Haarmann 1986, 258).

Diese relativierte Rolle der Sprache für die ethnische Identität wurde auch empirisch belegt. So bezieht sich beispielsweise eine Hypothese in der Untersuchung von Schnar (2010) genau auf den Aspekt der Sprache als „Gruppenzugehörigkeitsmarker (...) im Konzept der ethnischen Identität Russlanddeutscher" (Schnar 2010, 95). Die Untersuchungsergebnisse bestätigen diese Hypothese jedoch nicht, da die Befragten stärker ,ein abstammungsorientiertes ethnisches Selbstkonzept" vertreten (Schnar 2010, 96).

Alles in allem halte ich fest, dass auch in der Linguistik Identität zunehmend als Selbstkategorisierung von Individuen betrachtet wird (exemplarisch Gudykunst und Ting-Toomey 1990, 310). Wie oben dargelegt, kann sich sowohl die Identität (oder Teilidentitäten) auf den Sprachgebrauch von Individuen auswirken, aber auch andersherum kann die Sprache allgemein die soziale Wahrnehmung beeinflussen (vgl. McCann und Higgins 1990, 15-21). Im speziellen Kontext der sozialen Identität wird insbesondere angenommen, dass Sprache eine doppelte Rolle spielt. Zum einen wirkt sie sich kohäsiv nach innen aus durch Stärkung des Gruppenzusammenhaltes und der Gruppensolidarität (Ryan 1979, 153; Ager 2001, 84; Schnar 2010). Zum anderen aber hat sie eine Abgrenzungsfunktion nach außen (McCann und Higgins 1990, 1524; Schnar 2010, 22-23). Die Analyse von Haarmann (1986) ergibt jedoch, dass diese doppelte Rolle vor allem dann angenommen werden kann, wenn Sprache einen unerlässlichen Teil der Identität ausmacht.

Bisher bin ich auf den dynamischen Wechsel zwischen verschiedenen Identitäten oder verschiedenen Sprachen eines Individuums eingegangen, der zwar durch die (äußere) Situation bedingt ist, aber tendenziell durch das Individuum selbst initiiert und vollzogen wird. Für diese Arbeit ist jedoch die Rolle Dritter bei diesen Wechseln interessant, vor allem die 


\section{Umsetzung und Erfolg der Sprachlenkung}

identitätsbezogenen Konsequenzen eines Eingriffs in die Sprache von Personen(gruppen). Diese Frage wurde in allen vier von mir betrachteten Gebieten direkt oder indirekt aufgegriffen und ich unterscheide anhand der Beispiele aus dem Kapitel Forschungsstand grob zwei Tendenzen:

- Man nimmt an, dass man durch einen Eingriff in die Sprache einer Gruppe auch ihre Identität beeinflussen kann und führt entsprechend sprachliche Interventionen in der Praxis durch. Diese Ansicht ist bei vielen Sprachplanungsmaßnahmen aber auch im Bereich der Plansprachen zu beobachten. Entsprechend zieht sich das Thema durch weite Teile von Kapitel 2 und der zitierten Literatur durch. Zur Wiederholung nenne ich hier exemplarisch die Entwicklung einer Standardsprache, um das nationale Zusammengehörigkeitsgefühl zu entwickeln und zu stärken (Französisch, aber auch Hebräisch), Spracherhaltungsmaßnahmen wie für das Irische oder aber Zamenhofs Motive zur Entwicklung des Esperanto.

Zur Erklärung der (Miss-)Erfolge bei Sprachplanung, vor allem im Kontext der Sprachpflege oder des Spracherhalt, setzt Haarmann (1986) die von ihm postulierte relative Rolle der Sprache im Identitätskonstrukt ein. Da Sprache nicht immer einen unerlässlichen Teil der (ethnischen) Identität ausmacht, so ist Sprachplanung zum Erhalt der ethnischen Identität nicht zwangsläufig sinnvoll und in Konsequenz nicht immer erfolgreich (vgl. Haarmann 1986, 258-259). Auf diesen Aspekt gehe ich näher bei der Interpretation der Untersuchungsergebnisse in Kapitel 5 ein.

- Man greift in die Sprache einer Gruppe ein, ohne den Zusammenhang zwischen Sprache und Identität zu beachten. Auch für diesen Fall finden sich in den vier Gebieten jedoch Beispiele, die darauf hindeuten, dass Identitätsaspekte infolge eines Spracheingriffs aktiviert werden und sich dann auf den Eingriff selbst auswirken können. Beschrieben wird dieser Mechanismus beispielsweise für die erste Phase der LPLPEntwicklung in Abschnitt 2.1.2.1, in der die aus vornehmlich instrumentellen Gründen durchgeführte Sprachplanung in den Entwicklungsländern wenig erfolgreich war. Sie geriet auch deshalb in Kritik, da die jeweilige Kultur und Tradition, also insgesamt auch die Identität, der Zielgruppe, nicht beachtet wurden. Es führte dann zu einer allgemeinen Hinwendung zu den Identitätsaspekten im Kontext der Sprachplanung, die man in solchen Ansätzen wie den sprachlichen Menschenre- 
chen findet. Ein Beispiel für Kontrollierte Sprachen findet sich hingegen in der bereits zitierten Untersuchung von Lassen (2003), die u. a. ergab, dass ein Eingriff in die Sprache der Technischen Redaktion das Selbstverständnis von Technischen Redakteuren oder anderen Sprachexperten in Frage stellt (Lassen 2003, insb. 165-167), was ich als Identitätssalienz interpretiere. In der praktischen Literatur zur Terminologiearbeit findet man darüber hinaus Unternehmensberichte über eine Ablehnung des sprachlichen Eingriffs, die durch die aktive Zielgruppe mit Äußerungen wie ,das haben wir schon immer so gesagt" begründet wird (exemplarisch Leitner 2014, 19). Auch diese Berichte, insbesondere da das Pronomen wir verwendet wird, interpretiere ich als Hinweis auf Identitätsaspekte, obwohl es nicht ganz klar ist, auf welche Teilidentität (Unternehmen, Berufsgruppe, Abteilung etc.) sich diese Äußerungen beziehen.

Diese Perspektive macht noch einmal deutlich, dass ein Eingriff in die Sprache (also insbesondere auch die Sprachlenkung) aus der Sicht der Autoren idealtypisch zwei Ausprägungen annehmen kann:

- Sprachlenkung kann ein untergeordnetes Ziel von identitätsbezogenen Handlungszielen sein und zum Erhalt der Gruppenidentität beitragen. Dies bedeutet andererseits, dass man beispielsweise den Erhalt der Identität als Argument benutzen kann, um Sprachlenkung zu stützen oder zu rechtfertigen. Identitätssalienz ist somit eine Methode in der Umsetzung der Sprachlenkung. Dieser Mechanismus ist vor allem in identitätsbezogener Sprachlenkung stark ausgeprägt.

- Sprachlenkung kann die Gruppenidentität erst salient machen. Dabei nehme ich an, dass dies entweder durch die Bewusstmachung des eigenen Sprachgebrauchs erfolgt oder durch die Wahrnehmung der Autorengruppe als Eigen- oder Fremdgruppe geschehen kann. Diesen Mechanismus vermute ich eher bei instrumenteller Sprachlenkung.

Aufgrund des Forschungsstandes nehme ich für Sprachlenkung deshalb an, dass sowohl für identitätsbezogene wie auch instrumentelle Sprachlenkung das Konzept der Identitätssalienz zur Erklärung von Herausforderungen bei der Umsetzung herangezogen werden kann. 


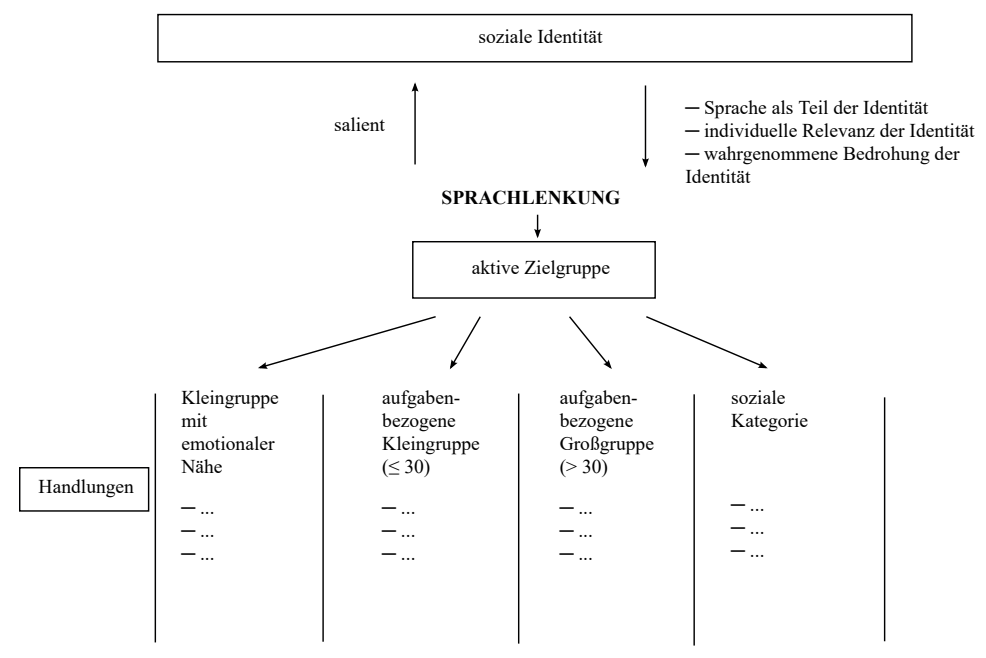

Abbildung 19.: Umsetzungsorientiertes Modell der Sprachlenkung

\subsection{Umsetzungsorientiertes Modell der Sprachlenkung}

Alle Vorüberlegungen aus diesem Kapitel, insbesondere Annahmen 1 und 2 in Abschnitt 4.4, ermöglichen mir, in diesem Abschnitt ein umsetzungsorientiertes Modell der Sprachlenkung vorzustellen, das drei besprochene Konstrukte: Gruppe, Identität sowie Handlungen berücksichtigt (Abbildung 19). Dieses Modell ist durch den besprochenen Forschungsstand beeinflusst, muss jedoch als weitestgehend deduktiv betrachtet werden. Es dient als Einstieg in die Untersuchung in Kapitel 5 und legt offen, mit welchem Vorverständnis von Sprachlenkung und Erfolgsfaktoren ich an die Untersuchung herangehe. Es hat also die Grundlagenfunktion für die Systematisierung in der primären Analyse sowie für die weiterführende Interpretation der Ergebnisse in Kapitel 5. Im Folgenden bespreche ich das Modell.

Den zentralen Aspekt des übergreifenden, umsetzungsorientierten Modells der Sprachlenkung bilden die aktiven Zielgruppen. Um ein besseres Verständnis des Umsetzungsprozesses zu schaffen und auf dessen Grundlage praktische Themen angehen zu können, wie eine Einschätzung der Umsetzbarkeit 
einer Maßnahme oder die Herleitung konkreter Vorgehensweisen, postuliere ich hier die Ausdifferenzierung der Sprachlenkung bzw. die Bündelung verschiedener Maßnahmen nach der Beschaffenheit der aktiven Zielgruppe. Hierfür bediene ich mich der vorgestellten Gruppenklassifikation nach Lickel, Hamilton, Lewis u. a. (2000) und Lickel, Hamilton und Sherman (2001), die zwei Merkmale - Gruppengröße und Gruppenziele - berücksichtigt. Für Sprachlenkung sehe ich folgende Gruppenarten für relevant:

- Kleingruppe mit Emotionaler Nähe (Familie)

- aufgabenbezogene Kleingruppe (Kleinunternehmen, Unternehmensabteilungen etc.)

- aufgabenbezogene Großgruppe (Mittelständisches/Großunternehmen, Berufsgruppe, Industriezweig, Beamte etc.)

- soziale Kategorie, wobei damit vor allem eine nichtaufgabenbezogene Großgruppe gemeint ist (Nation, Geschlecht, Altersgruppe)

Ich gehe davon aus, dass jede Sprachlenkungsmaßnahme sich zu einem gegebenen Zeitpunkt einer dieser Gruppenart zuordnen lässt, wobei dies wesentlich damit zusammenhängt, wie konkret und explizit die Ziele und die Zielgruppe in der Planungs- und Vorbereitungsphase angegeben wurden.

Die Einteilung nach Gruppenarten in Anlehnung an Lickel, Hamilton, Lewis u. a. (2000) und Lickel, Hamilton und Sherman (2001) verknüpft zwei Merkmale. Die Gruppengröße kann im Kontext der Sprachlenkung eine allgemeine Vorstellung davon geben, wie leicht alle Mitglieder der aktiven Zielgruppe erreichbar sind und, aus der Perspektive der Autoren, wie leicht eine Gruppe steuerbar ist. Das auf die aktive Zielgruppe bezogene Merkmal der Ziele (emotionale Nähe vs. Aufgabenbezogenheit) scheint für Sprachlenkung ebenfalls günstig zu sein, weil es mit den hier identifizierten Typen der Sprachlenkung aus Autorensicht (identitätsbezogen vs. instrumentell) weitestgehend korrespondiert. So nehme ich an, dass in beiden aufgabenbezogenen Gruppenarten eher instrumentelle Sprachlenkung stattfindet, während in den nichtaufgabenbezogenen Gruppenarten identitätsbezogene Sprachlenkung dominiert. Selbstverständlich sind es keine absoluten Annahmen, sondern lediglich Tendenzen. Die Frage der Übereinstimmung zwischen den Gruppenzielen und den Zielen der Autoren 


\section{Umsetzung und Erfolg der Sprachlenkung}

ist auch für die Umsetzbarkeit relevant, weil es ein Intergruppenkonflikt, wie in Abschnitt 4.6.2.3 theoretisch beschrieben, verursachen kann. ${ }^{120}$

Die Einteilung der Sprachlenkung nach Art der aktiven Zielgruppe ermöglicht eine systematische Verfeinerung der Betrachtung und bietet eine Alternative zur historisch gewachsenen, recht unsystematischen Aufteilung in die in dieser Arbeit beschriebenen vier Gebiete. Durch eine neue Bündelung verschiedener Maßnahmen kann es zudem zu einem besseren Austausch bisheriger Erkenntnisse zwischen diesen vier Gebieten kommen.

Der hier vorgeschlagenen Einteilung ist zudem zu entnehmen, dass ich es bei der Umsetzung der Sprachlenkung nicht für primär ausschlaggebend halte, welche Ebenen des Sprachsystems eine Maßnahme betrifft. Die systemlinguistischen Eigenschaften der Maßnahmen sehe ich im Kontext der Umsetzung als der Gruppenperspektive untergeordnet.

Die oberste Ebene des Modells in Abbildung 19 stellt die (soziale) Identität dar. Wie in Abschnitt 4.8 erläutert, wird im Kontext der Sprachlenkung die soziale Identität der Zielgruppe salient gemacht. Diese wird entweder durch die Autoren explizit thematisiert und in ein identitätsbezogenes Oberziel eingebettet. Oder die Sprachlenkung wirkt sich auf die Erhöhung der Selbstwahrnehmung aus, wobei der eigene Sprachgebrauch reflektiert oder aber die Autorengruppe als Fremdgruppe wahrgenommen wird, was beides mit der Bewusstmachung der eigenen Identität einhergeht. Dabei möchte ich betonen, dass die aktive Zielgruppe einen Eingriff in ihre Sprache nicht automatisch als Angriff auf ihre Identität empfinden muss. Im Modell behaupte ich lediglich, dass die eigene Identität bei den Mitgliedern der aktiven Zielgruppe kognitiv zugänglich gemacht wird.

Da ich annehme, dass die soziale Identität bei jeder Sprachlenkungsmaßnahme, egal ob identitätsbezogen oder instrumentell, salient gemacht wird, stellt die Identitätssalienz ein übergreifendes Merkmal dar, das alle Maßnahmen charakterisiert. Es steht also im Gegensatz zum Gruppenmerkmal, das zur Differenzierung der Sprachlenkung benutzt werden kann, wie ich weiter oben ausführte.

Sobald die soziale Identität salient wird, wirkt sie sich ihrerseits auf den Umsetzungsprozess aus, weil sie Intergruppenprozesse in Gang setzt. Aus der Erfolgsperspektive sehe ich insbesondere folgende drei Faktoren als entscheidend: $\mathrm{Ob}$ die (zu ändernde) Sprache einen unerlässlichen Teil der Identität ausmacht; ob die saliente (also durch Sprachlenkung betroffene) sozia-

120 Ein praktisches Beispiel ist das Thema der ideologisch-orientierten (value-oriented) und der instrumentell-orientierten (norm-oriented) Strömungen in Esperanto (2.2.4.2). 
le Identität für ein Individuum einen wichtigen Teil seiner Gesamtidentität ausmacht sowie ob die Sprachlenkung als Bedrohung der salienten Identität wahrgenommen wird. Dabei können diese Faktoren eine kontinuierliche und nicht einfach binäre Ausprägung einnehmen.

Man kann annehmen, dass eine Sprachlenkungsmaßnahme besonders schwer erfolgreich zu gestalten ist, wenn die betroffene Sprache einen unerlässlichen Teil der Identität ausmacht, diese Identität von den meisten Mitgliedern der Zielgruppe als wichtig empfunden wird und die Maßnahme als Identitätsbedrohung wahrgenommen wird. Hier kann u. a. das Phänomen der Reaktanz eine Rolle spielen, das auch für den sprachlichen Bereich angenommen wird (s. bspw. McCann und Higgins 1990, 17) Bedroht die Sprachlenkung im obigen Beispiel die Identität hingegen nicht und trägt möglicherweise sogar zur Verwirklichung der Oberziele bei (instrumentellen wie identitätsbezogenen gleichermaßen), so hat die Maßnahme bessere Erfolgsaussichten.

Die unterste Ebene in dem Modell stellen konkrete Handlungen dar, durch die Autoren das Sprachlenkungsziel erreichen können. Es handelt sich hierbei also um sog. Best Practices.

Ich gehe davon aus, dass nicht jede Handlung für jede Ausprägung der Sprachlenkung sinnvoll ist und postuliere daher die Auswahl von geeigneten Vorgehensweisen in Abhängigkeit vom Typ der aktiven Zielgruppe. In Abschnitt 4.7 ging ich allgemein auf die Möglichkeiten und Grenzen von Einstellungsänderungsstrategien (Persuasion, Anreize, Mere-Exposure-Effekt) zur Verhaltensänderung ein. An dieser Stelle bleibt die unterste Ebene des Modells jedoch noch unbesetzt, da konkretere Vorschläge erst nach der Literaturauswertung in Kapitel 5 möglich sind.

\subsection{Umsetzung und Erfolg der Sprachlenkung - Fazit}

In diesem Kapitel habe ich mich mit der zweiten Forschungsfrage dieser Arbeit befasst, wobei hier primär um die Begriffsbestimmung von Erfolg ging. Dabei wurde durch die Diskussion bestehender Ansätze aus den einschlägigen Gebieten noch einmal deutlich, dass die Frage nach dem SL-Erfolg bisher weder konzeptionell noch aus der Praxisperspektive systematisch erforscht wird. Dies hat verschiedene Folgen für diese Arbeit. 
Zum einen beruhen die in diesem Kapitel vorgeschlagenen Konzeptualisierungen im Unterschied zur Bestimmung der Sprachlenkung selbst weniger auf einer Synthese vorhandener linguistischer Erkenntnisse, sondern es handelt sich vielmehr um deduktiv hergeleitete Modellierungen, die auf extralinguistischen Instrumentarien beruhen.

Zum anderen hat das Kapitel erneut die Notwendigkeit verdeutlicht, die spezielle Forschungsfrage nach der Umsetzungswirksamkeit umzuformulieren, da aufgrund fehlender Modellierungen eine bewertende Analyse der Wirksamkeit nicht möglich ist. Darüber hinaus erfolgte aufgrund dieser mangelnden Theoretisierung in großen Teilen dieses Kapitels eine Vorstellung von extralinguistischen Grundlagen, die in Verbindung mit den vorgeschlagenen Modellierungen konzeptionell auf die Untersuchung im folgenden Kapitel 5 vorbereiten. 


\section{Untersuchung}

In diesem Kapitel erfolgt die Untersuchung von Umsetzungsmöglichkeiten für Sprachlenkung. Da die aktuelle Forschungslage zu diesem Gegenstand unbefriedigend ist, entscheide ich mich für eine qualitative Metaanalyse, mit der ich aus fragmentarischen Überlegungen eine umfassende Sicht auf den Gegenstand systematisch aufbaue.

Als Daten dienen mir hierfür Zitate aus der wissenschaftlichen einschlägigen Literatur, die ich beim Verfassen dieser Arbeit in einer Datenbank abgelegt habe. In diesen Zitaten werden Aussagen gemacht, welche Herausforderungen sich bei der Sprachlenkung stellen und wie man als SL-Autor vorgehen soll. Das Ziel der Untersuchung ist es, diese Zitate, die gebietsspezifisch erfasst sind, einerseits inhaltlich, andererseits gebietsübergreifend zu verallgemeinern, um so einen integrativen und mehrdimensionalen Überblick über die postulierten Probleme und Handlungsmöglichkeiten bei der Umsetzung von Sprachlenkung zu extrahieren. Ich gehe dabei in mehreren Schritten vor.

Zunächst bündele ich Zitate inhaltlich in übergreifende Einheiten, was in mehreren Abstraktionsphasen erfolgt. Dieser Schritt zielt hauptsächlich auf die Reduzierung der Datenmenge und auf das Aufdecken von übergeordneten Inhalten. Diese Inhalte bleiben in diesem Schritt jedoch an das jeweilige Gebiet gebunden. Die genaue Methode sowie ihre technische Implementierung ist in 5.1 beschrieben.

Die Bündelung aus dem ersten Schritt liefert Rohergebnisse, die ich im zweiten Untersuchungsschritt qualitativ analysiere, strukturiere und vor dem Hintergrund der im ersten Teil dieser Arbeit vorgestellten Konzepte, insbesondere aus der Sozialpsychologie, interpretiere. Das Ziel dieses Schrittes ist es, die Zusammenhänge innerhalb der aufgedeckten Kategorien von Herausforderungen und, getrennt davon, innerhalb von aufgedeckten Kategorien von Vorgehen, herauszuarbeiten. In diesem Schritt wird auch die Trennung in vier Gebiete weitestgehend aufgehoben. Dieser Untersuchungsschritt ist in 5.2 beschrieben.

Schließlich betrachte ich im letzten Schritt die in der Literatur postulierten Beziehungen zwischen Herausforderungen und Vorgehen, verschränke die- 


\begin{tabular}{|l|cl|}
\hline Gebiet & Schlagwort-ID & Schlagwort \\
\hline LPLP & 1 & $\begin{array}{l}\text { Mechanismen der Sprachlenkung: Linguistik, } \\
\text { AOP }\end{array}$ \\
& 6 & Erfolg Veränderung: Linguistik; AOP \\
& 12 & Erfolgsbedingung: Sprachlenkung: Linguistik \\
\hline PS & 148 & Erfolg: Esperanto \\
& 193 & Erfolg: Plansprachen \\
\hline KS & 216 & Erfolg: kontrollierte Sprachen \\
\hline TA & 318 & Terminologiearbeit: Erfolg \\
\hline
\end{tabular}

Tabelle 13.: Zusammenstellung der Datenbankschlagwörter zum Thema Erfolg

se mit den vorstrukturierten Rohergebnissen aus Schritt zwei und verfeinere sie weiter. Hier soll also das Hauptziel der Untersuchung erreicht werden, nämlich ein gebietsübergreifender, mehrdimensionaler Überblick über die postulierten Probleme und Handlungsmöglichkeiten bei Sprachlenkung. Das Hauptergebnis stelle ich in Form von Ablaufdiagrammen dar, die als grobe Handlungspläne für konkrete Sprachlenkungsmaßnahmen eingesetzt werden können. Dieser Untersuchungsschritt ist in 5.3 beschrieben.

\subsection{Datenerfassung}

Bei der Ausarbeitung des Forschungsstandes für diese Arbeit habe ich in einer Datenbank (vorwiegend direkte) Zitate zu verschiedenen Themen erfasst und sie inhaltlich verschlagwortet. ${ }^{121}$ Insbesondere versah ich entsprechende Zitate mit verschiedenen Schlagwörtern zum Thema Erfolg (s. Tabelle 13). Wie aus Tabelle 13 ersichtlich, sind Erfolgsschlagwörter und somit Zitate nach diesen Gebieten (LPLP, PS, KS, TA) getrennt geführt. Ein Zitat kann aber auch mehrfach verschlagwortet sein.

Als Zitatquellen benutzte ich einschlägige Literatur aus den vier Gebieten, die weitestgehend aus Kapitel 2 bekannt ist. Bei der Literaturauswahl ging es weniger um Repräsentativität, sondern darum, möglichst viele heterogene

121 Daraus ergab sich die Struktur von Forschungsstand. 
Quellen zu berücksichtigen, um auf diese Weise möglichst viele Facetten der Umsetzung aus der Literatur zu extrahieren. Ich war bestrebt, diese Quellenheterogenität sowohl im Querschnitt als auch im Längsschnitt und insbesondere unter diesen Aspekten zu erzielen:

- wissenschaftlicher Status: Ich berücksichtigte neben wissenschaftlich fundierten Monographien oder Aufsätzen auch Projektberichte oder Meinungsartikel. Darüber hinaus erfasste ich neben gut etablierter Literatur auch weniger verbreitete Quellen.

- Diachronie: Obwohl mein Augenmerk vor allem auf aktueller Literatur lag, flossen auch ältere Quellen in die Untersuchung mit ein.

Die Entscheidung, wann ausreichend Quellen in jedem Gebiet berücksichtigt wurden, kann trotz dieses Strebens nach möglichst großer Heterogenität als subjektiv betrachtet werden.

Obwohl ich in den Konzeptualisierungen in Kapitel 3 und Kapitel 4 eine Aufhebung der historisch gewachsenen Verteilung der Beschäftigung mit Sprachlenkung auf vier Gebiete postuliere, ist das Zitatkorpus aus pragmatischen Gründen nach vier Gebieten getrennt verschlagwortet. Ein Wechsel hin zu einer übergreifenden Perspektive setzt bei der Zitatauswertung, also im dritten Verallgemeinerungsschritt an und wird durch die Interpretation in Abschnitt 5.2 vollzogen.

Inhaltlich umfasst das Korpus Zitate, die sich sowohl auf systemlinguistische als auch nichtsystemlinguistische Aspekte des Erfolges beziehen. In der Kernuntersuchung sind jedoch weitestgehend lediglich nichtsystemlinguistische Zitate berücksichtigt.

\subsubsection{Zitatauswertung}

Das allgemeine Auswerteprinzip besteht in einer inhaltlichen Bündelung und einer anschließenden Verallgemeinerung von Zitaten in mehreren Schritten, s. Tabelle 14: Zitate werden zu Aussagen, Aussagen zu Kategorien und schließlich Kategorien zu Themen verallgemeinert. Da das Korpus nicht repräsentativ ist, wertete ich die Daten nicht quantitativ (z. B. aufgrund von Häufigkeit) aus. Das Hauptergebnis der Erfolgsuntersuchung beruht also nicht auf quantitativen Faktoren wie einer Tendenz in der Forschung sondern auf einer rein qualitativen Analyse.

Darüber hinaus flossen nicht alle mit einschlägigen Schlagwörtern versehenen Zitate in die Hauptuntersuchung mit ein. Die gesammelten Zitate sichtete 


\begin{tabular}{|c|c|c|c|c|c|c|}
\hline & $\begin{array}{l}\text { 1. Verallg.- } \\
\text { Schritt }\end{array}$ & & $\begin{array}{l}\text { 2. Verallg.- } \\
\text { Schritt }\end{array}$ & & $\begin{array}{l}\text { 3. Verallg.- } \\
\text { Schritt }\end{array}$ & \\
\hline Zitat & $\rightarrow$ & $\begin{array}{c}\text { Aussage } \\
\text { Paraphrase }\end{array}$ & $\rightarrow$ & $\begin{array}{l}\text { Kategorie } \\
\text { gebietsspezifisch }\end{array}$ & $\rightarrow$ & $\begin{array}{c}\text { Thema } \\
\text { gebietsübergreifend }\end{array}$ \\
\hline
\end{tabular}

Tabelle 14.: Prinzip der Datenauswertung

ich ein weiteres Mal, um ihre inhaltliche Relevanz für die Analyse sicherzustellen.

Inhaltlich war ich an zwei Arten von Aussagen zum Erfolg interessiert, die in Zitaten gemacht werden: Welche Probleme oder Herausforderungen sich bei der Umsetzung stellen und welche Vorgehen ${ }^{122}$ für möglich gehalten werden. Eines der Ziele der Auswertung und der anschließenden Interpretation war es dann aufzudecken, welche Vorgehen welchen Herausforderungen in der Literatur zugeordnet werden, um so eine Übersicht über die Forschungslage zu konkreten Handlungsmöglichkeiten der Autoren bei Sprachlenkung zu erhalten. Darüber hinaus sind in der Datenbank Strukturen angelegt, die die Auswertung einer weiteren Art von Aussagen ermöglichen: Was die aktive Zielgruppe selbst dazu motiviert, eine bestimmte sprachliche Neuerung anzunehmen. Diesen Aspekt habe ich in dieser Untersuchung jedoch nicht weiterverfolgt.

Im ersten Verallgemeinerungsschritt teile ich alle Zitate in einzelne Aussagen zu Herausforderungen und Vorgehen. Macht beispielsweise ein Zitat eine Aussage zu Herausforderungen und drei Aussagen zu Vorgehen, erfasse ich die Herausforderung als einen Datensatz in der Datenbanktabelle mit allen Herausforderungen und die Vorgehen als drei getrennte Datensätze in der Tabelle mit allen Vorgehen. Falls in einem Zitat eine Zuordnung von geeigneten Vorgehen zu festgestellten Herausforderungen postuliert wird, halte ich diese Verbindung zwischen den beiden Aussagen in einer weiteren Tabelle fest.

Methodisch kann die erste Segmentierung der Zitate in Einzelaussagen als in vivo betrachtet werden, da ich die Aussagen zwar paraphrasiere, die Paraphrase aber möglichst nah am Originalwortlaut wähle.

Im zweiten Schritt erfolgt eine Verallgemeinerung und Bündelung der Paraphrasen zu übergreifenden Kategorien, um Theoriebildung zu ermöglichen. Diese Kategorien sind zum einen nach Gebieten zum anderen auch nach Herausforderungen und Vorgehen getrennt. Während der erste Verallgemeinerungsschritt weitestgehend beschreibend ist, ist der zweite stärker interpreta-

122 Also im Kontext der vorangegangenen Modellierungen: Handlungen oder Teilhandlungen. 
tiv und erfolgt vor dem Hintergrund der besprochenen sozialpsychologischen Konstrukte der Identität, Gruppenprozesse und Einstellungen.

Schließlich bündele ich im dritten Verallgemeinerungsschritt die gebietsspezifischen Kategorien zu gebietsübergreifenden Themen.

Die so entstandene Liste von Themen ist nicht nur für alle vier Gebiete, sondern auch für Herausforderungen und Vorgehen gemeinsam und stellt somit einen Ausgangspunkt für eine integrative Betrachtung der Sprachlenkung dar.

\subsubsection{Technische Umsetzung}

Zur Erfassung, Pflege und Analyse der Zitate setze ich MySQL Workbench ein, ein relationales Datenbankmanagementsystem mit graphischer Benutzeroberfläche. Um ein besseres Verständnis für die Aufbereitung meiner Daten und das Auswertungsprinzip bei Lesern ohne Vorkenntnisse in relationalen Datenbankmanagementsystemen zu erzielen, gehe ich im folgenden Absatz allgemein auf den Aufbau einer relationalen Datenbank ein. Eine umfassende Einführung in das Thema kann diese Arbeit jedoch nicht leisten und ich verweise zur weiteren Lektüre beispielsweise auf Elmasri und Navathe (2009).

In einer relationalen Datenbank werden Daten in Tabellen gepflegt. Eine solche Tabelle kann zur Vereinfachung als eine reguläre Tabellenkalkulation aufgefasst werden, in der einzelne Datensätze in Zeilen abgelegt werden (sog. Tupeln), wobei Spalten einzelne Informationseinheiten (sog. Attribute) darstellen. Wichtig dabei ist, dass jeder Datensatz in einer gegebenen Tabelle eindeutig identifizierbar ist, was durch einen sog. Primärschlüssel realisiert wird. Der Primärschlüssel kann beispielsweise eine Zahl sein. Es handelt sich also um eine Datensatz-ID, die für jeden Datensatz in einer Spalte obligatorisch mitgeführt wird. Um die Datenbank besser zu strukturieren und übersichtlicher zu halten, können mehrere inhaltlich zusammengehörige Tabellen zu sog. Schemas zusammengefasst werden. Ein weiteres wichtiges Prinzip relationaler Datenbanken ist die Referenzierung der Daten aus anderen Tabellen über sog. Fremdschlüssel. Beziehen sich Daten in z. B. Tabelle 1 inhaltlich auf Daten in Tabelle 2, so enthält Tabelle 1 eine spezielle Spalte, in der entsprechende Primärschlüssel aus Tabelle 2 eingegeben sind. Aus der Sicht von Tabelle 1 sind also in dieser Spalte Fremdschlüssel enthalten. Der Vorteil einer solchen Referenzierung ist, dass die Daten nur an einer Stelle geändert werden müssen, um auch in anderen Tabellen immer auf dem neusten Stand zu sein. Schließlich ist es mithilfe sog. Sichten möglich, Daten aus verschie- 
denen Tabellen in einer Tabelle darstellen zu lassen. Diese neue Tabelle ist in der Datenbank physikalisch nicht enthalten, sondern wird für die aktuelle Abfrage dynamisch generiert. Dank Sichten können Zusammenhänge über mehrere Tabellen hinweg besser erkannt werden.

Die hier vorgestellte Datenbank liegt in der 3. Normalform vor (s. dazu Elmasri und Navathe 2009, 299-330) und enthält 7 Schemas:

- keywords: Erfassung von Schlagwörtern,

- zitate: Erfassung und Indexierung von allen Zitaten,

- analyse_Iplp: Analyse der mit Erfolg Veränderung: Linguistik; AOP und Erfolgsbedingung: Sprachlenkung: Linguistik verschlagworteten Zitate,

- analyse_ps: Analyse der mit Erfolg: Plansprachen und Erfolg: Esperanto verschlagworteten Zitate

- analyse_ks: Analyse der mit Erfolg: kontrollierte Sprachen verschlagworteten Zitate

- analyse_ta: Analyse der mit Terminologiearbeit: Erfolg verschlagworteten Zitate

- themen: Erfassung von gebietsübergreifenden Themen.

Da alle Analyse-Schemas den gleichen Aufbau haben, benutze ich im Folgenden stellvertretend Analyse_* sowie Beispiele aus dem Gebiet Plansprachen.

Im Folgenden beschreibe ich den Aufbau der Tabellen, die in den o.g. Schemas enthalten sind.

Schema 'keywords' Das Schema 'keywords' enthält nur eine Tabelle: 'keywords.list_keywords, ${ }^{123}$ in der alle Schlagwörter aufgelistet sind. ${ }^{124}$ Die Tabelle hat folgenden Aufbau (Spalten):

$$
\text { (keyword_id, }{ }^{125} \text { keyword). }
$$

123 Ich benutze für Tabellennamen die SQL-Gepflogenheit: Der erste Namenteil bezeichnet das übergeordnete Schema; nach dem Punkt folgt der eigentliche Tabellenname. Beispielsweise bedeutet 'keywords.list_keywords' die Tabelle list_keywords im Schema keywords.

124 Die Tabelle könnte auch direkt im Schema 'zitate' enthalten sein, was möglicherweise die Handhabbarkeit und die Lesbarkeit der Datenbank fördern würde. Ein selbstständiges 'keywords'-Schema könnte somit entfallen.

125 Primärschlüssel sind doppelt, Fremdschlüssel einfach unterstrichen. 
Schema 'zitate' Das Schema 'zitate' enthält zwei Tabellen: 'zitate' und 'zitate keywords'. In der Tabelle 'zitate.zitate' werden alle, nicht nur erfolgsrelevante Zitate erfasst, die ich bei der Ausarbeitung dieser Arbeit erfasste. ${ }^{126}$ Darüber hinaus enthält die Tabelle alle Angaben zum Zitat nach wissenschaftlichem Maßstab (Zitat-ID, das eigentliche Zitat, Zitatsseite, Bibtexkey ${ }^{127}$ ). Die Tabelle hat folgenden Aufbau (s. Tabelle 15):

$$
\text { (zitat_id, inhalt, seite, bibtexkey). }
$$

In der Tabelle 'zitate.zitate_keywords' wird jedem Zitat über seine ID eine Schlagwort-ID zugeordnet (eine n-n-Zuordnung ist somit möglich). Die Tabelle hat folgenden Aufbau:

$$
\text { (idzitate_keywords, id_zitate, } \underline{\text { id_keywords) }} \text {. }
$$

Schema 'analyse_*' Das Schema 'analyse *' dient zur Hauptuntersuchung der Zitate und besteht aus folgenden Tabellen (s. das EER-Diagramm in Abbildung 20):

- 'analyse_*ana': Hier ist für alle einschlägigen Zitate angegeben, ob sie in die Hauptuntersuchung aufgenommen werden oder nicht (zur_diss_ana=' 1 ' bzw. ' 0 '). Die Referenzierung der Zitate erfolgt über den Fremdschlüssel 'id_zitate'. Die Tabelle hat folgenden Aufbau (s. Tabelle 16):

$$
\left(\underline{\underline{\text { ana_id }}}, \underline{\text { id_zitate }}, z u r \_d i s s \_a n a\right) .
$$

- 'analyse_*ana_hvem': In dieser Tabellen sind nur diejenigen Zitate berücksichtigt, die in der Tabelle 'analyse *ana' mit zur_diss_ana=' 1 ' markiert wurden. Abhängig davon, ob sie eine Aussage zu Herausforderungen ( ' $h$ '), Vorgehen ( ' $v$ '), Erfolg ( ' $e$ ') oder Motiven (' $m$ ') enthalten, werden sie entsprechend mit ' 1 ' oder ' 0 ' markiert. Die Tabelle hat folgenden Aufbau (s. Tabelle 17):

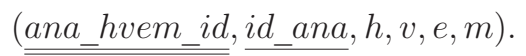

126 Allerdings ist im Anhang nur ein Auszug aus diesem Gesamtkorpus enthalten, der nur die erfolgsrelevanten Zitate umfasst.

127 Bibtexkey ist ein Schlüssel zur Zitat- und Literaturverwaltung mit BıвTEX. 


\begin{tabular}{|c|l|c|c|}
\hline zitat_id & inhalt & seite & bibtexkey \\
\hline \hline 877 & $\begin{array}{l}\text { "A broad tentative conclusion from this volu- } \\
\text { me seems to indicate that the relative success } \\
\text { of Esperanto, when measured against all ri- } \\
\text { vals, has little to do with linguistic structure. } \\
\text { Neither any of the "improvements" on Espe- } \\
\text { ranto, nor the naturalistic Interlingua and si- } \\
\text { milar projects, led to more widespread use or } \\
\text { recognition. On the contrary, the stability of } \\
\text { Esperanto over more than a century, and its } \\
\text { internal coherence in actual usage throughout } \\
\text { the world, probably helped it outdistance all } \\
\text { rivals. In any case, a dispassionate examina- } \\
\text { tion such as this volume contains, with con- } \\
\text { tributions from advocates of Ido and Interlin- } \\
\text { gua, is very useful.“ }\end{array}$ & Riain2003 & \\
\hline 946 & $\begin{array}{l}\text { „So müssen vor allem 'externe', ausser- } \\
\text { sprachliche Faktoren für das Ausbleiben ei- } \\
\text { nes Durchbruchs der Plansprachen verant- } \\
\text { wortlich gemacht werden, wie die fehlende } \\
\text { gesellschaftliche Akzeptanz, fehlendes Pres- } \\
\text { tige und, damit verbunden, mangelnde staat- } \\
\text { liche Stützung sowie Skepsis gegenüber ihrer } \\
\text { Praktikabilität.“ }\end{array}$ & Kuenzli2006 \\
\hline
\end{tabular}

Tabelle 15.: Beispiel: Tabelle 'zitate.zitate'

\begin{tabular}{|c|c|c|}
\hline ana_id & id_zitate & zur_diss_ana \\
\hline \hline 5 & 877 & 1 \\
\hline 137 & 946 & 1 \\
\hline
\end{tabular}

Tabelle 16.: Beispiel: Tabelle 'analyse_ps.ana' 


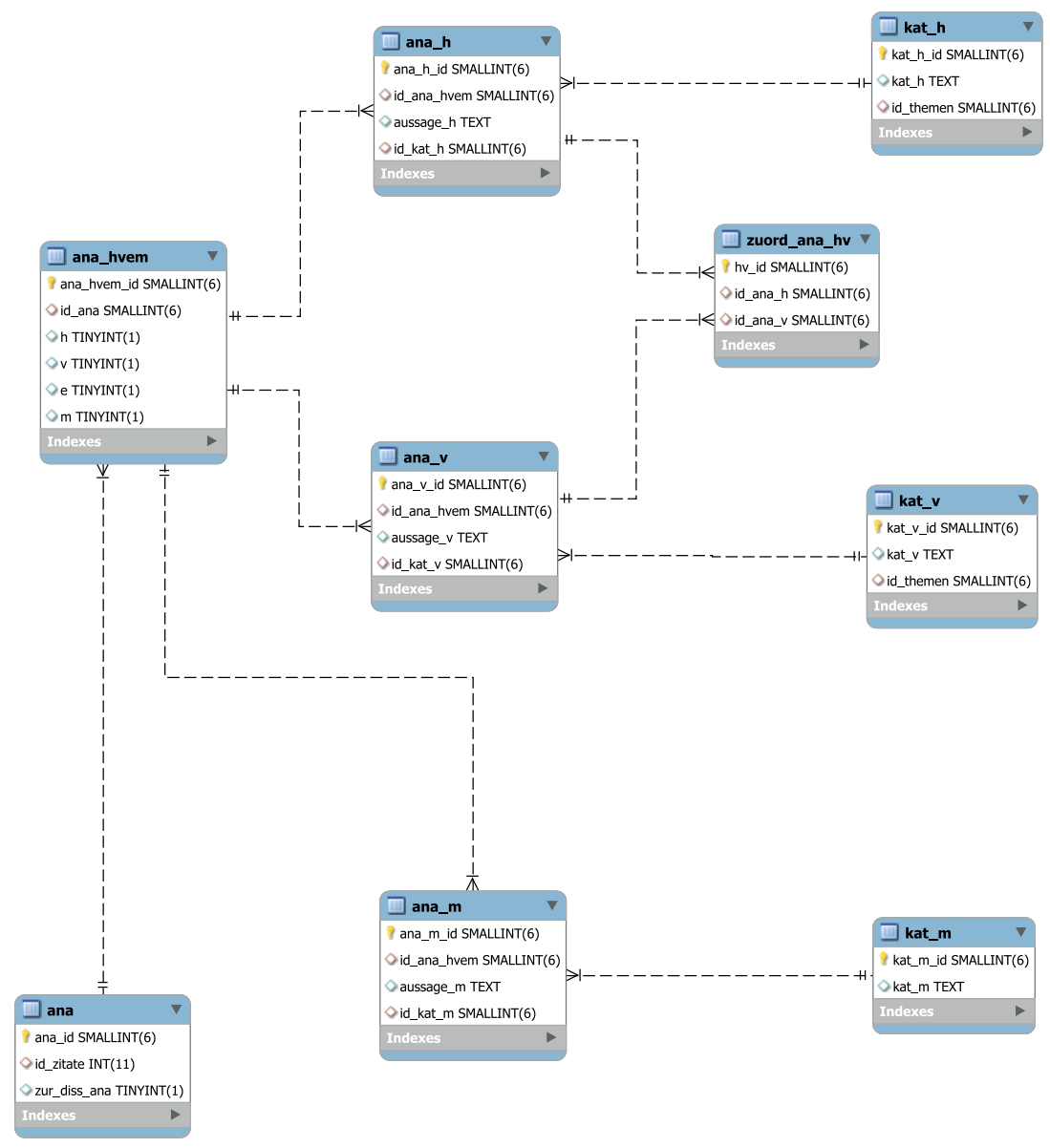

Abbildung 20.: EER-Diagramm von 'analyse_PS'

\begin{tabular}{|c|c|c|c|c|c|}
\hline ana_hvem_id & id_ana & $\mathrm{h}$ & $\mathrm{v}$ & $\mathrm{e}$ & $\mathrm{m}$ \\
\hline \hline 4 & 5 & 1 & 1 & 1 & 0 \\
\hline 121 & 137 & 1 & 0 & 1 & 0 \\
\hline
\end{tabular}

Tabelle 17.: Beispiel: Tabelle 'analyse_ps.ana_hvem' 


\begin{tabular}{|c|c|l|c|}
\hline ana_h_id & id_ana_hvem & aussage_h & id_kat_h \\
\hline \hline 2 & 4 & $\begin{array}{l}\text { Eine PS soll auch langfristig eta- } \\
\text { bliert sein. }\end{array}$ & 2 \\
\hline 158 & 4 & $\begin{array}{l}\text { Eine WHS soll sich gegen andere } \\
\text { WHS behaupten. }\end{array}$ & 6 \\
\hline 83 & 121 & Akzeptanz fehlt. & 15 \\
\hline 184 & 121 & Prestige fehlt. & 18 \\
\hline 185 & 121 & $\begin{array}{l}\text { Skepsis gegenüber Praktikabilität } \\
\text { einer WHS }\end{array}$ & 9 \\
\hline 186 & 121 & $\begin{array}{l}\text { mangelnde offizielle Unterstüt- } \\
\text { zung }\end{array}$ & 25 \\
\hline
\end{tabular}

Tabelle 18.: Beispiel: Tabelle 'analyse_ps.ana_h'

- 'analyse_*.ana_h': Diese Tabelle berücksichtigt nur diejenigen Zitate, die in der Tabelle 'analyse_*ana_hvem' mit ' $\mathrm{h}$ ' $={ }^{\prime} 1$ ' markiert wurden (die Werte in den Spalten 'v', 'e', ' $m$ ' sind dabei beliebig). Diese Referenz wird über den Fremdschlüssel 'id_ana_hvem' hergestellt. Die Aussage, also das Ergebnis der ersten Verallgemeinerung, wird als Paraphrase in der Spalte 'aussage $h$ ' angegeben. Enthält das ursprüngliche Zitat mehrere Aussagen, werden mehrere Datensätze (Zeilen) mit demselben Fremdschlüssel 'id_ana hvem' abgelegt. Außerdem wird über den Fremdschlüssel 'id_kat_h' festgehalten, welcher Herausforderungskategorie die Aussage zugeordnet ist. Die Tabelle hat folgenden Aufbau (s. Tabelle 18):

$$
\left(\underline{\underline{\text { ana_h_id}}}, \underline{i d \_a n a \_h v e m}, \text { aussage_h, id_kat_h}\right) .
$$

- 'analyse_*.ana_v': Diese Tabelle ist ein Pendant zur Tabelle 'analyse_*ana_h' für Zitate, die in 'analyse_*.ana_hvem' mit 'v'='1' markiert wurden (die Werte in den Spalten ' $h$ ', ' $e$ ', ' $m$ ' sind dabei beliebig). Die Tabelle hat folgenden Aufbau (s. Tabelle 19):

$$
\left(\underline{\underline{a n a \_v \_i d}}, \underline{i d \_a n a \_h v e m}, a u s s a g e \_v, \underline{i d \_k a t \_v}\right) .
$$




\begin{tabular}{|c|c|l|c|}
\hline ana_v_id & id_ana_hvem & aussage_v & id_kat_v \\
\hline \hline 2 & 4 & Sprachsystem verbessern. & 2 \\
\hline 159 & 4 & $\begin{array}{l}\text { Stabilität des Sprachsystems soll } \\
\text { gesichert werden. }\end{array}$ & 20 \\
\hline
\end{tabular}

Tabelle 19.: Beispiel: Tabelle 'analyse_ps.ana_v'

\begin{tabular}{|c|l|c|}
\hline kat_h_id & kat_h & id_themen \\
\hline \hline 2 & Langfristiger Gebrauch & 16 \\
\hline 6 & Sprachökologie: andere Plansprachen & 7 \\
\hline 9 & Funktionsfähigkeit einer PS infrage gestellt & 5 \\
\hline 15 & Akzeptanz fehlt & 5 \\
\hline 18 & Prestige fehlt & 5 \\
\hline 25 & Bottom-up-Verbreitung & 4 \\
\hline
\end{tabular}

Tabelle 20.: Beispiel: Tabelle 'analyse_ps.kat_h'

- 'analyse_*kat_h': Diese Tabelle listet alle Kategorien/Klassen von Herausforderungen auf, die sich aus der Verallgemeinerung der Paraphrasen in 'analyse_*.ana_h' ergeben. Darüber hinaus wird das übergreifende Thema, zu dem die Kategorie angehört, über den Fremdschlüssel 'id_themen' referenziert. Die Tabelle hat folgenden Aufbau (s. Tabelle 20):

$$
\text { (kat_h_id, } \left.k a t \_h, \underline{\underline{\text { id_themen }}}\right) \text {. }
$$

- 'analyse_*kat_v': Diese Tabelle listet alle Kategorien/Klassen von Vorgehen auf, die sich aus der Verallgemeinerung der Paraphrasen in 'analyse_*ana_v' ergeben. Darüber hinaus wird das übergreifende Thema, zu dem die Kategorie angehört, über den Fremdschlüssel 'id_themen' referenziert. Die Tabelle hat folgenden Aufbau (s. Tabelle 21):

$$
\text { (kat_v_id} \left., k a t \_v, \underline{\text { id_themen }}\right) \text {. }
$$




\begin{tabular}{|c|l|c|}
\hline kat_v_id & kat_v & id_themen \\
\hline \hline 2 & Sprachsystem normativ perfektionieren. & 16 \\
\hline 20 & Stabilität vor Perfektionierung. & 18 \\
\hline
\end{tabular}

Tabelle 21.: Beispiel: Tabelle 'analyse_ps.kat_v'

\begin{tabular}{|c|c|c|}
\hline hv_id & id_ana_h & id_ana_v \\
\hline \hline 1 & 2 & 2 \\
\hline 2 & 2 & 159 \\
\hline 3 & 158 & 2 \\
\hline 4 & 158 & 159 \\
\hline
\end{tabular}

Tabelle 22.: Beispiel: Tabelle 'analyse_ps.zuord_ana_hv'

- 'analyse_*.zuord_ana_hv': Diese Tabelle hält fest, welche Vorgehen in Verbindung mit welchen Herausforderungen in der Literatur genannt werden. Die Zuschreibung erfolgt nicht global pro Zitat, sondern pro Aussage und wird über die Zuordnung entsprechender Fremdschlüssel 'id_ana_h' (aus: 'analyse_*ana_h') und 'id_ana_v' (aus: 'analyse_*ana_v') realisiert. Die Tabelle hat folgenden Aufbau (s. Tabelle 22):

$$
\left(\underline{\underline{h v \_i d}}, \underline{i d \_a n a \_h}, \underline{\underline{i} \_\_n a \_v}\right) .
$$

Der Vollständigkeit halber sei noch erwähnt, dass das Schema zwei weitere Tabellen enthält, nämlich 'analyse_*.ana_m', als Pendant zur Tabelle 'analyse_*ana_h' für Zitate, die in 'analyse_*ana_hvem' mit ' $\mathrm{m}$ '='1' markiert sind, sowie Tabelle 'analyse_*.kat_m', die zur Erfassung von motivationsbezogenen Kategorien bestimmt war. Beide Tabellen sind wie angemerkt nicht Teil der Hauptuntersuchung.

Schema 'themen' : Das Schema 'themen' enthält nur eine Tabelle: 'themen.themen', in der alle gebietsübergreifenden Themen als Ergebnis der Datenauswertung enthalten sind. Die Tabelle hat folgenden Aufbau (s. Tabelle 23): 


\begin{tabular}{|c|l|}
\hline thema_id & thema \\
\hline \hline 4 & Gruppe \\
\hline 5 & Einstellungen \\
\hline 7 & Sprachökologie \\
\hline 16 & Gebrauch \\
\hline 18 & Sprachsystem allgemein \\
\hline
\end{tabular}

Tabelle 23.: Beispiel: Tabelle 'themen.themen'

(thema_id, thema).

\subsubsection{Abschließende Anmerkungen zur Datenerfassungsmethode}

Wie erwähnt, zielt die angewandte Methode zur Datenerfassung und -auswertung primär darauf, möglichst viele Handlungsmöglichkeiten zu identifizieren und weniger darauf, eine (quantitative) Tendenz aufzuzeigen, welche Vorgehen in der Literatur für wirksam gehalten werden. Erst im Interpretationsschritt werden die anhand der Literatur identifizierten Aussagen zum Umsetzungserfolg vor dem sozialpsychologischen Hintergrund interpretiert.

Die hier vorgestellte Methode ist nicht in Anlehnung an eine existierende Methode zur qualitativen Datenanalyse entstanden. Im Nachhinein stelle ich fest, dass sie trotz allgemeiner Parallelen weder der Grounded Theory (exemplarisch A. Strauss und Corbin 1996; Glaser und A. L. Strauss 1998) noch der Inhaltsanalyse nach Mayring, also den etablierten qualitativen Forschungsmethoden der Sozialwissenschaften, folgt (s. dazu bspw. Mayring 2002).

Vom Ziel her stelle ich eine gewisse Nähe zur Grounded Theory fest, da ich bei der Auswertung den Anspruch hatte, Einzelaussagen zum Zwecke der Theoriebildung zu verallgemeinern. Den größten Unterschied macht hier jedoch die Datenerfassung, da ich alle Zitate zunächst aufnahm und erst dann weitestgehend en bloc auswertete. Außerdem behandelte ich eine größere Datenmenge als bei der Grounded Theory gewöhnlich der Fall ist (vgl. Mayring 2002, 103-104). Im Vergleich zur Inhaltsanalyse nach Mayring (bspw. Mayring 2002) behandelte ich keine ganzen Werke, sondern mehrere kleinere Aus- 


\section{Untersuchung}

schnitte aus mehreren Hunderten von Werken (s. Anhang I: Verzeichnis der in der Untersuchung ausgewerteten Quellen). ${ }^{128}$ Da der Einsatz der Inhaltsanalyse nicht geplant war, so stelle ich im Nachhinein fest, dass mein Vorgehen vom fest vorgegebenen Untersuchungsaufbau einer Inhaltsanalyse abweicht (für Beispielbaupläne s. Mayring 2002, 114-121).

Zitate als Daten zu benutzen, um sich einen Überblick über die Forschungslage zu verschaffen, scheint naheliegend zu sein. Mir ist jedoch kein weiterer methodischer Ansatz bekannt, der den Umgang mit dieser Art von Daten wie in dieser Arbeit systematisiert. In Verbindung mit der vorgestellten Auswertungsmethode, die eine Reduktion der Datenkomplexität leistet, ermöglichen die Daten eine umfassende und vielseitige Analyse, die ich aufgrund ihrer Systematisierung aber auch Nachvollziehbarkeit/Transparenz sowie Intersubjektivität qualitative Metaanalyse nenne. Die Transparenz ist dadurch gewährleistet, dass die Methode und ihre technische Umsetzung offen gelegt sind und die Daten der Arbeit im Anhang beiliegen. Insbesondere soll der Zugriff auf die elektronische Form der Daten eine Nachuntersuchung ermöglichen. Die Nachvollziehbarkeit wird auch dadurch unterstützt, dass die Analyse kleinschrittig ist und der Schritt der Beschreibung (Aussagenparaphrase) und der der Interpretation (Kategorie- und Themenbildung) sowohl konzeptionell als auch datenbanktechnisch voneinander getrennt sind. Es soll daher nachvollziehbar sein, an welcher Stelle meine eigenen Gedanken stärker mit einfließen. Außerdem ist es dank der Implementierung als relationale Datenbank zu jeder Zeit möglich nachzuprüfen, welchen Originalzitaten die aufgestellten Kategorien oder Themen entstammen. Transparenz und insbesondere die Trennung zwischen Analyse und Interpretation trägt auch zur Intersubjektivität bei. Darüber hinaus lege ich zur Stärkung der Intersubjektivität meine Interpretationsgrundlage offen und beziehe mich im Interpretationsschritt und der anschließenden Vorstellung, Systematisierung und Diskussion der Ergebnisse in Abschnitt 5.2 auf die in Kapitel 4 vorgestellten Erkenntnisse der Sozialpsychologie.

Obwohl die Methode eine Reduktion der Datenkomplexität ermöglicht und so eine große Datenmenge verarbeitbar macht, bedarf sie weiterer Erläuterungen. Zum einen stellt sich die Frage nach der Eignung der verwendeten Quellen, da ich sie ungeachtet ihres wissenschaftlichen Status alle gleichberechtigt behandle. Die Entscheidung, neben fundierten wissenschaftlichen Quellen auch Meinungsartikel oder Einzelbeobachtungen im Korpus zu berücksichtigen, sehe ich als gerechtfertigt, weil es zurzeit wenige theoretische

128 Der Anhang befindet sich zum Download auf der Verlags-Webseite 
Anhaltspunkte zum Gegenstand Umsetzungserfolg von Sprachlenkung gibt. Da in dieser Arbeit mögliche Vorgehen zunächst einmal identifiziert werden sollen, spielt für das Korpus eine untergeordnete Rolle, wie gut eine Aussage wissenschaftlich gesichert ist. Allerdings sollen die gewonnenen Erkenntnisse dann zur Hypothesenbildung für empirische Zwecke einsetzbar sein, um eine abschließende Verifikation der aufgedeckten Faktoren durch empirische Untersuchungen zu ermöglichen.

Schließlich ergibt sich aus der Art der verwendeten Daten, dass sie nur eine textuelle Mikroperspektive auf erfolgreiche Umsetzung von Sprachlenkung wiedergeben können. Da ich größtenteils direkte Zitate verwende, spiegeln die Analyseergebnisse keine großen Zusammenhänge in einer Arbeit wider, die sich erst durch die Betrachtung ihrer gesamten argumentativen Kette ergeben würden. Auch implizite Aussagen, und insbesondere Zuordnungen von Herausforderungen und Vorgehen können so nicht berücksichtigt werden. Am Ende dieser Datenerfassung und -auswertung stehen also punktuelle Erkenntnisse, die ich als Rohergebnis auffasse. Offenbar stehen aber Herausforderungen und Vorgehen in vielfachen Beziehungen zueinander und sind hierarchisch angeordnet, wie die in Abschnitt 3.4.1.1 und Abbildung 15 vorgestellte Modellierung von Tätigkeiten, Handlungen und Teilhandlungen besagt. Diese Unzulänglichkeit gehe ich im folgenden Teil der Untersuchung an, in dem ich diese punktuellen Rohergebnisse interpretativ in eine Struktur setze.

\subsection{Rohergebnisse: Analyse und Interpretation}

Das Rohergebnis der Datenauswertung besteht aus Tabellen, die Zitate, Aussagen, Kategorien und Themen zu Herausforderungen und Vorgehen bei Sprachlenkung sowie weitere Metadaten enthalten. In den darauffolgenden Abschnitten setze ich mich mit diesem Rohergebnis auseinander, wobei mein Augenmerk hier noch nicht auf Zuordnungen von Herausforderungen und Vorgehen, sondern auf festgestellten Themen und Kategorien liegt.

Nach einer kurzen quantitativen Vorstellung in 5.2.1, die lediglich einen Überblick über die Menge der verarbeiteten Daten geben soll, setze ich mich mit dem Rohergebnis qualitativ auseinander. Ich strebe dabei keine reine Beschreibung an, sondern eine weitere Systematisierung und Interpretation, für die ich Konzepte benutze, die ich im ersten Teil dieser Arbeit vorgestellt habe.

Auf die Präsentation von extrahierten übergreifenden Themen in 5.2.2.1 folgt eine inhaltliche Auseinandersetzung mit den festgestellten Kategorien, 


\section{Untersuchung}

die ich aus mehreren Perspektiven beleuchte. Zunächst werte ich sie gebietsspezifisch und anschließend gebietsübergreifend aus, d.h. ich betrachte zunächst die Herausforderungen in einzelnen Gebieten (5.2.2.2) und vergleiche die so gewonnenen Erkenntnisse gebietsübergreifend (5.2.2.3). Gleich verfahre ich bei Vorgehen (entsprechend 5.2.2.4 und 5.2.2.5).

Diese weitere Systematisierung und Interpretation der Rohergebnisse hat das Ziel, eine Grundlage für die Analyse von Zuordnungen und den Aufbau von Handlungsplänen zu legen, die das Hauptergebnis der Untersuchung darstellen. Dies erfolgt in Abschnitt 5.3.

\subsubsection{Quantitative Vorstellung der Rohergebnisse}

Obwohl die Untersuchung qualitativ ist, sind in Tabelle 24 Eckdaten zusammengestellt, die eine Vorstellung über die Größe des Korpus und die Anzahl der verarbeiteten Daten geben.

Wie aus Tabelle 24 ersichtlich, untersuche ich in der Hauptanalyse 738 Zitate, wobei das Verhältnis einzelner Gebiete unausgewogen ist, da PS deutlich mehr als ein Viertel der Zitate ausmacht. Wie zu erwarten, erhöhte die Segmentierung der Zitate in einzelne Aussagen zunächst die Datenmenge: Betrachtet man die Anzahl der Aussagen zu Herausforderungen und Vorgehen zusammen, so hat sich die Gesamtanzahl von Informationseinheiten nahezu verdoppelt $(595+737=1332 \mathrm{im}$ Vergleich zu 738). Allerdings leistet der nächste Verallgemeinerungsschritt die gewünschte Reduktion der Datenkomplexität, so dass auf der Ebene der Kategorien nur entsprechend 192 Informationseinheiten (Herausforderungen) und 269 (Vorgehen) übrig bleiben. Ich beobachte zudem, dass auf der Ebene der H-Kategorien das Verhältnis von vier Gebieten recht ausgewogen ist, während bei V-Kategorien das Gebiet KS am stärksten vertreten ist, was sich auf der unteren Abstraktionsebene noch nicht so deutlich abzeichnet. Aus $461(192+269)$ Kategorien ergeben sich 18 gebietsübergreifende Themen.

Was die Anzahl der festgestellten Zuordnungen von Herausforderungen und Vorgehen betrifft, so ist das Verhältnis der vier Gebiete stark unausgewogen. Diese Tendenz bemerke ich sowohl auf der Ebene der Aussagen als auch der Kategorien. Ich beobachte, dass der Verallgemeinerungsschritt eine Komplexitätsreduktion bewirkt, so dass sich die Anzahl der Zuordnungen auf der Aussagenebene von 328 auf 255 auf der Kategorieebene verringert. Ich stelle jedoch fest, dass diese Reduktion bei Zuordnung deutlich schwächer ausfällt als allgemein bei Aussagen: Steht die Anzahl von Kategorien 


\begin{tabular}{|l|c|c|c|c|r|}
\hline & LPLP & PS & KS & TA & gesamt \\
\hline \hline Anzahl Zitate (nur Erfolg) & 154 & 299 & 145 & 204 & $\mathbf{8 0 2}$ \\
$\quad$ davon in der Hauptuntersuchung & 134 & 260 & 142 & 202 & $\mathbf{7 3 8}$ \\
Anzahl Aussagen (H) & 104 & 215 & 145 & 131 & $\mathbf{5 9 5}$ \\
Anzahl Aussagen (V) & 128 & 220 & 224 & 165 & $\mathbf{7 3 7}$ \\
Anzahl Kategorien (H) & 56 & 42 & 44 & 50 & $\mathbf{1 9 2}$ \\
Anzahl Kategorien (V) & 53 & 57 & 98 & 62 & $\mathbf{2 6 9}$ \\
Anzahl Themen & & & & & $\mathbf{1 8}$ \\
Anzahl Zuordnungen H-V (Aussagen) & 14 & 111 & 149 & 54 & $\mathbf{3 2 8}$ \\
$\quad$ auf Kategorieebene dargestellt & 12 & 84 & 111 & 48 & $\mathbf{2 5 5}$ \\
\hline
\end{tabular}

Tabelle 24.: Quantitative Übersicht über die Rohergebnisse

zu Anzahl von Aussagen im Verhältnis von ca. 1:3 ( $\frac{192}{595}=0$, 32 für Herausforderungen und entsprechend $\frac{269}{737}=0,36$ für Vorgehen), so beträgt dieses Verhältnis für Zuordnungen ca. 3:4 $\left(\frac{255}{328}=0,78\right)$. Die Komplexität wird für Zuordnungen durch den Verallgemeinerungsschritt nur halb so gut wie für alle Aussagen im Allgemeinen reduziert.

\subsubsection{Inhaltliche Vorstellung, Systematisierung und Diskussion der Rohergebnisse}

Die oberen Beschreibungen der Methode und der quantitativen Rohergebnisse spiegeln mein tatsächliches, induktives, Vorgehen bei der Analyse wider: von der speziellen Ebene der einzelnen Zitate über mehrere Abstraktionsschritte bis zur abstrakten Ebene der Themen. Aus Übersichtlichkeitsgründen entscheide ich mich in diesen Abschnitten indes für eine Betrachtung und Interpretation aus einer Top-down-Perspektive: Als Ausgangspunkt dienen mir die übergreifenden Themen, über die ich in die niedrigeren Abstraktionsebenen einsteige.

Die vollständigen Rohergebnisse aus der Datenbank sind in Anhang A und Anhang D zusammengestellt. ${ }^{129}$

129 Anhang A und Anhang D sind manuell aus vier Einzelsichten der MySQL-Datenbank zusammengesetzt, die jeweils die kat_h-Tabelle bzw. die kat_v-Tabelle eines Gebietes mit der Themen-Tabelle ('themen.themen') verbinden. 


\begin{tabular}{|r|l||r|l|}
\hline thema_id & Thema & thema_id & Thema \\
\hline \hline 1 & Identität & 2 & Instrumentalität \\
3 & allgemeine außersprachliche Fak- & 4 & Gruppe \\
& toren & & \\
5 & Einstellungen & 6 & Kosten \\
7 & Sprachökologie & 8 & Wissenschaftlichkeit \\
9 & Persuasion & 10 & Erlernbarkeit \\
11 & Arbeitsprozess & 12 & Machtmittel/Sanktionen \\
13 & Werkzeuge & 14 & Einhalten \\
15 & Bekanntmachen & 16 & Gebrauch \\
17 & Sonstiges & 18 & Sprachsystem allgemein \\
\hline
\end{tabular}

Tabelle 25.: Rohergebnis: übergreifende Themen

Die Vorstellung, Systematisierung und Diskussion erfolgt wie erwähnt in mehreren Schritten. Nach der Vorstellung der Themen werte ich die Ergebnisse bezüglich der Herausforderungen und bezüglich der Vorgehen getrennt aus, jeweils zunächst in den Gebieten einzeln und anschließend gebietsvergleichend.

\subsubsection{Vorstellung von Themen}

Die Verallgemeinerung von Kategorien zu gebietsübergreifenden Einheiten ergab 18 Themen, die in Tabelle 25 zusammengestellt und im Folgenden kurz erläutert sind.

- Identität: $\mathrm{Zu}$ diesem Thema werden Kategorien zusammengefasst, die verschiedene Aspekte der sozialen Identität einer Sprechergemeinschaft oder identitätsbezogene Oberziele der Sprachlenkung betreffen.

- Instrumentalität: Dieses Thema umfasst Kategorien, die sich zum einen auf die Tatsache beziehen, dass Sprachlenkung in (außersprachliche) Oberziele eingebettet ist. Darüber hinaus werden hier speziell instrumentelle Oberziele der Sprachlenkung (im Unterschied zu den identitätsbezogenen) behandelt.

- allgemeine außersprachliche Faktoren: Das Thema fasst Kategorien zusammen, die auf die Tatsache eingehen, dass Sprachlenkung allgemein 
immer in einen außersprachlichen Kontext eingebettet ist. Es kann sich dabei um Oberziele handeln oder aber auch Einflussfaktoren, die sich auf die Gestaltung und Erfolgsaussichten von Sprachlenkung auswirken.

- Gruppe: Kategorien, die zum Thema Gruppe zusammengefasst sind, machen zum einen auf Gruppenprozesse aufmerksam, die bei der Planung und Umsetzung von Sprachlenkung in Gang gesetzt werden. Andererseits sind hier auch verschiedene Eigenschaften von beteiligten Gruppen gesammelt.

- Einstellungen: Zum Thema Einstellungen sind verschiedene Kategorien gesammelt, die sich vorwiegend mit der Akzeptanz von SL befassen.

- Kosten: Hierzu gehört neben allgemeinen Beobachtungen zum finanziellen Aufwand von verschiedenen Aspekten von SL auch die Frage der Finanzierungssicherung.

- Sprachökologie: Das Thema Sprachökologie beruht auf der in 2.1.5.5 vorgestellten Theorie und betrachtet die neue Form, die durch Sprachlenkung geschaffen wird, im Netz von anderen, meist ungelenkt entstandenen Sprachformen.

- Wissenschaftlichkeit: Die hier zusammengefassten Kategorien betreffen Überlegungen, dass einzelne Aspekte der Sprachlenkung einer wissenschaftlichen Grundlage bedürfen.

- Persuasion: Das Thema umfasst verschiedene Aspekte der Überzeugungsarbeit, die neben Beschaffenheit und Inhalten von Argumenten auch auf die Mechanismen einer erfolgreichen Überzeugungsarbeit umfassen.

- Erlernbarkeit: Dieses Thema stellt verschiedene Überlegungen darüber zusammen, was sich günstig auf das Erlernen der neuen Form auswirkt.

- Arbeitsprozess: Unter dieses Thema fallen sowohl Überlegungen, wie sich SL auf die gewohnten Arbeitsprozesse der Betroffenen auswirkt, aber auch, welche Aufgaben bestimmte Gruppen innerhalb der Sprachlenkung übernehmen sollen. Das Thema ist also nicht konsequent aufgebaut und überlappt sich teilweise mit dem Thema Gruppe. 
- Machtmittel/Sanktionen: Hier sind Überlegungen zusammengefasst, welche formellen Machtmöglichkeiten man als Autor hat, um die Sprachlenkung in der aktiven Zielgruppe durchzusetzen.

- Werkzeuge: Hier sind Kategorien zusammengefasst, die den Einsatz von technischen Hilfsmitteln bei der Sprachlenkung betreffen.

- Einhalten: Dieses Thema umfasst Überlegungen, wie die Autoren das Einhalten der neuen Form durch die aktive Zielgruppe fördern oder gar erzwingen können.

- Bekanntmachen: Bei diesem Thema handelt es sich um Kategorien, die die Beobachtung betreffen, dass eine neue Form bei ihrer Einführung zunächst unbekannt ist und ihr Bekanntheitsgrad gesteigert werden muss.

- Gebrauch: Zu diesem Thema werden Kategorien zusammengefasst, die allgemein darauf eingehen, dass eine erfolgreiche Sprachlenkung nicht mit der Bereitstellung der neuen Form endet, sondern eine Etablierung der neuen Form im tatsächlichen Gebrauch bedeutet.

- Sonstiges: Hier werden alle Kategorien gesammelt, die sich keinem der übrigen Themen zuordnen lassen.

- Sprachsystem allgemein: $\mathrm{Zu}$ diesem Thema werden Überlegungen zum Aufbau und zur Beschaffenheit des Sprachsystems der neuen Form zusammengefasst. Wie erwähnt, liegt der Schwerpunkt dieser Arbeit auf nichtsystemlinguistischen Überlegungen und Zitate zu rein systemlinguistischen Aspekten wurden von mir nicht konsequent erfasst. Ich bin bei diesem Thema vor allem an systemlinguistischen Vorgehen-Kategorien interessiert, die nichtsystemlinguistischen Herausforderungs-Kategorien zugeordnet werden.

\subsubsection{Herausforderungen: Gebiete einzeln}

In diesem Abschnitt wende ich mich thematisch den Herausforderungen zu, die sich bei Sprachlenkung stellen. Ausgehend von den übergreifenden Themen, werte ich die Gebiete einzeln aus. Dabei gehe ich insbesondere auf die Themen ein, die auf unteren Abstraktionsebenen (v. a. Kategorien, aber in Einzelfällen auch Aussagen) stark vertreten sind. Da die Untersuchung nicht 
quantitativ ist, nehme ich die Anzahl der einem Thema zugeordneten Kategorien lediglich zum Anlass, die inhaltlichen Schwerpunkte auf den darunter liegenden Abstraktionsebenen genauer zu betrachten. Auf diese Weise verschaffe ich mir einen Überblick, wie stark ein Thema in einem Gebiet elaboriert ist.

In Tabelle 26 ist meine Bewertung der Themenelaboration in den vier Gebieten dargestellt. Dabei benutze ich folgende Abstufung: „ $+++{ }^{\prime}$ - facettenreichste Themen, d.h. solche, bei denen ich einen hohen Stellenwert im Korpus beobachte; , $++{ }^{\prime}-$ Themen, die elaboriert erscheinen, jedoch weniger als die facettenreichsten; „, “ - Themen, die im Korpus erwähnt sind, zu denen aber nur allgemeine Aussagen ${ }^{130}$ getroffen werden; ,_-“- - Themen, die ich im Korpus nicht feststelle.

Diese Tabelle dient mir in erster Linie zur Auswahl der zu beschreibenden Ergebnisse: Nur Themen, die mit , +++ “ und „,++ "bewertet sind, bespreche ich im Folgenden. Eine Ausnahme ist das Thema Sprachsystem, das ich erst bei der Auswertung von Zuordnungs-Tabellen ('analyse_*.zuord_ana_hv') aufgreife.

LPLP Die Analyse ergibt, dass die facettenreichsten Themen zu Herausforderungen in LPLP-Gebiet vor allem Identität, Gruppe und Einstellungen sind. Weniger elaboriert sind Instrumentalität, allgemeine außersprachliche Faktoren, Sprachökologie, Kosten und Machtmittel/Sanktionen. Nur allgemeine Aussagen werden hingegen zu den Themen Gebrauch, Wissenschaft, Persuasion, Erlernbarkeit und Arbeitsprozess gemacht. Keine Aussagen stelle ich zu Sprachsystem allgemein, Bekanntmachen, Einhalten und Werkzeuge fest.

Beim Thema Identität ergibt sich einerseits, dass eine Top-downSprachplanung die soziale Identität der (aktiven) Zielgruppe salient machen kann, was aber von Bedrohungsgefühlen oder Reaktanz begleitet sein kann. In diesem Zusammenhang ist auch die Herausforderung zu betrachten, dass sprachliche Menschenrechte berücksichtigt werden sollen. Andererseits stellt die Tatsache, dass Identität nicht zwangsläufig durch die Sprache konstituiert wird, eine weitere Herausforderung für die Sprachplanung dar.

Beim Thema Gruppe wird zunächst zwischen einer Top-down- und einer Bottom-up-Sprachplanung unterschieden, woraus ich auf die Existenz mehrerer beteiligter Gruppen, insbesondere einer Autorengruppe und einer Sprechergruppe (aktive Zielgruppe), schließe. Dies gibt mir Anlass, die festge-

130 Hier und in weiteren Abschnitten benutze ich Aussage im allgemeinen Sinne und nicht zur Bezeichnung von Werten einer Spalte in der benutzten MySQL-Datenbank. 


\section{Untersuchung}

\begin{tabular}{lllll}
\multicolumn{1}{c}{ LPLP } & PS & KS & TA \\
+++ & Identität & Gruppe & Gruppe & Aruppe \\
& Gruppe & Einstellungen & Arbeitsprozess \\
Einstellungen & & Werkzeuge & Werkzeuge \\
\hline++ & Instrumentalität & Identität & Identität & Einstellungen \\
außerspr. Faktoren & Sprachökologie & Arbeitsprozess & \\
Sprachökologie & Gebrauch & & \\
Kosten & & & \\
Machtmittel & & & Instrumentalität \\
\hline Gebrauch & außerspr. Faktoren & Instrumentalität & außerspr. Faktoren \\
Wissenschaftlichkeit & Kosten & außerspr. Faktoren & Kosten \\
Persuasion & Erlernbarkeit & Kosten & Sprachökologie \\
Erlernbarkeit & Machtmittel & Sprachökologie & Persuasion \\
Arbeitsprozess & Einhalten & Persuasion & Erlernbarkeit \\
& Bekanntmachen & Erlernbarkeit & Machtmittel \\
& Sprachsystem & Einhalten & Einhalten \\
& & Bekanntmachen & Bekanntmachen \\
& & Sprachsystem & Gebrauch \\
& & & Sprachsystem \\
\hline- & & & Identität \\
& Sprachsystem & Instrumentalität & Wissenschaftlichkeit & Wissenschaftlichkeit \\
Bekanntmachen & Wissenschaftlichkeit & Machtmittel & \\
Einhalten & Arbeitsprozess & Gebrauch & \\
Werkzeuge & Werkzeuge & & \\
& Persuasion & &
\end{tabular}

Tabelle 26.: Herausforderung: Bewertung der Elaboration von Themen 
stellten Kategorien hier mithilfe der Gruppenkonzepte aus der Sozialpsychologie weiter zu systematisieren.

Betrachtet man die aktive Zielgruppe, so ergibt sich einerseits, dass der Erfolg der Sprachplanung mit der vorhandenen Gruppenstruktur zusammenhängt: Explizit genannt werden dabei die Gruppengröße, aber auch die Disziplin und das Fortschrittsbedürfnis der Gruppenmitglieder, die ich als Merkmale von Gruppennormen interpretiere. Darüber hinaus wird das Engagement der Gruppenmitglieder genannt, was ich dem Gruppenzusammenhalt (Kohäsion) zuordne. Als Herausforderung wird außerdem nicht nur das Vorhandensein einer Gruppenstruktur gesehen, sondern auch der Eingriff in diese Struktur, der durch die Sprachplanung geschieht: Sprachplanung greift zum einen in den Alltag der Zielgruppe ein und lässt zum anderen eine Generationenlücke bezüglich der Sprachkompetenz entstehen. Dies interpretiere ich entsprechend als Eingriff in die Normen/Rollen, Eingriff in die Interaktionsstruktur und möglicherweise sogar in die Kohäsion der aktiven Zielgruppe. Diesem inhaltlichen Schwerpunkt ordne ich darüber hinaus auch die in den Ergebnissen direkt genannte Herausforderung zu, den Gruppenzusammenhalt zu stärken.

Was die Struktur der Autorengruppe betrifft, so wird die Eignung der Linguisten für die Statusplanung in Frage gestellt, was ich als Problem mit dem Status einzelner Gruppenmitglieder interpretiere.

Neben dieser Intragruppenperspektive lassen sich weitere Ergebnisse im Kontext der Intergruppenprozesse betrachten und systematisieren. So wird allgemein darauf hingewiesen, dass die Umsetzung der Sprachplanung mit den Beziehungen zwischen der Autoren- und der Zielgruppe zusammenhängt, wobei insbesondere die Übereinstimmung von Bedürfnissen beider Gruppen angesprochen wird. Ich interpretiere Bedürfnisse zu Zielen um und sehe diese Aussage im Kontext des in Abschnitt 4.6.2.3 beschriebenen wahrgenommenen Intergruppenkonfliktes, der besonders dann ausbleibt und nicht zur Bedrohung der sozialen Identität führt, wenn Gruppen in ihren Zielen übereinstimmen und zu deren Erreichung aufeinander angewiesen sind.

Darüber hinaus wird hier eine weitere Gruppendifferenzierung eingeführt: In die Sprechergruppe, die die Sprachpolitik bereits angenommen hat, und die Gruppe der potenziellen Sprecher. Es wird darauf hingewiesen, dass die Beziehung zwischen den beiden Gruppen für die Sprachplanung ebenfalls ausschlaggebend ist.

Was das Thema Einstellungen betrifft, so identifiziere ich zum einen Kategorien, die allgemeine Einstellungsaspekte betreffen, wofür stellvertretend 


\section{Untersuchung}

die Herausforderung der mangelnden Akzeptanz von Sprachplanung steht. Speziell wird in diesem Zusammenhang darauf eingegangen, dass die Relevanz der Sprachplanung in der aktiven Zielgruppe nicht erkannt ist, was ich als Problem auf der Ebene der kognitiven Einstellungskomponente interpretiere. Darüber hinaus wird darauf hingewiesen, dass (Sprach-)Einstellungen generell schwer änderbar sind und auch positive Einstellungen nicht automatisch zur Handlung (Annahme der Sprachpolitik) führen. Der zweite inhaltliche Block umfasst hingegen den Gruppenaspekt von Einstellungen, hinsichtlich der Bewertung sowohl von beteiligten Gruppen als auch von der Art, wie die Sprachplanung durchgeführt wird. So wird allgemein darauf hingewiesen, dass unterschiedliche Autorengruppen unterschiedliches Ansehen in der aktiven Zielgruppe genießen. Einerseits kann die Top-down-Sprachplanung (insbesondere Korpusplanung) andererseits aber auch eine Bottom-up-Planung durch Sprachaktivisten durch die gesamte Zielgruppe nicht akzeptiert sein. Dies zeigt noch einmal deutlich, dass die aktive Zielgruppe nicht homogen ist und die Bewertung der Sprachplanung insgesamt durch unterschiedliche Subgruppen der Gesamtzielgruppe unterschiedlich sein kann.

Auf Sprachplanung hat eine Reihe von allgemeinen außersprachlichen Faktoren einen Einfluss. Sprachplanung spiegelt speziell Machtverhältnisse in der Gesellschaft wider. Zudem ist die Einbettung von Sprachplanung in (außersprachliche) Oberziele ausschlaggebend, wobei, aus der Gruppenperspektive, unterschiedliche beteiligte Gruppen unterschiedliche Interessen verfolgen können. Das Zusammenspiel von diesen allgemeinen Faktoren ist also komplex und einzelfallspezifisch, so dass das Aufstellen von allgemeinen Sprachplanungsprinzipien schwierig ist.

Bei Instrumentalität wird darauf hingewiesen, dass diese nicht von der Identität getrennt betrachtet werden soll. Als instrumentelles Oberziel wird hierbei der Machterhalt durch die Eliten genannt. Andererseits wird darauf hingewiesen, dass die Sprachplanung (als Spezialfall der Kommunikationsverbesserung) nicht automatisch zum Erreichen von instrumentellen Oberzielen (hier: Produktivitätssteigerung) führt.

Beim Thema Sprachökologie wird allgemein darauf hingewiesen, dass andere Sprachen durchsetzungsfähig sind, was u.a. mit unterschiedlichem Sprachprestige oder, allgemeiner, mit unterschiedlichen Spracheinstellungen zusammenhängt.

Bei Kosten wird beobachtet, dass Sprachplanung zeit- und kostspielig ist und sich somit die Frage der Finanzierung stellt, was auch für eine Regie- 
rung eine Herausforderung ist. Dabei wird speziell darauf eingegangen, dass Statusplanung kostspieliger als Korpusplanung ist.

Schließlich ergibt sich zum Thema Machtmittel/Sanktionen, dass Statusplanung Autorität erfordert, über die aber die Autoren bei einer Bottom-upPlanung nicht verfügen. Andererseits ist die offizielle Unterstützung noch keine Erfolgsgarantie. Werden zudem offizielle Sanktionen eingesetzt, wird Sprachpolitik nur so lange eingehalten, wie diese Sanktionen andauern.

PS In dem zusammengestellten Korpus des Plansprachengebietes sind vor allem Gruppe und Einstellungen die facettenreichsten Herausforderungsthemen. Weniger elaboriert sind Identität, Sprachökologie und Gebrauch. Allgemeine Aussagen werden hingegen zu den Themen allgemeine außersprachliche Faktoren, Kosten, Erlernbarkeit, Machtmittel/Sanktionen, Einhalten, Bekanntmachen und Sprachsystem allgemein gemacht. Keine Aussagen stelle ich zu Instrumentalität, Wissenschaftlichkeit, Arbeitsprozess, Werkzeuge und Persuasion fest.

Wie in LPLP-Gebiet ergibt sich bei der Plansprachenkonstruktion ebenfalls ein heterogenes Bild verschiedener beteiligter Gruppen, wobei sich die meisten Aussagen auf die Situation des Esperanto beziehen. Ich identifiziere dabei zunächst aufgrund der Top-down- und Bottom-up-Unterscheidung die Autorengruppe und die aktive Zielgruppe. Des Weiteren wird darauf hingewiesen, dass sich innerhalb der aktiven Zielgruppe, die bei Esperanto ja mit der gesamten Weltbevölkerung gleichgesetzt werden kann, nur ein Teil der Menschen angesprochen fühlt. Aus diesem Grund erscheint mir eine weitere Differenzierung zwischen der aktuellen Sprechergruppe und der Gruppe von potenziellen Sprechern als sinnvoll. Auch bei PS kann man also die Entwicklungen aus der sozialpsychologischen Intra- und Intergruppenperspektive betrachten.

Was die Intragruppenperspektive auf die aktuellen Sprecher angeht, ist zunächst die Sprechervernetzung, wodurch ihre Interaktion sicherstellen sollte, eine Herausforderung. Vor dem Hintergrund der Gruppenstruktur interpretiere ich die Vernetzung als eine Möglichkeit, einzelne Individuen zu einer Gruppe zusammenzuschweißen, da Interaktion in der Sozialpsychologie mehrheitlich als ein konstitutives Gruppenmerkmal aufgefasst wird. In diesem Kontext können auch die genannten Diasporaeigenschaften der Esperantosprecher als Herausforderung betrachtet werden, vor allem was ihre räumliche Verteilung angeht. 


\section{Untersuchung}

Ist die Gruppe einmal konstituiert, so wird auf zwei verwandte Herausforderungen hingewiesen: Mangelnde Loyalität und starke Reformbestrebungen, die sich primär auf das Sprachsystem, aber auch auf die mit der Sprache verbunden Ideale, wie in 2.2.4.2 beschrieben, beziehen. Andererseits wird auch die Frage erörtert, wie die kollektive Weiterentwicklung der Sprache sichergestellt werden kann. Diese intergruppalen Herausforderungen lassen sich mit dem Modell der Gruppenentwicklung von Tuckman (1965) (4.6.2.2) interpretieren, wobei ich hier in der Untersuchung explizite Beispiele lediglich für die Phasen Herausbilden (Vernetzung) sowie Stürmen und Normenbilden (Reformbestrebungen/Loyalität) identifiziere.

Was die Intergruppenperspektive betrifft, so können einerseits Probleme in der Beziehung zwischen der Autoren- und aktuellen Sprechergruppe beobachtet werden, da das Sprachsystem den Bedürfnissen (Zielen) der Zielgruppe nicht entspricht bzw. nicht nachvollziehbar ist. Dies kann als Auslöser für einen Intergruppenkonflikt gesehen werden. Andererseits sind die Beziehungen zwischen der Gruppe der aktuellen und der potenziellen Sprecher eine Herausforderung, da sich, wie erwähnt, nur eine bestimmte Art von Menschen von Plansprachen (Welthilfssprachen) angezogen fühlt. Eine Ausweitung auf andere Individuen/Gruppen hängt allgemein mit den Eigenschaften der ursprünglichen/aktuellen Sprechergruppe zusammen.

Die Herausforderungen zu Einstellungen sind sowohl allgemeiner Natur, da auf die Trägheit der Menschen und die Angst vor Veränderung eingegangen wird. Zudem wird auch hier als Problem genannt, dass positive Einstellungen nicht automatisch mit einer Handlung einhergehen. Als spezifische Herausforderungen sehe ich die fehlende Akzeptanz und das geringe Prestige von Plansprachen. Wie bereits bei LPLP sehe ich auch speziell die kognitive Einstellungskomponente als problematisch, da die Relevanz von Welthilfssprachen nicht erkannt oder aber ihre Funktionsfähigkeit in Frage gestellt wird. Schließlich stellen eine ungünstige Berichterstattung in den Medien, sowie negative Einstellungen der Gruppe von potenziellen Sprechern weitere Herausforderungen dar.

Die Analyse ergibt drei Herausforderungen, die sich auf das Thema Identität beziehen. Zum einen kann eine Welthilfssprache als ein Angriff auf die (mit der Muttersprache assoziierte) Identität empfunden werden. So soll eine Welthilfssprache nicht zu einer Muttersprache werden, obwohl hier auch die Einheitlichkeitsbewahrung des Sprachsystems eine Rolle spielt. Ferner stellt sich die Frage, wie das Zusammengehörigkeitsgefühl der Sprecher, also eine Art Identität um die Sprache, geschaffen werden kann. 
Im Kontext der Sprachökologie wird darauf hingewiesen, dass andere Sprachen, sowohl Plansprachen als auch ethnische Sprachen, durchsetzungsfähig sind. Eine Plansprache hat zudem den Nachteil, dass ihr die üblichen Machtinstrumente einer ethnischen Sprache fehlen, wie beispielsweise die politische, militärische oder wirtschaftliche Bedeutung der dazugehörigen Sprechergruppe.

Schließlich wird bei Gebrauch thematisiert, dass der Erfolg einer Plansprache nicht mit einem (guten) Systementwurf gleichzusetzen ist, sondern auf dem tatsächlichen Sprachgebrauch beruht. Mit anderen Worten stellt sich die Frage, wie potenzielle Sprecher erreicht werden können. Dabei geht es auch darum, diesen Sprachgebrauch über einen längeren Zeitraum aufrechtzuerhalten. Aus der Sicht der potenziellen Sprecher ist bei einer neu konstruierten Plansprache ihr Gebrauch insofern eine Herausforderung, als sprachliche Vorbilder und Bezugspersonen fehlen, so dass die interessierten Sprecher in ihrem Gebrauch zunächst verunsichert sind.

KS Bei Kontrollierten Sprachen sind vor allem Gruppe, Einstellungen sowie Werkzeuge die facettenreichsten Herausforderungsthemen. Weniger elaboriert sind Identität und Arbeitsprozess. Allgemeine Aussagen werden hingegen zu den Themen Instrumentalität, allgemeine außersprachliche Faktoren, Kosten, Sprachökologie, Persuasion, Erlernbarkeit, Einhalten, Bekanntmachen und Sprachsystem allgemein gemacht. Keine Aussagen stelle ich zu Wissenschaftlichkeit, Machtmittel/Sanktionen und Gebrauch fest.

Beim Thema Gruppe findet sich wieder eine Differenzierung in Autorengruppe und aktive Zielgruppe und die zweite wird ebenfalls in die Gruppen der aktuellen und der potenziellen Sprecher aufgeteilt. Eine klare Bestimmung der aktiven Zielgruppe stellt dabei eine Herausforderung für die Autorengruppe dar.

Der Intragruppenperspektive ordne ich nur den allgemeinen Hinweis auf die Beschaffenheit der Zielgruppe zu. Die übrigen Kategorien betreffen hingegen die Intergruppenbeziehungen, vor allem zwischen der Autorengruppe und der aktiven Zielgruppe. So wird hauptsächlich die Zusammenarbeit zwischen diesen Gruppen als Herausforderung gesehen, von der aber beide profitieren können, da so einerseits die Bedürfnisse und Gepflogenheiten der Zielgruppe durch die Autorengruppe berücksichtigt, andererseits aber die KS-Entwickler bei der Korpusplanung unterstützt werden können. Wie aus den anderen Gebieten bekannt, stellt speziell die Nachvollziehbarkeit der topdown entstandenen Spezifikation für die aktive Zielgruppe eine Herausforde- 


\section{Untersuchung}

rung dar. Was die Beziehungen zwischen unterschiedlichen Subgruppen der aktiven Zielgruppe angeht, wird das Erreichen von potenziellen Sprechern als eine Herausforderung gesehen.

Das Thema Einstellungen umfasst wieder den allgemeinen Hinweis auf die Trägheit der Menschen und die Abneigung gegen Veränderungen. Ferner wird allgemein der Widerstand und mangelnde Akzeptanz von Kontrollierten Sprachen durch verschiedene Gruppen angesprochen. Die aktive Zielgruppe, in erster Linie Technische Redakteure, fühlen sich in ihrer Schreibkompetenz in Frage gestellt und können speziell mit einzelnen KS-Regeln unzufrieden sein. Weitere Probleme sind mangelndes Bewusstsein für Sprachprobleme, mangelnde Bereitschaft, eine KS zu entwickeln bzw. anzunehmen sowie mangelnde Überzeugung bei der Anwendung einer KS. Diese Probleme betreffen zwar die aktuelle Sprechergruppe, können sich aber auch auf weitere Gruppen beziehen wie die Gruppe von potenziellen Sprechern (z. B. andere Unternehmensbereiche), aber auch höhere Managementebenen, die ich als Gruppe der Auftraggeber bzw. Autoren auf der nächsthöheren Hierarchieebene sehe. Andererseits wird auch hier darauf hingewiesen, dass das Bewusstsein für oder das Wissen um Sprachprobleme nicht automatisch zur Entwicklung einer KS bzw. ihrer Annahme führt. Schließlich zähle ich zu den Einstellungsaspekten auch die (mangelnde) Vertrautheit mit dem KS-Konzept in allen betroffenen Gruppen.

Ein Thema, das in LPLP- und PS-Gebieten nicht behandelt wird, in KS aber eine wichtige Stellung einnimmt, sind Werkzeuge. Im Allgemeinen sollen sie beim Einführen und Einhalten der KS-Spezifikation unterstützend eingesetzt werden. Dabei sehe ich die Benutzerfreundlichkeit als übergreifende Herausforderung, die durch verwandte Kategorien spezifiziert wird. Hierzu gehören die Entwicklung von Werkzeugen unter praxisnaher Evaluation sowie ihre Integrierbarkeit in vorhandene Softwareumgebungen. Speziell bei einem Controlled Language Checker ist die Sicherstellung von Effektivität, Prüfadäquatheit und Personalisierbarkeit eine wichtige Erfolgsvoraussetzung.

Das Thema Identität ist deutlich weniger elaboriert als in den anderen Gebieten, dennoch greife ich es hier auf, da es einen neuen Aspekt zur Sprache bringt, nämlich unterschiedliche Auffassung von Sprachkompetenz der aktiven Zielgruppe, insbesondere Technischer Redakteure. Hinter dieser Herausforderung verbirgt sich der repetitive Stil, den eine KS erfordert bzw. erzwingt, der aber von der aktiven Zielgruppe als nicht geeignet für die Aufgabenbewältigung bzw. nicht als ihr eigener Stil empfunden werden kann. Da es sich um die Selbstauffassung von Technischen Redakteuren oder ande- 
ren Zielgruppen handelt, die durch eine Kontrollierte Sprache herausgefordert wird, habe ich diesen Punkt dem Thema Identität zugeordnet. Darüber hinaus wird darauf hingewiesen, dass eine KS politisch bzw. kulturell nicht neutral ist.

Beim Thema Arbeitsprozess stelle ich schließlich zwei Herausforderungen fest, die sich auf die Arbeit der aktiven Zielgruppe auswirken: Zum einen kann eine neu eingeführte KS den gewöhnlichen Arbeitsprozess behindern (verlangsamen, mehr Arbeitsschritte erfordern). Zum anderen kann die Einführung einer KS nur eine von vielen Arbeitsprozessänderungen sein, die gleichzeitig für eine oder mehrere beteiligte Zielgruppen eingeleitet werden.

TA Bei Terminologiearbeit sind vor allem Gruppe, Arbeitsprozess sowie Werkzeuge die facettenreichsten Herausforderungsthemen. Weniger elaboriert sind Einstellungen. Allgemeine Aussagen werden hingegen zu den Themen Instrumentalität, allgemeine außersprachliche Faktoren, Kosten, Sprachökologie, Persuasion, Erlernbarkeit, Machtmittel/Sanktionen, Einhalten, Bekanntmachen, Gebrauch und Sprachsystem allgemein gemacht. Keine Aussagen stelle ich zu Identität und Wissenschaftlichkeit fest.

Auch in Terminologiearbeit lassen sich ähnliche Gruppen identifizieren wie in den übrigen Gebieten: Autorengruppe (Arbeitsgruppe) und aktive Zielgruppe, die wieder in aktuelle und potenzielle Sprechergruppe zerfällt. Die Gruppe der potenziellen Sprecher kann weiter differenziert werden in interne und externe Sprecher, je nach der Zugehörigkeit z. B. zum gegebenen Unternehmen. Des Weiteren lassen sich auch hier die beiden sozialpsychologischen Perspektiven zur Systematisierung anwenden. So wird zum einen die Größe der aktiven Zielgruppe, zum anderen aber ihre Gepflogenheiten als Herausforderungen genannt. Ich interpretiere diese als Elemente der Gruppenstruktur, wobei ich den Hinweis auf Gepflogenheiten als Normen und Rollen sehe. Im Unterschied zu anderen Gebieten ergeben sich aus der Intragruppenperspektive explizite Herausforderungen bezüglich der Autorengruppe. Sie betreffen einerseits die Gruppenstruktur, da Verantwortlichkeiten unklar sein und Kompetenzen fehlen können, was sich entsprechend den Rollen und dem Status zuordnen lässt. Hinzu zähle ich darüber hinaus die genannte Rolle der Organisationsstruktur. Andererseits lassen die genannten Konflikte in der Arbeitsgruppe auf Herausforderungen in der Interaktion schließen und mit dem Modell von Tuckman (1965) als Phase Leisten interpretieren.

Auf der Intergruppenebene wird die Nachvollziehbarkeit der Autorenentscheidungen sowohl für die interne als auch die externe Zielgruppe erwähnt. 


\section{Untersuchung}

Darüber hinaus stellt sich die Frage, wie die Gepflogenheiten der Zielgruppe durch die Autorengruppe berücksichtigt werden können.

Das Thema Arbeitsprozesse sehe ich als das facettenreichste im TA-Gebiet. Dabei werden Arbeitsprozesse der Autorengruppe und der aktiven Zielgruppe angesprochen. Aus der Autorenperspektive spielt das Erzielen einer systematischen Vorgehensweise eine wichtige Rolle, worauf sich das Fehlen von Benchmarks oder anderen Qualitätskriterien (für außersprachliche Gesichtspunkte) erschwerend auswirkt. Innerhalb der Arbeitsgruppe werden ihre Koordination aber auch das Konsensfinden als Herausforderungen genannt. Im Gesamtkontext der Terminologiearbeit werden der Zeitaufwand und die mangelnde Unterstützung höherer Hierarchieebenen einer Organisation als Herausforderungen gesehen. Hinzu kommen die Schwierigkeiten, dass Terminologiearbeit nie abgeschlossen ist und früh in den Produktlebenszyklus eingebunden sein muss, eine nachträgliche Änderung von festgelegten Termini jedoch umständlich ist.

Weitere Herausforderungen zum Thema Arbeitsprozesse kann ich nicht eindeutig einer der Gruppen zuordnen. Dies führe ich auf den gemischten Ansatz zurück, bei dem eine aktive Beteiligung der Terminologienutzer am Terminologieaufbau angestrebt wird und in der Praxis die Benutzergruppe zur Autorengruppe hinzugezählt werden kann. Im Allgemeinen verursacht die Terminologiearbeit für alle Beteiligten Mehrarbeit. Gehen die Autoren wie gefordert systematisch vor, dann können sich weitere Herausforderungen für alle Beteiligten ergeben. Demnach können die Arbeitsprozesse unzureichend definiert oder impraktikabel sein (speziell: nicht in andere Prozesse integrierbar) oder aber nicht eingehalten werden.

Was das Thema Werkzeuge betrifft, so steht der Einsatz von Prüfwerkzeugen im Vordergrund. Eine Herausforderung ist dabei die Verfügbarkeit von geeigneten Werkzeugen. Stehen sie auch zur Verfügung, so stellt sich zuweilen das Problem, dass sie nicht genutzt werden. Zu weiteren Herausforderungen gehören, ähnlich wie bei KS, Benutzerfreundlichkeit und technische Integrierbarkeit.

Schließlich werden zum Thema Einstellungen der geringe Stellenwert der TA im Unternehmen, die geringe Handlungsbereitschaft oder speziell das mangelnde Bewusstsein für Sprachprobleme genannt. Auch hier wird beobachtet, dass positive Einstellungen nicht automatisch zu einer Handlung führen. 


\subsubsection{Herausforderungen: Gebiete vergleichend}

Nach einer Analyse der einzelnen Gebiete erfolgt in diesem Abschnitt eine integrative Gegenüberstellung der so gewonnenen Erkenntnisse mit dem Ziel, Aussagen festzuhalten, die sich auf mehrere Gebiete übertragen lassen. Die Ergebnisse der Einzelanalyse sind in Anhang B, nach inhaltlichen Aussagen geordnet, dargestellt. Ich beobachte dabei, dass die Themen Gruppe und Einstellungen in allen Gebieten besonders elaboriert sind, was mein primäres Forschungsinteresse bereits bei der Datenerfassung widerspiegelt. Das dritte Thema, dem ich in dieser Arbeit ein besonderes Augenmerk schenke - Identität - ist hingegen in unterschiedlichen Gebieten unterschiedlich stark elaboriert. Eine Streuung der Elaboration beobachte ich darüber hinaus bei den Themen Instrumentalität, Arbeitsprozess aber auch Machtmittel/Sanktionen. Zudem stelle ich fest, dass das Thema Werkzeuge nur in KS und TA vertreten ist.

Die Betrachtung der Elaboration benutze ich, wie eingangs in Abschnitt 5.2.2.2 erläutert, in erster Linie dazu, mir einen inhaltlichen Zugang zu den Rohergebnissen aus der Datenbank zu verschaffen. Die Knappheit oder gar das vollständige Fehlen eines Themas muss aber nicht bedeuten, dass es in einem Gebiet nicht behandelt wird oder irrelevant ist. Beispielsweise stelle ich fest, dass das Thema Persuasion in Gebiet PS fehlt. Es erscheint jedoch naheliegend, dass die Frage, wie man Menschen von einer Plansprache überzeugt, auch hier, ähnlich wie in den übrigen Gebieten, eine Rolle spielt.

In der vergleichenden Auswertung der Gebiete setze ich mich also stärker inhaltlich mit den in einer Einzelanalyse gewonnenen Aussagen auseinander und prüfe, in welchen Fällen es plausibel ist, eine Aussage allen vier Gebieten, ggf. leicht modifiziert, zuzuschreiben. Nach der vergleichenden Analyse stelle ich fest, dass dies in den meisten Fällen möglich ist. Die Ergebnisse dieser Systematisierung, Übertragung und Verallgemeinerung von Anhang B sind in Anhang $\mathrm{C}$ dargestellt. Viele der vorgeschlagenen Verallgemeinerungen sind leicht nachvollziehbar und ich beschränke mich deshalb in diesem Abschnitt lediglich auf die Beschreibung der Resultate in Anhang C, die aus meiner Sicht einer Erläuterung bedürfen.

Wie aus Anhang B ersichtlich, wird das Thema Identität in den einzelnen Gebieten unterschiedlich behandelt, sowohl was die Elaborierung als auch was den Inhalt betrifft. Ich halte fest, dass sich die Aussagen weitestgehend mit den Erkenntnissen des übergreifenden Modells der Sprachlenkung decken, das ich in Abschnitt 4.9 vorgeschlagen habe. Dies ist nicht überra- 


\section{Untersuchung}

schend, da ich in beiden Fällen auf dieselbe Literatur zurückgriff. Deshalb übernehme ich die Aussagen des Modells in Anhang C, wobei ich, zur Wiederholung, insbesondere das Salientmachen der sozialen Identität, ggf. ihre Bedrohung sowie die Rolle der Sprache in der Identitätskonstruktion für übergreifende Aspekte halte. Dass auch aufgabenbezogene Kompetenzen einen Teil der Identität ausmachen können, halte ich bei Sprachlenkung in aufgabenbezogenen Gruppen (Konstruktion von KS, TA) für wichtig.

Für das Thema Gruppe greife ich hier einige Punkte heraus. Zum einen sind in Anhang $\mathrm{C}$ alle identifizierten Gruppen zusammengetragen. Da die meisten von ihnen bereits in vorangegangenen Abschnitten besprochen sind, gehe ich hier lediglich auf die Unterscheidung zwischen den internen und externen Sprechern der aktiven Zielgruppe ein, weil ihre übergreifende Bestimmung zwar möglich, aber konzeptionell schwierig ist. Diese Unterscheidung stammt aus dem TA-Bereich und bezieht sich ursprünglich auf eine Organisation wie ein Unternehmen, der sowohl die Autorengruppe als auch die interne Sprechergruppe, aber nicht die externe Sprechergruppe angehört. Als Beispiel können hier die unternehmensinterne Arbeitsgruppe als Autoren, die Mitarbeiter eines Unternehmens als interne Zielgruppe und die Zulieferer als externe Zielgruppe genannt werden. Abstrakter betrachtet, setzt diese Unterscheidung also voraus, dass sich die interne Zielgruppe und die Autorengruppe zu einer übergreifenden Gruppe ${ }^{131}$ subsumieren lassen. Eine theoretische Grundlage liefert hierzu die Selbstkategorisierungstheorie von J. C. Turner (1987), die von mehreren hierarchisch organisierten Selbstkategorien (Teilidentitäten) ausgeht (kurz vorgestellt in Abschnitt 4.5.4). Ich schlage dann die Auffassung vor, dass die externe Zielgruppe nicht dieser übergreifenden Gruppe angehört und, weiter mit J. C. Turner (1987), diese beiden Gruppen sich erst auf der nächsthöheren Abstraktionsebene zu einer Gruppe verbinden lassen.

Ein weiterer Punkt, den ich hier erläutern möchte, bezieht sich auf die kritischen Phasen der Gruppenentwicklung. In Anhang B habe ich im PSBereich Herausbilden, Stürmen und Normenbilden und im TA-Bereich Leisten als kritische Phasen identifiziert. Ich schlage hierfür eine Uminterpretation vor. Die in Abschnitt 5.2.2.2 genannten Beispiele für Probleme in den Phasen Herausbilden, Stürmen und Normenbilden stammen aus der historischen Entwicklung der Esperantogemeinschaft. Wie in Abschnitt 2.2.4.3 ausgeführt, resultierten sie vor allem daraus, dass der Sprechergemeinschaft

131 Aus den in 4.6.1 vorgestellten gängigen Auffassungen von Gruppe soll eine solche übergreifende Gruppe auch als solche in der Wahrnehmung ihrer Mitglieder tatsächlich existieren. Diese übergreifende Gruppe soll also kein rein theoretisches Konstrukt sein. 
die entscheidende Rolle in der Weiterentwicklung der Sprache zugeteilt wurde und man deshalb von einer dezidierten Bottom-up-Ausrichtung sprechen kann. Bei einer Bottom-up-Ausrichtung müssen aber Rollen, Verantwortlichkeiten und Vorgehensweisen in der Autoren-Sprecher-Gruppe zunächst ausgehandelt werden, was anfangs zu kritischen Konflikten führen kann. Ich gehe davon aus, dass sich diese Aushandlung in der Autorengruppe bei Top-downSprachplanung weniger intensiv gestaltet, da der organisatorische Rahmen von Anfang an stärker vorgegeben ist. Hingegen sehe ich die Phase Leisten, auf die ich aus der TA-Literatur schließe, vor allem bei gemischten Ansätzen zur Sprachlenkung (gemischt top-down/bottom-up) als entscheidend, da bei Korpus- und Statusplanung verschiedene Positionen, Interessen und Ziele verschiedener Gruppen aufeinanderprallen können, was zu Konflikten führen kann.

Was den Konflikt zwischen verschiedenen Gruppen angeht, der der Intergruppenperspektive zugehört, stellte ich fest, dass verschiedene Gebiete auf einzelne Elemente des Intergruppenkonfliktes eingehen: So weisen LPLP und PS auf das Problem mit unterschiedlichen Zielen von beteiligten Gruppen hin, während KS die Bedeutung von Zusammenarbeit von verschiedenen Gruppen betont. Wie in Abschnitt 4.6.2.3 beschrieben, entscheidet erst das Zusammenspiel von beiden Elementen über das Ausbleiben eines Intergruppenkonfliktes, nämlich die Übereinstimmung von Zielen verschiedener Gruppen und die Notwendigkeit einer Zusammenarbeit, um diese Ziele zu erreichen. Diese Überlegungen sind allgemeingültig und entsprechend in Anhang C eingetragen.

Abschließend zum Thema Gruppe erwähne ich, dass ich für die aktive Zielgruppe zwar die Norm Disziplin und das Kohäsionsmerkmal Engagement übergreifend als erfolgsrelevante Elemente der Gruppenstruktur sehe, jedoch die Norm Fortschrittbedürfnis (LPLP) und die die Interaktion prägenden Diasporaeigenschaften (PS) nicht für verallgemeinerbar halte.

Die vergleichende Betrachtung des Themas Einstellungen ermöglichte mir die Identifizierung von Einstellungsobjekten, die ich zu einer weiteren Systematisierung von Erfolgsherausforderungen benutzte. Die Einstellungsherausforderungen können sich somit auf die neue Sprachform (Sprachsystem/Sprachgebrauch), die Autorengruppe, die aktuelle Zielgruppe, die Sprachlenkung (als Tätigkeit), aber auch auf Werkzeuge beziehen. Das Objekt Werkzeuge wird zwar nur in den Gebieten KS und TA besprochen, die konkreten Aussagen sind aber recht allgemeingültig 


\section{Untersuchung}

und können ggf. auf alle Hilfsmittel (wie Wörterbücher, Lehrwerke etc.) übertragen werden.

Ferner stelle ich fest, dass im Gebiet TA mit der Frage der Vertrautheit de facto der Mere-Exposure-Effekt (Abschnitt 4.7.2.3) beobachtet wird. Da es sich laut Sozialpsychologie um einen allgemeinen Effekt handelt, postuliere ich ihn übergreifend für alle vier Gebiete.

Das Thema Arbeitsprozesse ist besonders in TA aber auch in KS ausgeprägt. Obwohl viele Aussagen sich auf alle vier Gebiete verallgemeinern lassen, so stelle ich bei LPLP und TA gegensätzliche Positionen fest, was das Erzielen einer systematischen Vorgehensweise durch die Autorengruppe angeht. In TA ist eine systematische Vorgehensweise nahezu selbstverständlich und wird zu einer wesentlichen Voraussetzung für den Erfolg gemacht, während in LPLP pragmatischer davon ausgegangen wird, dass sich diese nicht immer erzielen lässt. Welche dieser beiden Positionen auf die übrigen zwei Gebiete übertragbar ist, lasse ich an dieser Stelle offen. Darüber hinaus stelle ich für TA weitere Aspekte fest, die möglicherweise ohne Weiteres nicht verallgemeinerbar sind.

Auch beim Thema Machtmittel/Sanktionen stelle ich schließlich ebenfalls zwei Positionen fest, die zwar nicht gegensätzlich, aber auch nicht vereinbar sind. In LPLP und teilweise in PS findet sich die Ansicht, dass Autorität für Sprachlenkung unabdingbar ist. In TA und in KS sehe ich hingegen eine Abschwächung dieser Aussage, wonach Sprachlenkung nicht zwangsläufig Autorität erfordert, aber von einer Top-down-Unterstützung (höherer Hierarchieebenen) durchaus profitieren kann.

\subsubsection{Vorgehen: Gebiete einzeln}

Nach der Analyse von Herausforderungen wende ich mich nun in den nächsten beiden Abschnitten den Vorgehen zu. Ich verfahre dabei ähnlich und betrachte in diesem Abschnitt zunächst die Rohergebnisse für einzelne Gebiete und vergleiche und verallgemeinere die so gewonnenen Erkenntnisse anschließend in Abschnitt 5.2.2.5.

LPLP Die Analyse ergibt, dass die facettenreichsten Themen zu den Vorgehen in LPLP-Gebiet vor allem Gruppe, Arbeitsprozess und Machtmittel/Sanktionen sind. Weniger elaboriert sind Identität, Instrumentalität, Einstellungen, Persuasion und Bekanntmachen. Nur allgemeine Aussagen werden hingegen $\mathrm{zu}$ den Themen allgemeine außersprachliche Faktoren, 


\begin{tabular}{|c|c|c|c|c|}
\hline & LPLP & PS & $\mathrm{KS}$ & TA \\
\hline \multirow[t]{5}{*}{+++} & Gruppe & Gruppe & Gruppe & Gruppe \\
\hline & Arbeitsprozess & Sprachsystem & Persuasion & Persuasion \\
\hline & Machtmittel & & Erlernbarkeit & Arbeitsprozess \\
\hline & & & Arbeitsprozess & \\
\hline & & & Werkzeuge & \\
\hline \multirow[t]{6}{*}{++} & Identität & Identität & Einstellungen & Einstellungen \\
\hline & Instrumentalität & Instrumentalität & Sprachsystem & Machtmittel \\
\hline & Einstellungen & Einstellungen & & Werkzeuge \\
\hline & Persuasion & Arbeitsprozess & & \\
\hline & Bekanntmachen & Bekanntmachen & & \\
\hline & & Gebrauch & & \\
\hline \multirow[t]{7}{*}{+} & außerspr. Faktoren & außerspr. Faktoren & außerspr. Faktoren & Identität \\
\hline & Kosten & Sprachökologie & Wissenschaftlichkeit & Instrumentalität \\
\hline & Wissenschaftlichkeit & Wissenschaftlichkeit & Machtmittel & außerspr. Faktoren \\
\hline & Sprachsystem & Persuasion & Bekanntmachen & Wissenschaftlichkeit \\
\hline & Einhalten & Erlernbarkeit & Gebrauch & Einhalten \\
\hline & & Machtmittel & & Bekanntmachen \\
\hline & & Einhalten & & Gebrauch \\
\hline \multirow[t]{5}{*}{-} & Sprachökologie & Kosten & Identität & Kosten \\
\hline & Erlernbarkeit & Werkzeuge & Instrumentalität & Sprachökologie \\
\hline & Werkzeuge & & Kosten & Erlernbarkeit \\
\hline & Gebrauch & & Sprachökologie & Sprachsystem \\
\hline & & & Einhalten & \\
\hline
\end{tabular}

Tabelle 27.: Vorgehen: Bewertung der Elaboration von Themen 


\section{Untersuchung}

Kosten, Wissenschaftlichkeit, Einhalten und Sprachsystem allgemein gemacht. Keine Aussagen stelle ich zu Sprachökologie, Erlernbarkeit, Werkzeuge und Gebrauch fest.

Wie in Abschnitt 5.2.2.1 erläutert, ist meine Handhabung des Themas Arbeitsprozess nicht konsequent. Dies hat zur Folge, dass ich in der Analyse der Vorgehen eine Überlappung der Themen Arbeitsprozesse und Gruppe feststelle. Ich entscheide mich deshalb, alle betreffenden Aussagen unter dem Thema Gruppe zu behandeln, weil die Gruppenperspektive eine Systematisierung der Arbeitsprozessaspekte ermöglicht.

Wie in den vorangegangenen Abschnitten identifiziere ich hier zwei Formen von Sprachplanung: top-down und bottom-up, woraus ich auf mehrere Gruppen schließe: die Autorengruppe und die aktive Zielgruppe. Dabei finden sich wieder allgemeine Hinweise darauf, dass beide Gruppen nicht homogen sind. So kann die aktive Zielgruppe in verschiedene Subgruppen zerfallen und die Autorengruppe in eine hierarchische Struktur eingebettet sein. Im Fall von Bottom-up-Sprachplanung übernimmt die aktive Zielgruppe gleichzeitig die Funktion einer Autorengruppe. Die meisten festgestellten Aussagen zu Handlungsmöglichkeiten (Vorgehen) lassen sich somit einer der beiden Formen der Sprachplanung zuordnen, wobei sie die Beziehungen innerhalb der betreffenden Gruppe, vor allem aber den Umgang mit der jeweils anderen Gruppe betreffen.

Was die Top-down-Sprachplanung betrifft, so wird aus der Intragruppenperspektive empfohlen, organisatorische Sprachplanungseinheiten in betreffenden Institutionen aufzubauen und ggf. die Verantwortung für die Sprachplanung auf diese zu übertragen. Was die Beziehungen zur aktiven Zielgruppe betrifft, so wird allgemein empfohlen, dass die Autoren die Gepflogenheiten der aktiven Zielgruppe berücksichtigen, wobei auf unterschiedliche Subgruppen unterschiedlich eingegangen werden soll. Darüber hinaus soll mit Sprachpolitik Fairness gegenüber der Zielgruppe gefördert werden. Andererseits stelle ich auch fest, dass die Autorengruppe ihre Entscheidungen der Zielgruppe autoritär mitteilen kann.

Bei der Bottom-up-Sprachplanung steht vor allem der Autorenaspekt der aktiven Zielgruppe im Mittelpunkt. So wird gefordert, dass Gruppen, die in der Machthierarchie übergeordnet sind, für die Mitarbeit gewonnen werden. Dies kann auch dann versucht werden, wenn die aktive Zielgruppe keine einheitliche Position bezüglich der Sprachplanung vertritt. ${ }^{132}$

132 In der Datenbank ist abzulesen, dass der Versuch, übergeordnete Gruppen zu erreichen, ohne selbst eine einheitliche Position zu vertreten als nicht erfolgreich angesehen wird. Da ich die Vorgehen nach behaupteter Wirksamkeit nicht auswerte, führe ich alle Vorgehen mit auf. 
Die Ergebnisse enthalten weitere Vorgehen, die ich für allgemeingültig halte und beiden Formen der Sprachplanung gleichermaßen zuordne: Systematisch vorgehen, wobei diesem das pragmatische Vorgehen gegenüber steht, Evaluationskriterien schaffen, Sprachplanung als Innovation auffassen und Statusplanung als Daueraufgabe behandeln. Diese Aussagen besitzen keinen wesentlichen Gruppenaspekt und könnten somit dem Thema Arbeitsprozesse zugeordnet werden. Ähnlich allgemein ist die Forderung, dass Sprachplanung zwei Phasen umfassen soll: Deskription und Präskription. Diese Forderung hat aber unterschiedliche Konsequenzen für die beiden Formen der Sprachplanung. Bei der Top-down-Ausrichtung sehe ich hier vor allem die erste Phase als wesentlich, weil ich daran ablese, dass zumindest eine rudimentäre Hinwendung zu der aktiven Zielgruppe notwendig ist. Andererseits bedeutet die zweite Phase für die Bottom-up-Ausrichtung, dass die aktive Zielgruppe nach Mitteln suchen muss, mit denen sie den Gebrauch fördern oder gar das Einhalten der neuen Form erzwingen kann. Diese Überlegung ist im Kontext der Machtmittel/Sanktionen wichtig.

Zusammenfassend stelle ich für die Themen Gruppe und Arbeitsprozesse fest, dass die zwei Formen der Sprachplanung - top-down und bottomup - idealtypisch sind und sich besser als ein Kontinuum auffassen lassen. Dabei stellen eine reine Top-down-Sprachplanung und eine reine Bottomup-Sprachplanung die beiden Pole dar. In der Mitte erreicht man den idealtypischen gemischten Ansatz, bei dem die Mitwirkung der Autorengruppe und der aktiven Zielgruppe in einem ausgewogenen Verhältnis stehen. Dieser ausgewogenen Mitte kann man sich von beiden Polen aus nähern: Top-down anfangen und die aktive Zielgruppe allmählich stärker mit einbeziehen oder aber bottom-up anfangen und hierarchisch übergeordnete Gruppen allmählich stärker mit einbeziehen. Diese Konzeptualisierung ist teilweise in den Aussagen beider Themen in Anhang D explizit zu finden.

Was das Thema Machtmittel/Sanktionen betrifft, so identifiziere ich in LPLP-Gebiet vor allem Handlungsmöglichkeiten eines Staates, dem verschiedene Mittel zur Durchsetzung einer Sprachpolitik zur Verfügung stehen. Dies ist eine spezielle Form der Top-down-Sprachplanung. Generell kann ein Staat Sprachplanung implizit oder explizit betreiben und eine autoritäre Staatspolitik wird allgemein als umsetzungsfördernd gesehen. Eine Verankerung der neuen Sprachform im Bildungssystem ist eine der Möglichkeiten, Sprachpolitik im Kindesalter zu fördern und diese Frühförderung wird als besonders erfolgversprechend gesehen. Darüber 


\section{Untersuchung}

hinaus kann ein Staat das Nichteinhalten einer Sprachpolitik gesetzlich sanktionieren.

Demgegenüber verfügt Bottom-up-Sprachplanung nicht über diese Machtmittel. Dieses Defizit versucht man entsprechend dadurch zu beheben, dass man, wie bereits erwähnt, höhere Hierarchieebenen (z. B. durch den Staat) oder aber durch fachliche Autoritäten für sich gewinnt. Diese Unterstützung kann entweder aktiv sein und durch die Bereitstellung von verschiedenen Mitteln (wie beispielsweise oben beschrieben) erfolgen oder aber in der Toleranz der von unten betriebenen Sprachplanung bestehen.

Obwohl beide Formen von Sprachplanung versuchen, von Machtmitteln und offiziellen Sanktionen Gebrauch zu machen, wird beobachtet, dass diese noch keine hinreichende Bedingung für den Erfolg von Sprachplanung darstellen.

Beim Thema Identität werden Vorgehen in Abhängigkeit von der angenommenen Wechselwirkung zwischen Sprache und Identität empfohlen. Nimmt man die Wechselwirkung an, so kann einerseits die soziale Identität dafür benutzt werden, Sprachplanung zu unterstützen: Werten die Autoren die soziale Identität auf, die mit betreffender Sprache in Beziehung steht, so erhoffen sie sich, dass eine Sprachplanungsmaßnahme automatisch mit aufgewertet wird. Andersherum kann die Bedeutung der Sprache für die soziale Identität durch die Autoren betont werden, wodurch eine entsprechende Sprachplanungsmaßnahme ebenfalls an Bedeutung gewinnt.

Eine andere Möglichkeit ist eine Entkoppelung beider Phänomene Sprache und Identität - voneinander, indem die emotionale/identitätsstiftende Funktion einer Sprache ausgeblendet und die rein instrumentelle Bedeutung einer Sprachplanungsmaßnahme herausgestellt wird.

Die dritte Möglichkeit stellt schließlich ein gemischter Ansatz dar, bei dem beide Aspekte der Sprache - Identität und Instrumentalität - im Auge behalten werden, wenngleich man bei Sprachplanung durchaus einen Schwerpunkt setzen kann.

Schließlich wird darauf hingewiesen, dass die Annahme einer Innovation, insbesondere also der Sprachplanung immer eine individuelle Komponente enthält, was ich vor dem Hintergrund der Selbstkategorisierungstheorie als Hinweis auf die unterste Ebene der sozialen Identität (individuelle Identität) interpretiere.

In Anknüpfung an die Wechselwirkung zwischen Sprache und Identität wird beim Thema Instrumentalität allgemein empfohlen, die instrumentelle Bedeutung der Sprachpolitik zu betonen, wobei aus der Sicht der Autoren der 
Machterhalt ein spezielles instrumentelles Oberziel darstellt. Zudem kann offiziell gestützte Sprachplanung auf die Domänen gerichtet sein, in denen die Sprache bereits gebraucht wird oder aber Domänen, die von der Sprache zum gegebenen Zeitpunkt nicht abgedeckt sind.

Zum Thema Einstellungen sollen allgemein positive Einstellungen in der Öffentlichkeit gefordert werden. Dies soll man zum einen durch die Schaffung des Bewusstseins für Sprachplanung erreichen. Diesen Punkt sehe ich als Element des eingeführten Modells der Elaborationswahscheinlichkeit (4.7.2.1), aus dem folgt, dass eine Einstellungsänderung durch Persuasion besonders dann erfolgversprechend ist, wenn Personen die Relevanz eines Themas für sich selbst erkennen.

Darüber hinaus ergibt die Analyse, dass Sprachplanung vor allem für öffentliche Domänen erfolgen soll, die ein höheres Prestige als private Domänen besitzen.

Zur Umsetzungsförderung soll allgemein Persuasion eingesetzt werden. Dabei sollen Botschaften einfach sein und wiederholt dargeboten werden. Als Argument soll speziell die Bedeutung der Sprachplanung für das Erreichen von außersprachlichen (Ober-)Zielen benutzt werden.

Schließlich wird zum Thema Bekanntmachen gefordert, dass Sprachpolitik wie ein kommerzielles Produkt vermarktet werden soll, wofür verschiedene Kommunikationskanäle und insbesondere verschiedene Medien zu nutzen sind.

PS Die Analyse ergibt, dass die facettenreichsten Themen zu Vorgehen in PS-Gebiet vor allem Gruppe und Sprachsystem sind. Weniger elaboriert sind Identität, Instrumentalität, Einstellungen, Erlernbarkeit, Arbeitsprozesse, Bekanntmachen und Gebrauch. Nur allgemeine Aussagen werden hingegen zu den Themen allgemeine außersprachliche Faktoren, Sprachökologie, Wissenschaftlichkeit, Persuasion, Machtmittel/Sanktionen und Einhalten gemacht. Keine Aussagen stelle ich zu Kosten und Werkzeuge fest.

Beim Thema Gruppe wird allgemein empfohlen, dass die Autoren aktive Schritte zum Aufbau einer Sprechergemeinschaft unternehmen sollen. Dies mag trivial erscheinen, die Geschichte der Plansprachenkonstruktion zeigt aber, dass es nicht immer selbstverständlich war. Diese Empfehlung steht auch in Verbindung mit der an einer anderen Stelle erwähnten Forderung, dass Sprachlenkung über eine reine Korpusplanung hinausgehen muss, um erfolgreich zu sein. Um den Aufbau einer Sprechergemeinschaft zu fördern, werden vor allem Maßnahmen zur Vernetzung der Sprecher, z. B. durch Zeit- 


\section{Untersuchung}

schriften oder Veröffentlichung von Kontaktdaten, insbesondere des Adresaro, ${ }^{133}$ genannt. Diese interpretiere ich als aktive Maßnahmen zur Förderung der Interaktion, die ein Merkmal der Gruppenstruktur ist und insgesamt als konstitutiv für Gruppen gilt. Zentral finde ich also die Interpretation, dass die Autoren Sprecher nicht isoliert gewinnen sollen, sondern sich von Anfang an um den Aufbau einer Gruppe (Gemeinschaft) mit allen konstitutiven Merkmalen (Interaktion, Selbstwahrnehmung, also Identität, als Gruppe und ggf. Kohäsion) bemühen sollen.

Ferner identifiziere ich in den Ergebnissen konkrete Empfehlungen zur Gestaltung von Beziehungen zwischen der Autoren- und der aktiven Zielgruppe (Intergruppenperspektive). Übergreifend gesehen, können zwei Führungsstile eingesetzt werden: autoritär und nichtautoritär. Weitere festgestellte Aussagen ordne ich vor allem dem nichtautoritären Stil zu.

Der nichtautoritäre Führungsstil wird durch Kompromissbereitschaft und diplomatisches Geschick charakterisiert. Diese sind besonders deshalb wichtig, da auf die Relevanz von Bottom-up-Verbreitung, Entscheidungstransparenz und diplomatischen Abstimmungen, insbesondere bei Entscheidungen über Korpusänderungen, verwiesen wird. Diese werden zudem durch den Verzicht des Autors auf seine Sonderstellung begünstigt. Dabei möchte ich herausstellen, dass dies nicht unbedingt einer Forderung nach einer ungelenkten, reinen Bottom-up-Ausrichtung gleichzusetzen ist, ${ }^{134}$ sondern lediglich als Empfehlung des nichtautoritären Führungsstils und eines gemischten Topdown-/Bottom-up-Ansatzes gesehen werden soll.

In den Rohergebnissen werden außerdem die Beziehung zwischen der $\mathrm{Au}-$ torengruppe und der Gruppe von potenziellen Sprechern angesprochen. Empfohlen wird die Aufnahme aller Personen ungeachtet ihrer politischen, religiösen oder gesellschaftlich-kulturellen Zugehörigkeit. Dieser Punkt schneidet das Thema Identität an, wobei ich zwei Interpretationsmöglichkeiten sehe. Die erste bette ich in die Selbstkategorisierungstheorie von J. C. Turner (1987) ein. Folglich ist die durch die Autoren aufzubauende Gruppenidentität der Sprecher auf einer sehr hohen Abstraktionsebene anzusiedeln, damit sich alle Ausprägungen der sozialen Identität darunter subsumieren lassen. Dabei nennt J. C. Turner (1987, 45-46) menschliches Wesen als höchst-

133 Zamenhof fügte der ersten Esperanto-Broschüre eine Lernverpflichtung bei, die Interessierte an ihn zurückschicken sollten (Dr. Esperanto 1887a, 7-8,24-26, 31). Die Liste der Unterzeichner und anderer Interessierten veröffentlichte er 1888 als sog. Adresaro (vgl. Forster 1982, 56-57).

134 Diese Forderung stammt aus der Esperanto-Forschung, die wirksame Schritte in der EsperantoEntwicklung identifizierte. Wie aber von Forster $(1982,56,59-61,81)$ beschrieben, behielt Zamenhof trotz eines impliziten Verzichts auf seine Sonderstellung als Autor seinen Einfluss in der EsperantoGemeinschaft. Auch später lassen sich in der Bewegung lenkende Personen oder Gremien identifizieren. 
mögliche Selbstkategorie. In der zweiten Interpretation wird dagegen das Betonen der instrumentellen Funktion der Sprache empfohlen. Dabei handelt es sich letztendlich um die Identitäts- oder Wertorientierung einer Plansprache/Welthilfssprache (Forster (1982), vgl. Abschnitt 2.2.4.2 in dieser Arbeit), die ich im Sinne meines Modells als Frage nach dem Typ der aufzubauenden Gruppe - Gruppe mit emotionaler Nähe oder aufgabenbezogene Gruppe - interpretiere.

Schließlich wird auf die Beziehungen zwischen der Autorengruppe und potenziellen Sprechern eingegangen, die bereits der Sprechergemeinschaft einer konkurrierenden Plansprache angehören. Dies ist ein neuer Typ beteiligter Gruppen. Die potenziellen Sprecher sollen für die (eigene) aktive Sprechergruppe gewonnen werden (,Kräfte vereinen“), was aber auf einer Übereinkunft aller Beteiligten beruhen soll. Dies interpretiere ich ebenfalls als einen Hinweis auf den nichtautoritären Führungsstil der Autoren.

Beim Thema Identität wird einerseits gefordert, dass eine WHS kulturell neutral sein soll, d. h. keine bestehende (ethnische) Identität bevorzugen soll. Andererseits sollen gleiche kulturelle Angebote wie bei ethnischen Sprachen (Fernsehen, Treffen, Kino, Radio) und Folklore geschaffen werden. Dies legt wiederholt die Interpretation nahe, dass der Aufbau einer Gruppe mit emotionaler Nähe um eine Welthilfssprache als Mittel der Wahl gesehen wird. Ferner wird empfohlen, dass eine WHS nicht als Ersatz für die Muttersprache behandelt werden soll. Die aufzubauende Identität soll also im Einklang mit der bestehenden sozialen Identität stehen und insbesondere keine Identitätsbedrohung hervorrufen. Dies soll vor allem durch die Verbreitung einer WHS als Hilfsmittel geschehen, was aber, im Widerspruch zur ersten Interpretation, den Aufbau einer aufgabenbezogenen Gruppe um die Welthilfssprache erfordert.

Beim Thema Instrumentalität werden zwei Möglichkeiten zu derer Förderung genannt: Organisation von Konferenzen, bei denen die WHS die Konferenzsprache ist sowie die Bereitstellung von Übersetzungen und Originalliteratur in der WHS. In erster Linie sollen dadurch praktische Möglichkeiten zum aktiven und passiven Gebrauch einer Sprache bereitgestellt werden, die auf vertrauten Möglichkeiten in natürlichen Sprachen beruhen. Die Literatur kann aber auch als Beispiel für Folklore gesehen werden und stellt somit einen Bezug zum Thema Identität dar.

Zum Thema Einstellungen beobachte ich, dass zum einen positive Einstellungen der Zielgruppe gegenüber einer Plansprache aber auch ihrer Weiterentwicklung gefördert werden sollen. Genannt werden speziell zum einen die 


\section{Untersuchung}

Stärkung der Loyalität und der Festlegung der Sprecher, zum anderen aber, und möglicherweise im Sinne einer Präzisierung, das Wecken und Erhalten von Enthusiasmus und Engagement der Sprecher. Hierdurch sehe ich vor allem die affektive und die konative Einstellungskomponente angesprochen. Zudem kann man versuchen, das Ansehen eines Projektes durch seine Umbenennung zu steigern.

Dem Einstellungsthema ordne ich zudem zwei weitere Überlegungen zu, bei denen es sich eigentlich um die Frage der Verhaltensänderung handelt. So können für bestimmte Zielgruppen finanzielle Begünstigungen beim Gebrauch einer Sprache oder beim Rückgriff auf die Gemeinschaftsinfrastruktur geschaffen werden. Hier handelt es sich also um eine Verhaltensänderung durch (äußere) Anreize. Eine Gegenposition vertritt allerdings die Empfehlung, auf den freiwilligen Gebrauch einer Plansprache zu setzen.

Empfehlungen für Arbeitsprozesse betreffen sowohl die Autorengruppe als auch die aktive Zielgruppe. Generell sollen Autoren die SL-Maßnahme mit einer kleineren Zielgruppe oder Domäne beginnen und sie allmählich auf weitere Sprecher ausweiten. Auf die Autoren beziehe ich ebenfalls die Empfehlung, die Arbeit gut zu strukturieren und eine entsprechende Organisation aufbauen. Schließlich sollen die Autoren die Verantwortung für die systemlinguistische Weiterentwicklung auf die Zielgruppe übertragen, was ich als Empfehlung einer gemischten Top-down-/Bottom-up-Ausrichtung mit Top-down-Beginn interpretiere.

Was das Bekanntmachen einer Plansprache betrifft, so wird generell beobachtet, dass die Autoren keine aktiven Schritte unternehmen müssen. Werden solche hingegen gemacht, so soll eine übergreifende Lobby aufgebaut und die öffentliche Präsenz gefördert werden. Zudem wird vorgeschlagen, eine WHS wie ein kommerzielles Produkt zu vermarkten.

Schließlich werden beim Thema Gebrauch zwei Positionen vertreten. So kann die Perfektionierung des Sprachsystems Vorrang vor dem eigentlichen Gebrauch haben oder aber andersherum. Entscheiden sich die Autoren für die zweite Möglichkeit, so sollen der kollektive Gebrauch gestärkt und die unmittelbare Einsatzfähigkeit der Plansprache gefördert werden.

KS Die Analyse ergibt, dass die facettenreichsten Themen zu Vorgehen im KS-Gebiet vor allem Gruppe, Persausion, Erlernbarkeit, Arbeitsprozess und Werkzeuge sind. Weniger elaboriert sind Einstellungen und Sprachsystem. Nur allgemeine Aussagen werden hingegen zu den Themen allgemeine auBersprachliche Faktoren, Wissenschaftlichkeit, Machtmittel/Sanktionen, Be- 
kanntmachen und Gebrauch gemacht. Keine Aussagen stelle ich zu Identität, Instrumentalität, Kosten, Sprachökologie und Einhalten fest.

Zum Thema Gruppe stelle ich fest, dass hauptsächlich auf die Gestaltung von Beziehungen zwischen der Autoren- und der Zielgruppe eingegangen wird. Auch hier ist die Unterscheidung zwischen einer Top-down- und einer Bottom-up-Ausrichtung zur weiteren Ergebnissystematisierung hilfreich. Diese beiden Pole sollen als Ausgangspunkte betrachtet werden und die vorgeschlagenen Vorgehen beziehen sich vor allem auf die Frage, wie man sich jeweils auf die Mitte hin, d. h. in Richtung eines gemischten Ansatzes bewegen kann.

Einerseits kann also die Entscheidung über den KS-Einsatz und entsprechende Werkzeuge top-down getroffen werden. Ist dies der Fall, so wird weiter gefordert, dass firmenspezifische, sowohl sprachliche als auch technische, Gepflogenheiten berücksichtigt werden. Hierzu werden mehrere spezifische Vorgehen vorgeschlagen, die ich nach dem aufsteigenden Grad der aktiven Beteiligung der Zielgruppe ordne. So können die Gepflogenheiten durch eine korpusbasierte Entwicklung und Verfeinerung der KS-Spezifikation erfolgen. Dabei kann diese Entwicklung alle Ebenen des Sprachsystems oder aber lediglich das Lexikon betreffen. Dies impliziert eine aktive Arbeit der Autoren und eine passive Beteiligung der Zielgruppe. Mehr aktive Beteiligung der Zielgruppe setzt die Empfehlung voraus, der Zielgruppe einen Einblick in alle (hauptsächlich technischen) Entwicklungen einer KS zu erlauben, selbst wenn sie selbst keinen aktiven Beitrag leisten soll. Eine aktive Rolle soll hingegen die Zielgruppe dann übernehmen, wenn ihr Feedback zu den von den Autoren vorgestellten Inhalten gefragt ist. Insbesondere wird auf die Bereitstellung von Feedbackmöglichkeiten im Internet eingegangen.

Wie bereits aus den Abschnitten zu Herausforderungen bekannt, wird auch hier beobachtet, dass die aktive Zielgruppe nicht homogen ist. Beginnt die KS-Entwicklung top-down, so sollen die Autoren viele verschiedene Subgruppen von Anfang an berücksichtigen, auf diese aber nach Möglichkeit wieder gruppenspezifisch eingehen. Speziell können unterschiedliche Kompetenzstufen der KS-Beherrschung eingeplant werden.

Fängt die KS-Konstruktion hingegen bottom-up an, so soll eine Kooperation mit der Meso- und Makroebene angestrebt werden.

Schließlich identifiziere ich in den Ergebnissen Aussagen zur Gestaltung der Beziehungen innerhalb der Autorengruppe, die ebenfalls heterogen sein kann. Demnach wird empfohlen, den Werkzeugentwicklern klare Vorgaben zu machen. 


\section{Untersuchung}

Beim Thema Persuasion liegt der Schwerpunkt auf Argumenten, die in verschiedenen Phasen der KS-Einführung durch die Autoren genutzt werden können. Die Argumente sind heterogen und bringen verschiedene Aspekte zur Sprache. Deshalb ordne ich sie mithilfe einer Kreuzklassifikation, die zum einen das Objekt des Argumentes, d. h. wen oder was das Argument inhaltlich anspricht, benutzt, und zum anderen das Thema des Argumentes, wobei ich auf die 18 Themen dieser Auswertung zurückgreife. Des Weiteren unterscheide ich, ob das Argument in der Vorbereitungsphase eingesetzt wird, um die betreffenden Personen von der KS-Einführung zu überzeugen, oder aber bestimmte Probleme der Umsetzungsphase behandelt.

In der Vorbereitungsphase können Argumente die aktive Zielgruppe, die passive Zielgruppe, höhere Hierarchieebenen der aktiven Zielgruppe sowie den Text an sich als Objekt haben. Für die aktive Zielgruppe betreffen die Argumente die leichte Erlernbarkeit einer KS, wobei der Kontrast einerseits zu einer Fremdsprache, andererseits aber zu einer formalen Sprache herausgestellt wird. Des Weiteren betreffen die Argumente die Arbeitsprozesse, die mit einer KS effizienter gestaltet werden können und den Einsatz weiterer sprachtechnologischer Anwendungen ermöglichen. Argumente für passive Zielgruppen betreffen zum einen die Leser der Texte, insbesondere die Kunden eines Unternehmens, deren Zufriedenheit durch den KS-Einsatz steigt. Ich ordne das Argument dem Thema Einstellungen zu. Darüber hinaus kann man für eine KS mit der Verbesserung der Übersetzungsprozesse (Thema: Arbeitsprozess) argumentieren. Was die höheren Hierarchieebenen der aktiven Zielgruppe betrifft, so beziehen sich die Argumente auf das Thema Kosten, wobei allgemeine wirtschaftliche Vorteile aber auch Sprachprobleme als quantitative Risikofaktoren genannt werden. Schließlich wird für das Objekt Text mit allgemeinen Qualitätsfaktoren oder Konsistenz argumentiert, was eventuell dem Thema Sprachsystem zuzuordnen ist bzw. eines allgemeineren linguistischen Themas bedürfte.

Die Argumente, die die Umsetzungsphase betreffen, sehe ich hauptsächlich auf die aktive Zielgruppe bezogen. Sie betreffen Themen wie Erlernbarkeit sowie Arbeitsprozesse, wobei entsprechend mit einem vertretbaren Schulungsaufwand, ggf. unter Einsatz eines CLC, und mit vertretbaren Produktivitätseinbußen in der Einführungsphase argumentiert werden kann.

Allgemein wird gefordert, dass die aktive Zielgruppe sich selbst von Vorteilen einer KS überzeugen soll. Was die Beschaffenheit der Argumente betrifft, so wird empfohlen, sowohl qualitative als auch quantitative Argumente zu nutzen. Des Weiteren sollen sie mit wissenschaftlich gesicherten Daten 
und realen Beispielen für Problemfälle aus der Welt der aktiven Zielgruppe untermauert sein. Bei der Auswahl der Beispiele soll zudem darauf geachtet werden, dass verschiedene Subgruppen ihre eigenen Beispiele benötigen können. Diese Aussagen lassen sich vor dem Hintergrund des Modells der Elaborationswahrscheinlichkeit (4.7.2.1) interpretieren: So werden vor allem die Instrumente der zentralen Route empfohlen: Betonung der persönlichen Relevanz des Themas durch Problemfallbeispiele sowie die Darbietung inhaltlicher Argumente. Die Wissenschaftlichkeit der Argumente ordne ich hingegen der Expertenheuristik, also der peripheren Route, zu. Beide Routen können eingesetzt werden, um die Selbstüberzeugung der Zielgruppe von der Einführung einer Kontrollierten Sprache zu unterstützen.

Beim Thema Erlernbarkeit spielen zunächst die KS-Regeln eine Rolle. Sie sollen zum einen einfach sein. Wichtig ist, dass die aktive Zielgruppe KSRegeln erlernt, bevor Werkzeuge (insb. CLC) eingesetzt werden, wobei sich die aktive Beherrschung in erster Linie auf die häufigsten Regeln beschränken kann.

Den eigentlichen Schwerpunkt beim Thema Erlernbarkeit bilden jedoch Überlegungen zur Gestaltung von KS-Schulungen. Grundsätzlich sollen Schulungen angeboten werden, wobei neben klassischen Präsenzschulungen auch Lernprogramme (Tutorials) entwickelt werden können. Zuallererst sollen Schulungen KS-Regeln, darüber hinaus aber weitere Inhalte vermitteln. Dazu gehören der neue Textverfassungsprozess, die Toolbedienung, aber auch die Oberziele der KS und ggf. der repetitive Schreibstil. Zudem soll die Schulung dazu genutzt werden, die KS-Benutzer zu motivieren oder gar zu begeistern, mit anderen Worten: Schulungen sollen positive Einstellungen gegenüber einer KS fördern. Die Autoren können hingegen das Benutzerfeedback zur Weiterentwicklung der KS nutzen. Aus organisatorischer Sicht wird schließlich der Einsatz kompetenter Schulungsleiter gefordert oder gar das vollständige Verzichten auf Schulungen dank CLC-Einsatz vorgeschlagen.

Die Empfehlungen zu Arbeitsprozessen beziehen sich vor allem auf die Autorengruppe. Zur aktiven Zielgruppe wird lediglich angemerkt, dass ihre bestehenden Arbeitsprozesse zum Zweck des KS-Einsatzes angepasst werden müssen.

Aus der Autorensicht beziehen sich die vorgeschlagenen Vorgehen einerseits auf die organisatorischen und andererseits auf die inhaltlichen Aspekte. Inhaltlich kann die Autorengruppe den Schwerpunkt auf die Bereitstellung und Einführung von KS-Software, KS-Spezifikation oder auf beides setzen. 


\section{Untersuchung}

Ein KS-Projekt kann also software-geleitet, spezifikationsgeleitet oder gemischt sein. Was die Arbeitsorganisation betrifft, so wird empfohlen, die KS (insb. die Regeln) in kleinen Schritten zu entwickeln. Speziell kann dies bedeuten, dass die KS zunächst in einer kleinen Gruppe eingeführt und getestet, und erst dann auf andere Gruppen ausgeweitet wird. Man kann dabei entweder eine streng systematische Vorgehensweise anstreben, oder aber eher pragmatisch vorgehen.

Zum Thema Werkzeuge wird der Einsatz von KS-spezifischen Tools allgemein empfohlen, wobei es je nach KS-Typ ein Controlled Language Checker, ein Predictive Editor, ein Reasoner oder der Conceptual-Authoring-Ansatz sein kann. In den Analyseergebnissen identifiziere ich ferner Überlegungen, die nicht KS-spezifisch sind, sondern für viele Computeranwendungen gelten. Dazu gehören Empfehlungen bezüglich der Rechenzeit, Entwicklungsverfahren, Benutzerfreundlichkeit, Bedienbarkeit, Funktionsadäquatheit sowie Integrierbarkeit in bestehende Softwareumgebungen und Arbeitsprozesse. Außer dem allgemeinen Vorschlag, Systemmeldungen in der KS anzubieten, stelle ich fest, dass sich weitere Hinweise speziell auf CLC beziehen und vorwiegend sein Korrekturverhalten betreffen. So kann unterschieden werden zwischen dem Anspruch an eine vollständige Autokorrektur und einem interaktiven Prüfmodus, bei dem ein CLC lediglich Korrekturvorschläge macht, die Entscheidungshoheit aber beim Anwender bleibt. Dabei soll die Anzahl der Fehlermeldungen überschaubar gehalten werden. In diesem Zusammenhang sollen hohe Präzisionswerte angestrebt werden, wobei der Recall-Wert durchaus niedrig bleiben kann. Weiterhin soll ein CLC modular aufgebaut sein, so dass verschiedene Ebenen des Sprachsystems einzeln geprüft werden können.

Beim Thema Einstellungen stelle ich fest, dass man grundsätzlich versuchen kann, eine KS ohne bestehende Akzeptanz in der aktiven Zielgruppe einzuführen. Die meisten Aussagen befassen sich jedoch damit, wie positive Einstellungen gegenüber einer KS gefördert werden können. Zum einen werden die Steigerung des sprachlichen Bewusstseins und die Vermittlung der Nützlichkeit einer KS für die eigene Arbeit genannt. Ich sehe diese als Einfluss auf die kognitive bzw. konative Einstellungskomponente, wodurch das Gefühl der eigenen Betroffenheit bzw. die persönliche Relevanz gesteigert werden sollen. Einstellungen der Zielgruppe können die Autoren also durch geschicktes Kommunizieren über die KS beeinflussen, vor allem durch die Betonung ihrer aufgabenbezogenen Relevanz. Darüber hinaus wird die Notwendigkeit einer Eingewöhnungsphase an eine KS erkannt. Ich deute dies als 
Hinweis auf die Nutzung des Mere-Exposure-Effekts, mit dem positive Einstellungen erreicht werden können.

TA Die Analyse ergibt, dass die facettenreichsten Themen zu Vorgehen in TA-Gebiet vor allem Gruppe, Persuasion und Arbeitsprozess sind. Weniger elaboriert sind Einstellungen, Machtmittel und Werkzeuge. Nur allgemeine Aussagen werden hingegen zu den Themen Identität, Instrumentalität, allgemeine außersprachliche Faktoren, Wissenschaftlichkeit, Einhalten, Bekanntmachen und Gebrauch gemacht. Keine Aussagen stelle ich zu Kosten, Sprachökologie, Erlernbarkeit und Sprachsystem allgemein fest.

Im Unterschied zu den bereits besprochenen Gebieten ergeben sich für Terminologiearbeit Überlegungen nicht nur zur Gestaltung der Intergruppenbeziehungen, sondern auch zur Gruppenstruktur, insbesondere der Autorengruppe.

Zum einen wird empfohlen, dass die Autorengruppe klein gehalten werden soll. Organisatorisch soll sie auf der Mesoebene (einer Organisation oder einer Gesellschaft) angesiedelt sein und Vertreter der aktiven Zielgruppe sowie nach Bedarf organisationsexterne Mitglieder umfassen. Eine spezielle Rolle in der Gruppe übernimmt dabei der Terminologiemanager. Für verschiedene Gruppenmitglieder werden zudem verschiedene Fähigkeiten gefordert, so sollen reguläre Mitglieder terminologierelevante Kompetenzen besitzen (Methoden der Terminologiearbeit, Terminologielehre aber auch Sachkompetenz). Dies bezieht sich auch auf die externen Mitglieder. Für Terminologiemanager wird hingegen speziell Führungskompetenz gefordert, die ich für relevant für die Übereinstimmung seiner offiziellen Rolle mit der Quasirolle halte. Die aufgabenbezogene Kompetenz sowie die Führungspersönlichkeit ordne ich folglich den sog. spezifischen Statusmerkmalen zu.

Zur Struktur der aktiven Gruppe wird lediglich angemerkt, dass Terminologiearbeit in allen relevanten Bereichen etabliert sein soll. Dies interpretiere ich wieder als Hinweis auf die Heterogenität der aktiven Zielgruppe.

Hinweise zur Gestaltung der Intergruppenbeziehungen betreffen in TA die Autoren- und die aktive Zielgruppe. Generell werden auch hier wieder die Möglichkeiten eines reinen Top-down-Ansatzes sowie der gemischte Ansatz mit Top-down-Beginn genannt. Ich beobachte zudem, dass eine reine Bottomup-TA oder der gemischte Ansatz mit Bottom-um-Beginn nicht explizit empfohlen werden. Was die Möglichkeiten der Bottom-up-Beteiligung betrifft, so stelle ich beim Thema Gruppe lediglich die Empfehlung zum korpusbasierten Terminologieaufbau fest. Weitere Überlegungen sind u.a. dem Thema Werk- 


\section{Untersuchung}

zeuge zugeordnet und umfassen Feedback-Möglichkeiten unter Einsatz von modernen Technologien.

Zum Thema Persuasion wird allgemein angemerkt, dass Überzeugungsarbeit notwendig ist. Argumente sollen generell die Vorteile der Terminologiearbeit für die Organisation darlegen. Dies erfolgt meistens durch das Aufzeigen, wie Terminologiearbeit zum Erreichen von Oberzielen beitragen kann. Als Oberziele werden vor allem die Kosten- und Qualitätsaspekte argumentativ genutzt. Für die Überzeugungsarbeit wird allgemein empfohlen, mit empirischen Daten zu arbeiten, die insbesondere Probleme mit einer uneinheitlichen Terminologie herausstellen. Im Kontext des Modells der Elaborationswahrscheinlichkeit geht es hier also um das Aufzeigen der eigenen Betroffenheit und der Relevanz von Terminologiearbeit für die aktive Zielgruppe.

Das Thema Arbeitsprozesse befasst sich vorwiegend mit den Aufgaben, die auf die Autorengruppe zukommen. Es wird darauf hingewiesen, dass Terminologiearbeit keine (rein) sprachwissenschaftliche Aufgabe ist und mehr als nur Katalogisierung der Terme umfasst. Folglich identifiziere ich neben der eigentlichen Terminologieausarbeitung auch Empfehlungen, die sich auf die organisatorischen Aspekte wie das Managementkonzept und BenchmarkEtablierung beziehen. Ferner stelle ich zwei unterschiedliche Positionen fest: Die eine fordert eine planvolle, systematische oder wissenschaftlich fundierte Vorgehensweise, die andere empfiehlt hingegen pragmatisches Vorgehen, das keine Perfektion anstrebt.

Das Managementkonzept hat eine hohe Priorität und soll z. B. vor der Softwareentwicklung Vorrang haben. Dazu gehört neben einer allgemeinen Workflowdefinition auch die konkrete Zuständigkeitsbestimmung. Die so definierten Arbeitsprozesse betreffen sowohl die Autorengruppe als auch die aktive Zielgruppe und sollen in die bestehenden möglichst leicht integrierbar sein; sie können aber auch eine Anpassung bestehender Prozesse erfordern. Darüber hinaus ist zu berücksichtigen, dass die inhaltliche Ausarbeitung der Terminologie früh im Produktlebenszyklus ansetzen muss. Trotz dieser vielfältigen Faktoren soll das Managementkonzept übersichtlich bleiben und über praktikable Zeitvorgaben verfügen.

Bei der eigentlichen Ausarbeitung der Terminologie soll Qualität vor Quantität stehen. Es wird allgemein auf eine gute Kommunikation in der Arbeitsgruppe als wichtigen Faktor hingewiesen. Speziell sollen Terminologiefestlegungen in der Arbeitsgruppe ausdiskutiert werden, bevor sie der aktiven Zielgruppe präsentiert werden. Alternativ kann man nichtfreigegebene Terminologie der aktiven Zielgruppe zur Verfügung stellen. 
Bei all diesen Aufgaben wird empfohlen, mit Terminologiearbeit klein anzufangen und den Umfang allmählich zu steigern. Dies betrifft sowohl den Umfang der Terminologie als auch die Überlegung, welche Subgruppen der aktiven Zielgruppe von Anfang an anvisiert werden. Es wird auch betont, dass die Vorgaben allen Beteiligten klar kommuniziert werden sollen.

Beim Thema Einstellungen wird allgemein aktive Akzeptanzförderung empfohlen. Dies soll durch die Sensibilisierung für Sprachprobleme sowie die Steigerung des Stellenwerts von Terminologiearbeit erreicht werden. Diese interpretiere ich als Maßnahmen zur Steigerung der persönlichen Relevanz von Terminologiearbeit. Darüber hinaus soll auch die Toolakzeptanz gefördert werden.

Zum Thema Machtmittel/Sanktionen wird einerseits beobachtet, dass die Autoren in der Lage sein müssen, Entscheidungen auch durchzusetzen. Damit hängt auch die Forderung zusammen, die Top-down-Unterstützung für Terminologiearbeit zu sichern. Als weitere Maßnahme wird vorgeschlagen, das Nichteinhalten der festgelegten Terminologie zu sanktionieren. Dabei wird nicht präzisiert, worin diese Sanktionierung bestehen sollte.

Schließlich wird auch für Terminologiearbeit der Einsatz von Werkzeugen empfohlen, da sie u.a. die Bottom-up-Beteiligung erleichtern. Vor der ToolEinführung soll ihre Eignung für das spezielle Szenario (z. B. ein Unternehmen) evaluiert werden. Dabei soll auf ihre Verfügbarkeit, die gegebenenfalls mit der Kostenfrage zusammenhängt, ihre (technische) Integrierbarkeit und Benutzerfreundlichkeit geachtet werden.

\subsubsection{Vorgehen: Gebiete vergleichend}

Parallel zu Abschnitt 5.2.2.3 steige ich in die vergleichende VorgehensAnalyse über den Vergleich von Themenelaborationstiefe in den einzelnen Gebieten ein.

Aufgrund der Einzelanalyse in Abschnitt 5.2.2.4 beobachte ich, dass insbesondere das Thema Gruppe in allen vier Gebieten stark elaboriert ist. Gleiches gilt für das Thema Arbeitsprozess, wobei es in PS geringfügig weniger stark elaboriert ist $\left(,+++^{\prime \prime}\right)$. Darüber hinaus hat das Thema Einstellungen übergreifend einen hohen Stellenwert.

Eine große Elaborationsstreuung beobachte ich für das Thema Identität. Es ist in Gebieten LPLP und PS sehr stark elaboriert, während es in TA nur schwach und in KS gar nicht vertreten ist. Dies ist nicht überraschend, da ich bereits im ersten Teil dieser Arbeit für die ersten beiden Gebiete eine stärkere identitätsbezogene Ausrichtung annahm. In den anderen beiden Gebieten 


\section{Untersuchung}

vermutete ich hingegen vorwiegend instrumentelle Ziele (s. 3.2.2.3). Diese Vermutung wird durch die Einzelanalyse nicht direkt bestätigt, da das Thema Instrumentalität in TA sehr schwach und in KS gar nicht vorhanden ist. Es ist aber in LPLP und PS, ähnlich wie Identität, recht stark elaboriert. Ein näherer Blick auf das Thema Persuasion, das in KS und TA sehr stark, in LPLP stark und in PS nur schwach elaboriert ist, zeigt aber, dass Argumente, mit denen Überzeugungsarbeit in KS und TA geleistet werden soll, vor allem instrumentelle Oberziele der Sprachlenkung betonen.

Darüber hinaus besteht eine Elaborationsdiskrepanz zwischen TA und KS einerseits und LPLP und PS andererseits beim Thema Werkzeuge: Es wird in den ersten beiden Gebieten ausführlich behandelt und ist in den letzten beiden nicht vorhanden. Dies beobachtete ich bereits bei Herausforderungsthemen. Schließlich stelle ich für das Thema Sprachökologie fest, dass es in PS nur schwach elaboriert und in den übrigen Gebieten gar nicht vorhanden ist. Dies ist insofern interessant, dass die Analyse der Herausforderungen eine Bedeutung dieses Themas in allen Gebieten zeigte. Ich halte also fest, dass die Sprachökologie durchaus als Herausforderung für Sprachlenkung gesehen wird, für die aber noch relativ wenige Handlungsmöglichkeiten postuliert werden.

Der Vergleich von Elaborationstiefe ermöglicht einen ersten Einblick in die Ergebnisse; die vergleichende Analyse zielt aber primär auf eine Übertragung und Verallgemeinerung von Einzelerkenntnissen. Ähnlich wie bei Herausforderungen prüfe ich, in welchen Fällen es plausibel ist, eine Aussage allen vier Gebieten, ggf. leicht modifiziert, zuzuschreiben. Nach der vergleichenden Analyse stelle ich fest, dass dies auch für Vorgehen in den meisten Fällen möglich ist. Die Ergebnisse dieser Systematisierung, Übertragung und Verallgemeinerung von Anhang E sind in Anhang F dargestellt. Auch hier sind viele der vorgeschlagenen Verallgemeinerungen leicht nachvollziehbar und ich beschränke mich im Folgenden lediglich auf die Beschreibung der Resultate, die aus meiner Sicht einer Erläuterung bedürfen.

Wie bereits erwähnt, ist das Thema Identität unterschiedlich stark elaboriert. Da es in KS komplett fehlt und in TA nur am Rande erwähnt wird, stellt sich die Frage, inwiefern die Aussagen aus den übrigen Gebieten verallgemeinerbar sind bzw. für andere Gebiete nützlich sein könnten. Ich nehme an, dass es sich bei Zielgruppen von Terminologiearbeit und Konstruktion von Kontrollierten Sprachen im Wesentlichen um aufgabenbezogene Gruppen handelt. In PS werden Vorgehen postuliert, die sich speziell auf die Identität von diesem Gruppentyp beziehen. Behält man die Unterscheidung nach 
Gruppentyp, so lassen sich entsprechende Aussagen kontextspezifisch verallgemeinern. Gleiches gilt auch für das Thema Instrumentalität.

Was das Thema Gruppe angeht, so beobachte ich, dass die Unterscheidung zwischen der Inter- und Intragruppenperspektive ein geeignetes Mittel zur weiteren Strukturierung der Aussagen darstellt. Hiernach identifiziere ich zunächst Aussagen zur Gruppenstruktur und stelle fest, dass LPLP und PS weitestgehend die aktive Zielgruppe betrachten, während TA den Schwerpunkt auf die Autorengruppe legt. Die Aussagen zu diesen beiden Gruppen lassen sich jedoch, leicht modifiziert und ggf. unter Berücksichtigung des Gruppentyps, weitestgehend verallgemeinern. Was die Intergruppenbeziehungen betrifft, so lasse ich an dieser Stelle offen, inwiefern sich die PS-Aussagen zu Beziehungen zwischen Gruppen von konkurrierenden Sprachprojekten (im Sinne der Sprachökologie also) verallgemeinern lassen. Der Versuch einer weiteren Strukturierung und Verallgemeinerung von Aussagen zu Beziehungen zwischen der Autoren- und der aktiven Zielgruppe führt hingegen zur Beobachtung, dass in allen vier Gebieten eine Unterscheidung zwischen einer reinen Top-down-SL, einer reinen Bottom-up-SL sowie gemischten Ansätzen (top-down anfangen und die Bottom-up-Beteiligung steigern und andersherum) vorhanden ist. Diese vier Ansätze postuliere ich folglich als vier (idealtypische) Formen der Sprachlenkung im Allgemeinen, die in der Gestaltung der Beziehungen zwischen der Autoren- und der aktiven Zielgruppe fußen. Die festgestellten Aussagen zu Vorgehen lassen sich somit diesen vier Formen der Sprachlenkung zuordnen, wobei die gemischten Ansätze danach abgestuft werden können, wie viel Beteiligung der jeweils anderen Gruppe angestrebt wird.

Beim Thema Einstellungen stelle ich fest, dass in allen Gebieten die Betonung der persönlichen Relevanz als Mittel zur Akzeptanzsteigerung gesehen wird, wobei bestimmte Empfehlungen ggf. nur für aufgabenbezogene Gruppen oder Gruppen mit emotionaler Nähe sinnvoll sind. Allgemein interpretiere ich dies als Empfehlung, Persuasion zur Einstellungsänderung einzusetzen. Des Weiteren stelle ich Empfehlungen zur Einstellungsänderung durch verschiedene Anreize (PS) sowie durch den Mere-Exposure-Effekt (KS) fest, die sich auf andere Gebiete ebenfalls übertragen lassen.

Zum Thema Persuasion beobachte ich, dass aktive Überzeugungsarbeit seitens der Autoren für notwendig gehalten wird. Einzelne Aussagen lassen sich dabei gut zum Modell der Elaborationswahrscheinlichkeit zusammensetzen. Ich postuliere dann den Umkehrschluss: Das Modell der Elaborationswahrscheinlichkeit kann eingesetzt werden, um geeignete Vorgehen für kon- 


\section{Untersuchung}

krete SL-Maßnahmen zu formulieren. Konkrete inhaltliche Argumente stelle ich vor allen in KS und TA fest. Sie betonen verschiedene instrumentelle Oberziele von SL und lassen sich als solche vor allem für aufgabenbezogene Zielgruppen verallgemeinern.

Beim Thema Erlernbarkeit sehe ich zur differenzierten Betrachtung der Vorgehen das Kriterium der Gruppengröße als notwendig, da die Idee von Schulungen, in denen die neue Form vermittelt wird, aus dem industriellen Kontext stammt. Die Aussagen zu Schulungen ordne ich somit kleineren, meist formellen bzw. aufgabenbezogenen Gruppen zu, während ich für größere Gruppen und soziale Kategorien Aussagen zu Lernmitteln zusammenstelle. Betrachtet man diese beiden Klassen näher, so stellt man fest, dass die Aussagen zu Großgruppen/sozialen Kategorien allgemeiner und auch in den Aussagen zu Kleingruppen enthalten sind.

Schließlich entferne ich beim Thema Arbeitsprozess weitestgehend alle Aussagen, die sich auf die Top-down- und Bottom-up-SL beziehen. Die Inhalte sind folglich danach strukturiert, ob eine systematische Vorgehensweise angestrebt werden soll oder nicht, wobei ich weiterhin zwischen organisatorischen und inhaltlichen Aspekten unterscheide.

\subsection{Hauptergebnis: Zuordnungen Herausforderung-Vorgehen}

Nach Vorüberlegungen zu gebietsspezifischen Herausforderungen und Vorgehen sowie Verallgemeinerungsmöglichkeiten wende ich mich in diesem Abschnitt nun der eigentlichen Forschungsfrage zu, welche Handlungsmöglichkeiten zur Umsetzung der Sprachlenkung der Autorengruppe zur Verfügung stehen, wobei ich sie in Verbindung mit den festgestellten Herausforderung setze. Hierfür analysiere ich die in den Zitaten postulierten Zuordnungen von den Vorgehen zu bestimmten Herausforderungen. Diese Analyse umfasst mehrere Schritte und basiert sowohl auf Daten aus der Datenbank als auch auf Ergebnissen in 5.2.2.3 und 5.2.2.5. Aus Übersichtlichkeitsgründen entscheide ich mich, im Hauptteil dieser Arbeit lediglich das Hauptergebnis zu präsentieren und erläutere in Abschnitt 5.3.1 mein Vorgehen exemplarisch am Thema Sprachökologie. Die Zwischenschritte bei den übrigen Themen sind in Anhang $\mathrm{H}$ dokumentiert. 


\subsubsection{Exemplarische Erläuterung der Analysemethode}

Wie in 5.1 erläutert, untersuchte ich Zitate u.a. darauf hin, ob darin eine ÄuBerung gemacht wird, welche Probleme bei Sprachlenkung mit welchen Vorgehen gelöst werden können. Diese Zuordnung ist nach dem ersten Abstraktionsschritt, d. h. auf der Ebene von Aussagen, in der Datenbank in den Tabellen *.zuord_ana_hv festgehalten. Für jedes der achtzehn Themen lässt sich diese Aussagen-Zuordnung z. B. gebietsweise ausgeben; Tabelle 28 zeigt die komplette Zuordnungsliste für das Thema Sprachökologie in PS.

Um die Analysekomplexität zu reduzieren, betrachte ich diese Zuordnungen auf der nächsthöheren Abstraktionsebene, der der Kategorien, und generiere hierfür eine entsprechende Sicht, s. Tabelle 29.

Diese Sicht umfasst alle Gebiete, wobei Zuordnungsduplikate entfernt sind. Ferner gebe ich für Herausforderungen und Vorgehen getrennt aus, welche Kategorien noch nicht zugeordnet sind, s. Tabelle $30 .{ }^{135}$ In diesen Tabellen erscheinen nur Kategorien, die über alle Themen hinweg nicht zugeordnet sind. Mit anderen Worten, wenn eine Kategorie, die zum Thema Sprachökologie gehört, einer Kategorie zum Thema beispielsweise Persuasion zugeordnet ist, dann erscheint sie in den Tabellen nicht. Ich bewerte dabei nicht, wie viele Zuordnungen für eine Kategorie bestehen, sondern lediglich die Tatsache, ob (mindestens) eine Zuordnung festgehalten ist oder nicht.

Diese drei Tabellen - die in der Literatur behaupteten KategorienZuordnungen sowie die isolierten Kategorien von Herausforderungen und Vorgehen - stellen für jedes Thema den Ausgangspunkt meiner interpretativen Analyse dar.

Im nächsten Schritt schlage ich für die isolierten Kategorien eine Zuordnung vor, wobei ich vorwiegend auf existierende Kategorien zugreife, in einzelnen Fällen aber neue Kategorien vorschlage. Ich strebe dabei einen Zuordnungsvorschlag für alle isolierten Kategorien an. Ich bevorzuge hierbei zwar wie in der Datenbank eine Zuordnung von den Vorgehen zu den Herausforderungen s. Anhang H, lasse aber auch in Vorbereitung auf den nächsten Analyseschritt bereits eine Verschachtelung von Herausforderungen bzw. von Vorgehen wie in Tabelle $31 \mathrm{zu}$. Diese Zuordnungsvorschläge sind andererseits nicht erschöpfend und ich ziehe es vor, eine Zuordnung innerhalb des betreffenden Themas zu finden. Beide Zuordnungstabellen, die aus der Datenbank generierte und die vorgeschlagene, kann man als Gesamtzuordnungstabelle betrachten.

135 Beim Thema Sprachökologie ergibt sich, dass es keine nichtzugeordneten Vorgehen-Kategorien gibt. 


\section{Untersuchung}

\begin{tabular}{|c|c|c|c|}
\hline ana_h_id & aussage_h & ana_v_id & aussage_v \\
\hline 22 & $\begin{array}{l}\text { Wie setzt man eine WHS gegen eine } \\
\text { ethnische Sprache durch? }\end{array}$ & 25 & $\begin{array}{l}\text { Da die Möglichkeit eines Eingriffs } \\
\text { in eine WHS ausgeprägter ist als bei } \\
\text { einer ethnischen Sprache, soll das } \\
\text { Sprachsystem einer WHS vollkom- } \\
\text { mener sein. }\end{array}$ \\
\hline 65 & $\begin{array}{l}\text { Konkurrenz verschiedener WHS- } \\
\text { Projekte als Verbreitungsbarriere }\end{array}$ & 64 & $\begin{array}{l}\text { Der Streit zw. Anhängern verschiede- } \\
\text { ner WHS führt zu negativem öffentli- } \\
\text { chem Bild der WHS-Bewegungen }\end{array}$ \\
\hline 66 & $\begin{array}{l}\text { Konkurrenz verschiedener WHS- } \\
\text { Projekte als Verbreitungsbarriere }\end{array}$ & 65 & $\begin{array}{l}\text { Die verschiedenen WHS sollen ver- } \\
\text { eint werden, damit die Unentschlosse- } \\
\text { nen gewonnen werden. }\end{array}$ \\
\hline 71 & $\begin{array}{l}\text { Konkurrenz verschiedener WHS- } \\
\text { Projekte als Verbreitungsbarriere }\end{array}$ & 69 & $\begin{array}{l}\text { Der Streit zw. Anhängern verschiede- } \\
\text { ner WHS führt zu negativem öffentli- } \\
\text { chem Bild der WHS-Bewegungen }\end{array}$ \\
\hline 74 & $\begin{array}{l}\text { Wie gewinnt man für das eigene Pro- } \\
\text { jekt Sprecher, wenn man auf die glei- } \\
\text { che Personengruppe wie andere Pro- } \\
\text { jekte (Esperanto) zielt }\end{array}$ & 72 & $\begin{array}{l}\text { Zamenhof war bei der Verbreitung des } \\
\text { Esperanto geduldig. }\end{array}$ \\
\hline 74 & $\begin{array}{l}\text { Wie gewinnt man für das eigene Pro- } \\
\text { jekt Sprecher, wenn man auf die glei- } \\
\text { che Personengruppe wie andere Pro- } \\
\text { jekte (Esperanto) zielt }\end{array}$ & 188 & $\begin{array}{l}\text { Ein Sprachentwurf soll auf soziale } \\
\text { Grundlage gestellt werden (Aufbau } \\
\text { einer Sprechergemeinschaft). }\end{array}$ \\
\hline 79 & $\begin{array}{l}\text { Wie/wo positioniert man Esperanto, } \\
\text { wenn Englisch bereits die wichtigste } \\
\text { Rolle spielt? }\end{array}$ & 78 & $\begin{array}{l}\text { Esperanto soll nicht versuchen, Eng- } \\
\text { lisch in seiner Rolle herauszufordern } \\
\text { oder zu ersetzen. }\end{array}$ \\
\hline 91 & $\begin{array}{l}\text { Bedeutung einer WHS weltweit auf- } \\
\text { bauen }\end{array}$ & 88 & $\begin{array}{l}\text { Die Bedeutung einer Sprache hängt } \\
\text { mit der Anzahl der Domänen, die sie } \\
\text { abdeckt, zusammen. }\end{array}$ \\
\hline 119 & $\begin{array}{l}\text { Wie etabliert man die eigene Sprache, } \\
\text { wenn andere Projekte bereits etabliert } \\
\text { sind? }\end{array}$ & 113 & $\begin{array}{l}\text { Positionierung einer WHS (Interlin- } \\
\text { gua) als rezeptives Mittel, das unmit- } \\
\text { telbar verständlich ist. }\end{array}$ \\
\hline 119 & $\begin{array}{l}\text { Wie etabliert man die eigene Sprache, } \\
\text { wenn andere Projekte bereits etabliert } \\
\text { sind? }\end{array}$ & 201 & $\begin{array}{l}\text { Ablehnung der ideologischen oder } \\
\text { emotionalen Seiten einer WHS und } \\
\text { ihre Positionierung in der Wissen- } \\
\text { schaft (Interlingua). }\end{array}$ \\
\hline 158 & $\begin{array}{l}\text { Eine WHS soll sich gegen andere } \\
\text { WHS behaupten. }\end{array}$ & 2 & Sprachsystem verbessern. \\
\hline 158 & $\begin{array}{l}\text { Eine WHS soll sich gegen andere } \\
\text { WHS behaupten. }\end{array}$ & 159 & $\begin{array}{l}\text { Stabilität des Sprachsystems soll gesi- } \\
\text { chert werden. }\end{array}$ \\
\hline 182 & $\begin{array}{l}\text { Konkurrenz zW. verschiedenen WHS- } \\
\text { Projekten als Verbreitungshindernis }\end{array}$ & 63 & $\begin{array}{l}\text { Der Streit zw. Anhängern verschiede- } \\
\text { ner WHS führt zu negativem öffentli- } \\
\text { chem Bild der WHS-Bewegungen }\end{array}$ \\
\hline
\end{tabular}

Tabelle 28.: Beispiel: Alle zugeordneten Aussagen im Gebiet PS; Thema: Sprachökologie 


\subsection{Hauptergebnis: Zuordnungen Herausforderung-Vorgehen}

\begin{tabular}{|c|c|c|c|c|}
\hline thema_h_id & kat_h & thema_v_id & kat_v & Gebiet \\
\hline 7 & $\begin{array}{l}\text { Die üblichen Machtinstrumente } \\
\text { einer Sprache fehlen }\end{array}$ & 18 & $\begin{array}{l}\text { Möglichst viele Domänen abde- } \\
\text { cken. }\end{array}$ & PS \\
\hline 7 & $\begin{array}{l}\text { Sprachökologie: andere Planspra- } \\
\text { chen }\end{array}$ & 4 & $\begin{array}{l}\text { Kräfte verschiedener } \text { WHS- } \\
\text { Bewegungen vereinen. }\end{array}$ & PS \\
\hline 7 & $\begin{array}{l}\text { Sprachökologie: andere Planspra- } \\
\text { chen }\end{array}$ & 4 & $\begin{array}{l}\text { Nichtautoritärer Führungsstil des } \\
\text { Autors (Kompromissbereitschaft, } \\
\text { diplomatisches Geschick). }\end{array}$ & PS \\
\hline 7 & $\begin{array}{l}\text { Sprachökologie: andere Planspra- } \\
\text { chen }\end{array}$ & 7 & $\begin{array}{l}\text { Andere Sprachprojekte aktiv an- } \\
\text { greifen und bekämpfen. }\end{array}$ & PS \\
\hline 7 & $\begin{array}{l}\text { Sprachökologie: andere Planspra- } \\
\text { chen }\end{array}$ & 9 & $\begin{array}{l}\text { Instrumentelle Funktion und prak- } \\
\text { tische Anwendung der Sprache } \\
\text { sachlich betonen. }\end{array}$ & PS \\
\hline 7 & $\begin{array}{l}\text { Sprachökologie: andere Planspra- } \\
\text { chen }\end{array}$ & 10 & $\begin{array}{l}\text { Sprachliches Material am Vertrau- } \\
\text { ten orientieren. }\end{array}$ & PS \\
\hline 7 & $\begin{array}{l}\text { Sprachökologie: andere Planspra- } \\
\text { chen }\end{array}$ & 11 & $\begin{array}{l}\text { Verantwortung für systemlinguisti- } \\
\text { sche Weiterentwicklung auf Spre- } \\
\text { cher übertragen }\end{array}$ & PS \\
\hline 7 & $\begin{array}{l}\text { Sprachökologie: andere Planspra- } \\
\text { chen }\end{array}$ & 16 & $\begin{array}{l}\text { Sprachsystem normativ perfektio- } \\
\text { nieren. }\end{array}$ & PS \\
\hline 7 & $\begin{array}{l}\text { Sprachökologie: andere Planspra- } \\
\text { chen }\end{array}$ & 18 & Stabilität vor Perfektionierung. & PS \\
\hline 7 & $\begin{array}{l}\text { Sprachökologie: andere Sprachen } \\
\text { (KS/natürlich) durchsetzungsfä- } \\
\text { hig }\end{array}$ & 11 & Pragmatisch vorgehen. & KS \\
\hline 7 & $\begin{array}{l}\text { Sprachökologie: andere Sprachen } \\
\text { durchsetzungsfähig }\end{array}$ & 12 & $\begin{array}{l}\text { Offizielle Sprachpolitik implizit } \\
\text { betreiben. }\end{array}$ & LPLP \\
\hline 7 & $\begin{array}{l}\text { Sprachökologie: ethnische Spra- } \\
\text { chen }\end{array}$ & 1 & $\begin{array}{l}\text { WHS als Hilfsmittel nicht als Er- } \\
\text { satz. }\end{array}$ & PS \\
\hline 7 & $\begin{array}{l}\text { Sprachökologie: ethnische Spra- } \\
\text { chen }\end{array}$ & 16 & $\begin{array}{l}\text { Sprachsystem normativ perfektio- } \\
\text { nieren. }\end{array}$ & PS \\
\hline
\end{tabular}

Tabelle 29.: Beispiel: Alle zugeordneten Kategorien, alle Gebiete; Thema: Sprachökologie

\begin{tabular}{|c|l|c|}
\hline thema_h_id & kat_h & Gebiet \\
\hline \hline 7 & Sprachökologie: unterschiedliches Prestige von Sprachen & LPLP \\
\hline 7 & Sprachökologie: Spracheinstellungen & LPLP \\
\hline 7 & $\begin{array}{l}\text { Sprachökologie: unkontrollierte Formen durchsetzungsfä- } \\
\text { hig }\end{array}$ & TA \\
\hline
\end{tabular}

Tabelle 30.: Beispiel: Isolierte (nicht zugeordnete) HerausforderungsKategorien, alle Gebiete; Thema: Sprachökologie 


\begin{tabular}{|c|l|c|l|}
\hline thema_h_id & kat_h & thema_h_id & kat_h \\
\hline \hline 7 & $\begin{array}{l}\text { Sprachökologie: unkontrol- } \\
\text { lierte Formen durchsetzungs- } \\
\text { fähig }\end{array}$ & 7 & $\begin{array}{l}\text { Sprachökologie: Sprachein- } \\
\text { stellungen }\end{array}$ \\
\hline 7 & $\begin{array}{l}\text { Sprachökologie: Sprachein- } \\
\text { stellungen }\end{array}$ & 7 & $\begin{array}{l}\text { Sprachökologie: unterschied- } \\
\text { liches Prestige von Sprachen }\end{array}$ \\
\hline
\end{tabular}

Tabelle 31.: Beispiel: Eigener Zuordnungsvorschlag für isolierte Kategorien, Thema: Sprachökologie; hier: Verschachtelung von Herausforderungs-Kategorien

Die Zuordnungen in den Gesamtzuordnungstabellen sind binär. Es liegt aber nahe, sie multidimensional betrachten und in einer komplexeren Struktur mit mehrfachen Abhängigkeiten darstellen zu wollen. Hierzu erfolgt eine Verschränkung der Gesamtzuordnungstabelle mit den Ergebnissen zunächst in 5.2.2.3 und dann in 5.2.2.5. Das Zwischenergebnis ist in einer reinen Textform festgehalten, wie in Tabelle 32, auf die ich in dieser Arbeit nicht weiter eingehe.

Das Hauptergebnis stelle ich grafisch dar, für das Thema Sprachökologie s. Abbildung 21. Es handelt sich dabei um eine Art Ablaufdiagramm mit fett hervorgehobenen Herausforderungen sowie Vorgehen in normaler Schriftstärke. Anfangskategorien befinden sich links (oben), beim Thema Sprachökologie ist es also Sprachökologie: andere Formen/Sprachen, und aufeinander folgende Kategorien sind mit Pfeilen verbunden. Handelt es sich bei den untergeordneten Kategorien um zusammenhängende Optionen, so sind sie auf einer Ebene angeordnet. Zusammenhängend bedeutet in diesem Fall nicht, dass es sich um sich gegenseitig ausschließende Alternativen handeln muss. Die Optionen können sich ausschließen, ergänzen oder aber nur in einem Spezialfall zutreffend sein. In manchen Fällen sind die Pfeile mit entsprechenden Einschränkungskriterien beschriftet, auf die ich in dieser Arbeit immer wieder zurückgreife, wie Gruppentyp oder Gruppengröße. Eine vollständige Ausarbeitung und Darstellung dieser Optionsbeziehungen untereinander oder eine Plausibilitätsprüfung von Optionskombinationen kann im Rahmen dieser Arbeit nicht geleistet werden. Die Diagramme sind vielmehr als deskriptive Sicht auf die Präskriptionsoptionen zu verstehen, die in der Forschung zu finden sind.

Jedes Diagramm bildet Kategorienabfolgen für ein bestimmtes Thema ab. Themen sind aber auch in vielfältiger Weise miteinander verbunden. Diese 


\begin{tabular}{|l|}
\hline Sprachökologie \\
\hline \hline Sprachökologie: andere Plansprachen (allgemein) -> \{Ver- \\
antwortung für systemlinguistische Weiterentwicklung auf \\
Sprecher übertragen. | Nichtautoritärer Führungsstil des Au- \\
tors (Kompromissbereitschaft, diplomatisches Geschick).\} \\
\hline Sprachökologie: andere Plansprachen -> Sprachökologie: \\
Spracheinstellungen -> Sprachökologie: unterschiedliches \\
Prestige von Sprachen -> \{Möglichst viele Domänen ab- \\
decken. | \{Instrumentelle Funktion und praktische Anwen- \\
dung der Sprache sachlich betonen. -> WHS als Hilfsmittel \\
nicht als Ersatz.\} | Sprachsystem normativ perfektionieren. \\
| Sprachliches Material am Vertrauten orientieren.\} \\
\hline Sprachökologie: Formen -> Sprachökologie: ungelenkte \\
Formen durchsetzungsfähig -> \{Andere Sprachprojekte \\
[und Sprachformen] aktiv angreifen und bekämpfen. | Kräf- \\
te verschiedener WHS-Bewegungen vereinen. | Pragma- \\
tisch vorgehen. | \{Offizielle Sprachpolitik implizit betrei- \\
ben. -> Mere-Exposure-Effekt nutzen. $\mid$ Sprachsystem nor- \\
mativ perfektionieren. | Stabilität vor Perfektionierung\} \\
\hline
\end{tabular}

Tabelle 32.: Beispiel: Zwischenergebnis: strukturierte Zuordnungen als Text

Verbindungen sind in den einzelnen Diagrammen als Verweise dargestellt (weiter mit ...). Ein themenübergreifendes Gesamtdiagramm ist theoretisch möglich, müsste aber mithilfe geeigneter Tools umgesetzt werden, um übersichtlich und benutzerfreundlich zu sein.

Die aufgestellten Ablaufdiagramme liefern für alle Themen einen multidimensionalen Überblick über die Forschungslage zu Sprachlenkungsproblemen und Lösungsansätzen. Die grafische Form ermöglicht dabei einen leichteren Zugang zu den Inhalten für den Leser. Diese leichtere Zugänglichkeit erfolgt in diesem Fall jedoch auf Kosten einer direkten Datenbankanbindung, da die Diagramme nicht aus der Datenbank generiert wurden und viele manuelle Schritte erforderten: Sie sind das Resultat einer interpretativen Auswertung der Datenbankinhalte, überdies erstellte ich die Grafiken von Hand. Darüber hinaus formulierte ich einzelne Kategorien teilweise um, um allgemeine Aussagen zu treffen (beispielsweise aus WHS als Hilfsmittel nicht als Ersatz. wurde Neue Sprachform als Hilfsmittel nicht als Ersatz., s. Abbildung 21). So ist in den Diagrammen nicht gekennzeichnet, ob eine Kategorie wörtlich aus der Datenbank stammt, paraphrasiert oder von mir neu vorgeschlagen wurde. Diese Datenbankentkoppelung kann für den Leser nachteilig sein, wenn be- 


\section{Untersuchung}

stimmte Kategorien/Inhalte in den Diagrammen als zu allgemein erscheinen und eine Vertiefung und weiterführende Recherche erwünscht ist.

In folgenden Abschnitten gehe ich auf einzelne Ablaufdiagramme ein. Es erfolgt dabei keine ausführliche Beschreibung der Inhalte, sondern ich lenke den Blick selektiv auf Aspekte, die aus meiner Sicht als wichtig erscheinen.

\subsubsection{Identität}

In der Datenbank finden sich lediglich sechs Zuordnungen zum Thema Identität. Identität-Herausforderungen werden entsprechend thematisch vor allem mit Vorgehen aus Identität und Erlernbarkeit aber auch Machtmittel/Sanktionen sowie Einstellungen verknüpft. Den größten Teil des Diagramms machen eigene Zuordnungsvorschläge aus, die sich vor allem auf Identität und Einstellungen beziehen.

Die Ausgangsprämisse stellt das Salientmachen von sozialer Identität durch Sprachlenkung dar (links oben). Die wichtigsten Herausforderungen sind dabei verschiedene Formen von Identitätsbedrohung, einschließlich der Reaktanz, bei deren Lösung der Stärkung der Bottom-up-Beteiligung eine wichtige Rolle zukommt. Eine andere Möglichkeit bietet das Ausblenden der identitätsstiftenden und das Betonen der instrumentellen Funktion der Sprache, was sich darin ausdrückt, dass die neue Form nicht als Ersatz für die bis jetzt gebrauchten Formen, sondern als parallel existierendes Hilfsmittel aufgefasst werden soll.

Eine weitere wichtige Herausforderung, die ich an dieser Stelle herausgreife, ist die bereits besprochene Beobachtung, dass Sprache für Identität nicht immer konstitutiv ist und somit die Sprachlenkung nicht immer als Mittel zur Identitätsbewahrung erfolgreich eingesetzt werden kann (Abschnitt 4.8). Dieser Herausforderung werden in der Datenbank keine Vorgehen direkt zugeordnet. Es liegt aber nahe, dass die SL-Autoren versuchen können, diese Verbindung zwischen Sprache und Identität und dadurch eine Legitimation für die SL-Maßnahme herzustellen. Mit anderen Worten, die Autoren können versuchen, die Zusammengehörigkeit (also die Identität) der Zielgruppe auf sprachlicher Grundlage aufzubauen oder zu stärken. Allgemein kann man hierzu wieder die Bottom-up-Beteiligung an SL einsetzen, was speziell unter dem Aspekt der Korpusplanung das Orientieren des Sprachmaterials am Vertrauten bedeuten könnte. Speziellere Vorgehen hängen vom Gruppentyp $\mathrm{ab}$, vor allem unter dem Aspekt der Aufgabenbezogenheit vs. der emotionalen Nähe. Es erscheint als wichtig, dass bei der Gestaltung der Sprachlenkung der 


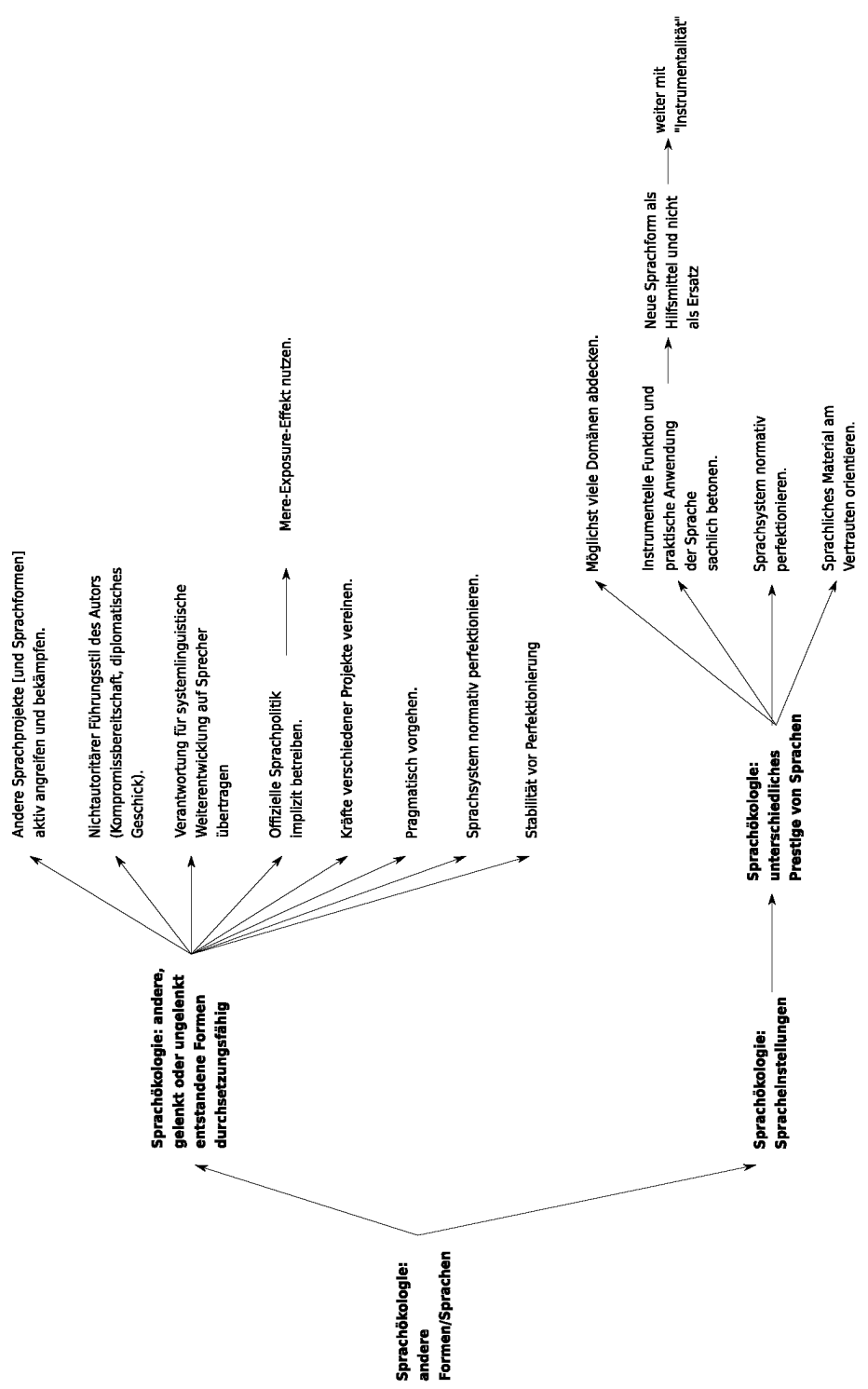

Abbildung 21.: Sprachökologie 


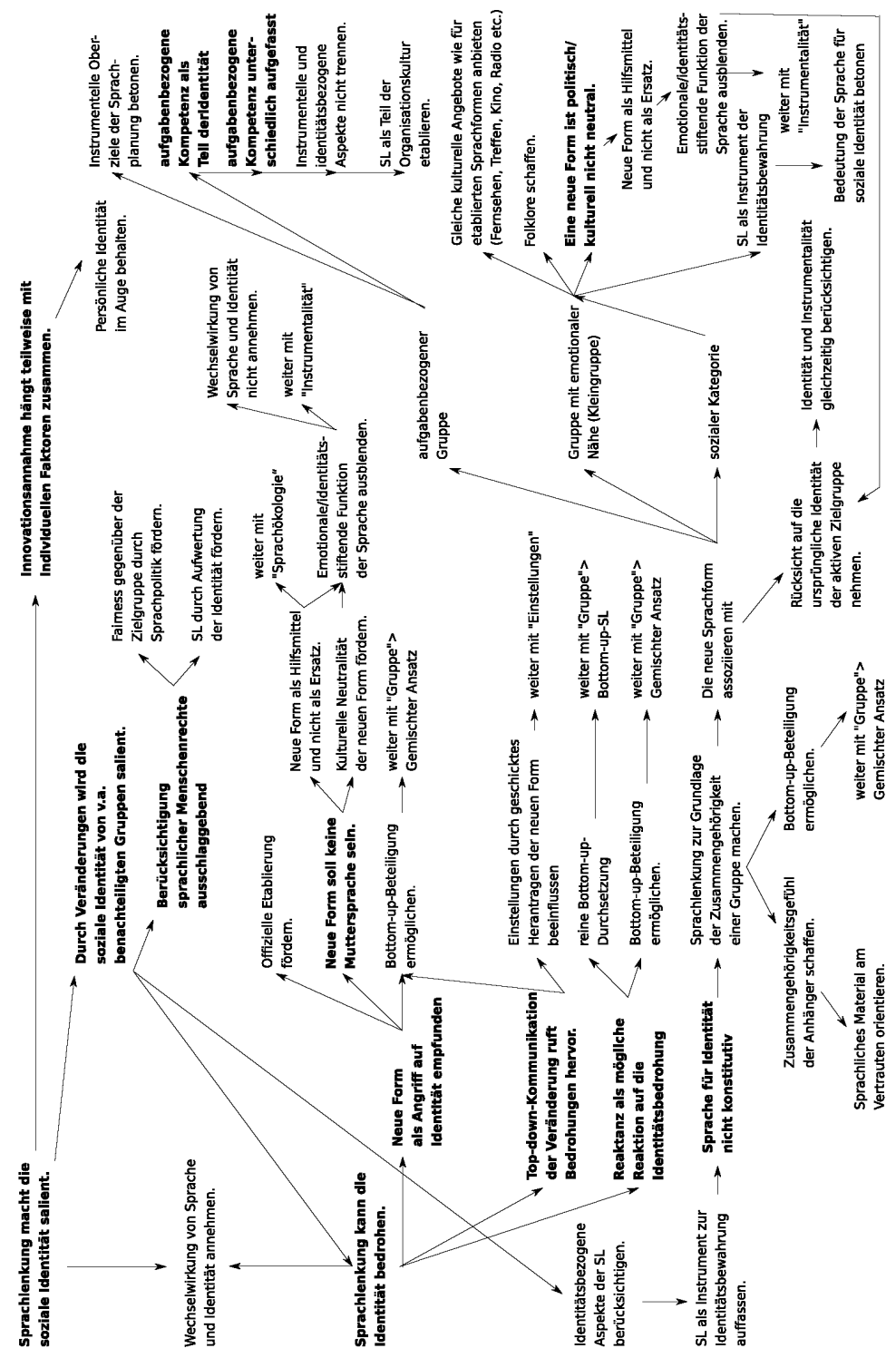

Abbildung 22.: Identität 
Aspekt des Gruppentyps beachtet wird, so dass beispielsweise bei einer aufgabenbezogenen Gruppe das Zusammengehörigkeitsgefühl nicht vornehmlich über emotionale/identitätsbezogene Aspekte der neuen Form hergestellt wird. Der ursprüngliche Gruppentyp soll also bei Sprachlenkung bewahrt werden.

Abschließend halte ich fest, dass SL nicht nur die soziale Identität, sondern auch die persönliche Identität salient macht (oben Mitte). Bei der Annahme der neuen Form spielen also auch individuelle Faktoren eine Rolle und die entsprechenden Mechanismen können nicht aus der kollektiven Perspektive der Sozialpsychologie beschrieben werden. Möglicherweise kann dieser individuelle Aspekt der Sprachlenkung mit Handlungs- und Motivationspsychologie und Erkenntnissen aus der Sprachlern- und -lehrforschung besser herausgearbeitet werden.

\subsubsection{Instrumentalität}

Bei diesem Thema gibt es in der Datenbank lediglich eine Zuordnung. Die meisten Zuordnungen im Diagramm sind also Eigenvorschläge, wobei Instrumentalität hauptsächlich mit anderen nicht zugeordneten Instrumentalitätskategorien und vereinzelt auch mit Identität, Wissenschaftlichkeit und Persuasion verknüpft wird.

Ich stelle fest, dass in der Datenbank zwei Auffassungen von Instrumentalität miteinander vermischt sind. In erster Linie kann Sprachlenkung als Instrument zum Erreichen von Oberzielen verstanden werden. Diese Auffassung stellt den Ausgangspunkt des Diagramms dar (links). Die erste Unterkategorie (oben) geht auf die übergreifende Herausforderung bei dieser Auffassung dar, d. h. die ungeklärte Frage, inwiefern mit SL Oberziele tatsächlich erreicht werden können. Hierzu gehören speziellere Aspekte wie der der Messbarkeit oder der Verflechtung von instrumentellen und identitäsbezogenen Oberzielen.

Weitere Unterkategorien repräsentieren die zweite Auffassung von Instrumentalität, nämlich instrumentelle Oberziele der Sprachlenkung, im Gegensatz zu identitätsbezogen, die beim vorangegangenen Thema (Identität) besprochen sind. Die Betonung von diesen instrumentellen Oberzielen, insbesondere von wirtschaftlichen Aspekten, ist vor allem bei aufgabenbezogenen Gruppen von Bedeutung. Zudem sollen Möglichkeiten zum Sprachgebrauch bereitgestellt werden, wobei sie sich nur auf bestimmte Domänen beziehen können. 


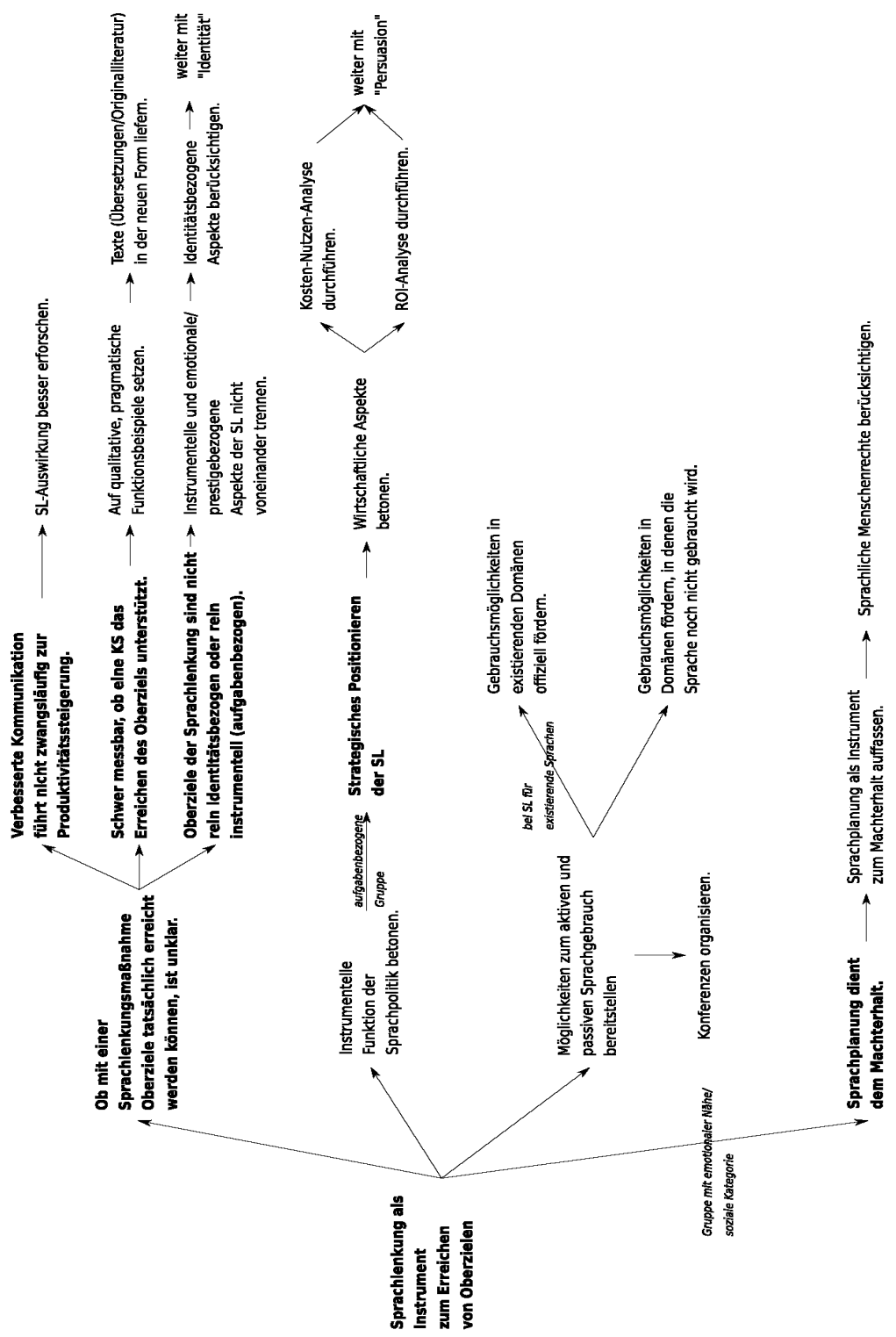

Abbildung 23.: Instrumentalität 


\subsubsection{Allgemeine außersprachliche Faktoren}

Die Herausforderungen zu diesem Thema sind vor allem mit Vorgehen aus Gruppe und vereinzelt auch aus Einstellungen, Persuasion, Arbeitsprozess und Bekanntmachen verknüpft. Die Eigenvorschläge betreffen meistens außersprachliche Faktoren.

Bei diesem Thema finden sich hauptsächlich Herausforderungen, denen, in den meisten Fällen, nur recht allgemeine Vorgehen zugeordnet sind. Demnach gehören zu den außersprachlichen Faktoren sowohl die generelle gesellschaftliche Situation wie auch spezielle situative Umstände. ${ }^{136}$ Da das Zusammenspiel von diesen (Erfolgs-)Faktoren komplex ist, wird das Aufstellen von allgemeinen SL-Prinzipien als impraktikabel gesehen, so dass man bei jeder konkreten Maßnahme pragmatisch vorgehen, gleichzeitig aber den konkreten außersprachlichen Kontext berücksichtigen soll.

Unter den Allgemeinen außersprachlichen Faktoren erscheinen wieder Oberziele von Sprachlenkung, die entweder instrumentell oder identitätsbezogen sein können. Dieser Aspekt wurde bereits beim Thema Instrumentalität angerissen. Die Überlappung wird noch deutlicher an der Herausforderung, wie gemessen werden kann, inwiefern SL das Erreichen von Oberzielen unterstützt (unten Mitte). Diese Herausforderung wird ebenfalls beim Thema Instrumentalität behandelt, wobei auf ein anderes Vorgehen gesetzt wird. Diese Überlappung zeigt, dass ich den Aspekt von SL-Oberzielen in der Datenbank nicht konsequent behandelt habe. Eine Lösung wäre das Verschieben aller Oberzielaspekte ins Thema Allgemeine außersprachliche Faktoren. Dies würde bedeuten, dass beim Thema Instrumentalität vor allem die zweite Auffassung von Instrumentalität, also in Abgrenzung zur Identität, behandelt werden sollte, während das Thema Allgemeine außersprachliche Faktoren die erste Auffassung von Instrumentalität im Sinne von Mittel zum Erreichen von Oberzielen (instrumentellen oder identitätsbezogenen gleichermaßen) übernehmen würde.

\subsubsection{Gruppe}

Das Thema Gruppe ist komplex und aus Übersichtlichkeitsgründen entschied ich, es in fünf Diagramme aufzuteilen. Die ersten drei Diagramme (Abbil-

136 Da dieses Diagramm auf der Auswertung von Kategorien beruht, die im zweiten Verallgemeinerungsschritt entstanden sind, sind im Diagramm keine konkreten Einflussfaktoren genannt. Diese sind in der Datenbank auf niedrigeren Abstraktionsebenen (Aussagen, konkrete Zitate) zu finden. 


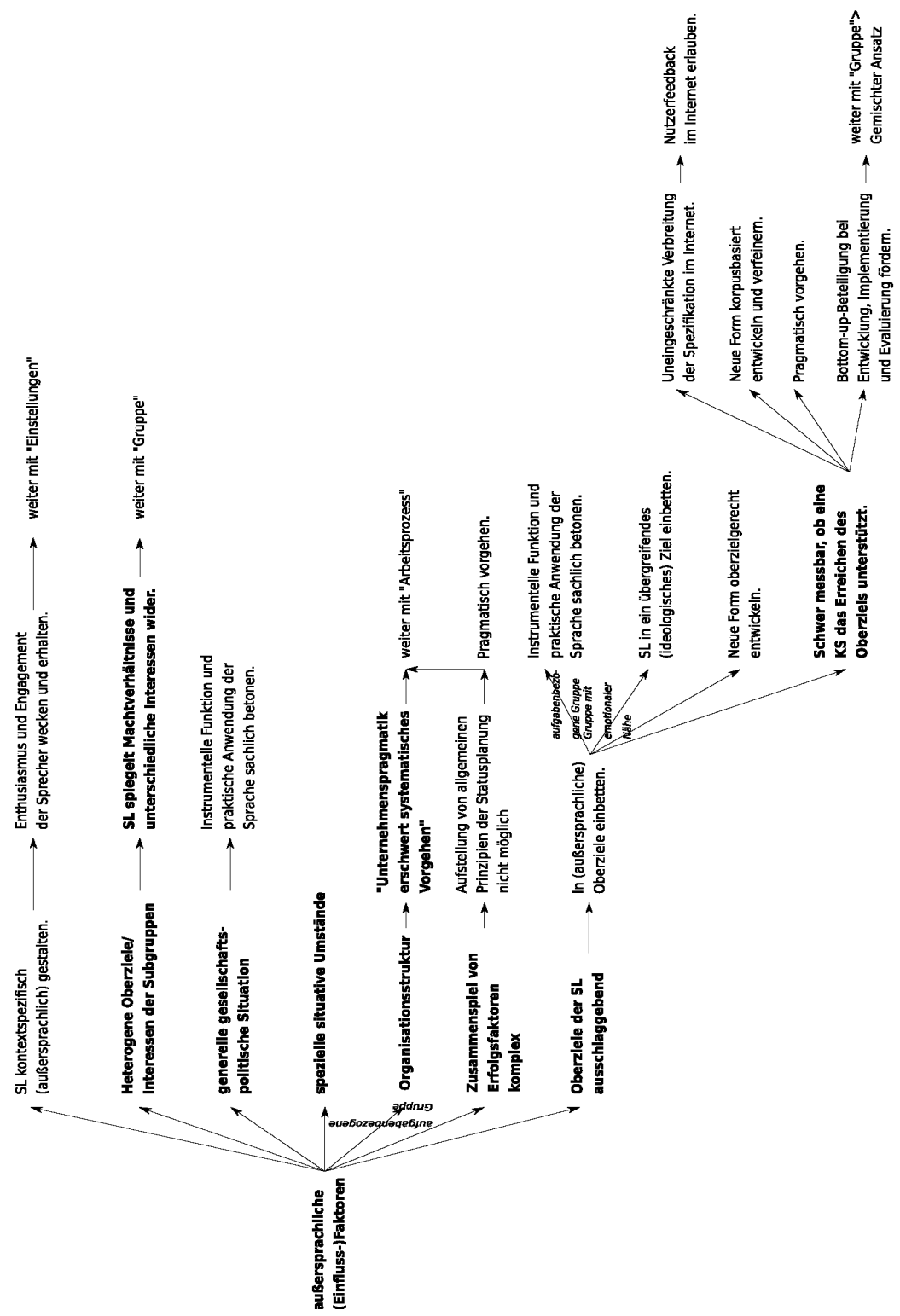

Abbildung 24.: Allgemeine außersprachliche Faktoren 
dung 25, Abbildung 26, Abbildung 27) behandeln die Intragruppenperspektive, insbesondere der Gruppenstruktur entsprechend: allgemein, Struktur der Zielgruppe und Struktur der Autorengruppe. Die letzten beiden bilden Kategorien zur Intergruppenperspektive ab, insbesondere was die Gestaltung der Beziehungen zwischen der Autoren- und der aktiven Zielgruppe betrifft, stellen die identifizierten Ausrichtungen von SL dar, entsprechend die reine Topdown- und die reine Bottom-up-SL (Abbildung 28) sowie den gemischten Ansatz, bei dem entweder top-down oder bottom-up begonnen werden kann (Abbildung 29).

Das Diagramm Intragruppenperspektive allgemein (Abbildung 25) behandelt drei Aspekte: die an der SL beteiligten Gruppen (oben), die Frage der Gruppenstruktur (Mitte) sowie die kritischen Phasen der Gruppenentwicklung (unten). Es ist insofern allgemein, dass sich die Inhalte weitestgehend auf alle beteiligten Gruppen beziehen. Unter Beteiligte Gruppen sind noch einmal die in Abschnitt 5.2.2.2 festgestellten Gruppen grafisch dargestellt, Ähnliches gilt für die Kritischen Phasen der Gruppenentwicklung (Abschnitt 4.6.2.2). Zur Gruppenstruktur wird allgemein als Herausforderung gesehen, dass SL in den Alltag bzw. die Gepflogenheiten der beteiligten Gruppen eingreift, sowohl was den Sprachgebrauch, oder aber möglicherweise die verwendeten Tools und allgemein die (aufgabenbezogenen) Arbeitsprozesse betrifft. Bei Sprachlenkung soll man sich im Allgemeinem dieses (vielfältigen) Eingriffs bewusst sein oder gar die Gepflogenheiten berücksichtigen. Insbesondere bei Arbeitsprozessen wird empfohlen, dass man bei Sprachlenkung die bestehenden Arbeitsprozesse nicht umgestaltet oder aber die Arbeitsprozesse gezielt an die Anforderungen der Sprachlenkung anpasst. Diese allgemeinen Überlegungen lassen sich auch auf andere genannte Gepflogenheiten wie Tools übertragen, auch wenn dies in der Datenbank nicht explizit enthalten ist.

Im Diagramm Intragruppenperspektive: aktive Zielgruppe (Abbildung 26) werden Herausforderungen dargestellt, die sich im Zusammenhang mit der Struktur der aktiven Zielgruppe stellen. Als Merkmale der Gruppenstruktur ergeben sich die Gruppengröße, Normen, Interaktion und Kohäsion. Als Erstes stellt sich Frage, wie man eine Gruppe(nstruktur) aufbauen kann. Es wird davon ausgegangen, dass dieser Aufbau aktiv unterstützt werden soll und die SL folglich über eine reine Korpusplanung hinausgehen muss. Als Instrument stehen dann die aus Identität bekannten Schritte zur Etablierung einer Gruppe mit emotionaler Nähe oder einer aufgabenbezogenen Gruppe zur Verfügung.

Was die Gruppengröße betrifft, so beobachte ich im Allgemeinen, dass das Problem der SL für größere Gruppen auf SL für kleinere Gruppen herunter- 


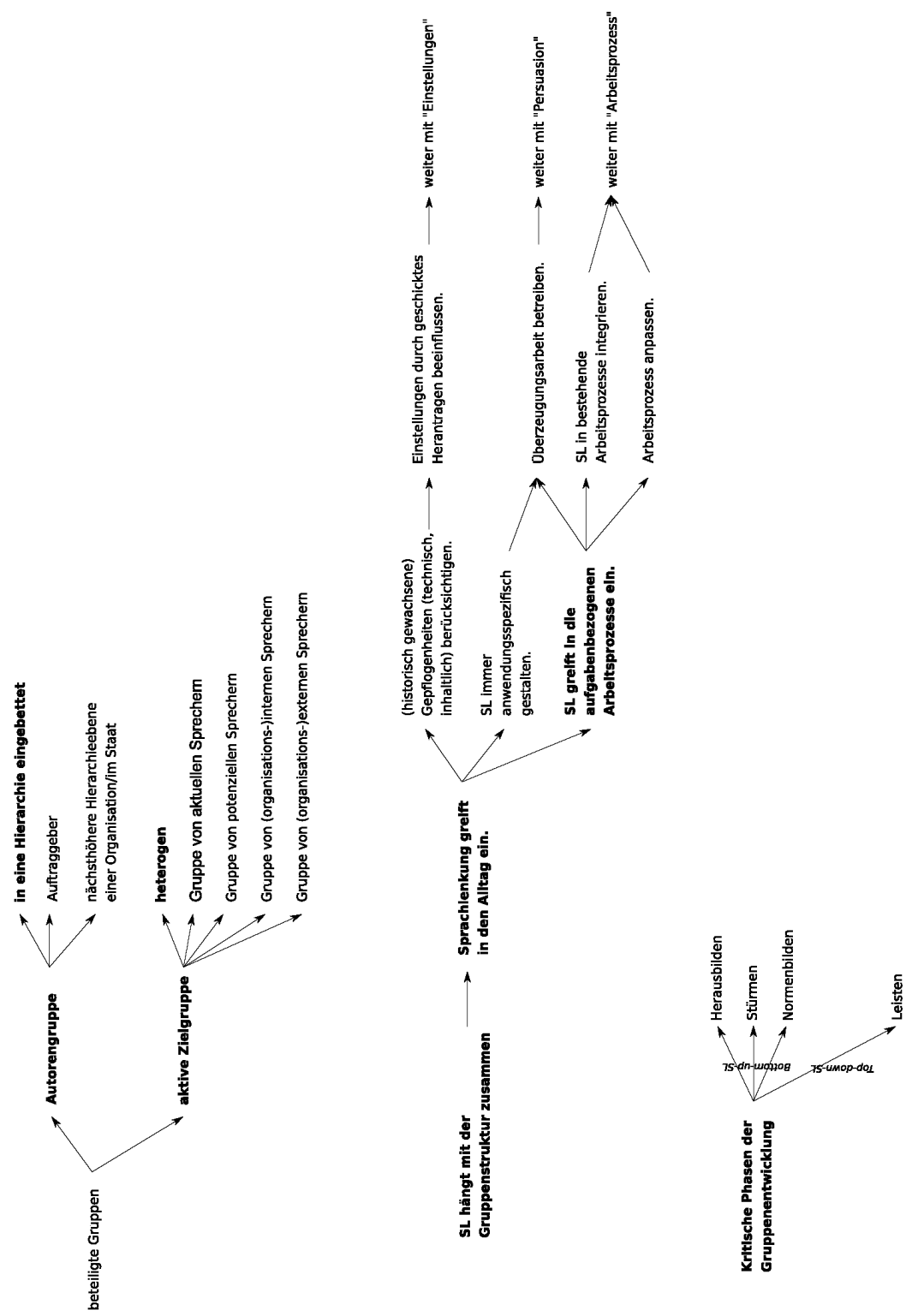

Abbildung 25.: Intragruppenperspektive allgemein 
gebrochen wird: Obwohl man von Anfang an die gesamte Zielgruppe im Sinn haben muss, soll die neue Form zunächst in einer kleineren Testgruppe eingeführt und etabliert und dann sukzessiv auf übrige Subgruppen ausgeweitet werden. Dabei kann man entweder einzelne Individuen gewinnen, oder aber ganze Gruppen, für die auch konkrete Vorgehen postuliert werden; so kann man im Sinne der Sprachökologie auf unterschiedliche Subgruppen unterschiedlich eingehen und gemäß dem Gruppentyp entsprechende Oberzieltypen betonen oder aber durch bestimmte Anreize, wie finanzielle Begünstigungen, die SL für die Zielgruppe attraktiver machen.

Beim Merkmal Interaktion handelt es sich zum einen um die Möglichkeiten zur Vernetzung von einzelnen Sprechern, wobei das Verfügbarmachen von Kontaktdaten, die Herausgabe von Zeitschriften oder das Organisieren von Konferenzen genannt werden. Zum anderen aber wird die Auswirkung von SL auf den etablierten Sprachgebrauch der aktiven Zielgruppe als Herausforderung gesehen. Um den historischen Sprachgebrauchsgepflogenheiten gerecht zu werden eignet sich der gemischte Ansatz, bei dem SL als Gruppenarbeit aufgefasst wird. Darüber hinaus soll bei der Korpusplanung die Gebräuchlichkeit berücksichtigt und ggf. auf eine strenge Normativität und Eineindeutigkeit der neuen Form verzichtet werden, so dass Synonyme und Homonyme erlaubt sein.

Schließlich stellen verschiedene Aspekte, die unter das Merkmal Kohäsion fallen, weitere Herausforderungen für SL dar. Es handelt sich dabei allgemein um die Frage, wie die Festlegung der aktiven Zielgruppe auf verschiedene Aspekte der SL erhöht werden kann, vor allem was die neue Sprachform oder aber die Art, wie sie elaboriert und weiterentwickelt wird, betrifft. Als Vorgehen werden beispielsweise das normative Perfektionieren des Sprachsystems, der Aufbau einer guten und effizienten Organisationsstruktur, die sich für SL einsetzt, sowie das uneingeschränkte Aufnehmen aller an SL-Interessierten in die aktive Zielgruppe vorgeschlagen. Bei bestimmten SL-Maßnahmen kann sich darüber hinaus die Tatsache als Herausforderung erweisen, dass die aktive Zielgruppe Eigenschaften einer Diaspora hat. Hierzu wird empfohlen, den Gruppenzusammenhalt durch verschiedene Vorgehen zu stärken, die auf die identitätsstiftende Funktion der SL setzen.

Die Herausforderungen bezüglich der Struktur der Autorengruppe sind in Diagramm Intragruppenperspektive: Autorengruppe (Abbildung 27) dargestellt. Wie bei der aktiven Zielgruppe identifiziere ich die Merkmale Gruppengröße und Interaktion, darüber hinaus aber auch Status und Rollen. Zur allgemeinen Zusammensetzung wird empfohlen, SL als Gruppenarbeit zu se- 


\section{Untersuchung}

hen und den gemischten Ansatz vorzuziehen, bei dem Vertreter der aktiven Zielgruppe und insbesondere auch (organisations-)externe Vertreter in der Arbeitsgruppe im engeren Sinne tätig sein sollen. Diese eigentliche Arbeitsgruppe soll möglichst klein gehalten werden (Gruppengröße), was gegen einen reinen Bottom-up-Ansatz (alle Mitglieder der Zielgruppe werden als gleichberechtigte Autoren behandelt) spricht.

Bei Status werden vor allem erforderliche spezifische Statusmerkmale genannt, in erster Linie die SL-bezogene (Sach-)Kompetenz für SL. Da Linguisten für Statusplanung nicht ausgebildet sind, soll SL als interdisziplinäre Aufgabe betrachtet werden, wobei Zuständigkeiten klar bestimmt sein sollen. Insbesondere wird für notwendig gehalten, innerhalb der Arbeitsgruppe einen Hauptverantwortlichen zu bestimmen, der über Führungskompetenz verfügen soll. Alle Mitglieder der Autorengruppe sollen sich außerdem durch Kompromissbereitschaft und diplomatisches Geschick auszeichnen. Was die Rollen in der Autorengruppe betrifft, so sollen die Aufgaben, Zuständigkeiten und Verantwortlichkeiten sowie der entsprechende Workflow klar definiert und festgelegt sein.

Beim Merkmal Interaktion wird vor allem auf die Konflikte bei Abstimmungen in der Arbeitsgruppe als Herausforderung hingewiesen, die durch besseres Kommunizieren gemildert werden können.

In Diagramm Intergruppenperspektive: reine Top-down-Sprachlenkung und reine Bottom-up-Sprachlenkung (Abbildung 28) sind Erkenntnisse zu den beiden ,reinen“ SL-Ansätzen zusammengetragen. Bei der idealtypischen Top-down-Sprachlenkung ist alleine die Autorengruppe für die Korpusund Statusplanung zuständig und arbeitet weitestgehend deduktiv. Bei der idealtypischen Bottom-up-Sprachlenkung sind alle Mitglieder der aktiven Zielgruppe gleichermaßen für Korpus- und Statusplanung zuständig und auch gleichberechtigt.

Bei der reinen Top-down-Sprachlenkung werden vor allem Herausforderungen genannt, die auf Beziehungen zwischen der Autorenund der Zielgruppe beruhen, insbesondere fehlende Akzeptanz von Autorenentscheidungen, was allgemein durch nähere Betrachtung von Einstellungen behoben werden kann. Nach der Verallgemeinerung von Teilaussagen füge ich als zweite Herausforderung den Intergruppenkonflikt hinzu, der durch einen gemischten Ansatz (mit Top-down-Beginn) gemindert werden kann. Außerdem wird die Nachvollziehbarkeit der neuen Form bei einem deduktiven Ansatz als Problem gesehen, das durch die Verringerung 
des systemlinguistischen Umfangs, aber auch durch eine kontextspezifische Korpusplanung gelöst werden kann.

Bei dem reinen Bottom-up-Ansatz kommt es hingegen zunächst auf eine kritische Anzahl an engagierten Sprechern an, wobei versucht werden kann, sowohl die Anzahl als auch das Engagement der Sprecher zu erhöhen. Insgesamt wird aber der Kerngedanke dieses Ansatzes als Herausforderung gesehen, die weitere Probleme nach sich zieht. Wenn jedes Mitglied der aktiven Zielgruppe gleichberechtigt ist, dann kann eine effiziente Korpus- und Statusplanung ohne eine einheitliche Position aller Mitglieder schwierig sein. Eine Abhilfe kann hier ein demokratisches Abstimmen über (alle) Vorschläge zur Korpus- und Statusplanung sein. Dieses Vorgehen kann zudem als Durchsetzungsinstrument eingesetzt werden, da einer Bottom-up-SL allgemein Machtmittel fehlen.

Insgesamt finden sich bei den „reinen“ Ansätzen kritische Herausforderungen, denen aber nicht viele wirksame Vorgehenskategorien zugeordnet sind, die mit dem „reinen“ Ansatz im Einklang stehen. Es wird dadurch nämlich deutlich, dass insgesamt ein gemischter Ansatz zur Lösung der festgestellten Probleme empfohlen wird.

Das letzte Diagramm zum Thema Gruppe: Intergruppenperspektive: gemischter Ansatz (Abbildung 29) elaboriert, wie die Probleme, die bei den ,reinen" SL-Ansätzen entstehen, mithilfe gemischter Ansätze bewältigt werden können. Der gemischte Ansatz mit Bottom-up-Beginn wird nur angerissen (rechts unten). Eine detaillierte Betrachtung stelle ich hingegen für den gemischten Ansatz mit Top-down-Beginn fest, bei dem das Vermeiden des Intergruppenkonfliktes die übergreifende Kategorie darstellt (links oben). Die zweite Herausforderung des reinen Top-down-Ansatzes (s. Diagramm Intergruppenperspektive: reine Top-down-Sprachlenkung und reine Bottom-upSprachlenkung) ist dem Intergruppenkonflikt in diesem Fall untergeordnet (links Mitte).

Die Unterkategorien, insbesondere Vorgehen, werden danach gebündelt, auf welches der in der Literatur behaupteten Elemente der Konfliktvermeidung - Übereinstimmung von Zielen oder der Zusammenarbeit beider Gruppen - sie sich beziehen. Eine Übereinstimmung aller Parteien bezüglich ihrer Bedürfnisse oder Oberziele wird als wichtige Voraussetzung gesehen. So muss sichergestellt werden, dass der Typ des SL-Oberziels aus der Sicht der Autoren und der Typ der aktiven Zielgruppe kompatibel sind. Dies beobachtete ich bereits bei Themen Identität und Instrumentalität. Erfüllt die neue, von den Autoren vorgeschlagene Sprachform hingegen die Bedürfnisse der 


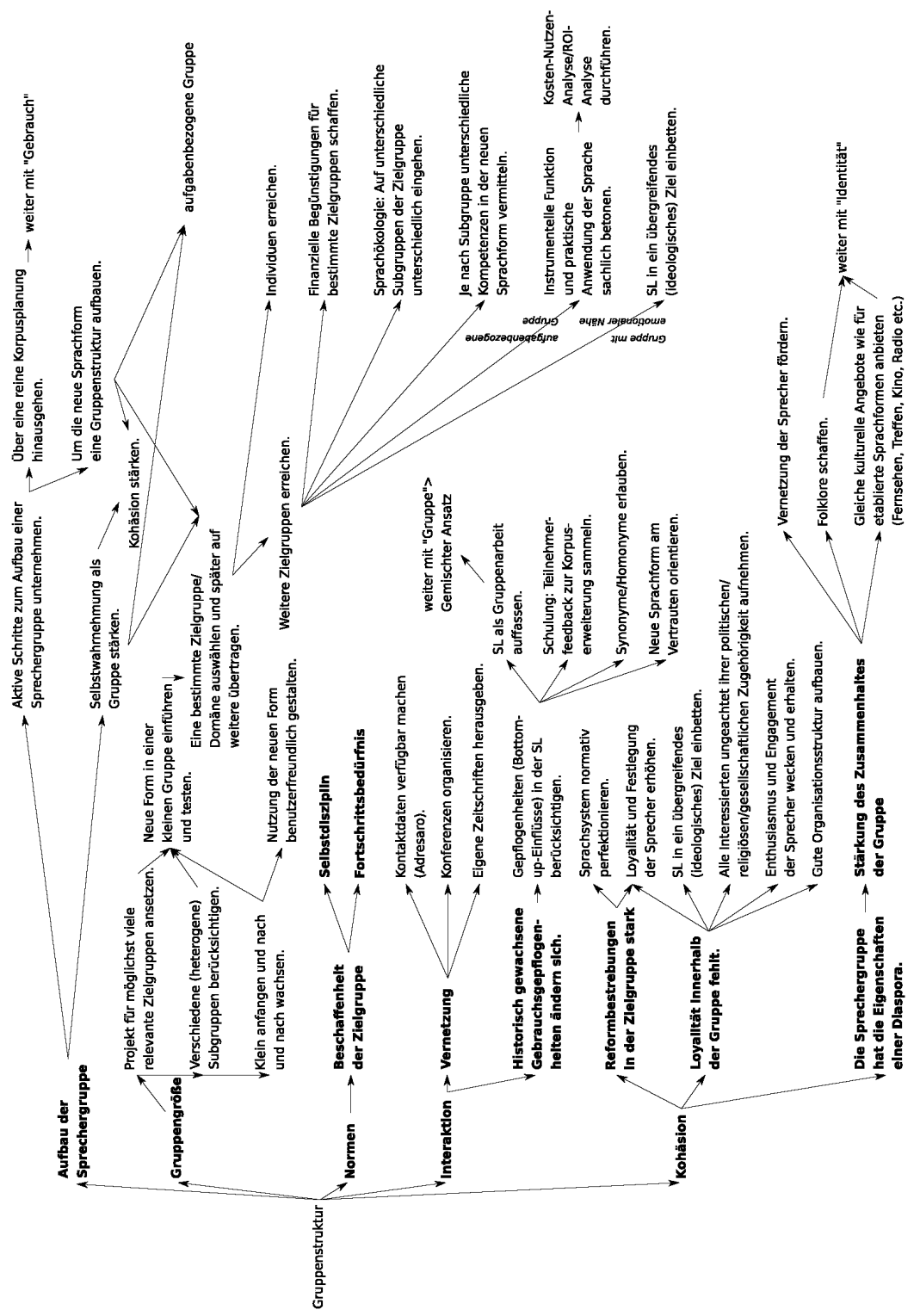

Abbildung 26.: Intragruppenperspektive: aktive Zielgruppe 


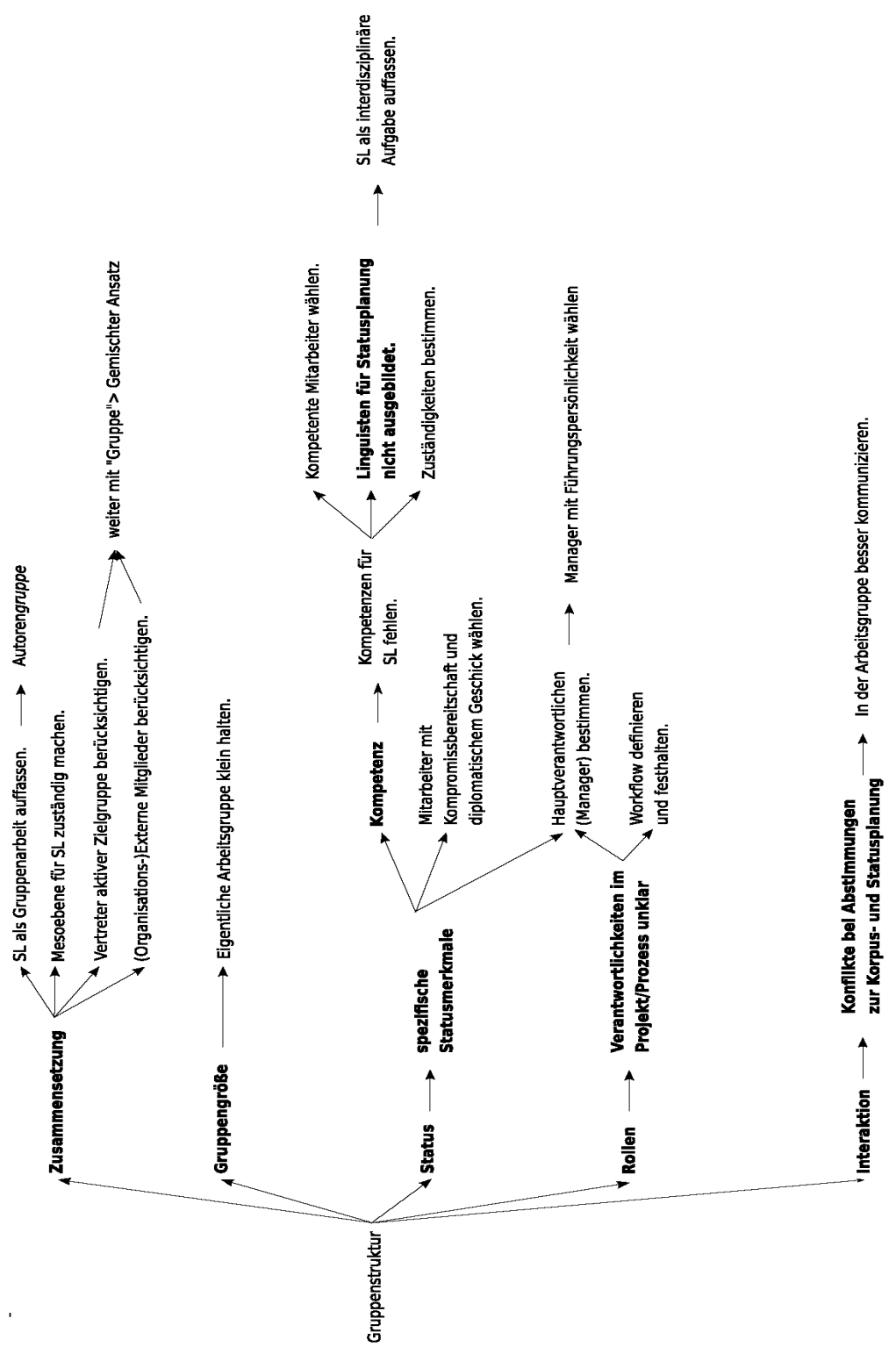

Abbildung 27.: Intragruppenperspektive: Autorengruppe 

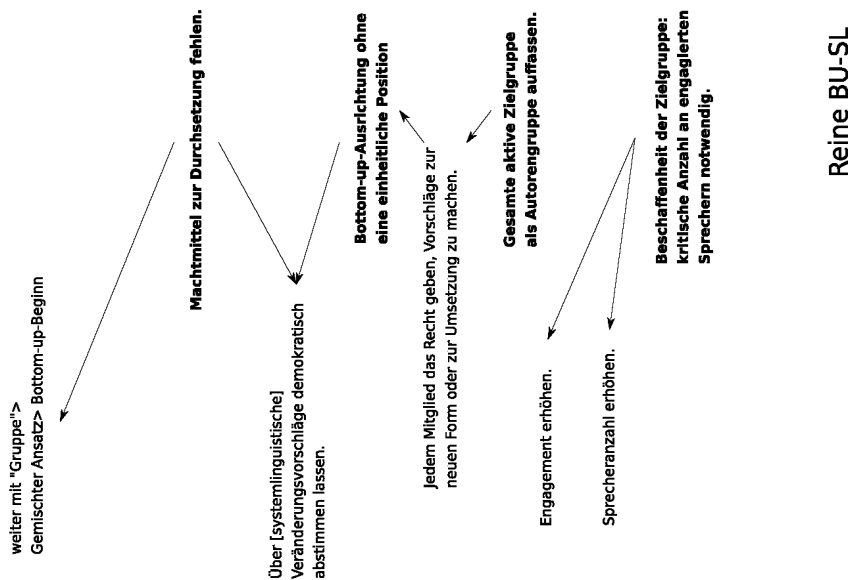

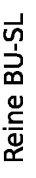




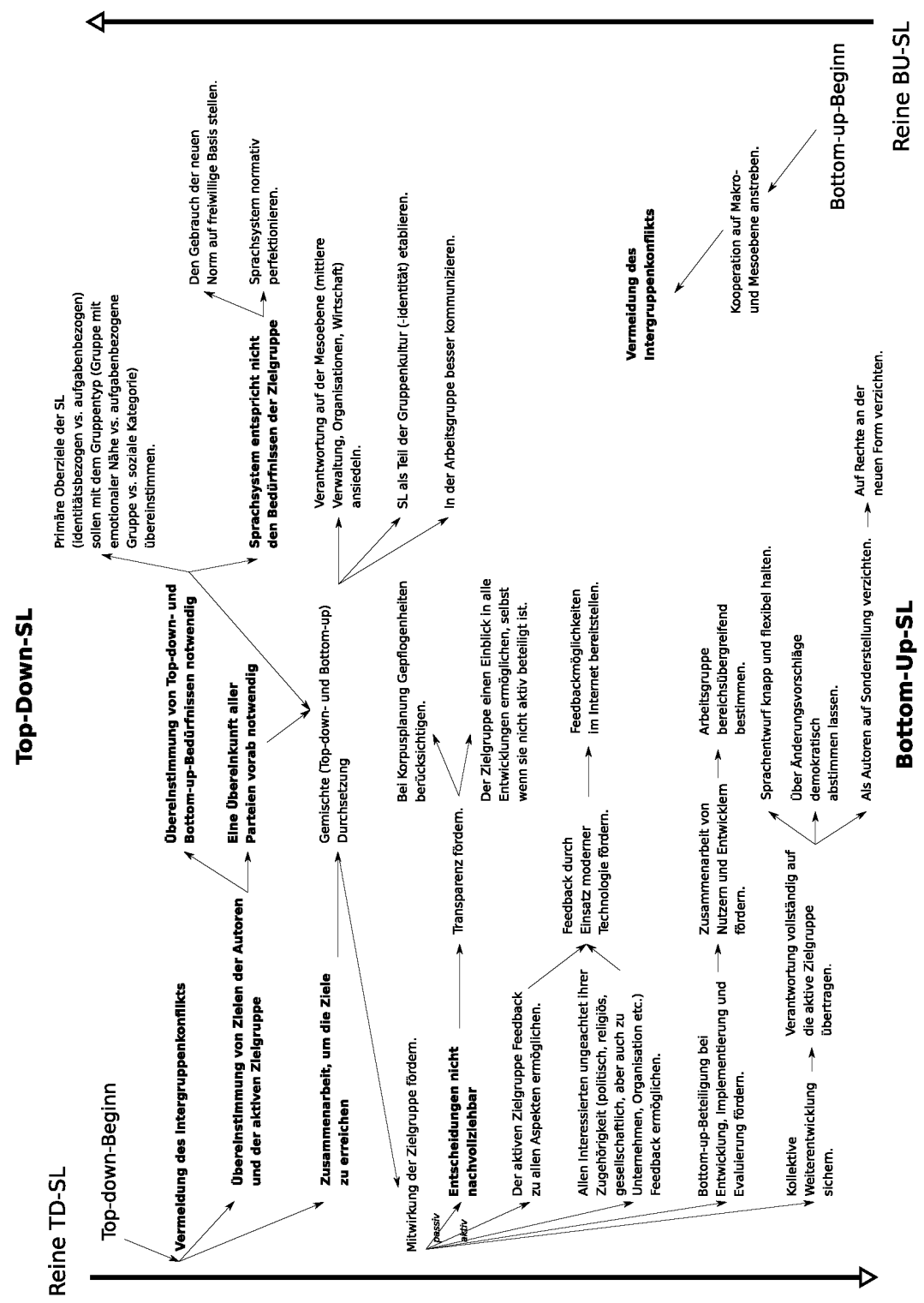

Abbildung 29.: Intergruppenperspektive: gemischter Ansatz 


\section{Untersuchung}

Zielgruppe nicht, so kann entweder das Sprachsystem verbessert oder aber sein Gebrauch auf freiwillige Basis gestellt werden.

Ein Mittel, mit dem sowohl die Zielübereinstimmung als auch die Zusammenarbeit erreicht werden können, ist dabei die Stärkung der Bottom-upBeteiligung bei Korpus- und Statusplanung. Bei Statusplanung handelt es sich um organisatorische Aspekte. Empfohlen werden hierzu die Verantwortungübertragung für SL auf die Mesoebene, eine bessere Kommunikation in der Arbeitsgruppe sowie die Etablierung von SL als Teil der Gruppenkultur. Ausführlicher behandelt werden Vorgehen bei der Korpusplanung, die sich weitestgehend danach abstufen lassen, wie aktiv die aktive Zielgruppe sein sollte (links Mitte). Eine passive Beteiligung bedeutet, dass Transparenz gefordert wird, indem die Gebrauchsgepflogenheiten dank Korpusarbeit durch die Autoren berücksichtigt werden. Die Zielgruppe kann auch passiv dadurch stärker eingebunden werden, wenn sie einen Einblick in alle Entwicklungen bekommt, ohne dass Feedbackmöglichkeiten vorgesehen sind. Im einfachsten Fall einer aktiven Mitwirkung kann sich die Zielgruppe an der Korpusplanung durch punktuelles Feedback beteiligen, wofür entsprechende Infrastruktur, z. B. im Internet, seitens der Autoren bereitgestellt werden muss. Diese Mittel können nur einem Teil der Zielgruppe zur Verfügung stehen oder aber uneingeschränkt allen Mitgliedern. Eine Steigerung der Bottom-up-Beteiligung kann dadurch erfolgen, dass die aktive Zielgruppe systematisch in das Entwerfen und Pflegen der neuen Form, allerdings unter Anleitung der Autoren, mit einbezogen wird. Die höchste Stufe der Bottom-up-Beteiligung wird erreicht, indem die Verantwortung für die Korpusplanung vollständig auf die aktive Zielgruppe übertragen wird, der Abstimmungsprozess demokratisch erfolgt und die Autorengruppe auf ihre Sonderstellung und ggf. auf die Rechte an der neuen Form komplett verzichtet. Es handelt sich bei dieser Stufe de facto um den reinen Bottom-up-Ansatz.

\subsubsection{Einstellungen}

In der Datenbank finden sich insgesamt 54 Zuordnungen von EinstellungHerausforderungen, das Thema wird also recht umfassend behandelt. Davon sind vor allem Persuasion und Gruppe besonders stark, sowie Werkzeuge, Erlernbarkeit und Arbeitsprozess stark vertreten. Vereinzelt werden Vorgehen aus Identität, Instrumentalität, Sprachökologie, Machtmittel, Einhalten und Bekanntmachen zugeordnet. Die Eigenvorschläge beziehen sich v. a. auf Einstellungen, aber auch Gruppe oder die anderen bereits genannten Themen. 


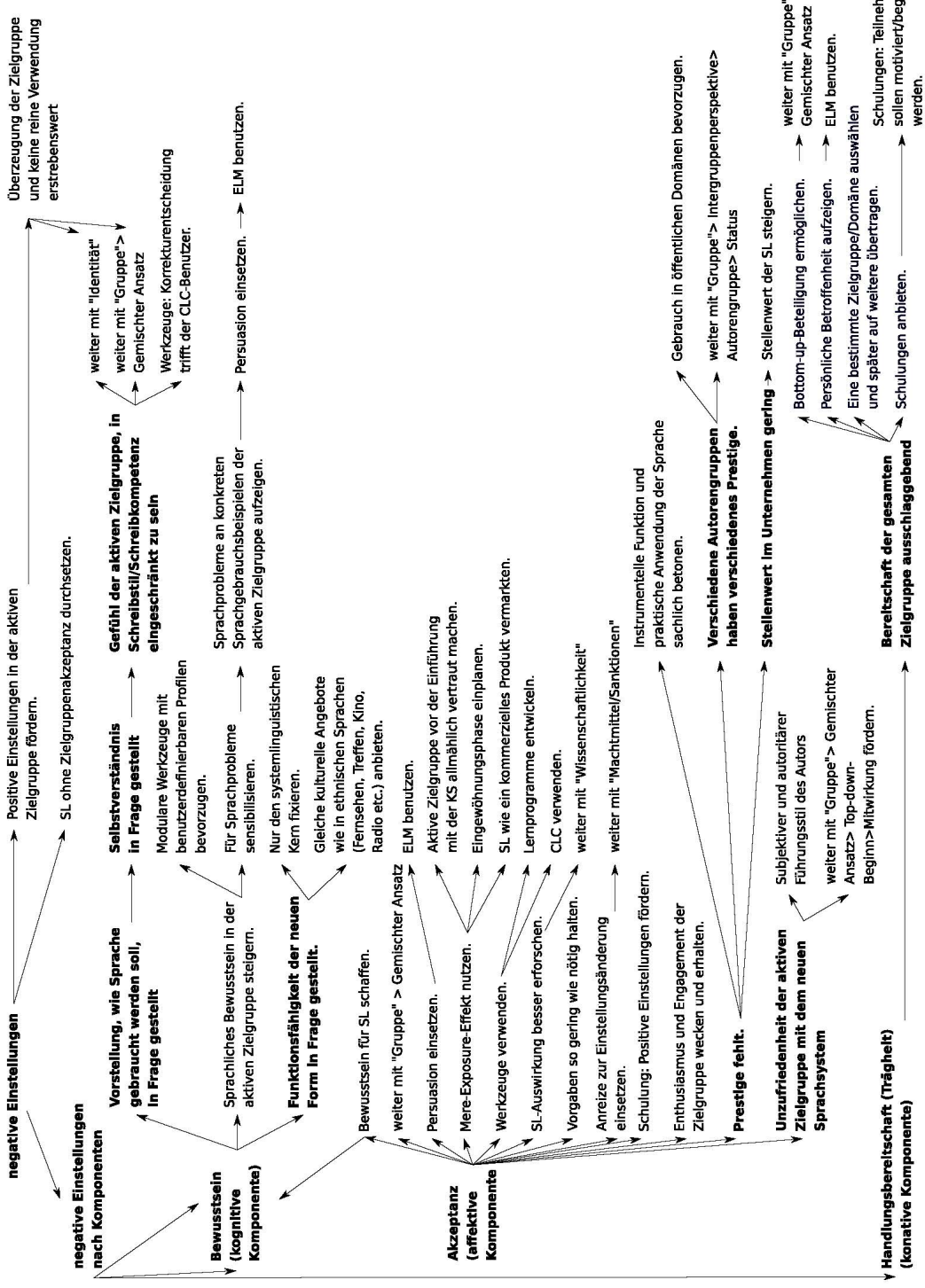

Abbildung 30.: Einstellungen 1 


\section{Untersuchung}

Im Diagramm Einstellungen 1 (Abbildung 30) werden Vorgehen zusammengetragen, mit denen negative Einstellungen der aktiven Zielgruppe der Sprachlenkung gegenüber behoben werden können. Allgemein wird es als notwendig gesehen, positive Einstellungen aktiv zu fördern; insbesondere soll die aktive Zielgruppe die neuen Form aus Überzeugung verwenden.

Weitere Vorgehen zur Förderung positiver Einstellungen lassen danach bündeln, ob sie zur Steigerung von Bewusstsein (kognitiv), Akzeptanz (affektiv) oder Handlungsbereitschaft (konativ) eingesetzt werden.

Die Einstellungsprobleme in der kognitiven Komponente beruhen zum einen auf der Beobachtung, dass durch SL die Vorstellung der aktiven Zielgruppe, wie Sprache zu gebrauchen ist, und dadurch auch ihr Selbstverständnis in Frage gestellt wird. Dem Gefühl des Diktiert- oder Eingeschränktseins kann man durch Berücksichtigung von Identitätsaspekten, einen gemischten SLAnsatz sowie günstige Werkzeugeingenschaften entgegenwirken. Eine weitere Herausforderung in der kognitiven Komponente bezieht sich auf die neue Form selbst, speziell ihre Wirksamkeit. Hierbei wird empfohlen, nur den Kern des Sprachsystems zu fixieren und sonst die neue Form flexibel zu halten sowie an kulturellen Verwendungsbeispielen die Funktionsfähigkeit der Form aufzuzeigen. Insgesamt wird auch die Steigerung des allgemeinen sprachlichen Bewusstseins und eine Sensibilisierung für Sprachprobleme empfohlen, was an konkreten Sprachgebrauchsbeispielen der aktiven Zielgruppe aufgezeigt werden soll und im Kontext von Persuasion und ELM gesehen werden kann.

Um die Akzeptanz zu stärken, wird auf verschiedene Mittel hingewiesen, die unter hier besprochene Themen fallen: Nutzung von Persuasion, MereExposure-Effekt, geeigneten Anreizen und Werkzeugen. Zudem sollen Enthusiasmus und Engagement geweckt und das sprachliche Bewusstsein erhöht werden. Akzeptanzprobleme können außerdem auf zwei speziellen Herausforderungen beruhen, nämlich dem Prestigeaspekt sowie einer Unzufriedenheit mit dem Sprachsystem. Das Prestige kann sich auf verschiedene Objekte beziehen, insbesondere die Oberziele, die Autorengruppe oder die SL insgesamt. Wobei vor allem die instrumentelle Funktion und die offiziellen Domänen als prestigeträchtig angesehen werden.

Für die konative Komponente ist die Handlungsbereitschaft der gesamten aktiven Zielgruppe ausschlaggebend. Diese soll gesteigert werden durch die Beteiligungsförderung der aktiven Zielgruppe an SL, das Aufzeigen der persönlichen Betroffenheit, was im Kontext des ELM eine Rolle spielt sowie 
durch das Herunterbrechen der SL-Durchsetzung auf einzelne Subgruppen und durch geeignete Schulungsmaßnahmen.

In Diagramm Einstellungen 2 (Abbildung 31) sind schließlich Aspekte zusammengetragen, die als Einschränkung der Vorgehen in Einstellungen 1 verstanden werden können. Obwohl die Steigerung der Handlungsbereitschaft als Förderung von positiven Einstellungen verstanden werden kann, so besteht weiterhin die Herausforderung, dass positive Einstellungen nicht automatisch zur Handlung führen. Als Abhilfe für diesen Aspekt werden insbesondere die Übersichtlichkeit der neuen Form sowie der Einsatz von Persuasion und Anreizen als wirksam gesehen, obwohl es auch notwendig sein kann, den Gebrauch der neuen Form auf freiwillige Basis zu stellen.

Ein wichtiger Einstellungsaspekt kommt zudem zutage, wenn die Durchsetzung von SL auf Subgruppen der aktiven Zielgruppe heruntergebrochen wird. Ein Problem kann hierbei die negative Bewertung verschiedener Aspekte der SL durch die aktuell zu erreichende Außengruppe sein. Mögliche Vorgehen umfassen u.a. die Verbesserung der Fremdbewertung durch die Umbenennung der konkreten Maßnahme, eine aktive Auseinandersetzung der Gruppe von aktuellen Sprechern mit der Gruppe von potenziellen Sprechern, den Übergang zum starken Bottom-up-Ansatz sowie Überzeugungsarbeit mithilfe wissenschaftlich gesicherter Daten. Eine weitere Lösung bietet die Annahme, dass auf unterschiedliche Subgruppen auch unterschiedlich eingegangen werden soll und insbesondere bei stark heterogenen Gruppen der Oberzieltyp der SL ggf. angepasst werden kann, um der Außengruppe gerecht zu werden. Dies kann aber zu weiteren Herausforderungen wie Akzeptanzproblemen seitens aktueller Sprecher oder, abstrakter ausgedrückt, einem Konflikt zwischen dem Oberzieltyp und dem Gruppentyp führen. Für diese von mir vorgeschlagenen Herausforderungen, stelle ich keine geeigneten Gegenmaßnahmen fest.

\subsubsection{Kosten}

Die wenigen Zuordnungen in der Datenbank beziehen sich auf Vorgehen aus Gruppe sowie Persuasion und Werkzeuge. Bei diesem Thema mache ich selbst keine Zuordnungsvorschläge.

Da die Frage der Wirtschaftlichkeit von SL nicht im Fokus dieser Arbeit steht, ist es auch nicht überraschend, dass das entsprechende Diagramm nur oberflächliche Zusammenhänge darstellt. Dennoch sind die beobachteten Herausforderungen relevant: Die präskriptive SL-Phase ist im Vergleich 

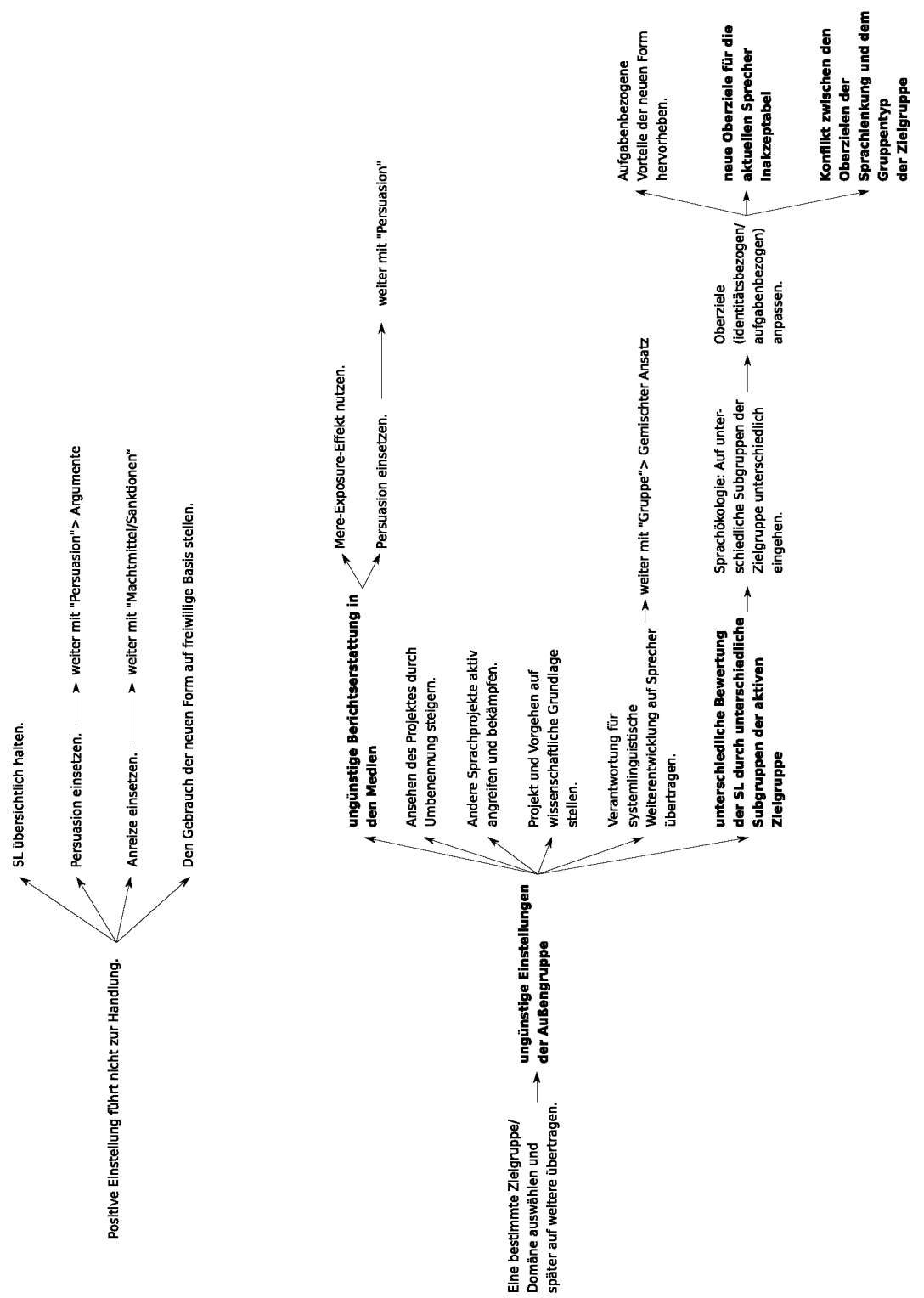

Abbildung 31.: Einstellungen 2 
zur deskriptiven sowie die Statusplanung im Vergleich zur Korpusplanung kostspieliger. Um die Finanzierung des Projektes zu sichern, können die Autoren ausschließlich auf die eigenen finanziellen Möglichkeiten setzen oder aber die Persuasion benutzen, um weitere Unterstützer zu gewinnen. Da SL insgesamt als kostspielig gesehen wird, können die Autoren versuchen, die Kosten in Verhältnis zum Nutzen zu setzen und dies als Argument zu gebrauchen.

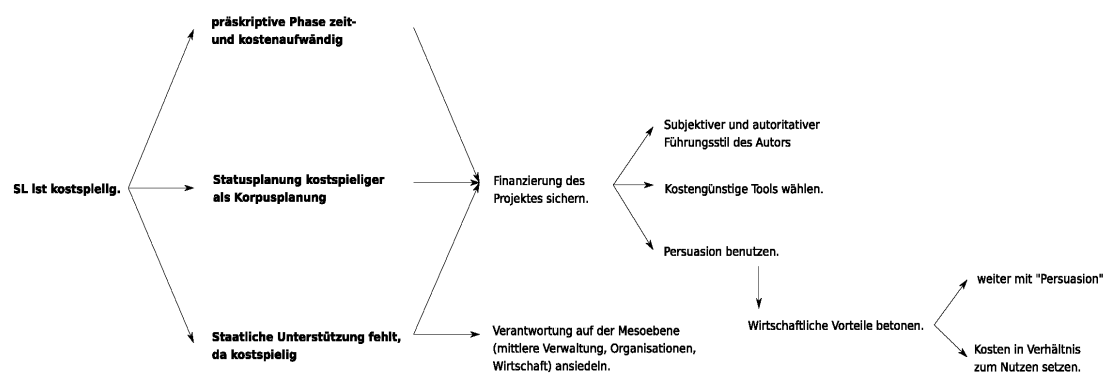

Abbildung 32.: Kosten

\subsubsection{Sprachökologie}

Die Zuordnungen zu Sprachökologie-Herausforderungen betreffen verschiedene Themen, beispielsweise Gruppe, Arbeitsprozesse, Gebrauch, Sprachsystem und vereinzelt auch Sprachökologie, Identität, Persuasion, Erlernbarkeit sowie Machtmittel/Sanktionen

Im Sinne des sprachökologischen Ansatzes (2.1.5.5) stellen bei der Etablierung der neuen Form andere, gelenkt oder ungelenkt entstandene sprach- 


\section{Untersuchung}

liche Formen eine Herausforderung dar. Um dem allgemein entgegenzuwirken können die Autoren versuchen, andere Sprachformen in ihrer Position abzuschwächen, beispielsweise durch einen direkten Angriff, einen nichtautoritären Führungsstil der Autoren bis hin zur Übertragung der Verantwortung für die sprachliche Weiterentwicklung auf die aktive Zielgruppe. Dieser expliziten Sprachlenkung steht die implizite Sprachlenkung gegenüber, die den Mere-Exposure-Effekt nutzen kann. Darüber hinaus können Autoren auf Zusammenarbeit mit anderen Projekten setzen und pragmatisch vorgehen und zudem kann die Stabilität der neuen Form vor der permanenten Perfektionierung Vorrang haben.

Eine spezielle Herausforderung im Kontext der Sprachökologie stellt die Bewertung von verschiedenen Sprachformen dar, insbesondere was ihr Prestige angeht. Um die neue Form gegenüber anderen Formen aufzuwerten, wird empfohlen, dass sie möglichst viele Domänen abdecken soll. Argumentativ soll man zudem insbesondere den instrumentellen Faktor betonen. Was die Korpusplanung betrifft, so soll sich das sprachliche Material am Vertrauten im Sinne von Altbewährtem orientieren und es soll stets perfektioniert und weiterentwickelt werden.

\subsubsection{Persuasion}

Die meisten Zuordnungen aber auch Eigenvorschläge hier sind innerhalb des Themas Persuasion zu verorten, betreffen aber auch vereinzelt Instrumentalität und außersprachliche Faktoren.

Wie in 5.2.2.5 erläutert, wird Überzeugungsarbeit als notwendig gesehen und ich strukturiere die Vorgehen zum Thema Persuasion mithilfe des Modells der Elaborationswahrscheinlichkeit. Empfehlungen, einfache und wissenschaftlich untermauerte Botschaften zu nutzen und diese auch zu wiederholen, zähle ich zur peripheren Route. Die zentrale Route bezieht sich sowohl auf die Fähigkeiten der zu überzeugenden Personen als auch auf ihre Motivation. Zu Fähigkeiten stelle ich in der Analyse keine Vorgehen fest. Der Motivationsaspekt zerfällt hingegen in zwei Elemente: Die Forderung, dass sich die aktive Zielgruppe selbst von SL-Vorteilen überzeugen soll, sowie in die eigentlichen Argumente, die die Autoren benutzen können.

Die Selbstüberzeugung beruht auf dem Aufzeigen der eigenen Betroffenheit und der persönlichen Relevanz der SL für die aktive Zielgruppe, was vor allem an verschiedenen Beispielen aus dem Sprachgebrauch der aktiven Zielgruppe erfolgen soll. 
Die Argumente können inhaltlich nach den hier besprochenen Themen gebündelt werden; sie betreffen insbesondere die Erlernbarkeit, Verständlichkeit, Kosten, Arbeitsprozess, Qualität, Einstellungen, oder generell die Oberziele der Sprachlenkung. Diese Unterkategorien sind nicht trennscharf, sondern auf vielfache Art miteinander verbunden, was im Diagramm durch Verbindungspfeile ausgedrückt ist. Außerdem stammen viele der Argumente aus den Gebieten KS und TA und betonen folglich die instrumentellen Oberziele der SL. Diese Argumente sehe ich als besonders für aufgabenbezogene Gruppen geeignet, was durch eine entsprechende Pfeilbeschriftung ausgedrückt ist.

\subsubsection{Erlernbarkeit}

Die Zuordnungen und Eigenvorschläge betreffen vor allem Erlernbarkeit. Weitere Zuordnungen beziehen sich auf Persuasion, Werkzeuge und Sprachsystem.

Die Erlernbarkeit der neuen Form kann durch verschiedene Vorgehen gesichert werden. Allgemeine Empfehlungen besagen, dass das Sprachsystem einfach gehalten werden und die aktive Zielgruppe die neue Form tatsächlich beherrschen soll, ggf. aber nicht in vollem Umfang. Eine wichtige Herausforderung stellt dabei die Lernmotivation dar, die ggf. fehlen kann. Dieser Motivationsaspekt berührt die individuelle Ebene der Sprachlenkung und ich stelle hierfür keine Vorgehenszuordnungen fest.

Neben diesen allgemeinen Aspekten stellen Überlegungen zu Lernmitteln den wesentlichen Teil des Diagramms dar. Lernmittel sollen zur Verfügung gestellt werden, wobei sie flexibel und personalisierbar sein sollen. Genannt werden vor allem die Möglichkeiten von Werkzeugen und Schulungen. Es wird empfohlen, Lernprogramme (Tutorials) anzubieten oder aber speziell Controlled Language Checker zu Lernzwecken einzusetzen, um den Schulungsaufwand zu minimieren oder Schulungen gar zu ersetzen. Dabei sollen die Benutzeroberfläche und die Systemmeldungen die neue Form verwenden.

Die inhaltlichen Überlegungen $\mathrm{zu}$ Lernmitteln beziehen sich, wie ausgeführt, ursprünglich auf Schulungen. Ich verallgemeinere sie aber auf alle Lernmittel. So wird empfohlen, dass nicht nur die neue Form selbst, sondern weitere Inhalte, wie wissenschaftliche Hintergründe, Oberziele, Toolkompetenz sowie, im Fall einer aufgabenbezogenen Gruppe, auch der neue Arbeitsprozess vermittelt werden. Der neue Arbeitsprozess kann beispielsweise einen neuen Textverfassungsprozess (Redaktionsprozess) oder aber einen neuen Schreibstil bedeuten, und ich nehme an, dass dies die 


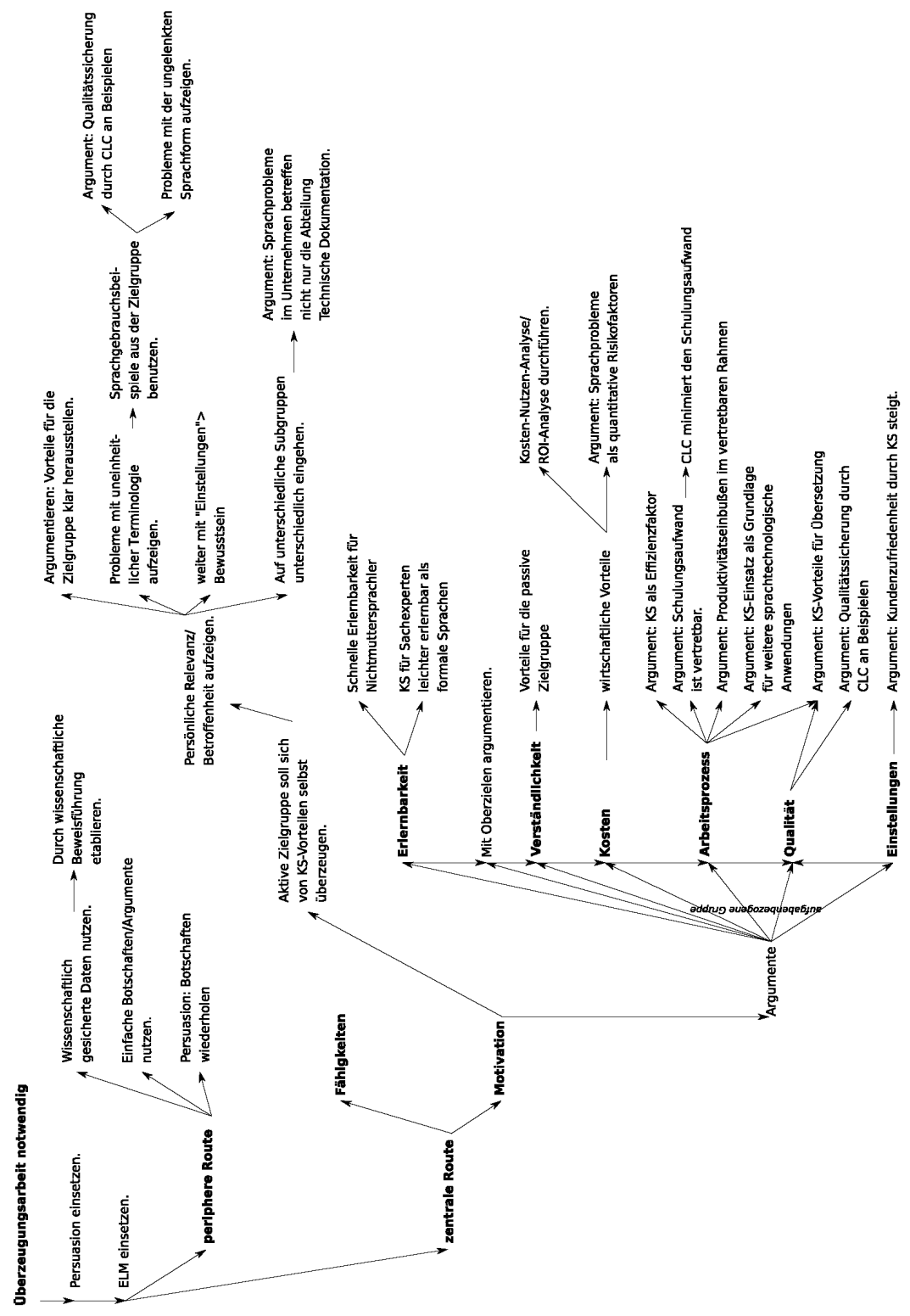

Abbildung 33.: Persuasion 


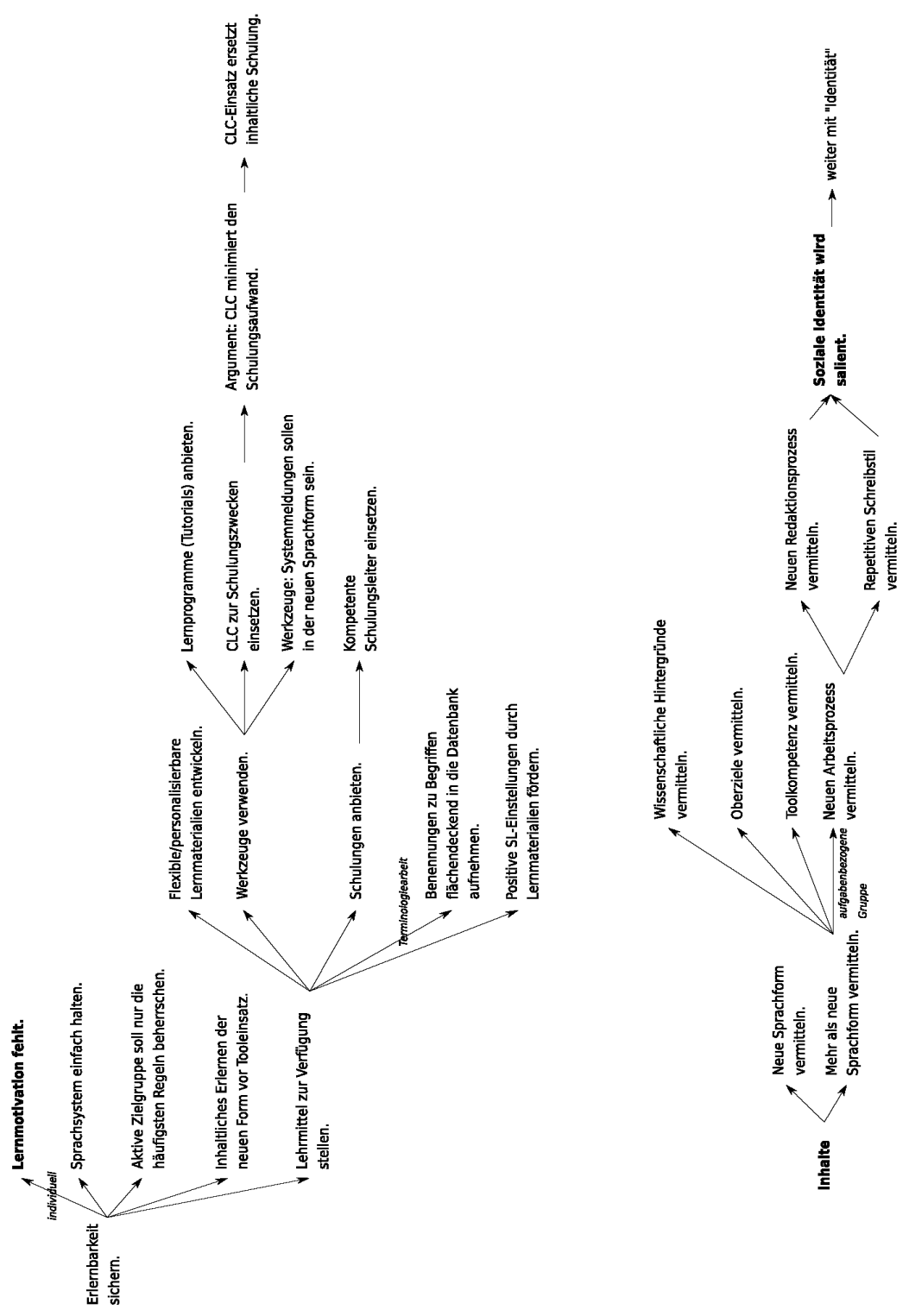

Abbildung 34.: Erlernbarkeit 


\section{Untersuchung}

soziale Identität einer aufgabenbezogenen Gruppe salient machen kann und mit dem Thema Identität im Zusammenhang steht.

\subsubsection{Arbeitsprozess}

Die Zuordnungen beziehen sich vor allem auf Arbeitsprozess, Werkzeuge, und Gruppe, vereinzelt aber auch Einstellungen, Wissenschaftlichkeit, Persuasion, Erlernbarkeit, Gebrauch. Weitere Zuordnungsvorschläge betreffen wieder das Thema Arbeitsprozess.

Auch das Thema Arbeitsprozess ist aus Übersichtlichkeitsgründen in zwei Diagramme aufgeteilt. Im ersten Diagramm (Arbeitsprozess 1, Abbildung 35) sind allgemeine Zuordnungen dargestellt, die sich auf die Gestaltung von Arbeitsprozessen der Autorengruppe (links oben) und der aktiven Zielgruppe (rechts unten) beziehen. Das zweite Diagramm (Arbeitsprozess 2, Abbildung 36) modelliert dann die spezielle Frage, wie die empfohlene systematische Vorgehensweise umgesetzt werden kann.

Im ersten Diagramm sind zwei Herausforderungen dargestellt, die die Arbeitsprozesse der Autorengruppe betreffen. Als Hauptschwierigkeit kann dabei der Zeitaufwand betrachtet werden, der mit SL verbunden ist, und speziell der Mehraufwand, da SL ggf. zusätzlich zu den laufenden Aufgaben auf die Autorengruppe zukommt. Um die Autorengruppe zeitlich zu entlasten, kann die aktive Zielgruppe stärker in SL im Sinne der Bottom-up-Beteiligung mit einbezogen werden. Damit im Zusammenhang steht darüber hinaus die Forderung, der aktiven Zielgruppe den noch nicht freigegebenen Entwurf der neuen Form zur Verfügung zu stellen, um durch die Rückmeldung die Verfeinerung und Weiterentwicklung der Form effizienter zu gestalten. Hierzu können außerdem spezielle Tools zum Einsatz kommen, die die Arbeitsprozesse beschleunigen. Außerdem wird empfohlen, die Arbeitsgruppe klein zu halten und trotz der Bottom-up-Beteiligung zentral zu steuern, um Produktivitätsverluste durch Abstimmungen gering zu halten.

Die zweite Herausforderung für die Arbeitsprozesse der Autorengruppe kann die Schwierigkeit darstellen, wenn Sprachlenkung im Zuge anderer Veränderungen in einer Zielgruppe eingeführt wird. Da allgemein eine Ablehnung von Veränderungen angenommen und von der sog. Trägheit der Menschen ausgegangen wird, spielen hier Überlegungen zum Thema Einstellungen eine wichtige Rolle.

Auf der Seite der aktiven Zielgruppe wird beobachtet, dass Sprachlenkung die eingebürgerten (Arbeits-)Abläufe verändert und ggf. verhindert, was sich 


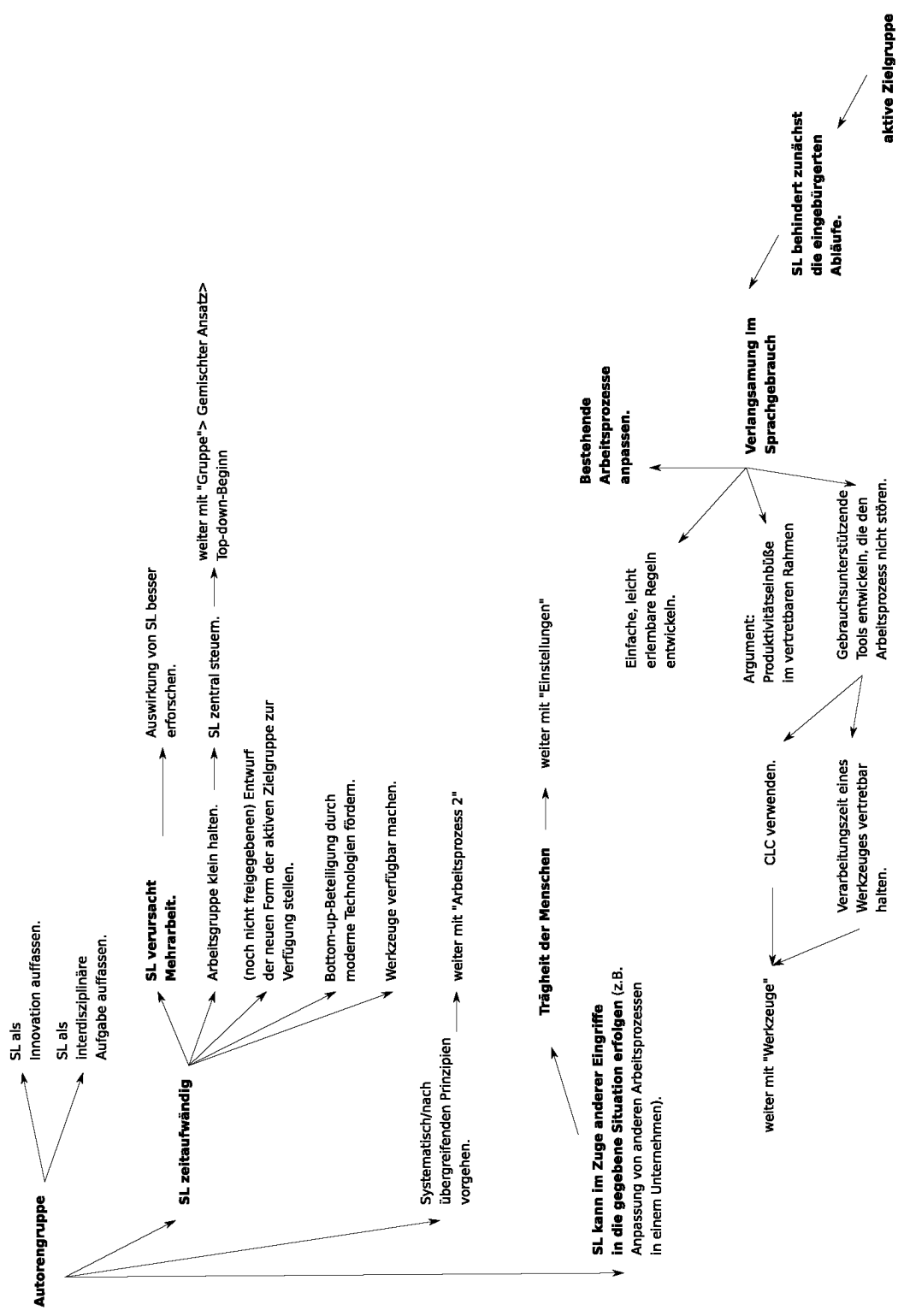

Abbildung 35.: Arbeitsprozess 1 


\section{Untersuchung}

u.a. in einer (anfänglichen) Verlangsamung im Sprachgebrauch niederschlägt. Um dem entgegenzuwirken, ist eine Anpassung von bestehenden (Arbeits-) Prozessen notwendig. Außerdem soll die neue Form leicht erlernbar sein und ihr Gebrauch durch unterstützende Tools wie CLC gefördert werden. Außerdem wird beobachtet, dass es sich oft lediglich um Anlaufschwierigkeiten handelt und sich die Produktivitätseinbußen im vertretbaren Rahmen halten, was ggf. als Argument verwendet werden kann.

Das zweite Diagramm behandelt wie erwähnt die Frage, wie eine systematische Vorgehensweise durch die Autorengruppe erreicht werden kann und welche Herausforderungen sich dabei stellen. Hiernach machen folgende Aspekte eine systematische Vorgehensweise aus: Definition und Festhalten von Arbeitsprozessen, Qualitätssicherung und Evaluation, organisatorische und inhaltliche Koordination der Arbeitsgruppe, Verfügbarmachen von Werkzeugen und Fortbestehen der Arbeitsprozesse. Ich greife im Folgenden einige dieser Punkte heraus.

Obwohl es naheliegend ist, dass Arbeitsprozesse klar definiert und die $\mathrm{Zu}-$ ständigkeiten bestimmt werden sollen, so wird beobachtet, dass diese Bestimmungen nicht immer vollständig umgesetzt werden und die SL somit de facto nicht vollständig systematisch erfolgt. Dies ist teilweise auf die (mangelnde) Praktikabilität der definierten Prozesse und Zuständigkeiten zurückzuführen. Deshalb wird empfohlen, dass man zwar ein systematisches und übersichtliches Managementkonzept im Voraus definieren soll, aber in der Umsetzung auch nach pragmatischen Gesichtspunkten handelt.

Die Qualität der SL soll der Quantität gegenüber eine übergeordnete Rolle spielen, hierfür müssen aber zunächst geeignete Benchmark-, Qualitäts- und Evaluationskriterien geschaffen werden, nicht zuletzt durch die Bestandsaufnahme und eine korpusbasierte Entwicklung der neuen Form. Darüber hinaus soll die Evaluation in jedem Umsetzungsschritt erfolgen.

Die Hauptherausforderung bei der inhaltlichen Abstimmung der Arbeitsgruppe über die Korpusplanung besteht in der Konsensfindung. Prinzipiell können Entscheidungen entweder autoritär oder demokratisch getroffen werden. Im zweiten Fall sollen inhaltliche Punkte grundsätzlich ausführlich ausdiskutiert werden, was Kompromissbereitschaft und diplomatisches Geschick der Beteiligten voraussetzt.

Werden SL-Werkzeuge eingesetzt, so wird empfohlen, ein Managementkonzept (Definition von Arbeitsprozessen, Zuständigkeiten etc.) zu entwickeln, bevor Softwarelösungen eingeführt werden. Generell können SL-Projekte danach unterschieden werden, in welchem Verhältnis die 
Korpusplanung zur Einführung von technologischen Lösungen steht. Einerseits kann die Korpusplanung übergeordnet sein und die Werkzeuge werden entsprechend der linguistischen Festlegungen implementiert. Auf der anderen Seite kann ein SL-Projekt softwaregeleitet sein, wenn die technischen Entwicklungen der Korpusplanung übergeordnet ist. Ein gemischter Ansatz, bei dem die technischen und sprachlichen Entwicklungen sich gegenseitig beeinflussen, ist ebenfalls möglich.

Schließlich wird darauf aufmerksam gemacht, dass eine systematische Arbeitsweise über einen längeren Zeitraum hinaus etabliert werden soll. Dies soll über eine feste Definition von Arbeitsprozessen erfolgen, wobei auf die Prozessintegrierbarkeit geachtet werden soll, was aber auch eine Anpassung von bestehenden Arbeitsprozessen bedeuten kann. Außerdem soll Korpusplanung an der Stelle ansetzen, wo neue Formen normalerweise ungelenkt entstehen, so dass Festlegungen möglichst zu einem frühen Zeitpunkt im Sprachgebrauch etabliert werden. Eine wichtige Herausforderung ist dabei, dass nachträgliche Änderungen von Festlegungen schwierig sein können. Deshalb wird empfohlen, mit SL klein anzufangen, die Korpusplanungsentscheidungen ausführlich in der Arbeitsgruppe auszudiskutieren, bevor sie zu Festlegungen werden, sowie den Gebrauch der neuen Form in einer kleinen Zielgruppe zu testen.

\subsubsection{Machtmittel/Sanktionen}

In der Datenbank findet sich lediglich eine Zuordnung. Die meisten Herausforderungen sind nicht zugeordnet und wurden von mir innerhalb des Themas Machtmittel gepaart.

Bei der Frage nach verfügbaren Machtmitteln zur Durchsetzung von SL ist die Unterscheidung zwischen einer Top-down-SL (links oben) und einer Bottom-up-SL (rechts unten) aufschlussreich.

Der Top-down-SL stehen verschiedene Machtmittel zur Verfügung, die insbesondere bei einer expliziten Sprachlenkung eingesetzt werden können. Zum einen kann das Nichteinhalten der neuen Norm mit verschiedenen Sanktionen belegt werden, wobei man hier auf das Phänomen achten muss, dass dann die neue Form möglicherweise nicht mehr eingehalten wird, sobald die Restriktionen wegfallen. Um dieses Phänomen zu umgehen, kann man den Gebrauch der neuen Form auf eine freiwillige Basis stellen oder aber versuchen, durch Einstellungsänderungen auch das Verhalten nachhaltig zu ändern. Zum anderen kann der Gebrauch der neuen Form von neuen Mitgliedern einer Gruppe 

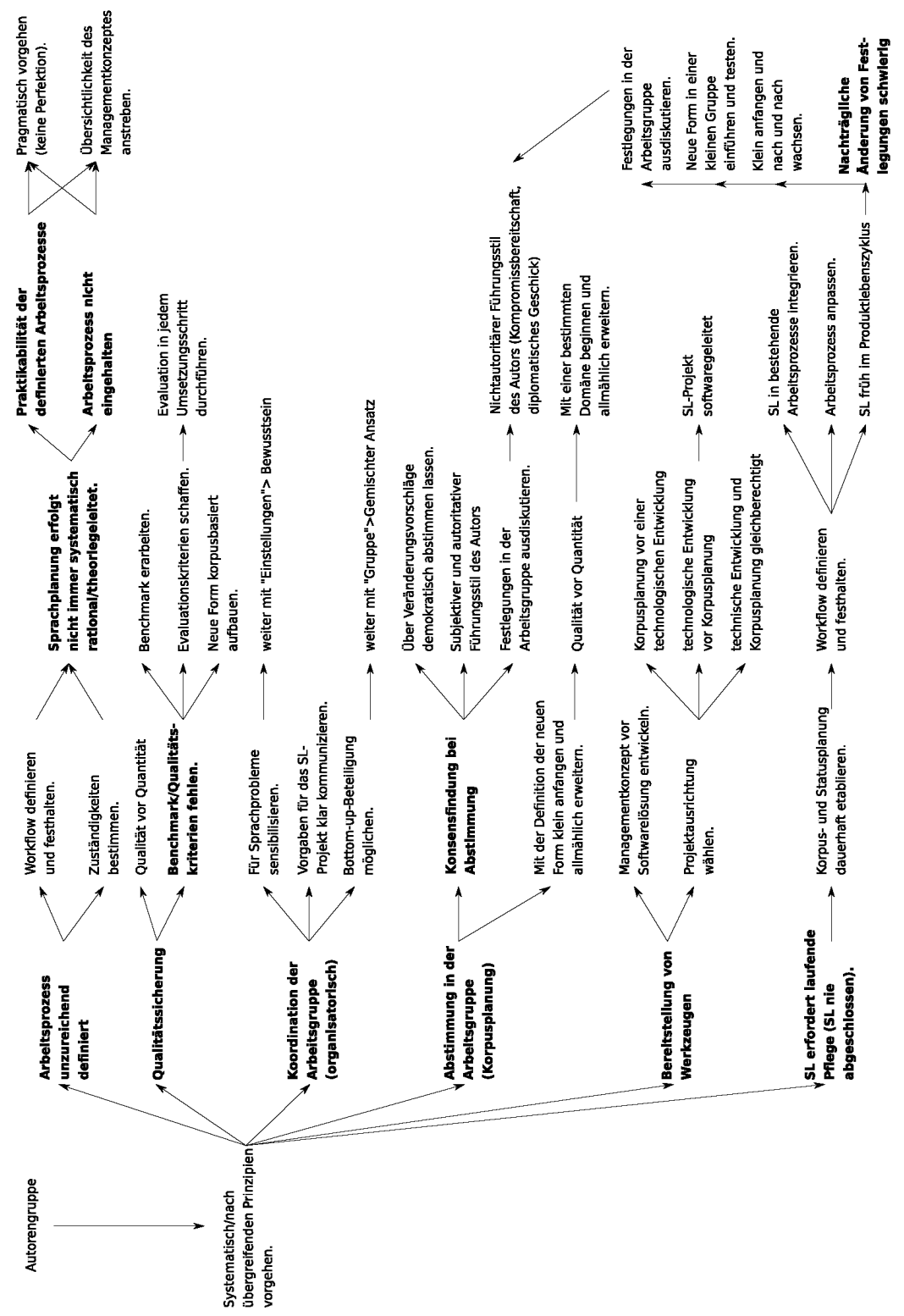

Abbildung 36.: Arbeitsprozess 2 


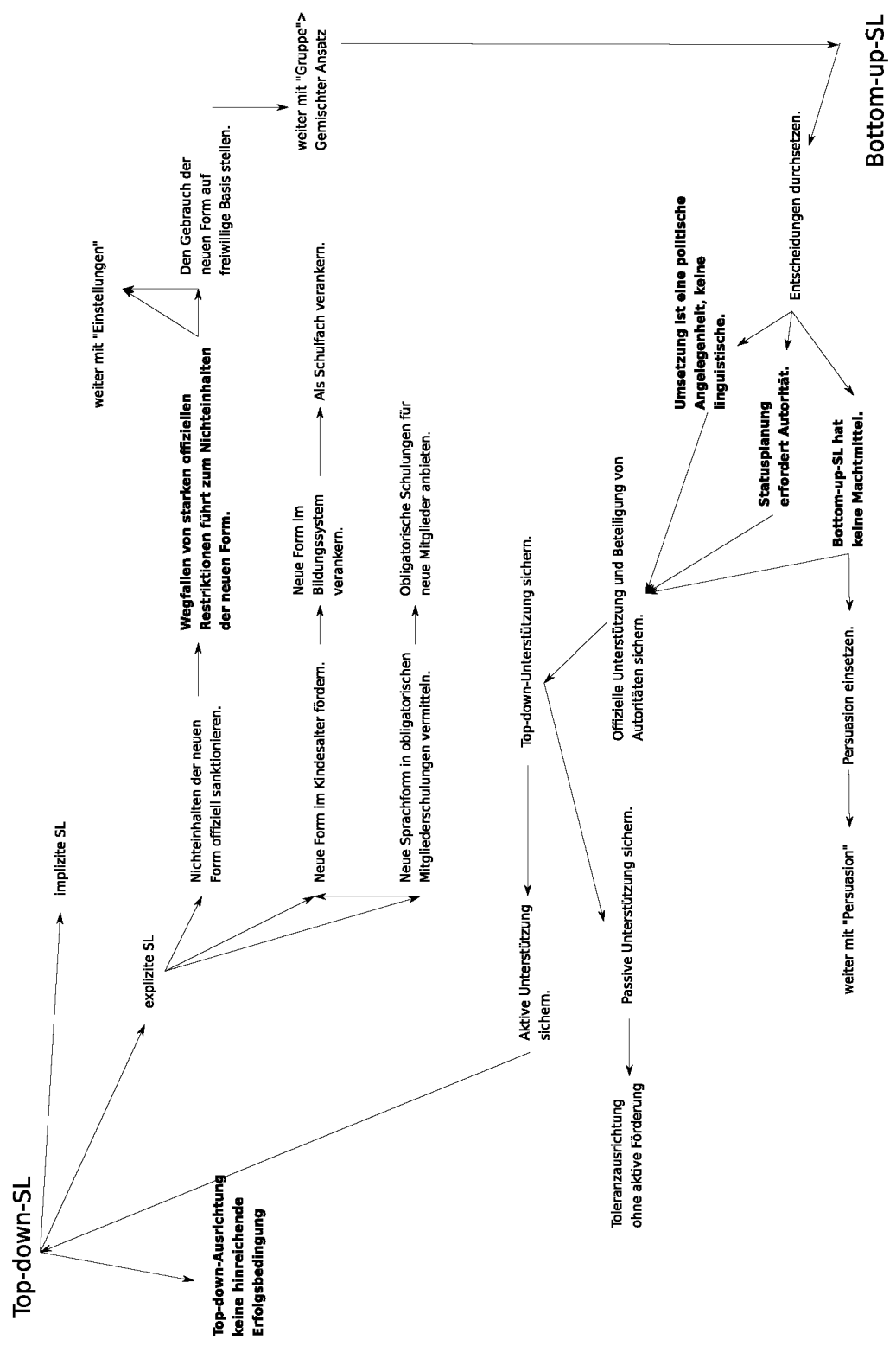

Abbildung 37.: Machtmittel/Sanktionen 


\section{Untersuchung}

als obligatorisch erwartet werden, wobei dies z. B. durch obligatorische Schulungen aktiv gefördert werden kann. Einen Spezialfall dessen stellt in diesem Kontext die Förderung des Gebrauchs im Kindesalter, die bei staatlicher Sprachlenkung durch die Verankerung der neuen Form im Bildungssystem ggf. als eigenständiges Fach unterstützt werden kann.

Bei einer reinen Bottom-up-Sprachlenkung stellt sich die Frage, wie Korpusplanungsentscheidungen durchgesetzt werden können. Als Herausforderungen sind hier also mangelnde Machtmittel einer Bottom-up-SL, die Frage, wie man auf anderen Wegen Autorität erlangen kann, sowie die Beobachtung, dass die Durchsetzung keine linguistische sondern eine politische Angelegenheit sei, zu nennen. Eine Möglichkeit, diese Probleme zu lösen, bietet die Persuasion. Sonst wird es als notwendig gesehen, offizielle Unterstützung und Beteiligung von Autoritäten sicherzustellen. Dabei kann entweder eine passive Top-down-Unterstützung mit sog. Toleranzausrichtung ohne aktive Förderung angestrebt werden, oder aber eine aktive Unterstützung, dank der die SL auf offizielle Machtmittel und Sanktionen zurückgreifen kann.

Wie im Diagramm zu erkennen ist, bedingen sich beide SL-Ausrichtungen (top-down und bottom-up) und können voneinander profitieren. Denn auch wenn in Theorie die Top-down-SL über Machtmittel verfügt, so stellen diese noch keine hinreichende Bedingung für den Erfolg einer Maßnahme dar, die von der gesamten aktiven Zielgruppe mit getragen werden muss.

\subsubsection{Werkzeuge}

In der Datenbank werden Werkzeuge-Herausforderungen Vorgehen aus folgenden Themen zugeordnet: Werkzeuge, Gruppe und vereinzelt Erlernbarkeit, Arbeitsprozess und Sprachsystem. Eigenzuordnungen beziehen sich auf weitere Werkzeuge-Vorgehen.

Werkzeuge können das Einführen und Durchsetzen der neuen Form unterstützen. Es kann sich dabei um speziell im Rahmen eines SL-Projektes entwickelte, aber auch um generische oder kommerzielle Lösungen handeln. Neben Empfehlungen zur Werkzeugart und ihren formalen Eigenschaften wird auf das Problem hingewiesen, dass verfügbare Werkzeuge von der aktiven Zielgruppe nicht genutzt werden. Für diese Herausforderung identifiziere ich keine geeignete Abhilfe.

Am ausführlichsten werden beim Thema Werkzeuge die Aspekte ihrer Benutzerfreundlichkeit behandelt. Diese kann unterstützt werden durch eine praxisnahe Anforderungsspezifikation und ggf. durch eine 
Bottom-up-Beteiligung bei der Entwicklung und Implementierung. Darüber hinaus spielen auch ihre Integrierbarkeit, Personalisierbarkeit, Funktionsadäquatheit und Bedienbarkeit (Benutzeroberfläche) eine zentrale Rolle, denen einzelne Vorgehensvorschläge zugeordnet sind. Ich greife an dieser Stelle zwei Punkte heraus. Bei Integrierbarkeit wird gefordert, dass ein Werkzeug den Arbeitsprozess nicht stören soll. Ich beobachte, dass dieser Forderung keine konkreten Umsetzungsvorschläge (Vorgehen) zugeordnet sind. Detaillierter wird hingegen auf Funktionsadäquatheit eingegangen, bei der vor allem die Prüf- und Korrekturverhalten eines CLC und die Rolle des Benutzers elaboriert werden.

\subsubsection{Einhalten}

In der Datenbank sind diesem Thema Vorgehen aus Werkzeuge, Erlernbarkeit und vereinzelt Identität, Einstellungen, Persuasion, Arbeitsprozess, Gebrauch, Sprachsystem zugeordnet.

Um das Einhalten der neuen Form zu erreichen, kann man zum einen auf ein freiwilliges Einhalten setzen, zum anderen aber auch Kontrollmöglichkeiten bereitstellen. Das freiwillige Einhalten kann mit dem Mere-ExposureEffekt unterstützt werden, wobei hier praktikable Zeitvorgaben und Eingewöhnungsphasen vorgesehen werden sollen. Darüber hinaus kann das Wissen über die neue Form verbreitet werden, wozu sowohl die Persuasion als auch Schulungen gebraucht werden können. Dadurch soll insbesondere die instrumentelle Funktion der neuen Form verbreitet werden, wobei zusätzlich die Vermittlung von formalen und wissenschaftlichen Hintergründen notwendig sein kann.

Um das Einhalten aktiv zu kontrollieren, kann der Gebrauch manuell oder maschinell geprüft werden. Je nach Prüfergebnis kann das Einhalten belohnt und das Nichteinhalten sanktioniert werden.

Wird die neue Form eingehalten und etabliert sie sich tatsächlich im Gebrauch, so kann der Sprachwandel einsetzen, der eine Herausforderung für die Einheitlichkeit der neuen Form darstellt. Um den Sprachwandel gering zu halten, kann die neue Form primär für instrumentelle Zwecke eingesetzt werden, ohne dass sie vorrangig die identitätsstiftende Funktion übernimmt. Die neue Form soll sich folglich nicht als Muttersprache etablieren. 


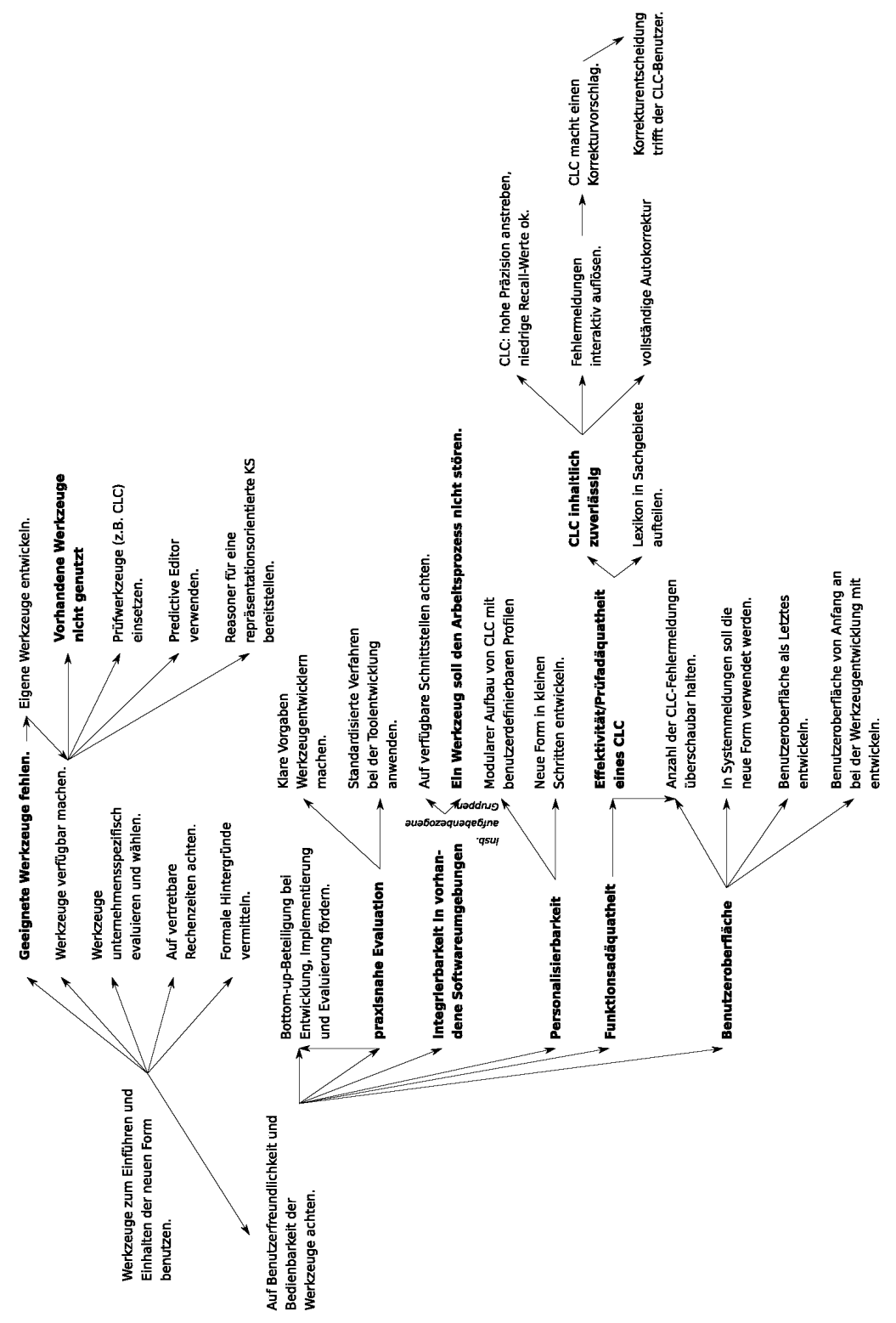

Abbildung 38.: Werkzeuge 


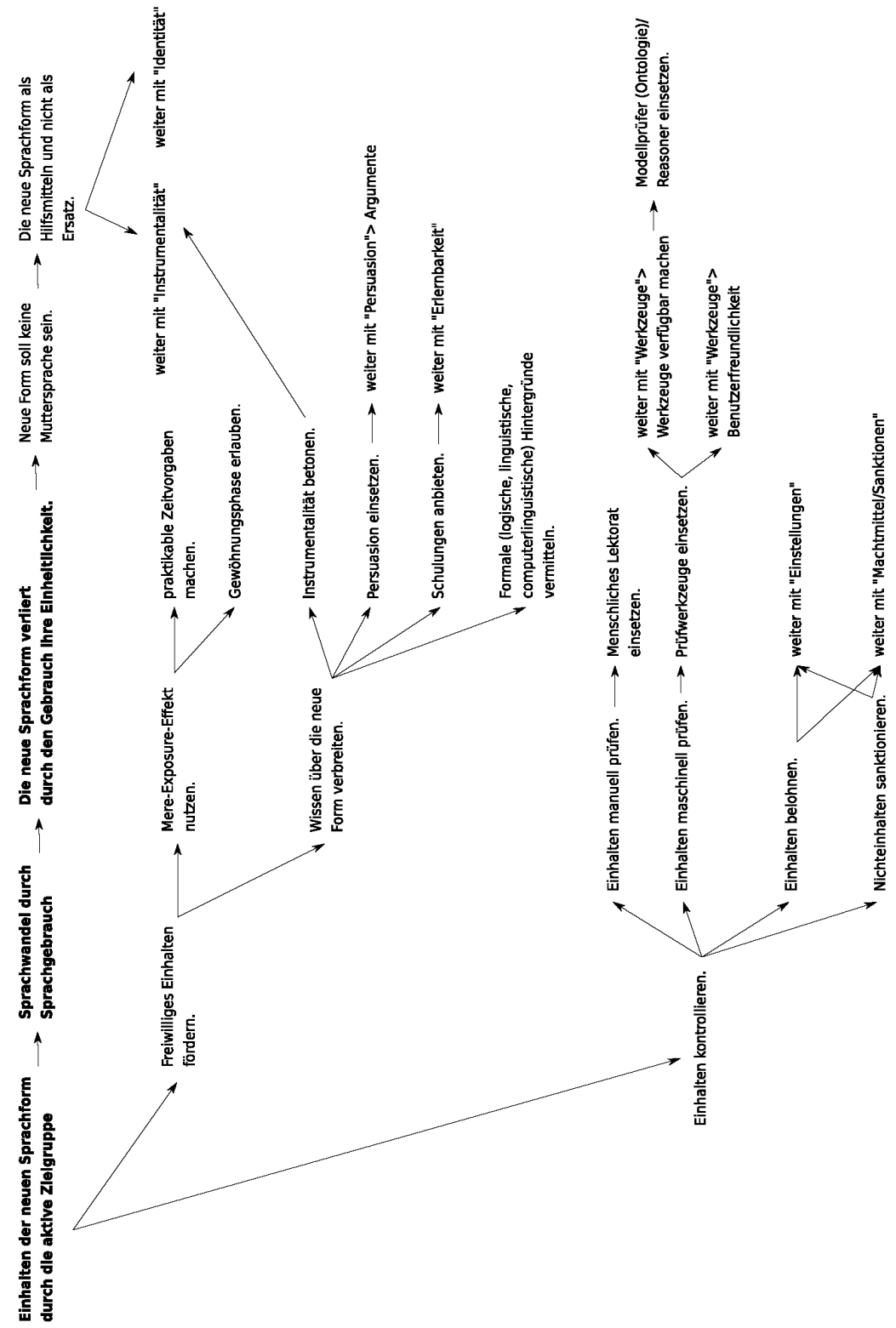

Abbildung 39.: Einhalten 


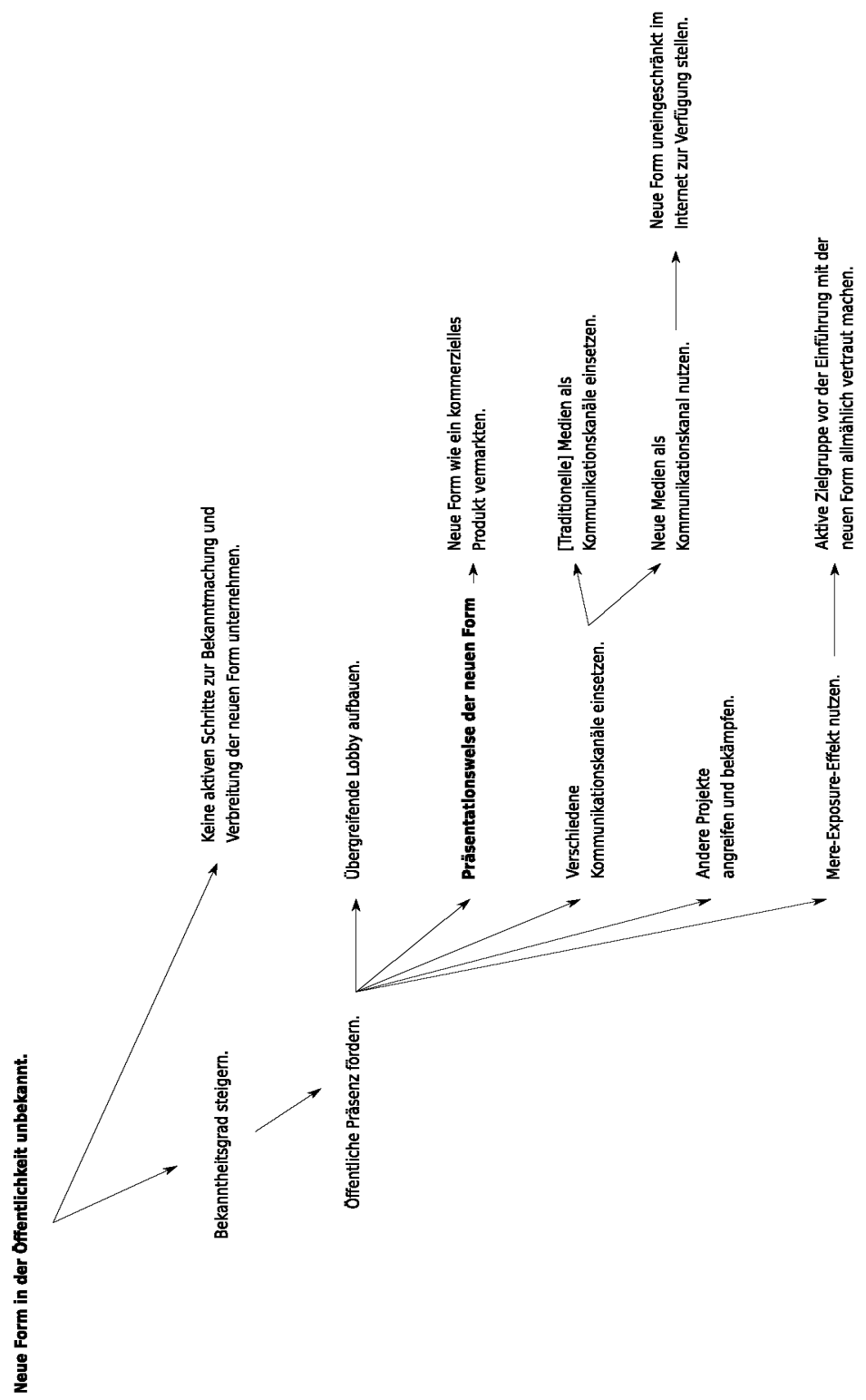

Abbildung 40.: Bekanntmachen 


\subsubsection{Bekanntmachen}

In der Datenbank findet sich nur eine Zuordnung, und zwar zu Sprachökologie-Vorgehen. Die Eigenvorschläge beziehen sich auf Bekanntmachen.

Bei diesem Thema ist die übergreifende Herausforderung, dass die neue Form in der Öffentlichkeit bzw. in der aktiven Zielgruppe unbekannt ist. Wenn keine Maßnahmen zur Bekanntmachung und Verbreitung der neuen Form unternommen werden, so endet SL mit Korpusplanung. Es wird jedoch empfohlen, aktive Schritte zu unternehmen, um den Bekanntheitsgrad zu steigern und die Präsenz der neuen Form in der Zielgruppe zu fördern. Hierzu empfiehlt man den Aufbau einer übergreifenden Lobby sowie die Vermarktung der neuen Form wie ein kommerzielles Produkt. Genutzt werden sollen verschiedene Kommunikationskanäle: Neben den traditionellen Kanälen wird auf neue Medien hingewiesen, insbesondere auf Möglichkeiten, die eine uneingeschränkte Verbreitung der neuen Form im Internet bietet. Öffentliche Aufmerksamkeit kann man zudem fördern, indem man sich mit anderen Formen oder Projekten aktiv auseinandersetzt. Dem Bekanntmachen kommt auch der Mere-Exposure-Effekt zunutze, insbesondere wird empfohlen, dass die aktive Zielgruppe bereits während der Korpusplanung mit der SL-Maßnahme vertraut gemacht wird, um diese Vertrautheit für die Statusplanung zu nutzen.

\subsubsection{Gebrauch}

In der Datenbank sind diesem Thema beinahe alle Vorgehen-Themen zugeordnet, wobei Gruppe am häufigsten vertreten ist. Die nicht vorhandenen Themen werden durch Eigenvorschläge vollständig ergänzt.

Bei diesem Thema kommt schließlich die Überlegung zum Ausdruck, dass es bei Sprachlenkung nicht einfach auf den Vorschlag der neuen Form im Sinne der Korpusplanung, sondern auf ihre Etablierung im Sprachgebrauch ankommt. Obwohl man eine neue Sprachform durchaus normativ perfektionieren kann, bevor sie für den Gebrauch freigegeben wird, wird auch gleichzeitig die Stabilität der neuen Form vor ihrer Perfektionierung gefordert. Allgemein sollte man bei SL also auf Gebrauch statt Perfektion setzen. Denn Statusplanung ist für erfolgreiche Sprachlenkung unabdingbar.

Eine Herausforderung für jede neue Form ist die Unsicherheit, wie diese im tatsächlichen Gebrauch verwendet werden soll. Um diese Unsicherheit abzubauen, kann nur ein kleiner Kern der neuen Form festgelegt sein und die Autoren sollen die normativen Eingriffe ins Sprachsystem gering halten. Zu- 


\section{Untersuchung}

dem sollen Gebrauchsbeispiele, z. B. in Form von Texten, vor der Statusplanung bereitgestellt sein. Diese Vorgehen öffnen jedoch die neue Form für den Sprachwandel, der die Einheitlichkeit der neuen Form verringern kann. Einen Gegenpol hierzu stellt dann der subjektive und autoritative Führungsstil der Autoren, die die oberste Autorität in Sachen Sprachgebrauch bleiben.

Neben der allgemeinen Beobachtung zur Statusplanung (links oben), dass SL über die reine Korpusplanung hinausgehen und als Innovation und interdisziplinäre Aufgabe aufgefasst werden muss, findet sich rechts davon eine Reihe von speziellen Vorgehen, die eingesetzt werden können, um die Statusplanung im Sinne der Gebrauchsförderung erfolgreich zu gestalten. Die konkreten Kategorien sind an dieser Stelle weniger interessant. Als wichtiger erscheint mir vielmehr die Beobachtung, dass diese Kategorien weitestgehend alle hier betrachteten Themen ${ }^{137}$ anschneiden. Diese Beobachtung führt mich zur Interpretation, dass die Etablierung des Sprachgebrauchs, also die Statusplanung, das übergreifende Thema für Sprachlenkung ist, das alle anderen hier behandelten Themen als Instrumente benutzt.

\subsection{Untersuchung - Fazit}

In diesem Kapitel habe ich eine qualitative Metaanalyse der Umsetzungsmöglichkeiten von Sprachlenkung durchgeführt. Mit anderen Worten habe ich den Forschungsstand bezüglich der postulierten Herausforderungen untersucht, die sich bei der Umsetzung von Sprachlenkung stellen, sowie der Handlungsmöglichkeiten, die die Autoren haben, um diese Herausforderungen zu bewältigen.

Als Ausgangsdaten für die Metaanalyse benutzte ich Zitate, die ich mit einer vorgeschlagenen Methode und unter Einsatz einer relationalen Datenbank in mehreren Schritten verallgemeinerte. Die Rohergebnisse dieser Auswertung unterzog ich einer weiteren Analyse und Interpretation, wofür die vorgestellten Konstrukte der Sozialpsychologie - Identität, Gruppe und Einstellungen - richtungsweisend waren. Das Hauptziel war dabei, eine gebietsübergreifende, netzartige Struktur von Handlungsmöglichkeiten aufzudecken.

Die Auseinandersetzung mit den Rohergebnissen hat gezeigt, dass sich die vorgestellten Theorien und Konstrukte aus der Sozialpsychologie gut eignen, um die Rohergebnisse in einschlägigen Themen zu strukturieren und weitere Zusammenhänge aufzudecken. Die Ergebnisse für diese Themen fallen auch

137 Alle Themen bis auf Kosten sind vertreten, was m. E. nicht als ein empirischer Hinweis auf eine geringe Relevanz dieses Themas, sondern als Lücke in meinen Daten interpretiert werden soll. 


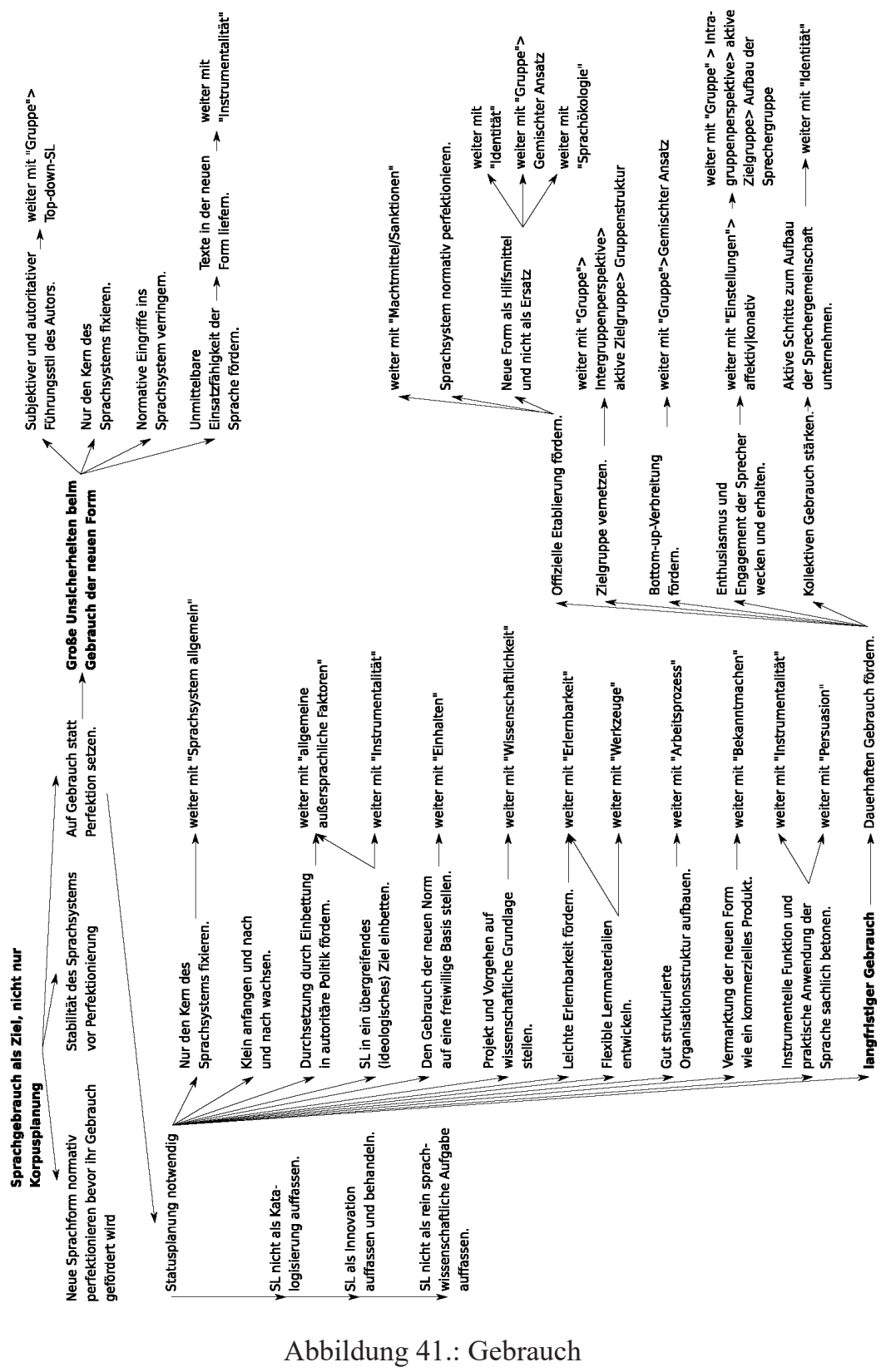




\section{Untersuchung}

ausführlicher aus, während andere Themen nur angerissen sind. Dies kann auch teilweise damit zusammenhängen, dass eine sozialpsychologische Interpretation bereits vor der Datenerfassung vorgesehen war und ich die Daten aus einer festen Forschungsperspektive erfasste. Dies stellt m. E. das Hauptergebnis an sich nicht in Frage, sondern zeigt, dass andere Diskurse nötig sind, um die übrigen Themen in allen Einzelheiten zu erforschen.

Das Hauptergebnis der Untersuchung sind 21 Ablaufdiagramme, die für 16 der 18 festgestellten übergreifenden Themen veranschaulichen, welche Herausforderungen sich bei der Umsetzung der Sprachlenkung stellen und wie Autoren entsprechend handeln können. Diese Ablaufdiagramme geben den Forschungsstand lediglich wieder, sind also beschreibend und nicht bewertend. Dies bedeutet, dass ich die festgestellten Vorgehen nicht weiter auf ihre Plausibilität oder Wirksamkeit hin untersuchte. Zwar ist in der Datenbank die behauptete Wirksamkeit von Vorgehen mit erfasst, eine Auswertung, Analyse und Diskussion der Wirksamkeit würde jedoch den Rahmen dieser Arbeit übersteigen. Die Daten stehen zur weiterführenden Forschung zur Verfügung. Somit sind die Diagramme als Bestandsaufnahme zu verstehen und die Wirksamkeitsbewertung bleibt als Desiderat.

Die Analyse ergibt, dass Gebrauch als übergeordnetes Thema betrachtet werden kann. Den Gebrauch der neuen Form (langfristig) zu sichern, stellt also die übergeordnete Handlung innerhalb der Sprachlenkung dar. Die übrigen 16 Themen (einschließlich Sprachsystem) können einerseits als Einflussfaktoren betrachtet werden, die sich auf die Sicherung des Gebrauchs erschwerend auswirken; andererseits aber können sie auch als Instrumente aufgefasst werden, mit denen der Gebrauch gefördert werden kann. Obwohl der Schwerpunkt der Untersuchung auf Statusplanung lag, verdeutlichen diese Ergebnisse, insbesondere die Bedeutung von sprachsystembezogenen Vorgehen für die Sicherung des Sprachgebrauchs, dass für die praktische Sprachlenkung eine Trennung von Korpus- und Statusplanung konzeptionell zwar möglich ist, umsetzungstechnisch aber nicht unbedingt die Methode der Wahl darstellen sollte. Betrachtet man nämlich die festgestellten vier idealtypischen SLFormen: reine Top-down- bzw. Bottom-up-SL sowie zwei gemischte Ansätze mit Top-down- bzw. Bottom-up-Beginn, so sieht man, dass der Einsatz von gemischten Ansätzen als Lösung für die Probleme der reinen Ansätze, insbesondere der Transparenz und Akzeptanz bei der Top-down-SL, aufgefasst werden kann. Denn bereits bei der Herausarbeitung des Korpus soll aus Statusplanungsgründen die aktive Zielgruppe mit involviert werden. Abstrakt betrachtet, kann für die Praxis also eine Aufhebung der konzeptionellen Tren- 
nung zwischen Korpus- und Statusplanung sowie eine engere Verflechtung beider Konzepte als eine weitere Handlungsmöglichkeit zur Sicherung des Sprachgebrauchs aufgefasst werden.

Im Vergleich mit den eigenen, in dieser Arbeit vorgestellten Konzeptualisierungen fällt insbesondere auf, dass die Untersuchungsergebnisse mit ihren 16 Themen mehr Einflussfaktoren aufdecken, als ich in dem weitestgehend deduktiv aufgestellten Umsetzungsorientierten Modell der Sprachlenkung in 4.9 postuliere, das in erster Linie lediglich auf Identität und teilweise auf Einstellungen eingeht. Andererseits nehme ich in diesem Modell an, dass sich die möglichen Vorgehen von Autoren nach dem Gruppentyp unterteilen lassen, was ich aber in der Untersuchung nur ansatzweise zeigen konnte. Das bereits erwähnte Desiderat der Wirksamkeitsbewertung müsste zukünftig erweitert werden, indem der Gruppentyp als Differenzierungskriterium mit untersucht wird.

Der Vergleich der 16 Themen mit den Kategorien, die ich zur weiterführenden Beschreibung der Sprachlenkung in 3.4.2 identifiziert und in Tabelle 9 zusammengefasst habe, zeigt, dass sich beide Ergebnisse nur geringfügig überlappen. Dies ist nicht weiter überraschend. Zum einen fußen sie auf anderen Perspektiven bei der Datenerhebung: Die Kategorien aus 3.4.2 sind das Resultat einer selektiven Erfassung des Forschungsstandes anhand der in Kapitel 2 vorgestellten Themenkomplexe. Diese Themenkomplexe spielten bei der Erfassung der Zitate für die Untersuchung eine geringe Rolle, denn ich nahm die Zitate anhand einer vagen Vorstellung vom SL-Erfolg durch nichtsystemlinguistische Faktoren in die Datenbank auf. Darüber hinaus verfolgen beide Auflistungen andere Zielsetzungen: Die Themen charakterisieren die Umsetzung, die Kategorien aus 3.4.2 dienen hingegen zur Beschreibung der Sprachlenkung an sich und können auch für eine feinere Unterteilung der Sprachlenkung eingesetzt werden. Vor diesem Hintergrund bietet es sich an, die Kategorien aus 3.4.2 auch daraufhin zu prüfen, ob sie parallel zum Gruppentyp die gesuchte feinere Differenzierung der identifizierten Vorgehen in jedem der 16 Themen leisten können. Das erwähnte Forschungsdesiderat wird also abermals erweitert.

Zusammenfassend lässt sich sagen, dass die Diagramme sowohl von wissenschaftlichem als auch praktischem Interesse sind. Als Ergebnis einer Metaanalyse stellen sie eine Grundlage für eine vertiefte Auseinandersetzung mit der Durchführung von Sprachlenkungsmaßnahmen dar. Insbesondere ermöglichen sie Hypothesenbildung und anschließende empirische Nachforschung und Validierung der hier erarbeiteten Inhalte, beispielsweise speziell 


\section{Untersuchung}

der Wirksamkeit von Vorgehen. Die in dieser Arbeit geleistete Einbettung in den sozialpsychologischen Diskurs schafft Voraussetzungen für den Einsatz von quantitativen Methoden empirischer Forschung. Eine Nachuntersuchung mit denselben Daten ist ebenfalls möglich, durch die besprochene technische Entkoppelung des Hauptergebnisses von der Datenbank jedoch erschwert.

Für Praktiker sind Diagramme ein guter Ausgangspunkt, um sich einen Überblick über Stolpersteine und Handlungsmöglichkeiten bei Sprachlenkungsmaßnahmen zu verschaffen und dabei auf die Erfahrung verschiedener Gebiete zurückzugreifen. Die Diagramme können also bei der Vorbereitung einer eigenen SL-Maßnahme hilfreich sein. Allerdings kann der Praktiker eine gute Fallunterscheidung vermissen, da die Diagramme ggf. redundante oder nichtzutreffende Inhalte für ein konkretes Szenario enthalten. Dies ist darauf zurückzuführen, dass diese Arbeit die Schaffung einer übergreifenden SL-Perspektive als Ziel hatte, die erst im Zuge weiterer wissenschaftlicher Erforschung und Rückmeldung aus der Praxis verfeinert werden kann. Die hier benutzten Kriterien des Gruppentyps und der Gruppengröße können hierbei einen Anhaltspunkt bieten. 


\section{Fazit}

Ziel vorliegender Arbeit war die Etablierung der Sprachlenkung als eines übergreifenden Forschungsgegenstandes, unter dem verschiedene praktische Maßnahmen zu subsumieren sind, die auf eine nachhaltige Veränderung des Sprachgebrauchs einer Gruppe von Menschen abzielen. Zu diesem Zweck verfolgte ich zwei allgemeine Forschungsfragen, die zum einen die Gegenstandbestimmung zum anderen aber den Erfolg von Sprachlenkung betrafen:

1. Mit welchen Mitteln lässt sich Sprachlenkung als Gegenstand übergreifend bestimmen?

2. Welche Möglichkeiten, den Erfolg der Sprachlenkung zu beeinflussen, werden in der Forschung genannt?

Diese allgemeinen Fragen lassen sich weiter spezifizieren, wobei sich der Schwerpunkt der zweiten Forschungsfrage im Verlauf dieser Arbeit aufgrund der Forschungslage verschoben hat.

Zur Beantwortung dieser Fragen ging ich synthetisch vor, indem ich zunächst vier verschiedene linguistische Gebiete - Sprachplanung und Sprachpolitik, Plansprachen, Kontrollierte Sprachen und Terminologien anhand deduktiv ausgewählter Aspekte betrachtete. Diese innerdisziplinären Erkenntnisse verband ich mit interdisziplinären Bezügen, wobei ich insbesondere auf die Handlungs- und Motivationspsychologie sowie die Sozialpsychologie zurückgriff. Dabei zeigte sich, dass beide Disziplinen einen wertvollen Beitrag zur Strukturierung und theoretischen Untermauerung der bruchstückhaften linguistischen Erkenntnisse leisten konnten.

In der bisherigen Forschung wird Sprachlenkung nur ansatzweise in ihrer vollen Breite erforscht. Als entscheidende Antwort auf meine erste Forschungsfrage halte ich fest, dass sich Sprachlenkung übergreifend bestimmen lässt, was ich anhand einer festlegenden Minimaldefinition vornahm. Eine integrative Sicht kann darüber hinaus mit den in der Linguistik bereits vorhandenen Mitteln vorgenommen werden: Wie ich gezeigt habe, können zu einer näheren Gegenstandcharakterisierung bekannte system-, sozio-, pragmalinguistische sowie extralinguistische Faktoren herangezogen werden. Diese 


\section{Fazit}

Faktoren können auch zu einer weiteren, systematischen Ausdifferenzierung der Sprachlenkung eingesetzt werden, die eine Alternative für die pragmatisch entstandene und historisch gewachsene Aufteilung der Forschung in die vier genannten Gebiete darstellen könnte. Diese Ausdifferenzierung konnte jedoch im Rahmen dieser Arbeit nicht geleistet werden. Zur ersten Forschungsfrage konstatiere ich zudem, dass alle hier betrachteten Gebiete einen nutzbringenden Beitrag zur Theoriebildung der Sprachlenkung leisten. LPLP ist zwar das Gebiet, das bereits übergreifende Definitionen hervorbrachte und dessen Konzeptualisierungen sich auf andere Gebiete anwenden lassen. Die anderen Gebiete aber machen auf Aspekte wie Instrumentalität, Systematik und Top-down-Präskriptivität aufmerksam, die in LPLP entweder noch nicht oder nicht mehr verfolgt werden. Eine Erkenntnissynthese bedeutet also für alle Gebiete einen Zugewinn.

Was die zweite Forschungsfrage angeht, so sehe ich die Forschungslage als insgesamt unbefriedigend. Diese Einschätzung bezieht sich sowohl auf die systematische, theoretische Fassung bzw. Messbarkeit von SL-Erfolg als auch auf die Formulierung von konkreten Umsetzungsempfehlungen. Insbesondere betreffen diese Unzulänglichkeiten die übergreifende SL-Forschung, aber auch in unterschiedlichem Maße die einzelnen Gebiete: Die Terminologiearbeit kann auf einige, bereits ein wenig verallgemeinerte Überlegungen zurückgreifen, während Kontrollierte Sprachen die Umsetzung der Sprachlenkung unter dem nichtsystemlinguistischen Erfolgsaspekt nur in einzelnen Projektberichten anschneiden, in der systematischen Forschung aber nicht weiter aufgreifen. Plansprachen können hingegen auf eine begrenzte Anzahl von Daten aus der Praxis zurückgreifen. Schließlich scheint die Frage nach der Lenkbarkeit der aktiven Zielgruppe durch die SL-Autoren, also dem, was ich in dieser Arbeit als Top-down-Erfolg bezeichne, in LPLP problematisch zu sein, weil dies im Konflikt zu LPLP-Leitansätzen wie den sprachlichen Menschenrechten steht. Um die Lücke um den theoretischen Erfolgsbegriff zu schließen, schlug ich in dieser Arbeit eigene Konzeptulisierungen vor, die zwar die linguistischen Vorüberlegungen im Hintergrund behalten, aber im Unterschied zu Überlegungen zur Gegenstandsbestimmung extralinguistisch geprägt sind und sich im wesentlichen auf Modellierungen aus den genannten psychologischen Gebieten stützen. Ich reduziere dabei den Begriff von Erfolg von Sprachlenkung auf den Erfolgsbegriff von einzelnen Maßnahmen. Das erste Modell ist dabei systematisierend und ermöglicht konzeptionell eine klare Trennung zwischen dem linguistischen und dem übergreifenden, nichtlinguistischen Erfolg. Als Überleitung von theoretischen zu praktischen As- 
pekten des SL-Erfolges schlug ich das zweite Umsetzungsmodell der Sprachlenkung vor. Das Modell diente als Befangenheitsoffenlegung und Interpretationsgrundlage für die darauffolgende Metaanalyse von Best Practice. Die ursprüngliche spezifische Forschungsfrage nach der Wirksamkeit von Autorenhandlungen musste aufgrund der beschriebenen Forschungslage modifiziert werden, denn im Unterschied zur allgemeinen Gegenstandbestimmung existieren bislang nicht genug theoretische fundierte Best-Practice-Kataloge, die ich auf ihre Verallgemeinerbarkeit, Übertragbarkeit und (kontextspezifische) Wirksamkeit hin untersuchen konnte. Aus dieser analytischen Forschungsfrage entstand also eine synthetische: nach den in der Literatur postulierten Handlungsmöglichkeiten für Autoren im Allgemeinen, ohne ihre Wirksamkeit zu bewerten. Neben den erwähnten theoretischen Definitionen und Konzeptualisierungen bleiben als Hauptresultat dieser Arbeit 21 induktiv entstandene Ablaufdiagramme, die das fragmentarische Wissen über Herausforderungen und entsprechende Handlungsmöglichkeiten bei Sprachlenkung aus den vier Gebieten zu einer Gesamtperspektive integrieren. Aus meiner Sicht stellen die Mechanismen der gemischten Top-down-/Bottom-up-Ansätze eine der wichtigsten Erkenntnisse aus den Diagrammen dar. Diese Ansätze werden in einem Kompromiss zwischen den Bedürfnissen der Zielgruppe und dem Lenkungswunsch der Autoren realisiert, was wiederum bedeutet, dass der Aspekt der Top-down-Erfolges in diesen Ansätzen nicht vollständig vernachlässigt werden kann. Die wissenschaftliche Erforschung von Top-downErfolg ist somit durchaus berechtigt und man sollte sich ihr in der Wissenschaft nicht verschließen. Dies betrifft speziell die Linguistik, in der häufig deskriptive Forschungsfragen im Vordergrund stehen. Denn im Unterschied zu der von Kloss (1969) postulierten Zuständigkeit von Nichtlinguisten für die Statusplanung zeigen die Ergebnisse der Untersuchung, dass die Berücksichtigung von Zielgruppenbedürfnissen bereits bei der Korpusplanung erfolgen muss, was aber eindeutig zur linguistischen Domäne gehört. Die Erforschung von Top-down-Erfolg alleine unter diesem Aspekt gehört also zur Aufgabe der (angewandten) Linguistik.

Obwohl vorliegende Arbeit praktisch motiviert war und sie insbesondere durch die Ablaufdiagramme wichtige Implikationen für die Praxis enthält, war ihr Anliegen hauptsächlich wissenschaftlicher und theoretischer Natur. Insbesondere verstehe ich sie als Beitrag zur (angewandt-)linguistischen Grundlagenforschung. Weitere Theoriebildung erachte ich dabei für nötig, was weiterhin aus einer übergreifenden Perspektive und im Dialog der einschlägigen Gebiete erfolgen soll. Als wichtigstes Desiderat sehe ich 


\section{Fazit}

zudem die empirische Validierung der Erkenntnisse, insbesondere der Ablaufdiagramme, mithilfe von qualitativen und quantitativen Methoden. Hierbei soll speziell eine Wirksamkeitsüberprüfung von festgestellten Handlungsmöglichkeiten erfolgen. Insbesondere sehe ich durch eine Anbindung an psychologische Konstrukte einen erleichterten Zugang zu quantitativen Methoden. Abgeleitet davon halte ich eine differenziertere Betrachtung dieser Handlungsmöglichkeiten für notwendig, da dies aufgrund der synthetischen Ausrichtung dieser Arbeit und der damit einhergehenden Ausblendung von Details nur unzureichend erfolgen konnte. Diese Ausdifferenzierung kann beispielsweise im Zuge der Wirksamkeitsüberprüfung und anhand der hier festgestellten system-, sozio-, pragma- und extralinguistischen Kategorien erfolgen. 


\section{Literatur}

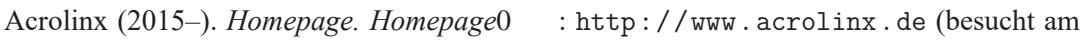
10. 02.2015)

Adams, Michael (2011a). „Owing language“. In: From Elvish to Klingon. Exploring invented languages. Hrsg. von Michael Adams. Oxford: Oxford University Press. Kap. Appendix $1,227-233$

Adams, Michael (2011b). „The spectrum of invention“. In: From Elvish to Klingon. Exploring invented languages. Hrsg. von Michael Adams. Oxford: Oxford University Press, 116

Adriaens, Geert und Lieve Macken (1995). „Technological evaluation of a controlled language application: precision, recall and convergence tests for SECC“. In: 6th International Conference on Theoretical and Methodological Issues in Machine Translation. (Leuven, 5.-7. Juli 1995), 123-141

Ager, Dennis (2001). Motivation in language planning and language policy. Clevedon: Multilingual Matters Ltd

Ager, Dennis (2005). „Image and prestige planning“. In: Current issues in language planning $6.6,1-43$

Ajzen, Icek (1991). „The theory of planned behavior“. In: Organizational behavior and human decision processes 50.2, 179-211

Ajzen, Icek und Martin Fishbein (1977). „Attitude-behavior relations: a theoretical analysis and review of empirical research“. In: Psychological bulletin 84.5, 888-918

Allen, Jeffrey und Christopher Hogan (2000). ,,Toward the development of a post-editing module for machine translation raw output. A controlled language perspective". Presented at the Third International Controlled Language Applications Workshop (CLAW2000), held in Seattle, Washington, 29-30 April 2000. Datei-Seitenzählung als Seitenangabe benutzt. 10Dhttp: //www . oocities .org/mtpostediting/allen-hogan-claw.doc

Almqvist, Ingrid und Anna Sågvall Hein (1996). „Defining ScaniaSwedish. A controlled language for truck maintenance“. In: Proceedings of the first international workshop on controlled language applications. The first international workshop on controlled language applications, CLAW 1996. (Katholieke Universiteit Leuven, Belgien, 26.-27. März 1996), $159-165$

Amtrup, Jan W. (2010). „Aspekte der Computerlinguistik“. In: Computerlinguistik und Sprachtechnologie. Eine Einführung. Hrsg. von Kai-Uwe Carstensen, Christian Ebert, Cornelia Ebert, Susanne Jekat, Ralf Klabunde und Hagen Langer. 3. überarbeitete und erweiterte Aufl. Heidelberg: Spektrum, Akademischer Verlag. Kap. 1.1, 1-17

Andersen, Henrik und Ellen Christoffersen (2006). „The Janus head article. On quality in the documentation process". In: Language at work 1 (1). PDF-Seitenzählung als Seitenangabe verwendet, o.S. $\square \square \square$ http : //ojs . statsbiblioteket.dk/index . php/law/ article/download/6235/5410 


\section{Literatur}

Angelov, Krasimir und Ramona Enache (2012). „Typeful ontologies with direct multilingual verbalization“. In: Controlled Natural Language. Second International Workshop, CNL 2010. (Marettimo Island, Italy, 13.-15. Sep. 2010). Hrsg. von Michael Rosner und Norbert E. Fuchs. Lecture Notes in Artificial Intelligence 7175. Berlin/Heidelberg: Springer, 1-20

Antia, Bassey E. (2015). „Language policy and terminology in south africa“. In: Handbook of terminology. Hrsg. von Hendrik J. Kockaert und Frieda Steurs. Bd. 1. Amsterdam/Philadelphia: John Benjamins Publishing Company, 467-489

Anton, Günter (2002). „Einige Bemerkungen zu Ido und zur Ido-Bewegung heute“. In: Plansprachen und ihre Gemeinschaften. Beiträge der 11. Jahrestagung der Gesellschaft für Interlinguistik e.V. (Berlin, 23.-25. Nov. 2001). Hrsg. von Detlev Blanke. Gesellschaft für Interlinguistik e.V., 22-26

Apeltauer, Ernst (2006). Grundlagen des Erst- und Fremdsprachenerwerbs. Eine Einführung. Fernstudieneinheit 15. Berlin u.a.: Langenscheidt

Archives of the Conlang Mailing List, 1991-94 (1991-1994). पण口 http : //www.glossopoeia.org/conlang (besucht am 05.03.2014)

Arntz, Reiner, Heribert Picht und Klaus-Dirk Schmitz (2014). Einführung in die Terminologiearbeit. 7., vollständig überarbeitete und aktualisierte Aufl. Hildesheim: Olms

ASD-STE100 (2013). Simplified Technical English. Specification ASD-STE100. International specification for preparation of maintenance documentation in a controlled language. ASD Industries Association of Europe

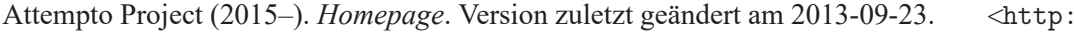
//attempto.ifi.uzh.ch/site/ (besucht am 11.02.2015)

Back, Otto (1979). „Über Systemgüte, Funktionsadäquatheit und Schwierigkeit in Plansprachen und in ethnischen Sprachen“. In: Terminologie als angewandte Sprachwissenschaft. Gedenkschrift für Univ.-Prof. Dr. Eugen Wüster. Hrsg. von Helmut Felber, Friedrich Lang und Wersig Gernot. München: K.G. Saur, 257-272

Back, Otto (1996). „Plansprachen“. In: Kontaktlinguistik. Ein internationales Handbuch zeitgenössischer Forschung. Hrsg. von Hans Goebl. Handbücher zur Sprach- und Kommunikationswissenschaft 12. Berlin: de Gruyter, 881-887

Baldauf, Richard B. (1994). „,[unplanned] language policy and planning“. In: Annual review of applied linguistics 14, 82-89

Bales, Robert F. (1950). Interaction process analysis: a method for the study of small groups. Cambridge, MA: Addison-Wesley

Barandovská-Frank, Vera (2002). „Über die Academia pro Interlingua“. In: Plansprachen und ihre Gemeinschaften November 2001 in Berlin. Beiträge der 11. Jahrestagung der Gesellschaft für Interlinguistik e.V. (Berlin, 23.-25. Nov. 2001). Hrsg. von Detlev Blanke. Gesellschaft für Interlinguistik e.V., 6-21

Barkowski, Hans (1995). „Deutsch als Zweitsprache“. In: Handuch fremdsprachenunterricht. Hrsg. von Karl-Richard Bausch, Herbert Christ und Hans-Jürgen Krumm. 3. überarbeitete und erweiterte Auflage. Tübingen/Basel: A. Francke, 360-365

Barthe, Kathy (1998). „GIFAS Rationalised French“. In: Proceedings of the second international workshop on controlled language application. CLAW 98. (Pittsburgh, Pennsylvania, 21.-22. Mai 1998), 87-102

Barzdins, Guntis (2014). ,FrameNET CNL: a knowledge representation and information extraction language“. In: Controlled Natural Language. 4th International Workshop, CNL 
2014. (Galway, Ireland, 20.-22. Aug. 2014). Hrsg. von Brian Davis, Kaarel Kaljurand und Tobias Kuhn. Lecture Notes in Artificial Intelligence 8625. Cham u.a.: Springer, 90-101

Baudouin de Courtenay, Jan (1907). ,Zur Kritik der künstlichen Weltsprachen“. In: Plansprachen. Beiträge zur Interlinguistik. Hrsg. von Reinhard Haupenthal. 1976. Aufl. Darmstadt: Wissenschaftliche Buchgesellschaft, 59-110

Bem, Daryl J. (1972). ,Self-perception theory“. In: Advances in experimental social psycho$\log y 6,1-62$

Benioff, David und Daniel Brett Weiss (2011-). Game of thrones. Fernsehserie. HBO

Berger, Joseph, Susan J. Rosenholtz und Jr. Morris Zelditch (1980). „Status organizing processes“. In: Annual review of sociology 6, 479-508

Bernardi, Raffaella, Diego Calvanese und Camilo Thorne (2007). „Expressing DL-Lite ontologies with Controlled English". In: Proceedings of the 20th international workshop on description logics DL'07. (Brixen/Bressanone, Italy, 8.-10. Juni 2007). Hrsg. von Diego Calvanese, Enrico Franconi, Volker Haarslev, Domenico Lembo, Boris Motik, Sergio Tessaris und Anni-Yasmin Turhan. Bozen/Bolzano: Bozen-Bolzano University Press, 195-202

Bernth, Arendse (1998a). „EasyEnglish: preprocessing for MT“. In: Proceedings of the second international workshop on controlled language application. CLAW 98. (Pittsburgh, Pennsylvania, 21.-22. Mai 1998), 30-41

Bernth, Arendse (1998b). „Panel discussion: standardization“. In: Proceedings of the second international workshop on controlled language application. CLAW 98. (Pittsburgh, Pennsylvania, 21.-22. Mai 1998), 192-193

Betz, Werner (1960). „Sprachlenkung und Sprachentwicklung“. In: Sprache und Wissenschaft. Vorträge gehalten auf der Tagung der Joachim Jungius-Gesellschaft der Wissenschaften. Göttingen: Vandenhoeck \& Ruprecht, 85-100

Blahuš, Marek (2011). „Toki Pona: eine minimalistische Plansprache“. In: Spracherfindung und ihre Ziele. Beiträge der 20. Jahrestagung der Gesellschaft für Interlinguistik e.V. (Berlin, 26.-28. Nov. 2010). Hrsg. von Sabine Fiedler. Gesellschaft für Interlinguistik e.V., 5155

Blamoutier, Françoise (1977). „Training in the drafting of texts for documentation in controlled language for the purposes of automatic translation". In: Overcoming the language barrier. Third European Congress on Information Systems and Networks. (Luxemburg, 3.-6. Mai 1977). Hrsg. von Commission of the European Communities. Bd. 1. München: Verlag Dokumentation, 495-511

Blanke, Detlev (1985). Internationale Plansprachen. Eine Einführung. Berlin: AkademieVerlag

Blanke, Detlev (1989). „Planned languages - a survey of some of the main problems“. In: Interlinguistics. Aspects of the science of planned languages. Hrsg. von Klaus Schubert. Hrsg. von Dan Maxwell. Trends in Linguistics. Studies and Monographs 42. Berlin: Mouton de Gruyter, 63-87

Blanke, Detlev (1997). „The term ,planned language“““. In: Esperanto, interlinguistics, and planned language. Hrsg. von Humphrey Tonkin. Lanham: University Press of America, $1-20$

Blanke, Detlev (1998). „Interlinguistik und Plansprachen“. In: Sitzungsberichte der LeibnizSozietät 21.2, 45-76 
Blanke, Detlev (2004). „Plansprachen“. In: Morphologie. Ein internationales Handbuch zur Flexion und Wortbildung. Hrsg. von Geert Booij, Christian Lehmann, Joachim Mugdan und Stavros Skopeteas. Handbücher zur Sprach- und Kommunikationswissenschaft 12.1. Berlin: Walter de Gruyter. Kap. 144, 1563-1573

Blanke, Detlev (2006). Interlinguistische Beiträge. Zum Wesen und zur Funktion internationaler Plansprachen. Hrsg. von Sabine Fiedler. Frankfurt a. M.: Peter Lang

Blanke, Detlev (2009a). „Causes of the relative success of Esperanto“. In: Language problems \& language planning 33.3, 251-266

Blanke, Detlev (2009b). „Sprachenpolitische Aspekte internationaler Plansprachen - unter besonderer Berücksichtigung des Esperanto“. In: Sprachenpolitik und Sprachkultur. Hrsg. von Detlev Blanke und Jürgen Scharnhorst. Frankfurt a. M.: Peter Lang, 205-246

Blanke, Detlev (2011). „,20 Jahre „Gesellschaft für Interlinguistik e.V“ (GIL)“. In: Spracherfindung und ihre Ziele. Beiträge der 20. Jahrestagung der Gesellschaft für Interlinguistik e.V. (Berlin, 26.-28. Nov. 2010). Hrsg. von Sabine Fiedler. Gesellschaft für Interlinguistik e.V., 115-149

Blanke, Detlev und Wera Blanke (1998). „Plansprachen als Fachsprachen“. In: Fachsprachen. Ein internationales Handbuch zur Fachsprachenforschung und Terminologiewissenschaft. Hrsg. von Lothar Hoffmann, Hartwig Kalverkämper und Herbert Ernst Wiegand. Bd. 14.1. Handbücher zur Sprach- und Kommunikationswissenschaft. Berlin: Walter de Gruyter. Kap. 91, 875-880

Blanke, Wera (1989). „Terminological standarstandard - its roots and fruits in planned languages“. In: Interlinguistics. Aspects of the science of planned languages. Hrsg. von Klaus Schubert. Hrsg. von Dan Maxwell. Trends in Linguistics. Studies and Monographs 42. Berlin: Mouton, 277-292

Bliss, Charles Kasiel (1949). International semantography: a non-alphabetical symbol writing readable in all languages. A practical tool for general international communication especially in science, industry, commerce, traffic, etc., and for semantical education, based on the principles of ideographic writing and chemical symbolism. Sidney: Institute for Semantography

Bock, Bettina M. (2014). In: Sprache barrierefrei gestalten. Perspektiven aus der Angewandten Linguistik. Hrsg. von Susanne J. Jekat, Heike Elisabeth Jüngst, Klaus Schubert und Claudia Villiger. TransÜD 69. Berlin: Frank \& Timme, 17-49

Boozer, Don (2011). ,Review of From Elvish to Klingon: exploring invented languages“. In: Fiat lingua, 1-9. पDDhttp : / / iatlingua . org/wp-content/uploads / 2011/11/ fl-000003-00.pdf

Borgius, Walther (1908). Warum ich Esperanto verließ. Eine Studie über die gegenwärtige Krisis und die Zukunft der Weltsprachen-Bewegung. Berlin: Liebheit \& Thiesen

Bormann, Artur (1958). „Grundzüge der Interlinguistik“. In: Plansprachen. Beiträge zur Interlinguistik. Hrsg. von Reinhard Haupenthal. 1976. Aufl. Darmstadt: Wissenschaftliche Buchgesellschaft, 278-296

Brändle, Diana (2010). „tekom-Studie: Erfolgreiches Terminologiemanagement im Unternehmen. Praxishilfe und Leitfaden: Grundlagen, Umsetzung, Kosten-Nutzen-Analyse, Systemübersicht. Rezension“. In: MDÜ. Fachzeitschrift für Dolmetscher und Übersetzer 4, 71-73

Bredenkamp, Andrew, Berthold Crysmann und Mirela Petrea (2000). „Building multilingual controlled language performace checkers". In: Proceedings of the third international 
workshop on controlled language applications. CLAW 2000. (Seattle, USA, 29.-30. Apr. 2000), 83-89

Brehm, Jack W. (1966). A theory of psychological reactance. New York/London: Academic Press

Breton, Tom (1995a). Allnoun: grammar with only one part of speech. Online. $\square \square$ Dhttp: //www . panix.com/ tehom/allnoun/allnoun8.faq

Breton, Tom (1995b). Allnoun: grammar with only one part of speech. Frequently asked

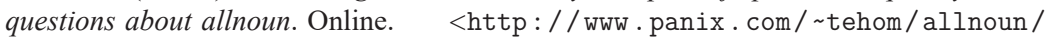
allnoun.faq

Brewer, Marilynn B. (1991). „The social self: on being the same and different at the same time." In: Personality and social psychology bulletin 17.5, 475-482

Brodbeck, Felix C. und Yves R. F. Guillaume (2010). „Arbeiten in Gruppen“. In: Enzyklopädie der Psychologie. Bd. 1: Arbeitspsychologie. Hrsg. von Uwe Kleinbeck und KlausHelmut Schmidt. III Wirtschafts-, Organisations- und Arbeitspsychologie. Göttingen u.a.: Hogrefe, 215-284

Brown, James Cooke (1960). „Loglan“. In: Scientific American 202.6, 53-63

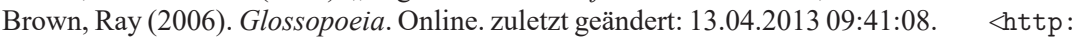
//www . carolandray.plus. com/Glosso/Glossopoeia.html

Brown, Rupert (2002). „Beziehungen zwischen Gruppen“. In: Sozialpsychologie. Hrsg. von Wolfgang Stroebe, Klaus Jonas und Miles Hewstone. 4., überarbeitete und erweiterte Auflage. Berlin/Heidelberg: Springer, 537-576

Bühler, Karl (1978). Sprachtheorie. Die Darstellungsfunktion der Sprache. Frankfurt a. M.: Ullstein

Bünzli, Alexandra und Stefan Höfler (2012). „Controlling ambiguities in legislative language“. In: Controlled Natural Language. Second International Workshop, CNL 2010. (Marettimo Island, Italy, 13.-15. Sep. 2010). Hrsg. von Michael Rosner und Norbert E. Fuchs. Lecture Notes in Artificial Intelligence 7175. Berlin/Heidelberg: Springer, 21-42

Buol, Bettina von (2000). Qualitätsgestützte, kooperative Terminologiearbeit. Aachen: Shaker

Cabré, M. Teresa (1999). Terminology: theory, methods, and applications. Amsterdam/Philadelphia: John Benjamins Publishing Company

Camilleri, John J., Gabriele Paganelli und Gerardo Schneider (2014). „A CNL for contractoriented diagrams“. In: Controlled Natural Language. 4th International Workshop, CNL 2014. (Galway, Ireland, 20.-22. Aug. 2014). Hrsg. von Brian Davis, Kaarel Kaljurand und Tobias Kuhn. Lecture Notes in Artificial Intelligence 8625. Cham u.a.: Springer, 135-146

Cardey, Sylviane, Peter Greenfield und Xiaohong Wu (2004). „Designing a controlled language for the maschine translation of medical protocols: the case of English to Chinese". In: Machine translation: from real users to research. 6th conference of the Association for Machine Translation in the Americas, AMTA 2004. (Washington, DC, USA, Sep.-Okt. 2004). Hrsg. von Robert E. Frederking und Kathryn B. Taylor. Lecture Notes in Artificial Intelligence 3265. Berlin u.a.: Springer, 37-47

Carlevaro, Tazio (1977). „Philosophiegeschichtliche Wurzeln und tiefenpsychologische Gründe des Plansprachenstreits (1907 - 1913)“. In: Grundlagenstudien aus Kybernetik und Geisteswissenschaft 17.4, 97-107

Carlevaro, Tazio (1998). „Das soziokulturelle Selbstverständnis der wichtigsten Plansprachen außer Esperanto“. In: Soziokulturelle Aspekte von Plansprachen. Beiträge der 7. 
Jahrestagung der Gesellschaft für Interlinguistik e.V. (Berlin, 7.-9. Nov. 1997). Hrsg. von Ulrich Becker. Gesellschaft für Interlinguistik e.V., 4-17

Carlson, Lauri, Shanshan Wang, Kun Ji und Igor Kudashev (2014). „TermFactory: terms in the web of data". In: Edition 10.1, 12-15

Cartwright, Dorwin und Alvin Zander (1968). Group dynamics. Research and theory. 3. Auflage. New York u.a.: Harper \& Row

Chaiken, Shelly, Akiva Liberman und Alice H. Eagly (1989). „Heuristic and systematic information processing within and beyond the persuasion context". In: Unintended thought. Hrsg. von James S. Uleman und John A. Bargh. New York/London: The Guilford Press, 212-252

Chan, Nelida (2015). „Language policies and terminology policies in canada“. In: Handbook of terminology. Hrsg. von Hendrik J. Kockaert und Frieda Steurs. Bd. 1. Amsterdam/Philadelphia: John Benjamins Publishing Company, 489-504

Choi, Hoon-Seok und John M. Levine (2004). „Minority influence in work teams: the impact of newcomers." In: Journal of experimental social psychology 40.2, 273-280

Chomsky, Noam (1972). Aspekte der Syntax-Theorie. Frankfurt a.M.: Suhrkamp

Christoffersen, Ellen und Margrethe H. Møller (2010). „Technical manuals for the elderly. Can controlled language principles help?“ In: Reconceptualizing lsp. Online proceedings. XVII European LSP Symposium 2009. (Aarhus, 2009). Hrsg. von Carmen Heine und Jan Engberg, 1-7. 미http://www . academia.edu/attachments/30165254/download_ file?st=MTQxMj YyMzEyMiw30S4yNDIuMTcwLjE00Q\%3D\%3D\&s=sidebar

Clark, Peter, William R. Murray, Phil Harrison und John Thompson (2010). „Naturalness vs. predictability: a key debate in controlled languages“. In: Controlled Natural Language. Workshop on Controlled Natural Language, CNL 2009. (Marettimo Island, Italy, 8.-10. Juni 2009). Hrsg. von Norbert E. Fuchs. Lecture Notes in Artificial Intelligence 5972. Berlin u.a.: Springer, 65-81

CLAW (1996). Proceedings of the first international workshop on controlled language applications. The first international workshop on controlled language applications, CLAW 1996. (Katholieke Universiteit Leuven, Belgien, 26.-27. März 1996)

CLAW (1998). Proceedings of the second international workshop on controlled language application. CLAW 98. (Pittsburgh, Pennsylvania, 21.-22. Mai 1998)

CLAW (2000). Proceedings of the third international workshop on controlled language applications. CLAW 2000. (Seattle, USA, 29.-30. Apr. 2000)

Cobarrubias, Juan (1983). „Language planning: the state of the art“. In: Progress in language planning: international perspective. Hrsg. von Joshua A. Fishman Juan Cobarrubias. Berlin: Mouton Publishers, 3-26

Combs, Mary Carol und Susan D. Penfield (2012). „Language activism and language policy“. In: The Cambridge handbook of language policy. Hrsg. von Bernard Spolsky. Cambridge: Cambridge University Press. Kap. 23, 461-474

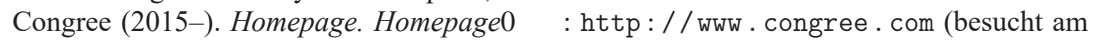
10. 02.2015)

Cooper, Robert L. (1989). Language planning and social change. Cambridge: Cambridge University Press

Costa, Rute (2006). „Plurality of theoretical approaches to terminology“. In: Modern approaches to terminological theories and applications. Hrsg. von Heribert Picht. Bern: Peter Lang, 77-89 
Couturat, Louis und Léopold Leau (1903). Historie de la langue universelle. Paris: Librairie Hachette

Crabbe, Stephen (2009). „Controlled languages for technical writing and translation“. In: Conference proceedings. The Ninth Portsmouth Translation Conference „The Translator as Writer". (Portsmouth, 7. Nov. 2009), 48-62. प0Dhttp : / / www . port . ac . uk / media/contacts-and-departments/slas/events/tr09-crabbe.pdf (besucht am 03. 02. 2014)

Cramer, Marcos (2013). „Proof-checking mathematical texts in controlled natural language“. Diss. Rheinischen Friedrich-Wilhelms-Universität Bonn

Cramer, Marcos, Bernhard Fisseni, Peter Koepke, Daniel Kühlwein, Bernhard Schröder und Jip Veldman (2010). „The Naproche project. Controlled natural language proof checking of mathematical texts“. In: Controlled Natural Language. Workshop on Controlled Natural Language, CNL 2009. (Marettimo Island, Italy, 8.-10. Juni 2009). Hrsg. von Norbert E. Fuchs. Lecture Notes in Artificial Intelligence 5972. Berlin u.a.: Springer, 170-186

Cranach, Mario Von, Guy Ochsenbein und Ladislav Valach (1986). „The group as a selfactive system: outline of a theory of group action“. In: European journal of social psychology $16.3,193-229$

Crystal, David (1998). Die Cambridge Enzyklopädie der Sprache. Studienausgabe. Frankfurt a.M./New York [Köln]: Campus Verlag [Parkland-Verlag]

Dalgarno, George (1661). Ars signorum: vulgo character universalis et lingua philosophica. Mikrofiche-Ausgabe 1989. English linguistics 1500-1800: 116. Menston: Scolar Press. London: Hayes

Dantuluri, Pradeep, Brian Davis, Pierre Ludwick und Siegfried Handschuh (2012). „Engineering a controlled natural language into Semantic MediaWiki“". In: Controlled Natural Language. Second International Workshop, CNL 2010. (Marettimo Island, Italy, 13.-15. Sep. 2010). Hrsg. von Michael Rosner und Norbert E. Fuchs. Lecture Notes in Artificial Intelligence 7175. Berlin/Heidelberg: Springer, 53-72

Davis, Brian, Pradeep Dantuluri, Laura Dragan, Siegfried Handschuh und Hamish Cunningham (2010). „On designing controlled natural languages for semantic annotation“. In: Controlled Natural Language. Workshop on Controlled Natural Language, CNL 2009. (Marettimo Island, Italy, 8.-10. Juni 2009). Hrsg. von Norbert E. Fuchs. Lecture Notes in Artificial Intelligence 5972. Berlin u.a.: Springer, 187-205

Davis, Brian, Ramona Enache, Jeroen van Grondelle und Laurette Pretorius (2012). „Multilingual verbalisation of modular ontologies using GF and lemon". In: Controlled Natural Language. Second International Workshop, CNL 2010. (Marettimo Island, Italy, 13.15. Sep. 2010). Hrsg. von Michael Rosner und Norbert E. Fuchs. Lecture Notes in Artificial Intelligence 7175. Berlin/Heidelberg: Springer, 167-184

Davis, Brian, Kaarel Kaljurand und Tobias Kuhn, Hrsg. (2014). Controlled Natural Language. 4th International Workshop, CNL 2014. (Galway, Ireland, 20.-22. Aug. 2014). Lecture Notes in Artificial Intelligence 8625. Cham u.a.: Springer

Denaux, Ronald, Vania Dimitrova, Anthony G. Cohn, Catherine Dolbear und Glen Hart (2010). „Rabbit to OWL: ontology authoring with a CNL-based tool“. In: Controlled Natural Language. Workshop on Controlled Natural Language, CNL 2009. (Marettimo Island, Italy, 8.-10. Juni 2009). Hrsg. von Norbert E. Fuchs. Lecture Notes in Artificial Intelligence 5972. Berlin u.a.: Springer, 246-264

DIN 2330 (2013). Begriffe und Benennungen - allgemeine Grundsätze. Berlin 
DIN 2331 (1980). Begriffssysteme und ihre Darstellung. Berlin

DIN 2342 (2011). Begriffe der Terminologielehre. Berlin

Dittmar, Norbert (1997). Grundlagen der Soziolinguistik. Tübingen: Niemeyer

Doherty, Stephen (2012). „Investigating the effects of controlled language on the reading and comprehension of machine translated texts: a mixed-methods approach." Diss. Dublin

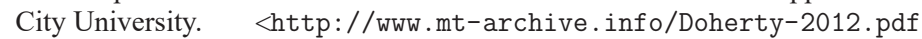

Dorsch, Friedrich, Hartmut Häcker und Kurt H. Stapf, Hrsg. (1994). Dorsch Psychologisches Wörterbuch. 12. überarbeitete und erweiterte Auflage. Bern u.a.: Hans Huber

Douglas, Shona und Matthew Hurst (1996). „Controlled language support for Perkins Approved Clear English (PACE)“. In: Proceedings of the first international workshop on controlled language applications. The first international workshop on controlled language applications, CLAW 1996. (Katholieke Universiteit Leuven, Belgien, 26.-27. März 1996), 93-105

Dr. Esperanto (1887a). Język międzynarodowy. Przedmowa i podręcznik kompletny por Po-

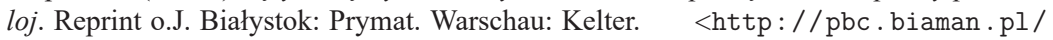
dlibra/doccontent?id=26013\&dirids=1

Dr. Esperanto (1887b). Meždunarodnyj jazyk. Predislovie i polnyi učebnik por Rusoj. Warschau: Kelter

Drewer, Petra (2008). „Terminologiemanagement. Methodische Grundlagen“. In: Terminologiearbeit für Technische Dokumentation. Hrsg. von Jörg Hennig und Marita Tjarks-Sobhani. tekom-Schriften zur technischen Kommunikation 12. Lübeck: Schmidt-Römhild, 54-69

Drewer, Petra und Wolfgang Ziegler (2011). Technische Dokumentation. Übersetzungsgerechte Texterstellung und Content-Management. Würzburg: Vogel Verlag

Drouin, Patrick (2006). ,Termhood: quantifying the relevance of a candidate term“. In: Modern approaches to terminological theories and applications. Hrsg. von Heribert Picht. Bern: Peter Lang, 375-391

Deutscher Terminologie-Tag e.V. (2010). Terminologiearbeit - Best Practices. Ordner

Duchêne, Alexandre und Monica Heller (2012). „Language policy in the workplace“. In: The cambridge handbook of language policy. Hrsg. von Bernard Spolsky. Cambridge: Cambridge University Press. Kap. 16, 323-334

Duličenko, Aleksandr Dmitrievič (1989). „Ethnic language and planned language“. In: Interlinguistics. Aspects of the science of planned languages. Hrsg. von Klaus Schubert. Hrsg. von Dan Maxwell. Trends in Linguistics. Studies and Monographs 42. Berlin: Mouton de Gruyter, 47-61

Dulichenko, Aleksandr Dmitrievič (1997). „Esperanto: a unique model for general linguistics“. In: Esperanto, interlinguistics, and planned language. Hrsg. von Humphrey Tonkin. Lanham: University Press of America, 67-69

Duval, Robert A. und Shelley Wicklund (1972). A theory of objective self awareness. New York u.a.: Academic Press

Eagly, Alice H. und Shelly Chaiken (1993). The psychology of attitudes. Fort Worth, TX: Harcourt Brace Jovanovich College Publishers

Ebenhoch, Peter (2014). „Strategische Aspekte von Terminologiearbeit“. In: Edition 10.2, $7-10$ 
Eckstein, Karina (2009). „Toolgestützte Terminologieextraktion“. In: Terminologiemanagement. Von der Theorie zur Praxis. Hrsg. von Felix Mayer und Uta Seewald-Heeg. Berlin: BDÜ, 108-120

Eco, Umberto (1995). The search for the perfect language. La ricerca della lingua perfetta nella cultura europea. Übers. von James Fentress. Oxford/Cambridge: Blackwell

Edwards, John (1994). Multilingualism. London: Routledge

Edwards, John (2012). „Language management agencies“. In: The Cambridge handbook of language policy. Hrsg. von Bernard Spolsky. Cambridge: Cambridge University Press. Kap. 21, 418-436

Edwards, John und Lynn MacPherson (1997). „Views of constructed languages, with special reference to Esperanto: an experimental study". In: Esperanto, interlinguistics, and planned language. Hrsg. von Humphrey Tonkin. Lanham: University Press of America, 93-116

Eichner, Heiner (2012). „Konstruierte Intersprachen: Herausforderung und Chance für die Sprachwissenschaft?" In: Zwischen Utopie und Wirklichkeit. Konstruierte Sprachen für die globalisierte Welt. Begleitband zur Ausstellung an der Bayerischen Staatsbibliothek (14. Juni bis 9. September 2012). Hrsg. von Andrea Pia Kölbl und Heiner Eichner. München: Allitera Verlag, 123-149

Eijk, Pim van der, Michiel de Koning und Gert van der Steen (1996). „Controlled language correction and translation". In: Proceedings of the first international workshop on controlled language applications. The first international workshop on controlled language applications, CLAW 1996. (Katholieke Universiteit Leuven, Belgien, 26.-27. März 1996), 64-73

Eisenberger, Naomi I., Matthew D. Lieberman und Kipling D. Williams (2003). „Does rejection hurt? An fMRI study of social exclusion“. In: Science 302.5643, 290-292

Elmasri, Ramez A. und Shamkant B. Navathe (2009). Grundlagen von Datenbanksystemen. Bachelorausgabe. 3. aktualisierte Aufl. München: Pearson Studium

Endres, Erik (2007). Reasoners for the Semantic Web. Kaon \& Kaon2. Seminar A.I. Tools, Universität des Saarlandes, Wintersemester 2006/07. प0 http : //www . dfki .de/ kipp/seminar_ws0607/reports/EricEndres-Reasoner . pdf (besucht am 13.01.2015)

Engelbrecht, Paula, Glen Hart und Catherine Dolbear (2010). ,Talking Rabbit: a user evaluation of sentence production“. In: Controlled Natural Language. Workshop on Controlled Natural Language, CNL 2009. (Marettimo Island, Italy, 8.-10. Juni 2009). Hrsg. von Norbert E. Fuchs. Lecture Notes in Artificial Intelligence 5972. Berlin u.a.: Springer, 56-64

Faber, Pamela und Clara Inés López Rodríguez (2012). „Terminology and specialized language“. In: A cognitive linguistics view of terminology and specialized language. Hrsg. von Pamela Faber. Berlin/Boston: De Gruyter Mouton, 9-31

Farrington, Gordon (1996). „AECMA Simplified English. An overview of the international aircraft maintenance language“. In: Proceedings of the first international workshop on controlled language applications. The first international workshop on controlled language applications, CLAW 1996. (Katholieke Universiteit Leuven, Belgien, 26.-27. März 1996), $1-21$

Fawcett, Helen (2005). Kontrollierte Sprache. semcon - Fachportal für Technische Dokumentation. 미 http : / / www . doku . info/doku_article_192.html (besucht am 07. 11.2014) 


\section{Literatur}

Fazio, Russell H. (1990). „Multiple processes by which attitudes guide behavior: the MODE model as an integrative framework". In: Advances in experimental social psychology 23, 75-109

Felber, Helmut (1979). „Die internationale terminologische Grundsatznormung. Rückblick und Ausblick“. In: Terminologie als angewandte Sprachwissenschaft. Gedenkschrift für Univ.-Prof. Dr. Eugen Wüster. Hrsg. von Helmut Felber, Friedrich Lang und Gernot Wersig. München u.a.: Saur, 61-69

Felber, Helmut (1998). „Weltweite terminologische Tätigkeiten zwischen 1965 und 1985 (Wüsters Lebenswerk)“. In: Eugen Wüster (1898 - 1977). Hrsg. von Erhard Oeser und Christian Galinski. Wien: TermNet, 69-104

Felber, Helmut und Gerhard Budin (1989). Terminologie in Theorie und Praxis. Forum für Fachsprachen-Forschung 9. Tübingen: Gunter Narr

Felber, Helmut und Magdalena Krommer-Benz (1979). „Das internationale Informationszentrum für Terminologie (Infoterm. Ein Brennpunkt der internationalen Terminologiearbeit“. In: Terminologie als angewandte Sprachwissenschaft. Gedenkschrift für Univ.-Prof. Dr. Eugen Wüster. Hrsg. von Helmut Felber, Friedrich Lang und Gernot Wersig. München u.a.: Saur, $70-86$

Felber, Helmut und Friedrich Hans Lang (1979). „Würdigung der Person und des Wissenschaftlers“. In: Terminologie als angewandte Sprachwissenschaft. Gedenkschrift für Univ.-Prof. Dr. Eugen Wüster. Hrsg. von Helmut Felber, Friedrich Lang und Gernot Wersig. München u.a.: Saur, 15-28

Fensel, Dieter (2004). Ontologies: a silver bullet for Knowledge Management and Electronic Commerce. Berlin u.a.: Springer

Festinger, Leon (1954). „A theory of social comparison processes“. In: Human relations 7.2, $117-140$

Festinger, Leon (1957). A theory of cognitive dissonance. Evanston, Ill: Row, Peterson \& Co.

Fiederer, Rebecca und Sharon O’Brien (2009). „Quality and machine translation: a realistic objective?" In: The journal of specialised translation (11), 52-74. प0口http : / / www . jostrans.org/issue11/art_fiederer_obrien.php

Fiedler, Sabine (2006). „Standardization and self-regulation in an international speech community: the case of Esperanto“. In: International journal of the sociology of language 177, $67-90$

Fiedler, Sabine (2011a), „Das Thema Plansprachen in der aktuellen sprachpolitischen Fachliteratur". In: Florilegium interlinguisticum. Festschrift für Detlev Blanke zum 70. Geburtstag. Hrsg. von Cyril Brosch und Sabine Fiedler. Frankfurt a. M.: Peter Lang, 79-105

Fiedler, Sabine (2011b). „Literarische Spracherfindungen aus interlinguistischer Sicht: die englischsprachigen Autoren J. Swift, G. Orwell und J.R.R. Tolkien und ihre fiktionalen Sprachen“. In: Spracherfindung und ihre Ziele. Beiträge der 20. Jahrestagung der Gesellschaft für Interlinguistik e.V. (Berlin, 26.-28. Nov. 2010). Hrsg. von Sabine Fiedler. Gesellschaft für Interlinguistik e.V., 9-29

Fischer, Lorenz und Günter Wiswede (2002). Grundlagen der Sozialpsychologie. ger. 2., überarb. und erw. Aufl. München u.a.: Oldenbourg, XVII, 769 S. : Ill., graph. Darst.

Fischer, Lorenz und Günter Wiswede (2009). Grundlagen der Sozialpsychologie. 3., völlig neu bearb. Aufl. Wolls Lehr- und Handbücher der Wirtschafts- und Sozialwissenschaften. München: Oldenbourg 
Fishbein, Martin und Icek Ajzen (1975). Belief, attitude, intention, and behavior. An introduc-

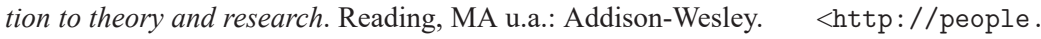
umass . edu/aizen/f\&a1975.html (besucht am 17.10.2015)

Fleischmann, Klaus (2009). „Aktive Terminologie - Workflows mit QuickTerm“. In: Edition $5.1,5-7$

Förster, Hans-Peter, Gerhard Rost und Michael Thiermeyer (2010). Corporate Wording. Die Erfolgsfaktoren für professionelle Kommunikation. Frankfurt a.M.: F.A.Z.-Institut für Management-, Markt- und Medieninformationen

Forster, Peter G. (1982). The Esperanto movement. Hrsg. von Joshua A. Fishman. Contributions to the Sociology of Language 32. The Hague u.a.: Mouton

Freudenthal, Hans (1960). Lincos: design of a language for cosmic intercourse. Studies in logic and the foundations of mathematics. Amsterdam: North-Holland Publishing Company

Fuchs, Norbert E., Hrsg. (2010). Controlled Natural Language. Workshop on Controlled Natural Language, CNL 2009. (Marettimo Island, Italy, 8.-10. Juni 2009). Lecture Notes in Artificial Intelligence 5972. Berlin u.a.: Springer

Fuchs, Norbert E., Stefan Höfler, Kaarel Kaljurand, Fabio Rinaldi und Gerold Schneider (2005). „Attempto Controlled English. A knowledge representation language readable by humans and machines“. In: Reasoning web. (Msida, Malta, 25.-29. Juli 2005). Hrsg. von Norbert Eisinger und Jan Małuszyński. Lecture Notes in Computer Science 3564. Pre-

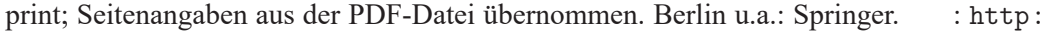
//attempto.ifi.uzh.ch/site/pubs/papers/drs_report.pdf

Fuchs, Norbert E., Kaarel Kaljurand und Tobias Kuhn (2008). „Attempto Controlled English for knowledge representation“. In: Reasoning web, fourth international summer school 2008. Hrsg. von Cristina Baroglio, Piero A. Bonatti, Jan Małuszyński, Massimo Marchiori, Axel Polleres und Sebastian Schaffert. Lecture Notes in Computer Science 5224. Berlin/Heidelberg: Springer, 104-124

Fuchs, Norbert E., Uta Schwertel und Rolf Schwitter (1999). Attempto Controlled English (ACE) language manual. Techn. Ber. Technical Report 99.03. Department of Informatics, University of Zurich. 매 http : / / attempto . ifi . uzh . ch / site / pubs / papers / ace3manual.pdf

Fuchs, Norbert E. und Rolf Schwitter (1996). „Attempto controlled english (ACE)“. In: Proceedings of the first international workshop on controlled language applications. The first international workshop on controlled language applications, CLAW 1996. (Katholieke Universiteit Leuven, Belgien, 26.-27. März 1996). Preprint; Seitenangaben aus der PDFDatei übernommen, 124-136. 매http://arxiv.org/pdf/cmp-lg/9603003v1.pdf

Galinski, Christian (2009). „History of ISO/TC 37 an Infoterm“. In: Edition 5.2, 19-23

Gallup, Sharlene (1993). „Caterpillar Technical English and automatic machine translation“. In: Stc proceedings, 421-424. 미내ttp: //www . stc . org/ConfProceed/1993/PDFs/ Pg421424.pdf

Gardner, Robert C. und Wallace E. Lambert (1959). „Motivational variables in second-language aquisition“. In: Canadian journal of psychology 13.4, 266-272

Gasser, Yvonne und Klaus Fleischmann (2008). „Mehr Spaß an Terminologie“. In: technische kommunikation 5, 52-55

Gaudin, François (1993). Socioterminologie. Des problèmes sémantiques aux pratiques institutionnelles. Mont-Saint-Aignan: Université de Rouen 


\section{Literatur}

Gerzymisch-Arbogast, Heidrun (1996). Termini im Kontext. Verfahren zur Erschließung und Übersetzung der textspezifischen Bedeutung von fachlichen Ausdrücken. Tübingen: Gunter Narr. प⿴囗口 http: //www . translationconcepts . org/pdf/Termini .pdf

Glaser, Barney G. und Anselm L. Strauss (1998). Grounded Theory. Strategien qualitativer Forschung. Bern u.a.: Huber

Gobbo, Federico (2014). „La malfacila tasko klasifiki planlingvojn“. In: esperanto. Oficiala organo de Universala Esperanto-Asocio (en oficialaj rilatoj kun UN kaj Unesko) 107 (1282), 164-165

Godden, Kurt (1998). „Controlling the business environment for controlled language“. In: Proceedings of the second international workshop on controlled language application. CLAW 98. (Pittsburgh, Pennsylvania, 21.-22. Mai 1998), 185-190

Godden, Kurt (2000). „The evolution of CASL controlled authoring at General Motors“. In: Proceedings of the third international workshop on controlled language applications. CLAW 2000. (Seattle, 29.-30. Apr. 2000), 14-19

Golden, Bernard (1981). „Kompara tipologio de planlingvoj kaj planlingvistoj““. In: Literatura foiro $12.66,15-19$

Goyvaerts, Patrick (1996). „Controlled English, curse or blessing? A user's perspective“. In: Proceedings of the first international workshop on controlled language applications. The first international workshop on controlled language applications, CLAW 1996. (Katholieke Universiteit Leuven, Belgien, 26.-27. März 1996), 137-142

Granström, Mats (2014). „Structured, standardised, multi-use terminology. Terminology in a wider sense". In: Rechte, Rendite, Ressourcen. Wirtschaftliche Aspekte des Terminologiemanagements. 14. Symposion des Deutschen Terminologie-Tages e.V. (Mannheim, 27.29. März 2014). Hrsg. von Petra Drewer, Felix Mayer und Klaus-Dirk Schmitz. Köln: Deutscher Terminologie-Tag e.V., 35-40

Greenwald, Anthony G. (1986). „Cognitive learning, cognitive response to persuasion, and attitude change“. In: Psychological foundations of attitudes. Hrsg. von Anthony G. Greenwald, Timothy C. Brock und Thomas M. Ostrom. New York u.a.: Academic Press, 147170

Grimshaw, Allen D. (1987). „Sociolinguistics versus sociology of language: tempest in a teapot or profound academic conundrum?" In: Soziolinguistik. Ein internationales Handbuch zur Wissenschaft von Sprache und Gesellschaft. Hrsg. von Ulrich Ammon. Berlin: Walter de Gruyter, 9-15

Grin, François (2003). Language policy evaluation and the European Charter for Regional or Minority Languages. Basingstoke: Palgrave Macmillan

Grin, François (2009). „Economics and language policy“. In: Handbook of language and communication: diversity and change. Hrsg. von Marlis Hellinger und Anne Pauwels. Handbooks of Applied Linguistics 9. Berlin/New York: Mouton de Gruyter, 271-297

Grin, François (2016). „Language policy and planning across levels“. Vortragsfolien, präsentiert auf Soillse 2016: Small Language Planning: Communities in Crisis. 2016-0606/2016-06-08, University of Glasgow

Grover, Claire, Alexander Holt, Ewan Klein und Marc Moens (2000). „Designing a controlled language for interactive model checking“. In: Proceedings of the third international workshop on controlled language applications. CLAW 2000. (Seattle, USA, 29.-30. Apr. 2000), 90-104 
Gudykunst, William B. und Stella Ting-Toomey (1990). „Ethnic identity, language and communication breakdowns“. In: Handbook of langauge and social psychology. Hrsg. von Howard Giles und W. P. Robinson. Reprint 1996. Chichester: John Wiley \& Sons, 309327

Gust, Dieter (2006). „Wirtschaftliche Terminologiearbeit in der Technischen Dokumentation". In: Edition 1.2, 16-20

Guy, Stephen und Rolf Schwitter (2014). „Architecture of a web-based predictive editor for Controlled Natural Language processing“. In: Controlled Natural Language. 4th International Workshop, CNL 2014. (Galway, Ireland, 20.-22. Aug. 2014). Hrsg. von Brian Davis, Kaarel Kaljurand und Tobias Kuhn. Lecture Notes in Artificial Intelligence 8625. Cham u.a.: Springer, 167-178

Haarmann, Harald (1986). Language in ethnicity. A view of basic ecological relations. Ebook, Reprint 2012. Contributions to the Sociology of Language 44. Berlin u.a.: De Gruyter Mouton

Hacker, Winfried (1985). Arbeitspsychologie. Psychische Regulation von Arbeitstätigkeiten. Bern u.a.: Hans Huber

Hacker, Winfried (2010). „Psychische Regulation von Arbeitstätigkeiten“. In: Enzyklopädie der Psychologie. Bd. 1: Arbeitspsychologie. Hrsg. von Uwe Kleinbeck und Klaus-Helmut Schmidt. III Wirtschafts-, Organisations- und Arbeitspsychologie. Göttingen u.a.: Hogrefe, 3-37

Haddock, Geoffrey und Gregory R. Maio (2007). „Einstellungen: Inhalt, Struktur und Funktionen“. In: Sozialpsychologie. Hrsg. von Klaus Jonas, Wolfgang Stroebe und Miles Hewstone. 5., vollständig überarbeitete Auflage. Berlin/Heidelberg: Springer, $187-223$

Haddock, Geoffrey und Gregory R. Maio (2014). „Einstellungen“. In: Sozialpsychologie. Hrsg. von Klaus Jonas, Wolfgang Stroebe und Miles Hewstone. 6., vollständig überarbeitete Auflage. Berlin/Heidelberg: Springer, 197-229

Hahn, Walter von (1983). Fachkommunikation. Entwicklung, linguistische Konzepte, betriebliche Beispiele. Berlin/New York: Walter de Gruyter

Haller, Johann (1996). MULTILINT. A technical documentation system with multilingual intelligence. Gehalten auf ASLIB 1996. पDDhttp://www.iai-sb.de/docs/aslib.pdf (besucht am 07.10.2014)

Haller, Johann (2000). Sprachtechnologie für die Automobildokumentation. Preprint. प्ण http://www.iai-sb.de/docs/otz.pdf (besucht am 07.10.2014)

Hamburger, Kurt (1954). „Li practic servicies de Esperanto. Unesco, Esperanto e occidentalinterlingue“. In: Universalaj lingvoj en Svislando: svisa enciklopedio planlingva. Hrsg. von Andreas Künzli. 2006. Aufl. La Chaux-de-Fonds: Svisa Esperanto-Soceto, 772-777

Hänig, Christian und Martin Schierle (2010). „Relationsextraktion aus Fachsprache - ein automatischer Ansatz für die industrielle Qualitätsanalyse“. In: Edition 6.1, 28-32

Haralambous, Yannis, Julie Sauvage-Vincent und John Puentes (2014). ,INAUT, a controlled language for the French coast pilot books Instructions nautiques". In: Controlled Natural Language. 4th International Workshop, CNL 2014. (Galway, Ireland, 20.-22. Aug. 2014). Hrsg. von Brian Davis, Kaarel Kaljurand und Tobias Kuhn. Lecture Notes in Artificial Intelligence 8625. Cham u.a.: Springer, 102-111

Harris, Zellig Sabbettai (1968). Mathematical structures of language. New York u.a.: Interscience Publishers 


\section{Literatur}

Harry, Ralph L. (1997). „Development of a language for international law: the experience of Esperanto“. In: Esperanto, interlinguistics, and planned language. Hrsg. von Humphrey Tonkin. Lanham: University Press of America, 173-182

Haugen, Einar (1959). „Planning for a standard language in modern Norway“. In: Anthropological linguistics $1.3,8-21$

Haugen, Einar (1966). Language conflict and language planning: the case of modern Norwegian. Cambridge, Massachusetts: Harvard University Press

Haugen, Einar (1983). „The implementation of corpus planning: theory and practice“. In: Progress in language planning. International perspective. Hrsg. von Joshua A. Fishman und Juan Cobarrubias. Berlin: Mouton, 269-289

Haupenthal, Reinhard (2005a). Johann Martin Schleyer (1831-1912). Pfarrer von Litzelstetten (1875-1885). Vortrag, gehalten am 29. Juni 2002 anlässlich des Patroziniums der Pfarrei St. Peter und Paul, Konstanz-Litzelstetten. Iltis, 2. korrigierte Auflage. Schliengen

Haupenthal, Reinhard (2005b). Über die Startbedingungen zweier Plansprachen. Schleyers Volapük (1879/80) und Zamenhofs Esperanto (1887). Iltis. Schliengen

Haupenthal, Reinhard (2012). „Was ist und zu welchem Zweck betreibt man Interlinguistik?“ In: Zwischen Utopie und Wirklichkeit. Konstruierte Sprachen für die globalisierte Welt. Begleitband zur Ausstellung an der Bayerischen Staatsbibliothek (14. Juni bis 9. September 2012). Hrsg. von Andrea Pia Kölbl und Heiner Eichner. München: Allitera Verlag, $11-32$

Haussteiner, Ingrid (2014). „Neue Wege der Terminologie- und Wissensvermittlung bei der Oesterreichischen Nationalbank". In: Edition 10.1, 24-26

Hayes, Phil, Steve Maxwell und Linda Schmandt (1996). „Controlled English advantages for translated and original English documents“. In: Proceedings of the first international workshop on controlled language applications. The first international workshop on controlled language applications, CLAW 1996. (Katholieke Universiteit Leuven, Belgien, 26.-27. März 1996), 84-92

Heald, Isobel (2000). „Evaluation and systematization of the procedure of choice of vocabulary in controlled languages". In: Proceedings of the third international workshop on controlled language applications. CLAW 2000. (Seattle, USA, 29.-30. Apr. 2000), 43-50

Heald, Isobel und Rémi Zajac (1998). „Compounds nouns in Simplified English“. In: Proceedings of the second international workshop on controlled language application. CLAW 98. (Pittsburgh, Pennsylvania, 21.-22. Mai 1998), 124-133

Hebling, Uta (2002). „Controlled Language am Beispiel des Controlled English“. CD-ROM. Diplomarbeit. Trier: Universität Heidelberg

Heckhausen, Jutta (2010). „Vorwort zur 4. Auflage“. In: Motivation und Handeln. Hrsg. von Jutta Heckhausen und Heinz Heckhausen. 4. überarbeitete und erweiterte Auflage. Berlin/Heidelberg: Springer, vii-viii

Heckhausen, Jutta und Heinz Heckhausen (2010). „Motivation und Handeln: Einführung und überblick“. In: Motivation und Handeln. Hrsg. von Jutta Heckhausen und Heinz Heckhausen. 4. überarbeitete und erweiterte Auflage. Berlin/Heidelberg: Springer, 1-9

Hellinger, Marlis und Anne Pauwels, Hrsg. (2009). Handbook of language and communication: diversity and change. Handbooks of Applied Linguistics 9. Berlin/New York: Mouton de Gruyter

Hertzler, Joyce Oramel (1965). A sociology of language. New York: Random House 
Herwatz, Rachel (2009). ,Webbasierte Terminologiearbeit - Terminologiearbeit unternehmensweit verteilen und pflegen“. In: Terminologiemanagement. Von der Theorie zur Praxis. Hrsg. von Felix Mayer und Uta Seewald-Heeg. Berlin: BDÜ, 82-92

Herzog, Gottfried (2009). ,106 Jahre Terminologienormung - ein großer Nutzen für die Wirtschaft". In: Edition 5.2, 15-18

Heyer, Gerhard (2010). „Text Mining und Text Services“. In: Edition 6.1, 7-10

Higgins, E. Tory (1987). ,Self-discrepancy: a theory relating self and affect“. In: Psychological review $94.3,319-349$

Höfler, Stefan (2012). „Legislative drafting guidelines: how different are they from controlled language rules for technical writing?" In: Controlled Natural Language. Third International Workshop, CNL 2012. (Zürich, 29.-31. Aug. 2012). Hrsg. von Tobias Kuhn und Norbert E. Fuchs. Lecture Notes in Artificial Intelligence 7427. Berlin/Heidelberg: Springer, $138-151$

Holmback, Heather, Serena Shubert und Jan Spyridakis (1996). ,Issues in conducting empirical evaluation of controlled languages". In: Proceedings of the first international workshop on controlled language applications. The first international workshop on controlled language applications, CLAW 1996. (Katholieke Universiteit Leuven, Belgien, 26.-27. März 1996), 166-177

Holterhus, Katharina und Stephanie Meister (2001). „Kontrollierte Sprachen in Technischen Dokumentationen“. Magisterarbeit. Universität Bielefeld

Hornberger, Nancy H. (2006). „Frameworks and models in language policy and planning“. In: An introduction to language policy: theory and method. Hrsg. von Thomas Ricento. Malden: Blackwell Publishing Ltd. Kap. 2, 24-41

Huijsen, Willem-Olaf(1998a). „Completeness of compositional translation. Volledigheid van compositioneel vertalen (met een samenvatting in het Nederlands)“. Drukkerij Elinkwijk B.V. Diss. Utrecht: Universität Utrecht

Huijsen, Willem-Olaf (1998b). „Controlled language - an introduction“. In: Proceedings of the second international workshop on controlled language application. CLAW 98. (Pittsburgh, Pennsylvania, 21.-22. Mai 1998), 1-15

Hult, Francis M. (2010). „Analysis of language policy discourses across the scales of space and time“. In: International journal of the sociology of language 202, 7-24

Humbley, John (1997). „Language planning and terminology planning“. In: Handbook of terminology management. Bd. 1: Basic aspects of terminology management. Hrsg. von Sue Ellen Wright und Gerhard Budin, 261-277

Hymes, Dell (1979). Soziolinguistik. Zur Ethnographie der Kommunikation. Frankfurt a.M.: Suhrkamp

Infoterm (2004). 30 years of Infoterm. Version 2004-06-21. प्ण \%7Bhttp : //www. infoterm. info/pdf/about_us/30_years_infoterm.pdf\%7D (besucht am 16.03.2015)

Infoterm (2015). International Information Centre for Terminology $\square \square \square$ : www . infoterm . info (besucht am 20.03.2015)

Ingsve, Hrakkar, Sebastian und Lajaki (2011). Tongues of Ice and Fire. Learn dothraki and

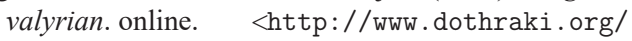

Ischreyt, Heinz (1965). Studien zum Verhältnis von Sprache und Technik. Institutionelle Sprachlenkung in der Terminologie der Technik. Sprache und Gemeinschaft: 4. Düsseldorf: Pädagogischer Verlag Schwann 


\section{Literatur}

ISO 15188 (2001). Project management guidelines for terminology standardization. ISO

Jackson, Howard (2011). „Invented vocabularies: the case of Newspeak and Nadsat“. In: From Elvish to Klingon. Exploring invented languages. Hrsg. von Michael Adams. Oxford: Oxford University Press, 49-73

Jacob, Henry (1948). „Sprachplanung“. In: Plansprachen. Beiträge zur Interlinguistik. Hrsg. von Reinhard Haupenthal. Bd. 1976. Darmstadt: Wissenschaftliche Buchgesellschaft, $217-233$

Janich, Nina (2004). Die bewusste Entscheidung. Eine handlungsorientierte Theorie der Sprachkultur. Tübingen: Gunter Narr

Janke, Regina (2013). Anforderungen an die Terminologieextraktion. Eine vergleichende Untersuchung der Bedürfnisse von Terminologen, Technischen Fachübersetzern und Technischen Redakteuren. Stuttgart: tcworld

Janton, Pierre (1978). Einführung in die Esperantologie. Hildesheim/New York: Georg Olms Jermołowicz, Renata (2003). „On the project of a universal language in the framework of the XVII century philosophy“. In: Studies in logic, grammar and rhetoric 6.19, 51-61

Jernudd, Björn H. (1983). „Evaluation of language planning: what has the last decade accomplished?" In: Progress in language planning. International perspectives. Hrsg. von Joshua A. Fishman Juan Cobarrubias. Berlin: Mouton, 345-378

Jernudd, Björn H. und Jyotirindra Das Gupta (1971). „Towards a theory of language planning". In: Can language be planned? Sociolinguistic theory and practice for developing nations. Hrsg. von Joan Rubin und Björn H. Jernudd. Honolulu: The University Press of Hawaii, 195-216

Jernudd, Björn und Jiři Nekvapil (2012). „History of the field: a sketch“. In: The Cambridge handbook of language policy. Hrsg. von Bernard Spolsky. Cambridge: Cambridge University Press. Kap. 2, 16-36

Jespersen, Otto (1930). „Interlinguistik - eine neue Wissenschaft“. In: Plansprachen. Beiträge zur Interlinguistik. Hrsg. von Reinhard Haupenthal. 1976. Aufl. Darmstadt: Wissenschaftliche Buchgesellschaft, 148-162

Johnson, Edward (1996). „LinguaNet. Controlling police communication“. In: Proceedings of the first international workshop on controlled language applications. The first international workshop on controlled language applications, CLAW 1996. (Katholieke Universiteit Leuven, Belgien, 26.-27. März 1996), 115-123

Johnson, Keith (2008). An introduction to foreign language learning and teaching. 2. Auflage. Harlow: Pearson Education Ltd.

Jonas, Klaus, Wolfgang Stroebe und Miles Hewstone, Hrsg. (2007). Sozialpsychologie. 5., vollständig überarbeitete Auflage. Berlin/Heidelberg: Springer

Jonas, Klaus, Wolfgang Stroebe und Miles Hewstone, Hrsg. (2014). Sozialpsychologie. 6. vollständig überarbeitete Auflage. Berlin/Heidelberg: Springer

Julià, Pere (1997). „Linguistic theory and international communication“. In: Esperanto, interlinguistics, and planned language. Hrsg. von Humphrey Tonkin. Lanham: University Press of America, 21-37

Jurkowski, Marian (1986). Od wieży babel do języka kosmitów. O językach sztucznych, uniwersalnych i międzynarodowych. Białystok: Krajowa Agencja Wydawnicza

Kaji, Hiroyuki (1999). „Controlled languages for machine translation: state of the art“. In: Machine Translation Summit VII - MT in the Great Translation Era. (Kent Ridge Digital 
Labs, Singapore, 13.-17. Sep. 1999), 37-39. प⿴囗口 पhttp : //www . mt-archive . info/ MTS-1999-Kaji.pdf

Kaljurand, Kaarel (2012). „General architecture of a controlled natural language based multilingual semantic wiki“. In: Controlled Natural Language. Third International Workshop, CNL 2012. (Zürich, 29.-31. Aug. 2012). Hrsg. von Tobias Kuhn und Norbert E. Fuchs. Lecture Notes in Artificial Intelligence 7427. Berlin/Heidelberg: Springer, 110-120

Kaljurand, Kaarel und Tanel Alumäe (2012). ,Controlled natural language in speech recognition based user interfaces“. In: Controlled Natural Language. Third International Workshop, CNL 2012. (Zürich, 29.-31. Aug. 2012). Hrsg. von Tobias Kuhn und Norbert E. Fuchs. Lecture Notes in Artificial Intelligence 7427. Berlin/Heidelberg: Springer, 79-94

Kamprath, Christine, Eric Adolphson, Teruko Mitamura und Eric Nyberg (1998). „Controlled language for multilingual document production: experience with Caterpillar Technical English". In: Proceedings of the second international workshop on controlled language application. CLAW 98. (Pittsburgh, Pennsylvania, 21.-22. Mai 1998). Preprint; Seitenangaben aus der PDF-Datei übernommen, 51-61

Kaplan, Robert B., Richard B. Baldauf Jr, Anthony J. Liddicoat, Pauline Bryant, MarieThérèse Barbaux und Martin Pütz (2000). „Current issues in language planning“. In: Current issues in language planning 1.2, 135-144

Kaplan, Robert B. und Richard B. Baldauf (1997). Language planning from practice to theory. Clevedon: Multilingual Matters Ltd

Katz, Daniel (1960). „The functional approach to the study of attitudes“. In: Public opinion quarterly 24.2, 163-204

KBA-Sheetfed Solutions (2016). KBA Rapida 106. Spitzenleistung durch Spitzentechnik. Version 05/2016-d. 미https : / / www . kba . com/fileadmin/user_upload / 01 Sheetfed/01_Produkte/PDF_Downloads/Ra_106/Rapida_106_d_web.pdf (besucht am 23.07.2016)

Keller, Nicole (2013). „Einfache Terminologieabstimmung mit Termflow“. In: Edition 9.2, 26-30

Keller, Nicole (2014). „Terminologie- und Wissensmanagement mit flashterm“. In: Edition $10.1,27-30$

Keller, Rudi (1982). „Zur Theorie des sprachlichen Wandels“. In: Zeitschrift für germanistische Linguistik 10.1, 1-27

Keller, Rudi (1990). Sprachwandel. Tübingen: Francke

Kimura, Goro Christoph (2011). „Eine Typologie interlingualer Kommunikationsmöglichkeiten“. In: Florilegium interlinguisticum. Festschrift für Detlev Blanke zum 70. Geburtstag. Hrsg. von Cyril Brosch und Sabine Fiedler. Frankfurt a. M.: Peter Lang, 29-46

Kimura, Goro Christoph und Sabine Fiedler (2013). „Interlinguistisch relevante Fachliteratur 2011/12“. In: Plansprachen - ideengeschichtliche Aspekte. Beiträge der 22. Jahrestagung der Gesellschaft für Interlinguistik e.V. (Berlin, 23.-25. Nov. 2012). Hrsg. von Sabine Fiedler. Gesellschaft für Interlinguistik e.V., 95-103

Kisa, Sonja Elen (2001-). Toki pona Ш口: http: //tokipona . org (besucht am 12. 03. 2014) Kittredge, Richard I. (2003). ,Sublanguages and controlled languages“. In: The Oxford handbook of computational linguistics. Hrsg. von Ruslan Mitkov. Oxford: Oxford University Press, 430-447

Klaesi, Ludwig (1945). „Kampf um Europa von der Schweiz aus gesehen: die Sprachenfrage und die neue Weltordnung aus der Sicht der Europa-Union“. In: Universalaj lingvoj en 
Svislando: svisa enciklopedio planlingva. Hrsg. von Andreas Künzli. Bd. 2006. La Chauxde-Fonds: Svisa Esperanto-Soceto, 762-767

Kleinbeck, Uwe (2010). „Handlungsziele“. In: Motivation und Handeln. Hrsg. von Jutta Heckhausen und Heinz Heckhausen. 4. überarbeitete und erweiterte Auflage. Berlin/Heidelberg: Springer, 285-307

Kloss, Heinz (1969). Research possibilities on group bilingualism. A report. Centre International de Recherches sur le Bilinguisme. Quebec: Centre Internat. de Recherches sur le Bilinguisme, 91

Kloss, Heinz (1998). The american bilingual tradition. Language in Education: Theory and Practice; 88. Washington: Center for Applied Linguistics/ Delta Systems Co

Knapp, Karlfried (2004). „Vorwort“. In: Angewandte Linguistik. Ein Lehrbuch. Hrsg. von Karlfried Knapp, Gerd Antos, Michael Becker-Mrotzek, Arnulf Deppermann, Susanne Göpferich, Joachim Grabowski, Michael Klemm und Claudia Villiger. Tübingen/Basel: A. Francke, xvii-xx

Kniele, Rupert (1889). Das erste Jahrzehnt der Weltsprache Volapük. Entstehung und Entwicklung von Volapük in den einzelnen Jahren, nebst Übersicht über den heutigen Stand der Weltsprache, Weltspracheklubs u.s.w. Ueberlingen a. B.: A. Schoy. पD口http : / / commons.wikimedia.org/wiki/File:Das_erste_Jahrzehnt_der_Weltsprache_ Volap\%C3\%BCk. djvu

Knops, Uus (1999). „Controlled language - issues in checkers’ design“. In: Machine Translation Summit VII - MT in the Great Translation Era. (Kent Ridge Digital Labs, Singapore, 13.-17. Sep. 1999), 40-45. पढ口 http : / / www . mt-archive . info/MTS-1999Knops.pdf

Knops, Uus (2000). ,Efficient roll-in and roll-out of controlled language applications. Position statement". In: Proceedings of the third international workshop on controlled language applications. CLAW 2000. (Seattle, USA, 29.-30. Apr. 2000), 134-135

Kotler, Philip und Gerald Zaltman (1971). „Social marketing: an approach to planned social change“. In: Journal of marketing 35, 3-12

Kotzin, Boris (1917). Geschichte und Theorie des Ido. Übers. von Dr. med. Wilhelm Winsch und Hans Reuß (aus Esperanto). Dresden: Ader \& Borel

Kraśko, Ryszard (1972). „Ludwik Zamenhof - twórca Esperanto“. In: Studia i materiały do dziejów miasta Białegostoku. Hrsg. von Jerzy Joka. Bd. 3. Prace Białostockiego Towarzystwa Naukowego 15. Białystok: Białostockie Towarzystwo Naukowe, 121-147

Kresic, Marijana (2006). Sprache, Sprechen und Identität. Studien zur sprachlich-medialen Konstruktion des Selbst. München: iudicium

Kuhn, Tobias (2010a). „An evaluation framework for controlled natural languages“. In: Controlled Natural Language. Workshop on Controlled Natural Language, CNL 2009. (Marettimo Island, Italy, 8.-10. Juni 2009). Hrsg. von Norbert E. Fuchs. Lecture Notes in Artificial Intelligence 5972. Berlin u.a.: Springer, 1-20

Kuhn, Tobias (2010b). „Controlled English for knowledge representation“. Diss. Universität Zürich

Kuhn, Tobias (2014). „A survey and classification of Controlled Natural Languages“. In: Computational linguistics 40.1, 121-170. पDDhttp: //www . mitpress journals . org/ doi/pdf/10.1162/COLI_a_00168 (besucht am 21.03.2014)

Kuhn, Tobias, Loïc Royer, Norbert E. Fuchs und Michael Schroeder (2006). „Improving text mining with controlled natural language: a case study for protein interactions“". In: Data 
integration in the life sciences. Third International Workshop, DILS 2006. (Hinxton, UK, 20.-22. Juli 2006). Hrsg. von Ulf Leser, Felix Naumann und Barbara Eckman. Lecture Notes in Computer Science 4075. Berlin u.a.: Springer, 66-81

Künzli, Andreas (2006). „Neutrale Universalsprachen als Alternative: einige pragmatische Überlegungen und Anmerkungen zum Thema“. In: Universalaj lingvoj en Svislando: svisa enciklopedio planlingva. Hrsg. von Andreas Künzli. La Chaux-de-Fonds: Svisa Esperanto-Soceto, 41-68

Kurtz, Gunde (2005). „Deutsch als „leicht zu erlernende Fremdsprache“““. In: Linguistik on-

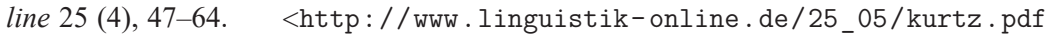
(besucht am 25.01.2014)

Kuznecov, Segej Nikolaevič (1989). „Interlinguistics: a branch of applied linguistics?“ In: Interlinguistics. Aspects of the science of planned languages. Hrsg. von Klaus Schubert. Hrsg. von Dan Maxwell. Trends in Linguistics. Studies and Monographs 42. Berlin: Mouton de Gruyter, 89-98

Lacy, Lee W. (2005). OWL. Representing information using the Web Ontology Language. Victoria, BC u.a.: Trafford

Lang, Friedrich (1959-1960). ,Wieselburg. Ein Knotenpunkt der internationalen Terminologiearbeit“. In: Sprachforum 3, 153-156

Lang, Friedrich Hans (1985). „Information und Dokumentation. Einleitung“. In: Terminologie und benachbarte Gebiete. 1965-1985. Gewidmet Helmut Felber. Hrsg. von Infoterm, $171-172$

Language Creation Society (2007-). Website $\square \square \square$ : http : / / conlang . org (besucht am 14. 03. 2014)

Lapenna, Ivo (1970). „The common language question before international organizations“. In: La monda lingvo-problemo 2, 83-102

Lapenna, Ivo (1971). „The common language question before international organizations“. In: La monda lingvo-problemo 3, 11-30

Large, J. Andrew (1985). The artificial language movement. Oxford u.a.: Blackwell u.a.

Lassen, Inger (2003). Accessibility and acceptability in technical manuals. Document Design Companion Series 4. Amsterdam/Philadelphia: John Benjamins Publishing Company, A survey of style and grammatical metaphor

Lasswell, Harold Dwight (1948). „The structure and function of communication in society“. In: The communication of ideas. A series of addresses. Hrsg. von Lyman Bryson. New York: Harper, 37-51

League of Nations (1922). „Esperanto as an international auxilary language“. In: Universalaj lingvoj en Svislando: svisa enciklopedio planlingva. Report of the general secretariat to the third assembly. Hrsg. von Andreas Künzli. 2006. Aufl. La Chaux-de-Fonds: Svisa Esperanto-Soceto, 561-578

Lehrbuch der Weltsprache Ido für Arbeiter (1923). Leipzig: Ido-Verlag

Lehrndorfer, Anne (1996). Kontrolliertes Deutsch. Linguistische und sprachpsychologische Leitlinien für eine (maschinell) kontrollierte Sprache in der Technischen Dokumentation. Tübingen: Gunter Narr

Lehtola, Aarno, Jarno Tenni und Catherine Bounsaythip (1998). „Definition of a controlled language based on augumented lexical entries“. In: Proceedings of the second international workshop on controlled language application. CLAW 98. (Pittsburgh, Pennsylvania, 21.-22. Mai 1998), 16-29 


\section{Literatur}

Leitner, Eva-Maria (2014). ,Terminologiearbeit - Voraussetzung für umfassendes Qualitätsmanagement in der Sprachdienstleistung“. In: Edition 10.2, 18-20

Leont'ev, Aleksej N. (1979). Tätigkeit, Bewußtsein, Persönlichkeit. Berlin: Volk und Wissen

Lewark, Eva-Maria und Ayten Turhan (2013). Online-Umfrage zu Terminologiearbeit und Acrolinx. Motivation - Vorgehensweise - Ergebnisse und Maßnahmen. Vortragsfolien der tekom-Jahrestagung 07.11.2013. 미http : / tagungen. tekom.de/fileadmin/tx _ doccon/slides/418_Fiducia_Umfrage_zu_Terminologiearbeit_und_Acrolinx. pdf (besucht am 08.06.2015)

Ley, Martin (2005). „Kontrollierte Textstrukturen. Ein (linguistisches) Informationsmodell für die technische Kommunikation“. Diss. Universität Gießen

L'Homme, Marie-Claude (2006). „A look at some Canadian contributions to terminology“. In: Modern approaches to terminological theories and applications. Hrsg. von Heribert Picht. Bern: Peter Lang, 55-75

Lickel, Brian, David L. Hamilton, Amy Lewis, Steven J. Sherman, Grażyna Wieczorkowska und A. Neville Uhles (2000). , Varieties of groups and the perception of group entitativity“. In: Journal of personality and social psychology 78.2, 223-246

Lickel, Brian, David L. Hamilton und Steven J. Sherman (2001). „Elements of a lay theory of groups: types of groups, relational styles, and the perception of group entitativity“. In: Personality and social psychology review 5.2, 129-140

Liddicoat, Anthony J. (2009). „Language planning and information and communication technologies“. In: Current issues in language planning 10.4, 355-360

Link, Lisa (2014). „Reguliertes Englisch in der Technischen Dokumentation. Unterschiedliche Ziele im Hinblick auf die Optimierung der Fachkommunikation“. In: Sprache barrierefrei gestalten. Perspektiven aus der Angewandten Linguistik. Hrsg. von Susanne J. Jekat, Heike Elisabeth Jüngst, Klaus Schubert und Claudia Villiger. TransÜD 69. Berlin: Frank \& Timme, 175-200

Linke, Angelika, Markus Nussbaumer und Paul R. Portmann (2001). Studienbuch Linguistik. 4. unveränderte Aufl. Tübingen: Niemeyer

Lins, Ulrich (2012). „Die ersten Hundert Jahre des Esperanto“. In: Zwischen Utopie und Wirklichkeit. Konstruierte Sprachen für die globalisierte Welt. Begleitband zur Ausstellung an der Bayerischen Staatsbibliothek (14. Juni bis 9. September 2012). Hrsg. von Andrea Pia Kölbl und Heiner Eichner. München: Allitera Verlag, 85-121

Linville, Patricia W. (1987). ,Self-complexity as a cognitive buffer against stressrelated illness and depression“. In: Journal of personality and social psychology 52.4, 663-676

Liver, Ricarda (1999). Rätoromanisch. Eine Einführung. Tübingen: Gunter Narr

Lo Jacomo, François (1989). „Optimization in language planning“. In: Interlinguistics. Aspects of the science of planned languages. Hrsg. von Klaus Schubert. Hrsg. von Dan Maxwell. Trends in Linguistics. Studies and Monographs 42. Berlin: Mouton de Gruyter, 121128

Löffler, Heinrich (2010). Germanistische Soziolinguistik. Berlin: Schmidt

Mangold, Max (1970). „Die phonetische Überlegenheit künstlicher Sprachen“. In: La monda lingvo-problemo 2, 129-137

Mannewitz, Cornelia (2002). ,Wer in aller Welt spricht Klingonisch?“ In: Plansprachen und ihre Gemeinschaften. Beiträge der 11. Jahrestagung der Gesellschaft für Interlinguistik e.V. (Berlin, 23.-25. Nov. 2001). Hrsg. von Detlev Blanke. Gesellschaft für Interlinguistik e.V., $107-114$ 
Manstead, Antony S. R. und Andrew G. Livingstone (2014). „Forschungsmethoden in der Sozialpsychologie“. In: Sozialpsychologie. Hrsg. von Klaus Jonas, Wolfgang Stroebe und Miles Hewstone. 6., vollständig überarbeitete Auflage. Berlin/Heidelberg: Springer, 2964

Maradan, Mélanie (2012). „Blissymbolics - von einer Pasigraphie zum System für die unterstützte Kommunikation“. In: Fachkommunikation - interlinguistische Aspekte. Beiträge der 21. Jahrestagung der Gesellschaft für Interlinguistik e.V. (Berlin, 18.-20. Nov. 2011). Hrsg. von Cyril Brosch und Sabine Fiedler, 61-68

Markus, Hazel (1977). ,Self-schemata and processing information about the self“. In: Journal of personality and social psychology 35.2, 63-78

Marrafa, Palmira, Raquel Amaro, Nuno Freire und Sara Mendes (2012). „Portuguese controlled language: coping with ambiguity“. In: Controlled Natural Language. Third International Workshop, CNL 2012. (Zürich, 29.-31. Aug. 2012). Hrsg. von Tobias Kuhn und Norbert E. Fuchs. Lecture Notes in Artificial Intelligence 7427. Berlin/Heidelberg: Springer, 152-166

Martin, George R. R. (1991-). A song of ice and fire. Bücherreihe

Massion, François (2009). „Terminologiemanagement: Luxus oder Muss? Von der Theorie zur Praxis“. In: Terminologiemanagement. Von der Theorie zur Praxis. Hrsg. von Felix Mayer und Uta Seewald-Heeg. Berlin: BDÜ, 27-34

Massion, François (2014). ,Terminologie als strategische Investition: ein Blick über die Zahlen hinaus“. In: Rechte, Rendite, Ressourcen. Wirtschaftliche Aspekte des Terminologiemanagements. 14. Symposion des Deutschen Terminologie-Tages e.V. (Mannheim, 27.29. März 2014). Hrsg. von Petra Drewer, Felix Mayer und Klaus-Dirk Schmitz. Köln: Deutscher Terminologie-Tag e.V., 133-143

Maxwell, Dan (1989). „Principles for constructing planned languages“. In: Interlinguistics. Aspects of the science of planned languages. Hrsg. von Klaus Schubert. Hrsg. von Dan Maxwell. Trends in Linguistics. Studies and Monographs 42. Berlin: Mouton de Gruyter, $101-119$

Mayer, Felix (2009). „Einleitung“. In: Terminologiemanagement. Von der Theorie zur Praxis. Hrsg. von Felix Mayer und Uta Seewald-Heeg. Berlin: BDÜ, 12-26

Mayer, Maurice (2010). „Corporate Language - Herr über die Sprachenvielfalt im Unternehmen bleiben“. In: Edition 6.1, 38-41

Mayrhofer, Manfred (1969). „Zur Problematik künstlicher Welthilfssprachen (Plansprachen)“. In: Plansprachen. Beiträge zur Interlinguistik. Hrsg. von Reinhard Haupenthal. 1976. Aufl. Darmstadt: Wissenschaftliche Buchgesellschaft, 308-319

Mayring, Philipp (2002). Einführung in die qualitative Sozialforschung. Eine Anleitung zu qualitativem Denken. 5. überarbeitete und neu ausgestattete Aufl. Weinheim/Basel: Beltz

McCann, C. Douglas und E. Tory Higgins (1990). „Social cognition and communication“. In: Handbook of langauge and social psychology. Hrsg. von Howard Giles und W. P. Robinson. Reprint 1996. Chichester: John Wiley \& Sons, 13-32

McGuire, William J. (1969). „,The nature of attitudes and attitude change“. In: Handbook of social psychology. Hrsg. von Gardner Lindzey und Elliott Aronson. 2. Auflage. Bd. 3. Reading, MA: Addison-Wesley, 136-314

McPike, Eugene F. (1925). Ido. The scientific international language. A lecture (revised) delivered before Members of the Faculty and Graduate Students of the University of Chicago, at the Harper Library, Chicago, January 22nd, 1925. Chicago 


\section{Literatur}

Means, Linda G., Patricia Chapman und Aulsan Liu (2000). „Training for controlled language processes". In: Proceedings of the third international workshop on controlled language applications. CLAW 2000. (Seattle, USA, 29.-30. Apr. 2000), 1-13

Means, Linda und Kurt Godden (1996). „The Controlled Automotive Service Langauge (CASL) project". In: Proceedings of the first international workshop on controlled language applications. The first international workshop on controlled language applications, CLAW 1996. (Katholieke Universiteit Leuven, Belgien, 26.-27. März 1996), 106-114

Mellish, Chris, Ehud Reiter und John Levine (1996). „NLG applications to technical documentation. A view through IDAS“. Selected papers. In: Hrsg. von Giovanni Adorni und Michael Zock. Lecture Notes in Computer Science 1036. Berlin u.a.: Springer, 368-382. 口DDhttp://dx.doi.org/10.1007/3-540-60800-1_40

Meyer, Ingrid, Douglas Skuce, Lynne Bowker und Karen Eck (1992). „Towards a new generation of terminological resources: an experiment in building a terminological knowledge base". In: Proceedings of the 14th conference on computational linguistics. COLING-92. (Nantes, 23.-28. Aug. 1992). Bd. 3, 956-960. पएD http : //delivery. acm.org/10.1145/1000000/993106/p956-meyer.pdf? ip=87. 173. $145.9 \& i d=993106 \&$ a c $=0 P E N \& k e y=4 D 4702 B 0 C 3 E 38 B 35 \% 2 E 4 D 4702 B 0 C 3 E 38 B 35 \%$ 2E4D4702B0C3E38B35 \% 2E6D218144511F3437 \& CFID $=498567376 \&$ CFTOKEN = 43491078 \& _ acm _ $=1428592432 \_7 d d 4758$ a186585575763391892a02874 (besucht am 09. 04.2015)

Meysmans, Jules (1911-1912). „Eine neue Wissenschaft“. In: Plansprachen. Beiträge zur Interlinguistik. Hrsg. von Reinhard Haupenthal. 1976. Aufl. Darmstadt: Wissenschaftliche Buchgesellschaft, 111-112

Moch, Gaston (1897). La question de la langue internationale et sa solution par l'Esperanto. Paris: Giard \& Brière. $\square \square$ http : / / anno . onb . ac . at/cgi-content/anno-buch? apm=0\&aid=100101\&zoom=1

Møller, Margrethe H. und Ellen Christoffersen (2006). „Building a controlled language le-

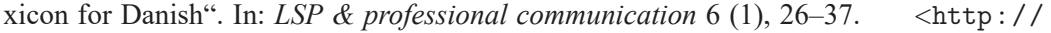
rauli.cbs.dk/index.php/LSP/article/view/2069/2068

Moore, Corinne B. (2000). „Controlled language at Diebold, Incorporated“. In: Proceedings of the third international workshop on controlled language applications. CLAW 2000. (Seattle, USA, 29.-30. Apr. 2000), 51-61

Moreland, Richard L. und John M. Levine (1982). „Socialization in small groups: temporal changes in individual-group relations." In: Advances in experimental social psychology $15,137-192$

Nahir, Moshe (1984). „Language planning goals: a classification“. In: Language problems \& language planning 8.3, 294-327

Namahn (2002). Controlled languages. A research note. URL nicht mehr aktiv. पण口http: //www. namahn.com/resources/documents/note-CL.pdf (besucht am 30. 05. 2008)

Neuberger, Oswald (1985). Arbeit. Begriff - Gestaltung - Motivation - Zufriedenheit. Stuttgart: Ferdinand Enke Verlag

Nijstad, Bernard A. und Daan Van Knippenberg (2007). „Gruppenpsychologie: grundlegende Prinzipien“. In: Sozialpsychologie. Hrsg. von Klaus Jonas, Wolfgang Stroebe und Miles Hewstone. 5., vollständig überarbeitete Auflage. Berlin/Heidelberg: Springer, 409-441 
Nijstad, Bernard A. und Daan Van Knippenberg (2014). „Gruppendynamik“. In: Sozialpsychologie. Hrsg. von Klaus Jonas, Wolfgang Stroebe und Miles Hewstone. 6., vollständig überarbeitete Auflage. Berlin/Heidelberg: Springer, 439-467

Njonko, Paul Brillant Feuto, Sylviane Cardey, Peter Greenfield und Walid El Abed (2014). „RuleCNL: a Controlled Natural Language for business rule specifications“. In: Controlled Natural Language. 4th International Workshop, CNL 2014. (Galway, Ireland, 20.22. Aug. 2014). Hrsg. von Brian Davis, Kaarel Kaljurand und Tobias Kuhn. Lecture Notes in Artificial Intelligence 8625. Cham u.a.: Springer, 66-77

Nuopponen, Anita (2014). „Tangled web of concept relations. Concept relations for ISO 1087-1 and ISO 704“. In: Proceedings. Terminology and Knowledge Engineering 2014. (Berlin, 19.-21. Juni 2014), 1-10.

http: //tke2014. sciencesconf .org/conference/tke2014/eda_en . pdf (besucht am 01.04.2015)

Nyberg, Eric, Teruko Mitamura und Willem-Olaf Huijsen (2003). „Controlled language for authoring and translation“. In: Computers and translation. A translator's guide. Hrsg. von Harold Somers. Benjamins Translation Library 35. Amsterdam/Philadelphia: John Benjamins Publishing Company, 245-281. 미http : / / site . ebrary . com / lib / academiccompletetitles/docDetail . action?doc ID $=10032038 \& \mathrm{p} 00=$ computers $\%$ 20translation (besucht am 06.02.2014)

O’Brien, Sharon (2003). „Controlling controlled English. An analysis of several controlled language rule sets". In: Controlled language translation. EAMT/CLAW 03. (Dublin, Ireland, 15.-17. Mai 2003). Preprint; PDF-Seitenzählung als Seitenangabe verwendet, o.S. प्Dhttp: //www.mt-archive.info/00/CLT-2003-Obrien.pdf

O'Brien, Sharon (2006). „Machine-translatability and post-editing effort: an empirical study using translog and choice network analysis“. Diss. Dublin City University

Ogden, Charles K. (1968). Basic English: international second language. Hrsg. von E. C. Graham. Mit einem Vorw. von L. W. Lockhart. New York: Harcourt, Brace \& World Inc. A revised and expanded version of The system of Basic English

Ogden, Charles K. und Ivor Armstrong Richards (1974). Die Bedeutung der Bedeutung. Eine Untersuchung über den Einfluss der Sprache auf das Denken und über die Wissenschaft des Symbolismus. 1. Aufl. Frankfurt a. M.: Suhrkamp

Okrand, Marc, Michael Adams, Judith Hendriks-Hermans und Sjaak Kroon (2011). „'Wild and whirling words': the invention and use of Klingon“. In: From Elvish to Klingon. Exploring invented languages. Hrsg. von Michael Adams. Oxford: Oxford University Press, $111-134$

Okrent, Arika (2009). In the land of invented languages: a celebration of linguistic creativity, madness and genius. 2010 paperback edition. New York: Spiegel \& Grau

Ölberg, Hermann (1954). ,Zur Grundlegung der Interlinguistik“. In: Plansprachen. Beiträge zur Interlinguistik. Hrsg. von Reinhard Haupenthal. 1976. Aufl. Darmstadt: Wissenschaftliche Buchgesellschaft, 243-254

OMG (2008). SBVR: semantics of business vocabulary and business rules $\square \square \square:$ http : / / www . omg. org/spec/SBVR/1.0/

Oostendorp, Marc van (2001). „Constructed language and linguistic theory“. In: Planned languages: from concept to reality. One the notions Possible, Impossible and Actual language. Hrsg. von Klaus Schubert. Brüssel: Hogeschool voor Wetenschap \& Kunst, 203221 


\section{Literatur}

Pace, Gordon J. und Michael Rosner (2010). „A controlled language for the specification of contracts“. In: Controlled Natural Language. Workshop on Controlled Natural Language, CNL 2009. (Marettimo Island, Italy, 8.-10. Juni 2009). Hrsg. von Norbert E. Fuchs. Lecture Notes in Artificial Intelligence 5972. Berlin u.a.: Springer, 226-245

Pace, Gordon J. und Michael Rosner (2014). „Explaining violation traces with finite state natural language generation models“. In: Controlled Natural Language. 4th International Workshop, CNL 2014. (Galway, Ireland, 20.-22. Aug. 2014). Hrsg. von Brian Davis, Kaarel Kaljurand und Tobias Kuhn. Lecture Notes in Artificial Intelligence 8625. Cham u.a.: Springer, 179-189

Pauwels, Anne (2012). „Language maintenance in/and cyberspace: a wake up call for dormant bilinguals". Konferenzbeitrag ICLASP13

Pauwels, Anne und Marlis Hellinger (2009). „Language and communication: diversity and change - an introduction“. In: Handbook of language and communication: diversity and change. Hrsg. von Marlis Hellinger und Anne Pauwels. Handbooks of Applied Linguistics 9. Berlin/New York: Mouton de Gruyter, 1-14

Pearson, Jennifer (1998). Terms in context. Amsterdam/Philadelphia: John Benjamins Publishing Company

Pei, Mario (1966). The story of language. 2. ed, 3. impr. London: Allen \& Unwin

Penn, Julia M. (1972). Linguistic relativity versus innate ideas. The origins of the Sapir-Whorf hypothesis in German thought. The Hague: Mouton

Pennycook, Alastair (2006). „Postmodernism in language policy“. In: An introduction to language policy: theory and method. Hrsg. von Thomas Ricento. Malden: Blackwell Publishing Ltd. Kap. 4, 60-76

Petersen, Lars-Erik, Dagmar Stahlberg und Dieter Frey (2006). „Selbstwertgefühl“. In: Handbuch der Psychologie. Hrsg. von Hans-Werner Bierhoff. Bd. 3: Handbuch der Sozialpsychologie und Kommunikationspsychologie. Göttingen: Hofgrefe, 40-48

Petty, Richard E. und John T. Cacioppo (1986). „,The elaboration likelihood model of persuasion“. In: Advances in experimental social psychology 19, 123-205

Peus, Heinrich (1913). Kurzer Abriß der Welthilfssprache Ido. Charlottenburg: Verlag des Deutschen Weltsprache-Bundes. 84

Pflaum, Rainer (2009). „Der konsequente Weg zur einheitlichen Terminologie“. In: Edition $5.1,14-17$

Phillipson, Robert (1992). Linguistic imperialism. Oxford: Oxford University Press

Phillipson, Robert (2006). „Language policy and linguistic imperialism“. In: An introduction to language policy: theory and method. Hrsg. von Thomas Ricento. Malden: Blackwell Publishing Ltd. Kap. 19, 346-361

Pich, Hans (2008). „Einführung von Terminologie-Management in Unternehmen: ein Praxisbericht“. In: Terminologiearbeit für Technische Dokumentation. Hrsg. von Jörg Hennig und Marita Tjarks-Sobhani. tekom-Schriften zur technischen Kommunikation 12. Lübeck: Schmidt-Römhild, 70-79

Picht, Heribert (2009). „Einige Gedanken zur geschichtlichen Entwicklung der Terminologie“. In: Edition 5.2, 5-9

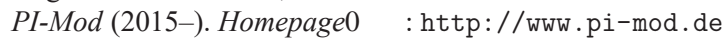

Piron, Claude (1989). „A few notes on the evolution of Esperanto“. In: Interlinguistics. Aspects of the science of planned languages. Hrsg. von Klaus Schubert. Hrsg. von Dan Max- 
well. Trends in Linguistics. Studies and Monographs 42. Berlin: Mouton de Gruyter, 129142

Pool, Jonathan (2006). „Can controlled languages scale to the web?“ In: 5th International Workshop on Controlled Language Applications, CLAW 2006. (Cambridge, Massachusetts, USA, 12. Aug. 2006). PDF-Seitenzählung als Seitenangabe verwendet, o.S. प्ण http: //www.mt-archive.info/CLAW-2006-Pool.pdf

Portnow, James (2011). „Gaming languages and language games“. In: From Elvish to Klingon. Exploring invented languages. Hrsg. von Michael Adams. Oxford: Oxford University Press, 135-159

Power, Richard (2012). „OWL Simplified English: a finite-state language for ontology editing“. In: Controlled Natural Language. Third International Workshop, CNL 2012. (Zürich, 29.-31. Aug. 2012). Hrsg. von Tobias Kuhn und Norbert E. Fuchs. Lecture Notes in Artificial Intelligence 7427. Berlin/Heidelberg: Springer, 44-60

Pratt-Hartmann, Ian und Allan Third (2006). „More fragments of language“. In: Notre dame journal of formal logic 47 (2), 151-177

Prentice, Deborah A. (1994). „Do language reforms change our way of thinking?“ In: Journal of language and social psychology 13.1, 3-19

Preux, Nathalie de (2005). „How much does using controlled language improve machine translation results?" In: Proceedings of the twenty-seventh international conference on translating and the computer. Translating and the Computer 27. (24.-25. Nov. 2005), 114. 매http://www.mt-archive.info/Aslib-2005-Preux.pdf

Pulman, Stephen G. (1996). „Controlled language for knowledge representation“. In: Proceedings of the first international workshop on controlled language applications. The first international workshop on controlled language applications, CLAW 1996. (Katholieke Universiteit Leuven, Belgien, 26.-27. März 1996), 233-242

Ramírez Polo, Laura (2012). „Use and evaluation of controlled languages in industrial environments and feasibility study for the implementation of machine translation." Diss. Universidad de Valencia. 미http: //www.mt-archive.info/10/Ramirez-2012.pdf

Ranta, Aarne (2004). „Grammatical framework. A type-theoretical grammar formalism“. In: Journal of functional programming 14 (02), 145-189. 미 Dttp : / / journals . cambridge.org/article_S0956796803004738

Ranta, Aarne (2011). Grammatical framework. Programming with multilingual grammars. CSLI Studies in Computational Linguistics. Stanford: CSLI Publications

Ranta, Aarne (2014). „Embedded controlled languages“. In: Controlled Natural Language. 4th International Workshop, CNL 2014. (Galway, Ireland, 20.-22. Aug. 2014). Hrsg. von Brian Davis, Kaarel Kaljurand und Tobias Kuhn. Lecture Notes in Artificial Intelligence 8625. Cham u.a.: Springer, 1-7

Ranta, Aarne, Ramona Enache und Grégoire Détrez (2012). „Controlled language for everyday use: the MOLTO phrasebook“. In: Controlled Natural Language. Second International Workshop, CNL 2010. (Marettimo Island, Italy, 13.-15. Sep. 2010). Hrsg. von Michael Rosner und Norbert E. Fuchs. Lecture Notes in Artificial Intelligence 7175. Berlin/ Heidelberg: Springer, 115-136

Rat für Deutschsprachige Terminologie (2005). Berufsprofil Terminologin Terminologe $\square \square$ Ш: http : / / ww . iim2 . fh-koeln . de/radt/images / veroeffentlichungen/RaDT_ Berufsprofil.pdf (besucht am 15.04.2015) 


\section{Literatur}

Rechtien, Wolfgang (2003). „Gruppendynamik“. In: Angewandte Sozialpsychologie: das Praxisbuch. Hrsg. von Ann Elisabeth Auhagen und Hans-Werner Bierhoff. Weinheim u.a.: Beltz PVU, 103-122

Reineke, Detlef (2014). „Terminologieaustausch“. In: Rechte, Rendite, Ressourcen. Wirtschaftliche Aspekte des Terminologiemanagements. 14. Symposion des Deutschen Terminologie-Tages e.V. (Mannheim, 27.-29. März 2014). Hrsg. von Petra Drewer, Felix Mayer und Klaus-Dirk Schmitz. Köln: Deutscher Terminologie-Tag e.V., 157-165

Remus, Robert und Khurshid Ahmad (2010). „Stimmungen in deutschsprachigen Nachrichten, Blogs und dem DAX“. In: Edition 6.1, 24-27

Reuther, Ursula (1998). „Controlling language in an industrial application“. In: Proceedings of the second international workshop on controlled language application. CLAW 98. (Pittsburgh, Pennsylvania, 21.-22. Mai 1998), 174-184

Reuther, Ursula (2003). „,Two in one - can it work? Readability and translatability by means of controlled language“. In: Controlled language translation. EAMT/CLAW 03. (Dublin, Ireland, 15.-17. Mai 2003). PDF-Datei ohne Seitenangaben, 124-132. प[Dhttp: //www . mt-archive.info/CLT-2003-Reuther.pdf

Reuther, Ursula und Antje Schmidt-Wigger (2000). „Designing a multi-purpose CL application“. In: Proceedings of the third international workshop on controlled language applications. CLAW 2000. (Seattle, USA, 29.-30. Apr. 2000), 72-82

Rey, Alain (1995). Essays on terminology. Amsterdam/Philadelphia: John Benjamins Publishing Company

Ricento, Thomas, Hrsg. (2006). An introduction to language policy: theory and method. Malden: Blackwell Publishing Ltd

Ricento, Thomas (2009). „Models and approaches in language policy and planning“. In: Handbook of language and communication: diversity and change. Hrsg. von Marlis Hellinger und Anne Pauwels. Handbooks of Applied Linguistics 9. Berlin/New York: Mouton de Gruyter, 211-240

Roelcke, Thorsten (2014), „Zur Gliederung von Fachsprache und Fachkommunikation“. In: Fachsprache 36.3-4, 154-178

Roulin, Corentin (2001). „Computer-assisted thesaurus management“. In: Handbook of terminology management. Bd. 2: Application-oriented terminology management. Hrsg. von Sue Ellen Wright und Gerhard Budin, 381-418

Rubin, Joan (1971). „Evaluation and language planning“. In: Can language be planned? Sociolinguistic theory and practice for developing nations. Hrsg. von Joan Rubin und Björn H. Jernudd. Honolulu: The University Press of Hawaii, 217-252

Rubin, Joan (1983). „Evaluating status planning: what has the past decade accomplished?“ In: Progress in language planning. International perspective. Hrsg. von Joshua A. Fishman und Juan Cobarrubias. Berlin: Mouton, 329-343

Ryan, Ellen Bouchard (1979). „Why do low-prestige language varieties persist?“ In: Language and social psychology. Hrsg. von Howard Giles und N. St. Clair Robert. Oxford: Basil Blackwell, 145-157

Rychtyckyj, Nestor (2006). „Standard language at Ford Motors. A case study in controlled language development and deployment“. In: 5th International Workshop on Controlled Language Applications, CLAW 2006. (Cambridge, Massachusetts, USA, 12. Aug. 2006). PDF-Seitenzählung als Seitenangabe verwendet, o.S. ODDhttp: //www . mt-archive . info/CLAW-2006-Rychtyckyj.pdf 
Sadler, Victor (1989). „Knowledge-driven terminography for machine translation“. In: Interlinguistics. Aspects of the science of planned languages. Hrsg. von Klaus Schubert. Hrsg. von Dan Maxwell. Trends in Linguistics. Studies and Monographs 42. Berlin: Mouton, 311-335

Sadler, Victor (1997). „Machine translation project reaches watershed“. In: Esperanto, interlinguistics, and planned language. Hrsg. von Humphrey Tonkin. Lanham: University Press of America, 191-197

Safwat, Hazem und Brian Davis (2014). „A brief state of the art of CNLs for ontology authoring“. In: Controlled Natural Language. 4th International Workshop, CNL 2014. (Galway, Ireland, 20.-22. Aug. 2014). Hrsg. von Brian Davis, Kaarel Kaljurand und Tobias Kuhn. Lecture Notes in Artificial Intelligence 8625. Cham u.a.: Springer, 190-200

Sakaguchi, Alicja (1998). Interlinguistik. Gegenstand, Ziele, Aufgaben, Methoden. Frankfurt a. M.: Peter Lang

Sapir, Edward, Leonard Bloomfield, Franz Boas, John L. Gerig und Georg Philip Krapp (1925). „Memorandum zum Problem einer internationalen Hilfssprache“. In: Plansprachen. Beiträge zur Interlinguistik. Hrsg. von Reinhard Haupental. 1976. Aufl. Darmstadt: Wissenschaftliche Buchgesellschaft, 133-147

Saulwick, Adam (2014). „Lexpresso: a Controlled Natural Language“. In: Controlled Natural Language. 4th International Workshop, CNL 2014. (Galway, Ireland, 20.-22. Aug. 2014). Hrsg. von Brian Davis, Kaarel Kaljurand und Tobias Kuhn. Lecture Notes in Artificial Intelligence 8625. Cham u.a.: Springer, 123-134

Saussure, René de (1918). La structure logique des mots: dans les langues naturelles, considérée au point de vue de son application aux langues artificielles. Bern: Imprimerie Büchler \& Cie

Scheffer, David und Julius Kuhl (2010). „Volitionale Prozesse der Zielverfolgung“. In: Enzyklopädie der Psychologie. Bd. 1: Arbeitspsychologie. Hrsg. von Uwe Kleinbeck und Klaus-Helmut Schmidt. III Wirtschafts-, Organisations- und Arbeitspsychologie. Göttingen u.a.: Hogrefe, 89-137

Schild, André (1957). „Interlingua - die aufkommende Welthilfssprache“. Prelego dum la 2a Internacia Kongreso de Interlingua en Basel. In: Universalaj lingvoj en Svislando: svisa enciklopedio planlingva. Hrsg. von Andreas Künzli. 2006. Aufl. La Chaux-de-Fonds: Svisa Esperanto-Soceto, 778-790

Schleyer, Johann Martin (1982). Volapük. die Weltsprache. Entwurf einer Universalsprache für alle Gebildete der ganzen Erde. Nachdruck der Ausgabe Sigmaringen 1880. beigebunden ist: Reinhard Haupenthal: Volapük-Bibliographie. Hildesheim u.a.: Georg Olms

Schlieben-Lange, Brigitte (1991). Soziolinguistik. Eine Einführung. 3. überarbeitete und erweiterte Aufl. Stuttgart: Kohlhammer

Schmidt, Christian und Ramona Mönch-Bläsing (2011). „Semantic vocabulary management". In: Edition 7.1, 13-15

Schmidt, Johann (1963). Geschichte der Universalsprache Volapük. Mit einem Nachw. von Reinhard Haupenthal. Edition Iltis, 2. Auflage 1998. Vergrößerter Faksimile-Druck des Original-Typoskripts Weißkrichen a. Ts. 1963. Saarbrücken. Literaturverzeichnis von Reinhard Haupenthal

Schmidt-Wigger, Antje (1998). „Grammar and style checking for German“. In: Proceedings of the second international workshop on controlled language application. CLAW 98. (Pittsburgh, Pennsylvania, 21.-22. Mai 1998), 76-86 


\section{Literatur}

Schmitz, Klaus-Dirk und Daniela Straub (2010). Erfolgreiches Terminologiemanagement im Unternehmen. Praxishilfe und Leitfaden: Grundlagen, Umsetzung, Kosten-Nutzen-Analyse, Systemübersicht. Stuttgart: TC und more

Schmitz, Klaus-Dirk und Daniela Straub (2016). Erfolgreiches Terminologiemanagement im Unternehmen. Praxishilfe und Leitfaden: Grundlagen, Umsetzung, Kosten-Nutzen-Analyse, Systemübersicht. 2. aktualisierte Auflage. Stuttgart: TC und more

Schnar, Natalie (2010). Sprache als Kriterium ethnischer Identität. Eine empirische Studie zum Stellenwert des Russischen im Ethnizitätskonzept russlanddeutscher Jugendlicher in der Diaspora Deutschland. Hamburg: Verlag Dr. Kovač

Schnieder, Lars, Christian Stein und Arno G. Schielke (2011). „Terminologiemanagementsysteme der nächsten Generation - Schlüssel für den Fachwortschatz“. In: Edition 7.1, 26-31

Schubert, Klaus (1989a). „An unplanned development in planned languages. A study of word grammar". In: Interlinguistics. Aspects of the science of planned languages. Hrsg. von Klaus Schubert. Hrsg. von Dan Maxwell. Trends in Linguistics. Studies and Monographs 42. Berlin: Mouton, 249-274

Schubert, Klaus (1989b). „Interlinguistics - its aims, its achievements, and its place in language science“. In: Interlinguistics. Aspects of the science of planned languages. Hrsg. von Klaus Schubert. Hrsg. von Dan Maxwell. Trends in Linguistics. Studies and Monographs 42. Berlin: Mouton, 7-44

Schubert, Klaus (1992). „Esperanto as an intermediate language for machine translation“. In: Computers in translation. A practical appraisal. Hrsg. von John Newton. London/New York: Routledge, 78-95

Schubert, Klaus (1997). „Ausdruckskraft und Regelmäßigkeit: Was Esperanto für automatische Übersetzung geeignet macht“". In: Esperanto, interlinguistics, and planned language. Hrsg. von Humphrey Tonkin. Lanham: University Press of America, 117-139

Schubert, Klaus (2001a). „Gestaltete Sprache. Plansprachen und die regulierten Sprachen der internationalen Fachkommunikation". In: Planned languages: from concept to reality. Hrsg. von Klaus Schubert. Brüssel: Hogeschool voor Wetenschap \& Kunst, 223-257

Schubert, Klaus (2001b). „Plansprachen zwischen Plan und Sprache. Einleitung“. In: Planned languages: from concept to reality. Hrsg. von Klaus Schubert. Brüssel: Hogeschool voor Wetenschap \& Kunst, 5-9

Schubert, Klaus (2003). „Plansprachen und internationale Fachkommunikation“. In: Plansprachen und Elektronische Medien. Beiträge der 12. Jahrestagung der Gesellschaft für Interlinguistik e.V. (Berlin, 6.-8. Dez. 2003). Hrsg. von Detlev Blanke. Gesellschaft für Interlinguistik e.V., 150-161

Schubert, Klaus (2007). Wissen, Sprache, Medium, Arbeit. Ein integratives Modell der einund mehrsprachigen Fachkommunikation. Tübingen: Gunter Narr

Schubert, Klaus (2009). „Kommunikationsoptimierung. Vorüberlegungen zu einem fachkommunikativen Forschungsfeld“. In: trans-kom. Zeitschrift für Translationswissenschaft und Fachkommunikation 2.1, 109-150. प⿴囗口 Dhttp : //www . trans-kom . eu/bd02nr01/ trans-kom_02_01_06_Schubert_Kommunikationsoptimierung.20090721.pdf

Schubert, Klaus (2011a). „Optimierung als Kommunikationsziel: bessere Sprachen“. In: Fach - Translat - Kultur. Bd. 1: Fach - Translat - Kultur. Hrsg. von Klaus-Dieter Baumann. 2 Bde. Berlin: Frank \& Timme, 363-392 
Schubert, Klaus (2011b). „Zum bewussten Eingreifen in die Sprache“. In: Florilegium interlinguisticum. Festschrift für Detlev Blanke zum 70. Geburtstag. Hrsg. von Cyril Brosch und Sabine Fiedler. Frankfurt a. M.: Peter Lang, 47-60

Schubert, Klaus (2013). „Bürgernahe Sprache. Überlegungen aus fachkommunikationswissenschaftlicher Sicht“. In: Synaps. A journal of professional communication 29, 48-57. 口प⿴https : / / www .nhh.no/Admin/Public/DWSDownload. aspx?File=\%2fFiles $\%$ 2fFiler $\% 2$ finstitutter $\% 2$ ffsk $\% 2$ fSynaps $\% 2 f 29-2013 \% 2$ fSchubert_29.pdf

Schubert, Klaus (2014). „Barrierefrei, reguliert, gelenkt. Prinzipien optimierenden Eingreifens in Sprache und Kommunikation“. In: Sprache barrierefrei gestalten. Perspektiven aus der Angewandten Linguistik. Hrsg. von Susanne J. Jekat, Heike Elisabeth Jüngst, Klaus Schubert und Claudia Villiger. TransÜD 69. Berlin: Frank \& Timme, 201-220

Schuchardt, Hugo (1904). „Bericht über die auf Schaffung einer künstlichen internationalen Hilfssprache gerichtete Bewegung“. In: Plansprachen. Beiträge zur Interlinguistik. Hrsg. von Reinhard Haupenthal. 1976. Aufl. Darmstadt: Wissenschaftliche Buchgesellschaft, $48-58$

Schüler, Julia und Veronika Brandstätter (2010). ,Zielbildung und Zielbindung“. In: Enzyklopädie der Psychologie. Bd. 1: Arbeitspsychologie. Hrsg. von Uwe Kleinbeck und KlausHelmut Schmidt. III Wirtschafts-, Organisations- und Arbeitspsychologie. Göttingen u.a.: Hogrefe, 39-88

Schwertel, Uta (2000). „Controlling plural ambiguities in Attempto Controlled English (ACE)“. In: Proceedings of the third international workshop on controlled language applications. CLAW 2000. (Seattle, USA, 29.-30. Apr. 2000), 105-119

Schwitter, Rolf(1998). ,Kontrolliertes Englisch für Anforderungsspezifikationen“. Diss. Zürich: Universität Zürich

Schwitter, Rolf(2010a). „Anaphora resolution involving interactive knowledge acquisition“. In: Controlled Natural Language. Workshop on Controlled Natural Language, CNL 2009. (Marettimo Island, Italy, 8.-10. Juni 2009). Hrsg. von Norbert E. Fuchs. Lecture Notes in Artificial Intelligence 5972. Berlin u.a.: Springer, 36-55

Schwitter, Rolf (2010b). „Controlled Natural Languages for knowledge representation“. In: Coling. 23rd International Conference on Computational Linguistics. (23.-25. Aug. 2010). Hrsg. von Chu-Ren Huang und Dan Jurafsky. Posters. Beijing: Chinese Information Processing Society of China, 1113-1121

Schwitter, Rolf (2012). „Working with events and states in PENG Light“. In: Controlled Natural Language. Second International Workshop, CNL 2010. (Marettimo Island, Italy, 13.-15. Sep. 2010). Hrsg. von Michael Rosner und Norbert E. Fuchs. Lecture Notes in Artificial Intelligence 7175. Berlin/Heidelberg: Springer, 154-174

Schwitter, Rolf und Marc Tilbrook (2006). „Writing RSS feeds in a machineprocessable Controlled Natural Language“. In: 5th International Workshop on Controlled Language Applications, CLAW 2006. (Cambridge, Massachusetts, USA, 12. Aug. 2006). PDF-Seitenzählung als Seitenangabe verwendet, o.S. प्ण http: //www.mt-archive. info/CLAW-2006-Schwitter.pdf

Seewald-Heeg, Uta (2009). „Terminologieverwaltungssysteme“. In: Terminologiemanagement. Von der Theorie zur Praxis. Hrsg. von Felix Mayer und Uta Seewald-Heeg. Berlin: BDÜ, 35-81

Shaw, David (2006). „Simplified Technical English in the 21st century. Summary“. In: 5th International Workshop on Controlled Language Applications, CLAW 2006. (Cambridge, 
Massachusetts, USA, 12. Aug. 2006). PDF-Seitenzählung als Seitenangabe verwendet,

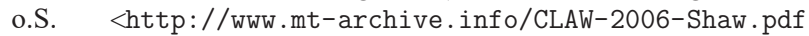

Sheremetyeva, Svetlana (2006). ,Integration of correction modules in a controlled language application“. In: 5th International Workshop on Controlled Language Applications, CLAW 2006. (Cambridge, Massachusetts, USA, 12. Aug. 2006). PDF-Seitenzählung als Seitenangabe verwendet, o.S. $\square \square \square$ http : / / www . mt-archive . info / CLAW-2006Sheremetyeva.pdf

Shiffman, Richard N., George Michel, Michael Krauthammer, Norbert E. Fuchs, Kaarel Kaljurand und Tobias Kuhn (2010). „Writing clinical practice guidelines in controlled natural language“. In: Controlled Natural Language. Workshop on Controlled Natural Language, CNL 2009. (Marettimo Island, Italy, 8.-10. Juni 2009). Hrsg. von Norbert E. Fuchs. Lecture Notes in Artificial Intelligence 5972. Berlin u.a.: Springer, 265-280

Shohamy, Elana Goldberg (2006). Language policy: hidden agendas and new approaches. London: Routledge

Siegel, Melanie und Christian Lieske (2015). „Beitrag der Sprachtechnologie zur Barrierefreiheit: Unterstützung für Leichte Sprache“. In: Trans-kom 8.1, 40-78

Simon, Bernd und Roman Trötschel (2007). „Das Selbst und die soziale Identität“. In: Sozialpsychologie. Hrsg. von Klaus Jonas, Wolfgang Stroebe und Miles Hewstone. 5., vollständig überarbeitete Auflage. Berlin/Heidelberg: Springer, 147-185

Six, Bernd und Brigitte Höcke-Pörzgen (1983). „Motivationstheorie und Handlungstheorie“. In: Enzyklopädie der Psychologie. Bd. 1: Theorie und Formen der Motivation. Hrsg. von Hans Thomae. IV Motivation und Emotion. Göttingen u.a.: Hogrefe, 227-290

Skutnabb-Kangas, Tove (2009). „Language planning and language rights“. In: Handbook of language and communication: diversity and change. Hrsg. von Marlis Hellinger und Anne Pauwels. Handbooks of Applied Linguistics 9. Berlin/New York: Mouton de Gruyter, 365397

Smart, Paul R., Jie Bao, Dave Braines und Nigel R. Shadbolt (2010). ,Development of a controlled natural language interface for Semantic MediaWiki“. In: Controlled Natural Language. Workshop on Controlled Natural Language, CNL 2009. (Marettimo Island, Italy, 8.-10. Juni 2009). Hrsg. von Norbert E. Fuchs. Lecture Notes in Artificial Intelligence 5972. Berlin u.a.: Springer, 206-225

Smith, Arden R. (2011). „Confounding Babel: international auxilary languages“. In: From Elvish to Klingon. Exploring invented languages. Hrsg. von Michael Adams. Oxford: Oxford University Press, 17-48

Sonnentag, Sabine, Doris Fay und Michael Frese (2004). „Handeln in Organisationen“. In: Enzyklopädie der Psychologie. Organisationspsychologie - Gruppe und Organisation. Hrsg. von Heinz Schuler. III Wirtschafts-, Organisations- und Arbeitspsychologie. Göttingen u.a.: Hogrefe, 251-291

Spaggiari, Laurent und Sylviane Cardey (2010). „A system to control language for oral communication“. In: Advances in natural language processing. 7th International Conference on Natural Language Processing IceTAL 2010. (Reykjavik, Iceland, 16.-18. Aug. 2010). Hrsg. von Hrafn Loftsson, Eiríkur Rögnvaldsson und Sigrún Helgadóttir. Lecture Notes in Computer Science 6233. Berlin u.a.: Springer, 393-400

Spears, Russell und Nicole Tausch (2014). ,Vorurteile und Intergruppenbeziehungen“. In: Sozialpsychologie. Hrsg. von Klaus Jonas, Wolfgang Stroebe und Miles Hewstone. 6., vollständig überarbeitete Auflage. Berlin/Heidelberg: Springer, 507-564 
Spolsky, Bernard (2004). Language policy. Cambridge University Press

Spolsky, Bernard (2009). Language management. Cambridge: Cambridge University Press

Spolsky, Bernard (2011). ,Achievements and prospects of the language policies in the world. A tentative taxonomy of language management agencies and an evaluation of their achievements and prospects“. In: Current status and prospects of the language policies in the world. The National Institute of the Korean Language, 1-28

Spolsky, Bernard (2012). „What is language policy?“ In: The Cambridge handbook of language policy. Hrsg. von Bernard Spolsky. Cambridge: Cambridge University Press. Kap. 1, 3-15

Spreeuwenberg, Silvie, Jeroen van Grondelle, Ronald Heller und Gartjan Grijzen (2012). „Using CNL techniques and pattern sentences to involve domain experts in modeling“. In: Controlled Natural Language. Second International Workshop, CNL 2010. (Marettimo Island, Italy, 13.-15. Sep. 2010). Hrsg. von Michael Rosner und Norbert E. Fuchs. Lecture Notes in Artificial Intelligence 7175. Berlin/Heidelberg: Springer, 175-193

Spreeuwenberg, Silvie und Keri Anderson Healy (2010). „SBVR's approach to controlled natural language“". In: Controlled Natural Language. Workshop on Controlled Natural Language, CNL 2009. (Marettimo Island, Italy, 8.-10. Juni 2009). Hrsg. von Norbert E. Fuchs. Lecture Notes in Artificial Intelligence 5972. Berlin u.a.: Springer, 155-169

Steenbergen, Jan van (2008). Multilingual mutterings. Classificatie van kunsttalen. Online. zuletzt geändert: 10.12.2008. [DDhttp://steen.free.fr/classificatie.html

Stein, Christian (2013). ,Interdisziplinäre Terminologie. Über die Konzeption einer Ontologie für den Exzellenzcluster „Bild Wissen Gestaltung“““. In: Edition 2, 15-19

Stocker, Frank (2002). „Wer spricht Esperanto? - eine soziologische Untersuchung zum Deutschen Esperanto Bund e.V.“ In: Plansprachen und ihre Gemeinschaften. Beiträge der 11. Jahrestagung der Gesellschaft für Interlinguistik e.V. (Berlin, 23.-25. Nov. 2001). Hrsg. von Detlev Blanke. Gesellschaft für Interlinguistik e.V., 37-52

Strasser, Gerhard F. (2012). „Ansätze zu internationaler Verständigung durch konstruierte Sprachen im 17. Jahrhundert". In: Zwischen Utopie und Wirklichkeit. Konstruierte Sprachen für die globalisierte Welt. Begleitband zur Ausstellung an der Bayerischen Staatsbibliothek (14. Juni bis 9. September 2012). Hrsg. von Andrea Pia Kölbl und Heiner Eichner. München: Allitera Verlag, 33-61

Strauss, Anselm und Juliet Corbin (1996). Grounded Theory. Grundlagen qualitativer Sozialforschung. Aus dem Amerikanischen übers. von Solveigh Niewiarra und Heiner Legewie. Weinheim: Beltz, PsychologieVerlagsUnion

Strehlow, Richard A. (2001a). „Terminology and indexing“. In: Handbook of terminology management. Bd. 2: Application-oriented terminology management. Hrsg. von Sue Ellen Wright und Gerhard Budin, 419-425

Strehlow, Richard A. (2001b). ,The role of terminology in retrieving information“. In: Handbook of terminology management. Bd. 2: Application-oriented terminology management. Hrsg. von Sue Ellen Wright und Gerhard Budin, 426-441

Strevens, Peter und Edward Johnson (1983). „SEASPEAK: a project in applied linguistics, language engineering, and eventually ESP for sailors“. In: The esp journal 2.2, 123-129. 口प⿴囗十 http://www. sciencedirect.com/science/article/pii/0272238093900020 (besucht am 10.02.2015) 


\section{Literatur}

Stroebe, Wolfgang (2007). „Strategien zur Einstellungs- und Verhaltensanderung“. In: Sozialpsychologie. Hrsg. von Klaus Jonas, Wolfgang Stroebe und Miles Hewstone. 5., vollständig überarbeitete Auflage. Berlin/Heidelberg: Springer, 225-263

Stroebe, Wolfgang (2014). „Strategien zur Einstellungs- und Verhaltensanderung“. In: Sozialpsychologie. Hrsg. von Klaus Jonas, Wolfgang Stroebe und Miles Hewstone. 6., vollständig überarbeitete Auflage. Berlin/Heidelberg: Springer, 231-268

Stroebe, Wolfgang, Miles Hewstone und Klaus Jonas (2014). „Einführung in die Sozialpsychologie“. In: Sozialpsychologie. Hrsg. von Klaus Jonas, Wolfgang Stroebe und Miles Hewstone. 6., vollständig überarbeitete Auflage. Berlin/Heidelberg: Springer, 1-28

Stürmer, Stefan (2009). Sozialpsychologie. Reinhardt

Sturz, Wolfgang (2014). „Von der Terminologieverwaltung zum Terminologiemanagement“. In: Edition 10.1, 20-23

Suchowolec, Karolina (2009). „Terminologiearbeit im Unternehmen. Einführung eines Gesamtkonzeptes“. Magisterarbeit. Technische Universität Dresden. unveröffentlicht

Suchowolec, Karolina (2011). „Vorlesung zur Terminologielehre. Folien der Vorlesung Terminologielehre, Hochschule Karlsruhe. Karolina Suchowolec und Ayten Turhan“. Sommersemester 2011; 6. Sitzung, 27.2011

Suchowolec, Karolina (2012), ,Terminologische Methodenarbeit im Kontext der Sprachlenkung“. In: Trans-kom 5.1, 29-39

Suchowolec, Karolina (2014). „Are style guides controlled languages? The case of Koenig \& Bauer AG“. In: Controlled Natural Language. 4th International Workshop, CNL 2014. (Galway, Ireland, 20.-22. Aug. 2014). Hrsg. von Brian Davis, Kaarel Kaljurand und Tobias Kuhn. Lecture Notes in Artificial Intelligence 8625. Cham u.a.: Springer, 112-122

Suchowolec, Karolina (o.D.). „The intricacy of a goal-based classification of Controlled Natural Languages“. In: demnächst

Święczkowska, Halina (2012). „Cognitive functions of language according to G. W. Leibniz“. In: Studies in logic, grammar and rhetoric 28.41, 45-62

Tajfel, Henri (1982). Gruppenkonflikt und Vorurteil. Entstehung und Funktion sozialer Stereotypen. Bern u.a.: Huber

Tajfel, Henri und John Turner (1986). „The social identity theory of intergroup behaviour“. In: Psychology of intergroup relations. Hrsg. von Stephen Worchel und William G. Austin. Chicago: Nelson, 7-24

Tajfel, Henry und John Turner (1979). „An integrative theory of intergroup conflict“. In: The social psychology of intergroup relations. Hrsg. von William G. Austin und Stephen Worchel. Monterey, CA: Books/Cole Publishing Company, 33-47

Tauli, Valter (1968). Introduction to a theory of language planning. Acta Universitatis Upsaliensis: Studia philologiae Scandinavicae Upsaliensia ; 6. Uppsala: Almqvist \& Wiksells

tekom-AG „Englisch für deutschsprachige Autoren“ (2014). Leitlinie regelbasiertes Schreiben. Englisch für deutschsprachige Autoren. Stuttgart: tekom

tekom-AG „Regelbasiertes Schreiben“ (2013). Leitlinie regelbasiertes Schreiben. Deutsch für die Technische Kommunikation. 2. erweiterte Aufl. Stuttgart: tekom

Temmerman, Rita (2000). Towards new ways of terminology description. The sociocognitive approach. Amsterdam/Philadelphia: John Benjamins Publishing Company

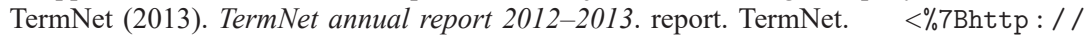
www . termnet.org/downloads/english/about_us/TN_AR_2012-2013.pdf\%7D (besucht am 17.03.2015) 
TermNet (2006-). Homepage TermNet. International network for terminology $\square \square \square$ : $\% 7$ Bhttp: //www . termnet.org\%7D (besucht am 20.03.2015)

Tesser, Abraham (1988). ,Toward a self-evaluation maintenance model of social behavior“. In: Advances in experimental social psychology 21, 181-227

Tewes, Uwe und Klaus Wildgrube, Hrsg. (1999). Psychologie-Lexikon. 2., überarbeitete und erweiterte Auflage. 1. Aufl. 1992. München/Wien: R. Oldenbourg Verlag

The Conlang Mailing List (1998-). Constructed languages list $\square \square \square$ : http : / / listserv . brown . edu/archives/cgi-bin/wa?A0=CONLANG (besucht am 25.01.2014)

Thorne, Camilo, Raffaella Bernardi und Diego Calvanese (2014). „Designing efficient controlled languages for ontologies“. In: Computing meaning. Hrsg. von Harry Bunt, Johan Bos und Stephen Pulman. Bd. 4. Text, speech and language technology 47. Dordrecht u.a.: Springer, 149-173

Thorne, Camilo und Diego Calvanese (2010). „Controlled english ontology-based data access". In: Controlled Natural Language. Workshop on Controlled Natural Language, CNL 2009. (Marettimo Island, Italy, 8.-10. Juni 2009). Hrsg. von Norbert E. Fuchs. Lecture Notes in Artificial Intelligence 5972. Berlin u.a.: Springer, 135-154

Thouin, Benoît (1982). ,The Meteo System“. In: Practical experience of machine translation. (London, 5.-6. Nov. 1981). Hrsg. von Veronica Lawson. Amsterdam u.a.: North-Holland Publishing Company, 39-44

Thouin, Benoît (1984). „Les conditions d'efficacité des banques de terminologie“. In: Actes du cinquième colloque OLF-STQ de terminologie. Terminologie et communication. (Montréal, 13.-15. Feb. 1984). Hrsg. von Office de la langue française. Société des traducteurs du Québec, 271-284

Tollefson, James W. (2011). „Language planning and language policy“. In: The Cambridge handbook of sociolinguistics. Hrsg. von Rajend Mesthrie. Cambridge: Cambridge University Press. Kap. 20, 357-376

Tonkin, Humphrey (1997a). „Introduction: planned language and LPLP“. In: Esperanto, interlinguistics, and planned language. Hrsg. von Humphrey

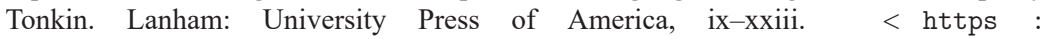
/ / books . google . de / books ? id=DdBjfQPprDoC\&pg=PR1\&hl=de\& source=gbs selected_pages\&cad $=2 \# v=$ onepage\&q\&f $=f$ alse (besucht am 11.08.2015)

Tonkin, Humphrey (1997b). „One hunderd years of Esperanto: a survey“. In: Esperanto, interlinguistics, and planned language. Hrsg. von Humphrey Tonkin. Lanham: University Press of America, 71-92

Tonkin, Humphrey (2001). „The role of literary language in Esperanto“. In: Planned languages: from concept to reality. Hrsg. von Klaus Schubert. Brüssel: Hogeschool voor Wetenschap \& Kunst, 11-35

Tonkin, Humphrey (2007). „Recent studies in Esperanto and interlinguistics: 2006“. In: Language problems \& language planning 31.2, 169-196

Tonkin, Humphrey (2009). „Arika Okrent. „In the land of invented languages“. Review“. In: Language problems \& language planning 33.3, 291-293

Tonkin, Humphrey (2011). „Plansprachen als Modelle der Sprachplanung“. In: Florilegium interlinguisticum. Festschrift für Detlev Blanke zum 70. Geburtstag. Hrsg. von Cyril Brosch und Sabine Fiedler. Frankfurt a. M.: Peter Lang, 61-68

Translation Centre for the Bodies of the European Union (1997-). InterActive Terminology

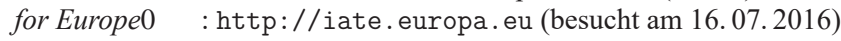




\section{Literatur}

Trubetzkoy, Nikolai Sergejewitsch (1939). „Wie soll das Lautsystem einer künstlichen internationalen Hilfssprache beschaffen sein?" In: Plansprachen. Beiträge zur Interlinguistik. Hrsg. von Reinhard Haupental. 1976. Aufl. Darmstadt: Wissenschaftliche Buchgesellschaft, 198-216

Tuckman, Bruce W. (1965). „Developmental sequence in small groups“. In: Psychological bulletin 63.6, 384-399

Turhan, Ayten (2011). ,9-Phasen-Modell der Terminologiearbeit nach Prof. Dr. Petra Drewer. Folien der Vorlesung Terminologielehre, Hochschule Karlsruhe. Karolina Suchowolec und Ayten Turhan“. Sommersemester 2011; 1. Sitzung, 16.03.2011

Turhan, Ayten und Oliver Collmann (2010). „FIDUCIA IT AG - Terminologie geht auf's Ganze“. In: Edition 6.1, 36-37

Turner, John C. (1987). Rediscovering the social group. Oxford u.a.: Blackwell

Wandruszka, Mario (1971). Interlinguistik: Umrisse einer neuen Sprachwissenschaft. ger. München: Piper

Weilandt, Annette (2014). „Prozessorientiertes Terminologiemanagement“. In: Edition 10.2, $11-13$

Weiner, E. S. C. und Jeremy Marshall (2011), „Tolkien’s invented languages“. In: From Elvish to Klingon. Exploring invented languages. Hrsg. von Michael Adams. Oxford University Press

Weisgerber, Leo (1959-1960). „Ein Markstein Angewandter Sprachwissenschaft: Begegnung mit Eugen Wüster". In: Sprachforum 3, 92-95

Welger, Helmut (1998). „Plansprachliche Gütekriterien und ihre Konkretisierung und Gewichtung bei Zamenhof". In: Soziokulturelle Aspekte von Plansprachen. Beiträge gehalten auf der 7. Jahrestagung der Gesellschaft für Interlinguistik e.V. (Berlin, 7.-9. Nov. 1997). Hrsg. von Ulrich Becker. Gesellschaft für Interlinguistik e.V., 34-41

Wenninger, Gerd, Hrsg. (2001a). Lexikon der Psychologie in fünf Bänden. Bd. 2: F bis L. Heidelberg: Spektrum Akademischer Verlag

Wenninger, Gerd, Hrsg. (2001b). Lexikon der Psychologie in fünf Bänden. Bd. 3: M bis Ref. Heidelberg: Spektrum Akademischer Verlag

Wersig, Gernot (1979). „Terminologieforschung und Informationswissenschaft - zwei Disziplinen in Kinderschuhen“. In: Terminologie als angewandte Sprachwissenschaft. Gedenkschrift für Univ.-Prof. Dr. Eugen Wüster. Hrsg. von Helmut Felber, Friedrich Lang und Gernot Wersig. München u.a.: Saur, 195-207

Werth, Lioba und Jennifer Mayer (2008). Sozialpsychologie. Heidelberg: Spektrum Akademischer Verlag

Wetzel, Michael (2014). „Multilinguale Taxonomien mit Coreon. Wissens- und Sprachmanagement in einer Lösung“. In: Rechte, Rendite, Ressourcen. Wirtschaftliche Aspekte des Terminologiemanagements. 14. Symposion des Deutschen Terminologie-Tages e.V. (Mannheim, 27.-29. März 2014). Hrsg. von Petra Drewer, Felix Mayer und Klaus-Dirk Schmitz. Köln: Deutscher Terminologie-Tag e.V., 41-51

Wieden, Wilfried (2011). „Wissensmanagement und Terminologie“. In: Edition. Fachzeitschrift für Terminologie 7.1, 8-15

Wilkins, John (1668). An essay towards a real character, and a philosophical language. London: Gellibrand. पDDhttp : / / books . google . de / books ? id=BCCtZjBtiEYC \& printsec $=$ frontcover\&hl=de\&source=gbs_ge_summary_r\&cad=0

Williams, Kipling D. (2001). Ostracism: the power of silence. New York: The Guilford Press 
Winkler, Kurt, Tobias Kuhn und Martin Volk (2014). „Evaluating the fully automatic multilanguage translation of Swiss avalanche bulletin“. In: Controlled Natural Language. 4th International Workshop, CNL 2014. (Galway, Ireland, 20.-22. Aug. 2014). Hrsg. von Brian Davis, Kaarel Kaljurand und Tobias Kuhn. Lecture Notes in Artificial Intelligence 8625. Cham u.a.: Springer, 44-54

Wojcik, Richard H. und James E. Hoard (1997). „Controlled languages in industry“. In: Survey of the state of the art in human language technology. Hrsg. von Giovanni Battista Varile und Antonio Zampolli. Hrsg. von Roland Cole, Joseph Mariani, Hans Uszkoreit, Annie Zaenen und Victor Zue. Studies in Natural Language Processing XII-XIII. Pisa/ Cambridge: Giardini Editori e Stampatori in Pisa/Cambridge University Press, 238-243

Wojcik, Richard H. und Heather Holmback (1996). „Getting a controlled language off the ground at Boeing“. In: Proceedings of the first international workshop on controlled language applications. The first international workshop on controlled language applications, CLAW 1996. (Katholieke Universiteit Leuven, Belgien, 26.-27. März 1996), 22-32

Wojcik, Richard, Heather Holmback und James Hoard (1998). „Boeing Technical English. An extension of AECMA SE beyond the aircraft maintenance domain“. In: Proceedings of the second international workshop on controlled language application. CLAW 98. (Pittsburgh, Pennsylvania, 21.-22. Mai 1998), 114-123

Wölbrink, Birgit (2008). „Tools für Terminologiemanagement“. In: Terminologiearbeit für Technische Dokumentation. Hrsg. von Jörg Hennig und Marita Tjarks-Sobhani. tekomSchriften zur technischen Kommunikation 12. Lübeck: Schmidt-Römhild, 80-92

Wood, Richard E. (1979). „A voluntary non-ethnic and non-territorial speech community“. In: Sociolinguistic studies in language contact: methods and cases. Hrsg. von William Francis Mackey. Bd. 6. Trends in linguistics: Studies and monographs. The Hague u.a.: Mouton, 433-450

Wright, Sue (2004). Language policy and language planning: from nationalism to globalisation. Basingstoke: Palgrave Macmillan

Wright, Sue Ellen und Gerhard Budin (1997). „Introduction“. In: Handbook of terminology management. Bd. 1: Basic aspects of terminology management. Hrsg. von Sue Ellen Wright und Gerhard Budin, 1-10

Wright, Sue Ellen und Leland D. Wright (1997). „Terminology management for technical translation“. In: Handbook of terminology management. Bd. 1: Basic aspects of terminology management. Hrsg. von Sue Ellen Wright und Gerhard Budin, 147-159

Wüster, Eugen (1931). Internationale Sprachnormung in der Technik. Besonders in der Elektrotechnik. 3. abermals ergänzte Aufl. 1970. Bonn: Bouvier

Wüster, Eugen (1955). „Die Benennungen „Esperantologie“ und „Interlinguistik“““. In: Plansprachen. Beiträge zur Interlinguistik. Hrsg. von Reinhard Haupenthal. 1976. Aufl. Darmstadt: Wissenschaftliche Buchgesellschaft, 271-277

Wüster, Eugen (1959-1960). „Das Worten der Welt“. In: Sprachforum 3/314, 183-204

Wüster, Eugen (1974). „Die allgemeine Terminologielehre - ein Grenzgebiet zwischen Sprachwissenschaft, Logik, Ontologie, Informatik und den Sachwissenschaften“. In: Germanistik online Datenbank. Internationales Referatenorgan mit bibliographischen Hinweisen 1 (1960) - 54 (2013), 61-106. पDD http : / / www . degruyter . com/view/OGERM/g74151698? rskey=3hRmPB\&result $=4$ (besucht am 23.03.2015) 
Wüster, Eugen (1979). Einführung in die allgemeine Terminologielehre und terminologische Lexikographie. Bd. 1: Textteil

Wüster, Eugen (1991). Einführung in die allgemeine Terminologielehre und terminologische Lexikographie. Mit einer Einl. von Richard Baum. 3 Aufl. Bonn: Romanistischer Verlag

Wyner, Adam, Krasimir Angelov, Guntis Barzdins, Danica Damljanovic, Brian Davis, Norbert Fuchs, Stefan Hoefler, Ken Jones, Kaarel Kaljurand, Tobias Kuhn, Martin Luts, Jonathan Pool, Mike Rosner, Rolf Schwitter und John Sowa (2010). „On controlled natural languages: properties and prospects“. In: Controlled Natural Language. Workshop on Controlled Natural Language, CNL 2009. (Marettimo Island, Italy, 8.-10. Juni 2009). Hrsg. von Norbert E. Fuchs. Lecture Notes in Artificial Intelligence 5972. Berlin u.a.: Springer, 281-289

Yamchi, Ana Arandan (2012). „Terminologie in der Technischen Dokumentation: Qualität planen und sichern“. In: Edition 8.1, 27-30

Zamenhof, Ludovic Lazarus (1905). „Letero pri la deveno de Esperanto. Brief an Nikolai Afrikanovitsch Borovko“. In: Żelazny, Walter. Ludwik Zamenhof. Życie i dzieło, recepcja i reminiscencje, wybór pism i listów. Übers. von Walter Żelazny (aus Esperanto). Kraków: Nomos, 103-106

Żelazny, Walter (2012). Ludwik Zamenhof. Życie i dzieło, recepcja i reminiscencje, wybór pism i listów. Kraków: Nomos

Zelewski, Stephan (2002). Wissensmanagement mit Ontologien. Eine einführende Darstellung. Techn. Ber. Universität GH Essen

Zelewski, Stephan (2005). „Einführung in das Themenfeld „Ontologien“ aus informationsund betriebswirtschaftlicher Perspektive". In: Ontologiebasierte Kompetenzmanagementsysteme. Grundlagen, Konzepte, Anwendung. Hrsg. von Stephan Zelewski, Yilmaz Alan, Adem Alparslan, Lars Dittmann und Thomas Weichelt. Berlin: Logos, 115-229

Zerfaß, Angelika (2009). „Terminologieaustausch“. In: Terminologiemanagement. Von der Theorie zur Praxis. Hrsg. von Felix Mayer und Uta Seewald-Heeg. Berlin: BDÜ, 93-107

Zimmermann, Dieter (1977). Sprachwissenschaft und Terminologiearbeit. Bd. 1: „Linguistische Normung “ zu Lasten der Verständigung unter Technikern. Arbeitsgruppe Vereinheitlichung, Sitzung vom 18. Oktober 1976 


\section{Anhang}

Der Anhang befindet sich zum Download auf der Verlags-Webseite. 


\section{SPRACHWISSENSCHAFT}

Band 1 Sergio Viaggio: A General Theory of interlingual Mediation. 408 Seiten. ISBN 978-3-86596-063-4

Band 2 Karl-Ernst Sommerfeldt: Regionalgeschichte im Spiegel der Sprache. 188 Seiten. ISBN 978-3-86596-077-1

Band 3 Ulla Fix: Stil - ein sprachliches und soziales Phänomen. Beiträge zur Stilistik. Herausgegeben von Irmhild Barz, Hannelore Poethe, Gabriele Yos. 462 Seiten.

ISBN 978-3-86596-138-9

Band 4 Anja Seiffert: Autonomie und Isonomie fremder und indigener Wortbildung am Beispiel ausgewählter numerativer Wortbildungseinheiten. 418 Seiten. ISBN 978-3-86596-141-9

Band 5 Ulla Fix: Texte und Textsorten - sprachliche, kommunikative und kulturelle Phänomene. 510 Seiten. ISBN 978-3-86596-179-2

Band 6 Juliana Goschler: Metaphern für das Gehirn. Eine kognitivlinguistische Untersuchung. 244 Seiten. ISBN 978-3-86596-188-4

Band 7 Maria Wirf Naro: La composition en allemand : structure sémantique et fonction littéraire. Mit einer ausführlichen Zusammenfassung in deutscher Sprache. 416 Seiten. ISBN 978-3-86596-284-3

Band 8 Bettina Bock/Ulla Fix/Steffen Pappert (Hg.): Politische Wechsel sprachliche Umbrüche. 404 Seiten. ISBN 978-3-86596-363-5

Band 9 Kerstin Preiwuß: Ortsnamen in Zeit, Raum und Kultur. Die Städte Allenstein/Olsztyn und Breslau/Wrocław. 470 Seiten. ISBN 978-3-86596-368-0

Frank \& Timme 


\section{SPRACHWISSENSCHAFT}

Band 10 Werner Holly: Sprache und Politik. Pragma- und medienlinguistische Grundlagen und Analysen, herausgegeben von Sonja Ruda und Christine Domke. 394 Seiten. ISBN 978-3-86596-438-0

Band 11 Hatice Deniz Canoğlu: Kanak Sprak versus Kiezdeutsch - Sprachverfall oder sprachlicher Spezialfall? Eine ethnolinguistische Untersuchung. 138 Seiten. ISBN 978-3-86596-483-0

Band 12 Ulla Fix: Sprache in der Literatur und im Alltag. Ausgewählte Aufsätze. 452 Seiten. ISBN 978-3-86596-369-7

Band 13 Anja Hennemann/Claudia Schlaak (Hg.): Korpuslinguistische Untersuchungen. Analysen einzelsprachlicher Phänomene. 202 Seiten. ISBN 978-3-86596-519-6

Band 14 Joachim Born/Wolfgang Pöckl (Hg.): „Wenn die Ränder ins Zentrum drängen ...“. Außenseiter in der Wortbildung(sforschung). 304 Seiten. ISBN 978-3-86596-449-6

Band 15 Ulla Fix: Sprache, Sprachgebrauch und Diskurse in der DDR. Ausgewählte Aufsätze. 744 Seiten. ISBN 978-3-86596-549-3

Band 16 Jenny Brumme/Sandra Falbe (ed.): The Spoken Language in a Multimodal Context. Description, Teaching, Translation. 316 Seiten. ISBN 978-3-7329-0021-3

Band 17 Inna Kozlova: De la búsqueda a la consulta. Resolución de problemas léxicos en producción textual en lengua extranjera. 374 Seiten. ISBN 978-3-7329-0049-7

Band 18 Gerd Antos/Ulla Fix/Bettina Radeiski (Hg.): Rhetorik der Selbsttäuschung. 262 Seiten. ISBN 978-3-86596-513-4 


\section{SPRACHWISSENSCHAFT}

Band 19 Carmela Pérez-Salazar/Inés Olza (eds.): Del discurso de los medios de comunicación a la lingüística del discurso. Estudios en honor de la profesora María Victoria Romero. 598 Seiten. ISBN 978-3-7329-0058-9

Band 20 Cordula Schwarze/Carmen Konzett (Hg.): Interaktionsforschung: Gesprächsanalytische Fallstudien und Forschungspraxis. 234 Seiten. ISBN 978-3-7329-0073-2

Band 21 Ingrid Wotschke: How Educated English Speak English. Pronunciation as Social Behaviour. 282 Seiten. ISBN 978-3-7329-0062-6

Band 22 Rudolf von Raumer: Geschichtliche Grammatik der Deutschen Sprache (1851). Faksimile der Handschrift und Transkription, herausgegeben von Hannelore Schwartze-Köhler. Mit einer Einleitung von Horst Haider Munske. 326 Seiten. ISBN 978-3-7329-0079-4

Band 23 Josef Klein: Grundlagen der Politolinguistik. Ausgewählte Aufsätze. 392 Seiten. ISBN 978-3-7329-0110-4

Band 24 Joanna Szczęk: Absageschreiben auf Bewerbungen. Eine pragmalinguistische Studie. 420 Seiten. ISBN 978-3-7329-0146-3

Band 25 Hartmut E. H. Lenk/Ulrike Richter-Vapaatalo (Hg.): Sie leben nicht vom Verb allein. Beiträge zur historischen Textanalyse, Valenz- und Phraseologieforschung. 228 Seiten. ISBN 978-3-7329-0098-5

Band 26 Anja Hennemann/Claudia Schlaak (Hg.): Politische und mediale Diskurse. Fallstudien aus der Romania. 296 Seiten. ISBN 978-3-7329-0074-9 


\section{SPRACHWISSENSCHAFT}

Band 27 Alejandro González Villar: Un análisis funcional y descriptivo de los marcadores pragmáticos y su traducción como herramienta en la construcción del diálogo ficticio. Estudio contrastivo alemán-catalánespañol en base a tres novelas de Hans Fallada. 436 Seiten. ISBN 978-3-7329-0183-8

Band 28 Josef Klein: Von Gandhi und al-Qaida bis Schröder und Merkel. Politolinguistische Analysen, Expertisen und Kritik. 432 Seiten. ISBN 978-3-7329-0123-4

Band 29 Sorin Gadeanu: Intelligente Laute: Ein System mit eigener Ordnung. Dynamisch-funktionale Kategorien zur „Phonetologie“ des Deutschen für Rumänischsprachige / Sunete inteligente: Un sistem cu ordine propie. Categorii dinamic-funcţionale ale ,fonetologiei“ limbii germane pentru vorbitorii de limba română. 344 Seiten. ISBN 978-3-7329-0229-3

Band 30 Juan Cuartero Otal/Juan Pablo Larreta Zulategui/

Christoph Ehlers (Hg.): Querschnitt durch die deutsche Sprache aus spanischer Sicht. Perspektiven der Kontrastiven Linguistik. 272 Seiten. ISBN 978-3-7329-0244-6

Band 31 Uwe Grund: Orthographische Regelwerke im Praxistest. Schulische Rechtschreibleistungen vor und nach der Rechtschreibreform. 248 Seiten. ISBN 978-3-7329-0279-8

Band 32 Enrico Garavelli/Hartmut E. H. Lenk (Hg.):

Verhüllender Sprachgebrauch. Textsorten- und diskurstypische Euphemismen. 174 Seiten. ISBN 978-3-7329-0213-2

Band 33 Bettina Radeiski: Denkstil, Sprache und Diskurse. Überlegungen zur Wiederaneignung Ludwik Flecks für die Diskurswissenschaft nach Foucault. 210 Seiten. ISBN 978-3-7329-0325-2

TFrank \& Timme 


\section{SPRACHWISSENSCHAFT}

Band 34 Coline Baechler/Eva Martha Eckkrammer/Johannes Müller-Lancé/ Verena Thaler (Hg.): Medienlinguistik 3.0 - Formen und Wirkung von Textsorten im Zeitalter des Social Web. 354 Seiten. ISBN 978-3-7329-0078-7

Band 35 Joanna Szczęk: Phraseologie der Farben. Phraseologisches Wörterbuch Deutsch-Polnisch/Polnisch-Deutsch. 130 Seiten. ISBN 978-3-7329-0337-5

Band 36 Dieter Cherubim: Sprachliche Aneignung der Wirklichkeit. Studien zur Sprachgeschichte des neueren Deutsch. 636 Seiten. ISBN 978-3-7329-0250-7

Band 37 Bernd Spillner: Error Analysis in the World. A Bibliography. 586 Seiten. ISBN 978-3-7329-0356-6

Band 38 Karolina Suchowolec: Sprachlenkung - Aspekte einer übergreifenden Theorie. 458 Seiten. ISBN 978-3-7329-0339-9

Band 39 Anna Gondek/Joanna Szczęk (Hg.): Kulinarische Phraseologie. Intra- und interlinguale Einblicke. 250 Seiten. ISBN 978-3-7329-0389-4 